
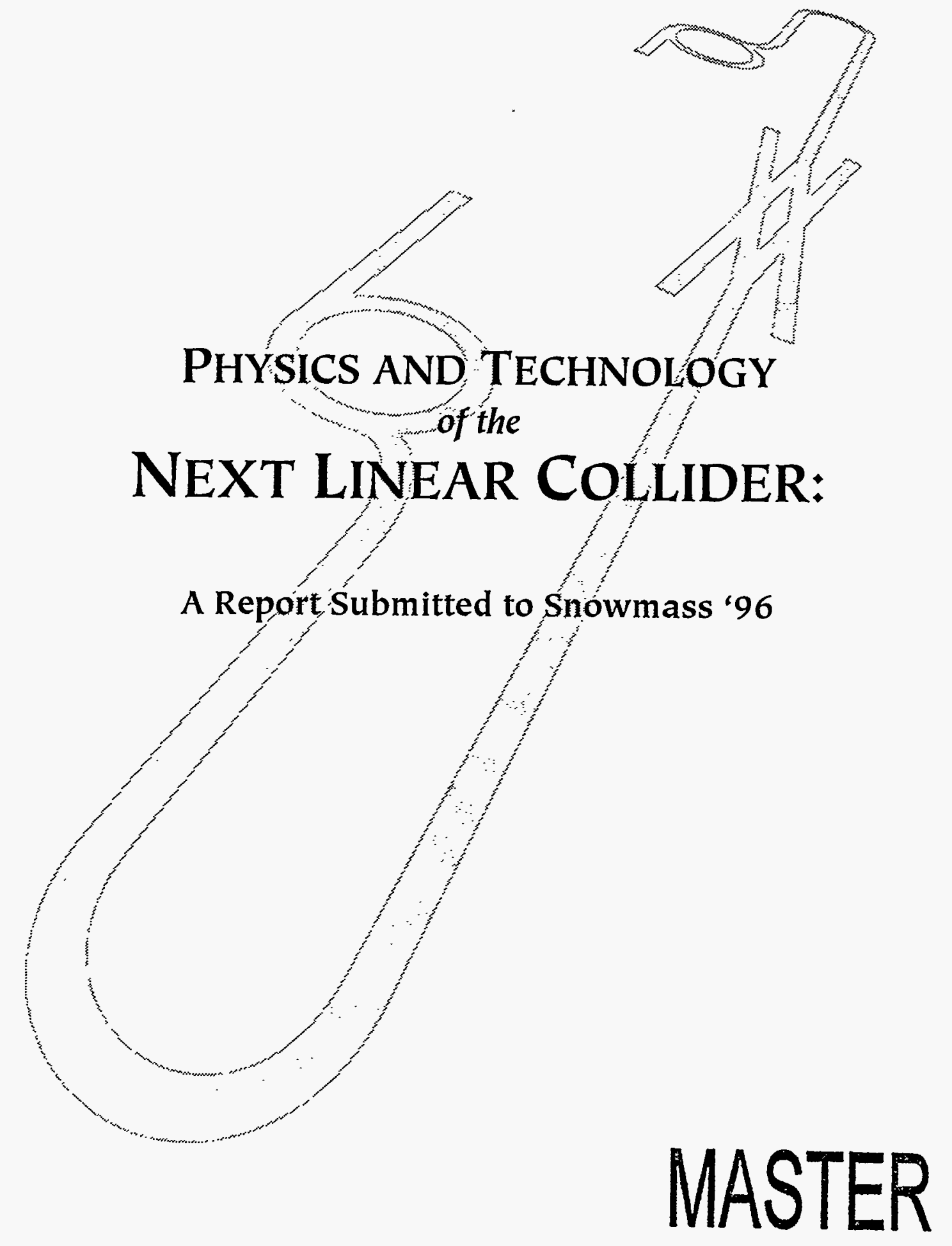

DISTRIBUTION OF THIS DOCUNEET IS UNLIMTED 
This document, and the material and data contained therein, was developed under sponsorship of the United States Government. Neither the United States nor the Department of Energy, nor the Leland Stanford Junior University, nor their employees, nor their respective contractors, subconfractors, or their employees, makes any warranty, express or implied, or assumes any liability of reponsibility for accuracy, completeness or usefulness of any information, apparatus, product or process disclosed, or represents that its use will not infringe privately owned rights. Mention of any product, its manufacturer, or suppliers shall not, nor is it intended to, imply approval, disapproval, or fitness for any particular use. A royalty-free, nonexclusive right to use and disseminate same for any purpose whatsoever, is expressly reserved to the United States and the University.

Inside Front Cover (clockwise from top):

"Flower-Petal" Microwave Mode Converters

X-Band Klystron

"Damped and Detuned" Accelerator Cell

\section{8-m-long X-Band Accelerator Section}

Inside Back Cover (clockwise from top left):

Fringe Pattern in Final Focus Test Beam (FFTB)

Spot-Size Monitor for F"Fi:

\section{X-Band RF Pulse Compressor}

FFTB Magnet, Precision Moving Stage, and Beam Position Monitor 


\section{DISCLAIMER}

Portions of this document may be illegible in electronic image products. Images are produced from the best available original document. 


\title{
PhySICS AND TECHNOLOGY of the NEXT LINEAR COLLIDER:
}

\author{
A Report Submitted to Snowmass '96
}

June, 1996

The NLC ZDR Design Group and

The NLC Physics Working Group

Prepared for the Department of Energy under contract number DE-AC03-76SF00515 by Stanford Linear Accelerator Center, Stanford University, Stanford, California. Printed in the United States of America. Available from National Technical Information Service, U.S. Department of Commerce, 5285 Port Royal Road, Springfield, Virginia 22161. 


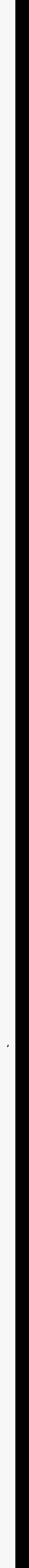




\section{The NLC Accelerator Design Group and The NLC Physics Working Group}

S. Kuhlman;

Argonne National Laboratory, Chicago, Illinois, USA

P. Minkowski;

University of Bern, Bern, Switzerland

W. Marciano, F. Paige;

Brookhaven National Laboratory, Upton, Long Island, New York, USA

V. Telnov;

Budker Institute for Nuclear Physics, Novosibirsk, Russia

J. F. Gunion, T. Han, S. Lidia;

University of California, Davis, California, USA

J. Rosenzweig;

University of California, Los Angeles, California, USA

J. Wudka;

University of California, Riverside, California, USA

N. M. Kroll;

University of California, San Diego, California, USA

D. A. Bauer, H. Nelson;

University of California, Santa Barbara, California, USA

H. Haber, C. Heusch, B. Schumm;

University of California, Santa Cruz, California, USA

M. Gintner, S. Godfrey, P. Kalyniak;

Carleton University, Ottawa, Canada

L. Rinolfi;

CERN, Geneva, Switzerland

A. Barker, M. Danielson, S. Fahey, M. Goluboff, U. Nauenberg, D. L. Wagner;

University of Colorado, Boulder, Colorado, USA

P. C. Rowson;

Columbia University, New York, New York, USA 
J. A. Holt, K. Maeshima, R. Raja;

Fermilab National Laboratory, Batavia, Illinois, USA

H. Baer, R. Munroe;

Florida State University, Tallahassee, Florida, USA

X. Tata;

University of Hawaii, Honolulu, Hawaii, USA

T. Takahashi, T. Ohgaki;

Hiroshima University, Hiroshima, Japan

R. van Kooten;

Indiana University, Bloomington, Indiana, USA

M. Akemoto, T. Higo, K. Higashi, K. Kubo, K. Oide, K. Yokoya;

KEK National Laboratory, Tsukuba, Japan

A. Jackson, W. A. Barletta, J. M. Byrd, S. Chattopadhyay, J. N. Corlett, W. M. Fawley, J. L. Feng, M. Furman, E. Henestroza, R. A. Jacobsen, K.-J. Kim, H. Li, H. Murayama, L. Reginato, R. A. Rimmer, D. Robin, M. Ronan, A. M. Sessler, D. Vanecek, J. S. Wurtele, M. Xie, S. S. Yu, A. A. Zholents;

Lawrence Berkeley National Laboratory, Berkeley, California, USA

L. Bertolini, K. Van Bibber, D. Clem, F. Deadrick, T. Houck, D. E. Klem, M. Perry, G. A. Westenskow;

Lawrence Livermore National Laboratory, Livermore, California, USA

B. Barakat;

Louisiana Tech University, Ruston, Louisiana, USA

A. J. Dragt, R. L. Gluckstern;

University of Maryland, College Park, Maryland, USA

S. R. Hertzbach;

University of Massachusetts, Amherst, Massachusetts, USA

P. Burrows, M. Fero;

Massachusetts Institute of Technology, Cambridge, Massachusetts, USA

M. Einhorn, G. L. Kane, K. Riles;

University of Michigan, Ann Arbor, Michigan, USA

G. Giordano;

University of Milano, Milan, Italy

G. B. Cleaver, K. Tanaka;

Ohio State University, Columbus, Ohio, USA 
J. Brau, R. E. Frey, D. Strom;

University of Oregon, Eugene, Oregon, USA

R. Hollebeek;

University of Pennsylvania, Philadelphia, Pennsylvania, USA

K. McDonald;

Princeton University, Princeton, New Jersey, USA

G. Couture;

Université de Quebec à Montreal, Montreal, Quebec, Canada

D. D. Meyerhofer;

University of Rochester, Rochester, New York, USA

F. Cuypers;

Paul Scherrer Institute

C. Adolphsen, R. Aiello, R. Alley, R. Assmann, K. L. Bane, T. Barklow, V. Bharadwaj, J. Bogart, G. B. Bowden, G. Bower, M. Breidenbach, K. L. Brown, D. L. Burke, Y. Cai, G. Caryotakis, R. L. Cassel, P. Chen, S. L. Clark, J. E. Clendenin, C. Corvin, F.J. Decker, A. Donaldson, R. Dubois, R. A. Early, K. R. Eppley, S. Ecklund, J. Eichner, P. Emma, L. Eriksson, Z. D. Farkas, A. S. Fisher, C. Foundoulis, W. R. Fowkes, J. Frisch, R. W. Fuller, L. Genova, S. Gold, G. Gross, S. Hanna, S. Hartman, S. A. Heifets, L. Hendrickson, R. H. Helm, J. Hewett, H. A. Hoag, J. Hodgson, J. Humphrey, R. Humphrey, J. Irwin, J. Jaros, R. K. Jobe, R. M. Jones, L. P. Keller, K. Ko, R. F. Koontz, E. Kraft, P. Krejcik, A. Kulikov, T. L. Lavine, Z. Li, W. Linebarger, G. A. Loew, R. J. Loewen, T. W. Markiewicz, T. Maruyama, T. S. Mattison, B. McKee, R. Messner, R. H. Miller, M. G. Minty, W. Moshammer, M. Munro, C. D. Nantista, E. M. Nelson, W. R. Nelson, C. K. Ng, Y. Nosochkov, D. Palmer, R. B. Palmer, J. M. Paterson, C. Pearson, M. E. Peskin, R. M. Phillips, N. Phinney, R. Pope, T. O. Raubenheimer, J. Rifkin, T. Rizzo, S. H. Rokni, M. C. Ross, R. E. Ruland, R. D. Ruth, A. Saab, H. Schwarz, B. Scott, J. C. Sheppard, H. Shoaee, S. Smith, W. L. Spence, C. M. Spencer, J. E. Spencer, D. Sprehn, G. Stupakov, H. Tang, S. G. Tantawi, P. Tenenbaum, F. Tian, S. Thomas, K. A. Thompson, J. Turner, T. Usher, A. E. Vlieks, D. R. Walz, J. W. Wang, A. W. Weidemann, D. H. Whittum, P. B. Wilson, Z. Wilson, M. Woodley, M. Woods, Y. T. Yan, A. D. Yeremian, F. Zimmermann;

Stanford Linear Accelerator Center, Stanford, California, USA

B. F. L. Ward, A. Weidemann;

University of Tennessee, Knoxville, Tennessee, USA

L. Sawyer, A. White;

University of Texas at Arlington, Arlington, Texas, USA

C. Baltay, S. Manly;

Yale University, New Haven, Connecticut, USA 

We present the prospects for the next generation of high-energy physics experiments with electron-positron colliding beams. This report summarizes the current status of the design and technological basis of a linear collider of center-of-mass energy $0.5-1.5 \mathrm{TeV}$, and the opportunities for high-energy physics experiments that this machine is expected to open.

Over the past two decades, particle physics experiments have made an increasingly precise confirmation of the "Standard Model" of strong, weak, and electromagnetic interactions. High-energy physicists feel confident that the basic structure of these once-mysterious interactions of elementary particles is now well understood. But the verification of this model has brought with it the realization that there is a missing piece to the story: although the structure of the weak interactions is based on a symmetry principle, we observe that symmetry to be broken, by an agent that we do not yet know. This agent, whatever its source, must provide new physical phenomena at the $\mathrm{TeV}$ energy scale.

The Large Hadron Collider (LHC) in Europe offers an entry into this energy regime with significant opportunity for discovery of new phenomena. An electron-positron collider at this next step in energy, the Next Linear Collider (NLC), will provide a complementary program of experiments with unique opportunities for both discovery and precision measurement. To understand the nature of the new phenomena at the TeV scale, to see how they fit together with the known particles and interactions into a grander picture, both of these facilities will be required.

In particular, electron-positron colliders offer specific features that are essential to understand the nature of these new interactions whatever their source. They allow precise and detailed studies of the two known particles that couple most strongly to these interactions, the $W$ boson and the top quark. They provide a clean environment for the discovery of new particles whatever their nature, and they provide special tools, such as the use of electron beam polarization, to dissect the couplings of those particles.

All of this would be merely theoretical if the next-generation linear collider could not be realized. But, in the past few years, the technology of the linear collider has come of age. The experience gained from the operation of the Stanford Linear Collider (SLC) has provided a firm foundation to the design choices for the NLC. The fundamental new technologies needed to construct the NLC have been demonstrated experimentally. Microwave power sources have exceeded requirements for the initial stage of the NLC, and critical tests assure us that this technology can be expected to drive beams to a center-of-mass energies of a $\mathrm{TeV}$ or more. Essential demonstrations of prototype collider subsystems have either taken place or are now underway: the Final Focus Test Beam has already operated successfully; a linear accelerator and a damping ring will be operated within the next year. A detailed feasibility study, the "Zeroth-Order Design Report" (ZDR), has shown that these components can be integrated into a complete machine design.

The Next Linear Collider can be constructed, and it will play an essential role in our understanding of physics at the $\mathrm{TeV}$ energy scale. 


\section{Contents}

Preface $\quad$ ix

1 The Next Linear Collider 1

1.1 Goals for the Next Linear Collider . . . . . . . . . . . . . . . . . 1

1.2 Accelerator Design Choices . . . . . . . . . . . . . . . . . . 4

1.2.1 The Stanford Linear Collider . . . . . . . . . . . . . 5

1.2 .2 Future Linear Colliders . . . . . . . . . . . . . . . 6

1.3 The Next Linear Collider $\ldots \ldots \ldots \ldots \ldots$

1.3.1 Technology Choice and Design Philosophy . . . . . . . . . . . . . . 9

1.3.2 Status Report on Technologies for the NLC . . . . . . . . . . . . . . . . . . . . .

1.4 Outlook for the Next Linear Collider . . . . . . . . . . . . . . 15

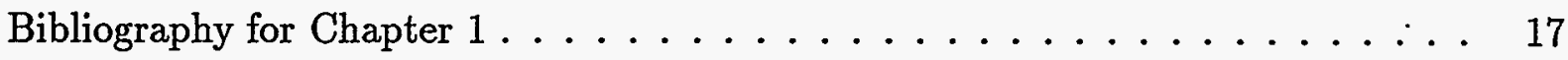

2 Physics Goals of the Next Linear Collider $\quad 19$

2.1 Introduction . . . . . . . . . . . . . . . 19

2.2 Standard Model Processes and Simulations . . . . . . . . . . . . . . 24

2.2 .1 Accelerator and Detector . . . . . . . . . . . . . . . . . . . . . . . . . . .

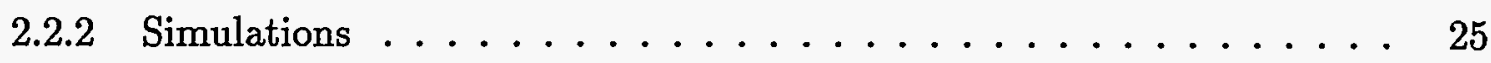

2.2.3 Standard Model Processes at the NLC . . . . . . . . . . 26

2.3 Top Quark Physics . . . . . . . . . . . . . . . . . 30

2.3.1 Top Production, Decay, and Measurement . . . . . . . . . 30

2.3.2 Threshold Physics. . . . . . . . . . . . . . . . . 32

2.3 .3 Top Couplings . . . . . . . . . . . . . . . . . 36

2.3.4 The Higgs-Top Yukawa Coupling . . . . . . . . . . . . . . 40

2.3.5 Top Physics Reach of NLC and Hadron Colliders . . . . . . . . 42

2.4 Higgs Boson Searches and Properties . . . . . . . . . . . . . . 44

2.4 .1 Introduction . . . . . . . . . . . . . . 44 
2.4.2 Present and Future Limits . . . . . . . . . . . . . 45

2.4.3 Standard Model Higgs . . . . . . . . . . . . . . . 48

2.4.4 Minimal Supersymmetric Standard Model Higgs . . . . . . . . . . 52

2.4.5 Determination of Properties of Higgs Bosons . . . . . . . . . . 55

2.4 .6 Summary . . . . . . . . . . . . . . . . 60

2.5 Supersymmetry . . . . . . . . . . . . . . . . 61

2.5.1 Supersymmetry Signals at the NLC . . . . . . . . . . . 63

2.5.2 Superparticle Mass Measurements . . . . . . . . . . . . . . 67

2.5.3 Supersymmetry Reach of the NLC and LHC . . . . . . . . . . 72

2.6 Anomalous Gauge Boson Couplings . . . . . . . . . . . . . . . . . 79

2.6 .1 Parametrization . . . . . . . . . . . . . . . 79

2.6.2 Present and Expected Pre-NLC Measurements . . . . . . . . . . 80

2.6.3 Measurements in $W$ Pair Production at the NLC . . . . . . . . 81

2.6.4 Measurements in Other Reactions at the NLC . . . . . . . . . . 82

2.6 .5 Conclusions . . . . . . . . . . . . . . . 83

2.7 Strong $W W$ Scattering $\ldots \ldots \ldots \ldots \ldots \ldots \ldots \ldots$

2.7.1 The Reaction $e^{+} e^{-} \rightarrow W^{+} W^{-} \ldots \ldots \ldots \ldots \ldots$

2.7.2 The Reaction $e^{+} e^{-} \rightarrow \nu \bar{\nu} W^{+} W^{-}$and $\nu \bar{\nu} \mathrm{ZZ} \ldots \ldots \ldots \ldots 8$

2.7.3 The Reaction $e^{+} e^{-} \rightarrow \nu \bar{\nu} t \bar{t} \ldots \ldots \ldots \ldots \ldots \ldots$

2.7.4 Statistical significances at $\mathrm{LHC}$ versus NLC $\ldots \ldots \ldots \ldots$

2.7 .5 Conclusion . . . . . . . . . . . . . . . . 93

2.8 New Gauge Bosons and Exotic Particles . . . . . . . . . . . . . . 94

$2.9 e^{-} e^{-}, e^{-} \gamma$, and $\gamma \gamma$ Interactions $\ldots \ldots \ldots \ldots \ldots \ldots$

2.9 .1 Higgs Boson Studies . . . . . . . . . . . . . . 100

2.9 .2 Other New Physics Signatures . . . . . . . . . . . . . . . . 102

2.9.3 Accelerator, Lasers, and the Interaction Region . . . . . . . . . 103

2.10 Precision Tests of QCD . . . . . . . . . . . . . . . . . . 105

2.10 .1 Precise Measurement of $\alpha_{s} \ldots \ldots \ldots \ldots \ldots \ldots$

2.10 .2 Energy Evolution Studies _ . . . . . . . . . . . . . . . 107

2.10.3 Symmetry Tests Using Beam Polarization . . . . . . . . . . . . 108

2.10.4 Gluon Radiation in $t \bar{t}$ Events; Anomalous Couplings . . . . . . . . . . 108

2.11 Design of the NLC Detector . . . . . . . . . . . . . . . . . 109

2.12 Physics Processes which Constrain Detector Performance . . . . . . . . . 113

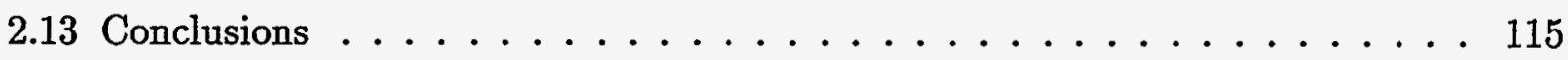

Bibliography for Chapter $2 \ldots \ldots \ldots \ldots \ldots \ldots \ldots \ldots$ 
3 A Zeroth-Order Design for the Next Linear Collider . 125

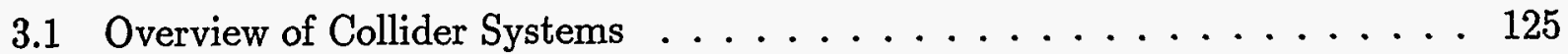

3.1.1 Injectors and Damping Rings . . . . . . . . . . . 125

3.1 .2 Bunch Length Compressors . . . . . . . . . . . . . . 127

3.1 .3 Main Linacs . . . . . . . . . . . . . . . . . . . . 128

3.1.4 The Beam Delivery System . . . . . . . . . . . . . . . 128

3.1 .5 Interaction Region $\ldots \ldots \ldots \ldots \ldots \ldots \ldots$

3.2 Polarized Electron Injector . . . . . . . . . . . . . . . . . . 131

3.3 Positron Source . . . . . . . . . . . . . . . . . . 134

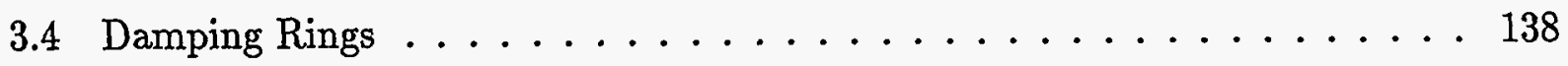

3.4.1 Main Damping Rings. . . . . . . . . . . . . . . . . . . . 140

3.4 .2 Positron Pre-Damping Ring . . . . . . . . . . . . . . . . . 142

3.5 Spin Rotators and Bunch Compressors . . . . . . . . . . . . 144

3.6 Main Linacs: Design and Dynamics . . . . . . . . . . . . . . . 148

3.6.1 Linac Layout and Site Requirements . . . . . . . . . . . 150

3.6.2 Operation and Tuning of the Main Linacs . . . . . . . . . 153

3.7 The RF System for the Main Linacs . . . . . . . . . . . . . 158

3.7 .1 Klystrons . . . . . . . . . . . . . . . . . 161

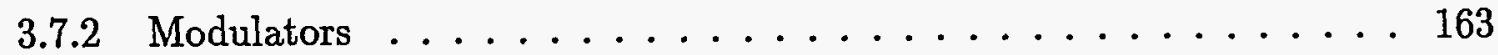

3.7.3 RF Pulse Compression and Power Transmission . . . . . . . . . 164

3.7 .4 Accelerator Structure . . . . . . . . . . . . . . . 165

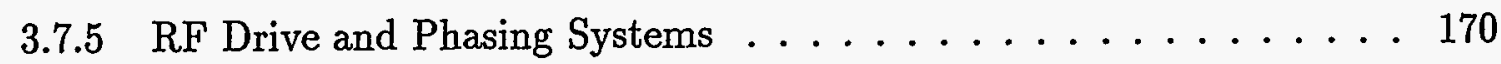

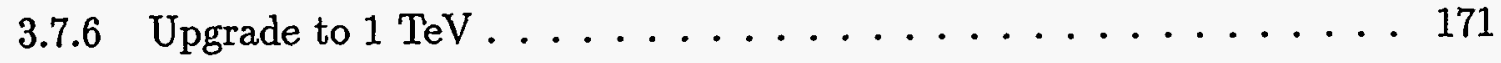

3.7 .7 Outlook . . . . . . . . . . . . . . 173

3.8 Beam Delivery. . . . . . . . . . . . . . . . 175

3.8 .1 Collimation System . . . . . . . . . . . . . . 175

3.8.2 IP Switch and Big Bend $\ldots \ldots \ldots \ldots \ldots \ldots \ldots \ldots$

3.8 .3 Final Focus $\ldots \ldots \ldots \ldots \ldots \ldots \ldots \ldots \ldots \ldots \ldots$

3.9 Interaction Region and Detector Backgrounds . . . . . . . . 185

3.9.1 Introduction . . . . . . . . . . . . . . . 185

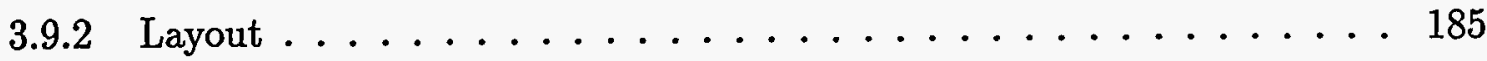

3.9.3 Backgrounds . . . . . . . . . . . . . . . . 186

3.9.4 Quad Support and the Optical Anchor . . . . . . . . . . . 192

3.9 .5 Conclusion . . . . . . . . . . . . . . . 193 
Bibliography for Chapter $3 \ldots \ldots$. . . . . . . . . . . . . . . . . 194

Appendix: Papers contributed to the NLC Physics Study 


\section{Chapter 1}

\section{The Next Linear Collider}

\subsection{Goals for the Next Linear Collider}

For the past 25 years accelerator facilities with colliding beams have been the forefront instruments used to study elementary particle physics at high energies (Fig. 1.1). Both hadronhadron and electron-positron colliders have been used to make important observations and discoveries. Direct observations of the $W^{ \pm}$and $Z^{0}$ bosons at CERN and investigations of the top quark at Fermilab are examples of physics done at hadron colliders. Electron-positron colliders provide well-controlled and well-understood experimental environments in which new phenomena stand out and precise measurements can be made. The discoveries of the charm quark and $\tau$ lepton at SPEAR, discovery of the gluon and establishment of QCD at PETRA and PEP, and precision exploration of electroweak phenomena at the SLC and LEP are highlights of the results produced by experiments at electron-positron colliders.

The ability to study nature with these two different kinds of instruments has proven essential to the advancement of our understanding of particle physics. This will remain true as we seek answers to questions posed at the $\mathrm{TeV}$ energy scale:

- What is the top quark, and what are its interactions?

- Why is the symmetry of the electroweak interaction broken, and what is the origin of mass?

- Do Higgs particles exist? If so, how many, and what are their structures and interactions?

- Is the world supersymmetric, and if so, what is its structure, and is this supersymmetry part of a larger unification of nature?

- Are quarks, leptons, and gauge bosons fundamental particles, or are they more complex?

- Are there other new particles or interactions, and what might nature contain that we have not yet imagined? 


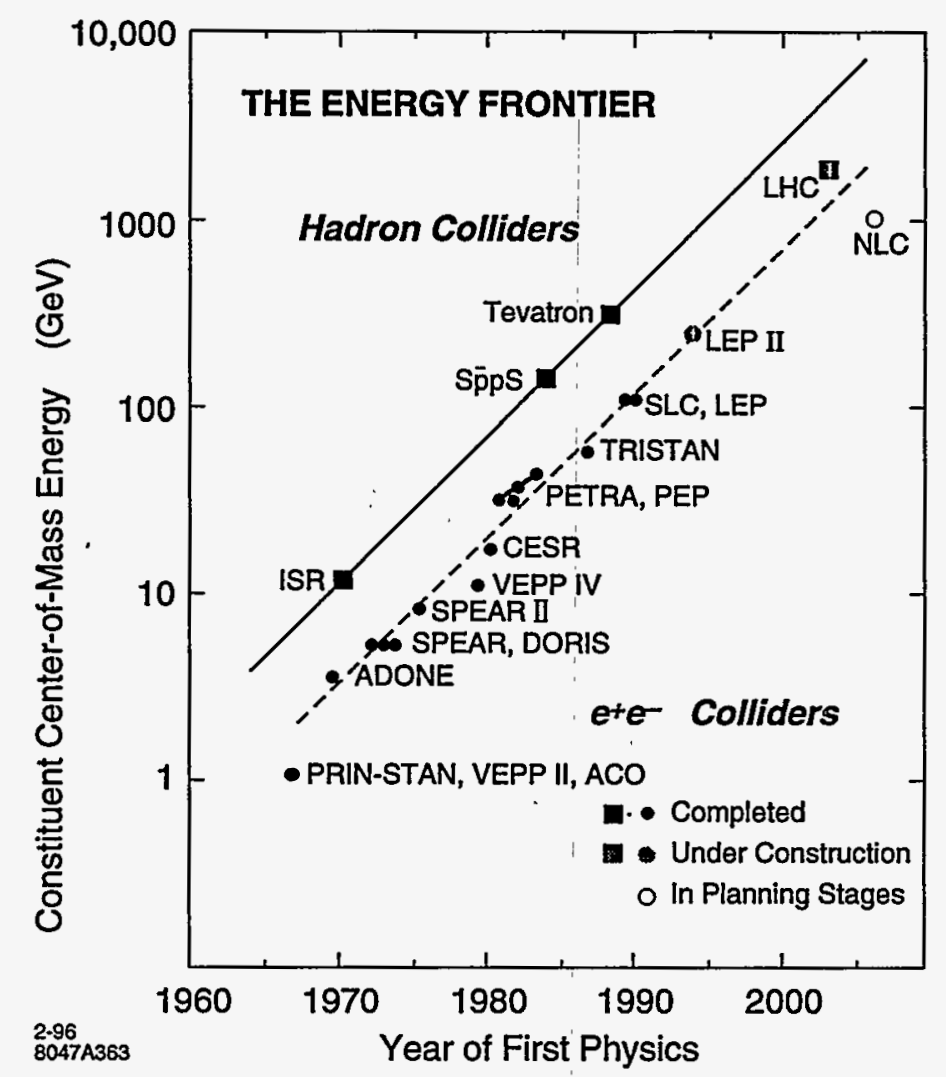

Figure 1.1: The energy frontier of particle physics. The effective constituent energy of existing and planned colliders and the year of first physics results from each.

The Large Hadron Collider (LHC) in Europe offers an entry into the TeV energy regime with significant opportunity for discovery of new phenomena. The planned participation in the design, construction, and utilization of this collider by nations around the world will make the LHC the first truly global facility for the study of particle physics. This will be an exciting and important step in the continuing evolution of our science.

The companion electron-positron collider at this next step in energy, the Next Linear Collider (NLC), will provide a complementary program of experiments with unique opportunities for both discovery and precision measurement. To understand the nature of physics at the $\mathrm{TeV}$ scale, to see how the new phenomena we expect to find there fit together with the known particles and interactions into a grander picture, both the LHC and the NLC will be required.

Studies of physics goals and requirements for the next-generation electron-positron collider began in 1987-88 in the United States [1, 2, 3], Europe [4, 5], and Japan [6, 7]. These regional studies have evolved into a series of internationally sponsored and organized workshops $[8,9,10]$ that continue to build an important consensus on the goals and specifications of the Next Linear Collider. This document is both a part of this process, and input to 
deliberations by the U.S. particle physics community that will take place this Summer at Snowmass, Colorado. To prepare for Snowmass, a series of workshops was held over the past year at locations throughout the United States. Working groups were established at a first meeting in Estes Park, Colorado to provide a framework for people to participate in the discussions of various topics in physics and experimentation at linear colliders. These groups continued to meet at subsequent workshops held at Fermilab, SLAC, and Brookhaven National Laboratory. This document contains a written summary from these workshops.

A picture has emerged of a high-performance collider able to explore a broad range of center of mass energies from a few hundred $\mathrm{GeV}$ to a $\mathrm{TeV}$ and beyond (Fig. 1.2). The goals of particle physics at the $\mathrm{TeV}$ scale require luminosities of approximately $10^{34} \mathrm{~cm}^{-2} \mathrm{sec}^{-1}$, and reliable technologies that can provide large integrated data samples. It is important that the beam energy spread remain well controlled, and that backgrounds created by lost particles and radiation from the beams be maintained at low levels. This will assure that the clean experimental environment historically offered by electron-positron colliders remains intact. Beam polarization is an additional tool available at a linear collider that provides new and revealing views of particle physics, and this too is a requirement for any future collider.

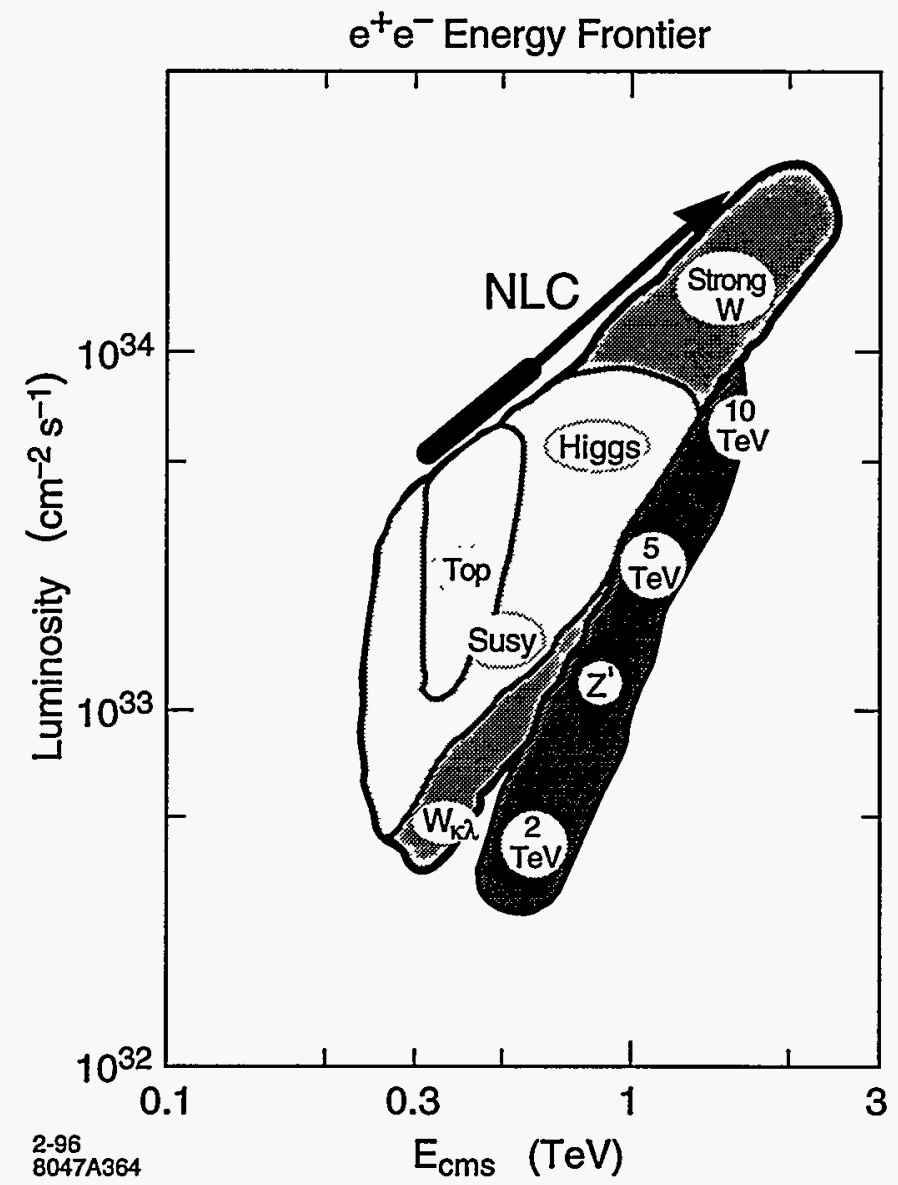

Figure 1.2: Physics goals for a TeV-scale $e^{+} e^{-}$collider. 
In this first chapter we introduce the accelerator physics and technologies of the Next Linear Collider, discuss design choices and philosophies, and provide a brief status report on the R\&D program that is being carried out in support of the NLC design effort. The second chapter of this document concentrates on the physics program of the NLC. The final chapter gives a more detailed overview of the accelerator design. A companion document, $A$ Zeroth-Order Design Report for the Next Linear Collider, that contains results from a rather extensive feasibility study of the NLC, has also been prepared [11]. This may be of further interest to readers.

\subsection{Accelerator Design Choices}

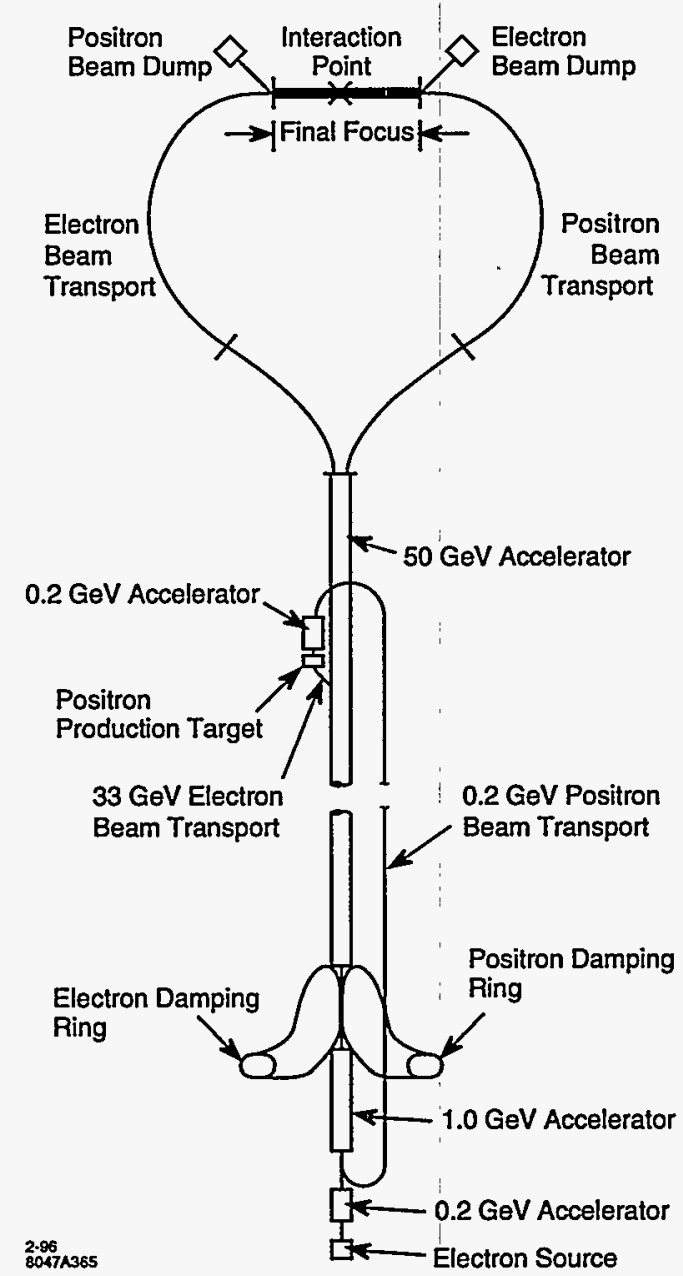

Figure 1.3: The Stanford Linear Collider (SLC). 


\subsubsection{The Stanford Linear Collider}

The Stanford Linear Collider (Fig. 1.3) was conceived and built to accomplish two goals: to study particle physics at the $100-\mathrm{GeV}$ energy scale, and to develop the accelerator physics and technology necessary for the realization of future high-energy colliders. The SLC was completed in 1987 and provided a first look at the physics of the $Z^{0}$ in 1989. In time, the luminosity provided by this machine has grown steadily (Fig. 1.4), and has allowed particle physicists to make unique and important studies of the $Z^{0}$ and its decays.

The design of the Next Linear Collider (NLC) presented in this document is intimately connected with experiences gained at the SLC. Our choices of technologies and philosophies of design have direct links to these experiences and considerable overlap with them. Lessons have been learned and techniques developed at the SLC that are relevant to the design and implementation of every part and system of the NLC:

- Injectors

- Stabilized high-power electron sources

- Polarized electrons

- High-power targets and positron capture

- Damping Rings

- Stabilized fast (50 ns) injection and extraction systems

- Sub-picosecond phase synchronization with linac rf systems

- Linear Accelerator

- Beam Acceleration

Management of large rf systems

Rf phase control

"Time-slot" compensation

Short-range longitudinal wake compensation

Multibunch beam loading compensation

- Emittance Preservation

Beam-based alignment

LEM-lattice/energy matching

BNS damping

Coherent wakefield cancellation

Dispersion-free steering

- Final Focus Systems

- Second-order chromatic optics and tuning

- Precision diagnostics

- Beam-beam control and tuning

- Experimentation

- Theory and modeling of backgrounds 
- Vulnerability of detector technologies

- Collimation-theory and implementation

- Systems Performance and Operation

- Precision instrumentation-BPMs and wirescanners

- Feedback theory and implementation

- Importance of on-line modeling and analysis

- Automated diagnostics and tuning

- Mechanical stabilization of supports and components

- Thermal stabilization of supports and components

- Reliability

- History monitoring (from seconds to years)

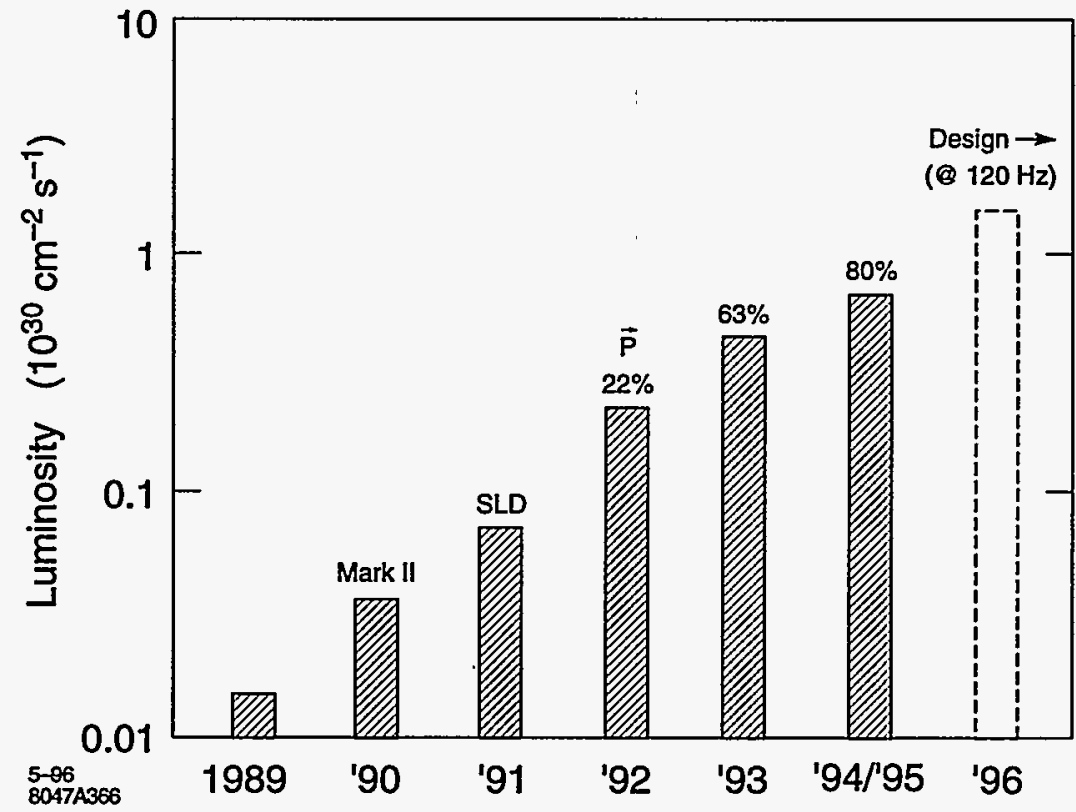

Figure 1.4: Performance of the SLC from early commissioning. Polarization of the electron beam is also shown.

\subsubsection{Future Linear Colliders}

The basic components of any linear collider are those already incorporated into the SLC; a generic collider complex is diagrammed in Fig. 1.5. The energy of such a future collider must be ten times that of the SLC, and a TeV-scale collider must be able to deliver luminosities that are several orders of magnitude greater than those achieved at the SLC. Trains of bunches of electrons and positrons are created, condensed in damping rings, accelerated to 


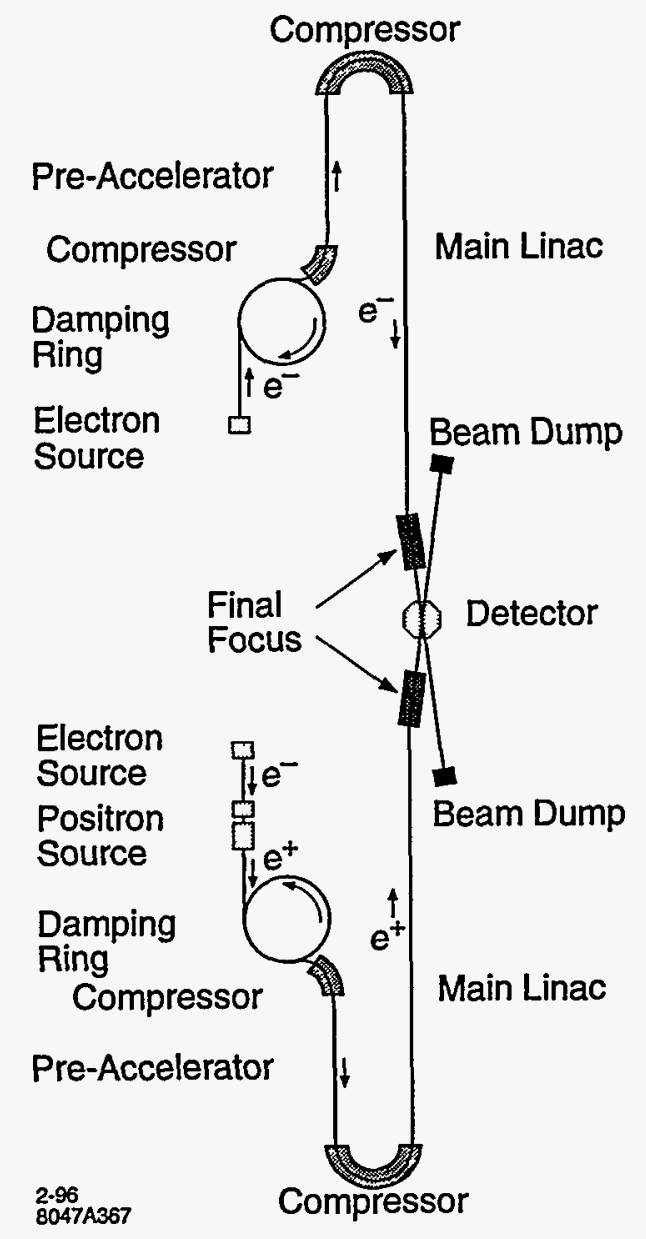

Figure 1.5: Schematic of a $\mathrm{TeV}$-scale linear collider.

high energy, focused to small spots, and collided to produce a luminosity given by

$$
L=\frac{n N^{2} H f}{4 \pi \sigma_{x}^{*} \sigma_{y}^{*}},
$$

where

$$
\begin{aligned}
n & =\text { number of bunches per train, } \\
N & =\text { number of particles per bunch, } \\
H & =\text { beam pinch enhancement } \\
f & =\text { machine repetition rate }
\end{aligned}
$$

and $\sigma_{x}^{*}$ and $\sigma_{y}^{*}$ are the horizontal and vertical beam dimensions at the collision point. Equation 1.1 can be written as

$$
L=\frac{1}{4 \pi E} \frac{N H}{\sigma_{x}^{*}} \frac{P}{\sigma_{y}^{*}}
$$


where $P$ is the average power in each beam. The factor $N / \sigma_{x}^{*}$ determines the number of beamstrahlung photons emitted during the beam-beam interaction, and since these photons will alter the effective spread in beam collision energies and can create backgrounds in experimental detectors, this factor is highly constrained. It is mainly the last ratio, $P / \sigma_{y}^{*}$, that can be addressed by accelerator technology; high luminosity corresponds to high beam power and/or small beam spots. These two parameters pose different, and in many cases contrary, challenges to the accelerator physicist, and several technologies that represent differing degrees of compromise between beam power and spot size are being developed. Table 1.1 summarizes the initial stage of the mainstream design choices.

Table 1.1: Linear collider design parameters $\left(\mathrm{E}_{\mathrm{cms}}=500 \mathrm{GeV}\right)$.

\begin{tabular}{|l|ccc|ccc|}
\hline \hline & $\begin{array}{c}\text { Frequency } \\
(\mathrm{GHz})\end{array}$ & $\begin{array}{c}\text { Gradient } \\
(\mathrm{MV} / \mathrm{m})\end{array}$ & $\begin{array}{c}\text { Total Length } \\
(\mathrm{km})\end{array}$ & $\begin{array}{c}\text { Beam Power } \\
(\mathrm{MW})\end{array}$ & $\begin{array}{c}\sigma_{y} \\
(\mathrm{~nm})\end{array}$ & $\begin{array}{c}\text { Luminosity } \\
\left(10^{33} \mathrm{~cm}^{-2} \mathrm{~s}^{-1}\right)\end{array}$ \\
\hline SuperC & 1.3 & 25 & 30 & 8.2 & 19 & 6 \\
S-Band & 3.0 & 21 & 30 & 7.3 & 15 & 5 \\
X-Band & 11.4 & 50 & 16 & 4.8 & 5.5 & 6 \\
2-Beam & 30.0 & 80 & 9 & 2.7 & 7.5 & 5 \\
\hline \hline
\end{tabular}

Each of the technologies in Table 1.1 is being pursued by physicists and engineers at laboratories around the globe. This strong international effort is remarkably well coordinated through collaborations that together provide a set of test facilities to address each of the important aspects of the collider design and implementation. A summary of the facilities presently in operation or under construction is given in Table 1.2.

Table 1.2: Linear collider test facilities around the world.

\begin{tabular}{|lllc|}
\hline \hline Facility & Location & \multicolumn{1}{c}{ Goal } & Operations \\
\hline SLC & SLAC & Prototype Collider & 1988 \\
ATF & KEK & Injector and Damping Ring & 1995 \\
TTF & DESY & SuperC Linac & 1997 \\
SBTF & DESY & S-band Linac & 1996 \\
NLCTA & SLAC & X-band Linac & 1996 \\
CTF & CERN & 2-Beam Linac & 1996 \\
FFTB & SLAC & Final Focus/IR & 1994 \\
\hline \hline
\end{tabular}




\subsection{The Next Linear Collider}

\subsubsection{Technology Choice and Design Philosophy}

The goal to reach 1 to $1.5-\mathrm{TeV}$ cms energy with luminosities of $10^{34} \mathrm{~cm}^{-2} \mathrm{~s}^{-1}$ or more and our experiences with the SLC, guide our choice of technologies for the NLC. We believe that the most natural match to these design goals is made with normal-conducting $\mathrm{X}$-band $(11.424 \mathrm{GHz})$ microwave components patterned after the S-band technology used in the SLC. A schematic of a section of the rf system of the NLC is shown in Fig. 1.6. Our choice of technology has required the development of new advanced rf klystrons and pulse-compression systems, but provides confidence that accelerating gradients of $50-100 \mathrm{MV} / \mathrm{m}$ can be achieved and used in the implementation of the collider. The technical risk of building a collider with new X-band technologies is perhaps greater than simply building a larger SLC at S-Band, but the goal to reach $1-1.5 \mathrm{TeV}$ is substantially more assured, and capital costs to reach these energies will be lower.

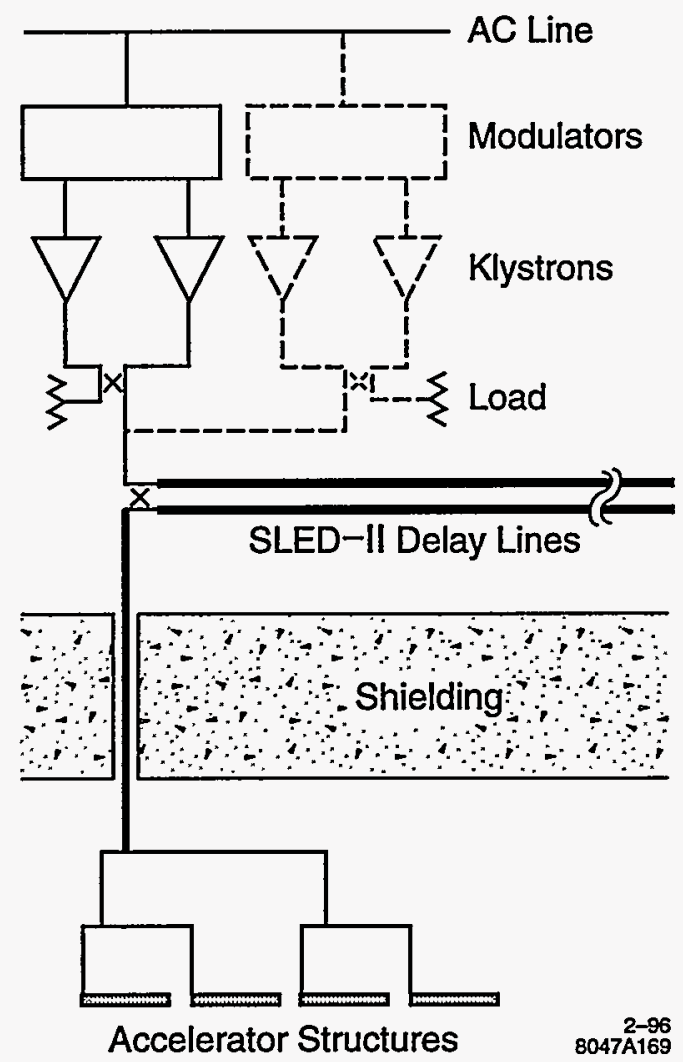

Figure 1.6: Normal-conducting rf system module in NLC main linacs. The dashed elements are expected to be necessary to reach $1 \mathrm{TeV} \mathrm{cms}$ energy.

The NLC is designed with nominal cms energy of $1 \mathrm{TeV}$. It is envisaged to be built with 
an initial rf system able to drive the beams to $0.5-\mathrm{TeV}$ cms energy, but with all infrastructure and beam lines able to support $1 \mathrm{TeV}$. The rf system design incorporates the ability to replace and add modulators and klystrons without access to the accelerator beam line (dashed lines in Fig. 1.6), so an unobtrusive, smooth, and adiabatic transition from $0.5 \mathrm{TeV}$ to $1 \mathrm{TeV}$ cms energy can be made with modest and expected improvements in X-band technology. This allows the collider to begin operation with the greatest of margins in cost and performance, and provides an excellent match to the anticipated physics goals at the energy frontier (Fig. 1.2). Our philosophy is akin to that taken previously in the construction of the SLAC linac which provided a $17-\mathrm{GeV}$ electron beam at its inauguration, was improved to $35 \mathrm{GeV}$, and with continued advances in S-band technology, now provides $50-\mathrm{GeV}$ electrons and positrons for the SLC.

The NLC design also incorporates multiple paths to further upgrade the cms energy to $1.5 \mathrm{TeV}$. The "trombone" shape of the collider layout would easily accommodate a straightforward albeit expensive increase in the length of the main accelerators without requiring extensive modification of the remainder of the complex. This final energy might also be accomplished by development of new, more efficient, X-band technologies; for example, gridded klystrons, cluster klystrons, or relativistic two-beam klystrons.

The highest-level parameters of the NLC are listed in Table 1.3. At each of the nominal 0.5 - and $1-\mathrm{TeV}$ cms energies, three sets of parameters define the operating plane of the collider. The expected luminosity is constant over the operating plane, but is achieved with differing combinations of beam current and spot size. This provides a region in parameter space where the collider can be operated. Construction and operational tolerances for the various subsystems of the collider are set by the most difficult portion of the operating region. For example, the more difficult parameters for the final focus are those of case (a) in Table 1.3 , for which the beam height is smallest. In contrast, preserving the emittance of the beam in the linac is more difficult in case (c), in which the beam charge is highest and the bunch length longest. This design philosophy builds significant margin into the underlying parameters of the collider.

An important element in the design strategy of the NLC is the use of the beam to measure and correct or compensate for errors in electrical and mechanical parameters of the accelerator. These techniques, many in extensive use at the SLC and FFTB, are able to achieve far greater accuracy than is possible during fabrication and installation of components. For example, the use of optical matching and beam-based alignment algorithms considerably loosen tolerances required on magnet strengths and positioning. These procedures require accurate measurement of the properties of the beam and extensive online modeling and control software. The existence of instrumentation suitable for these purposes is an important aspect of the readiness of technologies for the collider.

Additional performance overhead has been included in the designs of most subsystems of the NLC. Errors that we anticipate will occur during machine tuning operations have been taken into account. For example, the injector systems are designed to provide $20 \%$ more charge than is indicated in Table 1.3. Fabrication and alignment tolerances for main 
Table 1.3: High-level parameters and operating region in parameter space of the NLC.

\begin{tabular}{|l||ccc|ccc|}
\hline \hline & NLC-Ia & NLC-Ib & NLC-Ic & NLC-IIa & NLC-IIb & NLC-IIc \\
\hline Nominal CMS Energy (TeV) & & 0.5 & & & 1.0 & \\
Repition Rate (Hz) & & 180 & & & 120 & \\
Bunches Pulse & & 90 & & & 90 & \\
Bunch Separation (ns) & & 1.4 & & & 1.4 & \\
Bunch Charge $\left(10^{10}\right)$ & 0.65 & 0.75 & 0.85 & 0.95 & 1.10 & 1.25 \\
Beam Power (MW) & 4.2 & 4.8 & 5.5 & 6.8 & 7.9 & 9.0 \\
$\sigma_{x}$ at IP (nm) & 264 & 294 & 294 & 231 & 250 & 284 \\
$\sigma_{y}$ at IP (nm) & 5.1 & 6.3 & 7.8 & 4.4 & 5.1 & 6.5 \\
$\sigma_{z}$ at IP ( $\mu$ m) & 100 & 125 & 150 & 125 & 150 & 150 \\
Pinch Enhancement H & 1.4 & 1.4 & 1.5 & 1.4 & 1.4 & 1.5 \\
Beamstrahlung $\delta_{E}(\%)$ & 3.5 & 3.2 & 3.5 & 12.6 & 12.6 & 12.1 \\
No. Photons per $e^{-} / e^{+}$ & 0.97 & 1.02 & 1.16 & 1.65 & 1.77 & 1.74 \\
Max. Beam Energy (GeV) & 267 & 250 & 232 & 529 & 500 & 468 \\
Luminosity (10 ${ }^{33}$ ) & 5.8 & 5.5 & 6.0 & 10.2 & 11.0 & 10.6 \\
No. Klystrons & & 4528 & & & 9816 & \\
Klystron Peak Power (MW) & & 50 & & & 75 & \\
Pulse Compression Gain & & 3.6 & & & 3.6 & \\
Unloaded Gradient (MV/m) & & 50 & & & 85 & \\
Total Linac Length (km) & & 17.6 & & & 19.1 & \\
Beam Delivery Length (km) & & 10.4 & & & 10.4 & \\
Total Site Length (km) & & 30.5 & & & 30.5 & \\
Total Linac AC Power $(\mathrm{MW})$ & & 120 & & & 193 & \\
\hline \hline
\end{tabular}


linac structures are specified without assuming benefit from certain global tuning methods such as coherent wakefield cancellation. These are powerful techniques in routine practice at the SLC, but our philosophy is to use them only to provide operational margin. We also recognize that the beam-based tuning described above cannot be done with perfect accuracy. For example, we have analyzed the tuning procedure for the final focus and estimated a $30 \%$ increase in the spot size at the IP due to errors that we anticipate will occur in measuring and correcting aberrations inherent in the optics. (This is included in Table 1.3.) This layered approach to specification of collider performance is an important part of our design philosophy.

\subsubsection{Status Report on Technologies for the NLC}

Progress in development of X-band rf components has been impressive in recent years. Prototype klystrons now produce 50-MW pulses, over 1.5 microseconds long, with performance characteristics that are correctly modeled by computer codes. The most recent prototype produces $75-\mathrm{MW}$ pulses, one microsecond long. This exceeds the requirements of the initial $0.5-\mathrm{TeV}$ stage of the NLC, and indeed approaches the requirements for $1-\mathrm{TeV} \mathrm{cms}$ energy. Tests of rf pulse-compression transformers have exceeded most goals of the NLC, and highpower rf windows and mode converters that allow high-efficiency transfer of power between components have been successfully tested. Examples of some of these results are shown in Fig. 1.7.
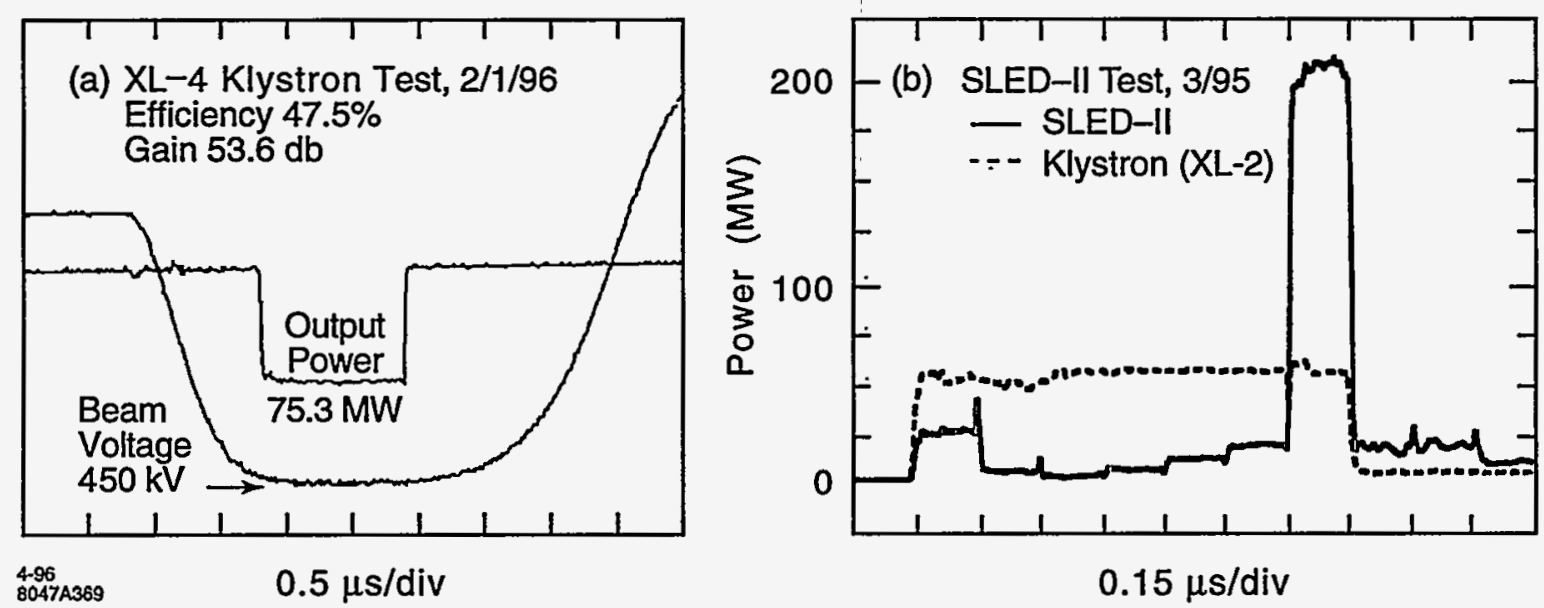

Figure 1.7: Results of tests of X-band rf components: (a) high-power klystrons, and (b) pulse compression systems.

The voltage gradient that can be used in a particle accelerator can be limited by the dark current created when electrons are drawn from the surfaces of the accelerator structures and captured on the accelerating rf wave. For a given rf frequency, there is a well-defined gradient beyond which some electrons emitted at rest will be captured and accelerated to relativistic velocities. This threshold gradient is about $16 \mathrm{MV} / \mathrm{m}$ at S-band, and scales to $64 \mathrm{MV} / \mathrm{m}$ at 
X-band. These are not actual limits to gradients that can be utilized in an accelerator since much of the charge is swept aside by the focusing quadrupoles of the machine lattice, but the dark current will grow rapidly above these values, and may adversely affect the primary beam or interfere with instrumentation needed for tuning. Gradients somewhat above the capture threshold are likely to be useful in practice, but the operational limits are not well known since no large-scale high-performance facility has been operated significantly above capture threshold. Expected thresholds of dark currents in S-band and X-band structures have been confirmed, and it has been proven that (unloaded) gradients as large as $70 \mathrm{MV} / \mathrm{m}$ can be used at X-band (Fig. 1.8).

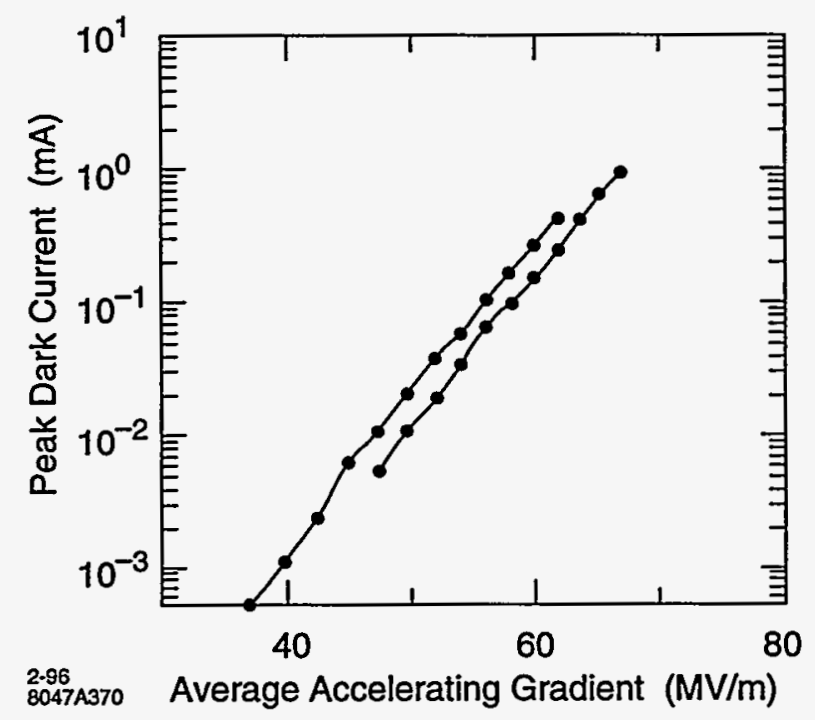

Figure 1.8: Processing of X-band accelerator structures to high gradient.

The electro-mechanical design of the structures of the main accelerator must not only produce the desired gradient, but must also minimize wakefields excited by the passage of the beam. The retarded electromagnetic fields left by each particle can disrupt the trajectories of particles that follow it through the accelerator. Many techniques to control the effects of the short-distance, intrabunch wakefields have been developed, tested, and put into use at the SLC. It will be necessary to also control long-range wakefields at the NLC in order to allow trains of closely spaced bunches to be accelerated on each rf pulse.

Structures in which wakefields are suppressed by careful tuning of their response to the passage of the beam have been developed, and tests have been performed at a facility (ASSET) installed in the SLAC linac (Fig. 1.9). Agreement with theoretical expectations is excellent and lends confidence to the design and manufacture of these structures. A more advanced design that further mitigates the long-range wakefields by coupling deflecting of modes to external energy-absorbing materials has been completed, and a prototype of this new structure is being readied for testing in ASSET as well.

Work remains to be done on X-band rf technologies, but with prototype components 

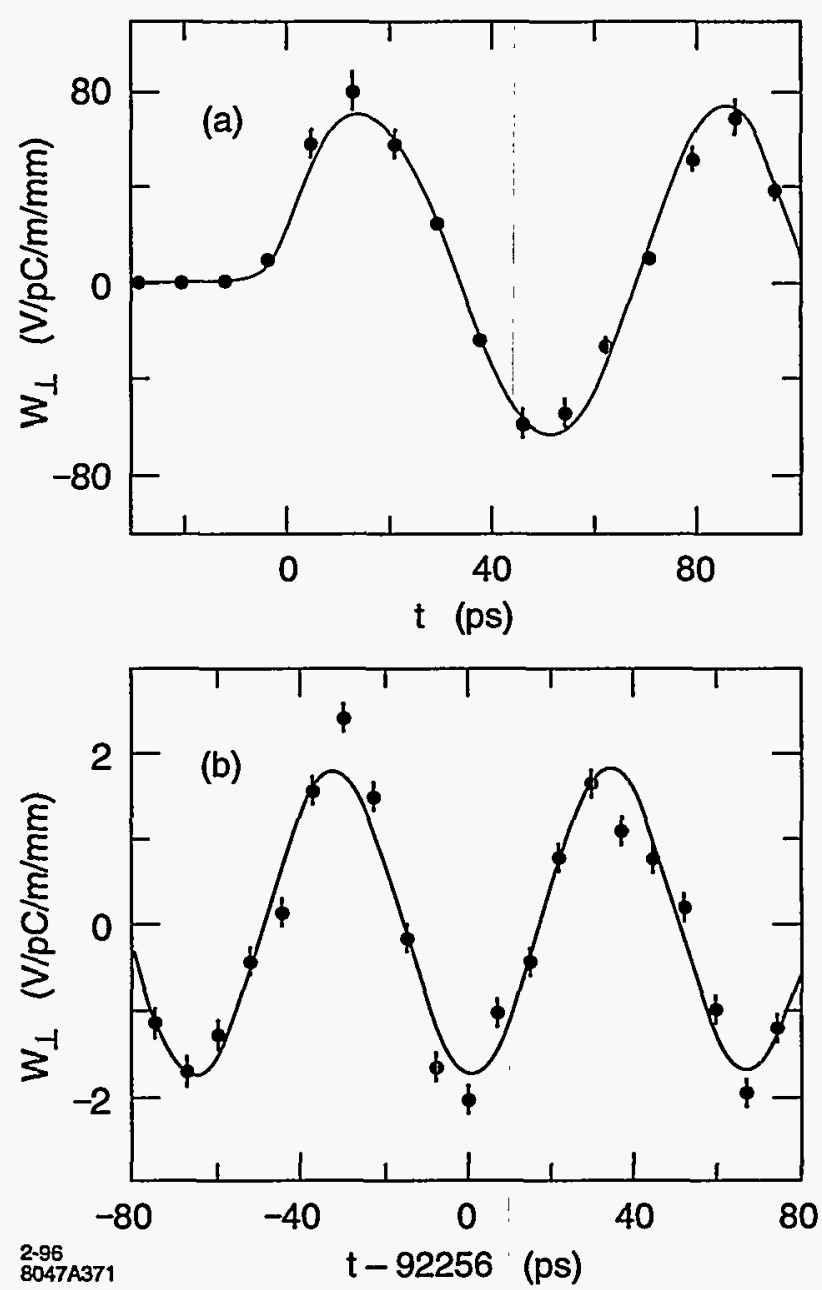

Figure 1.9: Measured and predicted transverse dipole wakefields in a 1.8-m-long X-band accelerator structure.

now in hand, tests of completely integrated systems have begun. A fully engineered test accelerator is under construction at SLAC that will allow optimization of rf systems and provide experience with beam operations at $\mathrm{X}$-band frequencies. This test accelerator will be a $40-\mathrm{m}$ long beam line containing six 1.8-m-long X-band structures powered by $50-75$ MW klystrons to an accelerating gradient of 50-85 MV/m. Commissioning of this facility has begun, and operations are expected to be underway by the end of this year (Table 1.2).

The spot sizes that must be produced at the interaction point of the NLC represent significant extrapolations from those achieved at the SLC. It is important to demonstrate that it is possible to demagnify a beam by the large factor needed in the NLC. An experiment has been performed by the Final Focus Test Beam (FFTB) Collaboration to show that such large demagnifications can be achieved. The FFTB is a prototype beam line installed in a channel located at the end of the SLAC linac at zero degrees extraction angle. The FFTB lattice is designed to produce a focal point at which the beam height can be demagnified by 
a factor of 380 to reduce the SLC beam $\left(\gamma \varepsilon_{y}=2 \times 10^{-6} \mathrm{~m}\right.$-rad) to a size smaller than 100 $\mathrm{nm}$. The demagnification factor of the FFTB beam line is well in excess of that needed for the NLC.

The FFTB optics are chromatically corrected to third order in the beam energy spread. (The SLC is corrected to second order.) All magnetic elements are mounted on precision stages that can be remotely positioned with step size of about 0.3 micron, and beam-based alignment procedures were developed that successfully place these elements to within 5-15 microns of an ideal smooth trajectory. New state-of-the-art instruments were developed and used to measure the FFTB beam positions and spot sizes. Following a brief shake-down run in August of 1993, data were taken with the FFTB during a three-week period in April and May of 1994. Beam demagnifications of 320 and spot sizes of $70 \mathrm{~nm}$ were controllably produced during this period. Measurements of these beams are shown in Fig. 1.10. The design of the NLC final focus follows that of the FFTB, and the experiences gained from the FFTB are incorporated into the tuning strategies for the NLC.

Important advances have also been made in instrumentation required to measure and control properties of the beams. The SLC control system has evolved dramatically over the past years to include extensive online modeling and automation of data analysis and tuning procedures. Scheduled procedures use sets of wire scanners to make complete measurements of the beam phase space, and provide recorded histories of machine performance. Online data-analysis packages are able to reconstruct fully coupled non-linear optical systems. Beam-based feedback and feedforward loops are in routine operation in the SLC with over 100 loops providing control of beam trajectories and energies. Beam position monitors have been developed for the FFTB that achieve pulse-to-pulse resolutions of $1 \mathrm{micron}$, and new position monitors have recently been installed that are able to measure beam motions of $100 \mathrm{~nm}$. The FFTB focal-point spot monitors have demonstrated techniques to measure beam sizes of $30-40 \mathrm{~nm}$, and extrapolation of these techniques to sizes as small as $10 \mathrm{~nm}$ is expected to be successful.

\subsection{Outlook for the Next Linear Collider}

As the SLC has systematically increased its luminosity, the accelerator physics and technologies of linear colliders have matured. Experiences and lessons learned from the task of making this first collider perform as an instrument for particle physics studies make a firm foundation on which to base the design and technology choices for the next linear collider. At the same time, essential demonstrations of new collider technologies have either taken place or soon will be underway. The experimental program with the FFTB is providing the experience needed to evaluate limitations to designs of final focus and interaction regions. The ability to demagnify beams by the amount required for the NLC has already been achieved. Microwave power sources have exceeded requirements for the initial stage of the NLC, and critical tests assure us that this technology can be expected to drive beams to center-of-mass energies of a $\mathrm{TeV}$ or more. Fully integrated test accelerators are presently under construction 

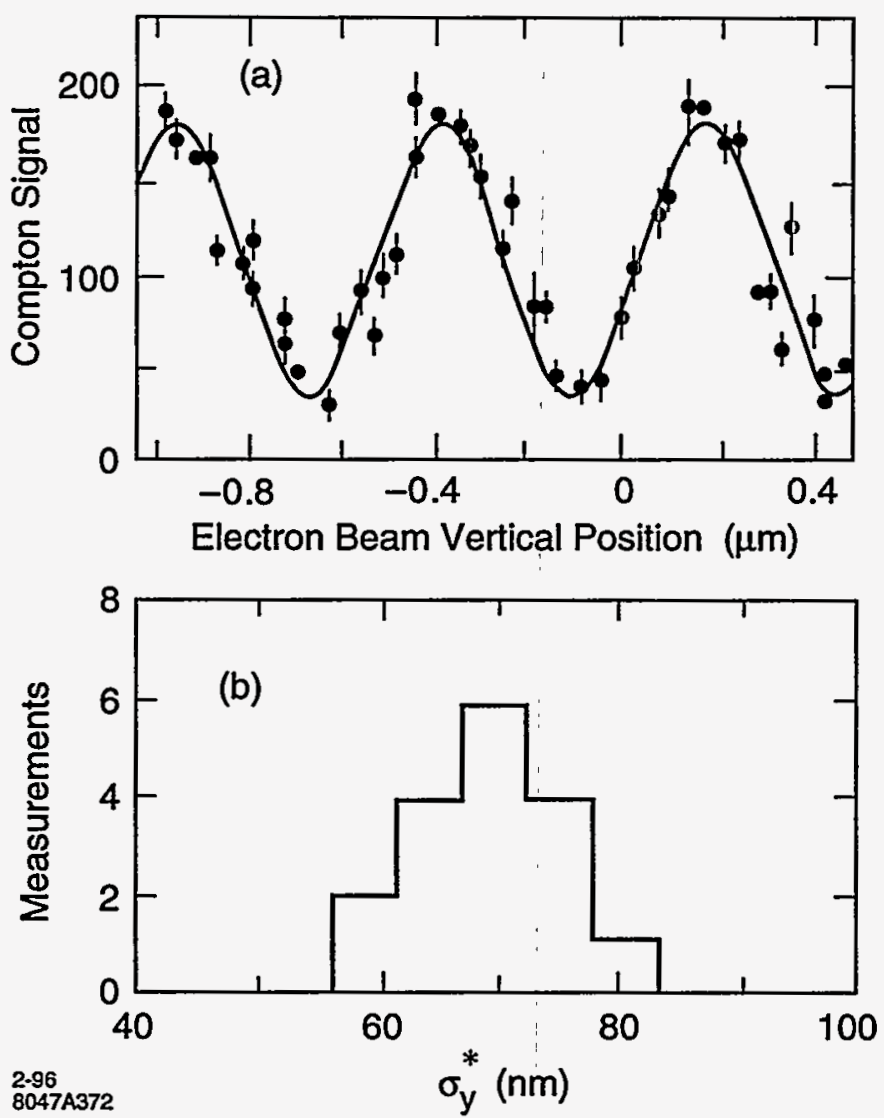

Figure 1.10: Measurement of 70-nm beam spots with a laser-Compton beam size monitor in the FFTB. (a) The rate of Compton scatters from a laser interference pattern used to determine the beam size, in this case $73 \mathrm{~nm}$. (b) Repeatability of spot measurement over periods of several hours.

at CERN, DESY, KEK, and SLAC that will soon provide answers to questions of technical optimization and costs of the major components of a TeV-scale collider.

Given the great international interest and commitment to the goals of a $\mathrm{TeV}$-scale highperformance $e^{+} e^{-}$collider, it is certain that the final design, construction, and utilization of such a collider will be a global effort. It is important that the scientific community put into place foundations for such a collaboration. The international character of the linear collider project is already reflected in the collaborations at work on the accelerator physics and technology of linear colliders, and in the process of international discussion and review of progress in the field [12]. It is essential that we continue to build on this base of understanding and cooperation, and make certain that all involved in this enterprise are full parties in its final realization. 


\section{Bibliography}

[1] C. Ahn et al. SLAC-Report-329, 1988.

[2] Proceedings of the 1988 DPF Summer Study: Snowmass '88, High Energy Physics in the 1990s, F. Gilman, ed., Snowmass, Colorado, 1988.

[3] Proceedings of the 1990 DPF Summer Study on High Energy Physics: Research Directions for the Decade, E. F. Berger, ed., Snowmass, CO, 1990.

[4] Proceedings of the 1987 LaThuile Meeting: Results and Perspectives in Particle Physics, M. Greco, ed., Gif-sur-Yvette, France, 1987.

[5] Workshop on Electron-Positron Collisions at $500 \mathrm{GeV}$ : The Physics Potential, DESY, 1990.

[6] Proceedings of the First Workshop on Japan Linear Collider (JLC I), S.Kawabata, ed., KEK, 1989.

[7] Proceedings of the Second Workshop on Japan Linear Collider (JLC II), KEK, 1990.

[8] Proceedings of the First International Workshop on Physics and Experiments with Linear Colliders, R. Orava, ed., Saariselka, Finland, 1991.

[9] Proceedings of the Second International Workshop on Physics and Experiments with Linear Colliders, F. Harris, et al., eds., Waikoloa, Hawaii, 1993.

[10] Proceedings of the Third International Workshop on Physics and Experiments with Linear Colliders, Iwate, Japan, 1995.

[11] "Zeroth-Order Design Report for the Next Linear Collider," SLAC Report 474 (Stanford University May 1996).

[12] International Linear Collider Technical Review Committee Report, 1995, G. Loew, ed. (Available from the editor.) 



\section{Chapter 2}

\section{Physics Goals of the Next Linear Collider}

\subsection{Introduction}

During the past several decades, significant advances have been made in elementary particle physics. We now have a renormalizable quantum field theory of strong and electroweak interactions, based on the principle of local $S U(3)_{c} \times S U(2)_{L} \times U(1)_{Y}$ gauge invariance. That theory properly describes the interactions of all known particles, incorporating the proven symmetries and successes of quantum electrodynamics, the quark model, and low energy V-A theory. It correctly predicted weak neutral currents, the now observed gluons and weak gauge bosons, and the special properties of the heavy fermions $\tau, b$, and $t$. Since it is a renormalizable theory, its predictions can be tested at the quantum loop level by high precision experiments. It has already confronted a wealth of data at the level of $1 \%$ or better without any significant evidence of inconsistency. Because of those impressive successes, the $S U(3)_{c} \times S U(2)_{L} \times U(1)_{Y}$ theory has been given the title "The Standard Model", a designation which establishes it as the paradigm against which future experimental findings and new theoretical ideas must be compared.

The Standard Model cannot be the final theory of Nature, but it does represent the completion of a major stage toward the uncovering of that theory. To make further progress, we must examine both the strengths and failings of this model and direct experimental effort toward the weakest points in its structure.

The Standard Model is based on the interactions of fermions and vector gauge bosons. The fermions are grouped into three generations of leptons and quarks which span an enormous mass range. Their newest member, the top quark, is exceptionally heavy. Why is the top so massive, or why are the other fermions so light? This question highlights the broader problem of why Nature chose to repeat the fermion generations three times and endow quarks and leptons with their observed pattern of masses and mixing. It is likely that future intense scrutiny of the top quark's properties will provide new insights regarding this 
important problem.

The vector bosons of the Standard Model are grouped into eight massless gluons of $S U(3)_{c}$ which mediate the strong interactions, plus the $W^{ \pm}, Z$, and $\gamma$ which are responsible for electroweak interactions. The $\mathrm{SU}(3)_{c}$ gauge theory, called quantum chromodynamics (QCD), taken on its own, is an ideal theory. It has no arbitrary or free parameters but can, in principle, explain all hadronic dynamics including confinement, asymptotic freedom, proton structure, and baryon and meson spectroscopy. Confirming those properties and uncovering additional more subtle features of QCD remains an important experimental and theoretical challenge.

In contrast with $\mathrm{QCD}$, the electroweak sector has many arbitrary parameters. Most stem from the Higgs mechanism which is used to break the $\mathrm{SU}(2)_{L} \times \mathrm{U}(1)_{Y}$ symmetry and endow particles with mass. In the simplest realization of this symmetry breaking, one introduces a scalar doublet $\phi$, the Higgs field, which obtains a vacuum expectation value $v$. This assumption introduces into the theory an electroweak mass scale $v \simeq 250 \mathrm{GeV}$. The masses of the $W$ and $Z$ bosons and the various quarks and leptons are proportional to $v$. Their disparity reflects extreme differences in their couplings to the scalar field $\phi$. It is true that this simple model with one Higgs field can parametrize all electroweak masses, quark mixing, and even CP violation. But it does not provide insight into any of these phenomena, or even into the basic fact that the electroweak gauge symmetry is spontaneously broken. The many unanswered questions associated with the Higgs field, or whatever more complicated structure leads to the breaking of the electroweak symmetry, call for experiments which thoroughly explore this sector.

An important testable prediction of the simple Higgs model is the existence of a neutral spin-0 remnant particle $H$, called the Higgs scalar. Its mass depends on the self-coupling $\lambda$ of the Higgs field through the relation

$$
m_{H}=\sqrt{2 \lambda} v,
$$

but it is unspecified as long as $\lambda$ is unknown. There is an experimental lower bound on $m_{H}$ of $65 \mathrm{GeV}$ from direct searches at LEP. That search reach is expected to be extended up to about $90 \mathrm{GeV}$ at LEP II. There is also an approximate upper bound on the Higgs mass $m_{H} \lesssim 800$ $\mathrm{GeV}$ from theoretical bounds on $\lambda$. For example, perturbative partial wave unitarity in high energy scattering of longitudinal $W$ bosons, $W_{L} W_{L} \rightarrow W_{L} W_{L}$ requires $|\lambda| \lesssim 8 \pi / 5$. This gives a large window in which to search. However, there is a much stronger upper bound which comes from the stronger assumption that the Higgs boson is a fundamental particle with no nonperturbative interactions up to the grand unification scale. This requires $m_{H} \lesssim 200$ $\mathrm{GeV}$; we will refer to a.Higgs boson satisfying this hypothesis as a 'light Higgs'.

Even more daunting than the problem of finding the Higgs boson $H$ in the context of the simple Higgs theory is the prospect that this theory is inadequate to correctly describe the weak interaction scale. This simplest theory has theoretical problems of self-consistency, particularly when it is extrapolated to a unified theory at high energies. Also, the fact that its pattern of couplings must be input without any explanation is a sign that this theory is 
only a parametrization of electroweak symmetry breaking rather than being a fundamental explanation of this phenomenon. This state of affairs has led to many speculations on the true symmetry breaking mechanism and, from there, to interesting new physics possibilities beyond the Standard Model with observable manifestations at high energy.

In order to build a theory in which electroweak symmetry is naturally broken by the expectation value of a fundamental Higgs field, it is necessary to incorporate supersymmetry (SUSY) at the weak interaction scale. That elegant boson-fermion symmetry allows a simple connection to gauge or string theory unification and provides a logic for the symmetrybreaking form of the Higgs potential. Achieving these goals, however, requires introducing novel partners for all Standard Model particles. It also requires at least two Higgs doublets and thus predicts five remnant scalars, $h, H, A, H^{ \pm}$. The $h$ should have a mass below about $150 \mathrm{GeV}$ and should be most similar to the Standard Model Higgs boson. Finding that particle and determining its properties may be our first window to supersymmetry. If supersymmetry does indeed appear below $1 \mathrm{TeV}$, there will be a wealth of supersymmetry partner spectroscopy waiting to be explored. Currently, supersymmetry has no direct experimental support. However, there are two very suggestive pieces of evidence that are in favor of this theory. The first is the values of the $S U(3) \times S U(2) \times U(1)$ coupling constants. These coupling constants are in just the relation predicted by a supersymmetric grand unified theory. The second is the tendency of the precision electroweak data to favor a light Higgs boson, which is an indication that the mechanism of electroweak symmetry breaking involves weakly-coupled fields.

Alternatively, one might imagine that there is no fundamental Higgs field, and that the electroweak symmetry is broken dynamically by fermion-antifermion condensation due to new strong forces at high energy. Scenarios ranging from $t \bar{t}$ condensation to complex extended technicolor models have been proposed. Their basic premise is very appealing, but no compelling model exists. Nevertheless, the generic idea of new underlying strong dynamics gives rise to testable consequences for anomalous top and gauge boson couplings and high energy scattering behavior.

This issue of whether the mechanism of electroweak symmetry breaking is weak-coupling or strong-coupling is the most important question in elementary particle physics today. The NLC should resolve it definitively. For the case in which this physics is weak-coupling, the NLC should have a rich experimental program involving the detailed study of Higgs bosons and supersymmetric particles. The precise spectrum and branching ratio determinations for these particles should give information which, like the values of the strong and electroweak coupling constants, can be extrapolated to the unification scale. This scenario offers the tantalizing possibility that experimental data collected at the NLC would be directly relevant to supergravity and superstring theories at very high energy. On the other hand, if the mechanism of electroweak symmetry breaking is strong-coupling, this could imply a new spectroscopy at the $\mathrm{TeV}$ energy scale which the NLC might access directly.

In addition to these two options which relate directly to the physics of electroweak sym-. metry breaking, there are many other possibilities for new physics at the $\mathrm{TeV}$ energy scale. 
These include larger gauge groups with additional $W^{\prime}$ and $Z^{\prime}$ gauge bosons, heavy new fermions, and additional scalars. Many of these possibilities are realized in specific models of electroweak symmetry breaking, so a broad-based search for new phenomena is an essential part of the experimental program devoted to this question. The most direct way to uncover such new particles and their associated phenomena is to search at very high energies above particle production threshold. Important indirect evidence can also be inferred from precision studies of Standard Model parameters such as $m_{W}, \sin ^{2} \theta_{W}$, and the couplings of heavy quarks and $W$ bosons to the $\gamma$ and $Z^{0}$.

For the exploration of all of these possibilities, which defines the next step in experimental high-energy physics, the Next Linear Collider (NLC) will play an essential role. We envisage this machine as an $e^{+} e^{-}$collider which operates initially at a center of mass energy of about $500 \mathrm{GeV}$ and can be upgraded to $1.5 \mathrm{TeV}$, providing a luminosity corresponding to $10^{4}$ events per year for a process with the point cross section for QED pair production. This machine will employ polarized electrons and offers the possibility of $e^{-} e^{-}, e \gamma$, and $\gamma \gamma$ collider options. With such a facility, it is possible to carry out crucial and unique experiments across the whole range of possibilities we have just described for the physics of the weak interaction scale. In this report, we will summarize the capabilities of the NLC to explore the physics of the weak interaction scale across this broad front. A design for the NLC is presented in an accompanying report [1].

In Section 2 of this chapter, we will summarize the basic conclusions of this report relevant to the physics studies, including the basic accelerator parameters of energy and luminosity. We will also describe the basic assumptions on detector performance that we will use to describe the physics capabilities of this machine. In Sections 11 and 12, after our discussion of the physics opportunities that the NLC will provide, we will give a more detailed description of a detector design and the constraints on the detector which come both from the physics goals and from the accelerator.

One of the first physics goals of the NLC will be the detailed study of the top quark at its threshold and just above. We will explain in Section 3 the special features of the $t \bar{t}$ threshold region which make it a unique laboratory for the precision measurement of the top mass and width, the QCD coupling of the top quark, and the possible couplings to the Higgs boson and other new particles. We will also describe how the NLC will make precision measurements of the couplings of top to electroweak gauge bosons, couplings which might contain signals of new strong interactions which connect top to the sector responsible for electroweak symmetry breaking.

Whether the electroweak gauge symmetry is broken by fundamental Higgs bosons or by new high-energy strong interactions, the NLC will bring important contributions to the experimental study of this sector. First of all, though the LHC and other facilities have the capability to find a light Higgs boson in many decay channels, the NLC is the only planned facility at which the existence of a light Higgs boson can be ruled out in a model-independent way. If the light Higgs boson is indeed present, we will show in Section 4 that the NLC will be able not only to discover this particle but also to characterize many of its interactions. 
We will show that the NLC has a unique capability to determine the couplings of the Higgs boson to $Z$ and $W$, to heavy quarks and leptons, and to photons. These measurements dovetail nicely with the expected measurement of the Higgs production cross section from gluon fusion at the LHC to give the complete phenomenological profile of this particle.

If the presence of a relatively light fundamental Higgs particle is accompanied by the appearance of supersymmetry at the $\mathrm{TeV}$ scale, the NLC can perform crucial experiments to characterize the new supersymmetric particles. We will show in Section 5 that the NLC can detect the supersymmetric partners of $W$ and $Z$ over essentially the complete range of parameters expected in the model. But, even more importantly, the NLC can measure the masses and mixing angles of these particles and, in so doing, determine the most important underlying parameters of supersymmetry. This determination of parameters will be essential not only for the exploration of the physics of fermion partners at $e^{+} e^{-}$colliders, but also for the extraction of detailed information about the underlying theory from the complementary signatures of supersymmetry seen at hadron colliders.

If electroweak gauge symmetry is broken by new forces at high energy, one can look for the signs of these forces in the couplings of $W$ bosons to the $\gamma$ and $Z$ and in the study of $W W$ scattering. We will show in Section 6 that the NLC is an ideal machine for the study of the gauge couplings of the $W$, capable of achieving parts per mil precision on the $W$ form factors. We will show in Section 7 that the NLC at the high end of its energy range can achieve constraints on $W W$ scattering comparable to those of the LHC, in an environment with a number of qualitative advantages. We will also show that the NLC also offers new windows into $W W$ interactions through the precision study of $e^{+} e^{-} \rightarrow W^{+} W^{-}$and through high-energy $t \bar{t}$ production.

Finally, these capabilities of the NLC to explore specific models of electroweak symmetry breaking are balanced by the ability of this facility to perform broad searches for novel fermions, scalars, and gauge bosons. We will describe the abilities of the NLC to search for exotic particles in $e^{+} e^{-}$annihilation in Section 8. In Section 9, we will show how this broad capability is extended further by the availability of $e^{-} e^{-}, e \gamma$, and $\gamma \gamma$ collisions. In Section 10 , we will show that the NLC will also contribute to the future program in the study of the strong interactions, in particular, through the precision measurement of $\alpha_{s}$.

Section 13 will present our conclusions. We will review the unique capabilities of the NLC and contrast its prospects with those of the next generation of hadron colliders. 


\subsection{Standard Model Processes and Simulations}

We begin by describing the basic assumptions underlying our study of the physics capabilities of the NLC. We will briefly discuss the expected energy and luminosity that the NLC will provide, the performance of the detector that we expect to have available, our simulation methods, and the magnitudes of the most important standard model background processes.

\subsubsection{Accelerator and Detector}

The NLC is envisaged as the first full-scale $e^{+} e^{-}$linear collider, a machine designed from the beginning with the goal of high-luminosity colliding beam physics and one which takes account of the lessons of its prototype, the SLC. The NLC will be designed for an initial energy of $500 \mathrm{GeV}$ in the center of mass, with an upgrade path to $1.5 \mathrm{TeV}$. It will provide a luminosity sufficient for a thorough experimental program on $e^{+} e^{-}$annihilation to standard and exotic particle pairs. It will provide a highly polarized $e^{-}$beam, and possibly also a polarized positron beam. Our basic assumptions on luminosity as a function of energy and on polarization are given in Table 2.1. These assumptions are justified in the description of the accelerator design given in Chapter 3 of this report and, at greater length, in [1].

Table 2.1: Basic Parameters of the Next Linear Collider

\begin{tabular}{|lrcc|}
\hline \hline Energy (GeV) & \multicolumn{3}{c|}{ Luminosity $\left(\mathrm{cm}^{-2} \mathrm{~s}^{-1}\right)$} \\
& $500 \mathrm{GeV}$ & $5 \times 10^{33}$ & \\
& $1000 \mathrm{GeV}$ & $1 \times 10^{34}$ & \\
& $1500 \mathrm{GeV}$ & $1 \times 10^{34}$ & \\
Polarization: & & & Initial \\
& & $80 \% e^{-}, 0 \% e^{+}$ & Possible \\
\hline \hline
\end{tabular}

The NLC experiments can be carried out with a standard $4 \pi$ multipurpose detector similar to those at LEP or SLC. In our concept of this detector, we include some innovations such as all-silicon tracking to minimize the effect of machine-related backgrounds, but for the most part the demands we have made on the detector are straightforwardly met. The essential performance assumptions we have made are shown in Table 2.2. Because of the small beam spot sizes at a linear collider, which allows us to bring a CCD vertex detector within $2 \mathrm{~cm}$ of the interaction point, the detector should have excellent $b$-tagging capabilities. The assumed curve of efficiency versus purity for $b$-tagging is shown in Fig. 2.1; the performance required has already been demonstrated in the SLD vertex detector. 
Table 2.2: Summary of the detector parametrization used in the simulations. Smeared quantities are denoted in the table by a subscript $s$.

\begin{tabular}{|c|c|c|}
\hline Particle & Energy & Momentum \\
\hline Electrons & $\frac{\delta E}{E}=\frac{12 \%}{\sqrt{E}}+1.0 \%$ & $P_{s}^{2}=E_{s}^{2}-m_{e}^{2}$ \\
& $E_{s}=E+\delta E$ & \\
\hline Photons & $\frac{\delta E}{E}=\frac{12 \%}{\sqrt{E}}+1.0 \%$ & $P_{s}=E_{s}$ \\
& $E_{s}=E+\delta E$ & \\
\hline Neutral Hadrons & $\frac{\delta E}{E}=\frac{45 \%}{\sqrt{E}}+2.0 \%$ & $P_{s}^{2}=E_{s}^{2}-m_{\pi}^{2}$ \\
& $E_{s}=E+\delta E$ & \\
\hline Charged Hadrons & $E_{s}^{2}=P_{s}^{2}+m_{\pi}^{2}$ & $\frac{\delta P_{x, y}}{P_{x, y}^{2}}=0.0005 \oplus \frac{0.0015}{P_{x, y} \sqrt{P}(\sin \theta)^{2.5}}$ \\
& & $\frac{\delta P_{z}}{P_{z}^{2}}=\frac{0.0015}{P_{x, y} \sqrt{P}(\sin \theta)^{2.5}}$ \\
& & $\left(P_{s}\right)_{i}=P_{i}+\delta P_{i}$ \\
\hline
\end{tabular}

\subsubsection{Simulations}

In the studies presented here, the detector model has been used in concert with a set of familiar and newly-written simulation programs. In general, the background processes were generated by PYTHIA 5.7 [2], except for the background from $e^{+} e^{-} \rightarrow W^{+} W^{-}$, which plays an especially important role at linear collider energies. For this process we used a new generator [3] which is based on the formalism for this reaction presented by Hagiwara et al. [4]. This generator computed the total amplitude for $W^{+} W^{-}$production and subsequent decay to four fermions, retaining the full spin correlations through the process. It did make the approximation of treating the $W$ 's as on-shell particles, but it properly treated the effects of initial state electron polarization, beamstrahlung, collinear multi-photon initial state bremsstrahlung, and a nonzero $W$ boson decay width. The same Monte Carlo program was used in the studies of nonstandard $W$ physics reported in Sections 6 and 7. Many of the other analyses used specialized generators at this level of sophistication to simulate the new physics processes. These are described in the various sections of this report. Except where it is reported otherwise, the hadronization of partons and subsequent decays were performed by JETSET 7.4 [2].

Four-vectors of stable particles emerging from the simulated reaction were adjusted by a detector resolution smearing routine, which implemented the parametrization summarized in Table 2.2. All quantities were parametrized as a function of theta. The smearing assumed Gaussian errors and populated tails out to $3.5 \sigma$. The parametrization assumed a dead cone about the beampipe of $150 \mathrm{mrad}(\cos \theta=0.99)$. The neutral particle and charged 


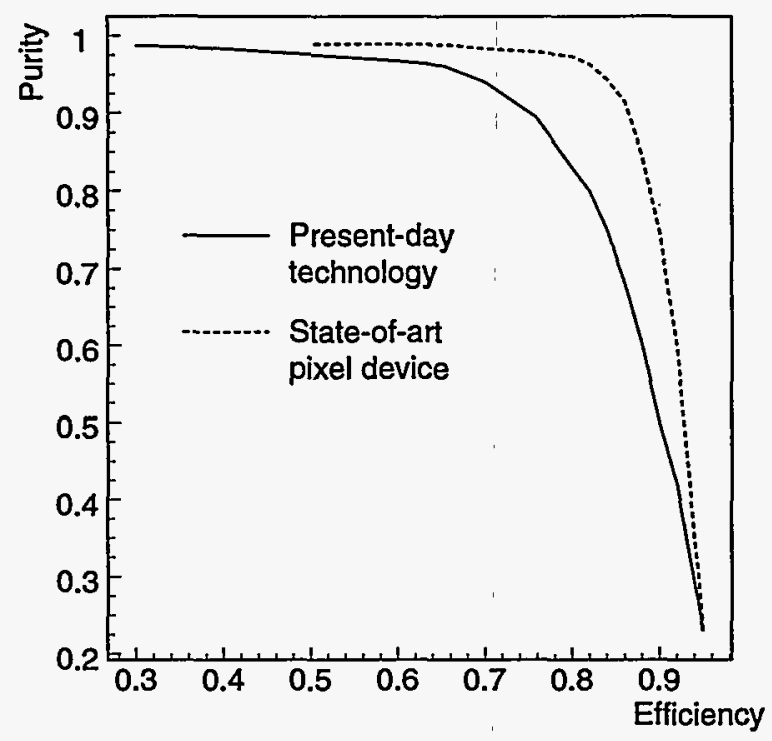

Figure 2.1: Efficiency versus purity relation for $b$-tagging with the NLC detector.

particle detection efficiencies were each taken to be $98 \%$. For neutral hadrons, the momentum directions were Gaussian smeared in a cone about the original direction with a half-width of $15 \mathrm{mrad}$ to simulate finite directional resolution. For photons and electrons, the directions were smeared by a cone of half-width $10 \mathrm{mrad}$.

\subsubsection{Standard Model Processes at the NLC}

Standard model processes, in addition to being interesting in their own right, are the background to searches for new physics at the NLC. Many of the standard model reactions at the NLC are familiar at lower energies and need only be extrapolated to higher energies. However, new processes, such as the pair production of gauge bosons, emerge as dominant reactions.

The cross sections of Standard Model processes at an $e^{+} e^{-}$collider are shown as a function of center of mass energy in Fig. 2.2 [5]. From left to right across this plot, the familiar $e^{+} e^{-}$ annihilation processes fall with energy according to the point cross section for $e^{+} e^{-} \rightarrow \mu^{+} \mu^{-}$ in QED,

$$
1 \mathrm{R}=\frac{4 \pi \alpha^{2}}{3 s}=\frac{87 \mathrm{fb}}{s\left(\mathrm{TeV}^{2}\right)} .
$$

At the same time, new processes involving pair production and multiple production of weak interaction vector bosons become important.

Another view of the standard model backgrounds is given in Fig. 2.3, where the cross sections for the dominant $e^{+} e^{-}$annihilation processes are shown as a function of the degree of longitudinal polarization. The curves were calculated using ISAJET 7.13 [6]. The peripheral 


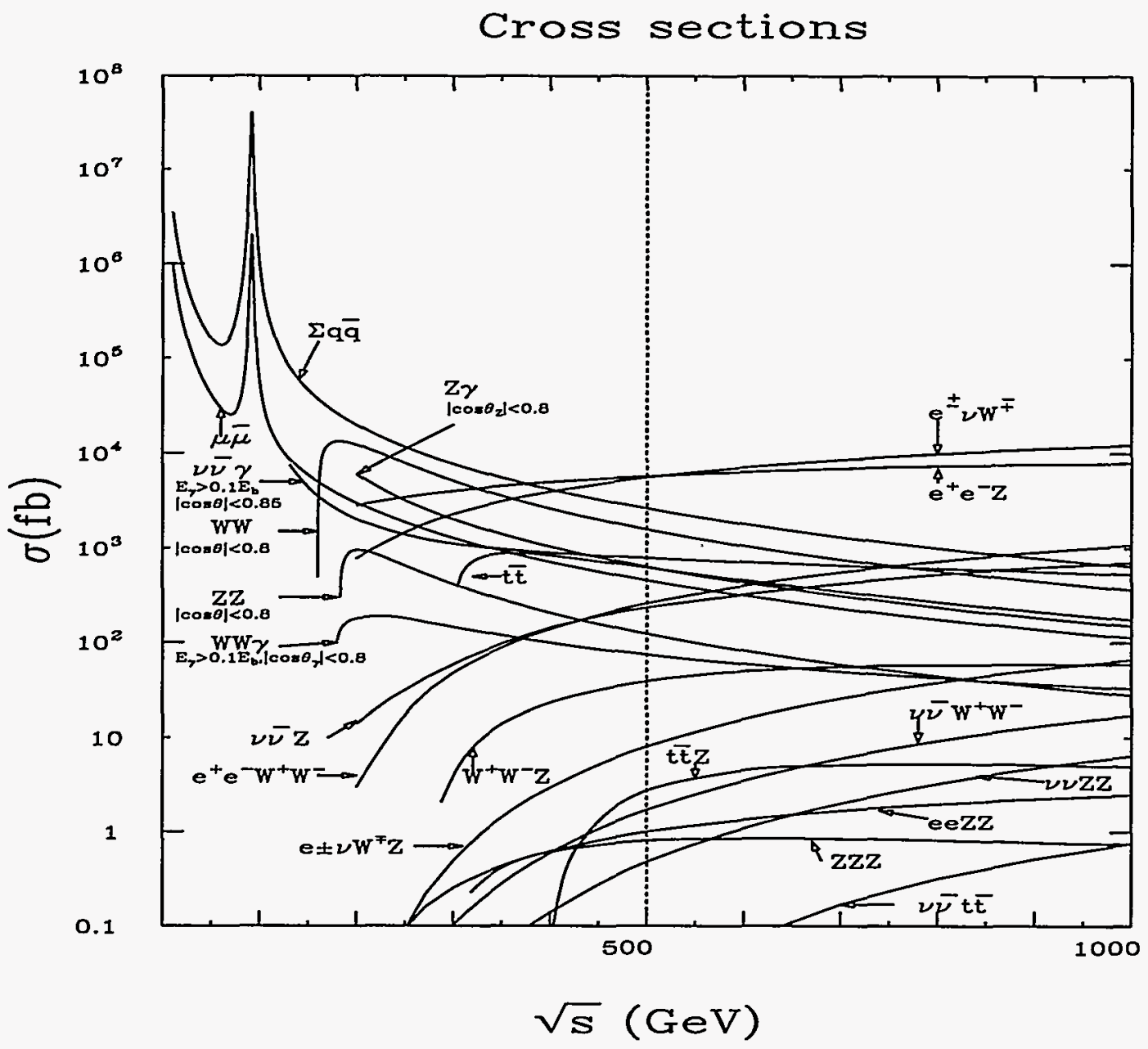

Figure 2.2: Cross sections for Standard Model physics processes in $e^{+} e^{-}$ annihilation, as a function of center of mass energy, from [5].

two photon, t-channel Bhabha scattering and $e^{+} e^{-} \rightarrow Z^{\circ} \gamma$ processes are not shown; the cross sections for these reactions are relatively independent of polarization. The reactions $e^{+} e^{-} \rightarrow$ $e^{+} \nu W^{-}, e^{-} \bar{\nu} W^{+}, e^{+} e^{-} Z^{0}$ are also not shown. The first of these is present only for left-handed $e^{-}$; the other two depend only weakly on $e^{-}$beam polarization. The most troublesome source of background in many of the physics analyses is the reaction $e^{+} e^{-} \rightarrow W^{+} W^{-}$, whose special role we have already pointed out. It is noteworthy that the cross section for this process can be reduced substantially by adjusting the electron beam polarization.

Cuts on other quantities, such as the acoplanarity and production angle will also be useful for removing standard model background. The distributions in these variables for standard model annihilation processes are shown in Figs. 2.4 and 2.5.

In general, the two photon and $e^{+} e^{-} \rightarrow Z^{0} \gamma$ processes are not important as backgrounds to annihilation processes because they may be removed easily from the data sample by low transverse momentum and multiplicity cuts [7]. The cross section for Bhabha scattering is 


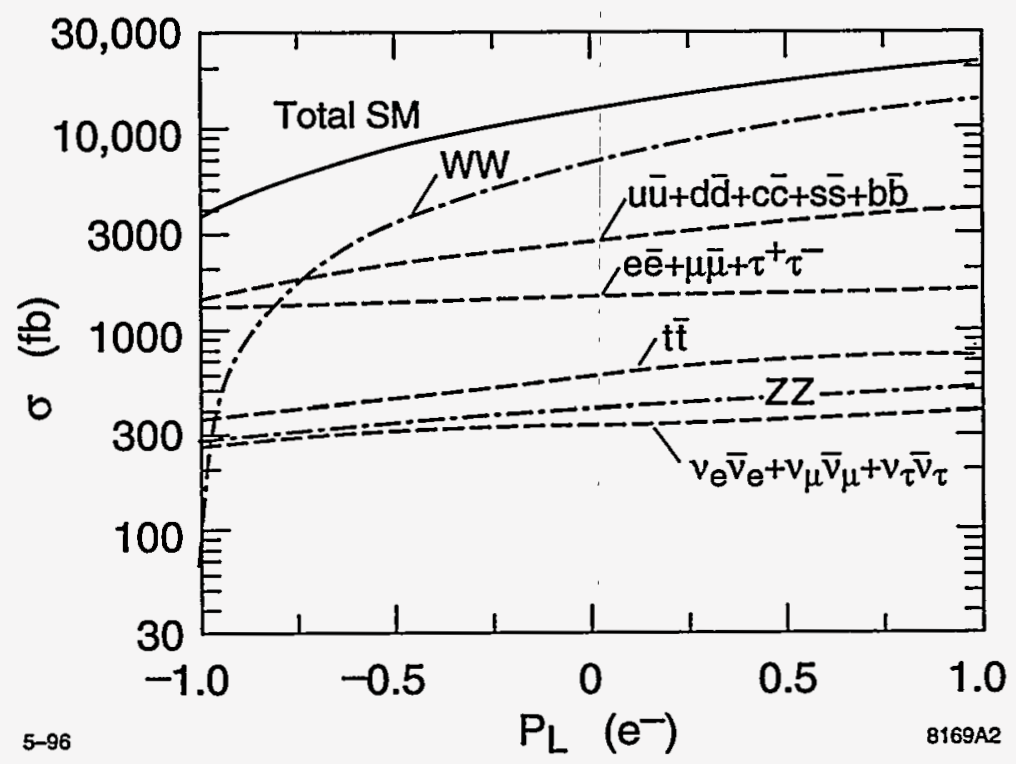

Figure 2.3: Cross sections for Standard Model physics processes in $e^{+} e^{-}$annihilation at $500 \mathrm{GeV}$, as a function of the electron longitudinal polarization.

very large in the forward direction but drops to a few units of $R$ at large angles. 


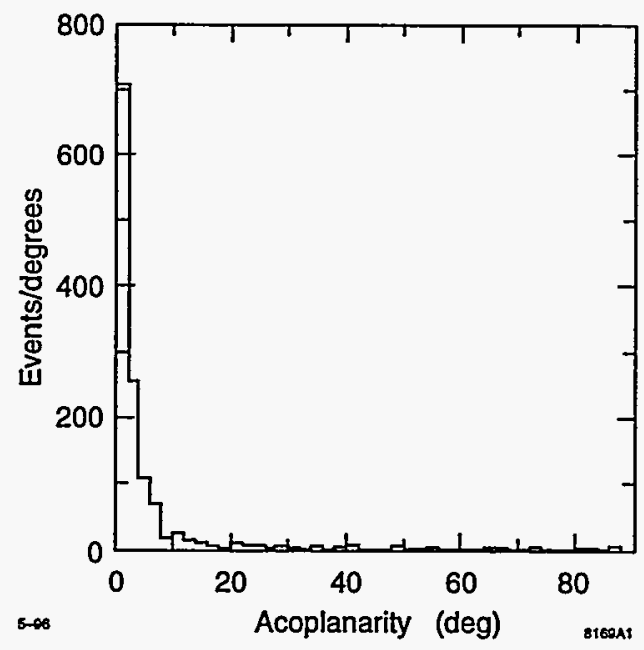

Figure 2.4: Expectation for the acoplanarity distribution in $e^{+} e^{-} \rightarrow$ $W^{+} W^{-}$events in which both $W$ bosons decay to hadrons.

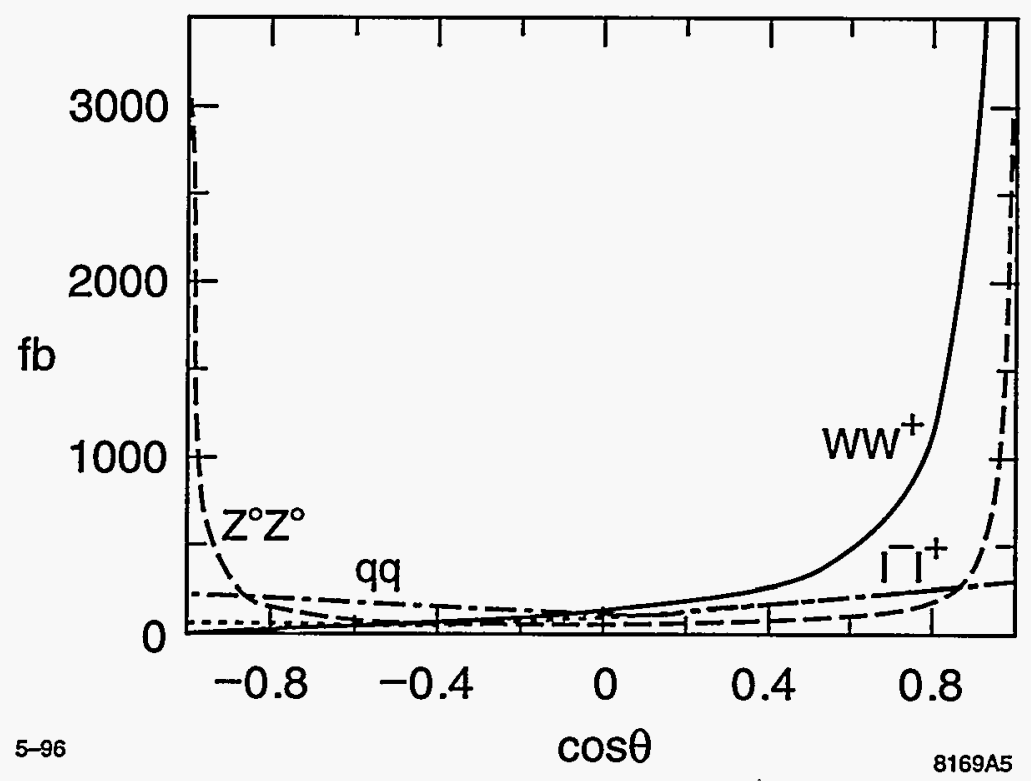

Figure 2.5: Expectation for the $\cos \theta$ distribution due to standard model processes. 


\subsection{Top Quark Physics}

The stage for the future of top physics has been set by the recent discovery [8] of top at Fermilab. The very large top mass, $m_{t} \approx 174 \pm 8 \mathrm{GeV} / \mathrm{c}^{2}$, forces one to consider the possibility that the top quark plays a special role in particle physics. At the very least, the properties of the top quark could reveal important information about the physics of electroweak symmetry breaking. In this context, the determination of the complete set of top quark properties should be an important goal. A high-energy future linear $e^{+} e^{-}$collider provides a very impressive tool to carry out a detailed top quark physics program.

The $t \bar{t}$ threshold region has a rich phenomenology which derives from its mix of toponium and continuum structure. Only in $e^{-} e^{+}$collisions can this threshold structure be properly resolved, making possible definitive measurements of the top mass and width and tests of the QCD potential at very short range. Above threshold, the NLC makes it possible to measure the complete set of top couplings to gauge bosons, both for neutral current ( $\gamma$ and $Z^{0}$ ) and charged current interactions. The high electron-beam polarization available at NLC plays an important role in such studies, simplifying the search for anomalous top couplings and CP violating effects. A complete understanding of electroweak symmetry breaking will require the measurement of the Higgs boson couplings to fermions; of these, the coupling to top is the most accessible. At the NLC, this quantity can be measured by direct $t \bar{t} H$ production above threshold and also, if the Higgs boson is light, by the effect of Higgs boson exchange on the threshold properties. Finally, the NLC provides a relatively clean final state and precise vertex detection which make it straightforward to study the decays of the top. All standard decay modes can be reconstructed with reasonable efficiency, and exotic decay modes, in those examples studied to date, can be readily identified.

The physics program for the top quark also imposes important constraints on the NLC design. The energy must be adjustable, to run both at the $t \bar{t}$ threshold and at a point in the continuum about $100 \mathrm{GeV}$ above threshold. The study of the threshold region requires that the center-of-mass energy spread be much smaller than the top quark width, and that tails in the energy distribution be understood. Experimenters must be able to determine both the absolute energy and the differential luminosity spectrum.

\subsubsection{Top Production, Decay, and Measurement}

The large mass of the top quark causes it to have a very large decay width, and this exerts a decisive influence on its phenomenology. In the Standard Model, the weak decay of top proceeds very rapidly via $t \rightarrow b W$, resulting in a total decay width given by

$$
\Gamma_{t} \approx(0.18)\left(m_{t} / m_{W}\right)^{3} \mathrm{GeV}
$$

For $m_{t}=180 \mathrm{GeV} / \mathrm{c}^{2}$ this lowest-order prediction is $\Gamma_{t}=1.71 \mathrm{GeV}$. After first-order QCD and electroweak corrections [9], this becomes $1.57 \mathrm{GeV}$. Hence, top decay is much more rapid than the characteristic time for hadron formation, for which the scale is $\Lambda_{\mathrm{QCD}}$. This implies 
that the phenomenology of top physics is fundamentally different than that of the lighter quarks. For example, there will be no top-llavored mesons. While we lose the familiar study of the spectroscopy of these states, we gain unique clarity in the ability to reconstruct the properties of the elementary quark itself. This may prove to be a crucial advantage toward uncovering fundamental issues.

The top decay also provides a natural cutoff for gluon emission. Indeed, in $t \bar{t}$ processes, the nonperturbative color strings appear in fragmentation only after the tops decay and form along the separating $b$ and $\bar{b}$ lines. Hard gluons emitted from the top and its product bottom quark can exhibit interference phenomena which are sensitive to the value of $\Gamma_{t}[10,11]$.

In the Standard Model, $\left|V_{t b}\right| \approx 1$, so that the decay mode $t \rightarrow b W$ completely saturates the decay width. Then the branching ratios are determined by the $W$ decay modes from the $b \bar{b} W^{+} W^{-}$intermediate state. This gives 6-jet, 4-jet + lepton, and 2-lepton final states in the ratio 4:4:1, or, including $Q C D$ corrections to the $W$ decay rates, $\mathrm{BR}\left(t \bar{t} \rightarrow b \bar{b} q q^{\prime} q q^{\prime}\right)=0.455$; $\mathrm{BR}\left(t \bar{t} \rightarrow b \bar{b} q q^{\prime} \ell \nu\right)=0.439 ; \mathrm{BR}(t \bar{t} \rightarrow \ell \nu \ell \nu)=0.106$, where $q=u, c, q^{\prime}=d, s$, and $\ell=e, \mu, \tau$.

The parton-like decay of top implies that, unlike other quarks, its spin is transferred to a readily reconstructable final state. Measurement of the $\bar{b} \bar{b} W^{+} W^{-}$final state therefore provides a powerful means of probing new physics manifested by top with helicity analyses. This is explored in Section 2.3.3. Another implication of the large $m_{t}$ is the Standard Model prediction that the decay $t \rightarrow b W$ produces mostly longitudinally polarized $W$ bosons; the degree of longitudinal polarization is given by $m_{t}^{2} /\left(m_{t}^{2}+2 m_{W}^{2}\right) \approx 72 \%$ for $m_{t}=180 \mathrm{GeV} / \mathrm{c}^{2}$. This reflects the fact that the longitudinally polarized $W$ bosons contain degrees of freedom from the electroweak symmetry breaking sector.

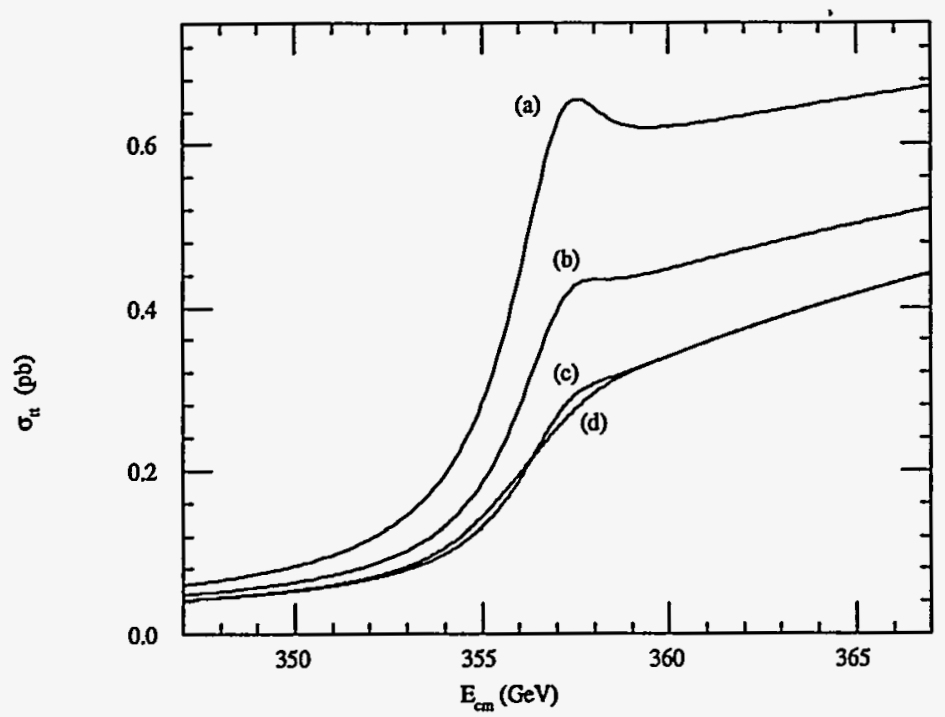

Figure 2.6: Production cross section for top-quark pairs near threshold for $m_{t}=180 \mathrm{GeV} / \mathrm{c}^{2}$. The ideal theoretical cross section is given by curve (a). In curves (b), (c) and (d), we add, successively, the effects of initial-state radiation, beamstrahlung, and beam energy spread. 
The $t \bar{t}$ cross section due to $s$-channel $e^{+} e^{-}$annihilation mediated by $\gamma, Z$ bosons increases abruptly just below threshold (see Fig. 2.6), reaches a maximum at roughly $50 \mathrm{GeV}$ above threshold, then falls roughly proportional to the point cross section, Eq. 2.2, as the energy increases. At $\sqrt{s}=500 \mathrm{GeV}$ the lowest-order total cross section for unpolarized beams is $0.54 \mathrm{pb}$; it is $0.74(0.34)$ for a fully left-hand (right-hand) polarized electron beam. Hence, in a design year of integrated luminosity $\left(50 \mathrm{fb}^{-1}\right)$ at $\sqrt{s}=500 \mathrm{GeV}$ we can produce 25,000 $t \bar{t}$ events. The cross sections for $t$-channel processes, resulting, for example, in final states such as $e^{+} e^{-} t \bar{t}$ or $\nu \bar{\nu} t \bar{t}$, increase with energy, but are still relatively small. We will discuss these processes in Section 7.3.

The emphasis of most event selection strategies has been to take advantage of the multijet topology of the roughly $90 \%$ of $t \bar{t}$ events with 4 or 6 jets in the final state. Therefore, cuts on thrust or number of jets drastically reduces the light fermion pair background. In addition, one can use the multi-jet mass constraints $M($ jet-jet $) \approx m_{W}$ and $M(3$-jet $) \approx m_{t}$. Simulation studies [12] have shown that multi-jet resolutions of $5 \mathrm{GeV} / \mathrm{c}^{2}$ and $15 \mathrm{GeV} / \mathrm{c}^{2}$ for the 2-jet and 3-jet masses, respectively, are adequate and readily achievable with LEP/SLC detectors. A detection efficiency of about $70 \%$ with a signal to background ratio of 10 was attained by selecting 6 -jet final states just above threshold. These numbers are typical also for studies which select the 4-jet $+\ell \nu$ decay mode.

Of the backgrounds considered in this study, that from $W$-pair production is the most difficult to eliminate. However, in the limit that the electron beam is fully right-hand polarized, the $W^{+} W^{-}$cross section is dramatically reduced. Thus it is possible to use the beam polarization to experimentally control and measure the background. We note, though, that the signal is also somewhat reduced by running with a right-handed polarized beam. A possible strategy might be to run with a right-handed polarized beam only long enough to make a significant check of the background due to $W$ pairs. Another important technique is that of precision vertex detection. The present experience with SLC/SLD can be used as a rather good model of what is possible at NLC. The small and stable interaction point, along with the small beam sizes and bunch timing, make the NLC ideal for pushing the techniques of vertex detection. This has important implications for top physics. Rather loose b-tagging, applied in conjunction with the standard topological and mass cuts mentioned above, should lead to substantially improved top event selection efficiencies and purities.

\subsubsection{Threshold Physics}

In Fig. 2.6 we show the cross section for $t \bar{t}$ production as a function of nominal center-of-mass energy for $m_{t}=180 \mathrm{GeV} / \mathrm{c}^{2}$. In this discussion, $m_{t}$ is the pole mass in QCD perturbation theory. The theoretical cross section, indicated as curve (a), is based on the results of Strassler and Peskin [13], using the $q \bar{q}$ potential of $Q C D$ with $\alpha_{s}\left(M_{Z}^{2}\right)=0.12$ and Standard Model couplings to $\gamma$ and $Z$. To this curve, the energy-smearing mechanisms of initial-state radiation, beamstrahlung, and beam energy spread, have been successively applied; curve (d) includes all effects. The beam effects were calculated using NLC design parameters. 
The threshold enhancement given by the predicted cross section curve of Fig. 2.6a reflects the Coulomb-like attraction of the produced $t \bar{t}$ state due to the short-distance QCD potential

$$
V(r) \sim-C_{F} \frac{\alpha_{s}(\mu)}{r},
$$

where $C_{F}=4 / 3$ and $\mu$ is evaluated at the scale of the Bohr radius of this toponium atom: $\mu \sim \alpha_{s} m_{t}$. The level spacings of the QCD potential, approximately given by the Rydberg energy, $\sim \alpha_{s}^{2} m_{t}$, turn out to be comparable to the widths of the resonance states, given by $\Gamma_{\theta} \approx 2 \Gamma_{t}$. Thus, the bound state exists, on average, only for approximately one classical revolution before one of the top quarks undergoes a weak decay. The level spacings of the QCD potential approximately given by the Rydberg energy, $\sim \alpha_{s}^{2} m_{t}$, turn out to be comparable to the widths of the resonance states, given by $\Gamma_{\theta} \approx 2 \Gamma_{t}$. Therefore the various toponium states become smeared together, as seen in Fig. 2.6, where only the bump at the position of the $1 \mathrm{~S}$ resonance is distinguishable. The infrared cutoff imposed by the large top width also implies [14] that the physics is independent of the long-distance behavior of the QCD potential. The assumed intermediate-distance potential is also found [12] to have a negligible impact. Hence, the threshold physics measurements depend only on the short-distance potential (Eq. 2.4) of perturbative QCD.

An increase of $\alpha_{s}$ deepens the QCD potential, thereby increasing the wave function at the origin and producing an enhanced $1 \mathrm{~S}$ resonance bump. In addition, the binding energy of the state varies roughly as the Rydberg energy $\sim \alpha_{s}^{2} m_{t}$. So the larger $\alpha_{s}$ has the combined effect of increasing the cross section as well as shifting the apparent position of the threshold to lower energy. The latter effect is also what is expected for a shift to lower $m_{t}$. Therefore, there exists a significant correlation between the measurements of $\alpha_{s}$ and $m_{t}$ from a threshold scan.

A number of studies have been carried out to simulate the measurement of the $t \bar{t}$ threshold cross section. Figure 2.7a depicts a threshold scan [12] for which an integrated luminosity of $1 \mathrm{fb}^{-1}$ has been expended at each of 10 energy points across the threshold, plus one point below threshold to measure backgrounds. A value of $m_{t}=150 \mathrm{GeV} / \mathrm{c}^{2}$ was used. No beam polarization was assumed. A fit of the data points to the theoretical cross section, including all radiative and beam effects discussed above, results in a sensitivity for the measurement of $m_{t}$ and $\alpha_{s}$ shown in Fig. 2.7b. The correlation between these two parameters is apparent. Even for the modest luminosity assumed here, the cross section measurement gives quite good sensitivity to these quantities. If no prior knowledge is assumed, the errors for $m_{t}$ and $\alpha_{s}$ are $200 \mathrm{MeV} / \mathrm{c}^{2}$ and 0.005 , respectively. Conversely, the single-parameter sensitivity for $m_{t}$ approaches $100 \mathrm{MeV} / \mathrm{c}^{2}$ if $\alpha_{s}$ is known to much better than $2 \%$ accuracy. We will describe a method for the precision measurement of $\alpha_{s}$ in Section 10.1. The theoretical systematic error due to uncertainties in the $t \bar{t}$ threshold cross section is of order $200 \mathrm{MeV}$.

For a quarkonium state, we expect the cross section at the $1 S$ peak to vary with the total width roughly as $\sigma_{1 S} \sim\left|V_{t b}\right| / \Gamma_{t}$, and therefore is very sensitive to the width, as indicated in Fig. 2.8 for rather wide variations in $\Gamma_{t}$ relative to the Standard Model expectation. (It is noted that the calculations of Figs. 2.6 and 2.8 use the uncorrected top width, so that the 

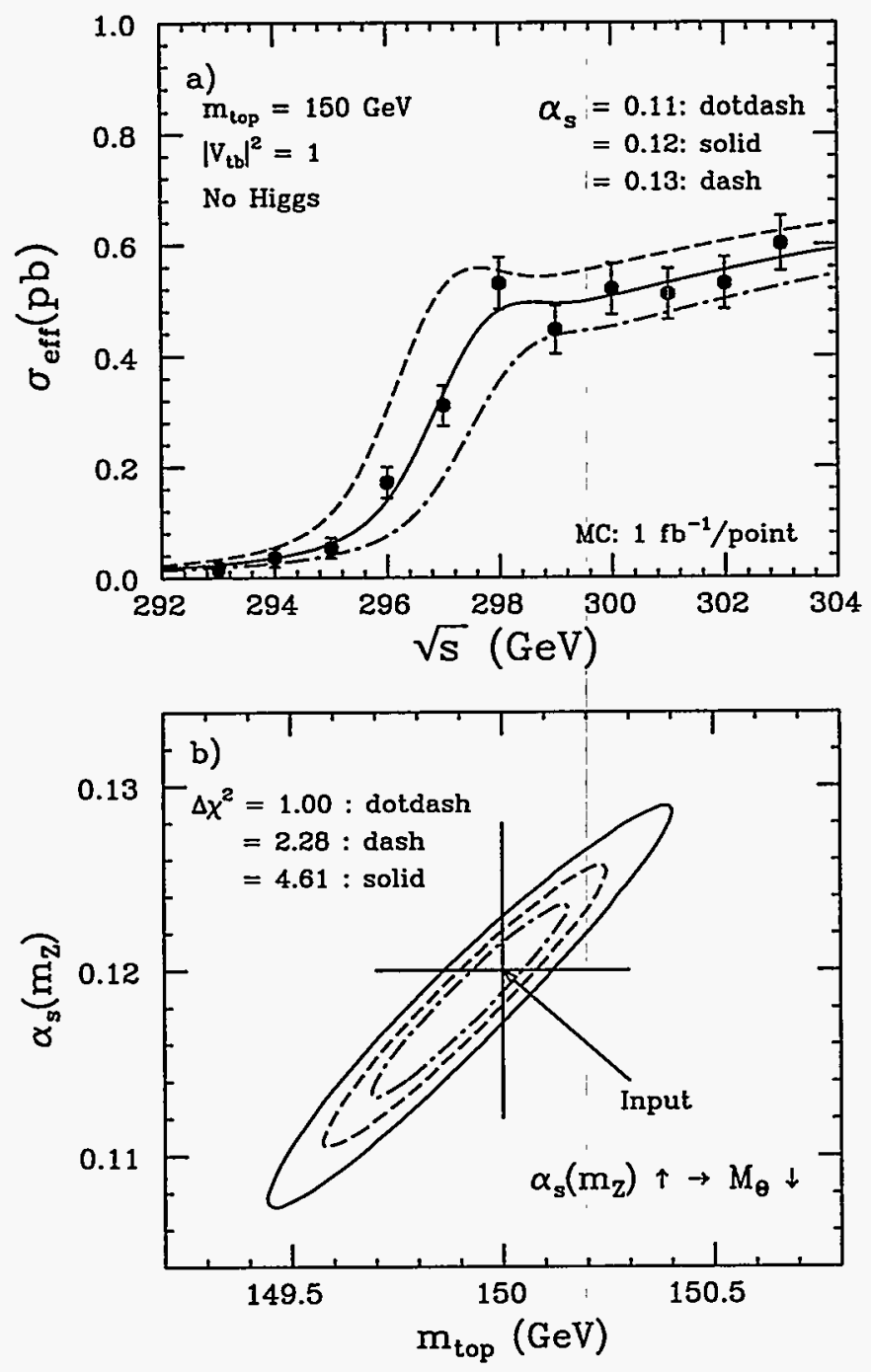

Figure 2.7: (a) Top threshold scan; (b) corresponding error ellipse for $m_{t}$ and $\alpha_{s}$. A value for $m_{t}$ of $150 \mathrm{GeV} / \mathrm{c}^{2}$ was assumed. 

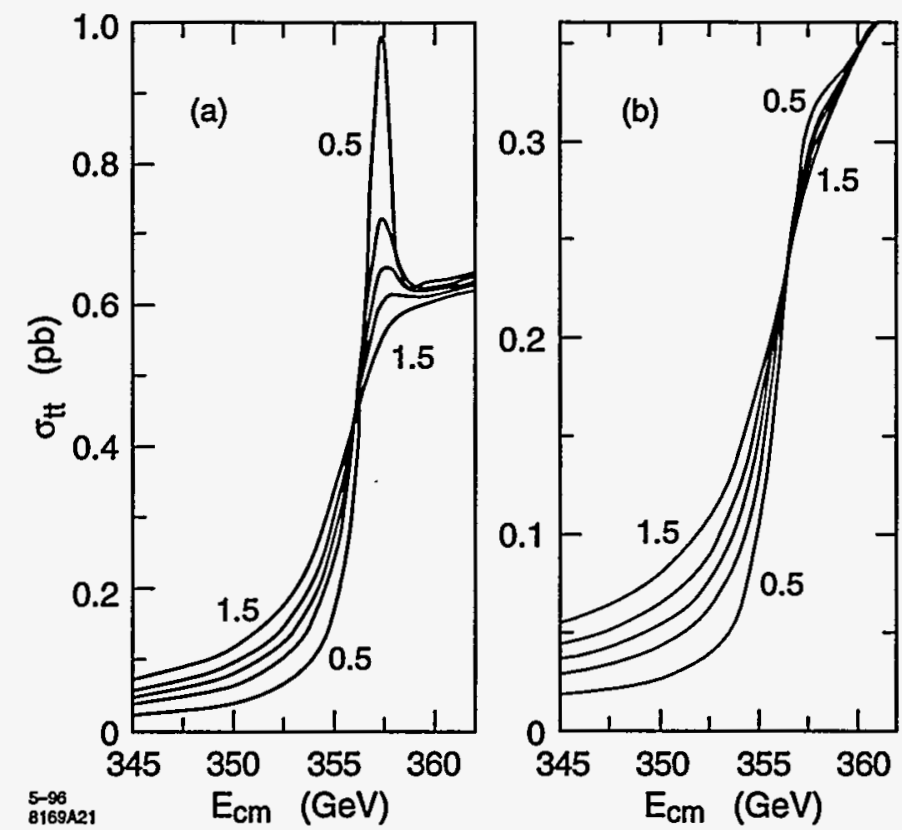

Figure 2.8: Variation of the $t \bar{t}$ threshold cross section with the top width for $m_{t}=180 \mathrm{GeV} / \mathrm{c}^{2}$. The curves correspond to values of $\Gamma_{t} / \Gamma_{S M}$ of $0.5,0.8,1.0,1.2$, and 1.5, presented in the order indicated, for (a) the theoretical cross section, and (b) the cross section after including radiative and beam effects.

resonance structure will be slightly more pronounced than what is shown.) After applying a correction for initial-state radiation and the beam-related energy spread, the width is affected as shown in Fig. 2.8. This implies that a scan strategy optimized for measuring $\Gamma_{t}$ would spend a relatively large fraction of running time below the 1S peak. The threshold physics, combining the cross section information with the momentum and asymmetry results, as discussed below, represents what is most likely the best opportunity to measure $\Gamma_{t}$.

In addition to the QCD potential, the $t-\bar{t}$ pair is also subject to the Yukawa potential associated with Higgs exchange:

$$
V_{Y}=-\frac{\lambda^{2}}{4 \pi} \frac{e^{-m_{H} r}}{r},
$$

where $m_{H}$ is the Higgs mass and $\lambda$ is the $t \bar{t}$-Higgs Yukawa coupling, $\lambda=m_{t} / v=\left[\sqrt{2} G_{F}\right]^{1 / 2} m_{t}$. Because of the extremely short range of the Yukawa potential, its effect is primarily to alter the wave function at the origin, and hence to shift the level of the cross section. This exciting possibility is discussed further in Section 2.3.4. The physics of the threshold cross section is, in summary, expected to depend on the following set of parameters:

$$
\sigma=\sigma\left(m_{t}, \alpha_{s}, \Gamma_{t}, m_{H}, \lambda\right) .
$$


As we have discussed, the lifetime of the toponium resonance is determined by the first top quark to undergo weak decay, rather than by the annihilation process. This has the interesting implication that the kinetic energy (or momentum) of the top quark as reconstructed from its decay products reflects the potential energy of the top in the QCD potential. Hence, a measurement of the momentum distribution will be sensitive to $\alpha_{s}$ and $\Gamma_{t}$. The theory [15] and phenomenology $[12,16]$ of this physics has been extensively studied. A convenient observable which has been used to characterize the distribution is the position of the peak in the reconstructed top quark momentum distribution. The position of this peak at a given center-of-mass energy is indeed found to be sensitive to $\Gamma_{t}$ and the other parameters in Eq. 2.6 .

Yet another, quite different observable has been studied $[17,12]$ to help further pin down the physics parameters at threshold. Top is produced symmetrically when produced in the $1 \mathrm{~S}$ state. The vector coupling of $t \bar{t}$ to the $\gamma$ and $Z$ can create $\mathrm{S}$ - and D-wave resonance states. On the other hand, the axial-vector coupling of the top quark to the $Z$ gives rise to $\mathrm{P}$-wave resonance states. Hence, there is naturally interference between $\mathrm{S}$ - and $\mathrm{P}$-waves which gives rise to a forward-backward asymmetry $\left(A_{F B}\right)$ proportional to $\beta \cos \theta$. Because of the large width of the resonance states, due to the large $\Gamma_{t}$, these states do overlap to a significant extent, and a sizable $A_{F B}$ develops. The value of $A_{F B}$ varies from about $5 \%$ to $12 \%$ across the threshold, with the minimum value near the $1 \mathrm{~S}$ resonance. Since the top width controls the amount of S-P overlap, we expect the forward-backward asymmetry to be a sensitive method for measuring $\Gamma_{t}$.

In summary, a data set of $50 \mathrm{fb}^{-1}$ at threshold would provide sensitivity to $m_{t}$ and $\alpha_{s}$ at the level of $120 \mathrm{MeV} / \mathrm{c}^{2}$ and 0.0025 , respectively. Similarly, the sensitivity to the total top decay width is $5-10 \%$. Accelerator and detector designs have become sufficiently stable to make possible calculations which incorporate the systematics associated with luminosity spectra and backgrounds. This would allow better determination of the limiting systematic errors at threshold, which are presently estimated to be at or below the sensitivities above. The measurement of the luminosity spectrum is discussed in more detail in Section 13.

\subsubsection{Top Couplings}

At the NLC, $e^{+} e^{-} \rightarrow t \bar{t}$ above threshold will provide a unique opportunity to measure simultaneously all of the top couplings. Due to its rapid weak decay, the top spin is transferred directly to the final state with negligible hadronization uncertainties, therefore allowing the helicity-dependent information contained in the Lagrangian to be propagated to the final state. This final state, expected to be dominated by $b W^{+} \vec{b} W^{-}$, can be fully reconstructed with good efficiency and purity, so that a complete helicity analysis can be performed.

The top neutral-current coupling can be generalized to the following expression for the $Z t \bar{t}$ or $\gamma t \bar{t}$ vertex factor:

$$
\mathcal{M}^{\mu(\gamma, Z)}=e \gamma^{\mu}\left[Q_{V}^{\gamma, Z} F_{1 V}^{\gamma, Z}+Q_{A}^{\gamma, Z} F_{1 A}^{\gamma, Z} \gamma^{5}\right]+\frac{i e}{2 m_{t}^{\prime}} \sigma^{\mu \nu} k_{\nu}\left[Q_{V}^{\gamma, Z} F_{2 V}^{\gamma, Z}+Q_{A}^{\gamma, Z} F_{2 A}^{\gamma, Z} \gamma^{5}\right]
$$


This expression reduces to the familiar Standard Model tree level expression when we set the form factors to $F_{1 V}^{\gamma}=F_{1 V}^{Z}=F_{1 A}^{Z}=1$, with all others zero. The quantities $Q_{A, V}^{\gamma, Z}$ are the usual SM coupling constants: $Q_{V}^{\gamma}=\frac{2}{3}, Q_{A}^{\gamma}=0, Q_{V}^{Z}=\left(1-\frac{8}{3} \sin ^{2} \theta_{W}\right) /\left(4 \sin \theta_{W} \cos \theta_{W}\right)$, and $Q_{A}^{Z}=-1 /\left(4 \sin \theta_{W} \cos \theta_{W}\right)$. The non-standard couplings $F_{2 V}^{\gamma, Z}$ and $F_{2 A}^{\gamma, Z}$ correspond to the electroweak magnetic and electric dipole moments, respectively. While these couplings are zero at tree level in the Standard Model, the analog of the magnetic dipole coupling is expected to attain a value of order $\alpha_{s} / \pi$ due to corrections beyond leading order. On the other hand, the electric dipole term violates $\mathrm{CP}$ and is expected to be zero in the Standard Model through two loops [18]. Such a non-standard coupling necessarily involves a top spin flip, hence is proportional to $m_{t}$.
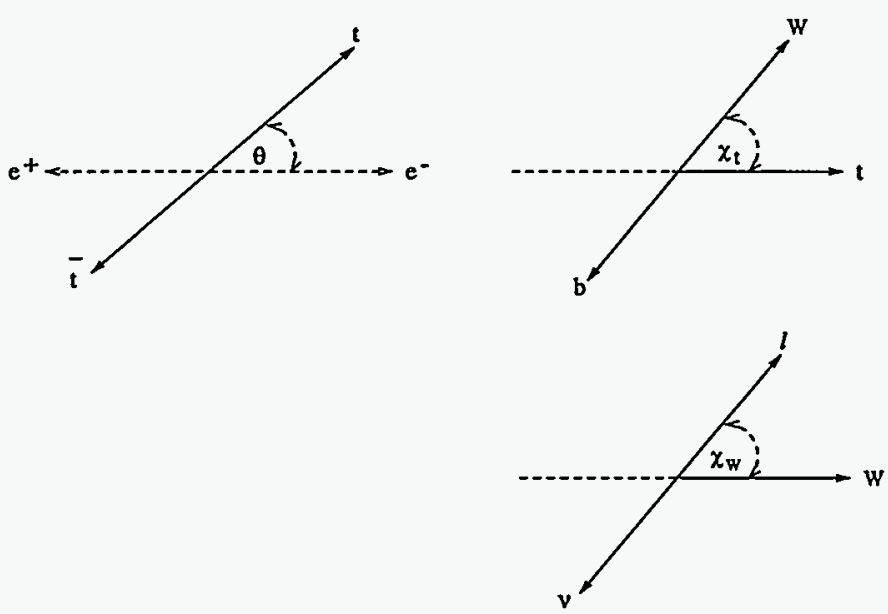

Figure 2.9: Definitions of helicity angles. (a) Production angle $\theta$ in $t \bar{t}$ rest frame; (b) $\chi_{t}$ measured in the top rest frame as shown; and (c) $\chi_{W}$ in the $\mathrm{W}$ rest frame.

The form factors can be measured through their distinct dependencies on the helicities of the $e^{-}, e^{+}, t$, and $\bar{t}$, which can be accessed experimentally through the beam polarization and the angular distributions in the final state. The production and decay angles can be defined as shown in Fig. 2.9. The angle $\chi_{W}$ is defined in the $W$ rest frame. The analogous statement holds for the definition of $\chi_{t}$. Experimentally, all such angles, including the angles corresponding to $\chi_{t}$ and $\chi_{W}$ for the $\bar{t}$ hemisphere, are accessible. Given the large number of constraints available in these events, full event reconstruction is entirely feasible. To reconstruct $\theta$ one must also take into account photon and gluon radiation. Photon radiation from the initial state is an important effect, which, however, represents a purely longitudinal boost which can be handled within the framework of final-state mass constraints. Gluon radiation can be more subtle. Jets remaining after reconstruction of $t$ and $\bar{t}$ can be due to gluon radiation from $t$ or $b$, and the correct assignment must be decided based on the kinematic constraints and the expectations of QCD. 


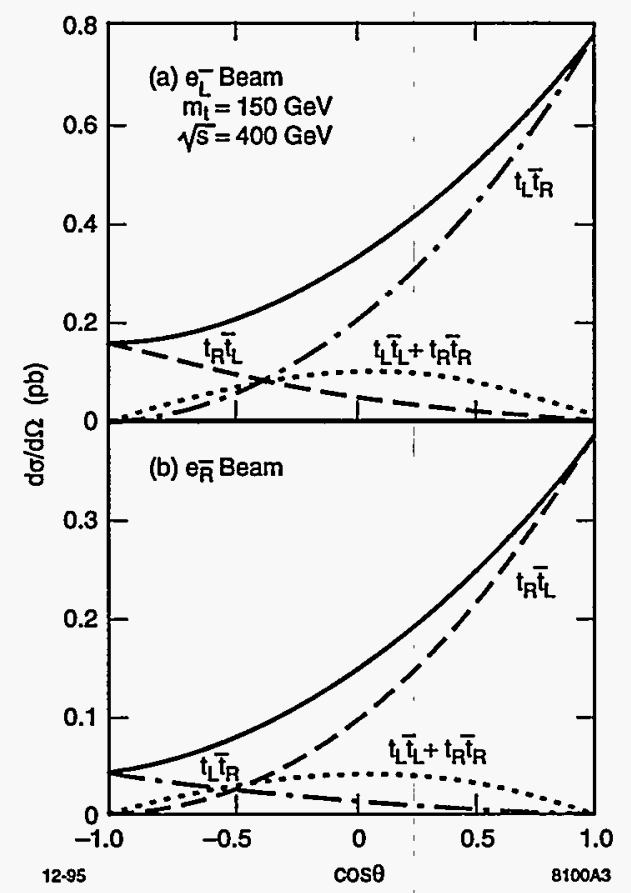

Figure 2.10: Production angle for $t \bar{t}$ for the possible final-state helicity combinations, as indicated, for $100 \%$ polarized beams with (a) lefthand polarized electrons, and (b) right-hand polarized electrons. The complete cross sections are the solid curves.

The distributions of the production angle $\theta$ for the SM in terms of the various helicity states are given in Fig. 2.10 for left and right-hand polarized electron beam. We see, for example, that for a left-hand polarized electron beam, top quarks produced at forward angles are predominantly left handed, while forward-produced top quarks are predominantly right handed when the electron beam is right-hand polarized. These helicity amplitudes combine to produce the following general form for the angular distribution [19]:

$$
\frac{d \sigma}{d \cos \theta}=\frac{\beta_{t}}{32 \pi s}\left[c_{0} \sin ^{2} \theta+c_{+}(1+\cos \theta)^{2}+c_{-}(1-\cos \theta)^{2}\right],
$$

where $c_{0}$ and $c_{ \pm}$are functions of the form factors of Eq. 2.7, including any non-standard couplings. The helicity structure of the event is highly constrained by the measurements of beam polarization and production angle.

For the measurement of the decay form factors, there are two alternative methods that might provide higher statistics. The first is to measure the top quark decay distributions using polarized beams at the $t \bar{t}$ threshold, making use of the fact that the spin of the nonrelativistic top quarks follows the spin of the incident electron and positron [20]. The second is to analyze the polarization of top quarks above threshold using the beam axis boosted to the top frame; this gives a very high polarization for the decay analysis [21]. 
For the top charged-current coupling we can write the $W t b$ vertex factor as

$$
\mathcal{M}^{\mu, W}=\frac{g}{\sqrt{2}} \gamma^{\mu}\left[P_{L} F_{1 L}^{W}+P_{R} F_{1 R}^{W}\right]+\frac{i g}{2 \sqrt{2} m_{t}} \sigma^{\mu \nu} k_{\nu}\left[P_{L} F_{2 L}^{W}+P_{R} F_{2 R}^{W}\right]
$$

where the quantities $P_{L, R}$ are the left-right projectors. In the Standard Model, we have $F_{1 L}^{W}=1$ and all others zero. The form factor $F_{1 R}^{W}$ represents a right-handed, or $V+A$, charged current component. As mentioned earlier, the case where the $W$ is longitudinally polarized is particularly relevant for heavy top, and the $\chi_{t}$ and $\chi_{W}$ distributions are sensitive to this behavior.

We now outline an analysis [22] to measure or set limits on the various form factors mentioned above. We consider a modest integrated luminosity of $10 \mathrm{fb}^{-1}, m_{t}=180 \mathrm{GeV} / \mathrm{c}^{2}$, and $\sqrt{s}=500 \mathrm{GeV}$. Electron beam polarization is assumed to be $\pm 80 \%$. The decays are assumed to be $t \rightarrow b W$. In general, one needs to distinguish $t$ from $\bar{t}$. The most straightforward method for this is to demand that at least one of the $\mathrm{W}$ decays be leptonic, and to use the charge of the lepton as the tag. (One might imagine using other techniques, for example with topological secondary vertex detection one could perhaps distinguish $b$ from $\bar{b}$.) So we assume the following decay chain:

$$
t \bar{t} \rightarrow b \vec{b} W W \rightarrow b \bar{b} q \bar{q}^{\prime} \ell \nu
$$

where $\ell=e, \mu$. The branching fraction for this decay chain is $29 \%$.

Since the top production and decay information is correlated, it is possible to combine all relevant observables to ensure maximum sensitivity to the couplings. In this study, a likelihood function is used to combine the observables. We use the Monte Carlo generator developed by Schmidt [23], which includes $t \bar{t}(g)$ production to $\mathcal{O}\left(\alpha_{s}\right)$. Most significantly, the Monte Carlo correctly includes the helicity information at all stages. The top decay products, including any jets due to hard gluon radiation, must be correctly assigned with good probability. The correct assignments are rather easily arbitrated using the $\mathrm{W}$ and top mass constraints. When the effects of initial-state radiation and beamstrahlung are included, it has been shown [19] that the correct event reconstruction can be performed with an efficiency of about $70 \%$. The overall efficiency of the analysis, including branching fractions, reconstruction efficiency, and acceptance, is about $18 \%$.

After simple, phenomenological detection resolution and acceptance functions are applied, the resulting helicity angles (see Fig. 2.9) are then used to form a likelihood which is the square of the theoretical amplitude for these angles given an assumed set of form factors. Table 2.3 summarizes some of the results of this analysis. The upper and lower limits of the top quark couplings in their departures from the Standard Model values are given at $68 \%$ and $90 \%$ CL. All couplings, with real and imaginary parts, can be determined in this way. The right-handed charged-current coupling is shown both for unpolarized and $80 \%$ left-polarized electron beam, whereas the other results assume $80 \%$ left-polarized beam only. We see that even with a modest integrated luminosity of $10 \mathrm{fb}^{-1}$ at $\sqrt{s}=500 \mathrm{GeV}$, the sensitivity to the form factors is quite good, at the level of $5-10 \%$ relative to Standard Model couplings. In 
terms of absolute units, the $90 \%$ CL limit of $F_{2 A}^{Z}$ at 0.15 , for example, corresponds to a $t-Z$ electric dipole moment of $8 \times 10^{-18} \mathrm{e}-\mathrm{cm}$.

Table 2.3: Results from the global top quark form factor analysis described in the text, for a data sample of $10 \mathrm{fb}^{-1}$ and $\sqrt{s}=500 \mathrm{GeV}$.

\begin{tabular}{|c|c|r|r|}
\hline \hline Form Factor & $\begin{array}{c}\text { SM Value } \\
\text { (Lowest Order) }\end{array}$ & $\begin{array}{r}\text { Limit } \\
68 \% \mathrm{CL}\end{array}$ & $\begin{array}{r}\text { Limit } \\
90 \% \mathrm{CL}\end{array}$ \\
\hline$F_{1 R}^{W}(P=0)$ & 0 & \pm 0.13 & \pm 0.18 \\
$F_{1 R}^{W}(P=80 \%)$ & 0 & \pm 0.06 & \pm 0.10 \\
$F_{1 A}^{Z}$ & 1 & $1 \pm 0.08$ & $1 \pm 0.13$ \\
$F_{1 V}^{Z}$ & 1 & $1 \pm 0.10$ & $1 \pm 0.16$ \\
$F_{2 A}^{\gamma}$ & 0 & \pm 0.05 & \pm 0.08 \\
$F_{2 V}^{\gamma}$ & 0 & \pm 0.07 & ${ }_{-0.11}^{+0.13}$ \\
$F_{2 A}^{Z}$ & 0 & \pm 0.09 & \pm 0.15 \\
$F_{2 V}^{Z}$ & 0 & \pm 0.07 & \pm 0.10 \\
$\Im\left(F_{2 A}^{Z}\right)$ & 0 & \pm 0.06 & \pm 0.09 \\
\hline \hline
\end{tabular}

\subsubsection{The Higgs-Top Yukawa Coupling}

The coupling strength of the Higgs boson to a fermion is proportional to the fermion's mass. The Higgs-top coupling is consequently large and may be unique among the Higgsfermion couplings in that it is accessible to direct measurement. Such measurements have been contemplated at LHC [24], but they require efficient vertex tagging in high-luminosity running. The environment at NLC is much cleaner, but the luminosity requirements are comparable. With the availability of large data sets $\left(>50 \mathrm{fb}^{-1}\right)$, several approaches are tractable at NLC: (1) for light to moderate mass Higgs bosons, the $t \bar{t}$ production crosssection near threshold is sensitive to the Higgs contribution to the $t \bar{t}$ potential; (2) for relatively light Higgs, the yield of $t \bar{t} H$ events measures the Higgs-top coupling; and (3) for Higgs masses exceeding the $t \bar{t}$ threshold, the Higgs boson resonance can appear in $t \bar{t} Z$ events and exhibit the Higgs-top coupling.

Threshold measurements have been discussed above for their intrinsic interest and sensitivity to basic top parameters. Here we note that the presence of an additional attractive, short range force arising from Higgs exchange increases the modulus of the toponium wavefunction at the origin, and thereby enhances the cross-section. Fig. 2.11 shows the distinctive energy dependence of the Higgs enhancement factor, which peaks at the 1S state [25]. Fujii et al.[12] have simulated a threshold scan of 10 points, spaced at $1 \mathrm{GeV}$ intervals, to de- 


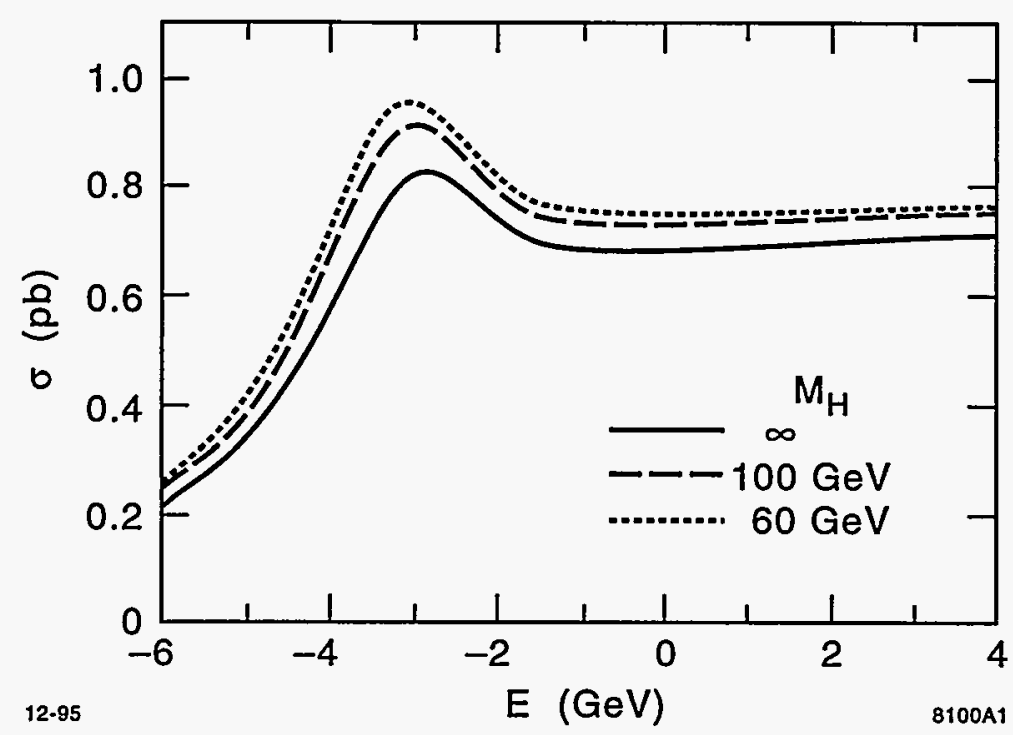

Figure 2.11: Theoretical cross section as a function of Higgs mass for $m_{t}=180 \mathrm{GeV} / \mathrm{c}^{2}$.

termine the sensitivity to the Higgs-top coupling strength. Their results imply that a $10 \%$ measurement is possible with $100 \mathrm{fb}^{-1}$ evenly distributed over the 10 points for $M_{H}=100$ $\mathrm{GeV}$. The enhancement is roughly inversely proportional to the Higgs mass, so the scan would yield a $20 \%(30 \%)$ measurement for a $200(300) \mathrm{GeV}$ Higgs mass. An optimized scan will do better.

The $t \bar{t} H$ events almost always result from "Higgs-strahlung", radiation of the Higgs boson from one of the top quarks, so their yield provides a measure of the square of the Higgs-top coupling. The cross-section for the process is small [26], in the $\mathcal{O}(1) \mathrm{fb}$ range for a $500 \mathrm{GeV}$ NLC and $M_{H}<100 \mathrm{GeV}$. Detection of the events is challenging; they typically contain 8 jets, including $4 b$ jets. The process $e^{+} e^{-} \rightarrow t \bar{t} Z$ occurs at comparable rate, and along with $e^{+} e^{-} \rightarrow t \bar{t} j j$ constitutes the important background. Preliminary studies $[12,27]$ show that a $100 \mathrm{fb}^{-1}$ sample at $500 \mathrm{GeV}$ will give a $\leq 15 \%$ measurement of the Higgs-top coupling for $M_{H} \leq 100 \mathrm{GeV}$. At $\sqrt{s}=1000 \mathrm{GeV}$, the sensitivity extends to over $200 \mathrm{GeV}$ for a measurement of similar accuracy.

The cross-section for $e^{+} e^{-} \rightarrow t \bar{t} Z$ is about $5 \mathrm{fb}$ between 500 and $1000 \mathrm{GeV}$ center-of-mass energies. When the Higgs mass is above the $t \bar{t}$ threshold, this cross section is enhanced by the process $e^{+} e^{-} \rightarrow Z^{0} H^{0}$, with Higgs decay to $t \bar{t}$. Fujii et al. [28] have studied the process for $m_{t}=130 \mathrm{GeV}$, and concluded that, with an integrated luminosity of $60 \mathrm{fb}^{-1}$ at $\sqrt{s}=600$ $\mathrm{GeV}$, one could measure the top-Higgs coupling within $10 \%$ for a $300 \mathrm{GeV}$ Higgs. For Higgs masses above $2 m_{t}$, the cross-section is lower, and the increased width of the Higgs will make isolating a signal in the $t \bar{t}$ invariant mass distribution more difficult. Even so, one could measure the Higgs-top coupling for a $400 \mathrm{GeV}$ Higgs produced at $\sqrt{s}=1000 \mathrm{GeV}$ within about $35 \%$ with a data sample of $100 \mathrm{fb}^{-1}$.

The Higgs-strahlung process is also sensitive to deviations from the Standard Model 
involving extended Higgs sectors. The $t \bar{t} H$ final state can result from Higgs emission from the $t$ (or $\bar{t}$ ), or from the intermediate $Z$. Interference between these sub-processes can give rise to large CP violating effects in extended Higgs models. This was studied in Ref. [29] for a range of two-Higgs doublet models, and it was found that for roughly $100 \mathrm{fb}^{-1}$ of data at $\sqrt{s}=800 \mathrm{GeV}$ it would be possible to observe a significant $\mathrm{CP}$ asymmetry in a number of final-state observables.

The Higgs-top coupling can be determined in the case that the Higgs is very heavy $\left(M_{H}>400 \mathrm{GeV} / \mathrm{c}^{2}\right)$ by measuring the rate of the process $e^{+} e^{-} \rightarrow \nu \bar{\nu} t \bar{t}$ At $\sqrt{s}=1500 \mathrm{GeV}$, the cross section for this process is about $2 \mathrm{fb}$ in the absence of a Higgs, but will be enhanced by more than a factor of two for Higgs masses in the range $400-1000 \mathrm{GeV} / \mathrm{c}^{2}$. Preliminary studies by Fujii [28] show that care is required to eliminate radiative $t \bar{t}, e^{+} e^{-} t \bar{t}$, and $t \bar{t} Z$ backgrounds, but suggest that the Higgs-top coupling can be measurable up to $m_{H}=1$ $\mathrm{TeV} / \mathrm{c}^{2}$. The case of a very heavy Higgs boson is discussed in more detail in Section 7.3.

\subsubsection{Top Physics Reach of NLC and Hadron Colliders}

Table 2.4 summarizes the top physics reach of the NLC and several hadron colliders. The Tevatron Upgrade $\left(\mathrm{TeV}^{*}\right)$ will establish the baseline for top quark physics in the LHC/NLC era, and will address many subjects of interest in top physics. Its reach has been studied in a report by Amidei et al. [30]. The Atlas TDR [24] provides some information on the top physics reach at LHC; this subject will certainly be developed further in the future. The table at best represents what has been studied to date. If a particular measurement at a particular machine has not yet been analyzed, the corresponding entry has been left blank. An " $\mathrm{X}$ " marks measurements that cannot be made at a particular machine, by virtue of excessive backgrounds, insufficient signal, or unavailable production mechanisms.

The table demonstrates how crucial a role the NLC plays in obtaining a complete picture of top quark physics. NLC will provide the definitive top mass measurement. It will provide the only direct measure of the top width; at hadron colliders, the total width can be inferred only from a $V_{t b}$ measurement using the assumption that the top has no unobserved exotic decays. The NLC will measure the axial-vector and vector electroweak couplings, some of the charged-current couplings (expressed here as CKM elements), the top-Higgs coupling, and the flavor specific strong coupling. Hadron colliders will also measure the charged current couplings (although $V_{t d}$ is probably impossible at both hadron and $e^{+} e^{-}$colliders), and the strong and electromagnetic couplings, but not the couplings to the $Z$. The LHC may probe the top-Higgs coupling by isolating $t \bar{t} H$ events, but only with difficulty. The NLC can measure the top decay form factors, checking for longitudinal $W$ production and searching for right-handed $W$ 's, as can the hadron colliders. Only the NLC can measure the electroweak magnetic and electric dipole moments, because they depend on the neutral current production mechanism. We should note that LHC can be sensitive to top-associated $\mathrm{CP}$ violation through more complicated effective interactions [31,32]. Rare decays with distinctive signatures can be sought in either environment, with the advantage to hadron 
Table 2.4: Top Physics at Future Facilities

\begin{tabular}{|c|c|c|c|c|c|}
\hline Quantity & $\begin{array}{c}\mathrm{TeV}^{*} \\
\left(1 \mathrm{fb}^{-1}\right)\end{array}$ & $\begin{array}{c}\text { TeV33 } \\
\left(10 \mathrm{fb}^{-1}\right)\end{array}$ & $\begin{array}{c}\text { LHC } \\
\left(100 \mathrm{fb}^{-1}\right)\end{array}$ & $\begin{array}{c}\operatorname{NLC}(\sqrt{s}=360) \\
\left(50 \mathrm{fb}^{-1}\right)\end{array}$ & $\begin{array}{c}\operatorname{NLC}(\sqrt{s}=500) \\
\left(50 \mathrm{fb}^{-1}\right)\end{array}$ \\
\hline$\Delta m_{t}$ & $3.5 \mathrm{GeV} / \mathrm{c}^{2}$ & $2.0 \mathrm{GeV} / \mathrm{c}^{2}$ & $2 \mathrm{GeV} / \mathrm{c}^{2}$ & $0.20 \mathrm{GeV} / \mathrm{c}^{2}$ & \\
\hline$\Delta \Gamma_{t}$ & & & & $6-8 \%$ & \\
\hline$\Delta a_{t}$ & $\mathrm{X}$ & $\mathrm{X}$ & $\mathrm{X}$ & & $4 \%$ \\
\hline$\Delta v_{t}$ & $\mathrm{X}$ & $\mathrm{X}$ & $\mathrm{X}$ & & $5 \%$ \\
\hline$\Delta V_{t b}$ & $14 \%$ & $6 \%$ & $\mathrm{X}$ & & \\
\hline$\Delta V_{t s}$ & $\mathrm{X}$ & $\mathrm{X}$ & $\mathrm{X} ?$ & $?$ & $?$ \\
\hline$\Delta V_{t d}$ & $\mathrm{X}$ & $\mathrm{X}$ & $\mathrm{X}$ & $\mathrm{X}$ & $\mathrm{X}$ \\
\hline$\Delta \lambda_{t}$ & $\mathrm{X}$ & $\mathrm{X}$ & $?$ & $14 \%$ & $20 \%$ \\
\hline$\Delta \alpha_{s}^{t}$ & $\left(\sigma_{t \bar{t}}=11 \%\right)$ & $\left(\sigma_{t \bar{t}}=4 \%\right)$ & & 0.005 & \\
\hline$\Delta B\left(t \rightarrow b W^{0}\right)$ & $4 \%$ & $1.3 \%$ & & $1 \%$ & \\
\hline$\Delta B\left(t \rightarrow b W_{R}\right)$ & $2 \%$ & $0.6 \%$ & & & $2 \%$ \\
\hline$\Delta \delta$ & $\mathrm{X}$ & $\mathrm{X}$ & $\mathrm{X}$ & & $<0.3 e \hbar / 2 m_{t}$ \\
\hline$\Delta d$ & $\mathrm{X}$ & $\mathrm{X}$ & $\mathrm{X}$ & & $<4 \times 10^{-18} \mathrm{e}-\mathrm{cm}$ \\
\hline$B\left(t \rightarrow H^{+} b\right)$ & $<15 \%$ & $<6 \%$ & $<1.4 \%$ & & $<2 \%$ \\
\hline$B\left(t \rightarrow \tilde{t} \tilde{\chi}^{0}\right)$ & & & & & $<1 \%$ \\
\hline$B(t \rightarrow c \gamma)$ & $<0.3 \%$ & $<0.04 \%$ & & & \\
\hline$B(t \rightarrow c Z)$ & $<1.5 \%$ & $<0.4 \%$ & $<5 \times 10^{-5}$ & & $<$ few $\cdot 10^{-4}$ \\
\hline$B\left(t \rightarrow c h^{0}\right)$ & & & & $<1 \%$ & \\
\hline
\end{tabular}

decays by virtue of the large statistical samples anticipated. The more exotic decays, e.g. $t \rightarrow \tilde{t} \tilde{\chi}^{0}$, are more sensitively sought in the clean environment of the NLC. 


\subsection{Higgs Boson Searches and Properties}

\subsubsection{Introduction}

Despite the extraordinary success of the Standard Model (SM) in describing particle physics up to the highest energy available today, the mechanism responsible for electroweak symmetry breaking (EWSB) has yet to be determined. In particular, the Higgs boson $[33,34,35]$ predicted in the minimal Standard Model and the theoretically attractive Supersymmetric (SUSY) Grand Unified Theory (GUT) extensions thereof have yet to be observed. If EWSB does indeed derive from nonzero vacuum expectation values for elementary scalar Higgs fields, then one of the primary goals of constructing future colliders must be to completely delineate the Higgs boson sector. In particular, it will be crucial to discover all of the physical Higgs bosons and determine their masses, widths and couplings. Conversely, if a fundamental Higgs boson does not exist, it is essential to demonstrate this unambiguously.

The EWSB mechanism in the Standard Model is phenomenologically characterized by a single Higgs boson $\left(h_{S M}\right)$ in the physical particle spectrum. The mass of the $h_{S M}$ is undetermined by the theory, but its couplings to fermions and vector bosons are completely determined. In SUSY theories, there are two Higgs doublets with vacuum expectation values $v_{1}, v_{2}$. These contribute mass terms for the gauge bosons proportional to $\left(v_{1}^{2}+v_{2}^{2}\right)$, masses for down-type fermions proportional to $v_{1}$, and masses for up-type fermions proportional to $v_{2}$. In the Minimal Supersymmetric Standard Model (MSSM) [36, 37] these two doublets give rise to five physical Higgs bosons: $h^{0}$, the lighter of the two $C P$-even states; $H^{0}$, the heavier $C P$-even state; the $C P$-odd $A^{0}$ boson, and a pair of charged bosons $H^{ \pm}$. The mass of the minimal SM Higgs boson is unspecified, but in the MSSM, there are tree-level relations which determine the spectrum of masses in terms of one of the boson masses (e.g., the mass of the $A^{0}$ ) and the ratio of the vacuum expectation values, $v_{2} / v_{1}$. The CP-even and CP-odd neutral Higgs bosons have nontrivial mixing angles $\alpha$ and $\beta$, respectively, which affect their couplings and decays. In particular, $v_{2} / v_{1}=\tan \beta$. Both masses and couplings receive further radiative corrections which are functions of the SUSY Higgs mass parameter, $\mu$, the scale of mass at which SUSY is broken, $M_{S U S Y}$, the mass of the top quark, and the $A_{i}$ parameters of the soft supersymmetry-breaking interaction. More general models of the Higgs sector, which also include electroweak singlets, are also possible in SUSY theories. Finally in non-supersymmetric models with two Higgs doublets (2HDM), the Higgs bosons may have mixed $C P$ character.

Supersymmetry has exciting implications for the discovery potential of the Higgs bosons that it predicts. In the MSSM, considering renormalization group improved radiative corrections and assuming $m_{t}=180 \mathrm{GeV}$ with the stop mass less than $1 \mathrm{TeV}$, the lightest Higgs boson must have mass $M_{h^{0}} \lesssim 130 \mathrm{GeV}$. An even more sweeping statement can be made [38] that $M_{h^{0}} \lesssim 150 \mathrm{GeV}$ for any SUSY theory with a grand unification at high energy which includes the elementary Higgs fields. 


\subsubsection{Present and Future Limits}

The best direct limits on the SM Higgs boson come from searches at LEP, with the present limit [39] being $M_{h_{S M}}>65.2 \mathrm{GeV}$ at $95 \%$ confidence level (C.L.). These limits can also be interpreted in the framework of the MSSM to exclude the lightest SUSY Higgs with mass less than approximately $45 \mathrm{GeV}$. Electroweak radiative corrections including the top quark and the Higgs boson affect precision electroweak measurements, and global fits [40] using data from LEP, SLC, the Tevatron, and neutrino scattering give the relatively weak limit implying that $M_{h_{S M}}<300 \mathrm{GeV}$ (95\% C.L.).

\section{LEP2}

The limit on the Higgs boson mass will be improved in the near future with the operation of LEP2. With an integrated luminosity of $150 \mathrm{pb}^{-1}$ in each of the four LEP detectors, expected from one year of running at design luminosity, the $5 \sigma$ discovery reach can be increased [41] to about $95 \mathrm{GeV}$ with running at center-of-mass energies of $192 \mathrm{GeV}$ scheduled for 1997. At the same energy and luminosity, the process $e^{+} e^{-} \rightarrow h A$ can be discovered (excluded) at a cross section of $65(30) \mathrm{fb}$, when supersymmetric decay channels are closed. The resultant exclusion region in the MSSM parameter space can be found in Fig. 2.13. The possibility of running at $205 \mathrm{GeV}$, which would result in an extension of limits close to the MSSM bound, is currently being investigated.

\section{Upgraded Tevatron}

The associated production of a Higgs boson and a $W$ or $Z$ boson, with the Higgs decaying to $b \vec{b}$ and the $W$ or $Z$ decaying leptonically, is a possible way to detect the Higgs in the mass range $60-130 \mathrm{GeV}$, at a high luminosity Tevatron collider [30]. The Higgs decay gives rise to 2 jets, thus one will use $b$ tagging to reduce the large $W+2$ jet background. It appears that the present $b$ tagging capability at CDF is more than adequate to reduce this background (at moderate Run II luminosities, e.g., $10^{32} \times \mathrm{cm}^{-2} \mathrm{sec}^{-1}, 1 \mathrm{TeV} \times 1 \mathrm{TeV}$ ) if this capability is extended to larger rapidities (as is planned in Run II for both CDF and D0). After $b$ tagging, the largest background at Higgs masses below $100 \mathrm{GeV}$ is QCD production of $W+b \bar{b}$ and top backgrounds for masses above $100 \mathrm{GeV}$. Figure 2.12 shows the dijet mass distribution for the sum of all these backgrounds, plus the $W+H$ signal for $10 \mathrm{fb}^{-1}$. An observation of the Higgs for masses below $100 \mathrm{GeV}$ is possible after the Main Injector upgrade, and is within reach of the present Run II accelerator after several years of data-taking. For higher mass Higgs bosons, these statistics are too low; one would need about $25 \mathrm{fb}^{-1}$ to observe the 120 $\mathrm{GeV}$ Higgs. This study assumed an approximate $20 \%$ improvement in dijet mass resolution obtained from applying a clustering algorithm that reduces the effect of gluon radiation at large angles to the jet. This dijet mass resolution and jet clustering is crucial in seeing the Higgs. It has been argued that the $h \rightarrow \tau^{+} \tau^{-}$and $Z \rightarrow \nu \bar{\nu}$ channels can be used to improve these results [43]. 

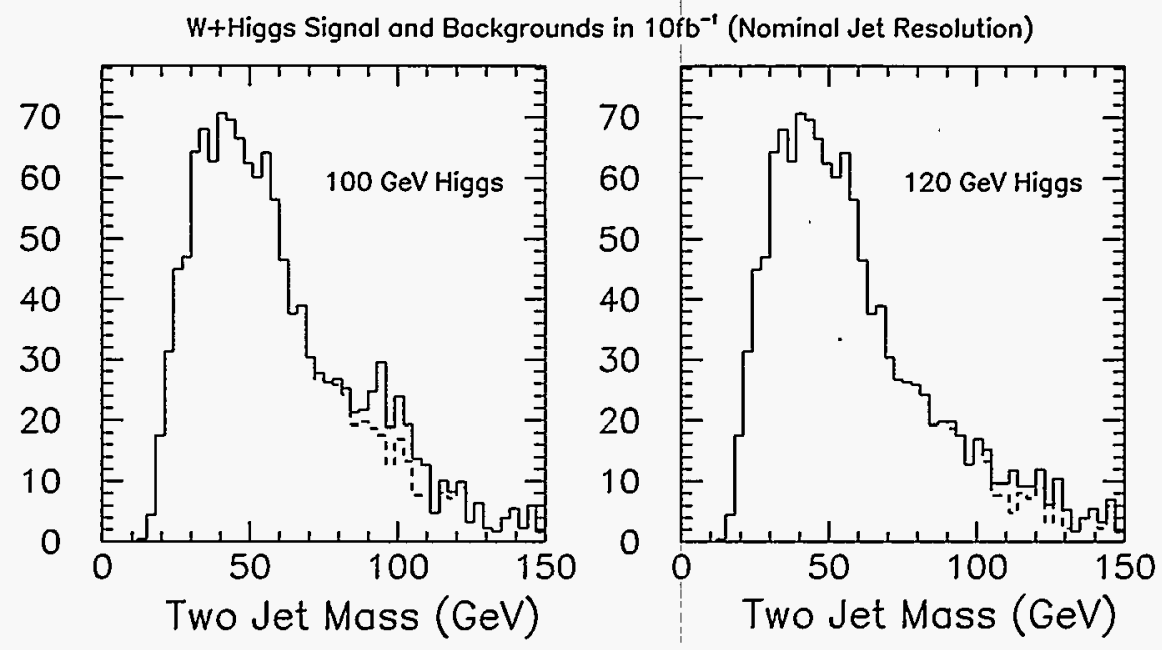

Figure 2.12: The signal plus background mass distributions for the $W H$ process with $10 \mathrm{fb}^{-1}$ of data at $2 \mathrm{TeV}$. The solid line is signal+background, the dashed line the sum of all backgrounds.

\section{Large Hadron Collider}

At the Large Hadron Collider (LHC), detection of the SM $h_{S M}$ is possible through the process $g g \rightarrow h_{S M} \rightarrow \gamma \gamma$ for $M_{h_{S M}}<150 \mathrm{GeV}$ and through $g g \rightarrow h_{S M} \rightarrow Z Z^{(*)} \rightarrow 4 \ell$ for $M_{h_{S M}}>130 \mathrm{GeV}$. A heavy $h_{S M}$ is also detectable in the reaction $W W \rightarrow h_{S M}$, with the Higgs decaying to $Z Z$ and also, possibly, to $W W$. The $\gamma \gamma$ channel that is crucial for a light $h_{S M}$ demands an excellent electromagnetic calorimeter, and much attention has been devoted to this in the LHC detector designs. For $M_{h_{S M}}<120 \mathrm{GeV}$, it will also be possible to detect $t \bar{t} h_{S M}$ and (possibly) $W h_{S M}$ with $h_{S M} \rightarrow b \bar{b}$, provided that the high $b$-tagging efficiency and purity projections are realized. Detection of the $h_{S M}$ in the intermediate mass region when $M_{h_{S M}}<2 M_{W}$ generally requires accumulating data for at least a year when the LHC is run at full luminosity. This should be contrasted with $e^{+} e^{-}$collisions, where the $e^{+} e^{-} \rightarrow Z h_{S M}$ mode will allow detection in the same mass region in a matter of a few hours, assuming full instantaneous luminosity.

In the case of the MSSM for large $M_{A^{0}}$, the $h^{0}$ is similar to the Standard Model Higgs $h_{S M}$. As for the $h_{S M}$, the $h^{0}$ is straightforward to detect at an $e^{+} e^{-}$collider. On the other hand, the $H^{0}$ and $A^{0}$ do not resemble the Standard Model Higgs boson, and so one must separately consider their production process. We will show below that the observability of $H^{0}$ and $A^{0}$ at an $e^{+} e^{-}$collider depends only on the beam energy: for $\sqrt{s}>2 M_{A^{0}}-20 \mathrm{GeV}$, these particles are found in the reaction $e^{+} e^{-} \rightarrow Z^{*} \rightarrow H^{0} A^{0}$.

The story at the LHC is much more complex. The reactions which can be used to detect the Higgs particles of the MSSM, and their limits of applicability in parameter space, are displayed in Fig. 2.13 [35]. This figure represents the limit of the LHC capability, summing the results of two detectors in a multi-year run at design luminosity. For values of $M_{A}>200$ $\mathrm{GeV}$, the LHC can detect the $H^{0}$ and $A^{0}$ only in certain specific decay channels, shown in 
the figure, whose availability depends on the value of $\tan \beta$. Since this figure summarizes a great deal of analysis, we must point out at least a few of the assumptions which are used. The channel $A^{0}, H^{0} \rightarrow \tau^{+} \tau^{-}$can be used only when the branching ratio to $\tau$ is enhanced by a large value of $\tan \beta$. For small $\tan \beta$, modes with $b \bar{b}$ or $t \bar{t}$ in the final states require $b$ tagging capabilities that will be challenging in the detection environment of the LHC. In addition, it should be noted that the process $A^{0}, H^{0} \rightarrow t \bar{t}$ has so far been studied only at the level of the comparison of cross sections for signal and background, and that, since the signal is $2-10 \%$ of the background, an excellent knowledge of the $g g \rightarrow t \bar{t}$ cross section is required. Finally, though the $h^{0}$ should be detected for the generic situation illustrated in Fig. 2.13, there are regions of the full parameter space of the MSSM where the $h^{0}$ would not be observed [42]. Thus, it is unlikely that the whole MSSM Higgs spectrum would be observed at the LHC, and it is not possible to rule out the MSSM if none of its Higgs bosons are seen at the LHC.

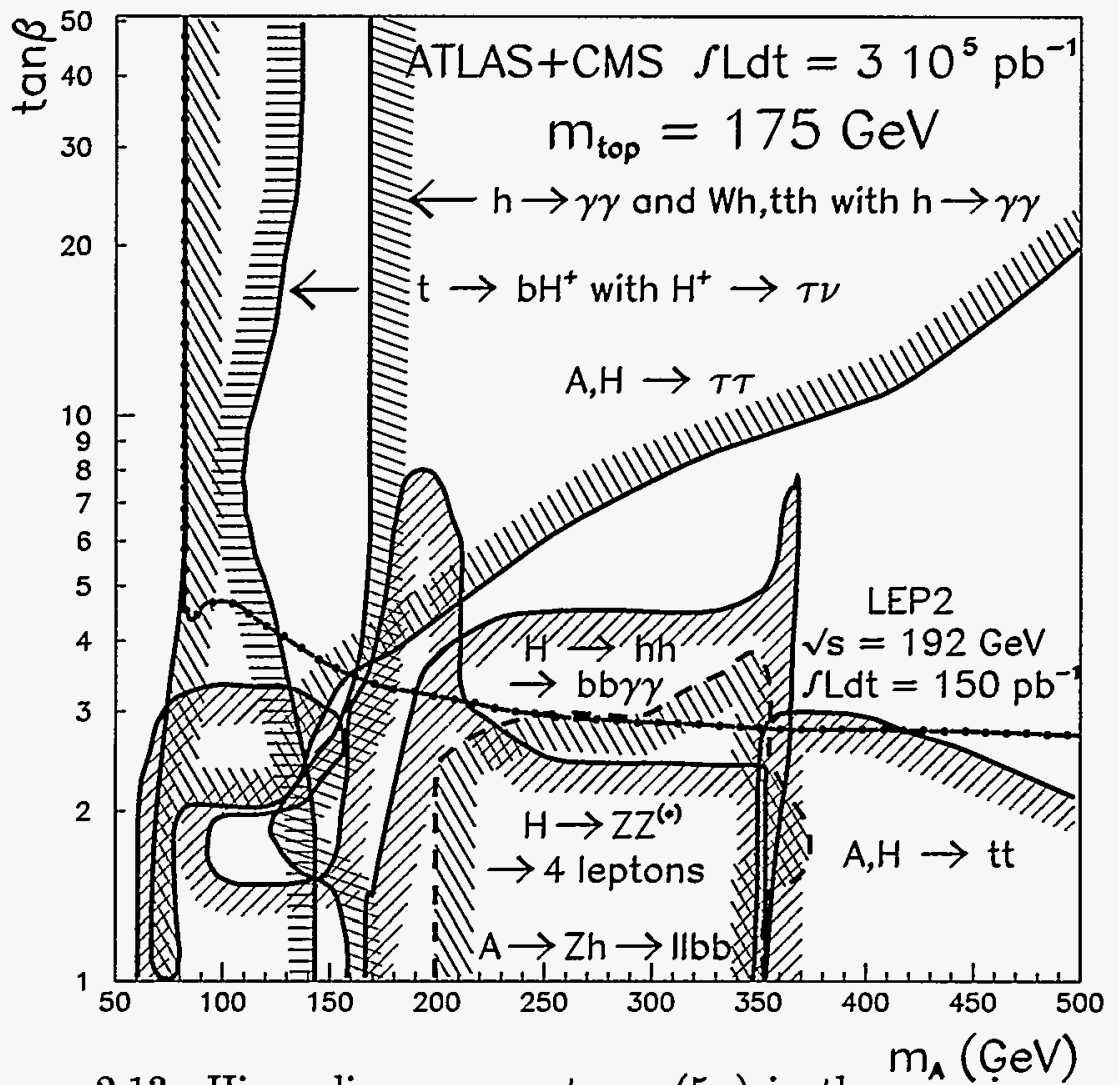

Figure 2.13: Higgs discovery contours $(5 \sigma)$ in the generic parameter space of the MSSM for ATLAS+CMS at the LHC, for a multi-year run at design luminosity, $300 \mathrm{fb}^{-1}$ per detector, from [35]. Renormalization group improved radiative corrections are included for $M_{h^{0}}$ and $M_{H^{0}}$, assuming $m_{\bar{t}}=1 \mathrm{TeV}$ and no squark mixing. 


\subsubsection{Standard Model Higgs}

The main production processes for the SM Higgs in $e^{+} e^{-}$annihilation are $e^{+} e^{-} \rightarrow Z H$ and the gauge boson fusion processes $e^{+} e^{-} \rightarrow \nu \bar{\nu} H$ (WW fusion) and $e^{+} e^{-} \rightarrow e^{+} e^{-} H$ ( $Z Z$ fusion). The cross sections for these processes are shown in Fig. 2.14. With a typical integrated luminosity of $10 \mathrm{fb}^{-1}$ at $\sqrt{s}=500 \mathrm{GeV}$ with $M_{H}=150 \mathrm{GeV}$, about 1000 signal events would be expected before cuts and branching ratios. Handy "rules of thumb" are that the peak for $Z H$ production occurs at $\sqrt{s} \approx M_{Z}+\sqrt{2} M_{H}$ and that the crossover for equal cross sections from the fusion and bremsstrahlung mechanisms occurs at $\sqrt{s} \approx 0.6 M_{H}+400 \mathrm{GeV}$.
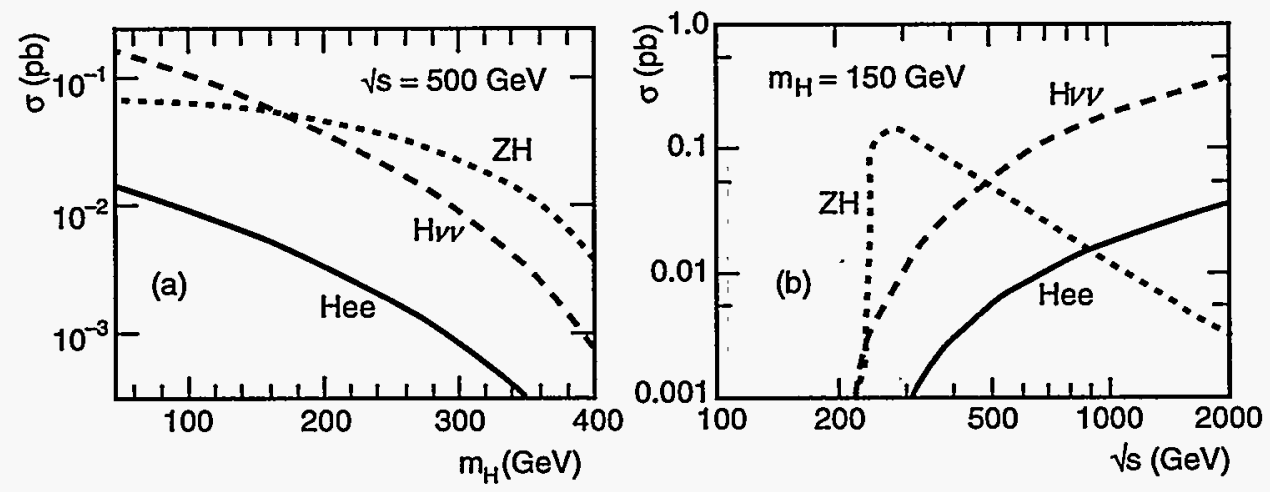

Figure 2.14: Cross section for Standard Model Higgs boson production.
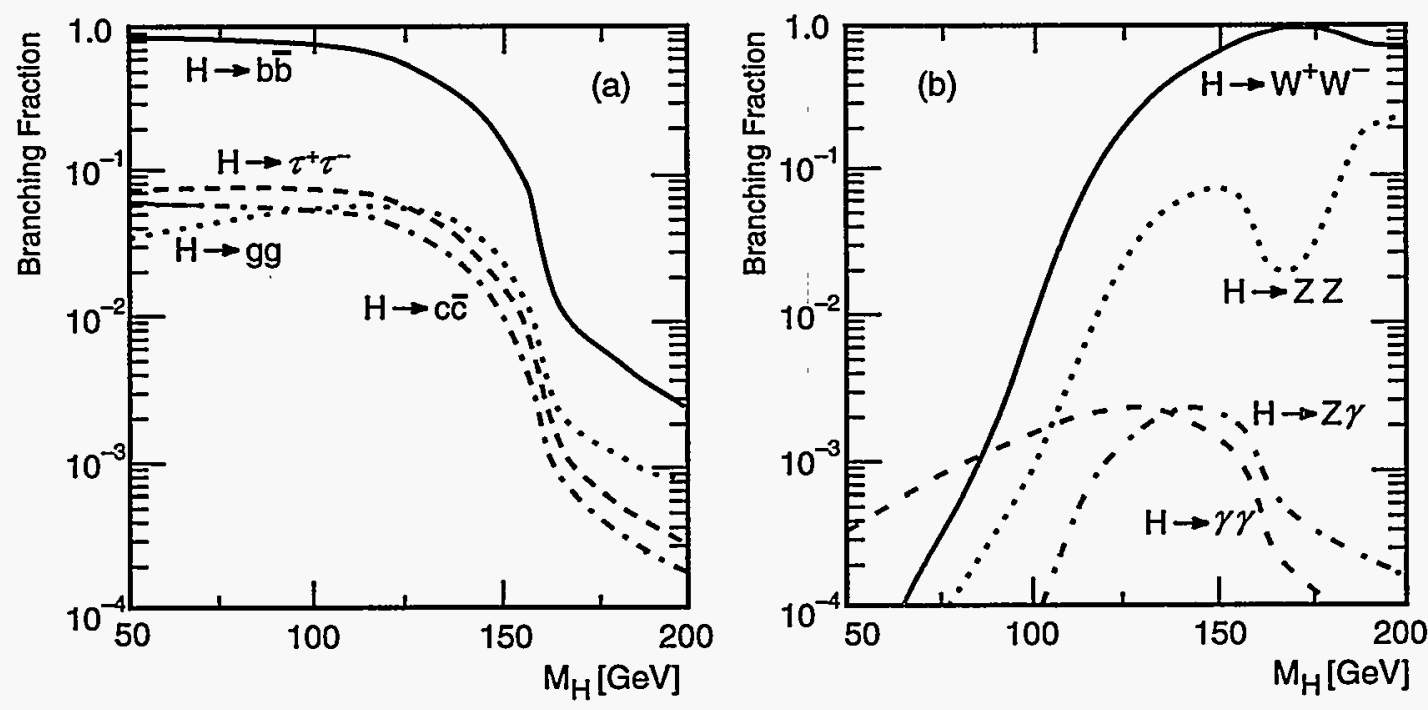

Figure 2.15: Branching fractions of a SM Higgs boson.

The decay modes of the Standard Model Higgs depend strongly upon its mass. The branching ratios of the Standard Model Higgs are shown in Fig. 2.15. A very interesting 
region is the intermediate mass Higgs with $M_{Z}<M_{H}<2 M_{W}$, which at a hadron collider is relatively more difficult to detect than a heavy Higgs boson. Almost all of the decays, both those to fermions and those into pairs of gauge bosons are identifiable in $e^{+} e^{-}$experiments, and it should be possible to measure individual branching ratios. For an intermediate mass Higgs, the dominant decay channel is clearly $H^{0} \rightarrow b \bar{b}$, with the branching ratio for $H^{0} \rightarrow$ $W^{+} W^{-(*)}$ growing with increasing mass (even for $E_{c m}<2 M_{W}$ where one of the $W$ 's must be off shell). This latter channel remains dominant for heavy Higgs bosons, and is joined by $Z Z$ and $t \bar{t}$ modes when kinematically accessible.

\section{Signal Topologies and Backgrounds}

Typical signal topologies in the intermediate mass range are shown in Fig. 2.16. The associated production $e^{+} e^{-} \rightarrow Z^{0} H^{0}$, is followed by standard decays of the $Z^{0}\left(10 \% \ell^{+} \ell^{-}, 20 \%\right.$ $\nu \bar{\nu}$, and $70 \% q \bar{q})$ and decays of the $H^{0}$ mostly into $b \bar{b}$, occasionally into $\tau^{+} \tau^{-}$, and more rarely into $c \bar{c}$ and $g g$. After straightforward cuts, the most serious backgrounds are due to irreducible Standard Model processes, $e^{+} e^{-} \rightarrow Z Z, Z \nu \bar{\nu}$, and $W e \nu$.

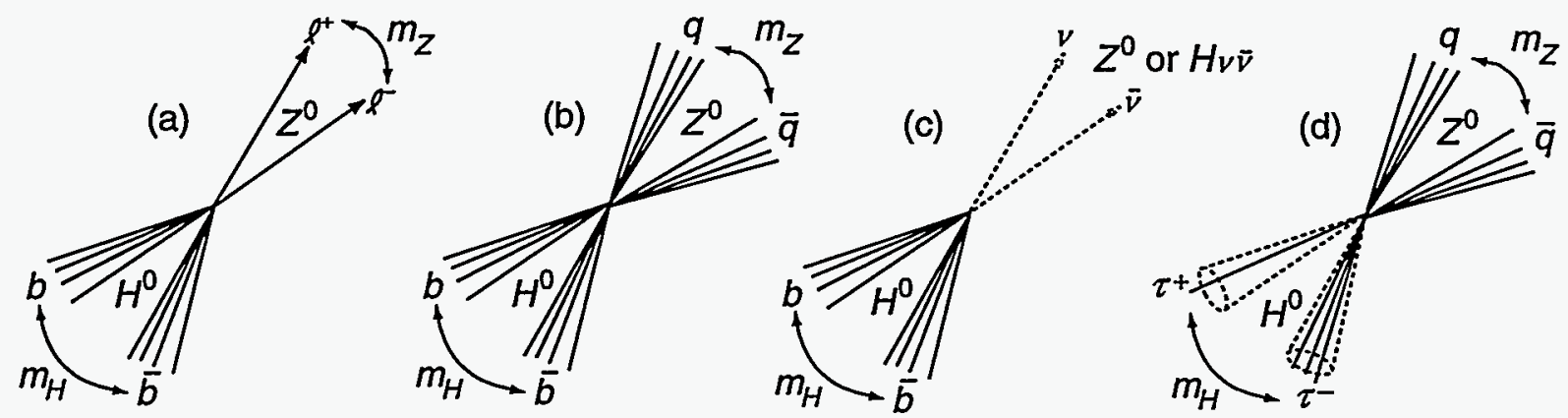

Figure 2.16: Important signal topologies for an intermediate mass SM Higgs boson.

\section{Experimental Studies}

The detection of Higgs bosons in $e^{+} e^{-}$collisions at high energy has been studied extensively in simulations $[48,45,46]$. The detector simulations that have been employed usually emulate LEP/SLC-type detectors using smeared four-vectors, while some consider simulations of a more ambitious JLC-type detector [47]. Most studies include the effects of beamstrahlung, the radiation of photons in the intense electromagnetic fields of the beam-beam collision. Like more standard initial-state radiation, this effect is usually taken into account in kinematic fits by allowing for an unknown missing momentum along the beam axis.

In the topology of Fig. 2.16a, one can first identify two leptons with invariant mass close to the mass of the $Z^{0}$, and then investigate the remaining hadronic mass or use kinematic 
constraints to study the missing or recoil mass in the event:

$$
M_{m i s s}=\sqrt{\left(\sqrt{s}-E_{\ell^{+}}-E_{\ell^{-}}\right)^{2}-\left(\vec{p}_{\ell^{+}}+\vec{p}_{\ell^{-}}\right)^{2}} .
$$

This quantity has a large peak at the $Z^{0}$ mass from the irreducible background process $e^{+} e^{-} \rightarrow Z^{0} Z^{0} ; Z^{0} \rightarrow \ell^{+} \ell^{-} ; Z^{0} \rightarrow q \bar{q}$. If $M_{H} \approx M_{Z}$, the signal and the $Z Z$ background are kinematically equivalent, and one would need $b$-quark tagging to distinguish the signal. Since $\operatorname{Br}\left(Z^{0} \rightarrow b \bar{b}\right) \simeq 20 \%$, while $B r\left(H^{0} \rightarrow b \bar{b}\right) \simeq 85 \%$ at this mass, an analysis in this worst case would require $50 \mathrm{fb}^{-1}$ of data.

The four-jet topology of Fig. 2.16b has been considered in a number of studies, in particular, in a comprehensive study by Janot [48] at $\sqrt{s}=500 \mathrm{GeV}$ which assumed an integrated luminosity of $10 \mathrm{fb}^{-1}$. After selection cuts, for $M_{H}=110 \mathrm{GeV}$, a small signal is observed above the background, which comes mainly from $e^{+} e^{-} \rightarrow W^{+} W^{-}, Z^{0} Z^{0}$, and $q q(\gamma)$. This signal is greatly enhanced, as shown in Fig. $2.17 \mathrm{~b}$, by requiring that at least one of the jets forming the Higgs signal peak come from a tagged $b$ quark. The vertex-tagger is assumed to have the conservative performance $\epsilon_{b \bar{b}}=50 \%, \epsilon_{c \bar{c}}=2.5 \%, \epsilon_{c \bar{s}}=0.3 \%$, and $\epsilon_{q \bar{q}}=0.1 \%$, where the numbers give the efficiency for tagging a particular quark combination. The importance of $b$ tagging is even greater as one moves up in mass.
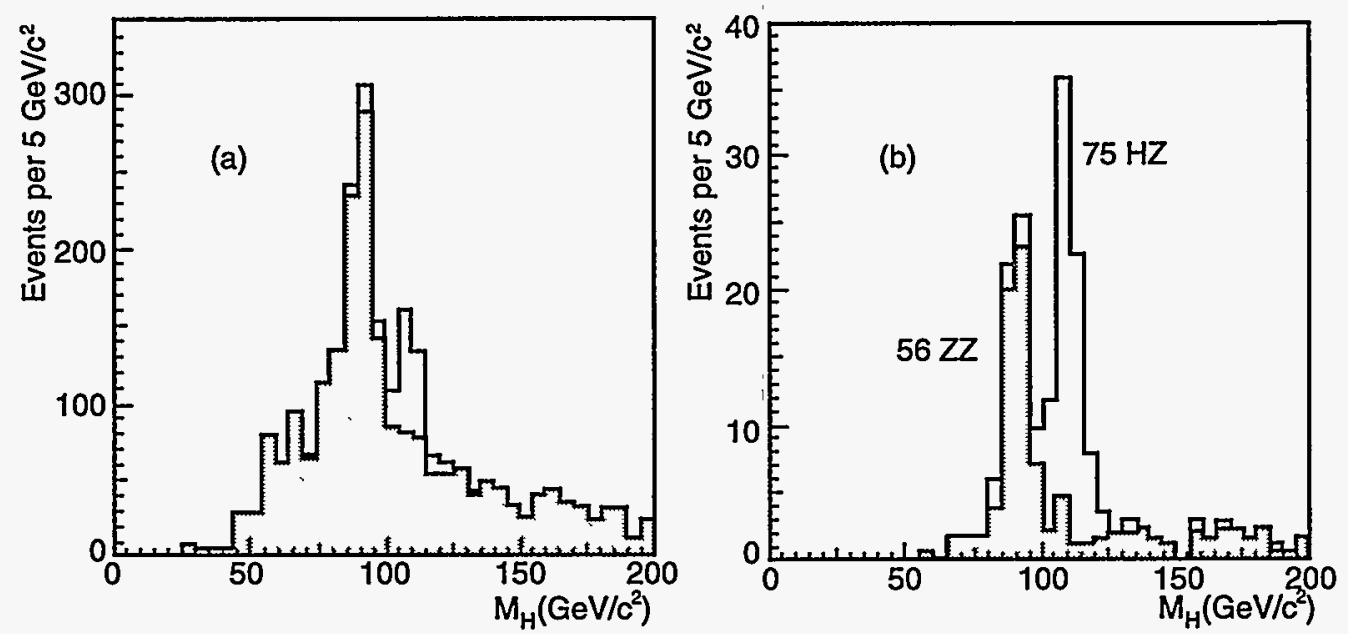

Figure 2.17: Distribution of the invariant mass of the Higgs jet pair in the four-jet topology (a) before and (b) after b-quark tagging, for all known backgrounds (shaded histograms) and for the signal $\left(M_{H}=\right.$ $110 \mathrm{GeV}$ ) (adapted from Ref. 10).

The missing energy topology shown in Fig. 2.16c can arise either from the $Z H$ process, with $\left(Z^{0} \rightarrow \nu \bar{\nu}\right)\left(H^{0} \rightarrow b \bar{b}\right)$, or from $W W$ fusion. The resultant events will have large missing energy, transverse momentum, and mass, plus the presence of acoplanar jets. This distinctive signature offsets the loss of the $Z$-mass constraint. The last topology of Fig. 2.16d can be isolated by tagging two $\tau$ leptons either from their one- or three-prong decays recoiling 
against a reconstructed $Z^{0}$ decaying into $q \bar{q}$. Using a kinematically-constrained fit the missing neutrinos from the $\tau$ decay can be taken into account.

The examples given so far rely primarily on the large branching ratio for $H^{0} \rightarrow b \bar{b}$. As the $H^{0}$ gets heavier, other decay modes begin to become important. For example, for $M_{H}=140 \mathrm{GeV}, B r\left(H^{0} \rightarrow W^{*} W\right) \simeq 45 \%$. The mode $\left(Z^{0} \rightarrow q \bar{q}\right)\left(H^{0} \rightarrow W^{*} W\right)$ has been investigated [49] by demanding a six-jet event with a reconstructed $Z^{0}$ hadronic decay, one jet pair reconstructing to $M_{W}$, and the last pair peaking at $m<M_{W}$, depending on $M_{H}$. Similarly, for $M_{H}=160 \mathrm{GeV}$, the decay $H^{0} \rightarrow W^{+} W^{-}$dominates, and for production via fusion, $e^{+} e^{-} \rightarrow H \nu \bar{\nu}$, the result is an acoplanar pair of reconstructed $W$ bosons and a total visible mass peaking at the $H$ mass which is expected to be well above background without the need for $b$-tagging.

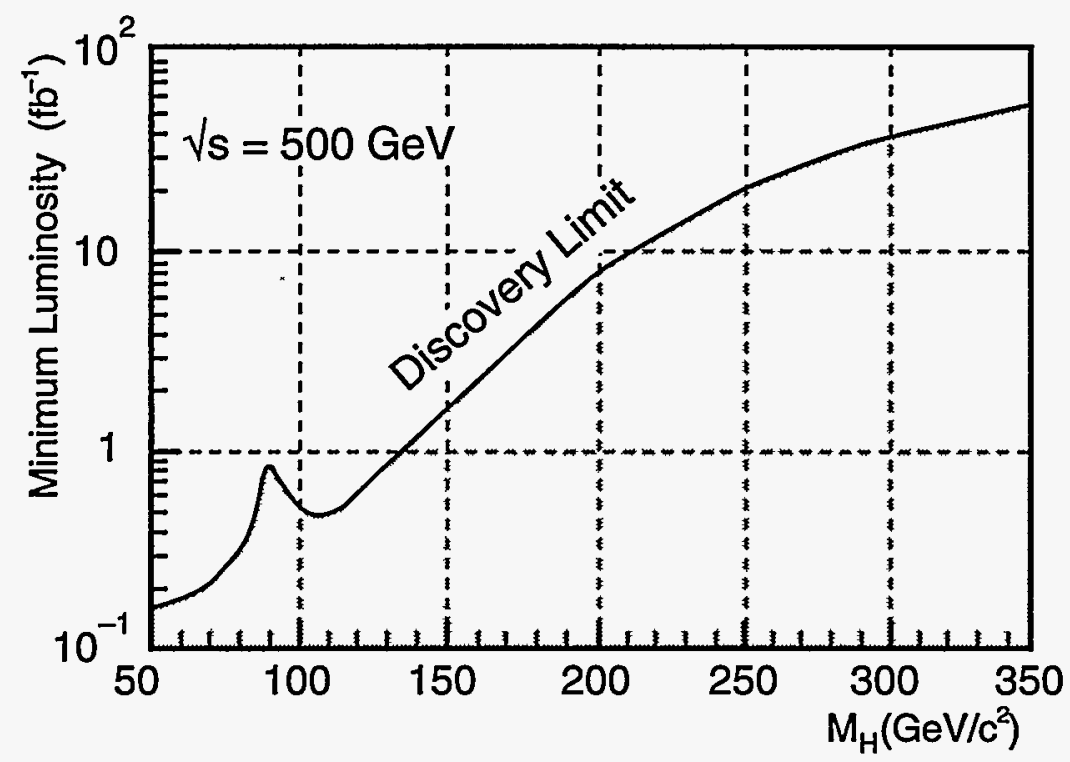

Figure 2.18: Minimum luminosity needed to discover a SM Higgs boson at a center-of-mass energy of $500 \mathrm{GeV}$.

Thus, studies have shown that with a detector similar to the LEP/SLC detectors, with $b$-quark vertex tagging provided by silicon microvertex detectors using present technology, an intermediate-mass SM Higgs boson cannot escape detection at an $e^{+} e^{-}$linear collider at $\sqrt{s}=500 \mathrm{GeV}$. Figure 2.18 shows an estimate [48] of the minimum luminosity required to discover at the $5 \sigma$ level a SM Higgs boson of a particular mass. An integrated luminosity of only $5 \mathrm{fb}^{-1}$ would be adequate to cover the entire intermediate mass range, while $20 \mathrm{fb}^{-1}$ would allow a reach in mass up to about $\sqrt{s} / 2$.

Higher $\sqrt{s}$ would of course allow one to probe for the existence of much heavier Higgs bosons, mostly through $W W$ fusion and decay into pairs of vector bosons. At center of mass energies of 1-2 TeV, different backgrounds such as $e^{+} e^{-} W^{+} W^{-}$and $e^{+} \nu_{e} W^{-} Z^{0}$ need to be addressed. Even though there exist older studies [7, 50] of searches for heavy Higgs bosons in $1-2 \mathrm{TeV} e^{+} e^{-}$collisions, these investigations need to be updated with more detailed 
simulations and to keep abreast of theoretical developments [51] regarding backgrounds and the decay of heavy Higgs bosons. For very large Higgs boson masses, this study becomes a part of the general problem of studying $W W$ scattering at high energies; we discuss this problem in some detail in Section 7.2.

\subsubsection{Minimal Supersymmetric Standard Model Higgs}

In the framework of the MSSM, production of the lightest $C P$-even state $h^{0}$ is similar to that of the SM higgs boson. It is produced by the $Z h$ and $W W$ fusion processes just described, with $h^{0}$ replacing $h_{S M}$. In addition, new modes of production also open up, involving the heavy Higgs bosons $H^{0}$ and $A^{0}$. The various production processes for $h^{0}$ and $H^{0}$ in $e^{+} e^{-}$ annihilation depend on the mixing angles $\alpha$ and $\beta$ as indicated in Table 2.5. Notice the sum rule: One process in each line always has a substantial rate. As $m_{A} \rightarrow \infty$ in the MSSM, $\cos (\beta-\alpha) \rightarrow 0$, and only the processes in the left-hand column of the table occur. In this limit, the rates of the $h^{0}$ production processes are identical to those for the Standard Model Higgs.

Table 2.5: Dependence of the cross section on Higgs boson mixing angles for various Higgs boson production processes in the MSSM.

\begin{tabular}{|c|c|}
\hline \hline $\sin ^{2}(\beta-\alpha)$ & $\cos ^{2}(\beta-\alpha)$ \\
\hline$h^{0} Z^{0}$ & $H^{0} Z^{0}$ \\
$h^{0} \nu \bar{\nu}$ & $H^{0} \nu \bar{\nu}$ \\
$H^{0} A^{0}$ & $h^{0} A^{0}$ \\
\hline \hline
\end{tabular}

The phenomenology of the SUSY Higgs bosons varies in a smooth way as $M_{A}$ is varied. The contours of Higgs mass over the MSSM parameter space are shown in Fig. 2.19 [52]. If $M_{A}<125 \mathrm{GeV}$, then $A^{0}$ and $h^{0}$ are close in mass; if $M_{A}>125 \mathrm{GeV}$, then $M_{A} \simeq M_{H}$ and we begin to approach the large $M_{A}$ limit. However, if $M_{A}<230 \mathrm{GeV}$, then all of the MSSM Higgs bosons should still be observable at the NLC with $\sqrt{s}=500 \mathrm{GeV}$. If $M_{A}>230 \mathrm{GeV}$, it is possible that, at the $\sqrt{s}=500 \mathrm{GeV}$ stage of the NLC, only the lightest SUSY Higgs $h^{0}$ may be observable and it would have production rates virtually indistinguishable from those of a minimal Standard Model Higgs boson. The remaining Higgs states could be discovered at higher $\sqrt{s}$, and there are also precision tests available, to be described later, which could distinguish a Standard Model Higgs from a supersymmetric Higgs. However, since the $h^{0}$ will result in decay topologies similar to that of the SM Higgs, if this lightest $h^{0}$ is not observed, then the MSSM is categorically ruled out. If the $h^{0}$ is not seen below $150 \mathrm{GeV}$, the more general supersymmetry models incorporating grand unification are also excluded. 

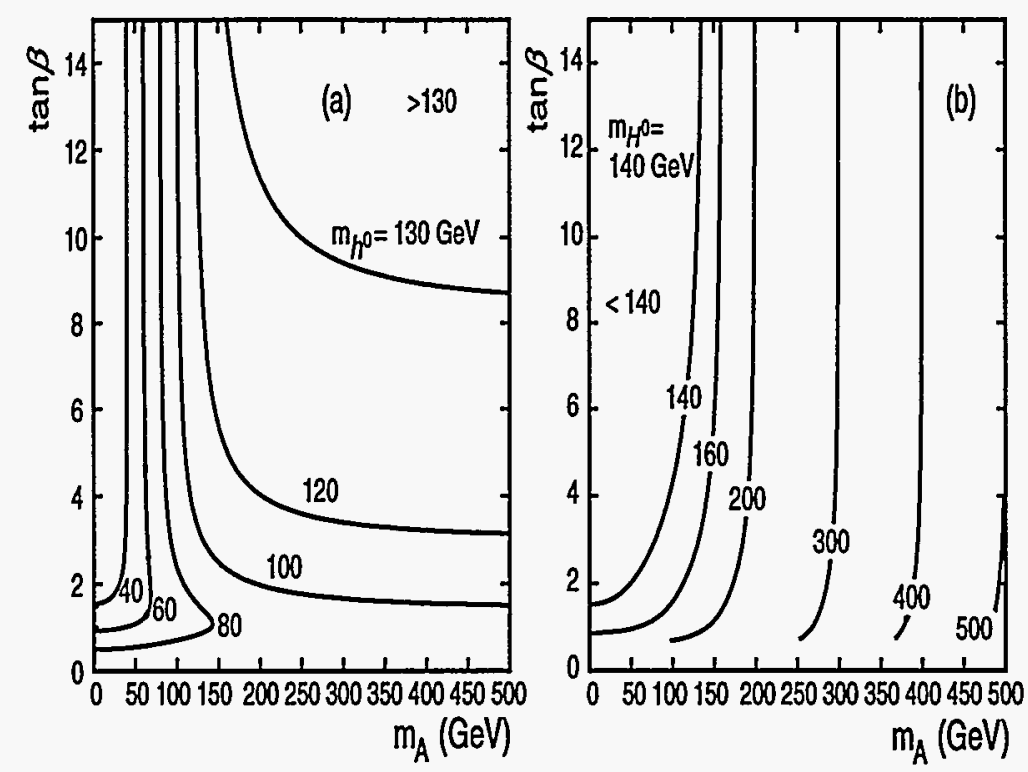

Figure 2.19: Contours of the values of (a) $M_{h}$ and (b) $M_{H}$ in the MSSM for $m_{t}=180 \mathrm{GeV}$, assuming $m_{S U S Y}=1 \mathrm{TeV}$ and maximal top squark mixing.

In general, for an intermediate mass boson, the branching ratio for a minimal SM Higgs boson to $b \vec{b}$ is very close to that of the light $C P$-even state $h^{0}$. For large values of $\tan \beta$, the other neutral MSSM Higgs bosons decay predominantly into $b \bar{b}$, with a $3 \%$ branching ratio into $\tau^{+} \tau^{-}$. This simple pattern becomes more complex for smaller values of $\tan \beta$ [51] with modes such as $H^{0}, A^{0} \rightarrow t \bar{t}$ (for $M_{A^{0}} \approx M_{H^{0}}>2 m_{t}$ ) and $H^{0} \rightarrow h^{0} h^{0}$ and $A^{0} \rightarrow Z h^{0}$ (for $M_{A^{0}} \approx M_{H^{0}}<2 m_{t}$ ) becoming more important. Despite more complicated cascade decays into lighter Higgs states, the bottom line remains clear: There should be plenty of jets from $b$-quarks to tag and elucidate signals. A case in point is the spectacular decay of $H^{0} A^{0}$ into six $b$ jets $H^{0} \rightarrow h^{0} h^{0}, h^{0} \rightarrow b \bar{b}$. Most final states decay into at least four $b$-quark jets, underlining the overwhelming importance of $b$-tagging in experimental studies. An interesting case deserving further study in simulations is the heavy Higgs decay into $t \bar{t}^{(*)}$.

\section{Experimental Studies}

All of the topologies of Fig. 2.16 can be explored in the MSSM with the $h^{0}$ taking the role of the Standard Model Higgs boson. A repetition of the analyses described earlier either would observe a single $h^{0}$ similar to that of the SM Higgs, or, if $\sin ^{2}(\beta-\alpha) \simeq 0.5$, would observe both the $h^{0}$ and $H^{0}$ states as shown in Fig. 2.20a [48].

An identical preselection for a four-jet topology can be used to search for $H A$. We require in this case that all four jets in $\left(H^{0} \rightarrow b \bar{b}\right)\left(A^{0} \rightarrow b \bar{b}\right)$ be tagged as $b$ jets. For large enough $M_{A}$ and $\tan \beta$, we have $M_{A} \simeq M_{H}$, and we can demand that the two jet-pair masses of the 

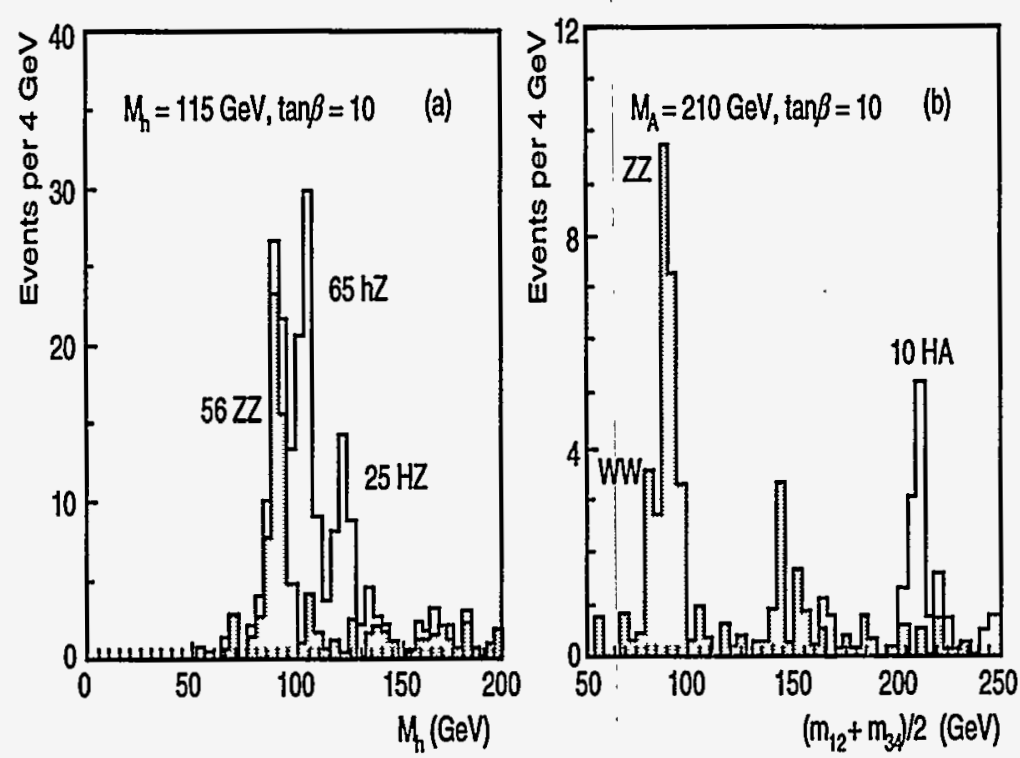

Figure 2.20: (a) Jet masses recoiling from a reconstructed $Z^{0}$ after $b$ tagging for $\sin ^{2}(\beta-\alpha) \simeq 0.5$, and integrated luminosity of $10^{-1} \mathrm{fb}$; (b) average of the two jet-pairs closest in invariant mass in an identified $b \bar{b} b \bar{b}$ final state with $M_{A}=210 \mathrm{GeV}$ (adapted from [48]).

possible combinations to be close to equal. Then a signal as shown in Fig. $2.20 \mathrm{~b}$ is possible. Since all the neutral SUSY Higgs decay into $\tau^{+} \tau^{-}$at some level, it is possible to observe all three MSSM states in a single analysis by looking at the invariant mass of both the $\tau^{+} \tau^{-}$and $q \bar{q}$ in the $\tau^{+} \tau^{-} q \bar{q}$ final state. In Fig. 2.21, we show the regions of plane of $M_{A}$ versus $M_{h}$ in which is it possible to observe all three neutral Higgs states of the MSSM at a linear collider with $\sqrt{s}=500 \mathrm{GeV}$, or, conversely, the region where only the $h^{0}$ can be observed [53].

In theories with multiple Higgs doublets such as the MSSM, searches for the charged Higgs bosons $H^{ \pm}$are also important. An $e^{+} e^{-}$collider should be able to better resolve the hadronic decays of the $H^{ \pm}$compared to a hadronic collider, and all of the expected final states $H^{+} H^{-} \rightarrow c \bar{s} \bar{c} s, t \bar{b} \bar{t} b$, plus the easier topologies of $c \bar{s} \tau^{-} \nu$ and $\tau^{+} \nu \tau^{-} \bar{\nu}$ should be observable. A detailed simulation analysis [54] at $\sqrt{s}=500 \mathrm{GeV}$ with an integrated luminosity of $10 \mathrm{fb}^{-1}$, which makes heavy uses of $b$-tagging, has shown that one can establish a signal over the $e^{+} e^{-} \rightarrow t \bar{t}$ and $W^{+} W^{-}$background in all of these channels. The results show a detection sensitivity for charged Higgs bosons up to about $210 \mathrm{GeV}$ at $\sqrt{s}=500 \mathrm{GeV}$, independent of decay mode. These conclusions can be strengthened further by adding the decay $t \rightarrow b H^{+}$. The bottom line is that if the $A^{0}$ is lighter than about $200 \mathrm{GeV}$, then the $H^{ \pm}$should be observable also.

If one is considering SUSY Higgs bosons, one should allow for the possibility of their decay into other SUSY particles. As an example, it is possible for $h^{0}$ or $H^{0}$ to decay into a pair of the lightest neutralinos (mixtures of fermionic partners of the $Z$ and $\gamma$ ) that would be stable and neutral. The result would be an "invisible" decay of the Higgs. This topology 


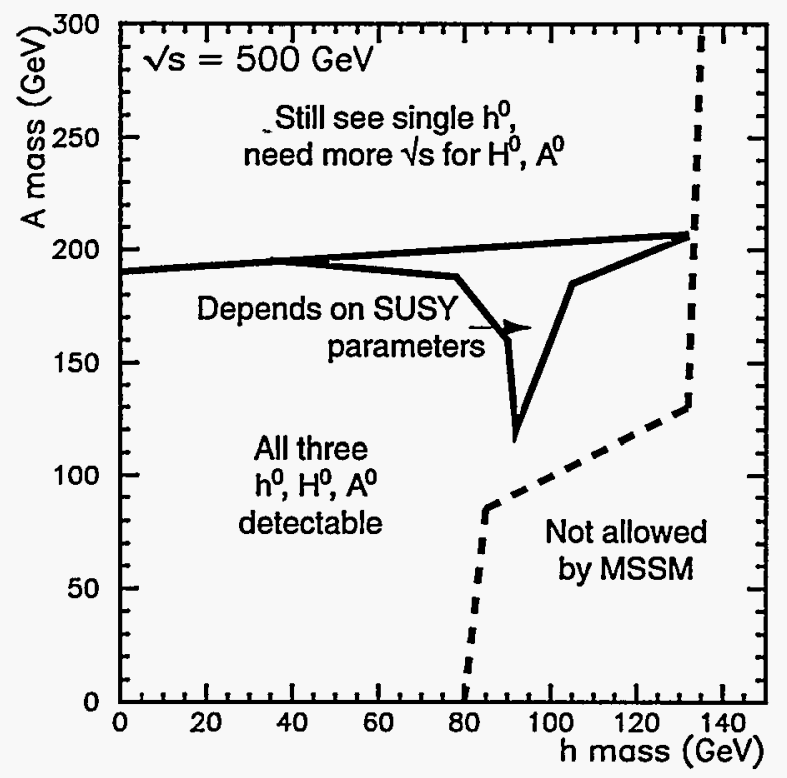

Figure 2.21: Regions of simultaneous detectability of $h^{0}, H^{0}$, and $A^{0}$ at center-of-mass energy of $500 \mathrm{GeV}$ [53].

can be identified in the same way as described previously by studying the missing mass in a $h Z$ or $H Z$ event where the $Z$ decays into a pair of electrons or muons. Both missing mass resolution and backgrounds (smaller direct $Z \nu \bar{\nu}$ cross section) improve with lower centerof-mass energy, and such an analysis would benefit from running at $\sqrt{s} \simeq 300 \mathrm{GeV}$. The possibility of SUSY Higgs decays into other SUSY particles [51] such as SUSY partners of quarks and leptons warrant further experimental simulations.

\subsubsection{Determination of Properties of Higgs Bosons}

\section{Mass Measurement}

To measure the mass of one or more of the possible Higgs bosons, one would probably optimize the running conditions to have smaller center-of-mass energy, to improve momentum resolution and to go to the peak of the cross section. For an intermediate mass Higgs, $\sqrt{s}=200-300 \mathrm{GeV}$ is appropriate. Under these conditions, one can precisely measure the recoil mass in $e^{+} e^{-} \rightarrow Z^{0} h^{0}$ events opposite to the reconstructed leptonic decay $Z^{0} \rightarrow e^{+} e^{-}$or $\mu^{+} \mu^{-}$. Other modes, such as the four-jet topology, can also be employed. In all cases, kinematic fitting would be used to constrain the leptons or jets from a $Z^{0}$ to reconstruct to $M_{Z}$ and to allow for missing $E_{\gamma}$ along the beam axis. A typical jet-jet mass resolution of $\sigma_{M} \simeq 2.0 \mathrm{GeV}$ can be achieved assuming the excellent momentum resolution of $\sigma_{p_{t}} / p_{t}=1 \times 10^{-4} \oplus 0.1 \%$ envisaged for the JLC detector [47]. For our NLC detector design, we could achieve $\sigma_{M} \simeq 3.9 \mathrm{GeV}$, as shown in Fig. 2.22. The differences between detector designs are much smaller when kinematic constrained fitting is included in the analysis. The 
JLC-type detector has been estimated to provide a estimated precision on the Higgs mass of approximately $0.1 \%$ for $\sqrt{s}=300 \mathrm{GeV}, \int L \cdot d t=30 \mathrm{fb}^{-1}$, and a $2.0 \%$ full width beam energy spread [55]. On the other hand, the NLC-type detector gives $\Delta M_{h} \leq 160 \mathrm{MeV}$ up to $M_{h} \simeq 160 \mathrm{GeV}$ with $50 \mathrm{fb}^{-1}$ at $\sqrt{s}=500 \mathrm{GeV}$.

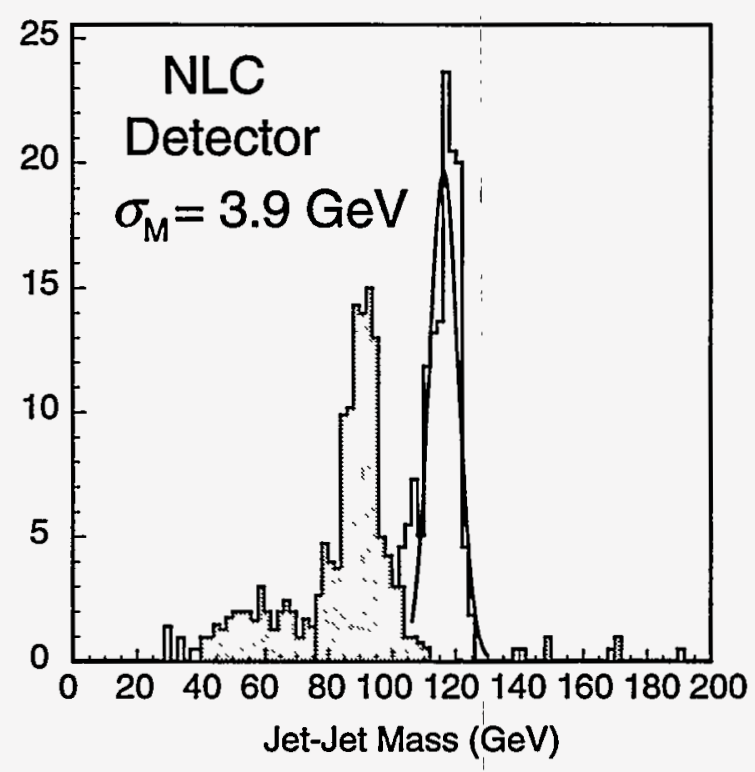

Figure 2.22: Mass resolution of $\sigma_{M} \simeq 3.9 \mathrm{GeV}$ in the jet-jet invariant mass for jets from Higgs decay assuming the performance of a NLC detector (see text) for a simulated signal (open histogram) of a $120 \mathrm{GeV}$ SM Higgs boson and all known backgrounds (shaded histogram) at $300 \mathrm{GeV}$ with $10 \mathrm{fb}^{-1}$.

Once $M_{H}$ is known precisely, it can be used as an input to check the experimental measurements of branching ratios and the production cross section with Standard Model predictions. Its value can also be compared to the theoretical value obtained from precision electroweak measurements, combined with the measurements of $M_{W}$ and $m_{t o p}$ expected from a linear collider.

\section{Cross Section Measurement}

Measuring the production cross section of the Higgs provides one way of disentangling a SM Higgs boson from a SUSY Higgs, if one can observe the cross section suppression due to mixing

$$
\sigma\left(e^{+} e^{-} \rightarrow h^{0} Z^{0}\right)=\sin ^{2}(\beta-\alpha) \sigma\left(e^{+} e^{-} \rightarrow h_{S M}^{0} Z^{0}\right) .
$$

A distinct advantage of $e^{+} e^{-}$linear colliders over hadronic colliders is the ability to almost unambiguously tag the $Z^{0}$ in $h Z$ events and being 'able to study all of the decays $h^{0} \rightarrow X$ with small backgrounds. Both total absolute cross sections and individual Higgs branching ratios can then be measured. By using leptonic decays of the $Z^{0}$ and kinematical fitting, 
the absolute production cross section can be measured [55] with a precision of $7 \%$ with an integrated luminosity of $30 \mathrm{fb}^{-1}$ and to $5 \%$ with $50 \mathrm{fb}^{-1}$. However it should be kept in mind that for a large area of SUSY parameter space, the SUSY Higgs cross section is less than $10 \%$ different from the SM Higgs cross section.

\section{Spin-Parity and CP Determination}

In principle, the spin and parity of the Higgs boson can be found by studying both the production angular distribution of the Higgs and also the resulting angular distribution of the decay products of the $Z^{0}$ in its rest frame in $H Z$ events. In the high energy limit, Table 2.6 shows the expected angular distribution of scalar (e.g., $\left.h^{0}, H^{0}\right)$ and pseudoscalar (e.g., $A^{0}$ ) Higgs bosons. In the table, $\theta$ is the production angle of the Higgs boson and $\theta_{*}$ is the polar angle of the fermions from $Z$ decay measured in the $Z$ rest frame. In practice, however, a purely CP-odd Higgs boson couples to $Z Z$ only at the one-loop level, and then the $Z A$ cross section would be very small. For a Higgs boson that is a mixture of CP-even and $\mathrm{CP}$-odd components, the production would mainly be sensitive to the CP-even part, and the angular distributions would not reveal the CP-odd component [56].

Table 2.6: Expected angular distributions for Higgs bosons with different spin-parity.

\begin{tabular}{|c|c|c|}
\hline \hline & Scalar, $0^{++}$ & Pseudoscalar, $0^{-+}$ \\
\hline$d \sigma\left(e^{+} e^{-} \rightarrow H Z^{0}\right) / d \cos \theta$ & $\propto \sin ^{2} \theta$ & $\propto\left(1-\sin ^{2} \theta\right)$ \\
$d \sigma\left(Z^{0} \rightarrow f \bar{f}\right) / d \cos \theta_{*}$ & $\propto \sin ^{2} \theta_{*}$ & $\propto\left(1 \pm \cos \theta_{*}\right)^{2}$ \\
\hline \hline
\end{tabular}

A much better way to determine the Higgs' $C P$ character is with polarized $\gamma \gamma$ collisions $[57,58]$. In this technique, which we will discuss in detail in Section 9.2, the Higgs boson is produced as an $s$-channel resonance. Then it is possible to study the angular correlations of the decay products of the resonance in decays such as $H \rightarrow \tau^{+} \tau^{-}$and $t \bar{t}$. By spin analyzing the subsequent decays $t \rightarrow b \ell \nu$ for top quarks from heavy Higgs boson decay, and $\tau \rightarrow \pi \nu$ or $\tau \rightarrow \rho \nu$ for $\tau$ 's from intermediate mass Higgs decay, a $C P$-even state and $C P$-odd state can be distinguished $[56,59]$. This $C P$ state separation is much better in the angular correlations between top quark decay products.

\section{Branching Ratio Measurements}

The measurement of the branching ratios of any observed Higgs boson is an essential ingredient to understand the nature of the symmetry breaking and to make predictions about other aspects of the Higgs sector. This is especially when only a single neutral Higgs is observed, which might be either the Standard Model Higgs or the lightest neutral Higgs from SUSY. 
The clean environment in $e^{+} e^{-}$annihilation permits one to tag a $Z^{0}$ in one hemisphere, and then observe the decay $h^{0} \rightarrow X$ in any decay mode in the opposite hemisphere. An example of such an analysis [49] at $\sqrt{s}=400 \mathrm{GeV}$, simulating an SLD-like detector, first identifies a $Z^{0}$ in a $H Z$ event and then considers those decays where the recoiling Higgs decays into jets. The Higgs decays to two jets can be separated by flavor by counting the number of tracks with a significantly large impact parameter: $b_{\text {norm }}=b / \sigma_{b}>3$, where $b$ is the impact parameter and $\sigma_{b}$ is the error on $b$. The decay $h \rightarrow W W^{(*)}$ is identified by demanding that the event be consistent with containing six jets, and that a jet pair with invariant mass close to the $W$ mass is found. With $50 \mathrm{fb}^{-1}$ of data, $B r(h \rightarrow b \bar{b})$ can be measured to a statistical precision of $7 \%$, and branching ratios into $W W^{*}$ and $(c \bar{c}+g g)$ to $24 \%$ and $39 \%$ respectively. These relative errors are shown superimposed upon the Standard Model values in Fig. 2.23a. The figures also shows the variation in branching ratios that one would expect from the variation of $\tan \beta$. In the MSSM, it is very difficult to arrange such a large variation in $\tan \beta$ without a compensatory variation in $\alpha$, but the figure shows the utility of this measurement in Higgs studies in a more general context. For the comparison of the Standard Model Higgs boson to the MSSM, one should consult Fig. 2.23b, where the branching ratio of a light Higgs boson into $b \bar{b}$ is compared for these two possibilities over the MSSM parameter space.
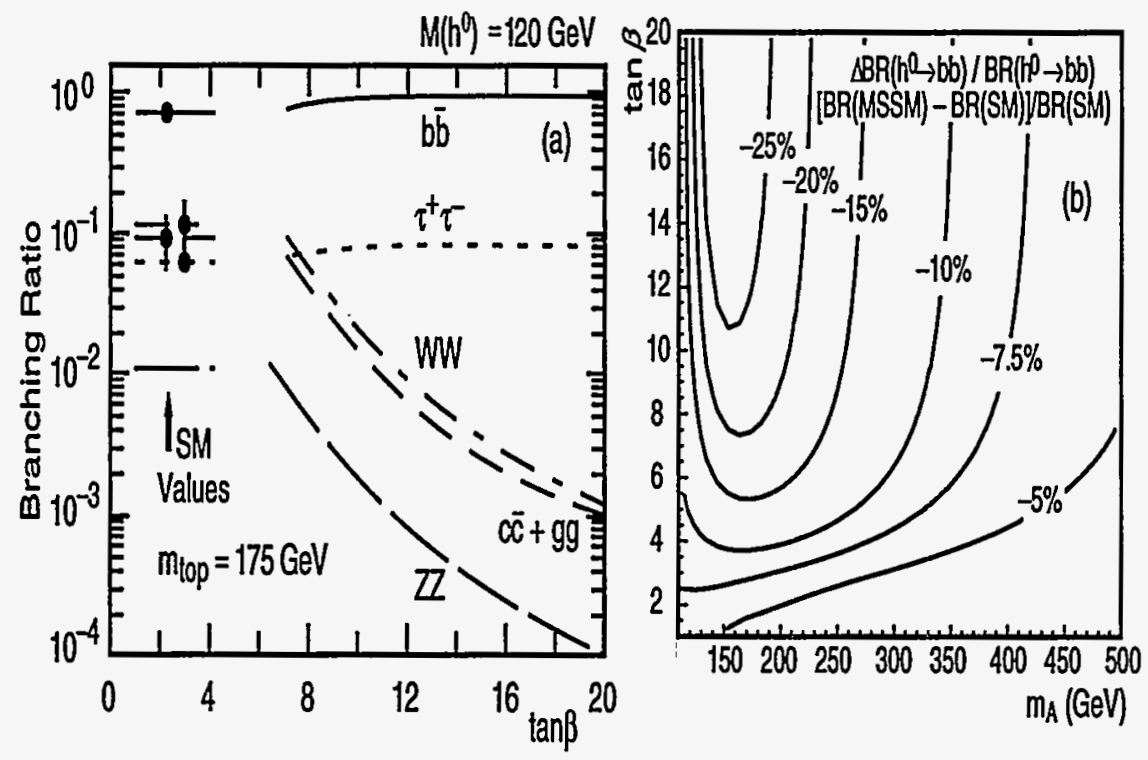

Figure 2.23: (a) Expected errors on the Standard Model branching fractions compared to those predicted for a $120 \mathrm{GeV} h^{0}$ MSSM Higgs boson; (b) contour lines of fractional deviation of $\operatorname{Br}\left(h^{0} \rightarrow b \bar{b}\right)\left(m_{t}=\right.$ $\left.175 \mathrm{GeV}, m_{\text {SUSY }}=1 \mathrm{TeV}\right)$.

An interesting quantity [61] is the ratio of branching ratios to $c \bar{c}$ versus $b \bar{b}$. At tree level,

$$
\frac{B r(h \rightarrow c \bar{c})}{B r(h \rightarrow b \bar{b})} \approx \frac{m_{c}^{2}}{m_{b}^{2}} \cdot\left(\frac{M_{h}^{2}-M_{A}^{2}}{M_{A}^{2}-M_{Z}^{2}}\right)^{2}
$$


where $m_{c}$ and $m_{b}$ are the $c$ and $b$ quark masses respectively. (We should note that this formula can receive substantial radiative corrections in some regions of the MSSM parameter space.) If the branching ratios indicated are measured along with $M_{h}$, it is possible to estimate $M_{A}$. In a simulation study of this measurement [62] at $\sqrt{s}=300 \mathrm{GeV}, H Z$ events are selected for each decay mode of the $Z^{0}$, and the decay mode of the Higgs is determined using threedimensional impact parameters. Flavor tagging is performed by selecting charged tracks that satisfy $b / \sigma_{b} \geq 2.5$ and counting the number in each jet from the Higgs decay. For $M_{H}=120 \mathrm{GeV}, 50 \mathrm{fb}^{-1}$ of data, and assuming $90 \%$ polarization of the electron beam, the statistical error on the ratio of branching ratios $\operatorname{Br}(h \rightarrow c \bar{c}+g g) / B r(h \rightarrow b \bar{b})$ would be $20.4 \%$, varying with the Higgs mass as shown in Fig. 2.24a. This does not include a substantial systematic uncertainty from $m_{c} / m_{b}$, which we believe will be reduced in the next few years through lattice gauge theory calculations. Then, as shown in Fig. 2.24b, this measurement could be sensitive to $A^{0}$ masses up to $400 \mathrm{GeV}$, well above the maximum kinematic reach of a $500 \mathrm{GeV}$ collider. Observation of the $A^{0}$ in this way would help to plan the next step in energy.
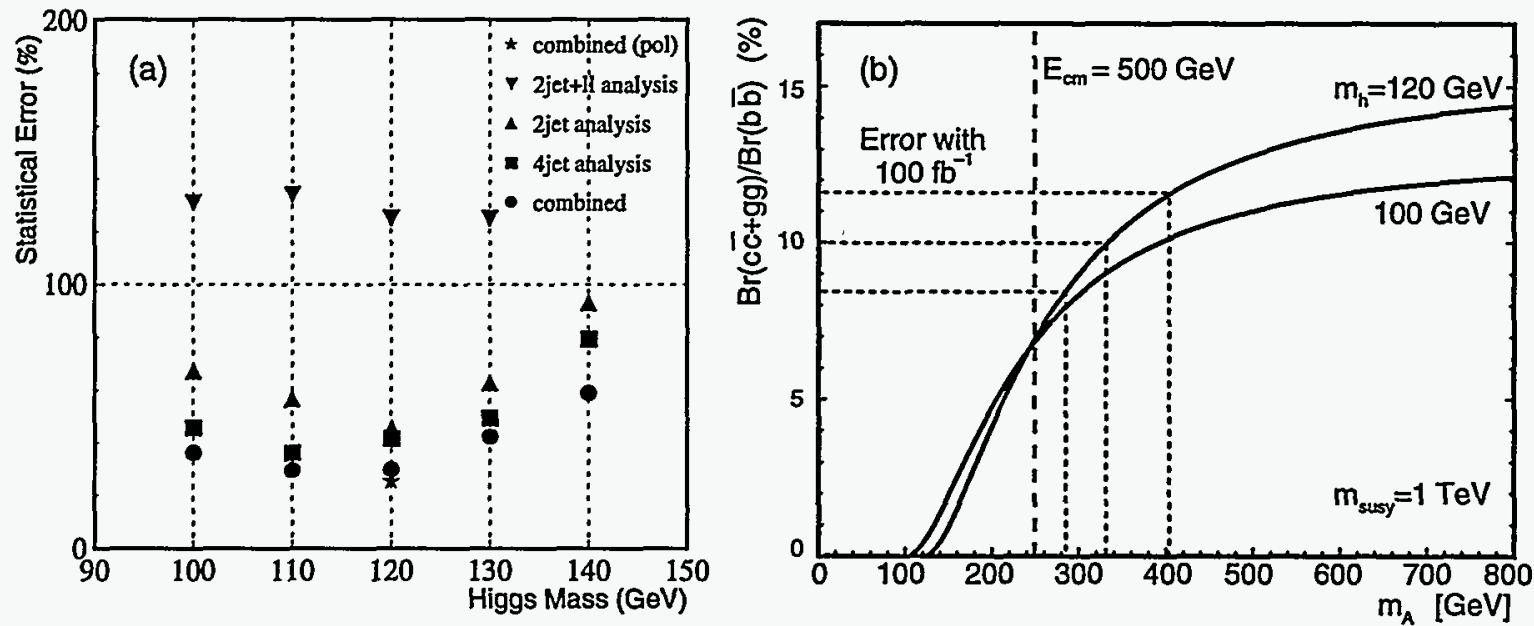

Figure 2.24: (a) Statistical error with $50 \mathrm{fb}^{-1}$ of data on $\operatorname{Br}(h \rightarrow$ $c \bar{c}+g g) / B r(h \rightarrow b \bar{b})$ as a function of Higgs mass; (b) implications for estimation of $A^{0}$ mass.

For the decay $h \rightarrow \gamma \gamma$, it is difficult to measure the branching ratio at an $e^{+} e^{-}$collider because this mode is relatively rare. However, it should be possible to measure the absolute partial width $\Gamma(h \rightarrow \gamma \gamma)$ by exploiting the ability of an electron collider to be run as a $\gamma \gamma$ collider. This measurement is discussed in Section 9.1.

\section{Determination of Higgs Total Width}

In the preceding sections, we have indicated many ways in which measurements at the NLC can distinguish between the Standard Model Higgs and the light Higgs $h^{0}$ of the MSSM. There are also a number of quantities at the LHC which are sensitive to this difference, as 
outlined in [35]. However, to obtain the complete set of partial width of the Higgs boson in a model-independent way, measurements from LHC must be combined it with data both from $e^{+} e^{-}$and $\gamma \gamma$ collisions at the NLC. A possible procedure is the following. First determine $B r(b \bar{b})$ from $Z h$ events and combine with $\sigma(W W \rightarrow h) \cdot B r(b \bar{b})$ both measured at the NLC to obtain the $W W h$ coupling. Alternatively, a measurement of $\sigma\left(e^{+} e^{-} \rightarrow Z h\right)$ at the NLC gives the $Z Z H$ coupling and the ratio of the $W W h$ and $Z Z h$ couplings given by $M_{W}^{2} / M_{Z}^{2}=\cos ^{2} \theta_{W}$ also gives the $W W h$ coupling. This coupling and a measurement of $\sigma(W h) \cdot B r(\gamma \gamma)$ at the LHC can be used to determine $B r(\gamma \gamma)$. Therefore one can combine $B r(b \bar{b})$ with the $\gamma \gamma$ collider measurement of $\sigma(\gamma \gamma \rightarrow h) \cdot B r(b \bar{b})$ to obtain $\Gamma(h \rightarrow \gamma \gamma)$. We can then finally compute the total width $\Gamma_{h}^{\text {tot }}=\Gamma(h \rightarrow \gamma \gamma) / B r(\gamma \gamma)$ and $\Gamma(h \rightarrow b \bar{b})=B r(b \bar{b}) \Gamma_{h}^{\text {tot }}$. A simpler route exists using only $e^{+} e^{-}$data when the Higgs boson is heavy enough that the branching ratio to $W W^{*}$ is relatively large, so that it can be measured accurately. In this case, we can simply measure $B r\left(h \rightarrow W W^{*}\right)$ and infer $\Gamma_{h}^{\text {tot }}=\Gamma\left(h \rightarrow W W^{(*)}\right) / B r\left(W W^{(*)}\right)$. Although the accumulation of errors may be significant, the basic point is that data from all three colliders or from the NLC alone can be combined to complete a model-independent determination of the properties of a light Higgs boson.

\subsubsection{Summary}

From the studies described, the discovery of a Standard Model intermediate-mass Higgs boson at an $e^{+} e^{-}$linear collider at $\sqrt{s}=500 \mathrm{GeV}$ can be easily achieved with an integrated luminosity of only $10 \mathrm{fb}^{-1}$. Such a machine allows the detection of at least the lightest MSSM Higgs $h^{0}$, if not all three SUSY neutral states. If the lightest Higgs is not observed, then not only is the Minimal Supersymmetric Standard Model ruled out, but also the general idea that the Higgs boson is a fundamental particle up to the unification scale is called into question. If the $A^{0}$ is not kinematically accessible at $\sqrt{s}=500 \mathrm{GeV}$, then the measurement of $h^{0}$ branching ratios can give hints of the values of $M_{A}$ and $\tan \beta$ and tell us where to go next in energy. For definitive evidence, $\sqrt{s}>2 M_{A}$ would still be needed. For Higgs bosons above the intermediate mass range, their decay into pairs of vector bosons makes them straightforward to detect at the NLC as at the LHC; however, more up-to-date experimental simulations are needed. Just as important as its ability to discover the Higgs boson is the ability of the linear collider to make precision measurements of the properties and couplings of a Higgs boson. Even if the Higgs boson is discovered earlier at LEP2 or at the LHC, we will need the NLC to learn its complete story. 


\subsection{Supersymmetry}

To build a complete unified theory with a fundamental Higgs boson, one is led to introduce supersymmetry, the symmetry between fermions and bosons in space-time. Supersymmetry is the only known principle with sufficient structure to allow the construction of grand unified theories in which fundamental scalar particles can naturally be very light compared to the unification scale. Supersymmetric unification models explain the values of the Standard Model coupling constants as measured at $Z^{0}$ energies, and also incorporate a mechanism of electroweak symmetry breaking associated with the heavy top quark. General reviews of supersymmetric models can be found in $[36,37,63,64]$. Supersymmetry also offers the more speculative but tantalizing possibility of a connection between phenomena observable at collider energies and string theory and other profound mathematical theories of the fundamental forces $[65,66]$.

In this section, we will examine the manner in which supersymmetry (SUSY) might manifest itself at a $0.5-1.0 \mathrm{TeV} e^{+} e^{-}$Linear Collider (NLC). Our discussion here is part of a broader, and continuing, investigation. At present, our study is being carried out within the supersymmetry scenario based on the minimal supergravity model with gauge coupling unification and radiative electroweak symmetry breaking (SUGRA). We have calculated most of the relevant cross sections and angular distributions for the production and decay of supersymmetric particles, and we include a report of them as an appendix [67]. Because of the difficulty of knowing where and how supersymmetry will manifest itself, we must study the phenomenology of supersymmetry over a wide range of its parameters. In this study, we have chosen five points in the parameter space of the SUGRA model which illustrate qualitatively different possibilities for the spectrum of new particles [67]. Because of the power of the experimental tools offered by the NLC, our goals are much more ambitious than simply to discover the existence of supersymmetry. We would like to measure the masses of supersymmetric particles with precision, and determine the underlying values of the basic parameters of the theory. In the most optimistic scenario, the extrapolation of these parameters to the unification scale would give evidence into the details of the fundamental unified model [68].

The number of supersymmetric particles is quite large. Hence, it is typical that many of these particles will be produced in the same data sample at a particular energy centerof-mass energy. One of the properties of $e^{+} e^{-}$linear colliders is that the electron can be longitudinally polarized and its orientation can be changed at will. Already, the SLC provides an electron beam with $77 \%$ polarization. We expect that, in the future, this magnitude can be increased substantially. The ability to have electron beams with high longitudinal polarization is very useful to discriminate between the various supersymmetric signals and to understand and remove the Standard Model background processes [69]. This can be seen by examining the standard model cross sections as a function of polarization, shown already in Fig. 2.3, and comparing these to the polarization-dependence of the cross sections for the supersymmetric production processes, shown for two representative points in Fig. 2.25. Having a $90 \%$ longitudinally polarized electron in the right handed mode will reduce the production 

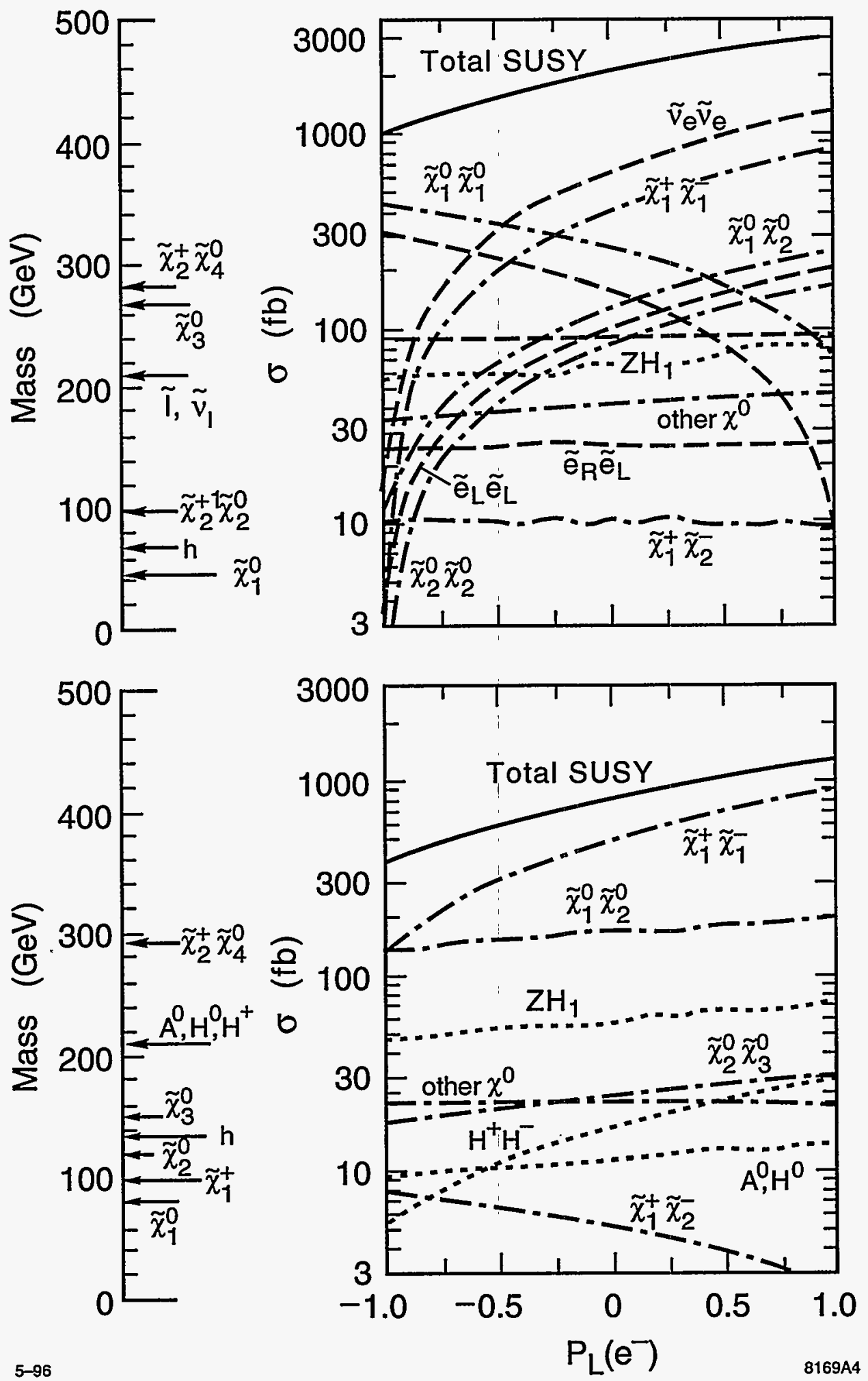

$5-96$

Figure 2.25: The cross section of Supersymmetric particle production at $\mathrm{E}_{\mathrm{cm}}=0.5 \mathrm{TeV}$ as a function of the electron polarization for points 3,4 in our parameter space. 
rate of Standard Model background processes such as $W^{+} W^{-}$pair production by an order of magnitude while enhancing some of the supersymmetric particle signals. The left-handed supersymmetric particles, like the Standard Model background, are also suppressed, while the right-handed ones are enhanced. This is seen in Fig. 2.25. Hence, by varying the polarization we can determine which of the supersymmetric particles are giving us a particular signal. Polarization of the positrons could give an additional advantage. For example, if we could collide totally right-handed electrons with totally left-handed positrons, all of the standard model backgrounds from $e^{+} e^{-}$annihilation processes would disappear, while the supersymmetric signals from $\tilde{e}_{R}^{-} \tilde{e}_{R}^{+}$production would remain. (Some background would also remain due to two-photon reactions [70].) An additional handle on our ability to discriminate among the various supersymmetric signals is their different angular distributions [67]. These distributions for a typical case are shown in Fig. 2.26. A third powerful discriminating tool is the adjustment of the center-of-mass energy. Once one has an estimate of the masses of the lightest supersymmetric particles, it is advantageous to decrease the energy of the collider so that only these lightest states are produced, measure their properties at this lower energy, and then increase the energy of the collider systematically.

\subsubsection{Supersymmetry Signals at the NLC}

In our study, we have generated signal and background processes using the simulation program ISAJET [6]. This program allows for both electron and positron longitudinal polarization. The influence of the detector is accounted for by smearing the generated momenta and directions of the particles produced in the simulation with resolution functions as described in Section 2.

The spectrum of supersymmetric particles for the five parameter sets that we have chosen for detailed study are exhibited in Table 2.7. These spectra are computed consistently from a supergravity model with $m_{t}=180 \mathrm{GeV}$. The values.of the underlying parameters for these scenarios is given in [67].

In each of the five cases, the lowest mass supersymmetric particle is the $\chi_{1}^{0}$. In the class of models we discuss, there is a conserved R-parity which implies that this particle is stable. It then passes through the detector without leaving a signal. This particle is in all cases sufficiently massive that it carries away significant missing energy. On the other hand, Standard Model background processes can mimic this signal because, as a result of their peaked differential cross-section in the forward and backward directions [67], many of the particles in the final state go along the beam direction. Also it is possible that neutrinos can carry away a sizable portion of the energy, or that the event is mismeasured due to the detector resolution.

In Fig. 2.27 we show the expected observed energy for the Standard Model processes $e^{+} e^{-} \rightarrow W^{+} W^{-}, Z^{0} Z^{0}, q \bar{q}$ after a requirement that at least three particles be present in each hemisphere. This allows us to avoid including the $e \nu W$ final state and most of the events where the W's and Z's decay into leptons and neutrinos; only a few events with $\tau$ in the 

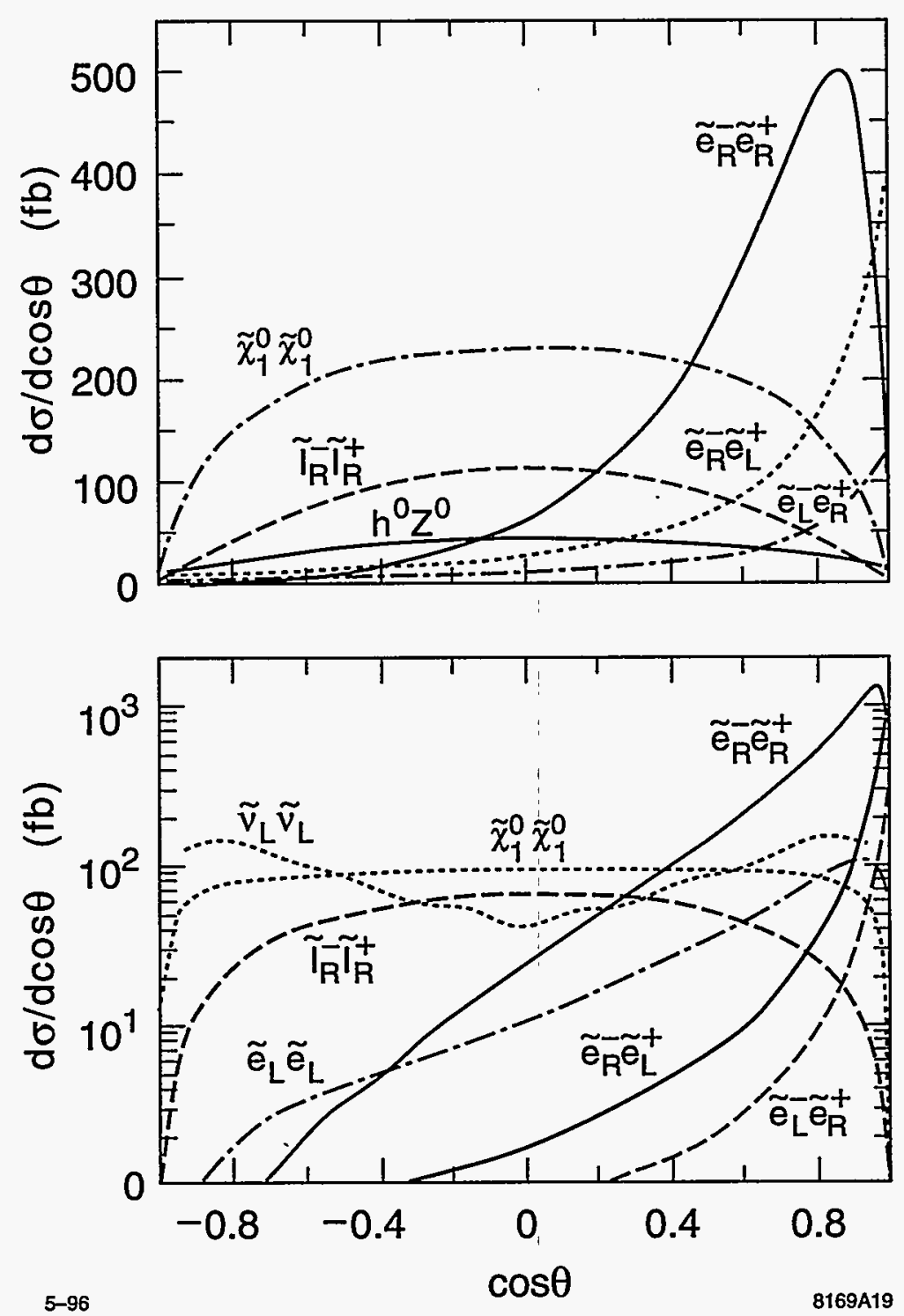

Figure 2.26: The differential cross section of supersymmetric particle production for an $80 \%$ polarized right handed electron at $\mathrm{E}_{\mathrm{cm}}=0.5$ and $1.0 \mathrm{TeV}$ for point 2 in our parameters list. 
Table 2.7: Supersymmetric particle masses at five representative points in the parameter space of phenomenological supergravity models.

\begin{tabular}{|c|r|r|r|r|r|}
\hline \hline Parameter Set & 1 & 2 & 3 & 4 & 5 \\
\hline$\chi_{1}^{0}$ & 85.87 & 128.51 & 44.41 & 77.83 & 57.00 \\
$\chi_{2}^{0}$ & 175.24 & 257.05 & 96.73 & 115.29 & 111.21 \\
$\chi_{3}^{0}$ & 514.96 & 549.08 & 267.81 & 146.84 & 440.03 \\
$\chi_{4}^{0}$ & 523.78 & 556.07 & 284.18 & 292.40 & 460.12 \\
$\chi_{1}^{ \pm}$ & 175.12 & 257.02 & 96.10 & 96.06 & 109.82 \\
$\chi_{2}^{ \pm}$ & 522.82 & 555.72 & 282.52 & 292.45 & 457.09 \\
$h^{0}$ & 84.86 & 92.24 & 68.82 & 130.58 & 102.15 \\
$H^{0}$ & 766.47 & 698.24 & 389.39 & 201.72 & 619.21 \\
$A^{0}$ & 762.32 & 693.30 & 381.75 & 200.00 & 616.45 \\
$H^{ \pm}$ & 765.70 & 697.05 & 388.81 & 214.75 & 620.52 \\
$\tilde{q}_{L}$ & 605.23 & 670.84 & 317.23 & 1000.00 & 464.04 \\
$\tilde{b}_{L}$ & 516,58 & 621.43 & 272.31 & 1000.00 & 384.70 \\
$\tilde{t}_{1}$ & 417.65 & 537.12 & 265.55 & 923.13 & 179.85 \\
$\tilde{q}_{R}$ & 605.23 & 670.84 & 317.23 & 1000.00 & 464.04 \\
$\tilde{b}_{R}$ & 597.15 & 655.70 & 313.40 & 1000.00 & 457.79 \\
$\tilde{t}_{2}$ & 547.20 & 655.21 & 328.15 & 1099.35 & 495.72 \\
$\tilde{l}_{\bar{L}}^{\tilde{\nu}_{-}}$ & 425.96 & 238.35 & 215.72 & 1000.00 & 320.69 \\
$\tilde{\nu}_{L}$ & 421.43 & 230.16 & 206.63 & 1000.00 & 314.65 \\
$\tilde{l}_{R}$ & 408.80 & 156.97 & 206.54 & 1000.00 & 307.45 \\
$\tilde{g}^{-}$ & 552.19 & 760.16 & 298.15 & 900.00 & 428.03 \\
\hline \hline
\end{tabular}




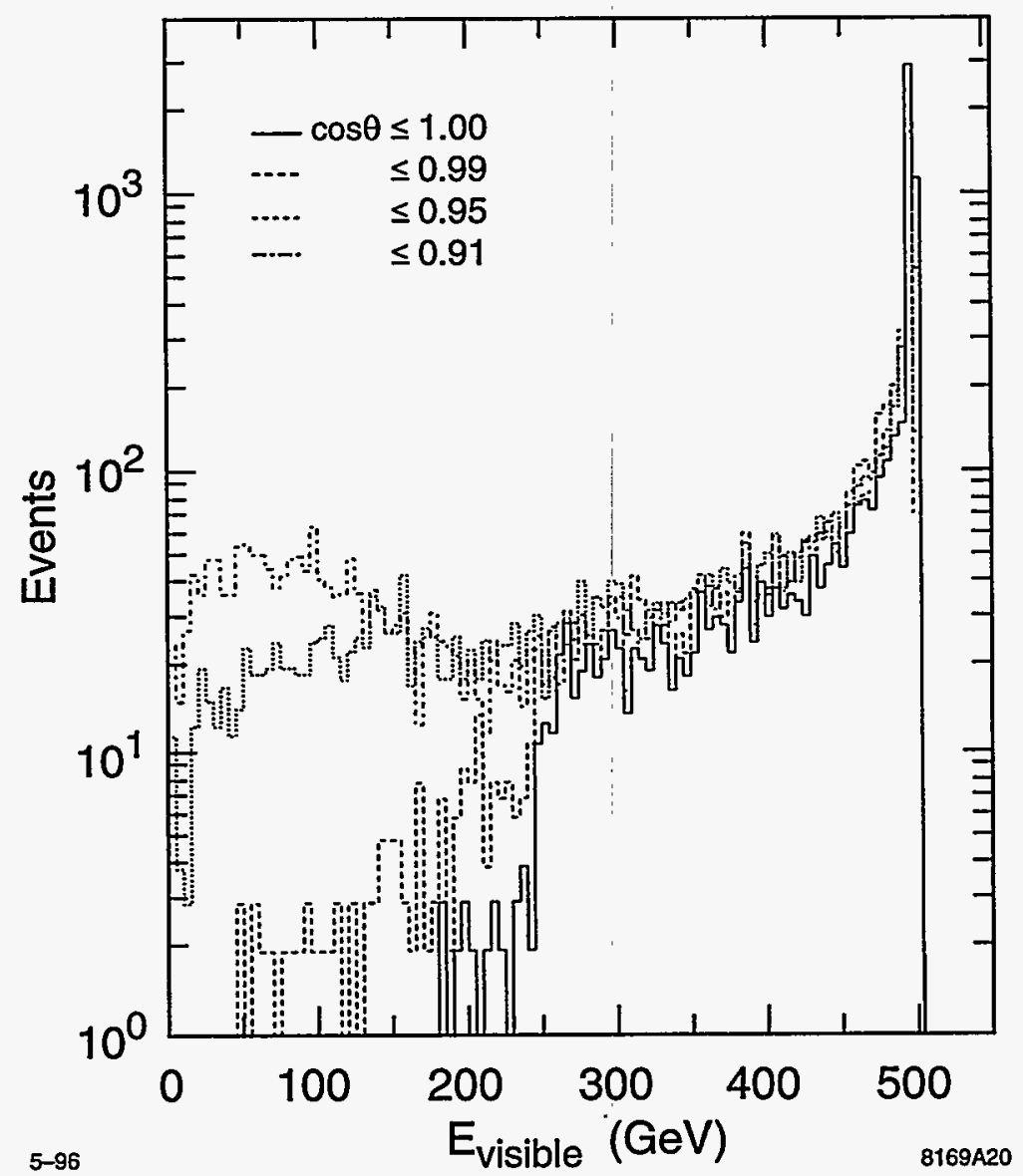

Figure 2.27: The Visible Energy for Standard Model final states $W^{+} W^{-}, Z^{0} Z^{0}, q \bar{q}$ for various calorimetry coverage. We require that there be at least 3 particles in each hemisphere. 
final state remain. The figure shows the effect of various assumptions about the calorimetric coverage in $\cos (\theta)$, to determine how much energy is lost in the beam direction. We note that the tail of events with low visible energy $(\mathrm{E} \approx 100 \mathrm{GeV}$ or less) begins to increase noticeably for $\cos (\theta)<0.97$ or so. Hence good calorimetric coverage is imperative if we want to use the low visible energy as a signal for supersymmetric particles. We do not smear the particles in this plot to determine the true loss of particles down the beam direction. The detector design presented in this report has calorimetric coverage down to $\cos (\theta)=0.99$, which, as we will see, is sufficient to allow us to see the signal due to supersymmetric particles.

We have made a study of the signals seen in the various sets of SUGRA parameters [67]. We show here some of the results for the signal to background ratios for the SUGRA parameter set 4 . In this scenario, the main signals are due to the pair-production of the charginos $\chi_{1}^{ \pm}$and the neutralinos $\chi_{1}^{0}, \chi_{2}^{0}$. The visible (observed) energy for these signals and the others [67] is shown in Fig. 2.28. The signal is in the region of $100 \mathrm{GeV}$ visible energy; hence our requirement that there needs to be calorimetric coverage down to small angles. In Fig. 2.29 we show the visible energy distribution, properly normalized with the relative cross section for background Standard Model events and signal from supersymmetric events. A small bump can be observed in the region of small visible energy. To isolate the signal due to chargino $\left(\chi_{1}^{+} \chi_{1}^{-}\right)$production, we require that there be at least five hadrons in each hemisphere. After additional cuts in the data to enhance the signal, we are able to obtain a signal to background ratio of 12 to 1 as shown in Fig. 2.30. This signal is then used to determine the masses of the $\chi_{1}^{ \pm}$and the $\chi_{1}^{0}$ as described below. Similarly, with appropriate cuts we obtain a signal for $\chi_{2}^{0} \chi_{1}^{0}$ production with no background from the Standard Model processes, but, as shown in Fig. 2.31, a background of $\approx 10 \%$ from $\chi_{1}^{+} \chi_{1}^{-}$. (We expect to reduce this background with further analysis.) The complete observed signal is shown in Fig. 2.32. This can then be used to determine the masses of the $\chi_{2}^{0}$ and $\chi_{1}^{0}$. It is interesting to check that the two determinations of the $\chi_{1}^{0}$ mass agree. This self consistency would give us confidence that we are seeing the consequences of a consistent model and would encourage us to use the resulting model parameters to predict the masses of the other particles. The levels of signal to background seen in this analysis are typical for scenarios of supersymmetric particle production processes in $\mathrm{e}^{+} \mathrm{e}^{-}$colliders.

\subsubsection{Superparticle Mass Measurements}

We will now discuss in more detail the measurement of the masses, spins, and cross-sections of the various possible supersymmetric signals. Some very beautiful studies on these issues have already been reported in $[68,71,72]$. These papers indicate that, indeed, linear collider experimentation provides very powerful methods by which to measure the production and decay parameters of the various supersymmetric particles. This should allow us to uncover which of the various supersymmetric models is the correct one. One recent study [68] has shown that, for slepton or chargino pair-production, we can use the upper and lower limits of the energy spectrum of the secondaries from supersymmetric particle decays to determine these particle masses. In addition, we can use the angular distribution of the signal to 


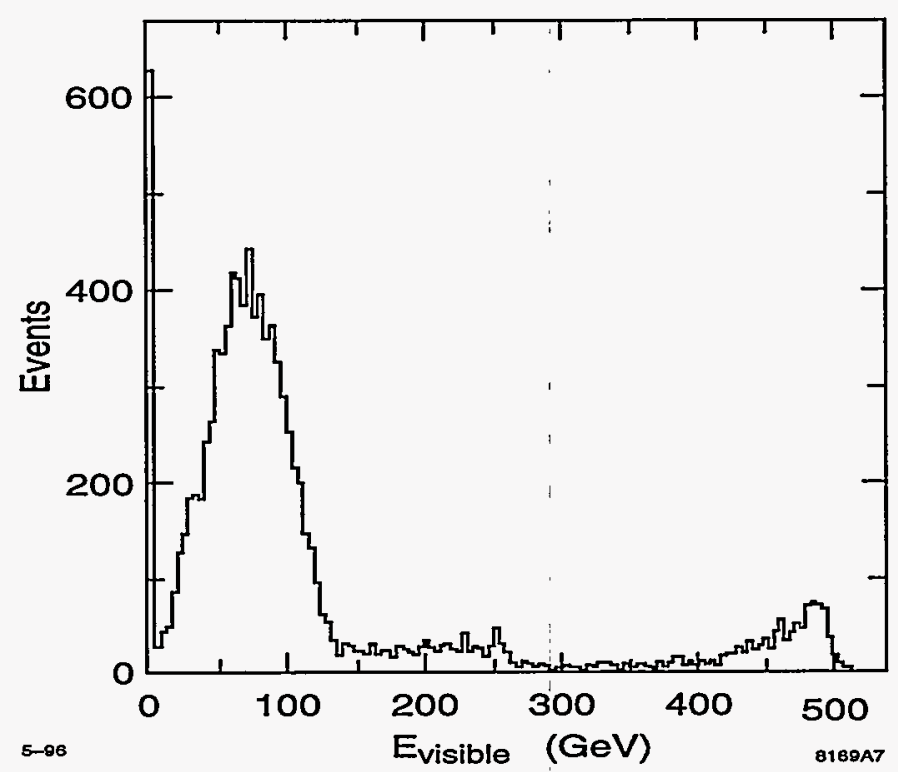

Figure 2.28: The visible energy for the supersymmetric processes defined by the SUGRA parameter set 4 after detector resolution smearing. The peak at 0 is due to $\tilde{\chi}_{1}^{0} \tilde{\chi}_{1}^{0}$ and $\tilde{\chi}_{2}^{0} \tilde{\chi}_{1}^{0} \rightarrow \nu \bar{\nu} \tilde{\chi}_{1}^{0} \tilde{\chi}_{1}^{0}$ processes. The broad small peak at $500 \mathrm{GeV}$ is due to $Z^{0} h^{0}, H^{+} H^{-}$, and $H^{0} A^{0}$ final states.

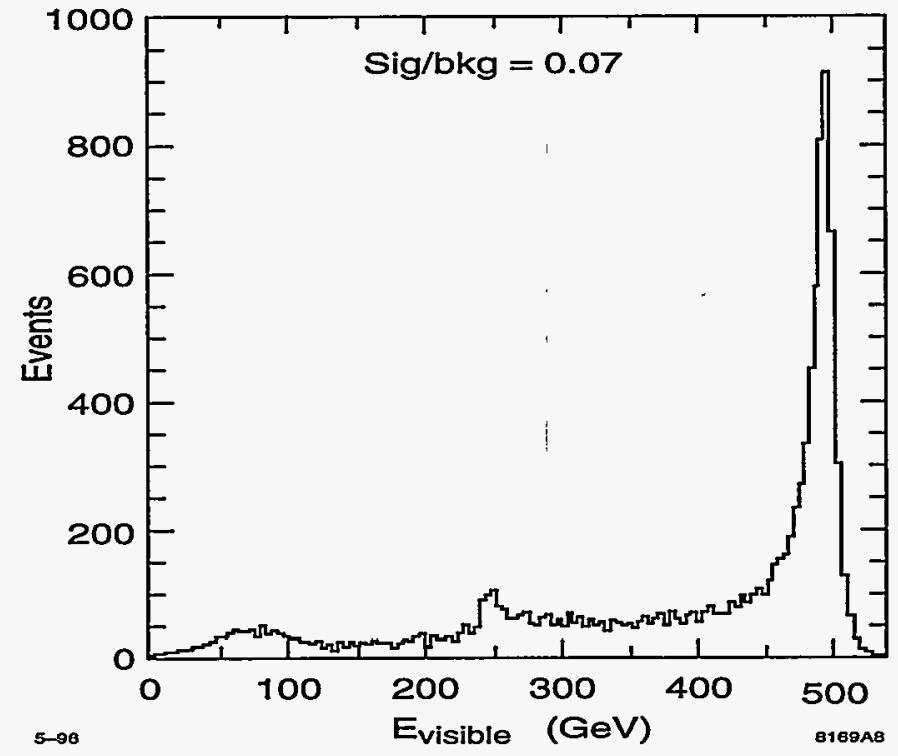

Figure 2.29: The visible energy for the normalized (relative crosssection) SUSY processes associated with SUGRA parameters 4 and the Standard Model processes. The smearing due to detector resolution is included. 


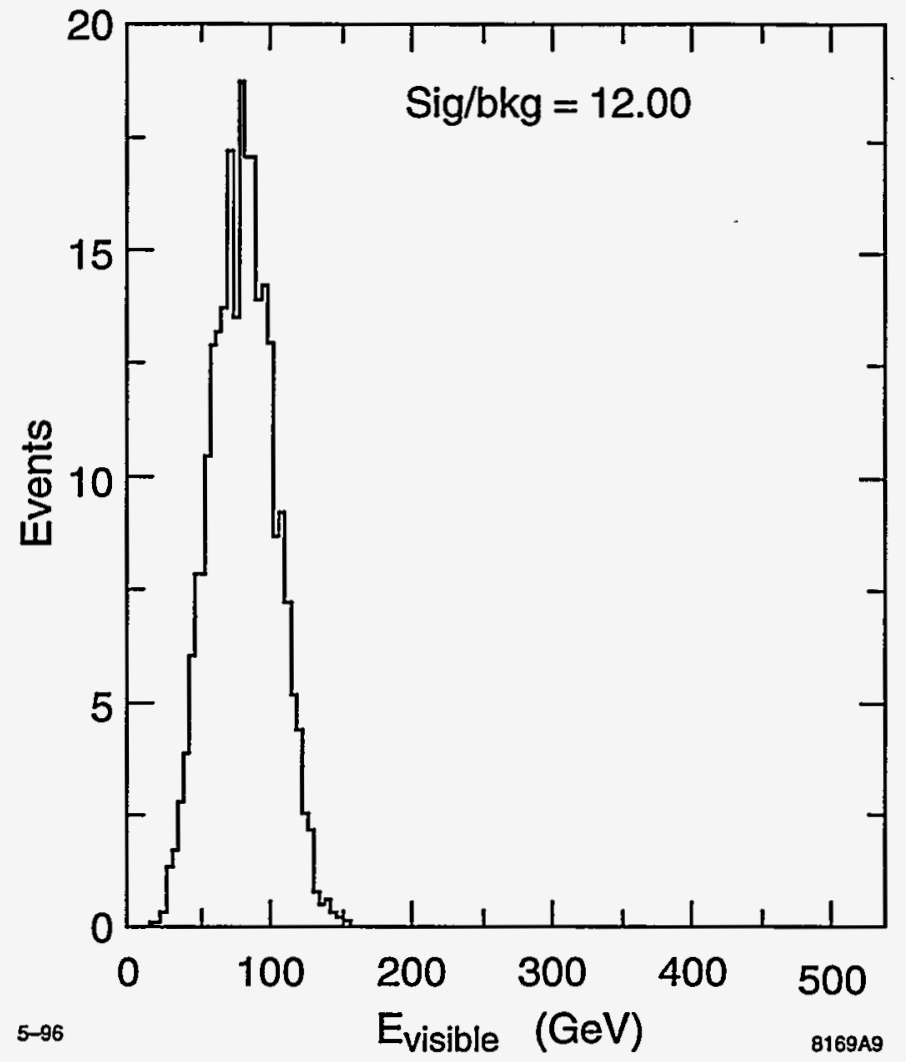

Figure 2.30: The visible energy for the normalized (relative crosssection) SUSY process $e^{+} e^{-} \rightarrow \chi_{1}^{+} \chi_{1}^{-}$associated with parameter set 4 and Standard Model processes after cuts to enhance the signal over background. The cuts are that there be only 1 broad jet with $>5$ particles and with $E_{v i s}<80 \mathrm{GeV}$ in each hemisphere.

say something about the spin of the sparticles producing these distributions. A threshold scan will also differentiate between scalars and fermions by determining whether the energy dependence follows a a $\beta$ or a $\beta^{3}$ law.

In this study, we expand on this work by increasing the number of cases that have been studied and attempt to determine how much these measurements constrain the possible region of parameter space. Here we will describe how well we can determine the masses of the supersymmetric particles using the simulated resolution parameters in our detector design. For brevity, we will only consider here the parameters sets 3 and 5 . In case 3 , the most important supersymmetry cross section for an incident $95 \%$ left-hand polarized electron beam is that of of sneutrino pair production, $\tilde{\nu}_{e} \tilde{\nu}_{e}$, as shown in Fig. 2.25. The branching ratio for $\tilde{\nu}_{e} \rightarrow e^{-} \chi_{1}^{+}$is $61 \%$. The $\chi_{1}^{+}$decays mostly to $W^{+} \chi_{1}^{0}$. Hence, $5-10 \%$ of the time we can have a final state signal $e^{\mp} e^{ \pm} \mu^{ \pm}+2$ jets. This signal will have hardly any Standard Model background. The energy distribution of the $e^{\mp}$ can be used to determine the $\tilde{\nu}_{e}$ mass. The $e^{\mp}$ energy spectrum, based on a $20 \mathrm{fb}^{-1}$ data sample, is shown in Fig. 2.33. The background 

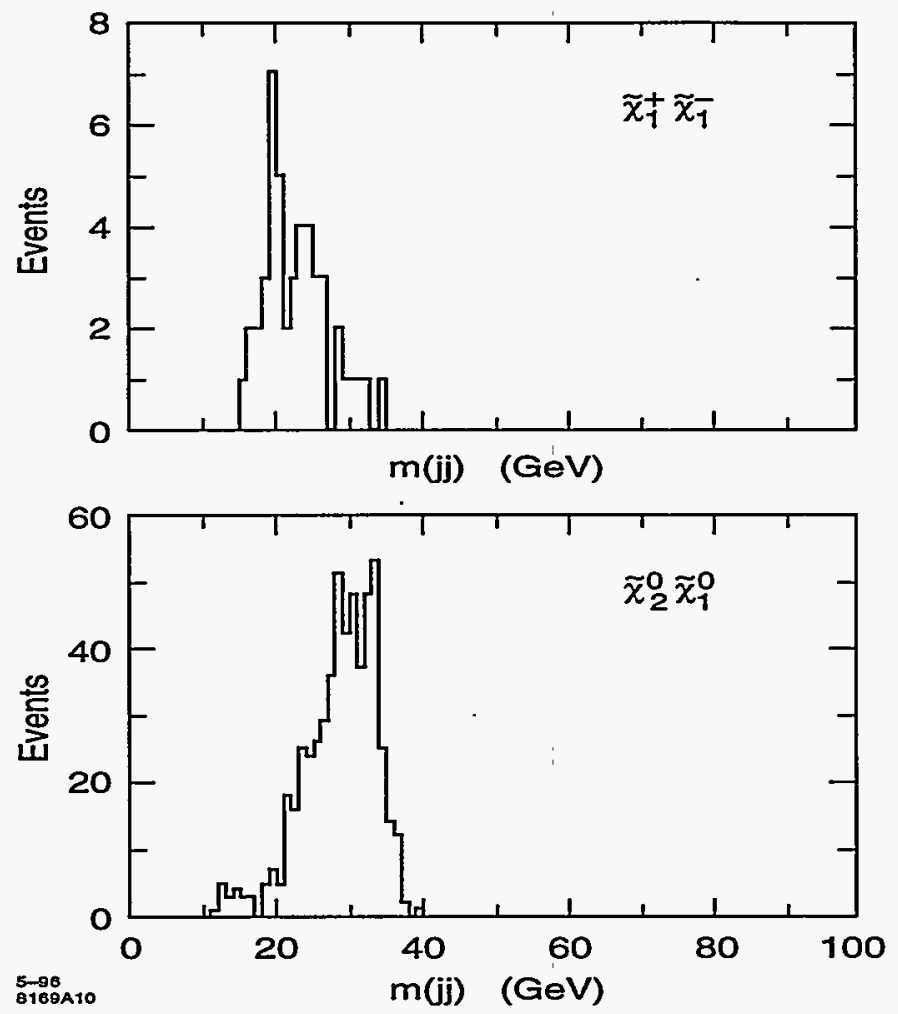

Figure 2.31: The 2-jet mass distribution for the processes $e^{+} e^{-} \rightarrow$ $\chi_{1}^{+} \chi_{1}^{-}\left(\chi_{1}^{0} \chi_{2}^{0}\right) \rightarrow q \bar{q} \chi_{1}^{0} q \bar{q} \chi_{1}^{0}\left(\chi_{1}^{0} q \bar{q} \chi_{1}^{0}\right)$. The cuts require 2 jets with more than 1 particle in each and both jets in one hemisphere only. $E_{v i s i b l e}<$ $125,70 \mathrm{GeV}$ for the two jets, and $\cos (\theta)<0.85$ for the thrust axis of the event.

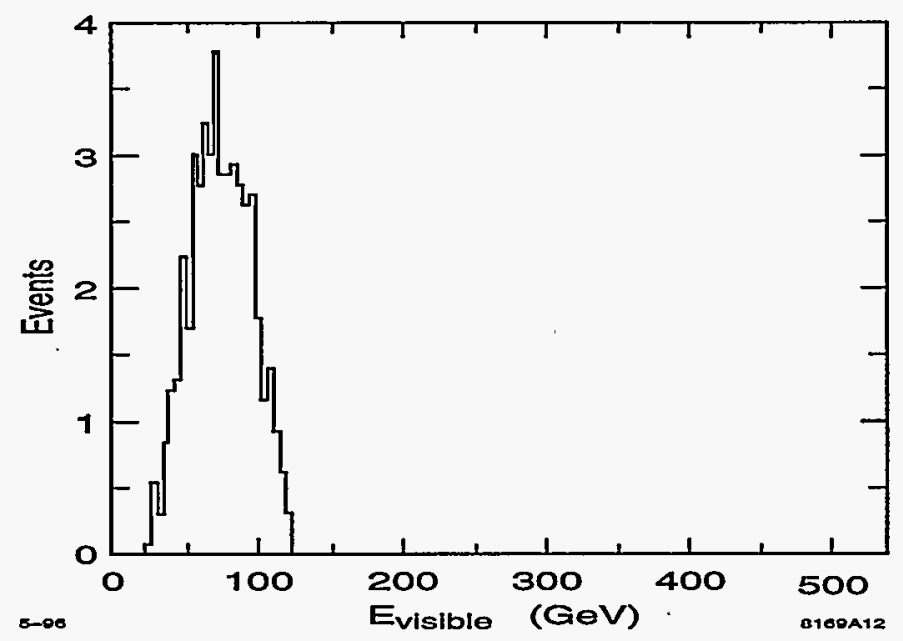

Figure 2.32: The visible energy distribution for the SUSY process $e^{+} e^{-} \rightarrow \chi_{2}^{0} \chi_{1}^{0}$ associated with parameter set 4 and the Standard Model background processes, after the cuts defined in the previous figure to enhance the signal over background. 
is shown by the dotted line. A fit to this energy spectrum leads to the following values for the masses:

$$
\begin{aligned}
& M_{\bar{\nu}_{e}}=207.5 \pm 2.5(4) \mathrm{GeV} \\
& M_{\chi_{1}^{+}}=97.0 \pm 1.2(2) \mathrm{GeV}
\end{aligned}
$$

at the $68 \%$ (90\%) confidence level. These results should be compared with the input values, given in the table, of 206.6 and $96.1 \mathrm{GeV}$ respectively.

Another interesting signal is the $\tilde{e}_{L}^{+} \tilde{e}_{L}^{-}$process in order to determine the mass of the $\tilde{e}_{L}$ (left-handed slepton). The useful signal is due to the decay chain $\tilde{e}_{\bar{L}}^{-} \rightarrow e^{-} \chi_{2}^{0} \rightarrow e^{-} Z^{0} \chi_{1}^{0} \rightarrow$ $e^{-} \mu^{-} \mu^{+} \chi_{1}^{0}$. This leads to a final state with one electron, one positron, four muons, and a small visible energy. We can also consider the analogous decay chain for $\tilde{\mu}_{L}^{+} \tilde{\mu}_{\bar{L}}^{-}$. We considered these two possibilities together by isolating final states with 6 leptons and missing energy, in which the highest energy leptons are either $e^{+} e^{-}$or $\mu^{+} \mu^{-}$. This analysis assumes that $\tilde{e}_{\bar{L}}$ and $\tilde{\mu}_{\bar{L}}$ have the same mass; with higher statistics, a mass splitting would be apparent. The results for an effective 1 year run $\left(50 \mathrm{fb}^{-1}\right)$ is shown in Fig. 2.34. The fit to the lepton energy spectrum gives the following values for the masses:

$$
\begin{aligned}
& M_{\tilde{e}_{L}}=221.6 \pm 5.6(8) \mathrm{GeV} \\
& M_{\chi_{2}^{0}}=94.7 \pm 5.3(10) \mathrm{GeV}
\end{aligned}
$$

at the $68 \%$ (90\%) confidence level, which are quite close to the input values of 215.7 and $96.8 \mathrm{GeV}$ in spite of the low statistics in this sample. Since $M_{\tilde{\nu}}-M_{\tilde{e}_{\tilde{L}}}$ is determined by just $S U(2)$ symmetry, these measurements lead to a model-independent constraint on the parameter $\tan (\beta)$ according to the equation:

$$
M_{\tilde{\nu}}^{2}-M_{\tilde{e}_{L}}^{2}=M_{W}^{2} \cos (2 \beta) .
$$

In the case of SUGRA parameter 5 we have a series of signals whose masses can be determined. This point includes a low mass stop squark $\tilde{t}_{1}$, a chargino $\chi_{1}^{+}$, neutralinos $\chi_{1}^{0}$ and $\chi_{2}^{0}$, and the light Higgs boson $h^{0}$. We measure the $\chi_{1}^{+}$and $\chi_{1}^{0}$ masses by studying the production process $\mathrm{e}^{+} \mathrm{e}^{-} \rightarrow \chi_{1}^{+}+\chi_{1}^{-}$. For a $95 \%$ left handed polarized electron the crosssection is $\approx 0.75 \mathrm{pb}$ so that for a 1 year run at our standard luminosity we get over $10^{4}$ events. The $\chi_{1}^{+}$decays into the 3 body final states $q \bar{q} \chi_{1}^{0}$ and $e \nu \chi_{1}^{0}$, with the branching ratios predicted for the Standard Model $W$ boson decay into the similar channels. Hence, about $68 \%$ of the time it will decay into two hadronic jets $+\chi_{1}^{0}$. To isolate this signal we use similar cuts to those discussed above associated with Fig. 2.30. The resulting $E_{j j}$ values, the energies of each of the 2 jet systems from the $\tilde{\chi}_{1}^{+}$decays, has no sharp end point behavior due to the 3 body nature of its decay. Hence we cannot easily use the $E_{j j}$ spectrum to determine the masses. Since the combined mass of the 2 jets, $M_{j j}$ does not vary much in this case, and since we have a large sample of events, we can force two body kinematics on this process by selecting a slice of $\mathrm{M}_{j j}$ around a given value, which in our case is chosen to be $30 \mathrm{GeV}$. Hence the $\mathrm{E}_{j j}$ distribution follows approximately the two body kinematics of the 
process $\tilde{\chi}_{1}^{+} \rightarrow \tilde{\chi}_{1}^{0}+(j j)(30 \mathrm{GeV})$. The $\mathrm{E}_{j j}$ distribution and the best mass fit to the data is shown in Fig. 2.35. The result is:

$$
\begin{gathered}
M_{\tilde{\chi}_{1}^{+}}=107.5 \pm 6.5 \mathrm{GeV} \\
M_{\tilde{\chi}_{1}^{0}}=55.0 \pm 3.5 \mathrm{GeV}
\end{gathered}
$$

at the $68 \%$ confidence level. This is to be compared with the input values of 109.8 and 57.0 $\mathrm{GeV}$ respectively.

Finally, for parameter point 5 , we have also studied determining the mass of the $\tilde{t}_{1}$ (stop) quark. Here we note that the process $e^{+} e^{-} \rightarrow \tilde{t}_{1}^{+} \tilde{t}_{1}^{-} \rightarrow b \tilde{\chi}_{1}^{+} \bar{b} \tilde{\chi}_{1}^{-}$occurs with a $100 \%$ branching ratio. Since this cross section hardly depends on the electron polarization we study this case with a $95 \%$ right handed polarized electron $\left(P_{L}\left(e^{-}\right)=-0.9\right)$ to minimize the background from $W W$ pair production [67]. We isolate the events with $\geq 5$ jets, and we select from these events with two tagged $b$ 's, and no isolated leptons or $\tau$ jets. Finally we require a missing mass $>140 \mathrm{GeV}$. For our standard 1 year run we obtain a SUSY signal of 286 events with a $W W$ background of 36 events. The energy distribution of the $b$-jets is shown in Fig. 2.36. This distribution depends on the mass of the $\tilde{t}_{1}$ and the mass of the $\tilde{\chi}_{1}^{+}$. The masses we obtain are:

$$
\begin{aligned}
& M_{\bar{t}_{1}}=182 \pm 11 \mathrm{GeV} \\
& M_{\bar{\chi}_{1}^{+}}=114 \pm 8 \mathrm{GeV}
\end{aligned}
$$

to be compared with the input values of 180 and $110 \mathrm{GeV}$ respectively. Other interesting work on squark mass determination [73] has also been carried out.

We hope that in this short presentation we have indicated the effectiveness of an 0.51.0 $\mathrm{TeV} e^{+} e^{-}$Linear Collider in determining the masses of the Supersymmetric particles. We have not discussed how to determine the spin of these. This we propose to accomplish where possible by looking at their production angular distribution and by looking at their production behavior as a function of the electron longitudinal polarization. This work will continue in order to determine further what additional parameters need to be determined to be able to guarantee that the signals we observe are due to supersymmetric particles.

\subsubsection{Supersymmetry Reach of the NLC and LHC}

To conclude, we make a brief comparison of the relative reach capabilities of a $0.5-1.5 \mathrm{TeV}$ $e^{+} e^{-}$Linear Collider and the CERN LHC $p p$ collider as to their ability to determine whether the observed signals are due to supersymmetric particles. First of all, the NLC, operating at $\mathrm{E}_{\mathrm{cm}} \geq 250-300 \mathrm{GeV}$ should be able to search for the light Higgs boson, $h^{0}$, over the entire parameter space range of the minimal supersymmetry model. If the NLC does not observe the $h^{0}$, then this model must be ruled out. In addition, since the $h^{0}$ is expected to behave very nearly like a Standard Model Higgs boson, even if it is discovered, it may be difficult to tell if it is a SUSY or Standard Model Higgs. Hence, discovery of the $h^{0}$ alone may not be 
sufficient evidence for supersymmetry. On the other hand, the NLC has a substantial ability to discover many of the superpartners.

In Fig. 2.37, we show our estimates of the reach of NLC and LHC into the SUGRA parameter space, defined by underlying mass parameters $m_{0}$ and $m_{1 / 2}$. In the top figure, we have plotted the contours corresponding to $\tilde{\ell}_{R}$ and $\tilde{\chi}_{1}^{ \pm}$masses of 250,500 and $750 \mathrm{GeV}$, approximately representing the reach of $\mathrm{NLC}(0.5 \mathrm{TeV}), \mathrm{NLC}(1.0)$ and $\mathrm{NLC}(1.5)$ in observing these supersymmetric particles. We also show the reach for supersymmetry recently calculated [74] for the CERN LHC assuming $10 \mathrm{fb}^{-1}$ of integrated luminosity. Comparing the two figures we note that the reach of the LHC is larger than that of the NLC at 500 $\mathrm{GeV}$, but its reach is comparable to that of the NLC at $1 \mathrm{TeV}$. It is important to note that the reactions at NLC and LHC typically access different particles in the supersymmetry spectrum, so the experiments at these colliders should be considered cooperative rather than competitive. In addition, precision measurements of particle properties such as mass, spin, and mixing angles will be much easier at the NLC [68] than at the LHC. The LHC might be able to provide complementary information via squark and gluino production channels which may not be accessible at the NLC. 

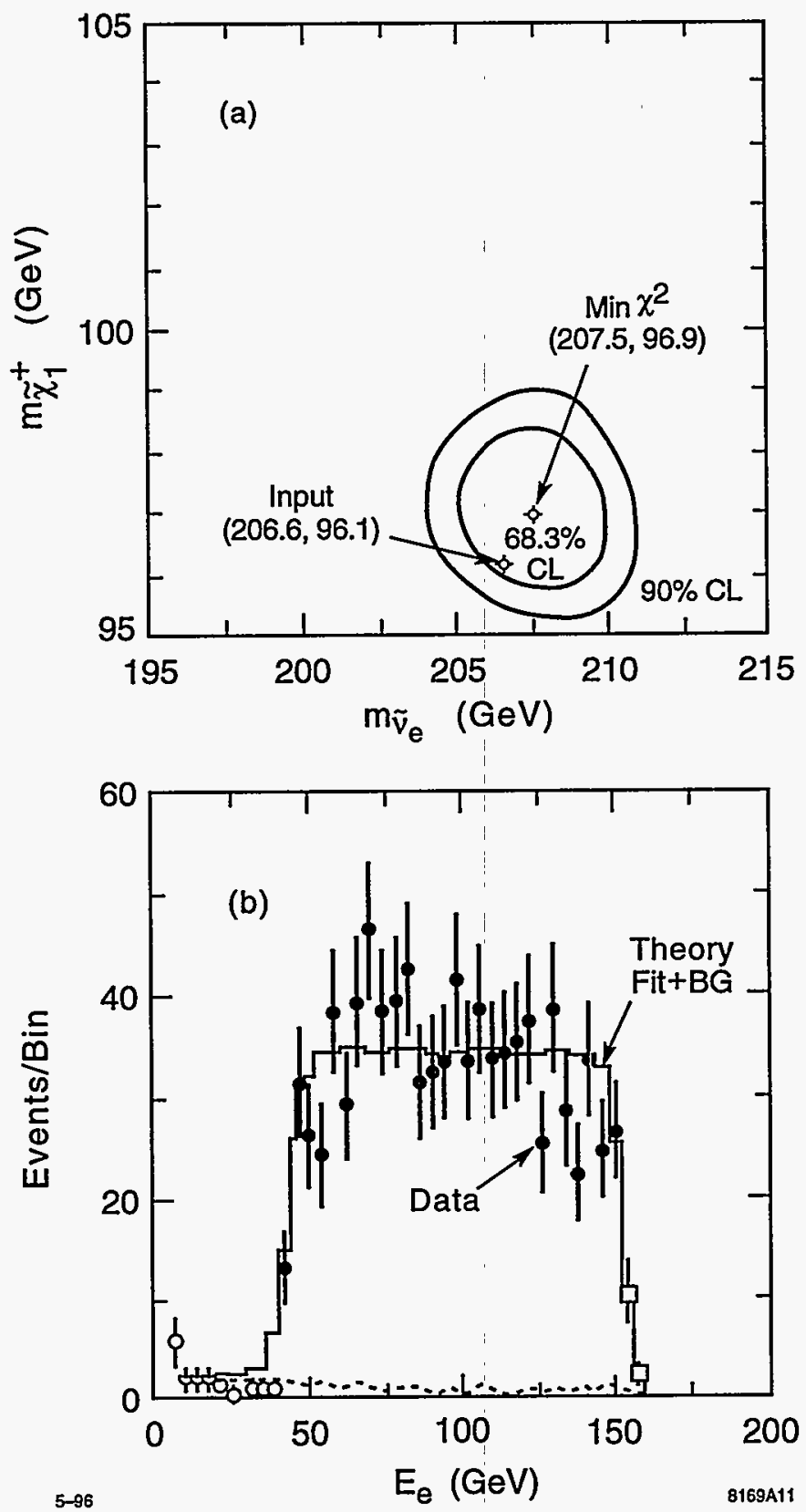

Figure 2.33: The electron energy distribution in the process $e^{+} e^{-} \rightarrow$ $\tilde{\nu}_{e} \tilde{\nu}_{e} \rightarrow e^{-} \chi_{1}^{+} e^{+} \chi_{1}^{-} \rightarrow e^{-} \mu^{+-} \chi_{1}^{0} e^{+} \chi_{1}^{0}+2$ jets and the fit that determines the mass of the $\tilde{\nu}_{e}=207.5 \pm 2.5 \mathrm{GeV}$ and the mass of the $\chi_{1}^{+}=97.0$ $\pm 1.2 \mathrm{GeV}$ at the $68 \%$ C.L. 

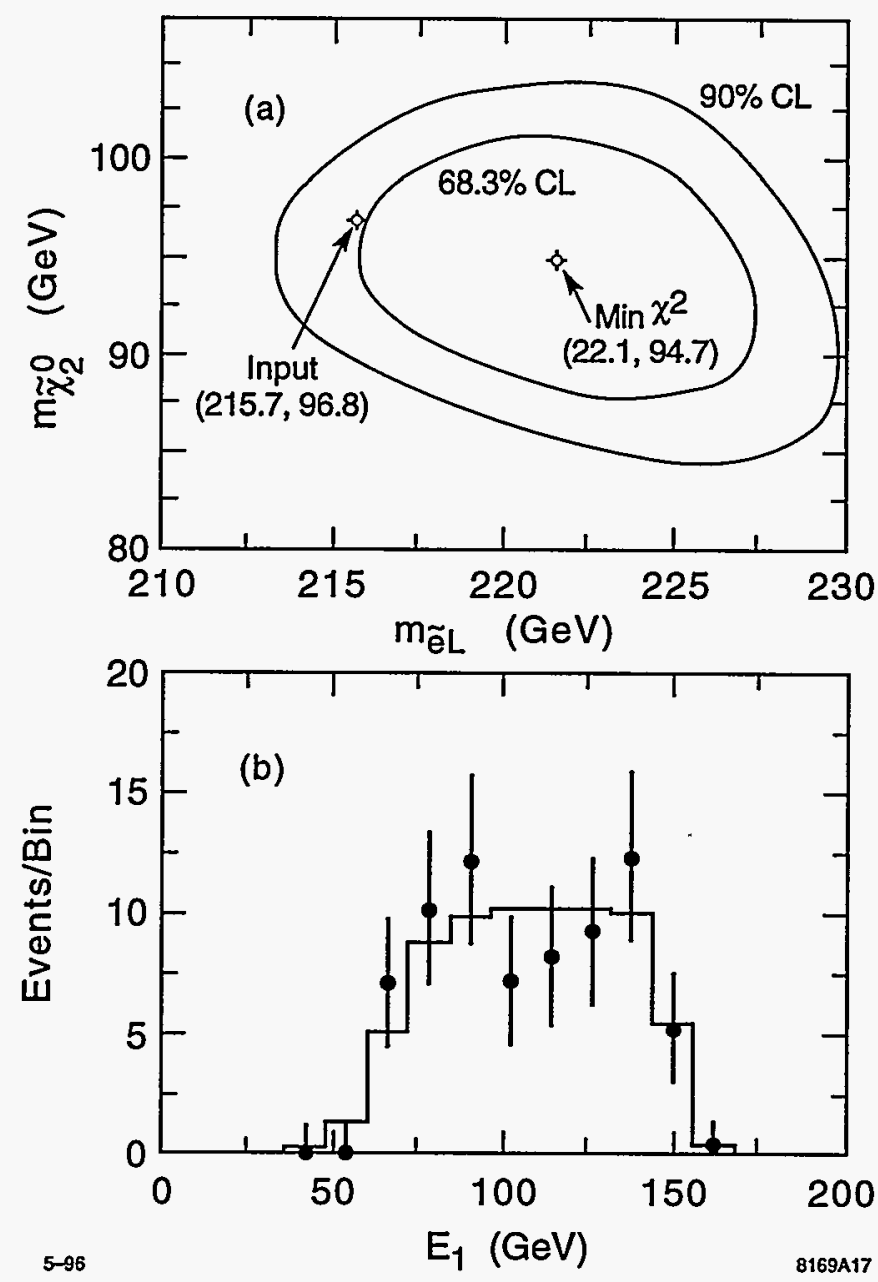

Figure 2.34: The energy distribution of the two highest energy leptons in the process $e^{+} e^{-} \rightarrow \tilde{\ell}_{L}^{-} \tilde{\ell}_{L}^{+} \rightarrow \ell^{-} \tilde{\chi}_{2}^{0} \ell^{+} \tilde{\chi}_{2}^{0} \rightarrow \ell^{-} Z^{0} \chi_{1}^{0} \ell^{+} Z^{0} \chi_{1}^{0} \rightarrow \operatorname{six}$ leptons plus missing energy, and the fit that determines the mass of the $\tilde{\ell}_{L}$ and the $\chi_{2}^{0}$. Only those events were considered in which the highest energy leptons were $e^{+} e^{-}$or $\mu^{+} \mu^{-}$. The fit gives the mass values $M_{\bar{\ell}_{L}}=221.6 \pm 5.6 \mathrm{GeV}$ and $M_{\tilde{\chi}_{2}^{0}}=94.7 \pm 5.3 \mathrm{GeV}$ 

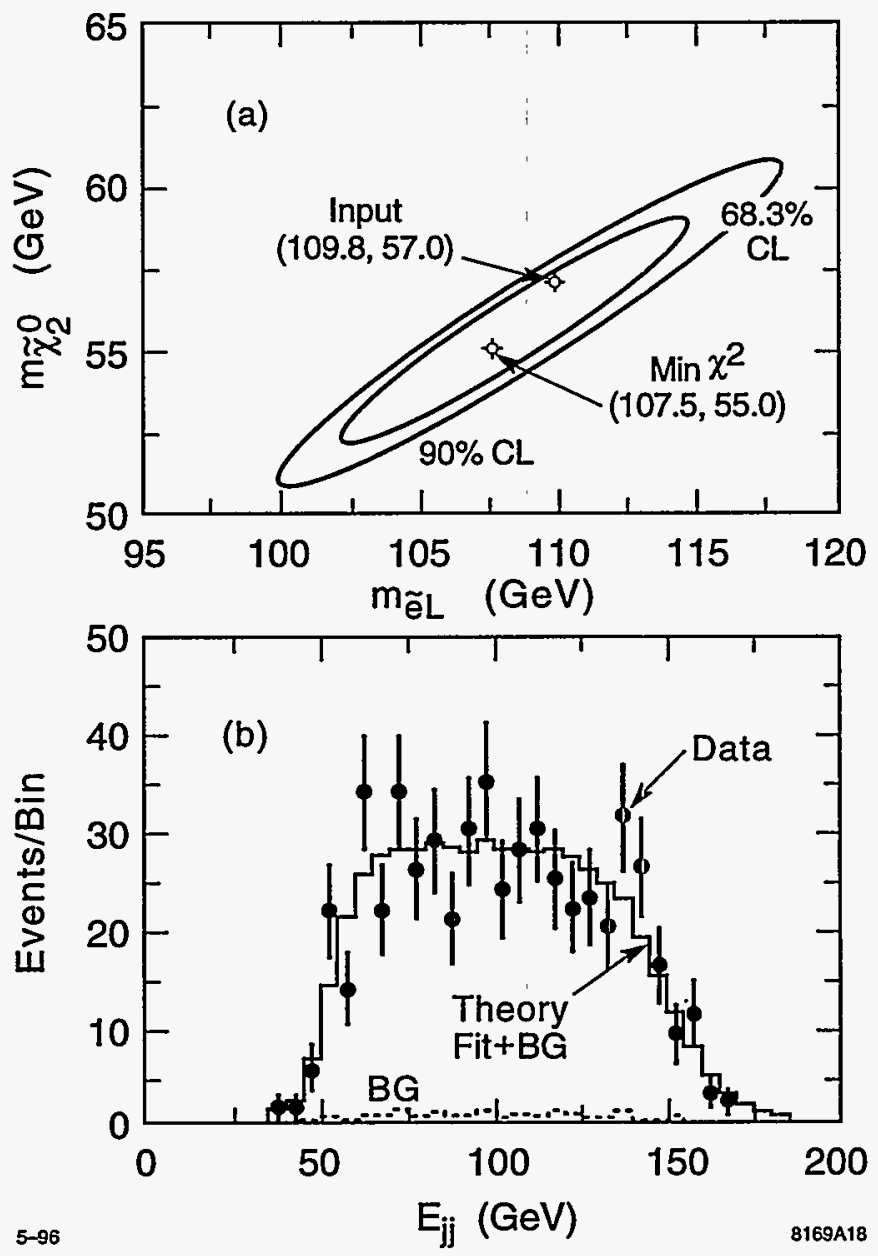

Figure 2.35: The quark pair energy distribution in the process $e^{+} e^{-} \rightarrow$ $\tilde{\chi}_{1}^{+} \tilde{\chi}_{1}^{-} \rightarrow q \bar{q} \tilde{\chi}_{1}^{0} q \bar{q} \tilde{\chi}_{1}^{0}$ and the fit to the $\tilde{\chi}_{1}^{+}, \tilde{\chi}_{1}^{0}$ masses. The fit gives the mass values $M_{\tilde{\chi}_{1}^{+}}=107.5 \pm 6.5 \mathrm{GeV}$ and $M_{\tilde{\chi}_{1}^{0}}=55.0 \pm 3.5 \mathrm{GeV}$. 

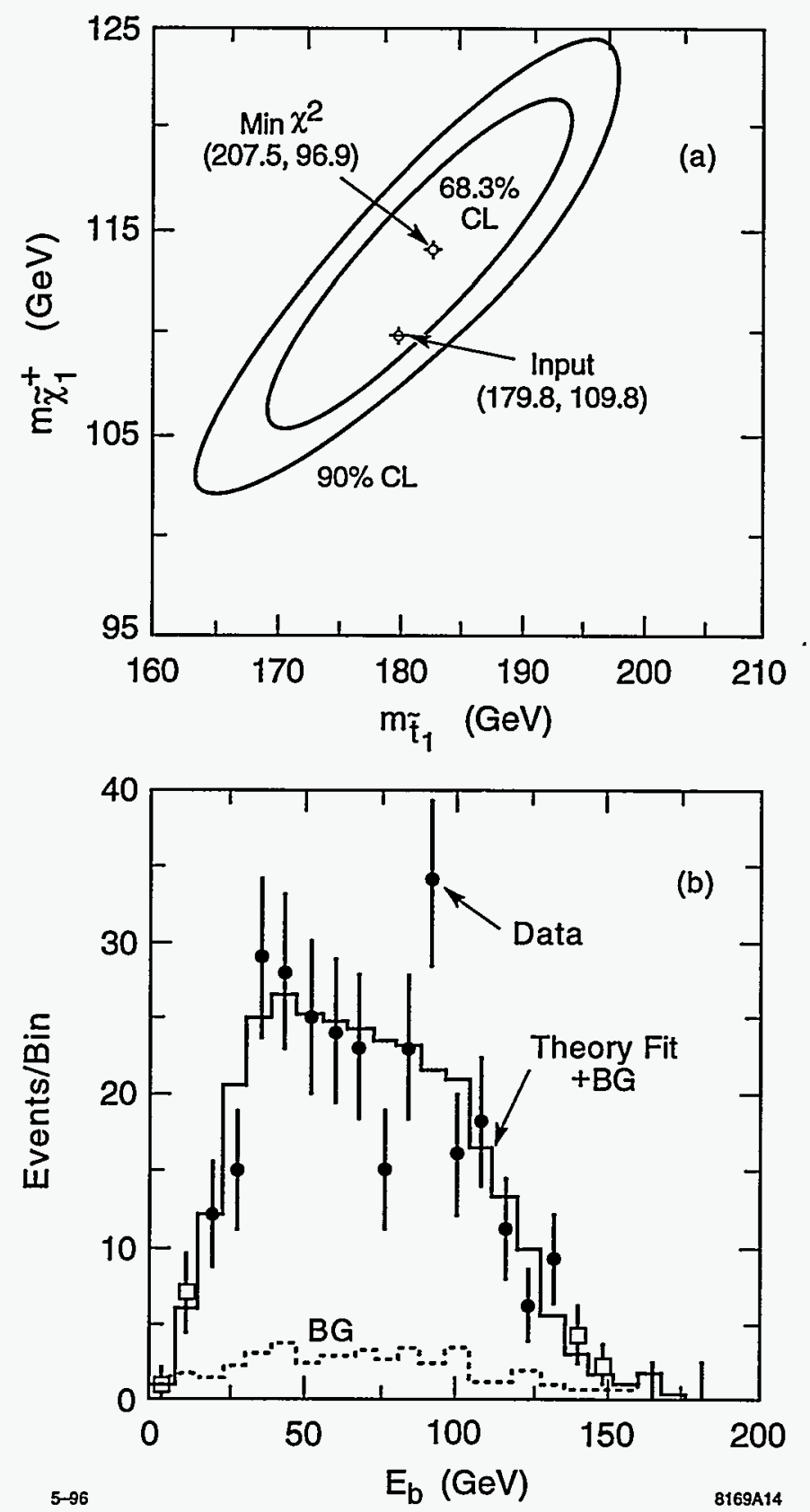

Figure 2.36: The b-jet energy distribution in the process $e^{+} e^{-} \rightarrow \tilde{t}_{1} \overline{\tilde{t}_{1}} \rightarrow$ $b \tilde{\chi}_{1}^{+} \bar{b} \tilde{\chi}_{1}^{-}$and the fit to the $\tilde{t}_{1} \tilde{\chi}_{1}^{+}$masses. The fit gives the mass values $M_{\tilde{t}_{1}}=182 \pm 11 \mathrm{GeV}$ and $M_{\tilde{\chi}_{1}^{+}}=114 \pm 8 \mathrm{GeV}$. 

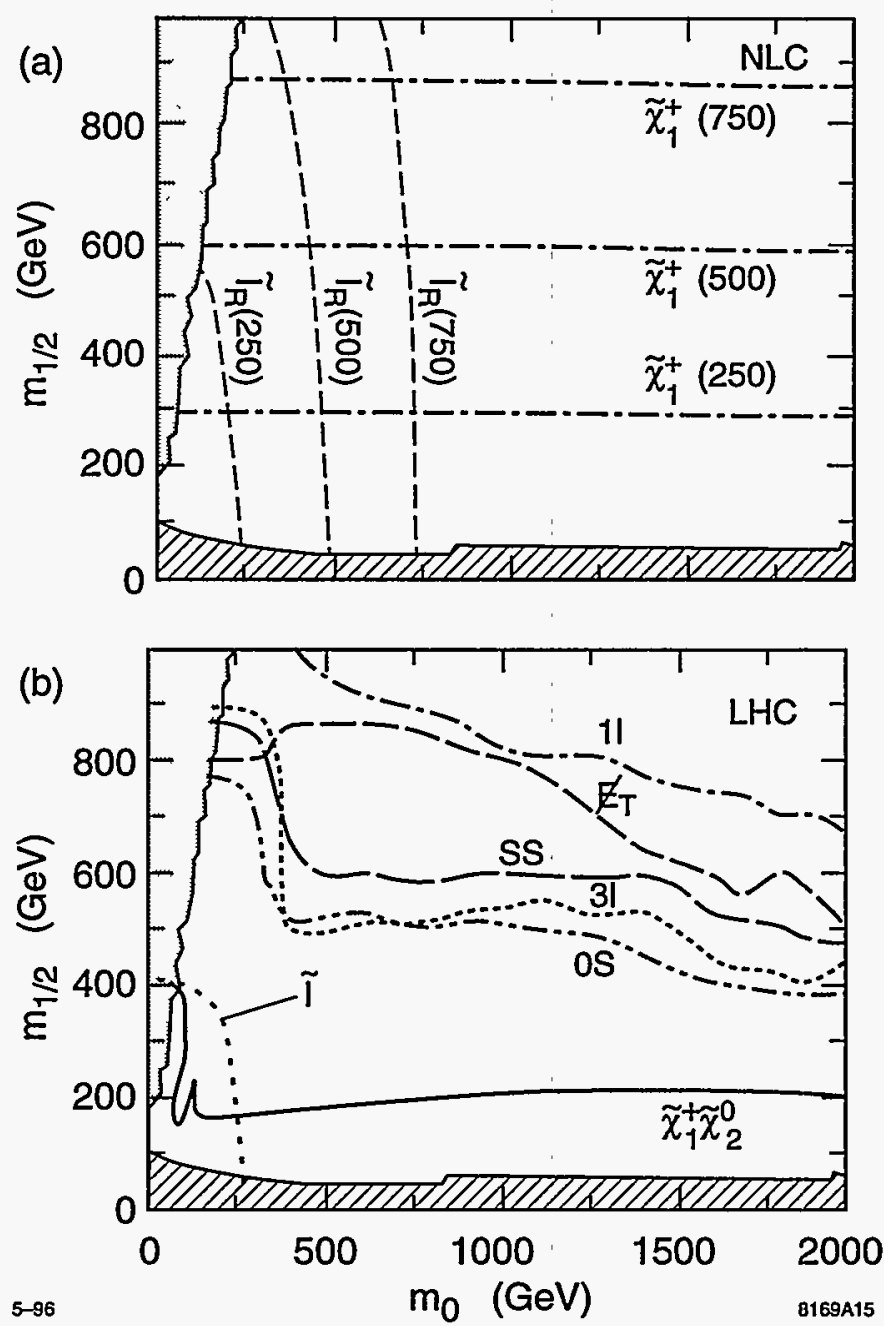

Figure 2.37: Comparison of the reach of the NLC(0.5 TeV), NLC(1.0), NLC(1.5) presented in the top figure, and the LHC assuming an integrated luminosity of $10 \mathrm{fb}^{-1}$ in the bottom figure. The contours are labeled $\tilde{\ell}$ for the slepton reach, $\tilde{\chi}_{1}^{+} \tilde{\chi}_{1}^{0}$ for the $\tilde{\chi}_{1}^{+} \tilde{\chi}_{1}^{0} \rightarrow 3 \ell$ reach, $1 \ell$ for the reach via lepton + jets $+\not_{T}$ events, $\not_{T}$ for events with multi-jets $+\not_{T}$, SS for same-sign dileptons + jets $+Z_{T}$ and $3 \ell$ for trilepton + jets $+H_{T}$. 


\subsection{Anomalous Gauge Boson Couplings}

Although the Standard Electroweak Model has been verified to astounding precision in recent years at LEP and SLC, one important component has not been tested directly with significant precision: the non-Abelian self couplings of the weak vector gauge bosons. Deviations of non-abelian couplings from expectation would signal new physics, perhaps arising from unexpected loop corrections involving propagators of new particles. In addition, as will be discussed in Chapter 8, precise measurements of $W W V$ couplings, where $V=\gamma$ or $Z$, can provide important information on the nature of electroweak symmetry breaking. Recent results from CDF and D0 indicate the presence of triple gauge boson couplings, but have not yet reached a precision better than order unity [75]. Upcoming measurements at LEP II, at an upgraded Tevatron and at the LHC will improve upon this precision considerably, but cannot the match the expected precision of a $500 \mathrm{GeV} \mathrm{NLC,} \mathrm{much} \mathrm{less} \mathrm{that} \mathrm{of} \mathrm{a} 1.0$ or 1.5 TeV NLC. There exist indirect constraints on the anomalous couplings from the precision electroweak measurements at the $Z^{0}$ resonances, in particular, from the fact that loop diagrams involving weak vector bosons are seen to take the values expected in the Standard Model. However, the ambiguities in the calculation of these diagrams call for more direct measurements [76].

In this brief report we restrict attention mainly to measurement of possibly anomalous $W W V$ couplings via the process $e^{+} e^{-} \rightarrow W^{+} W^{-}$, but much work has been done on other processes that involve non-abelian couplings in $e^{+} e^{-}$annihilation, including $Z Z \gamma, Z \gamma \gamma$, $W W Z Z$, and $W W W W$ [75]. In addition, many of these couplings can also be measured independently using the $e^{-} e^{-}, e^{-} \gamma$ and $\gamma \gamma$ options for the NLC. We will describe one common parametrization of anomalous $W W V$ couplings, summarize present and expected pre-NLC measurements of $W W V$ couplings, and discuss in more detail what can be done at the NLC.

\subsubsection{Parametrization}

In parametrizing anomalous couplings, we follow the notation of Ref. [4] in which the generic effective Lagrangian for the $W W V$ vertex is written:

$$
\begin{aligned}
L_{W W V} / g_{W W V}= & i g_{1}^{V}\left(W_{\mu \nu}^{\dagger} W^{\mu} V^{\nu}-W_{\mu}^{\dagger} V_{\nu} W^{\mu \nu}\right)+i \kappa_{V} W_{\mu}^{\dagger} W_{\nu} V^{\mu \nu}+\frac{i \lambda_{V}}{M_{W}^{2}} W_{\lambda \mu}^{\dagger} W_{\nu}^{\mu} V^{\nu \lambda} \\
& -g_{4}^{V} W_{\mu}^{\dagger} W_{\nu}\left(\partial^{\mu} V^{\nu}+\partial^{\nu} V^{\mu}\right)+g_{5}^{V} \epsilon^{\mu \nu \rho \sigma}\left(W_{\mu}^{\dagger} \partial_{\rho} W_{\nu}\right) V_{\sigma}+\tilde{\kappa}_{V} W_{\mu}^{\dagger} W_{\nu} \tilde{V}^{\mu \nu} \\
& +\frac{i \tilde{\lambda}_{V}}{M_{W}^{2}} W_{\lambda \mu}^{\dagger} W_{\nu}^{\mu} \tilde{V}^{\nu \lambda}
\end{aligned}
$$

where $W_{\mu \nu} \equiv \partial_{\mu} W_{\nu}-\partial_{\nu} W_{\mu}, V_{\mu \nu} \equiv \partial_{\mu} V_{\nu}-\partial_{\nu} V_{\mu},\left(A \partial_{\mu} B\right) \equiv A\left(\partial_{\mu} B\right)-\left(\partial_{\mu} A\right) B$, and $\tilde{V}_{\mu \nu} \equiv$ $\frac{1}{2} \epsilon_{\mu \nu \rho \sigma} V^{\rho \sigma}$. The normalization factors are defined for convenience to be $g_{W W \gamma} \equiv-e$ and $g_{W W Z} \equiv-e \cot \theta_{W}$. The 7 coupling parameters defined in Eq. 2.11 for each of $\gamma$ and $Z$ include the $\mathrm{C}$ and $\mathrm{P}$ violating couplings $g_{5}^{V}$ as well as the CP-violating couplings $g_{4}^{V}, \tilde{\kappa}_{V}, \tilde{\lambda}_{V}$. In most studies and in this one, such terms are neglected. At tree level in the Standard 
Model $g_{1}^{V}=\kappa_{V}=1$ and $\lambda_{V}=g_{4}^{V}=g_{5}^{V}=\tilde{\kappa}_{V}=\tilde{\lambda}_{V}=0$. The couplings in Eq. 2.11 should properly be written as form factors with momentum-dependent values. This complication is of little importance at an $e^{+} e^{-}$collider where the $W W$ center of mass energy is well defined, but it must be borne in mind for other processes, particularly those measurable at hadron colliders.

We follow the common convention in defining $\Delta g_{1}^{Z} \equiv g_{1}^{Z}-1$ and $\Delta \kappa_{V} \equiv \kappa_{V}-1$. The $W$ electric charge fixes $g_{1}^{\gamma}\left(q^{2} \rightarrow 0\right) \equiv 1$. In perhaps more familiar notation, one can express the $W$ magnetic dipole moment as $\mu_{W} \equiv \frac{e}{2 M_{W}}\left(1+\kappa_{\gamma}+\lambda_{\gamma}\right)$ and the $W$ electric quadrupole moment as $Q_{W} \equiv-\frac{e}{M_{W}^{2}}\left(\kappa_{\gamma}-\lambda_{\gamma}\right)$.

In any model with new physics at high energy that couples to the $W$ boson, the anomalous couplings will be induced at some level. A useful way to represent this effect is to write an $S U(2) \times U(1)$-invariant effective Lagrangian to represent the effects of the new physics, and then to couple this to the weak vector bosons by gauging the symmetry. In the literature, this has been done using both linear and nonlinear effective Lagrangians $[76,77,78]$. Typically, the anomalous couplings predicted in such models are suppressed by factors of $M_{W}^{2} / \Lambda^{2}$, where $\Lambda$ is a multi-TeV scale, or by factors $\alpha_{w} / 4 \pi$. These lead to typical values of the anomalous couplings below $10^{-2}$ and make it difficult to observe these couplings before directly observing the new physics itself. In the Standard Model one expects loop contributions of $O\left(10^{-3}\right.$ [79]. In the supersymmetry model, one might expect loop contributions, depending on the values of the supersymmetry parameters [80].

For the present discussion, we use a linear realization with the additional constraint of equal couplings for the $\mathrm{U}(1)$ and $\mathrm{SU}(2)$ terms in the effective Lagrangian that contribute to anomalous triple gauge boson couplings. This gives an effective lagrangian with two free parameters, which we will take to be $K_{\gamma}$ and $\lambda_{\gamma}$. This restriction is called the 'HISZ scenerio' [81]. This restriction of the parameter space has recently been applied to comparative studies of the anomalous $W$ couplings at colliders [75]. It is important to note, however, that studies of $e^{+} e^{-} \rightarrow W^{+} W^{-}$at the NLC can also test this hypothesis by independently determining the $\gamma$ and $Z$ couplings to the $W$ [82].

\subsubsection{Present and Expected Pre-NLC Measurements}

The only present direct measurements of $W W V$ couplings come from the CDF and D0 Experiments [75] at the Tevatron, which have searched for $W W, W Z$, and $W \gamma$ production. The $W W$ and $W Z$ searches have yielded $O(1)$ candidates and the $W \gamma$ searches have yielded $O(10)$ candidates, consistent with expectation. These observations have led to limits on the corrections to the coupling parameters of order unity. For example, D0 sets a $95 \%$ CL range $-1.8<\Delta \kappa_{\gamma}<1.9$ assuming $\lambda_{\gamma}=0$ or a range $-0.6<\lambda_{\gamma}<0.6$ assuming $\kappa_{\gamma}=0$ (both limits assume $\Lambda=1 \mathrm{TeV}$ in the appropriate form factors).

One expects significant improvement at LEP II [83], once the accelerator exceeds the $W$-pair threshold energy. Techniques similar to those described below will be applied to $e^{+} e^{-} \rightarrow W^{+} W^{-}$events at c.m. energies ranging from threshold at $\approx 160 \mathrm{GeV}$ to $\approx 195 \mathrm{GeV}$. 
Assuming no anomalous couplings are observed after an integrated luminosity of $500 \mathrm{pb}^{-1}$, $95 \%$ CL limits on individual couplings (all others set to zero) of $O(0.1)$ are expected.

After the Main Injector upgrade has been completed, it is expected that the Tevatron will collect $O(1-10) \mathrm{fb}^{-1}$ of data. (Further upgrades in luminosity are also under discussion.) If $10 \mathrm{fb}^{-1}$ is achieved, it is expected [75] that limits on $\Delta \kappa_{\gamma}$ and $\lambda_{\gamma}$ will be obtained that are competitive with those from LEP II with $500 \mathrm{pb}^{-1}$.

Finally, one expects the LHC accelerator to turn on sometime before the NLC and to look for the same signatures considered at the Tevatron. The planned luminosity and c.m. energy, however, give the LHC a large advantage over even the Main Injector Tevatron in probing anomalous couplings. The ATLAS Collaboration has estimated [24] that with 100 $\mathrm{fb}^{-1}$, one can obtain (in the HISZ scenario) $95 \%$ CL limits on $\Delta \kappa_{\gamma}$ and $\lambda_{\gamma}$ in the range 5-10 $\times 10^{-3}$. We should note that these studies do not yet include helicity analysis on the $W$ bosons.

\subsubsection{Measurements in $W$ Pair Production at the NLC}

The fact that the expected LHC limits improve dramatically upon those of a high-luminosity Tevatron indicates the importance of center-of-mass energy. This reflects the fact that, in the effective Lagrangian description, the anomalous couplings arise from higher-dimension effective interactions.

The NLC has an added advantage over hadron colliders in reconstructing $W$ pair events due to absence of spectator partons. To a good approximation, full energy and momentum conservation constraints can be applied to the visible final states. Thus an $e^{+} e^{-} \rightarrow W^{+} W^{-}$ event can ideally be characterized by five angles: the production angle $\Theta_{W}$ of the $W^{-}$with respect to the electron beam, the polar and azimuthal decay angles $\theta^{*}$ and $\phi^{*}$ of one daughter of the $W^{-}$in the $W^{-}$reference frame, and the corresponding decay angles $\bar{\theta}^{*}$ and $\bar{\phi}^{*}$ of one of the $W^{+}$daughters. In practice, initial-state photon radiation and final-state photon and gluon radiation (in hadronic $W$ decays) complicate the picture. So does the finite width of the $W$. Nevertheless, for the studies below, we will characterize $e^{+} e^{-} \rightarrow W^{+} W^{-}$events by these five angles and fit distributions in the angles to obtain values of anomalous couplings.

At high energies, the $e^{+} e^{-} \rightarrow W^{+} W^{-}$process is dominated by $t$-channel $\nu_{e}$ exchange, leading primarily to very forward-angle $W^{\prime}$ 's where the $W^{-}$has an average helicity near minus one. This makes the bulk of the cross section difficult to observe with precision. However, the amplitudes affected by the anomalous couplings are not forward peaked; the central and backward-scattered $W$ 's are measurably altered in number and helicity by the couplings. $W$ helicity analysis through the decay angular distributions provides a powerful probe of anomalous contributions. Most studies, including those discussed here, restrict attention to events for which $\left|\cos \Theta_{W}\right|<0.8$. Not surprisingly, the most powerful channel to use is one in which one $W$ decays leptonically (to $e \nu$ or $\mu \nu$ ) and the other hadronically $(\approx 30 \%$ of all $e^{+} e^{-} \rightarrow W^{+} W^{-}$events) Although one gains statistics when both $W^{\prime}$ 's decay hadronically, one loses considerable discriminating power from being unable to purely tag the charge of 
the $W$ daughter quarks. The channel in which both $W$ 's decay leptonically suffers from both poor statistics and kinematic ambiguities due to two undetected neutrinos. For the leptonic $v s$ hadronic channel, the lepton energy carries important information for kinematic reconstruction. For simplicity, most studies have not yet attempted to incorporate $W \rightarrow \tau \nu$ decays.

Figure 2.38 (taken from Ref. [84]) shows $95 \%$ CL exclusion contours in the plane $\lambda_{\gamma}$ vs $\Delta \kappa_{\gamma}$ in the HISZ scenario for different c.m. energies and integrated luminosities $\left(0.5 \mathrm{fb}^{-1}\right.$ at $190 \mathrm{GeV}, 80 \mathrm{fb}^{-1}$ at $500 \mathrm{GeV}$, and $190 \mathrm{fb}^{-1}$ at $1500 \mathrm{GeV}$ ). These contours are based on ideal reconstruction of $W$ daughter pairs produced on mass-shell with no initial-state radiation. The contours represent the best one could possibly do. A previous study [82] assuming a very high-performance detector but including initial-state radiation and a finite $W$ width found some degradation in these contours, primarily due to efficiency loss when imposing kinematic requirements to suppress events far off mass-shell or at low effective c.m. energies. Nevertheless, one attains a precision of $O\left(10^{-3}\right)$ at $\mathrm{NLC}(500)$ and $O\left(\right.$ few $\left.\times 10^{-4}\right)$ at $\mathrm{NLC}(1500)$. Another nice feature of coupling studies at NLC is the ability to disentangle couplings in models more general than HISZ via tuning of the electron beam polarization, as shown in Ref. [82].

A study [85] undertaken for this workshop has examined the effects of detector resolution on achievable precisions. One might expect a priori that the charged track momentum resolution would be most critical since the energy spectrum for the $W$-daughter muons peaks at a value just below the beam energy, falling off nearly linearly with decreasing energy. One might also expect the hadron calorimeter energy resolution to be important in that it affects the energy resolution of jets to be identified with underlying $W$-daughter quarks. Indeed, one finds that these resolutions do matter, but that the nominal resolution parameters assumed for the purposes of this workshop are quite adequate. A significant source of degradation in sensitivity to anomalous couplings comes from initial state photon radiation, aggravated by beamsstrahlung, the presence of which is difficult to establish in a single lepton-jets event, given the undetected neutrino. In this respect, the four-jets channel in which both $W$ 's decay hadronically, is more promising. Further investigation of this channel is in progress.

In summary, the studies reviewed in this section confirm the power of the NLC to extract anomalous couplings. We expect some degradation in coupling parameters precision from the ideal case due to the underlying physical phenomena of initial state photon radiation and the finite $W$ width and a smaller degradation from the imperfection of matching detected particles to primary $W$ daughters, but these effects are not serious and should be straightforward to incorporate in a real measurement.

\subsubsection{Measurements in Other Reactions at the NLC}

In addition, the NLC allows measurements of non-Abelian gauge boson couplings in other channels [75]. The process $e^{+} e^{-} \rightarrow Z \gamma$ probes $Z Z \gamma$ and $Z \gamma \gamma$ couplings, and processes such as $e^{+} e^{-} \rightarrow W W Z$ probe quartic couplings. The $W W \gamma$ and $W W Z$ couplings can be probed 
independently via the processes $e^{+} e^{-} \rightarrow \nu \bar{\nu} \gamma$ and $e^{+} e^{-} \rightarrow \nu \bar{\nu} Z$, respectively.

Similar measurements can be carried out at $e^{-} e^{-}, e^{-} \gamma$ and $\gamma \gamma$ colliders, where the expected reduction in luminosity is at least partly compensated by other advantages [82, 86]. For example, the process $\gamma e^{-} \rightarrow W^{-} \nu_{e}$ probes the $W W \gamma$ coupling, independent of $W W Z$ effects. The polarization asymmetry in this reaction reverses as the energy of the collisions is varied, and the location of this zero-crossing provides a sensitive probe of $\lambda_{\gamma}$ [87]. The reaction $\gamma \gamma \rightarrow W^{+} W^{-}$also separates effects of the $\gamma$ couplings from those of the $Z$, and also probes the 4-boson $W W \gamma \gamma$ vertex. The power of this facility is enhanced by its ability to polarize both incoming beams [82].

\subsubsection{Conclusions}

Although there will have been a number of measurements of anomalous coupling parameters from LEP II, the Tevatron, and the LHC before the turn-on of the NLC, the precisions on the values of the couplings attainable with the NLC will quickly overwhelm the previous measurements. Moreover, the higher the accessible energy at NLC, the more dramatic the improvement will be. Figure 2.39 (taken from Ref. [88]) shows a useful comparison among these accelerators. The enormous potential of the NLC is apparent. 

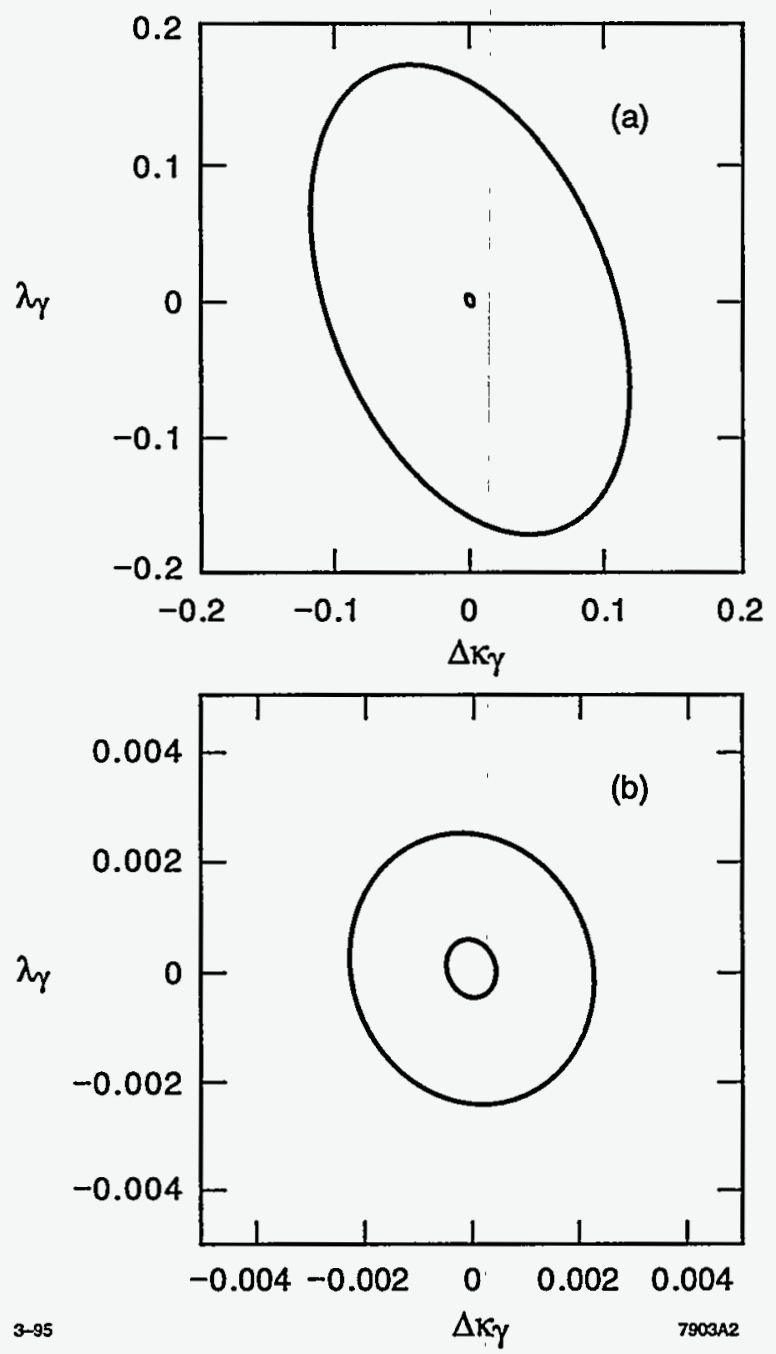

Figure 2.38: $95 \%$ CL contours for $\Delta \kappa_{\gamma}$ and $\lambda_{\gamma}$ in the HISZ scenario. The outer contour in (a) is for $\mathrm{E}_{\mathrm{CM}}=190 \mathrm{GeV}$ and $0.5 \mathrm{fb}^{-1}$. The inner contour in (a) and the outer contour in (b) is for $\mathrm{E}_{\mathrm{CM}}=500 \mathrm{GeV}$ with $80 \mathrm{fb}^{-1}$. The inner contour in (b) is for $\mathrm{E}_{\mathrm{CM}}=1.5 \mathrm{TeV}$ with $190 \mathrm{fb}^{-1}$. 


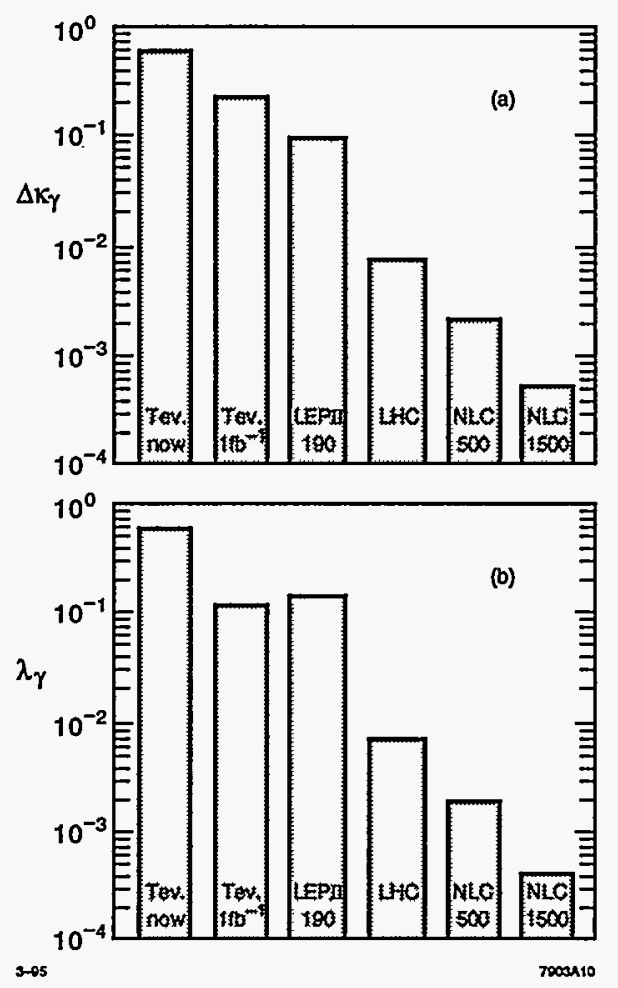

Figure 2.39: Comparison of representative 95\% CL upper limits on $\Delta \kappa_{\gamma}$ and $\lambda_{\gamma}$ for present and future accelerators. 


\subsection{Strong $W W$ Scattering}

In this section we examine how well an $e^{+} e^{-}$linear collider with a center-of-mass energy of 500-1500 GeV can study the strongly interacting Higgs sector. We also compare the estimated sensitivity of such a collider with that of the LHC.

\subsubsection{The Reaction $e^{+} e^{-} \rightarrow W^{+} W^{-}$}

Strong electroweak symmetry breaking affects the reaction $e^{+} e^{-} \rightarrow W^{+} W^{-}$through anomalous couplings at the $W^{+} W^{-} \gamma$ and $W^{+} W^{-} Z$ vertices and through enhancements in $W_{\mathrm{L}}^{+} W_{\mathrm{L}}^{-}$ production due to $I=J=1$ resonances. Here we have used the symbol $W_{L}$ to denote a longitudinally polarized $\mathrm{W}$ boson. Anomalous couplings at the three-gauge boson vertices are related to the chiral Lagrangian parameters $L_{9 L}$ and $L_{9 R}$ [77]. A technipion form factor $F_{T}$ is used to parameterize [89] the strong $W_{\mathrm{L}}^{+} W_{\mathrm{L}}^{-}$interaction in the $I=J=1$ state; it is analogous to the rho-dominated pion form factor in $e^{+} e^{-} \rightarrow \pi^{+} \pi^{-}$.

Whether one is measuring trilinear vector boson couplings or searching for an enhancement in $W_{\mathrm{L}}^{+} W_{\mathrm{L}}^{-}$production, the experimental goal is the same: disentangle the $W^{+} W^{-}$ polarization states, and in particular isolate the polarization state $W_{\mathrm{L}}^{+} W_{\mathrm{L}}^{-}$. We shall describe the results of a study that utilizes a final-state helicity analysis of all observable final-state variables in order to isolate $W_{\mathrm{L}}^{+} W_{\mathrm{L}}^{-}$production [3].

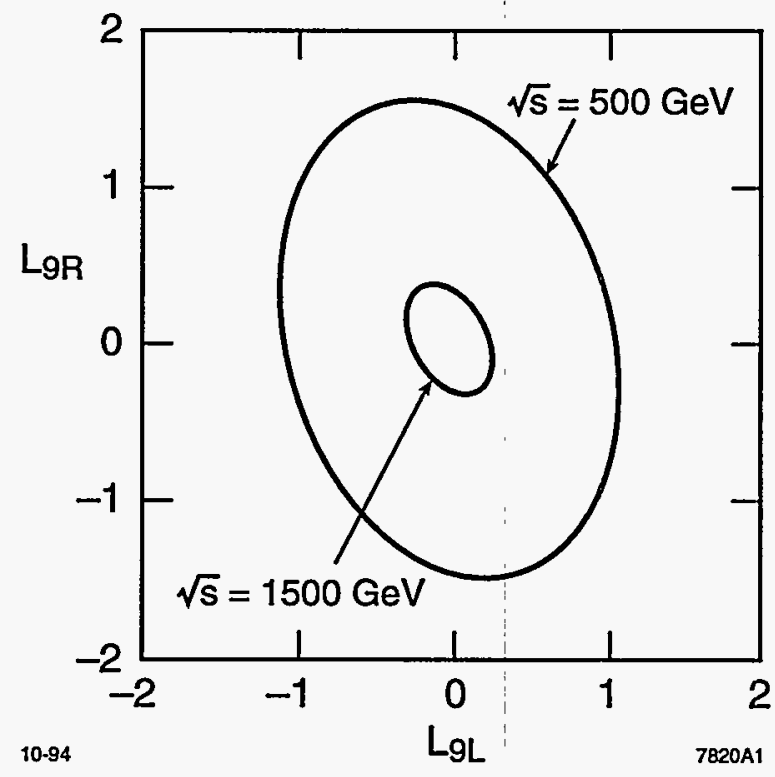

Figure 2.40: The 95\% confidence level contours for $L_{9 L}$ and $L_{9 R}$ at $\sqrt{s}=500 \mathrm{GeV}$ with $80 \mathrm{fb}^{-1}$, and at $\sqrt{s}=1500 \mathrm{GeV}$ with $190 \mathrm{fb}^{-1}$. The outer contour is for $\sqrt{s}=500 \mathrm{GeV}$. In each case the initial state electron polarization is $90 \%$. 
The maximum likelihood method is used to fit for chiral Lagrangian parameters or for the real and imaginary parts of the technipion form factor. Figure 2.40 shows the $95 \%$ confidence level contours for the chiral Lagrangian parameters $L_{9 L}$ and $L_{9 R}$ at $\sqrt{s}=500 \mathrm{GeV}$ and at $\sqrt{s}=1500 \mathrm{GeV}$. The parameters $L_{9 L}$ and $L_{9 R}$ are normalized such that values of $\mathcal{O}(1)$ are expected if the Higgs sector is strongly interacting.

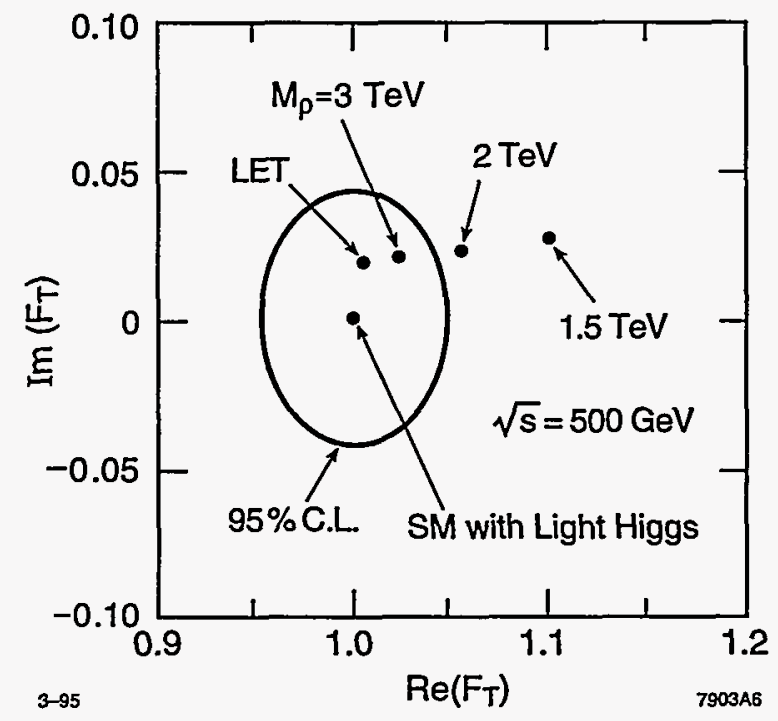

Figure 2.41: 95\% confidence level contours for the real and imaginary parts of $F_{T}$ at $\sqrt{s}=500 \mathrm{GeV}$ with $80 \mathrm{fb}^{-1}$. The values of $F_{T}$ for various technirho masses are indicated.

Figure 2.41 shows the $95 \%$ confidence level contour for the real and imaginary parts of $F_{T}$ at $\sqrt{s}=500 \mathrm{GeV}$. The use of beam polarization is essential to obtain a strong constraint on $\operatorname{Im}\left(F_{T}\right)$. Also indicated are values of $F_{T}$ for various technirho masses. The infinite technirho mass point is labelled Low Energy Theorem (LET) since in this limit there is residual $W_{\mathrm{L}}^{+} W_{\mathrm{L}}^{-}$scattering described by the same low energy theorems that govern low energy $\pi^{+} \pi^{-}$scattering. We see that the NLC at $\sqrt{s}=500 \mathrm{GeV}$ can exclude technirho masses up to about $2.5 \mathrm{TeV}$ and can discover technirho resonances with masses of more than 1.5 TeV. The significance of the $1.5 \mathrm{TeV}$ technirho signal would be $6.7 \sigma$. A $1.0 \mathrm{TeV}$ technirho would produce a $17.7 \sigma$ signal. For comparison, the minimal technicolor model predicts a technirho mass of about $2.0 \mathrm{TeV}$.

Figure 2.42 contains confidence level contours for the real and imaginary parts of $F_{T}$ at $\sqrt{s}=1500 \mathrm{GeV}$. The non-resonant LET point is well outside the light Higgs $95 \%$ confidence level region and corresponds to a $4.5 \sigma$ signal. The labeling of points here deserves some comment. We use the model of [89] to describe the form factor $F_{T}$. In this model, as the vector resonance mass is taken to infinity, its effect on the form factor decreases, and what is left behind is the residual scattering predicted by the LET. The values for high-mass technirho indicate this decoupling. With this understanding, the $6 \mathrm{TeV}, 4 \mathrm{TeV}$ and $3 \mathrm{TeV}$ 


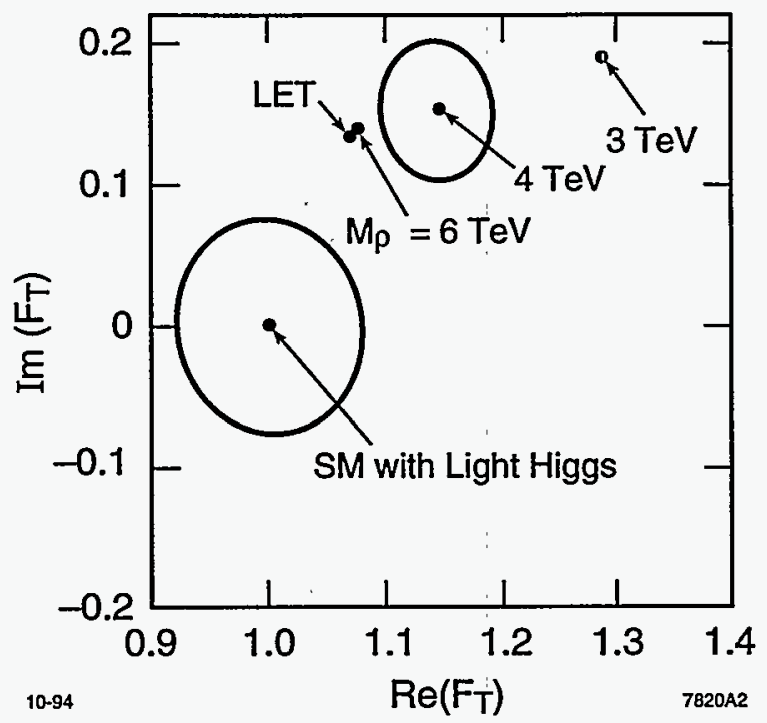

Figure 2.42: Confidence level contours for the real and imaginary parts of $F_{T}$ at $\sqrt{s}=1500 \mathrm{GeV}$ with $190 \mathrm{fb}^{-1}$. The contour about the light Higgs value of $F_{T}=(1,0)$ is $95 \%$ confidence level and the contour about the $M_{\rho}=4 \mathrm{TeV}$ point is $68 \%$ confidence level.

technirho points correspond to $4.8 \sigma, 6.5 \sigma$, and $11 \sigma$ signals, respectively. A $2 \mathrm{TeV}$ technirho would produce a $37 \sigma$ signal.

It might appear that the value of $F_{T}$, and hence the significance of technirho signals, would be very sensitive to the technirho width when $\sqrt{s}$ is much less than the technirho mass. In the model we have considered, however, this is not true. The results presented above were obtained assuming that $\Gamma_{\rho} / M_{\rho}=0.22$. If, for example, the technirho width is reduced to $\Gamma_{\rho} / M_{\rho}=0.03$ then the $1 \mathrm{TeV}$ signal at $\sqrt{s}=500 \mathrm{GeV}$ is reduced from $17.7 \sigma$ to $16.3 \sigma$, the $1.5 \mathrm{TeV}$ signal at $\sqrt{s}=500 \mathrm{GeV}$ is reduced from $6.7 \sigma$ to $6.4 \sigma$, and the $4 \mathrm{TeV}$ signal at $\sqrt{s}=1500 \mathrm{GeV}$ is reduced from $6.5 \sigma$ to $6.3 \sigma$.

\subsubsection{The Reaction $e^{+} e^{-} \rightarrow \nu \bar{\nu} W^{+} W^{-}$and $\nu \bar{\nu} \mathrm{ZZ}$}

The important gauge boson scattering processes $W_{\mathrm{L}}^{+} W_{\mathrm{L}}^{-} \rightarrow W_{\mathrm{L}}^{+} W_{\mathrm{L}}^{-}$and $W_{\mathrm{L}}^{+} W_{\mathrm{L}}^{-} \rightarrow Z_{L} Z_{L}$ are studied at the NLC with the reactions $e^{+} e^{-} \rightarrow \nu \bar{\nu} W^{+} W^{-}$and $e^{+} e^{-} \rightarrow \nu \bar{\nu} Z Z$. We describe the results that Barger et al. [90] have obtained by analyzing these processes.

Barger et al. use several models to test the effectiveness of their analysis of $e^{+} e^{-} \rightarrow$ $\nu \bar{\nu} W^{+} W^{-}$and $\nu \bar{\nu} Z Z$. In addition to the Standard Model Higgs boson with mass $m_{H}=$ $1 \mathrm{TeV}$, they use a Chirally-Coupled Scalar (CCS) model, a Chirally-Coupled Vector (CCV) model, and the LET model. They utilize a series of cuts to produce an event sample that is rich in the final states $\nu \bar{\nu} W_{\mathrm{L}}^{+} W_{\mathrm{L}}^{-}$and $\nu \bar{\nu} Z_{L} Z_{L}$. Figure 2.43 shows the $M_{W W}$ and $M_{Z Z}$ distributions after all cuts. The $1 \mathrm{TeV}$ Higgs scalar resonance stands out in both the $\nu \bar{\nu} W W$ 


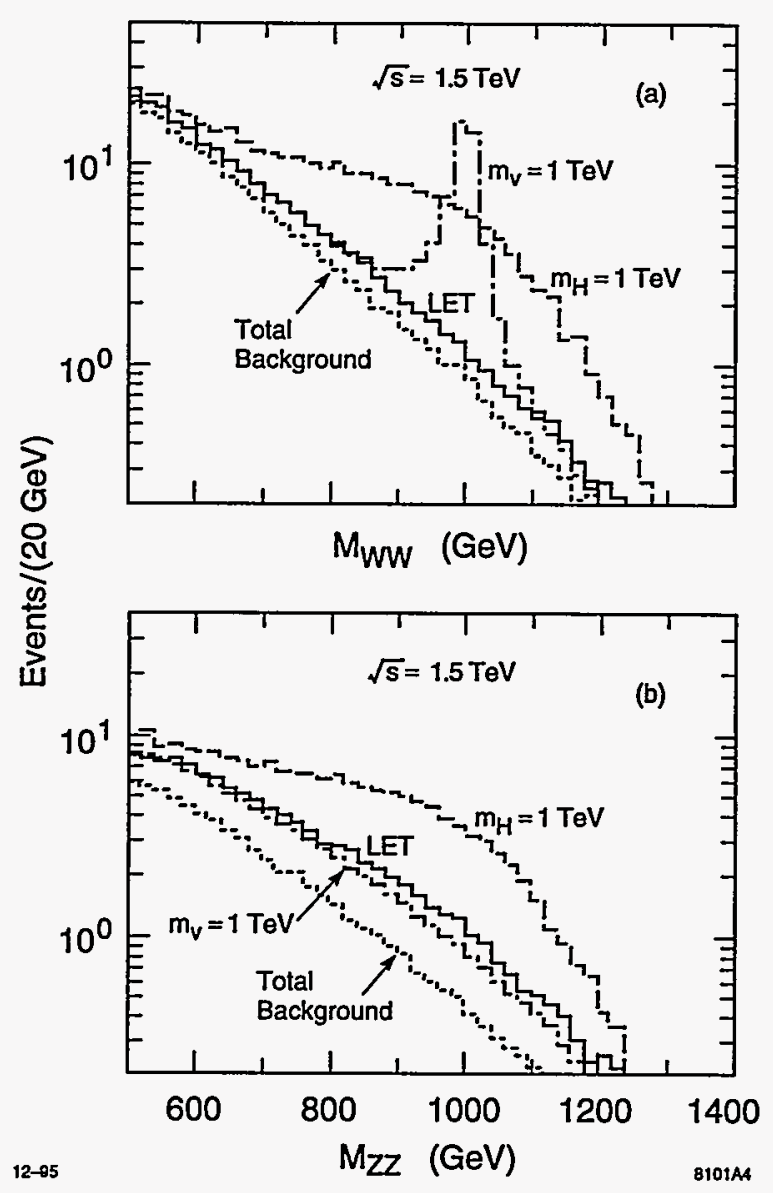

Figure 2.43: Expected numbers of $W^{+} W^{-}, Z Z \rightarrow(j j)(j j)$ signal and background events after all cuts for $200 \mathrm{fb}^{-1}$ luminosity at $\sqrt{s}=1.5 \mathrm{TeV}$ : (a) $e^{+} e^{-} \rightarrow \nu \bar{\nu} W^{+} W^{-}$, (b) $e^{+} e^{-} \rightarrow \nu \bar{\nu} Z Z$. The dotted histogram shows total SM background. The solid, dashed and dotdashed histograms show signal plus background for the LET, SM, and CCV models, respectively; CCS model results are close to the SM case.

and $\nu \bar{\nu} Z Z$ final states. The $1 \mathrm{TeV}$ vector resonance is prominent in the $M_{W W}$ distribution (of course such a resonance would have been seen earlier as a $16 \sigma$ signal at $\sqrt{s}=0.5 \mathrm{TeV}$, in $\left.e^{+} e^{-} \rightarrow W^{+} W^{-}\right)$. The LET signal is larger for the final state $\nu \bar{\nu} Z Z$ than it is for $\nu \bar{\nu} W W$.

The statistical significance of the signals for the different models is given in Table 2.8 assuming $100 \%$ initial-state $e^{-}$polarization at $\sqrt{s}=1.5 \mathrm{TeV}$ and $200 \mathrm{fb}^{-1}$ luminosity. Note that the statistical significance of the LET signal is $8.5 \sigma$ in the $\nu \bar{\nu} Z Z$ channel.

The $I=2$ Goldstone boson scattering channel can be probed at the NLC through the reaction $e^{-} e^{-} \rightarrow \nu \nu W^{-} W^{-}$. Barger et al. [91] have studied this reaction and they obtain 
Table 2.8: Signal and background for $e^{+} e^{-} \rightarrow \nu \bar{\nu} W^{+} W^{-}$and $e^{+} e^{-} \nu \bar{\nu} Z Z$ with $100 \%$ initial state electron polarization, from Ref. [90].

\begin{tabular}{|l|c|c|c|c|}
\hline \hline Signal $(S)$ or & SM & Scalar & Vector & LET \\
Background $(B)$ & $M_{H}=1 \mathrm{TeV}$ & $M_{S}=1 \mathrm{TeV}$ & $M_{V}=1 \mathrm{TeV}$ & \\
\hline$S\left(e^{+} e^{-} \rightarrow \nu \bar{\nu} W^{+} W^{-}\right)$ & 330 & 320 & 92 & 62 \\
$B$ (backgrounds) & 280 & 280 & 7.1 & 280 \\
$S / \sqrt{B}$ & 20 & 20 & 35 & 3.7 \\
$S\left(e^{+} e^{-} \rightarrow \nu \bar{\nu} Z Z\right)$ & 240 & 260 & 72 & 90 \\
$B$ (backgrounds) & 110 & 110 & 110 & 110 \\
$S / \sqrt{B}$ & 23 & 25 & 6.8 & 8.5 \\
\hline \hline
\end{tabular}

Table 2.9: Signal and background for $e^{-} e^{-} \rightarrow \nu \bar{\nu} W^{-} W^{-}$with $100 \%$ initial state electron polarization, from Ref. [91].

\begin{tabular}{|l|c|c|c|c|}
\hline \hline Signal $(S)$ or & SM & Scalar & Vector & LET \\
Background $(B)$ & $M_{H}=1 \mathrm{TeV}$ & $M_{S}=1 \mathrm{TeV}$ & $M_{V}=1 \mathrm{TeV}$ & \\
\hline$S\left(e^{-} e^{-} \rightarrow \nu \bar{\nu} W^{-} W^{-}\right)$ & 110 & 140 & 140 & 170 \\
$B$ (backgrounds) & 710 & 710 & 710 & 710 \\
$S / \sqrt{B}$ & 4.0 & 5.2 & 5.4 & 6.3 \\
\hline \hline
\end{tabular}

the statistical significances shown in Table 2.9. An initial state electron polarization of $100 \%$ has been assumed for both beams.

\subsubsection{The Reaction $e^{+} e^{-} \rightarrow \nu \bar{\nu} t \bar{t}$}

If there is no light Higgs boson, the process $t \bar{t} \rightarrow W^{+} W^{-}$violates unitarity in the multi$\mathrm{TeV}$ energy region. It is natural then to ask if strong symmetry breaking can be detected through the process $W^{+} W^{-} \rightarrow t \bar{t}$. This process would be studied at the NLC by observing the reaction $e^{+} e^{-} \rightarrow \nu \bar{\nu} t \bar{t}$.

The total cross sections for $e^{+} e^{-} \rightarrow e^{+} e^{-t} t \bar{t}$ and $e^{+} e^{-} \rightarrow \nu \bar{\nu} t \bar{t}$ have been calculated by Kauffman [92] for an $e^{+} e^{-}$center-of-mass energy of $2 \mathrm{TeV}$ (Fig. 2.44). The cross sections for $\sqrt{s}=1.5 \mathrm{TeV}$ should be similar. For $\sqrt{s}=2 \mathrm{TeV}$ and $200 \mathrm{fb}^{-1}$ luminosity we would have 1200 events from $e^{+} e^{-} \rightarrow e^{+} e^{-} t \bar{t}$ and 60 (400) events from $e^{+} e^{-} \rightarrow \nu \bar{\nu} t \bar{t}$ for $m_{H}=$ 0.1 (1.0) TeV. A $1 \mathrm{TeV}$ Higgs boson therefore produces a $30 \%$ increase in the sum of the 


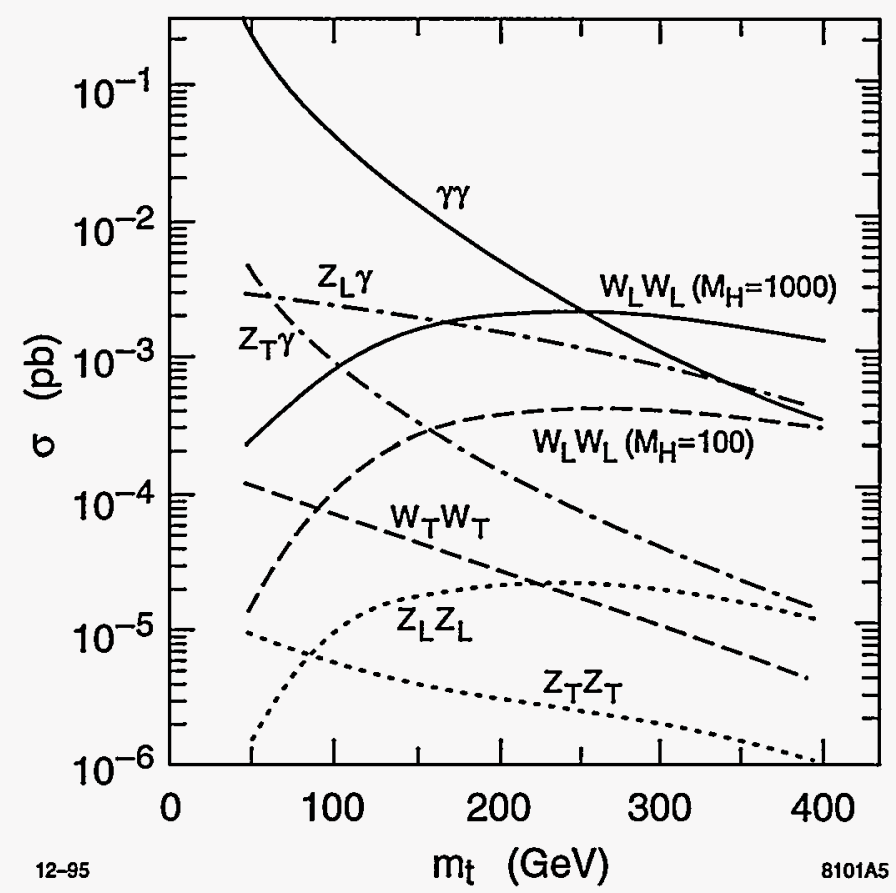

Figure 2.44: Contributions from various subprocesses to the total cross sections for $e^{+} e^{-} \rightarrow e^{+} e^{-} t \bar{t}$ and $e^{+} e^{-} \rightarrow \nu \bar{\nu} t \bar{t}$. The contributions are plotted as a function of the top quark mass $m_{t}$. The $e^{+} e^{-}$center-ofmass energy is $2 \mathrm{TeV}$.

cross sections for $e^{+} e^{-} \rightarrow e^{+} e^{-} t \bar{t}$ and $e^{+} e^{-} \rightarrow \nu \bar{\nu} t \bar{t}$ before any cuts are applied. The same $1 \mathrm{TeV}$ Higgs boson produces only a $0.3 \%$ increase in the sum of the cross sections for $e^{+} e^{-} \rightarrow e^{+} e^{-} W^{+} W^{-}$and $e^{+} e^{-} \rightarrow \nu \bar{\nu} W^{+} W^{-}$.

The strong symmetry breaking signal can be further enhanced by performing a helicity analysis on the $t \bar{t}$ final state to isolate the polarization states $t_{L} \bar{t}_{L}$ and $t_{R} \bar{t}_{R}$ corresponding to helicity-flip pair production. This can be done by observing the $W$ energy distribution in $t$ decays. Projecting out these helicity combinations would be the analog of projecting out the $W_{\mathrm{L}}^{+} W_{\mathrm{L}}^{-}$and $Z_{L} Z_{L}$ final states in gauge boson scattering.

\subsubsection{Statistical significances at $\mathrm{LHC}$ versus $\mathrm{NLC}$}

The statistical significances of strong symmetry breaking signals at the NLC and LHC are summarized in Table 2.10. The LHC results are taken from the ATLAS design report [24]. If an entry is blank it may mean that the process is insensitive to the corresponding model or that the analysis has not been done; no distinction is made between the two possibilities. The entries for the direct gauge boson scattering processes at the NLC $\left(W^{+} W^{-} \rightarrow Z Z\right.$ and $W^{-} W^{-} \rightarrow W^{-} W^{-}$) assume that the electron beam always has $90 \%$ left-handed polarization. For both the NLC and LHC results it was assumed that the $1.5 \mathrm{TeV}$ technirho had a width 
of $0.33 \mathrm{TeV}$.

Table 2.10: Statistical significances of strong symmetry breaking signals at the NLC and LHC.

\begin{tabular}{|l|l|c|c||c|c|c|}
\hline \hline Collider & Process & $\begin{array}{c}\sqrt{s} \\
(\mathrm{TeV})\end{array}$ & $\begin{array}{c}\mathcal{L} \\
\left(\mathrm{fb}^{-1}\right)\end{array}$ & $\begin{array}{c}M_{\rho}= \\
1.5 \mathrm{TeV}\end{array}$ & $\begin{array}{c}M_{H}= \\
1 \mathrm{TeV} .\end{array}$ & LET \\
\hline NLC & $e^{+} e^{-} \rightarrow W^{+} W^{-}$ & .5 & 80 & $7 \sigma$ & - & - \\
NLC & $e^{+} e^{-} \rightarrow W^{+} W^{-}$ & 1.0 & 200 & $35 \sigma$ & - & - \\
NLC & $e^{+} e^{-} \rightarrow W^{+} W^{-}$ & 1.5 & 190 & $366 \sigma$ & - & $5 \sigma$ \\
NLC & $W^{+} W^{-} \rightarrow Z Z$ & 1.5 & 190 & - & $22 \sigma$ & $8 \sigma$ \\
NLC & $W^{-} W^{-} \rightarrow W^{-} W^{-}$ & 1.5 & 190 & - & $4 \sigma$ & $6 \sigma$ \\
& & & 100 & - & $14 \sigma$ & - \\
LHC & $W^{+} W^{-} \rightarrow W^{+} W^{-}$ & 14 & 100 & - & $3 \sigma$ & $6 \sigma$ \\
LHC & $W^{+} W^{+} \rightarrow W^{+} W^{+}$ & 14 & 100 & - & - & - \\
LHC & $W^{+} Z \rightarrow W^{+} Z$ & 14 & 100 & $7 \sigma$ & - \\
\hline \hline
\end{tabular}

Some points about Table 2.10 are worth noting:

1. Although the LHC can access larger $W^{+} W^{-}$center-of-mass energies, the statistical significance of the NLC signal is always larger. In most cases, this is a small effect, but it makes a significant point: Electron beam polarization, smaller backgrounds, and the utilization of the full $e^{+} e^{-}$center-of-mass energy for vector resonances has allowed the NLC to more than make up for its lower range of $W^{+} W^{-}$center-of-mass energies.

2. The NLC has a special ability to detect vector resonances. At $\sqrt{s}=500 \mathrm{GeV}$ the technirho mass reach of the NLC is equal to that of the LHC. At $\sqrt{s}=1500 \mathrm{GeV}$ the NLC is sensitive to strong interaction effects in $I=J=1 W^{+} W^{-}$scattering even when they are nonresonant.

3. Signal significances for $W^{+} W^{-} \rightarrow t \bar{t}$ were not included in Table 2.10 because detector simulations have not yet been performed. From the discussion in Section 2.7.3, however, it appears that this is a promising reaction for the study of strong symmetry breaking at the NLC. It is probably very difficult to study $W^{+} W^{-} \rightarrow t \bar{t}$ at LHC due to the large background from $g g \rightarrow t \bar{t}$.

Finally it is important to remember that the significances shown in Table 2.10 include statistical errors only. Systematic errors have largely been ignored in analyses so far, both for the LHC and for the NLC. 


\subsubsection{Conclusion}

An $e^{+} e^{-}$linear collider with $\sqrt{s}=500-1500 \mathrm{GeV}$ would be an effective partner to the LHC in the study of strong symmetry breaking. It provides important complementary capabilities for the discovery of vector resonances and the extraction of chiral Lagrangian parameters. The NLC and LHC are expected to have similar statistical errors for scalar resonance and non-resonant signals in gauge boson scattering; however, the physics environments at the two machines are very different, and the systematic errors for the NLC analyses are probably smaller. The NLC is probably the only machine that can study $W^{+} W^{-} \rightarrow t \bar{t}$. 


\subsection{New Gauge Bosons and Exotic Particles}

In many extensions to the Standard Model, in particular those involving an enlargement of the $S U(3)_{c} \times S U(2)_{L} \times U(1)_{Y}$ gauge group or compositeness, new particles are expected to exist beyond those associated with either supersymmetry or technicolor. The properties and signatures of exotic particles have recently been reviewed in [93] In almost all cases, these particles can be directly produced and have their properties analyzed in $e^{+} e^{-}$collisions given a sufficiently large center-of-mass energy. If such energies are not available, the indirect influence of the existence of many kinds of exotic particles can also be examined through precision measurements at the NLC.

Perhaps the most well-studied of all exotic particles is the new neutral gauge boson, $Z^{\prime}$, which is a basic ingredient of all theories with extended gauge sectors. (When the additional group factor is non-abelian, new charged gauge bosons, $W^{\prime}$, may also be present.) If such a particle exists and is accessible at the $\mathrm{TeV}$ scale, it is possible that it may first be directly produced via the Drell-Yan process at the LHC, provided the $Z^{\prime}$ couples to both quarks and leptons as it does in most models. If the $Z^{\prime}$ does not couple to leptons it cannot be produced at the NLC; in this case, searches at the LHC would also be difficult, since the effects of the $Z^{\prime}$ would have to be observed as perturbations of di-jet mass distributions. If the $Z^{\prime}$ couples only to leptons, then the NLC provides the unique means to study it.

It is possible that a $Z^{\prime}$, though it exists, may not be seen at the LHC due to its mass and/or the nature of its couplings to fermions. In this case the NLC can extend the search reach for some models; in addition the NLC may be able to determine both the mass and couplings of the $Z^{\prime}$. A 'lucky' scenario would be one where the mass of the $Z^{\prime}$ is less than the NLC center-of-mass energy; in this case, by sitting on-resonance and repeating the LEP/SLC experimental program, we can determine all of the $Z$ 's couplings to fermions. A much more likely scenario is that $M_{Z^{\prime}}>\sqrt{s}$ so that deviations in the cross section and associated asymmetries for various flavors could be used to look for indirect $Z^{\prime}$ effects in a manner similar to the observation of the $Z$ at PEP, PETRA, and TRISTAN below the resonance. In some cases, this indirect 'reach' can be as large as $M_{Z^{\prime}} \simeq 10 \sqrt{s}$. Figure 2.45 shows a comparison of the indirect $Z^{\prime}$ search capability at LEP and the NLC as well as the direct production reach at the Tevatron and LHC for several extended models.

If a $Z^{\prime}$ exists, we would like to know how it couples to the various flavors so that we can identify the correct extended gauge structure. If the $Z^{\prime}$ is seen at the LHC and its mass is not much larger than about $1 \mathrm{TeV}$, several analyses have shown [93] that the LHC and a $\sqrt{s}=500 \mathrm{GeV}$ NLC will nicely complement each other in extracting fermion coupling information. If the $Z^{\prime}$ is more massive, then LHC cannot perform coupling analyses using any of the presently available techniques either due to backgrounds or statistical limitations. However, if the mass of the $Z^{\prime}$ is less than about $2 \mathrm{TeV}$, then the NLC running in the $\sqrt{s}=0.5-1 \mathrm{TeV}$ range can obtain significant coupling information even if the $Z^{\prime}$ is missed by the LHC and its mass is a priori unknown. (This value goes up to $M_{Z^{\prime}} \simeq 3 \mathrm{TeV}$ if $\sqrt{s}=1.5$ $\mathrm{TeV}$ energies are available.) A representative example of such an analysis is shown in Fig. 2.46 [94]. Similar results can be obtained for different values of the input parameters as well 


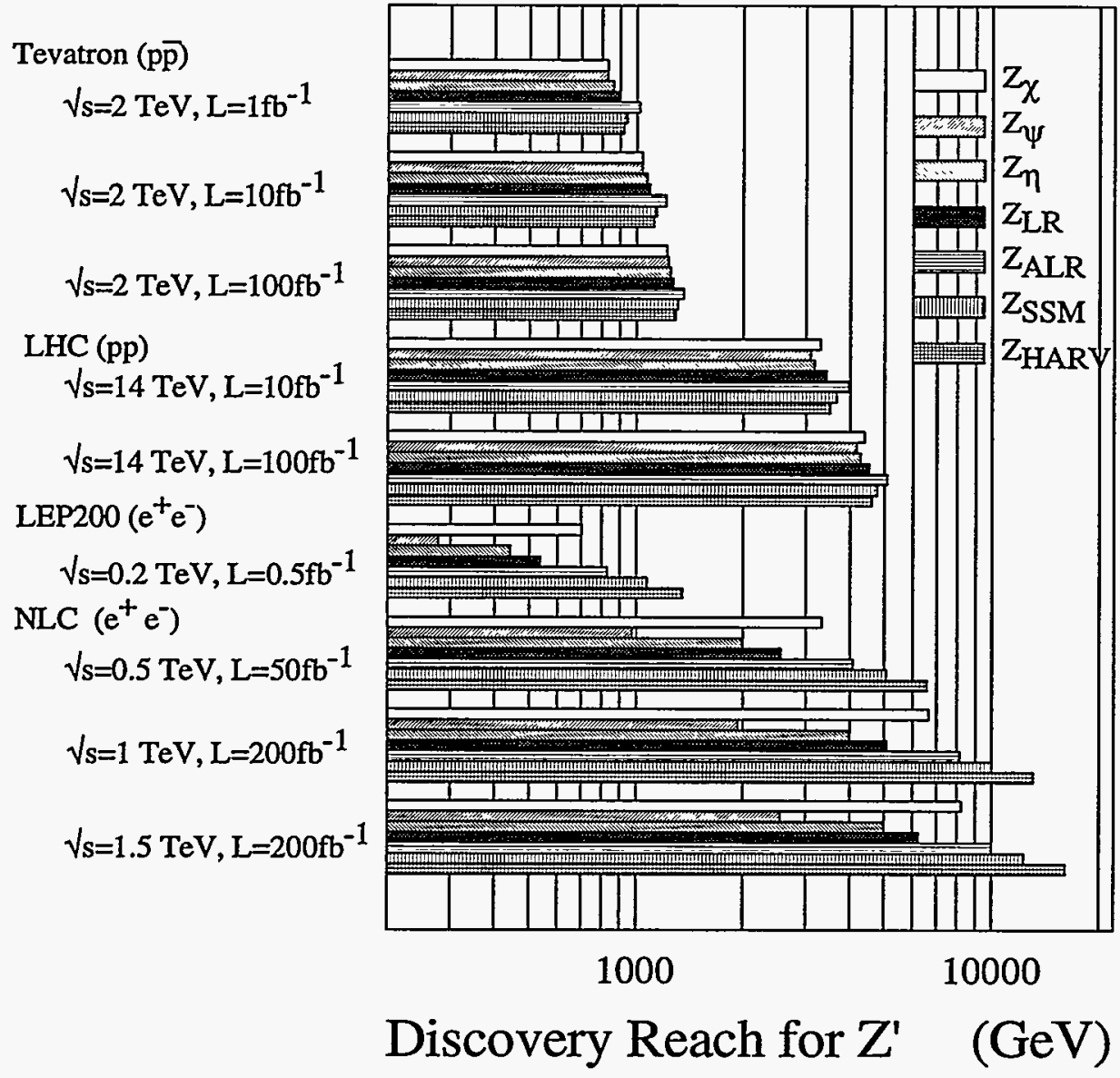

Figure 2.45: Tevatron and LHC bounds are based on 10 events in the $e^{+} e^{-}+\mu^{+} \mu^{-}$channels; decays to SM final states only is assumed. LEP and NLC bounds are $99 \%$ CL using the observables $\sigma_{l}, R^{\text {had }}, A_{L R}^{l}$ and $A_{L R}^{\text {had }}$. 


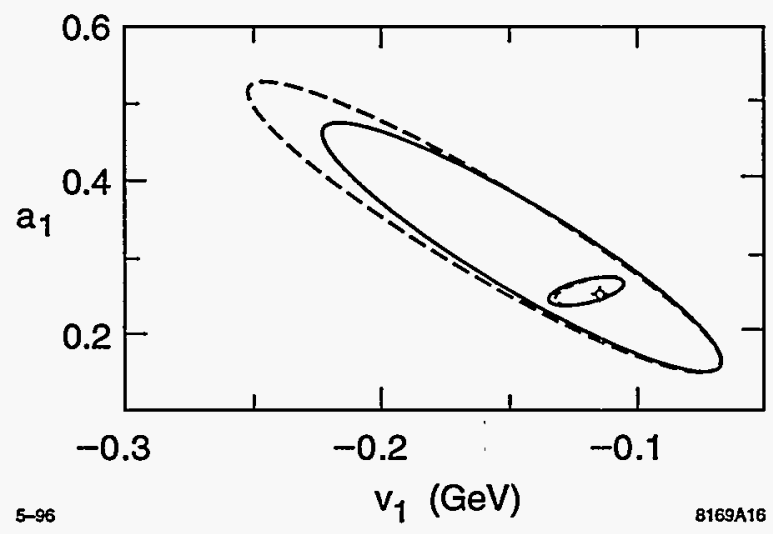

Figure 2.46: Extracted leptonic couplings of a $1.53 \mathrm{TeV} Z^{\prime}$ at the NLC, at $95 \% \mathrm{CL}$, using data at $0.5\left(70 \mathrm{fb}^{-1}\right), 0.75\left(100 \mathrm{fb}^{-1}\right)$, and $1 \mathrm{TeV}(150$ $\left.\mathrm{fb}^{-1}\right)$. All leptonic observables were used and generation independence was assumed. The large ovals are applicable when $M_{Z^{\prime}}$ is unknown a priori and must be obtained from the fit; the solid (dashed) curve corresponds to electron beam polarization $P=90(80) \%$. The small ovals correspond to the scenario in which $M_{Z^{\prime}}$ is known (for example, from the LHC); the two curves represent the same two cases. The dot represents the input values of the couplings.

as for the other fermion flavors (although fewer observables are available), thus allowing the structure of the extended gauge model to be determined by comparing the extracted values of these couplings with specific model predictions.

In generic Standard Model extensions, the new gauge bosons are usually accompanied by additional fermionic and/or bosonic degrees of freedom the most common of which are either vector-like or singlets with respect to the Standard Model electroweak group. In addition to new quarks and leptons, some of these particles, such as leptoquarks (LQs) and diquarks, may have atypical $B$ and $L$ number assignments. The production and decay of both spin- 0 and spin-1 LQs at $e p, e^{+} e^{-}$, and $p p / p \bar{p}$ colliders has been studied by many groups $[93,94]$. Allowing for both helicities in their interactions with ordinary quarks and leptons there are 14 possible species of LQs with reasonably strict constraints applicable to their intergenerational couplings. (Low energy constraints also force LQ couplings to be chiral.) At $e p$ colliders, LQ production rates are proportional to the squares of unknown Yukawa couplings so they may easily be missed. By contrast, at hadron colliders, the cross section is the same for all LQ types of a given spin but the signal depends upon how the LQ decays. Thus it will not be possible at hadron colliders to uniquely determine which LQ type is being produced. On the other hand, at $e^{+} e^{-}$machines, the production cross sections and asymmetries are determined solely by the $\mathrm{LQ}$ electroweak quantum numbers and spin. Several studies have shown [93] that the LQ species can found and identified in both $e^{+} e^{-}$ and $\gamma e$ collisions. In the first case the 'ID reach' is $\simeq 0.45 \sqrt{s}$; this improves to $\simeq 0.8 \sqrt{s}$ in the $\gamma e$ mode if the Yukawa couplings are of order electromagnetic strength. In a manner 
similar to the $Z^{\prime}$ searches discussed above, $t$ and $u$ channel LQ exchange can be probed for indirectly via the process $e^{+} e^{-} \rightarrow q \bar{q}$ provided the Yukawa couplings are sufficiently large. For Yukawa couplings of electromagnetic strength, the reach can exceed $6 \mathrm{TeV}$ at the NLC.

Table 2.11 shows a comparison of the search capabilities of LEPII, the Tevatron, the LHC and NLC for a number of different exotic particles. As is clear from the above, great care should be exercised when comparing the capabilities of different machines to find and explore the properties of exotics. The results in the table for $e^{+} e^{-}$colliders are indirect bounds for $Z$ 's but represent direct production limits at hadron colliders. Limits on all other particles in the case of $e^{t} e^{-}$colliders are for direct production except where noted. It is important to remember that in many other cases $e^{+} e^{-}$colliders provide important indirect bounds as in both the $Z^{\prime}$ and LQ examples. For a $W^{\prime}$ in the Left-Right Symmetric Model $\left(W_{R}\right)$, a number of additional assumptions are required before search reaches can be quoted for hadron colliders; these are: $(i)$ equal left- and right-handed gauge couplings and CKM matrices, (ii) right-handed neutrinos appear only as missing $p_{t}$, (iii) only Standard Model final states are accessible in decays. The possibility of surrendering any or all of these assumptions has been analyzed and was found to lead to downward, potentially drastic, alterations in search expectations via the Drell-Yan process. Searches for new quarks at hadron colliders are also subject to decay mode assumptions and are species dependent. In some cases, for example, flavor changing neutral current decays are at least as important as the more conventional charged current ones. 
Table 2.11: Limits on various exotic particles, in GeV. Run II is $2 \mathrm{fb}^{-1}$ at $2 \mathrm{TeV}$; NLC is 200 $\mathrm{fb}^{-1}$; LHC is $14 \mathrm{TeV}$ and $100 \mathrm{fb}^{-1}$. $Z^{\prime}$ limits at hadron colliders assume only SM final states are accessible. $Z^{\prime}$ limits at $e^{+} e^{-}$colliders are indirect. * is the Tevatron limit assuming the standard $W^{\prime}$ search assumptions. ${ }^{\dagger}$ limit applies when none of the conditions in text are fulfilled; ${ }^{t \dagger}$ result using $Z^{\prime}$ bound and LR model relationships. ${ }^{+}$is from single production. The limit is for first generation spin- 0,1 LQs at HERA and the Tevatron including Run $1 \mathrm{~b}$ estimate; similar numbers will hold for the 2nd generation case at hadron colliders. In the dilepton, diquark and LQ cases, EM strength Yukawas are assumed. ${ }^{++}$are indirect limits from $e^{+} e^{-} \rightarrow q \bar{q}$ with these EM strength Yukawas. ${ }^{* *}$ combines several search modes. ${ }^{\ddagger}$ is from $e^{+} e^{-} \rightarrow 2 \gamma .{ }^{*}$ implies the limit is highly decay mode dependent. The value of ' 60 ' as the present limit for the last four entries reflect approximate LEP1.4 null searches. New lepton limits are for both charged and neutral cases. Many entries are estimates.

\begin{tabular}{|l|c|c|c|c|c|}
\hline \hline Particle & Present & LEPII & TeV(Run II) & LHC & NLC $(1 \mathrm{TeV})$ \\
\hline$Z_{S M}^{\prime}$ & 650 & 1070 & 1020 & 4760 & 10000 \\
$Z_{x}^{\prime}$ & 425 & 703 & 910 & 4380 & 6670 \\
$Z_{\psi}^{\prime}$ & 415 & 278 & 910 & 4180 & 1940 \\
$Z_{\eta}^{\prime}$ & 440 & 443 & 940 & 4280 & 3980 \\
$Z_{L R}^{\prime}(\kappa=1)$ & 445 & 538 & 970 & 4520 & 5090 \\
$Z_{A L R M}^{\prime}$ & 420 & 820 & 1100 & 5080 & 8150 \\
$W_{R}$ & $720^{\star}, 300^{\dagger}$ & 90 & $990,400^{\dagger}$ & $5310,1500^{+\dagger}$ & $800^{+}$ \\
LQ(spin-0) & 180 & $90,160^{+}, 700^{++}$ & $300,400^{++}$ & $1400,1700^{++}$ & $500,900^{+}, 6000^{++}$ \\
LQ(spin-1) & 300 & $90,160^{+}$ & $440,500^{++}$ & $2200,2500^{++}$ & $500,900^{+}, ?^{++}$ \\
Axigluon & $200-930$ & $150 ?$ & 1160 & $5000 ?$ & 800 \\
$E_{6}$ diquark & $280-350$ & 90 & $200-570$ & 5000 & 500 \\
$q^{*}$ & $90-750^{* *}$ & $90,180^{+}$ & 820 & $5000-6000 ?$ & $500,900^{+}$ \\
$e^{*}$ & $60,127^{\ddagger}$ & $90,180 ?^{+}$ & $500 ?$ & 4000 & $500,900^{+}$ \\
new leptons & 60 & 90 & $? ?$ & 1000 & 500 \\
new quarks & 60 & 90 & $*$ & $*$ & 500 \\
dileptons & 60 & 90 & - & - & $900^{+}$ \\
\hline \hline
\end{tabular}




\section{$2.9 e^{-} e^{-}, e^{-} \gamma$, and $\gamma \gamma$ Interactions}

Up to this point, we have concentrated on the reactions available at the NLC in $e^{+} e^{-}$ collisions. However, it is possible with a linear electron collider to study more general processes, and, in fact, to collide $e^{-}, e^{+}$, and $\gamma$ beams in any combination. A photon beam with substantial brightness at high energy can be created by Compton backscattering of a laser beam from the electron beam. At least for the $e^{-}$and $\gamma$ combinations, the polarizations of the two beams can be controlled independently. The energy spectrum of the backscattered Compton photon beam depends on the relative electron and laser beam polarization in the manner shown in Fig. 2.47. Thus, polarization is also useful in tuning the $\gamma$ energy spectrum, for example, to produce a peak at a fixed energy.

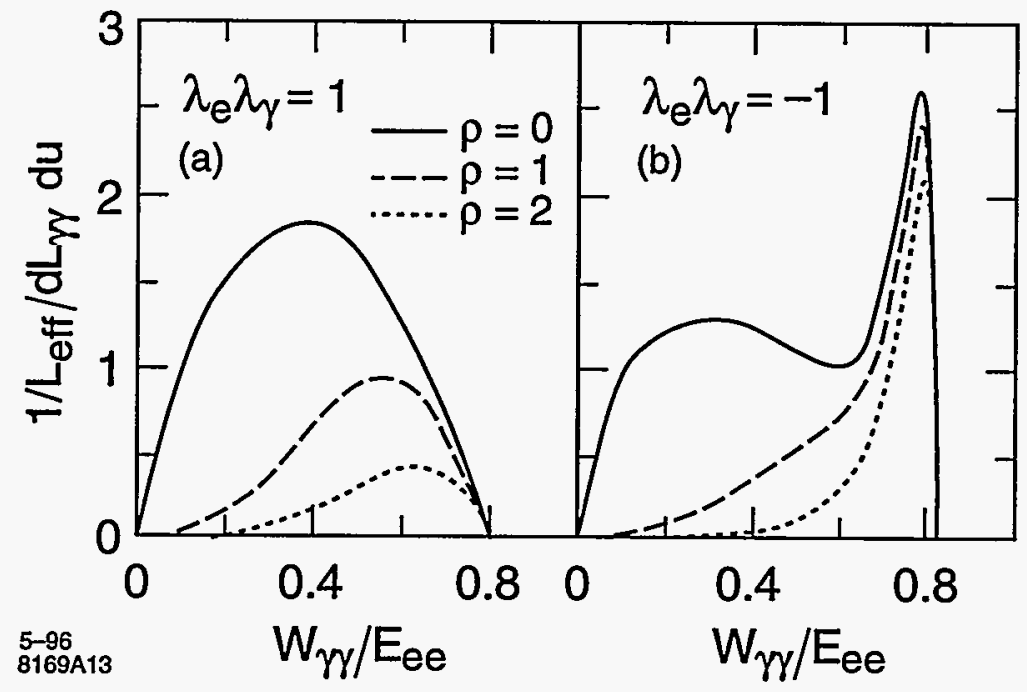

Figure 2.47: $\gamma \gamma$ luminosity for two different combinations of photon and electron polarization, and several values of $\rho$, the ratio of the intrinsic spreads of the photon and electron beams.

The availability of these additional initial states at the NLC adds significantly to the overall physics program. We have already noted their role in the study of Higgs bosons, anomalous electroweak couplings, and strong $W W$ scattering. The exotic quantum numbers of the $e^{-} e^{-}$initial state and the quasi-hadronic nature of the photon also permit searches not available to $e^{+} e^{-}$annihilation. Table 2.12 illustrates the variety of physics issues that have been discussed for each of these modes. The physics expectations for $e^{-} e^{-}$and $\gamma \gamma$ colliders have been recently been reviewed in specialized workshops $[95,96]$.

In this report, we give special consideration to the possibility of measuring the Higgs boson coupling to $\gamma \gamma$ by creating the Higgs boson as a resonance in $\gamma \gamma$ scattering. We will then review a number of other physics topics which are special to $e^{-} e^{-}$or $\gamma \gamma$ reactions. We conclude this section with a discussion of the expectations for the luminosity and the interaction region design for these reactions. 
Table 2.12: A listing of topics that will be investigated at a TeV-level linear collider. Check marks show which different initial states contribute prominently to their study, highlighting the unique contributions that $e^{-} e^{-}, e^{-} \gamma$, and $\gamma \gamma$ options will be able to make.

\begin{tabular}{|l|ccccccccc|}
\hline \hline & QCD & $t \bar{t}$ & H & SUSY & $\begin{array}{c}\text { Anomalous } \\
\text { Couplings }\end{array}$ & $e^{*}$ & $\nu_{M}$ & $Z^{\prime}$ & $X^{--}$ \\
\hline$e^{-} e^{-}$ & & & $\sqrt{ }$ & $\sqrt{ }$ & $\sqrt{ }$ & $\sqrt{ }$ & $\sqrt{ }$ & $\sqrt{ }$ & $\sqrt{ }$ \\
$e^{+} e^{-}$ & $\sqrt{ }$ & $\sqrt{ }$ & $\sqrt{ }$ & $\sqrt{ }$ & $\sqrt{ }$ & & & $\sqrt{ }$ & \\
$e^{-} \gamma$ & $\sqrt{ }$ & & $\sqrt{ }$ & $\sqrt{ }$ & $\sqrt{ }$ & $\sqrt{ }$ & & & $\sqrt{ }$ \\
$\gamma \gamma$ & $\sqrt{ }$ & $\sqrt{ }$ & $\sqrt{ }$ & $\sqrt{ }$ & $\sqrt{ }$ & & & & \\
\hline \hline
\end{tabular}

\subsubsection{Higgs Boson Studies}

A photon linear collider provides a clean method to search for an intermediate mass Higgs boson, via the reactions $\gamma \gamma \rightarrow H \rightarrow b \bar{b}$ or ZZ. It is complementary to searches using hadron and $e^{+} e^{-}$machines, being sensitive to different models and couplings. More importantly, a $\gamma \gamma$ linear collider permits a direct measurement of the two-photon width of the Higgs. The coupling of the Higgs boson to two photons involves loops in which any charged fermion or boson with couplings to the Higgs must contribute. The dominant contributions come from species heavier than that Higgs boson. Thus, a measurement of the two-photon width is quite sensitive to new physics even at higher mass scales. Supersymmetric models, and other extensions of the Standard Model with more complicated Higgs sectors typically predict Higgs spectra and two-photon couplings which differ substantially from those of the Standard Model [97]. In the minimal supersymmetric model (MSSM), the heavy neutral bosons $A^{0}$ and $H^{0}$ can be studied up to higher masses than in the $e^{+} e^{-}$mode [98]. In technicolor models, in which Higgs bosons are composite, there are often light CP-odd bound states with cross sections for $\gamma \gamma$ production similar to those of elementary Higgs bosons [99]. Finally, the dependence of the Higgs production on the photon polarization tests the CP properties of the Higgs boson, and may reveal CP violation in the Higgs sector [57, 100].

For a Standard Model Higgs boson with mass below about $300 \mathrm{GeV}$, the beam energy spread of a $\gamma \gamma$ collider is much greater than the total width of the Higgs boson, and so the number of $H \rightarrow X(=b \bar{b}, W W, Z Z)$ events expected is

$$
N_{H \rightarrow X}=\left.\frac{d L_{\gamma \gamma, J_{z}=0}}{d W_{\gamma \gamma}}\right|_{M_{H}} \frac{8 \pi^{2} \Gamma(H \rightarrow \gamma \gamma) B(H \rightarrow X)}{M_{H}^{2}},
$$

where $W_{\gamma \gamma}$ is the two-photon invariant mass and the initial-state photons have the same helicity. Since the Higgs boson has spin zero, the initial photons must be in a $J_{Z}=0$ state. Event rates for Higgs production and decay into the three primary final states of interest are shown in Fig. 2.48 [101]. 


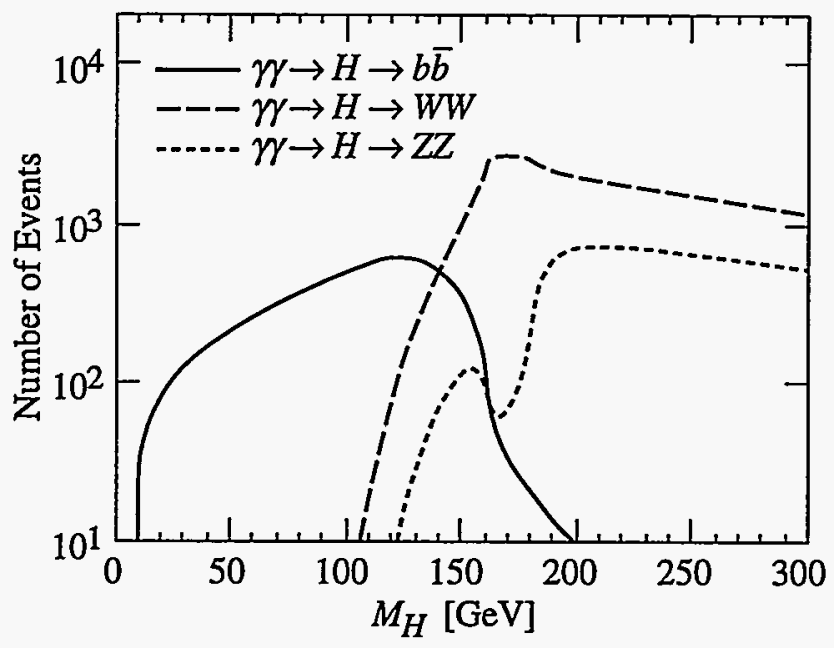

Figure 2.48: Event rates for $\gamma \gamma \rightarrow H \rightarrow b \vec{b}$ and $\gamma \gamma \rightarrow H \rightarrow W W, Z Z$, assuming a luminosity function of $4 \times 10^{-2} \mathrm{fb}^{-1} / \mathrm{GeV}$ and $\lambda_{1} \lambda_{2}=1$.

In the intermediate mass region $(<150 \mathrm{GeV})$ the Higgs decays dominantly to $b \bar{b}$. With vertex tagging to remove the backgrounds from light quark production, the most important backgrounds arise from continuum production of heavy quarks. These backgrounds are quite large, but can be actively suppressed by exploiting the polarization dependence of their crosssections, which are dominated by the $J_{z}= \pm 2$ configurations of initial photons, and by the use of angular cuts. Additional event shape and jet width cuts must be used to suppress radiative processes [102].

Several additional potential backgrounds might fake the presence of a Higgs boson with mass close to the $Z$ mass. The most important of these is the reaction $e \gamma \rightarrow e Z \rightarrow e b \bar{b}$, initiated by a residual electron left over from the original Compton backscatter. To avoid this background, it will be necessary to sweep these spent electrons away from the interaction point. The process $\gamma \gamma \rightarrow f \bar{f} Z$, where the $f \bar{f}$ go down the beam pipe and the $Z$ decays to $b \bar{b}$ provides a background of the same order of magnitude as that of $a \approx 90 \mathrm{GeV}$ Higgs signal [103]. These backgrounds are less important if the Higgs boson mass is already known to be separated from that of the $Z$ by at least the experimental mass resolution, for example, from the observation of the Higgs in $e^{+} e^{-} \rightarrow Z H$.

For Higgs boson masses above about $150 \mathrm{GeV}$, the dominant Higgs decay is to $W W$, where one of the $W$ 's may be virtual. However, the large continuum cross section is not easily suppressed, so the $W W$ final state will be a difficult one to use for doing Higgs physics. Fortunately, the $Z Z$ or $Z Z^{*}$ decay channel can be utilized. Except for Higgs masses between the $W W$ and $Z Z$ thresholds, the Higgs has a branching fraction into this channel of approximately $1 / 3$, while the Standard Model cross section for $\gamma \gamma \rightarrow Z Z$ is small. Hadronic decays of the $Z$ bosons predominate, but the huge $\gamma \gamma \rightarrow W W$ cross section results in a large number of background events in which both $W$ 's are misidentified as $Z$ 's. Thus, to tag the Higgs unambiguously, it is necessary to require that at least one $Z$ decays leptonically. For 
Higgs masses above about $350 \mathrm{GeV}$, the $\gamma \gamma \rightarrow Z Z$ continuum background becomes large and makes detection of the Higgs very difficult [104].

Detailed Monte Carlo studies $[101,105]$ indicate that it should be possible to measure the two-photon width of a Higgs to a precision better than $10 \%$ over most of the mass range up to $250 \mathrm{GeV}$. Measurements of this quality should distinguish among many of the competing models for Higgs production. Further discrimination will be supplied if several Higgs bosons can be detected and their $\Gamma_{\gamma \gamma}$ values obtained.

For $e^{-} e^{-}$collisions, we note that the Standard Model Higgs signals from the reactions $e^{-} e^{-} \rightarrow e^{-} e^{-} H, e^{-} e^{-} Z H$, and $e^{-} \nu_{e} W^{-} H$ have cross sections of order $10 \%$ of the leading modes for the $e^{+} e^{-}$initial state. Moreover, in the intermediate mass range where $H \rightarrow b \bar{b}$ decays dominate, there are effective cuts which lead to very clean data samples [106]. For $m_{H}>200 \mathrm{GeV}$, the process $e^{-} e^{-} \rightarrow e^{-} e^{-} H$ via $\mathrm{ZZ}$ fusion becomes a very favorable discovery channel for the Higgs: It overtakes the process $e^{+} e^{-} \rightarrow Z H$ in cross-section with rising $\sqrt{s}$, and the background rejection is more effective than in $e^{+} e^{-} \rightarrow H \nu \nu$ via $W W$ fusion because there is no missing momentum and both electrons can be detected [107]. In certain models with extended gauge symmetries, $e^{-} e^{-}$collisions also offer the possibility of double Higgs production $\left(H^{-} H^{-}\right)$[108] or doubly charged Higgs production $\left(H^{--}\right)$[109].

\subsubsection{Other New Physics Signatures}

Experiments on $e^{-} e^{-}, e^{-} \gamma$, and $\gamma \gamma$ collisions can also give additional insight into many of the other physics topics we have discussed. In the study of the top quark, for example, the $e^{-} \gamma$ reactions offers the possibility of creating top quarks singly in a well-controlled way through $e^{-} \gamma \rightarrow \nu \bar{t} b$. This reaction has been suggested as a method for obtaining a very precise value of $V_{t b}$, with an accuracy better than $3 \%$ [110]. The $\gamma \gamma$ and $e^{-} \gamma$ colliders are also powerful tools for tests of QCD in the study of the photon structure function and other manifestations of the photon hadronic structure [111].

In supersymmetric theories, the reaction $e^{-} e^{-} \rightarrow \tilde{e}^{-} \tilde{e}^{-}$is a promising mode for the discovery and study of the selectron. In regions of parameter space where the neutralinos are heavy or degenerate, this reaction has the advantage of exceptionally low background [112]. In addition, since it proceeds by neutralino exchange, the cross section for this reaction can be used to measure the neutralino masses and mixings. If the selectron is heavy, the kinematic reach for its discovery can be extended by searching for single selectron production via $e^{-} \gamma \rightarrow \tilde{e} \tilde{\gamma} \rightarrow e \tilde{\gamma} \tilde{\gamma}$ [113].

We have discussed the utility of $e^{-} \gamma$ and $\gamma \gamma$ collisions in the study of anomalous $W$ couplings at the Section 6.4. In Section 7.2, we have pointed out the importance of the reaction $e^{-} e^{-} \rightarrow \nu \bar{\nu} W^{-} W^{-}$in the general program of studies of a strongly interacting Higgs sector. We should also point out the possibility of studying $W W$ scattering at $\gamma \gamma$ colliders. The probability for a photon to branch into a virtual $W^{+} W^{-}$is large at high energies. Thus, it is interesting to observe the process $\gamma \gamma \rightarrow W^{+} W^{-} W^{+} W^{-}$and to use the spectator $W^{\prime} \mathrm{s}$ at relatively low transverse momentum to define the kinematics of a hard $W W$ scattering 
process. It has been shown that it is possible to extract a significant signal for the scattering of longitudinal $W$ bosons in $\gamma \gamma$ collisions at $1.5 \mathrm{TeV}[111,114]$.

In the study of exotic particles and extended gauge groups, there are many interesting reactions which are probed most easily in $e^{-} e^{-}$scattering. If heavy Majorana neutrino exist and mix with the Standard Model neutrinos, the reaction $e^{-} e^{-} \rightarrow W^{-} W^{-}$might have a significant cross section, estimated at 150 events $/ 100 \mathrm{fb}^{-1}$ at a $1 \mathrm{TeV}$ NLC [115]. This process would directly probe lepton number violating couplings. If the gauge group is just $S U(2) \times U(1)$, the signal should appear only in the specific polarization state $e_{L} e_{\bar{L}}$. Unified models in which the three generations are embedded into a single representation of the gauge group predict the existence of gauge bosons with dilepton quantum numbers. These can be discovered as s-channel peaks in $e^{-} e^{-}$scattering [116]. An $e^{-} \gamma$ collider can also be sensitive to dilepton gauge bosons over almost the whole mass region less than $\sqrt{s}$, down to coupling strengths as small as $10^{-4} \alpha$ [117]. Other exotic states such as leptoquark bosons can be studied in $e^{-} \gamma$ and $\gamma \gamma$ reactions in a manner which complements their observation in $e^{+} e^{-}$ annihilation [118]. In particular, models of leptoquark bosons typically predict zeros in the angular distribution for production in $e^{-} \gamma$, and the identification of these features can be a powerful tool in discriminating different models [119].

Finally, Møller scattering at high energy is an ideal way to search for lepton compositeness and other sources of lepton contact interactions. For example, with $50 \mathrm{fb}^{-1}$ of data and $90 \%$ polarized beams, the measurement of $e^{-} e^{-} \rightarrow e^{-} e^{-}$can establish a $95 \%$ confidence limit on the electron compositeness parameter $\Lambda_{L L}^{+}$of $140 \mathrm{TeV}$ [120]. Similarly, the sensitivity to the presence of a $Z^{\prime}$ boson is somewhat greater in Møller scattering than in Bhabha scattering at the same energy and luminosity $[121,122]$, though $e^{+} e^{-}$annihilation offers many additional channels to study. The $e^{-} \gamma$ option allows for searches for excited electrons via $e^{-} \gamma \rightarrow e^{*} \rightarrow e^{-} \gamma$ which are sensitive almost up to the kinematic limit for couplings of size $10^{-5} \alpha$, and the $\gamma \gamma$ option offers similar sensitivity up to masses of $0.7 \sqrt{s}$ for excited lepton searches in $\gamma \gamma \rightarrow e e^{*}, \mu^{*}, \tau \tau^{*}[123]$.

\subsubsection{Accelerator, Lasers, and the Interaction Region}

As will be explained in Chapter 3 , the NLC will include a bend before the interaction region to remove muon and other lower-energy background in the beam. Thus, it is possible to have two interaction regions, one on each side of the linear accelerator. It is natural to design one of these interaction region to be optimized for $e^{-} \gamma$ and $\gamma \gamma$ collision. Since these modes require $e^{-} e^{-}$beams to allow complete control of the polarization [124], it is natural to carry out $e^{-} e^{-}$experiments also in this separate region and also consider a final focus configuration optimized for $e^{-} e^{-}$running. The detailed design of this interaction region has been begun in [1]. We now summarize some of the design considerations.

Of the three reactions to be studied in this interaction region, $e^{-} e^{-}$is the easiest to realize. The major difference from $e^{+} e^{-}$is that the like charges of the beams causes a repulsion which tends to reduce the luminosity by about a factor of 3 relative to the $e^{+} e^{-}$ 
design if no adjustment is made in the final focus. In the $\gamma \gamma$ and $e^{-} \gamma$ cases, there is an inefficiency in converting an electron beams to a photon beam and preserving the tiny spot size required to achieve high luminosity. On the other hand, photon beams do not have a strong beam-beam interaction with beamstrahlung radiation and $e^{+} e^{-}$pair creation, so it is possible to recover part of what is lost by colliding more intense beams and by making the beams round rather than flat, as required for $e^{+} e^{-}$operation. For an $e^{+} e^{-}$collider capable of producing a luminosity of $5 \times 10^{33} \mathrm{~cm}^{-1} \mathrm{sec}^{-1}$, a reasonable goal would be to obtain luminosities of $2.5 \times 10^{33} \mathrm{~cm}^{-1} \mathrm{sec}^{-1}$ in $e^{-} e^{-}, 3 \times 10^{33} \mathrm{~cm}^{-1} \mathrm{sec}^{-1}$ in $e^{-} \gamma$, and $10^{33}$ $\mathrm{cm}^{-1} \mathrm{sec}^{-1}$ in $\gamma \gamma$. In the $\gamma$ reactions, this goal refers to the luminosity within a $10 \%$ band width in center of mass energy.

To produce a $\gamma$ beam by Compton backscattering from an electron beam, the final focus must accommodate a system of mirrors designed to focus light from intense laser beams onto Compton conversion points located about $5 \mathrm{~mm}$ from the interaction point. The laser required for the Compton conversion must have tens of kilowatts of average power, compressed to a peak power of 1 Terawatt in a pulse matched to the electron bunch. A wavelength of about 1 micron is required, with close to $100 \%$ polarization. The laser pulse timing should match that of the electron beam. Such a laser could be built by either combining diode pumping and chirped pulse amplification in solid state lasers or by a free electron laser driven by an induction linac and using chirped pulse amplification. We note that the SLAC experiment E-144 [125] has succeeded in creating Terawatt laser pulses, at a repetition rate of about $1 \mathrm{~Hz}$, and has demonstrated their collisions with the beam spot of the Final Focus Test Beam.

Round beams are required both to maximize the luminosity and to match the laser beam profile at the conversion point. Focussing with $\beta_{x}^{*}=\beta_{y}^{*} \approx 0.5 \mathrm{~mm}$ would produce a beam spot size $\sigma_{x}^{C P}=718 \mathrm{~nm}, \sigma_{y}^{C P}=91 \mathrm{~nm}$ at the conversion point. For these conditions, the Compton conversion efficiency is about $65 \%$ per beam, so the luminosity in $\gamma \gamma$ collisions is necessarily less then $40 \%$ of the geometric luminosity. Only about $20 \%$ of this is in the spectral peak at high energy shown in Fig. 2.47, so the resulting luminosity in high-energy $\gamma \gamma$ collisions is about $10 \%$ of the geometrical expectation. After Compton scattering, the degraded electrons continue towards the interaction point and must be deflected when $e^{-} e^{-}$ or $e^{-} \gamma$ collisions are not desired. The simplest way of doing this is to bring the beams together with a small vertical offset $\left(\approx 1 \sigma_{y}\right)$, and then rely on the mutual repulsion of the electrons to bend these away. There are alternative proposals which involve using a small sweeping magnet or plasma lens near the interaction point; these will require more study to see if they can be implemented without degrading luminosity and detector performance. The dispersion of the degraded electrons also requires a somewhat larger crossing angle (currently estimated as $30 \mathrm{mrad}$ ) at the interaction point than is planned for $e^{+} e^{-}$collisions.

While the inclusion of the hardware of the interaction region puts some special requirements on the second detector, the physics goals demand performance comparable to that in $e^{+} e^{-}$. Certain backgrounds may be more severe, especially those due to the spent electron beams. Luminosity monitoring will require special, low-angle detectors for Møller scattering in $e^{-} e^{-}$mode and for $\gamma \gamma \rightarrow \ell^{+} \ell^{-}$. 


\subsection{Precision Tests of QCD}

Tests of QCD are both enriching and essential to the program of measurements to be made at the NLC. Since QCD is our theory of strong interactions it would be irresponsible not to test it at the highest energy scales available in different hard scattering processes. In addition, the precise determination of the strong coupling $\alpha_{s}$ is key to a better understanding of high energy physics. For example, the current precision of $\alpha_{s}\left(m_{z}^{2}\right)$ measurements, limited to 5$10 \%$, results in the dominant uncertainty on our prediction of the energy scale at which grand unification of the strong, weak and electromagnetic forces takes place [126]. Measurements of hadronic event properties at high energies, combined with existing lower energy data, would allow one to test the gauge structure of QCD by searching for anomalous 'running' of observables, such as the 3-jet event rate, and to set limits on models which predict such effects, for example those involving light gluinos. Gluon radiation in $t \bar{t}$ events is expected to be strongly regulated by the large mass and width of the top quark; $t \bar{t} g$ events will hence provide an exciting new domain for QCD studies. Conversely, measurements of gluon radiation patterns in $t \bar{t} g$ events may provide valuable additional constraints on the top quark decay width. In addition, searches could be made for anomalous chromo-electric and chromomagnetic moments of top quarks, which modify the rate and pattern of gluon radiation and for which the phase space increases as the c.m. energy is raised. Finally, polarized electron beams could be exploited to allow tests of symmetries using multi-jet final states.

\subsubsection{Precise Measurement of $\alpha_{s}$}

Tests of QCD can be quantified in terms of the consistency of values of the yardstick $\alpha_{s}\left(m_{z}^{2}\right)$ measured in different experiments. Measurements of $\alpha_{s}\left(m_{z}^{2}\right)$ have been performed over a range of $Q^{2}$ from roughly 1 to $10^{4} \mathrm{GeV}^{2}$ [127], and are consistent within the errors; an average yields $\alpha_{s}\left(m_{z}^{2}\right)=0.117 \pm 0.006$, implying that QCD has been tested to a precision of about $5 \%$, which is modest compared with the achievement of sub- $1 \%$ level tests of the electroweak theory. This is due primarily to the theoretical uncertainties that dominate the measurements. These uncertainties are due to both the restriction of perturbative QCD calculations to low order, and non-perturbative ('hadronization') effects that are presently incalculable in QCD. We consider whether a measurement of $\alpha_{s}\left(m_{z}^{2}\right)$ at the 1\%-level of precision is possible at the NLC by extrapolation of a recent measurement from $e^{+} e^{-}$annihilation at the $Z^{0}$ resonance by the SLD Collaboration, based on 15 hadronic event shape observables measured with a sample of 50,000 hadronic events [128]:

$$
\alpha_{s}\left(m_{Z}^{2}\right)=0.1200 \pm 0.0025 \text { (exp.) } \pm 0.0078 \text { (theor.) } .
$$

The experimental error is composed of statistical and systematic components of about \pm 0.001 and \pm 0.002 respectively, and the theoretical uncertainty has components of \pm 0.003 and \pm 0.007 arising from hadronization and missing higher order terms, respectively.

Based on this experience, we can estimate the errors to be expected for a similar measurement of $\alpha_{s}$ at a $500 \mathrm{GeV}$ NLC: 
Statistical error: At design luminosity, the NLC would deliver roughly 200,000 $q \bar{q}$ $(\mathrm{q}=\mathrm{u}, \mathrm{d}, \mathrm{s}, \mathrm{c}, \mathrm{b})$ events per year implying that a statistical error on $\alpha_{s}\left(m_{z}^{2}\right)$ of about \pm 0.0005 could be obtained. Cuts for rejection of $\mathrm{W}^{+} \mathrm{W}^{-}$and $t \bar{t}$ backgrounds, based on kinematic information as well as beam polarization and b-tagging, will not substantially reduce the $q \bar{q}$ sample size.

Systematic error: This results primarily from the uncertainty in modeling the jet resolution of the detector. The situation may be improved at the NLC both from an improved calorimeter and from the naturally improved calorimeter energy resolution for higher energy jets. It is not unreasonable to suppose that the current systematic error of \pm 0.002 could be reduced by a factor of two, but more convincing demonstration of this point would require a simulation of the detector, as well as the event selection and analysis cuts [129].

Hadronization uncertainty: Since jets of hadrons, rather than partons, are observed in detectors, it is necessary to correct hadronic distributions for any smearing and bias effects that occur in the hadronization process. Such corrections are usually estimated from Monte Carlo simulations incorporating hadronization models. In $Z^{0}$ decays, they are typically at the level of $10 \%$ [128]. One can argue that non-perturbative corrections to jet final states in $e^{+} e^{-}$annihilation can be parametrized in terms of inverse powers of $\sqrt{s}$ [130], and that for a generic observable $X$ the ratio of non-perturbative to perturbative QCD contributions is dominated by a term of the form:

$$
\frac{\delta X^{\text {non-pert }}}{X^{\text {pert }}} \sim \frac{\log s}{\sqrt{s}} .
$$

Increasing $Q$ from $91 \mathrm{GeV}$ to $500 \mathrm{GeV}$ decreases this ratio by a factor of 5 , implying that hadronization corrections should be of order $2 \%$ at NLC. Assuming that these corrections can be estimated to better than $\pm 50 \%$, the hadronization uncertainty on $\alpha_{s}\left(m_{z}^{2}\right)$ should be less than \pm 0.001 .

Uncertainty due to missing higher orders: Currently perturbative QCD calculations of hadronic event measures are available complete up to $\mathcal{O}\left(\alpha_{s}^{2}\right)$. Since the data contain knowledge of all orders one must estimate the possible bias inherent in measuring $\alpha_{s}\left(m_{z}^{2}\right)$ using the truncated QCD series. It is customary to estimate this from the dependence of the fitted $\alpha_{s}\left(m_{z}^{2}\right)$ on the QCD renormalization scale, yielding a large and dominant uncertainty of about \pm 0.007 [128]. At $500 \mathrm{GeV}$ this uncertainty will be reduced only slightly to \pm 0.006 .

From this simple analysis it seems reasonable to conclude that achievement of the luminosity necessary for 'discovery potential' at the NLC will result in a $q \bar{q}$ event sample of sufficient size to measure $\alpha_{s}\left(m_{z}^{2}\right)$ with a statistical uncertainty of better than $1 \%$. Construction of detectors superior in performance to those in operation today should enable reduction of systematic errors to the $1 \%$ level. Hadronization effects should be significantly smaller and imply a sub-1\% uncertainty. The missing ingredient for an overall $1 \%$-level $\alpha_{s}\left(m_{z}^{2}\right)$ measurement at $500 \mathrm{GeV}$ is the calculation of $\mathcal{O}\left(\alpha_{s}^{3}\right)$ perturbative QCD contributions, which should be actively pursued. 


\subsubsection{Energy Evolution Studies}

The non-Abelian gauge structure of QCD implies that the strong coupling decreases roughly as $1 / \ell n s$. Existing hadronic final states data from $e^{+} e^{-}$annihilation at the PETRA, PEP, TRISTAN, SLC and LEP colliders span the range $14 \leq \sqrt{s} \leq 91 \mathrm{GeV}$, although hadronization uncertainties are large on the data below $25 \mathrm{GeV}$ [127]. A $1.5 \mathrm{TeV}-\mathrm{NLC}$ would increase the lever-arm in $1 / \ell n s$ by almost a factor of two, allowing detailed study of the energy evolution of QCD observables proportional to $\alpha_{s}$, such as the rate of 3-jet production $R_{3}$. This would provide not only a test of the fundamental structure of the $\mathrm{SU}(3)_{c}$ group, but also a search-ground for new physics that might produce 'anomalous' running. One such possibility is the existence of a light, electrically neutral colored fermion that couples to gluons, for example, a light gluino. The existence of such a particle would manifest itself via a modification of gluon vacuum polarization contributions involving fermion loops, effectively increasing the number of light fermion flavors $N_{f}$ entering into the QCD $\beta$-function. For the case of a light gluino, which leads to a $10 \%$ increase in the value of $R_{3}$ at $500 \mathrm{Gev}$, a $1 \%$ measurement of $\alpha_{s}$ would allow this effect to be detected with a significance of many standard deviations.

However, data from experiments at different $e^{+} e^{-}$colliders contributing to this analysis, some of which were recorded more than 10 years ago, were treated differently by the various experimental groups, and have relatively large systematic errors that are at least partly uncorrelated from point to point. It is clear that the precision of searches for anomalous running of QCD observables at NLC would be improved significantly if new data were taken at lower c.m. energies with the same detector and analysis procedures. Table 2.13 shows the number of $q \bar{q}$ events delivered per day at various c.m. energies by the NLC operating at design luminosity; more luminosity would be delivered per day than was recorded in total by the original lower energy colliders! The ability to maintain high luminosity at low center of mass energies presents a formidable challenge to the design of the collider, but even running at lower luminosity could deliver substantial data samples, especially at the $Z^{0}$, although the high event rates would present extreme requirements on the triggering and data processing capabilities of the detector.

Table 2.13: Number of $q \bar{q}$ events per day delivered by NLC at design luminosity

\begin{tabular}{|c|r|}
\hline \hline CM Energy $Q(\mathrm{GeV})$ & $q \bar{q}$ events/day \\
\hline 500 & 1750 \\
91 & $20,000,000$ \\
60 & 75,000 \\
35 & 150,000 \\
\hline \hline
\end{tabular}




\subsubsection{Symmetry Tests Using Beam Polarization}

For polarized $e^{+} e^{-}$annihilation to three hadronic jets one can define the triple-product $\overrightarrow{S_{B}} \cdot\left(\overrightarrow{k_{1}} \times \overrightarrow{k_{2}}\right)\left(B=\gamma, Z^{0}\right)$, which correlates the boson polarization vector $\overrightarrow{S_{B}}$ with the normal to the three-jet plane defined by $\overrightarrow{k_{1}}$ and $\overrightarrow{k_{2}}$, the momenta of the highest- and the secondhighest-energy jets respectively. The triple-product is even under reversal of $C P$, and odd under $T_{N}$, where $T_{N}$ reverses momenta and spin-vectors without exchanging initial and final states. Standard Model $T_{N^{-}}$odd contributions of this form at the $Z^{0}$ resonance have been investigated [131] and are found to be of order $\beta \sim 10^{-5}$; the first experimental study of this quantity has been made by SLD [132], yielding limits: $-0.022<\beta<0.039$. Above the $Z^{0}$ the dominant Standard Model contributions remain smaller than 2 parts in $10^{5}$, presenting a background-free observable for new contributions from beyond the Standard Model, for example, due to rescattering of new gauge bosons that couple only to baryon number [133].

\subsubsection{Gluon Radiation in $t \bar{t}$ Events; Anomalous Couplings}

The large mass and decay width of the top quark serve to make the study of gluon radiation in $t \bar{t}$ events a new arena for testing QCD. The mass $m_{t}$ acts as a cutoff for collinear gluon radiation, and the width $\Gamma_{t}$ acts as a cutoff for soft gluon radiation, allowing reliable perturbative QCD calculations to be performed. We have noted in Section 3.1 the influence of the large top quark width on the rate and pattern of gluon radiation in $t \bar{t}$ events.

The existence of anomalous couplings of top quarks to gluons could manifest itself via a modification of the rate and pattern of emitted gluon radiation, beyond effects such as those just discussed. A parametrization of anomalous couplings in the strong-interaction Lagrangian may be written:

$$
\mathcal{L}^{q \bar{q} g}=g_{s} \bar{q} T_{a}\left(\gamma_{\mu}+\frac{i \sigma_{\mu \nu} k^{\nu}}{2 m_{t}}\left(\kappa-i \tilde{\kappa} \gamma_{5}\right)\right) q G_{a}^{\mu}
$$

where $\kappa$ and $\tilde{\kappa}$ represent anomalous 'chromomagnetic' and 'chromoelectric' dipole moments, respectively. The chromoelectric moment gives rise to $\mathrm{CP}$-violating effects. Their effects on gluon radiation have been calculated to the leading order in $\alpha_{s}$ [134]. At $\sqrt{s}=500 \mathrm{GeV}$, limits of $|\kappa| \leq 0.03$ appear reachable; at $\sqrt{s}=1.5 \mathrm{TeV}$, one may additionally constrain $|\tilde{\kappa}| \leq 0.2$. These should be compare to a limit of $|\kappa| \leq 0.1$ which would be obtained from a measurement of the $t \bar{t}$ cross section at the Tevatron with 10 times the current integrated luminosity. A similar limit on $\kappa$ from $\mathrm{LHC}$ would require a $t \bar{t}$ cross section measurement to better than $20 \%$. 


\subsection{Design of the NLC Detector}

Now that we have described the expected physics program of the NLC, we must discuss in more detail what experimental facilities should be necessary. In this section, we will present a sample design of an NLC detector and discuss the general issues which constrain this design. This detector takes into account the particular features of the NLC accelerator and its machine-related backgrounds. A more complete discussion of the machine/detector interaction is given at the end of Chapter 3 .

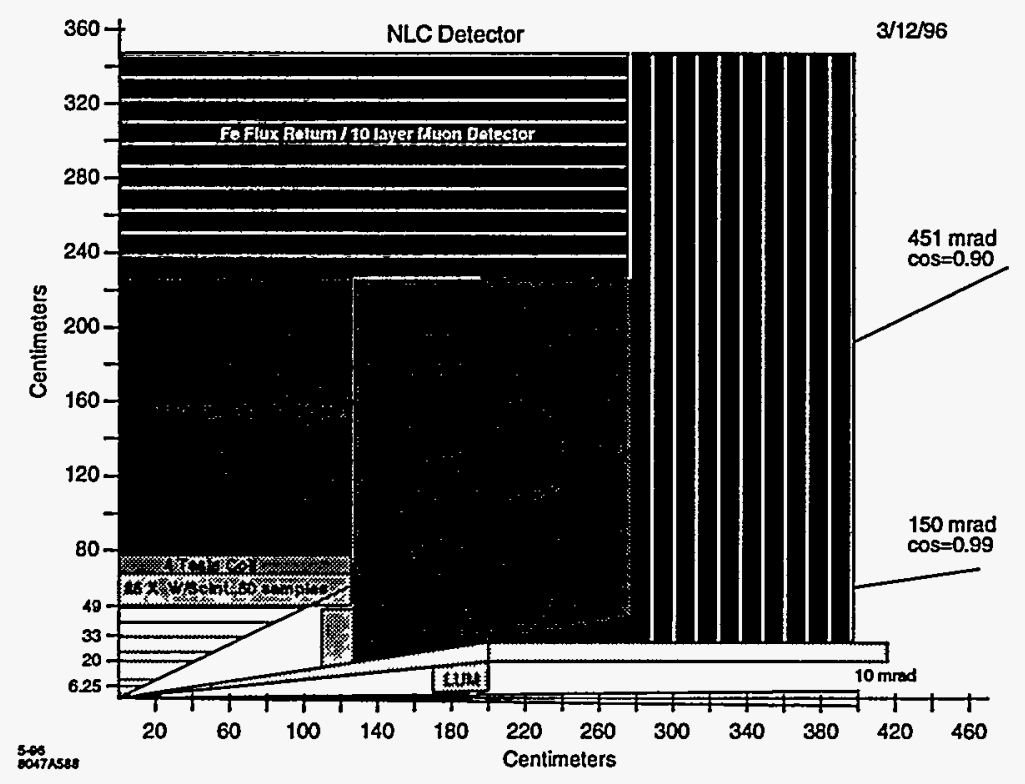

Figure 2.49: NLC Detector.

A general plan of a detector for the NLC is shown, in quadrant view, in Fig. 2.49. This detector is at a very early conceptual design stage. The architecture is a "standard" solenoid, and is just one possible approach. A 3 or 4 layer CCD vertex detector surrounds a beryllium vacuum pipe. The momentum measurement is carried out by layers of silicon microstrip detectors in a 4 Tesla field, which is produced by a superconducting solenoid outside of the electromagnetic calorimeter. A hadronic calorimeter is located outside the coil, and the structure is wrapped in laminated iron for a flux return and muon identification system. The final focus quadrupoles are located inside the detector at an $L^{*}$ of $2 \mathrm{~m}$. A summary of the components and expected performance of the detector is given in Table 2.14.

The small volume 4 Tesla field is well matched to the expected backgrounds, cost, and technology. The small size of the tracking system eases the mechanical problem of stabilizing the final focus elements relative to one another at the required nanometer level. However, this design increases the complexity of the elements inside the detector. For example, the final quadrupoles, envisioned as permanent magnets, would have to be shielded by a small super conducting solenoid. The tracking system is modeled as 5 layers of Si microstrips 
Table 2.14: Basic Components of the NLC Detector

\begin{tabular}{|l|c|}
\hline \hline Vertex & $3-5$ CCD Layers \\
& $2.6 \oplus\left(13.7 / p \sin ^{3 / 2} \theta\right)$ \\
\hline Tracking & $5 \mathrm{Si}$ Layers \\
& $|\cos \theta| \leq 0.9$ \\
& $\delta p_{T} / p_{T}=2 \times 10^{-4}$ at $100 \mathrm{GeV}$ at $\cos \theta=0.9$ \\
\hline Coil & $4 \mathrm{~T}, 2-3 X_{0}$ \\
\hline EM Calorimeter & $25 X_{0}, 50$ samples \\
& $\sigma_{E} / E=\left(\frac{12 \%}{\sqrt{E}}\right) \oplus 1 \%$ \\
\hline Hadronic Calorimeter & Fe Scintillator \\
& $6 \lambda, 100$ samples \\
& $\sigma_{E} / E=\left(\frac{45 \%}{\sqrt{E}}\right) \oplus 2 \%$ \\
\hline Return Flux & $6 \lambda, 10$ samples \\
\hline \hline
\end{tabular}

with 5 micron spatial resolution. The expected momentum resolution, including multiple scattering, is shown in Figure 2.50. Agreement that this performance is a reasonable match to the physics goals is probably the most important question for the overall detector design. The cylindrical layers extend to $\cos \theta=0.9$. This leaves sufficient space for the ends of the support structure, electronics, cabling, and cooling. End cap trackers could be added for improved coverage. Experience with SLC/SLD confirms the idea that backgrounds from a linear collider can be very severe for conventional wire chambers. A silicon strip system would be extremely robust.

The electromagnetic calorimeter would be inside the coil, 25 radiation lengths thick, and with angular segmentation in the range of 30 to $40 \mathrm{mrad}^{2}$. Possible structures include tungsten silicon-diode sampling towers and BGO or perhaps more exotic crystals. The crystals would be interesting not so much for their good resolution but for their lack of. interconnections. The calorimetry is extended by endcaps to $\cos \theta=0.99$.

The superconducting coil has an inner radius of $70 \mathrm{~cm}$ and a half length of $1.2 \mathrm{~m}$. Since there are only weak constraints on the thickness of the coil, given that it is outside the electromagnetic calorimeter, and since it is a relatively small coil, it should be a reasonable coil to design and build. This design will be made more challenging, however, by its required integration with the quadrupole shields and supports.

The hadronic calorimeter is assumed to be an iron scintillator sampling system, $6 \lambda$ thick and 100 samples. At this time, no magnetic modeling of the structure has been done. It is 


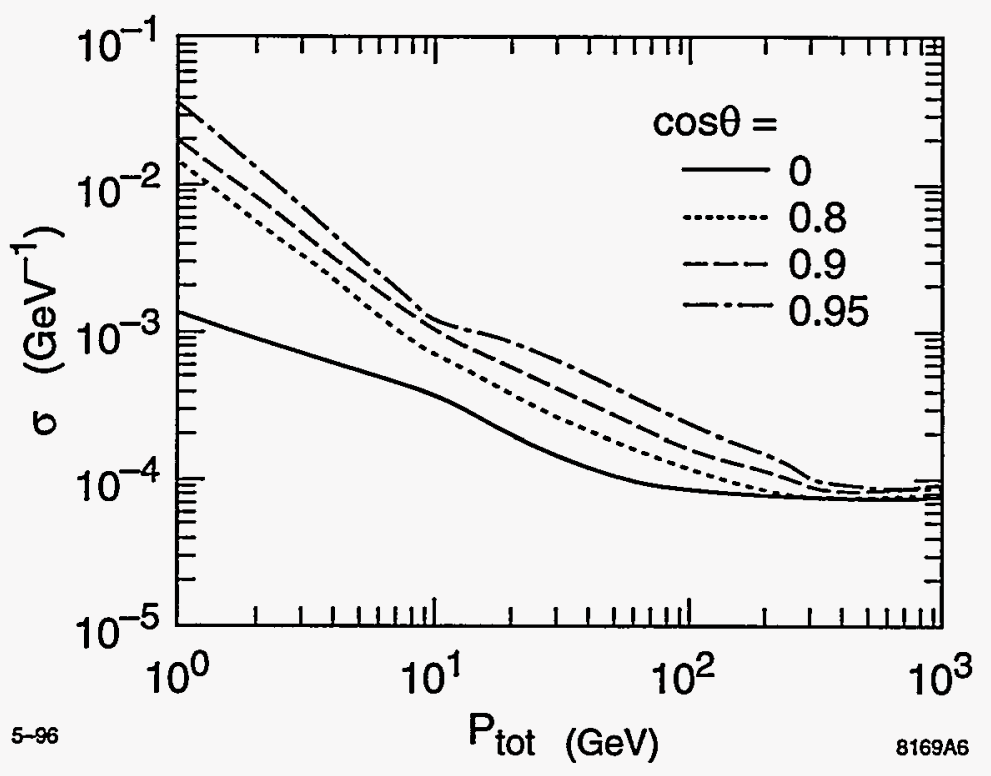

Figure 2.50: NLC Detector tracking parameters.

unclear whether the advantages of an iron based calorimeter in containing the magnetic flux would be outweighed by the structural problems.

Finally, the detector is wrapped in a flux return $6 \lambda$ thick with 10 layers for muon detectors. Questions of overall geometry for internal access and vibration control are completely open.

The crossing of the two beams at the interaction point produces an irreducible $e^{+} e^{-}$ pair background at small $p_{\perp}$. These particles can be kept away from a small radius vertex detector by the relatively high 4 Tesla magnetic field. A reasonable vertex detector for this environment would be a successor to the SLD vertex detector using large area CCDs with 20 micron square pixels. The CCDs could be read out in a time equivalent to a small number of beam crossings, and the beams could be suppressed subsequent to a trigger if necessary. At SLD the innermost layer of the vertex detector, at $r=2.5 \mathrm{~cm}$ with $B=0.6 \mathrm{~T}$, sees $0.4 \mathrm{hits} / \mathrm{mm}^{2}$ summed over its 19 machine pulse readout time. Vertex hits are successfully attached to tracks extrapolated from a conventional drift chamber whose closest wire is at $r=22 \mathrm{~cm}$. Thus a conservative estimate for the tolerable background is $1 \mathrm{hit} / \mathrm{mm}^{2}$. Were the NLC vertex detector to extrapolate hits from its own high resolution outer layers into an inner layer, the much smaller search area would allow a much larger $\left(10-20 \mathrm{hits} / \mathrm{mm}^{2}\right)$ background to be tolerated.

Fig. 2.51 shows the hit density from the $e^{+} e^{-}$pair background, per bunch train (a series of 90 bunch collisions), at a radius of $3 \mathrm{~cm}$ and at $2 \mathrm{~cm}$. The results are shown as a function of $z$ for a 4 Tesla field. At $r=3 \mathrm{~cm}$ the background is nonexistant out to $z=45 \mathrm{~cm}$. At $r=2 \mathrm{~cm}$ the background is similar to that tolerated by an existing detector out to $z=17$ $\mathrm{cm}$, a length adequate to provide vertexing coverage well beyond the $\cos \theta=0.90$ currently used in the physics simulations. 


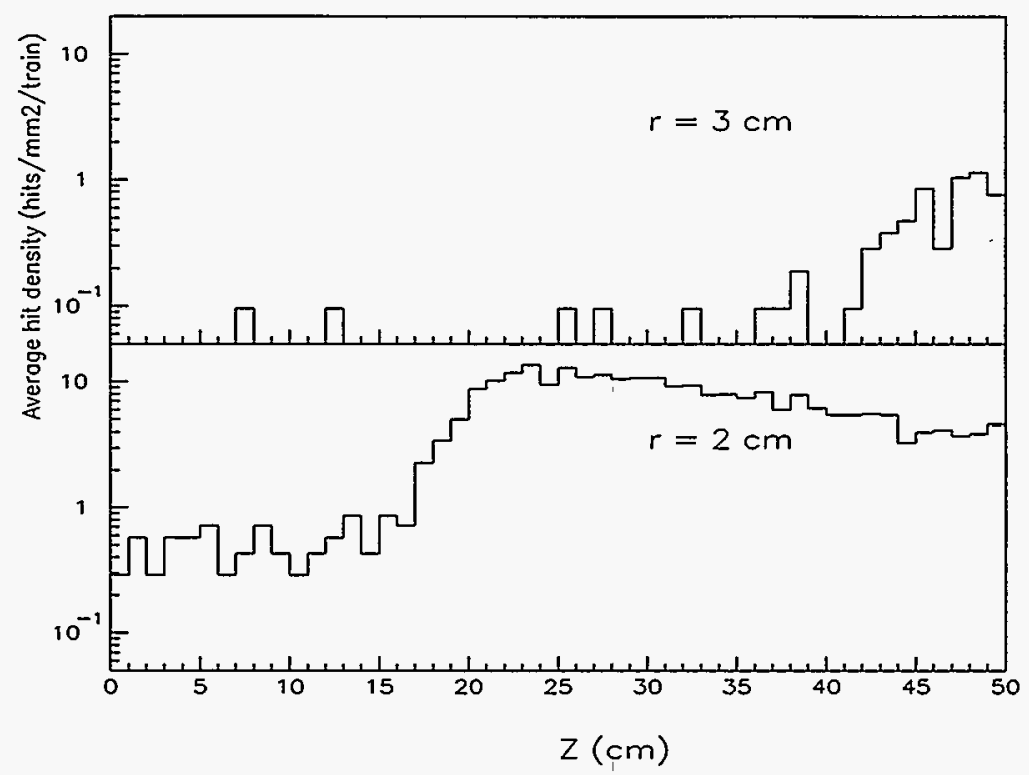

Figure 2.51: Electron pair hit density per $\mathrm{mm}^{2}$ per train of 90 bunches, computed with the Monte Carlo program ABEL. As the pairs leave the IP in a 4 Tesla field, hits are scored (a) at $r=3 \mathrm{~cm}$, and (b) at $r=2$ $\mathrm{cm}$.

In addition to the irreducible background of pairs emanating from the interaction point, the vertex detector will see a background of very low energy backscattered particles resulting from the interaction of the pairs that have been curled up by the field and gone forward to strike the quad faces, septum, and luminosity monitor. In the current interaction region design the calculated rate is 2.0 hits $/ \mathrm{mm}^{2} /$ train at $r=3 \mathrm{~cm}$, flat in $z$, and roughly constant down to $r=2 \mathrm{~cm}$, then rising at lower radii. This background rate, already sufficiently low at $r=2 \mathrm{~cm}$ for efficient vertexing, is very sensitive to the details of the interaction region design. Efforts to reduce the flux of these soft particles, by, for instance, the use of low- $Z$ coatings, are underway.

As experience in the collider operation is gained, one may want to consider vertex detection at smaller radii. Even at the very aggressive radius of $1 \mathrm{~cm}$, in a 4 Tesla field the pair background hit rate remains below $10 / \mathrm{mm}^{2} /$ train out to $z=3 \mathrm{~cm}$. Moreover, since the tracking system radius largely drives the scale of the entire detector, a small tracker permits consideration of relatively small $L^{*}$ as a possible future upgrade of the final focus design. The small $L^{*}$ is plausible because the axial extent of the tracker is small, about $1 \mathrm{~m}$ for $\cos \theta<0.9$. This leads to significant improvements in required tolerances in the final focus system. 


\subsection{Physics Processes which Constrain Detector Per- formance}

In general, the requirements of the NLC physics program do not put strong constraints on the detector design and performance. For most physics analyses, the performance of the detectors constructed for SLC and LEP would be quite sufficient. These detectors already include the basic requirements for the study of $e^{+} e^{-}$annihilation at high energy: calorimetry with good energy resolution, segmentation, and charged lepton identification; tracking with high momentum resolution, good forward angular acceptance and overall granularity; and excellent secondary vertex tagging. However, it is always interesting to consider advanced detectors with improved performance in one or another of these areas. In this section, we enumerate those physics topics which put exceptional or unusual demands on the detector and which might motivate a more advanced detector design.

A basic benchmark for detector performance is that given by standard model Higgs production in the all-hadronic mode: $e^{+} e^{-} \rightarrow Z h \rightarrow j j+b \bar{b}$. This sets a standard of hadronic energy resolution, angular coverage for high acceptance, and $b$-tagging efficiency. The recognition of the $Z$ in its hadronic decay is required, more generally, for high-statistics branching ratio determinations for the Higgs boson.

For the Higgs boson of the Minimal Supersymmetric Model, it is possible for the Higgs boson to decay by mechanisms very different from those of the Standard Model. The interesting mode $e^{+} e^{-} \rightarrow h A \rightarrow b \bar{b} b \bar{b}$ places a premium on angular coverage and efficiency of $b$-tagging. More generally, it is important in this theory to be able to recognize the production of the Higgs boson in $e^{+} e^{-} \rightarrow Z h$ making as few assumptions as possible on the final state to which the Higgs decays. Such a model-independent search for the Higgs boson is possible if the $Z$ can be reconstructed as an $\ell^{+} \ell^{-}$pair superimposed on any reasonable Higgs boson final state. This analysis constrains the momentum resolution and two-track separation of the tracking device.

Finally, though the decay of the Higgs to $\gamma \gamma$ is rare, one might hope to observe the branching ratio for $h \rightarrow \gamma \gamma$ from the process $e^{+} e^{-} \rightarrow Z h$. This would demand a high-quality electromagnetic calorimeter, one with at least 3 times better resolution than is called for in our design [135].

For studies of supersymmetry, we have shown that calorimeter coverage down to $\cos \theta=$ 0.99 is essential to reduce the standard model backgrounds to events with low visible energy. In addition, we have seen that the mass measurements for supersymmetric particles typically involve the measurement of sharp kinematic endpoints in lepton or jet energy distributions. The calorimetric energy resolution must be sufficiently good that the measurement of these endpoints is limited only by initial state radiation and beamstrahlung.

For studies of the $W$ boson vertices in $e^{+} e^{-} \rightarrow W^{+} W^{-}$, it is necessary to completely reconstruct the final state kinematics by measurement of a lepton +2 jet system. This would seems to demand high-quality tracking. However, it has been shown that the tracking requirements are less severe if kinematic fitting is used to determine the jet energies [85]. 
In the study of $W W$ scattering, the reactions $e^{+} e^{-} \rightarrow \nu \bar{\nu} W W$ and $e^{+} e^{-} \rightarrow \nu \bar{\nu} Z Z$ give signatures for distinct models of a strongly interacting Higgs sector, as explained in Section 7.2. Thus, it is necessary to distinguish these processes experimentally. Because the event rate is rather small, it is necessary to rely on detection of $W$ and $Z$ in their hadronic decay modes. Thus, it is important that the calorimetric mass resolution for $W$ and $Z$ be sufficiently small that these particles can be distinguished on the basis of their masses. The analysis discussed in Section 7.2, which assumed a calorimeter resolution essentially the same as that of our model detector, found and incorporated a probability of about $10 \%$ for confusing each $W$ for a $Z$ or vice versa. In addition, this analysis requires good forward coverage for high-energy electrons and positrons, to remove background from the processes $e^{+} e^{-} \rightarrow e^{+} e^{-} W^{+} W^{-}$and $e^{+} e^{-} \rightarrow e \nu W Z$ which are induced by virtual photons.

Finally, we note that the study of the top quark threshold is affected by the energy spread of the $e^{+} e^{-}$collisions, which is determined by the accelerator design. For this study to be carried out optimally, the beam energy spread from beamstrahlung should be kept to the level of initial state radation, about $3 \%$, and the intrinsic beam energy spread of the accelerator should be kept to $0.1 \%$. These requirements are quite reasonable for the machine design discussed in Chapter 3. In addition, since the differential luminosity spectrum depends on the beam parameters, it is necessary to measure this directly. This requires the measurement of acollinearity angles in small-angle Bhabha scattering to better than $1 \mathrm{mrad}$ for $\theta \sim 200$ $500 \mathrm{mrad}$. The detector design in Section 11 includes an appropriate electromagnetic shower detector placed inside the synchrotron radiation mask. 


\subsection{Conclusions}

The Standard Model of strong and electroweak interactions represents a tremendous scientific achievement. It provides an elegant and well tested description of fundamental forces based on underlying local symmetry concepts. However, despite those successes, some basic questions remain unanswered. Addressing those issues should guide us to a deeper understanding and better appreciation of Nature's laws.

What is the origin of electroweak symmetry breaking and mass generation? That outstanding problem drives high energy physics and establishes its experimental goals. Fortunately, resolution of that puzzle appears to be within reach of the next generation of hadron and lepton colliders, the LHC and the NLC.

In this report, we have described some of the physics goals and capabilities of the NLC, emphasizing both its unique features and complementarity to the LHC program planned at CERN. The envisioned project would start with an initial variable center-of-mass energy up to about $500 \mathrm{GeV}$ and be upgradable to $1-1.5 \mathrm{TeV}$. It would feature a highly polarized $e^{-}$ beam as well as $e^{-} e^{-}, \gamma e^{-}$and $\gamma \gamma$ collider options.

The first phase of the NLC guarantees extensive physics capabilities and interesting discovery potential. Sitting at and above the $t \bar{t}$ threshold will allow unprecedented precision measurements of the top quark's mass, partial decay widths, gauge and Higgs boson couplings etc. Its very large mass may be a signal of the top quark's special role in elementary particle physics and electroweak symmetry breaking. We must, therefore, explore its properties and search for additional anomalous features. In that endeavor, the LHC will be a top quark factory, capable of producing and studying samples of order $10^{7}$ tops in a number of characteristic signatures. The NLC will complement such studies as a precision instrument which is sensitive to the broad range of top couplings to strong and weak gauge bosons. Similarly, studies of gauge boson pair production at the NLC will provide high precision measurements of triple-gauge boson couplings at the level of $1-0.1 \%$ (with increasing centerof-mass energy). At that level of sensitivity anomalous properties could become unveiled, particularly if there is new strong dynamics at $\sim 1 \mathrm{TeV}$.

If a fundamental Higgs scalar exists and has mass below $350 \mathrm{GeV}$, it will be found and studied in the first phase of the NLC via $e^{+} e^{-} \rightarrow Z H$. That mass reach is extremely broad and encompasses a most important domain. If the Standard Model is perturbative up to grand-unification energies, then $m_{H} \lesssim 200 \mathrm{GeV}$. If supersymmetry is manifested at the electroweak scale, then the lightest scalar satisfies $m_{h}<150 \mathrm{GeV}$. The first stage of the NLC will be definitive in confirming or negating those expectations. A higher mass Higgs ( $>350$ $\mathrm{GeV}$ ) would be easily accessed at the LHC or higher energy phases of NLC. Additional Higgs scalars of SUSY scenarios, or pseudo-Goldstone bosons of dynamical symmetry breaking schemes, would be unveiled in pair-production as their thresholds are passed at the NLC. The clean environment of that facility would allow detailed studies of their decay patterns. A compelling feature of $e^{+} e^{-}$colliders is the ability to not only produce new particles, but to thoroughly explore their properties. 
If supersymmetry manifests itself near the electroweak scale, a plethora of new particles, the supersymmetric partners of quark, leptons, and gauge bosons, awaits discovery. Unfolding that entire spectrum and its properties will require the full capabilities of the LHC and NLC. The first direct evidence for production of gluinos and squarks should be found at LHC, if not already at the Tevatron. On the other hand, the study of color-singlet superpartners is best carried out at $e^{+} e^{-}$colliders. Since these particles are typically lighter, they determine the decay patterns of the particles produced in strong interactions. Thus, the NLC will be essential for unraveling the entire spectrum and precisely measuring the new particle properties. The study of superpartners will be greatly facilitated by use of the high $e^{-}$polarization both for suppressing backgrounds and enhancing signals. High precision determinations of the SUSY parameters will provide important insight and constraints on the mechanism of SUSY breaking, as well as a potential new window to the physics of grand unification and superstrings.

If new strong dynamics is responsible for electroweak symmetry breaking, it may be difficult to uncover and fully explore. In such a scenario, the NLC will be an extremely valuable instrument. It will probe the new dynamics via $W^{+} W^{-} \rightarrow W^{+} W^{-}$and $W^{+} W^{-} \rightarrow$ $t \bar{t}$ scattering, and through anomalous gauge boson couplings and other probes in $e^{+} e^{-} \rightarrow$ $W^{+} W^{-}$. Such studies will become ever more revealing as the higher energy upgrades are attained. In addition, new strong dynamics is likely to imply a wealth of spectroscopy at very high energies. The NLC, particularly its highest energy phase, should be a very important facility for discovering and studying such states.

The new energy domain opened up by the NLC will also allow systematic searches for new particles such as $Z^{\prime}$ bosons, heavy fermions, leptoquarks, etc. If such particles are found, the $e^{-}$polarization will be extremely useful for deciphering their properties.

The physics capabilities of the NLC can be greatly expanded by the $e^{-} e^{-}, \gamma e^{-}$, and $\gamma \gamma$ collider options possible at such an accelerator. Those collision modes will complement $e^{+} e^{-}$studies and could prove particularly useful to provide new observables of supersymmetric particles, to study the Higgs boson as an $s$-channel resonance, and to explore $W^{-} W^{-}$ scattering.

During the twentieth century, elementary particle physics emerged as a scientific discipline. Since its inception, progress and advancement have followed our ability to probe short distances via high energy collisions. By pushing to even higher energies, we should be able to continue the advancement. In this report, we have reviewed the questions that are now at the frontier of particle physics, and we have shown that the NLC has a central role is answering every one. We thus view the NLC as a forefront facility for particle physics, an essential tool for probing the hidden symmetries of Nature, a well tuned instrument for the start of our next millennium. 


\section{Bibliography}

[1] "Zeroth Order Design Report for the Next Linear Collider," SLAC Report 474 (Stanford University, May 1996).

[2] T. Sjöstrand, Comp. Phys. Comm. 82, 74 (1994).

[3] T. Barklow, preprint SLAC-PUB-7087, to appear in the proceedings of the Workshop on Physics and Experiments with Linear Collider, LCWS95.

[4] K. Hagiwara, K. Hikasa, R. D. Peccei, D. Zeppenfeld, Nucl. Phys. B282, 253 (1987).

[5] A. Miyamoto, in Physics and Experiments with Linear $e^{+} e^{-} e$ Colliders, F. A. Harris, S. L. Olsen, S. Pakvasa, and X. Tata, eds. (World Scientific, Singapore, 1993).

[6] F. Paige and S. Protopopescu, in Supercollider Physics (Snowmass 1986), D. Soper, ed. (World Scientific, Singapore, 1986); H. Baer, F. Paige, S. Protopopescu, and X. Tata, in Proceedings of the Workshop on Physics at Current Accelerators and Supercolliders, J. Hewett, A. White, and D. Zeppenfeld, eds. (Argonne National Laboratory, 1993).

[7] C. Ahn et al., Opportunities and requirements for Experimentation at High Energy $e^{+} e^{-}$ Colliders, SLAC-report-329, May 1988.

[8] F. Abe, et al. (CDF Collaboration), Phys. Rev. Lett. 74, 2626 (1995); S. Abachi, et al. (D0 Collaboration), Phys. Rev. Lett. 74, 2632 (1995).

[9] M. Jezabek, J. H. Kuhn, and T. Teubner, Z. Phys. C56, 653 (1992).

[10] G. Jikia, Phys. Lett. B257, 196 (1991).

[11] V. A. Khoze, L. H. Orr, and W.J. Stirling, Nucl. Phys. B378, 413 (1992).

[12] K. Fujii, T. Matsui, and Y. Sumino, Phys. Rev. D50, 4341 (1994).

[13] M. Strassler and M. Peskin, Phys. Rev. D43, 1500 (1991).

[14] V. Fadin and V. Khoze, JETP Lett. 46, 525 (1987), Sov. J. Nucl. Phys. 48, 309 (1988).

[15] Y. Sumino, K. Fujii, K. Hagiwara, H. Murayama, and C.-K. Ng, Phys. Rev. D47, 56 (1993); M. Jezabek, J. Kuhn, and T. Teubner, Z. Phys. C56, 653 (1992). 
[16] P. Igo-Kemenes, M. Martinez, R. Miquel, and S. Orteu, in Physics and Experiments with Linear $e^{+} e^{-}$Colliders, F. A. Harris, S. L. Olsen, S. Pakvasa, and X. Tata, eds. (World Scientific, Singapore, 1993).

[17] H. Murayama and Y. Sumino, Phys. Rev. D47, 82 (1993).

[18] W. Bernreuther and M. Suzuki, Rev. Mod. Phys. 63, 313 (1991).

[19] G. A. Ladinsky and C. P. Yuan, Phys. Rev D49, 1994 (4415); and references therein.

[20] R. Harlander, M. Jezabek, J. H. Kuhn, and T. Teubner, Phys. Lett. B346, 137 (1995);

R. Harlander, M. Jezabek, J. H. Kuhn, and M. Peter, hep-ph/9604328.

[21] S. Parke and Y. Shadmi, preprint FERMILAB-PUB-96/042-T.

[22] R. Frey, preprint SLAC-PUB-7075, to appear in the proceedings of the Workshop on Physics and Experiments with Linear Colliders, LCWS95.

[23] C. R. Schmidt, preprint hep-ph/9504434.

[24] ATLAS Collaboration, Technical Proposal. CERN/LHC/94-43.

[25] R. Harlander, M. Jezabek, and J. H. Kühn, preprint hep-ph/9506292.

[26] A. Djouadi; J. Kalinowski and P. M. Zerwas, Z. Phys. C54, 255 (1992).

[27] P. Comas, R. Miquel, M. Martinez, and S. Orteu, preprint CERN-PPE/96-40, to appear in the proceedings of the Workshop on Physics and Experiments with Linear Colliders, LCWS95.

[28] K. Fujii, to appear in the proceedings of the 1995 SLAC Summer Institute.

[29] S. Bar-Shalom, D. Atwood, G. Eilam, R. Mendel, and A. Soni, Phys. Rev. D53, 1162 (1996).

[30] D. Amidei and R. Brock, eds., Future ElectroWeak Physics at the Fermilab Tevatron, FERMILAB-PUB-96/046.

[31] C. R. Schmidt and M. E. Peskin, Phys. Rev. Lett. 69, 410 (1992).

[32] C.-P. Yuan, Mod. Phys. Lett. A10, 627 (1995).

[33] P. W. Higgs, Phys. Lett. 12, 132 (1964).

[34] J. F. Gunion, H. E. Haber, G. L. Kane and S. Dawson, The Higgs Hunter's Guide (Addison-Wesley, Redwood City, 1990). 
[35] J. F. Gunion, A. Stange and S. Willenbrock, in Electroweak Symmetry Breaking and New Physics at the TeV Scale, T. Barklow, S. Dawson, H. Haber, and J. Seigrist, eds. (World Scientific, Singapore, 1996).

[36] H. P. Nilles, Phys. Repts. 110, 1 (1984).

[37] H. Haber and G. Kane, Phys. Repts. 117, 75 (1985).

[38] T. Moroi and Y. Okada, Phys. Lett. B295, 73 (1992); G. L. Kane, C. Kolda, J. D. Wells, Phys. Rev. Lett. 70, 2686 (1993).

[39] J. F. Grivaz, preprint LAL-95-83 (1995), to appear in the proceedings of the International Europhysics conference on High Energy Physics, Brussels, 1995.

[40] D. Abbaneo, et al.(LEP Electroweak Working Group) and E. Etion, et al.(LEP Heavy Flavor Group), preprint LEPEWWG/96-01.

[41] M. Carena and P. Zerwas et al., Higgs Physics, to appear in the proceedings of the LEP2 Workshop, CERN Yellow Report, eds. G. Altarelli et al.

[42] G. L. Kane, G. D. Kribs, S. P. Martin, and J. D. Wells, Phys. Rev. D53, 213 (1996).

[43] S. Mrenna and G. L. Kane, preprint hep-ph/9406337.

[44] P. Janot, in Physics and Experiments with Linear $e^{+} e^{-}$Colliders, F. A. Harris, S. L. Olsen, S. Pakvasa, and X. Tata, eds. (World Scientific, Singapore, 1993).

[45] J. Gunion, in Physics and Experiments with Linear $e^{+} e^{-}$Colliders, F. A. Harris, S. L. Olsen, S. Pakvasa, and X. Tata, eds. (World Scientific, Singapore, 1993).

[46] S. Komamiya, in Physics and Experiments with Linear Colliders, R. Orava, P. Eerola, and M.Nordberg, eds. (World Scientific, Singapore, 1992); H.E. Haber, ibid.; J.-F. Grivaz, in $e^{+} e^{-}$Collisions at $500 \mathrm{GeV}$ : The Physics Potential, P. M. Zerwas, ed., DESY$92-123$.

[47] Y. Fujii, in Physics and Experiments with Linear $e^{+} e^{-}$Colliders, F. A. Harris, S. L. Olsen, S. Pakvasa, and X. Tata, eds. (World Scientific, Singapore, 1993); JLC-1 Design Report, KEK Report 92-16.

[48] P. Janot, in Physics and Experiments with Linear Colliders F. A. Harris, World Scientific, 1993, eds. F.A. Harris et al., Waikoloa, Hawaii.

[49] M. D. Hildreth, T. L. Barklow, and D. L. Burke, Phys. Rev. D49, 3441 (1993).

[50] P. Burchat, D. Burke, and A. Petersen, Phys. Rev. D38 (1988) 2735; J. Alexander, et al., in High Energy Physics in the 1990s (Snowmass 1988), S. Jensen, ed. (World Scientific, Singapore, 1989); F. Richard, in Proceedings of the Workshop on Physics at Future Accelerators (La Thuile), CERN 87-07, 1987. 
[51] A. Djouadi, W. Kilian, and P. Ohmann, preprint hep-ph/9512312, to appear in the proceedings of the Workshop on Physics and Experiments with Linear Collider, LCWS95.

[52] M. Carena, J. R. Espinosa, M. Quiros, and C. E. M. Wagner, Phys. Lett. B335, 209 (1995); M. Carena, M. Quiros, and C. E. M. Wagner, Nucl. Phys. B461, 407 (1996); H. E. Haber, R. Hemplling, and A. H. Hoang, preprint CERN-TH-95-216.

[53] A. Sopczak, Charged Higgs Boson Discovery Potential at a $500 \mathrm{GeV} e^{+} e^{-}$Linear Collider, CERN-PPE/93-197; A. Sopczak, in $e^{+} e^{-}$Collisions at $500 \mathrm{GeV}$ : The Physics Potential, P. M. Zerwas, ed., DESY-93-123C.

[54] J. Rosiek and A. Sopczak, Phys. Lett. B341, 419 (1995).

[55] K. Kawagoe, in Physics and Experiments with Linear $e^{+} e^{-}$Colliders, F. A. Harris, S. L. Olsen, S. Pakvasa, and X. Tata, eds. (World Scientific, Singapore, 1993).

[56] B. Grządkowski and J. F. Gunion, Phys. Lett B350, 218 (1995).

[57] J. F. Gunion and B. Grządkowski, Phys. Lett. B294, 361 (1992).

[58] J. F. Gunion and J. G. Kelly, Phys. Lett. B333, 220 (1994).

[59] M. L. Stong, preprint hep-ph/9504345.

[60] H. Haber, preprint hep-ph/9505240.

[61] J. Kamoshita, Y. Okada, and M. Tanaka, preprint hep-ph/9512307.

[62] I. Nakamura and K. Kawagoe, preprint hep-ex/9604010; I. Nakamura, to appear in the proceedings of the Workshop on Physics and Experiments with Linear Colliders, LCWS95.

[63] H. E. Haber, in Recent Directions in Particle Theory (TASI-92), J. Harvey and J. Polchinski, eds. (World Scientific, Singapore, 1993).

[64] H. Murayama, in Physics with High Energy Colliders, S. Yamada and T. Ishii, eds. (World Scientific, Singapore, 1995).

[65] M. E. Peskin, preprint hep-ph/9604339, to appear in the proceedings of the Yukawa International Seminar YKIS'95.

[66] R. Arnowitt and P. Nath, contributed paper in this volume.

[67] H. Baer, R. Dubois, S. Fahey, S. Manly, R. Munroe, U. Nauenberg, X. Tata, D. L. Wagner, contributed paper in this volume.

[68] T. Tsukamoto, K. Fujii, H. Murayama, M. Yamaguchi, and Y. Okada, Phys. Rev. D51, 3153 (1995). 
[69] H. Murayama, I. Watanabe,and K. Hagiwara, HELAS: Helicity Amplitude Subroutines for Feynman Diagram Evaluations, KEK-91-11 (1992).

[70] R. Becker and C. Vander Velde, in Physics and Experiments with Linear Colliders, F. A. Harris, S. L. Olsen, S. Pakvasa, and X. Tata, eds. (World Scientific, Singapore, 1993).

[71] K. Fujii, T. Tsukamoto, and M. M. Nojiri, preprint hep-ph/9511338, to appear in the proceedings of the Workshop on Physics and Experiments with Linear Collider, LCWS95.

[72] J. L. Feng, H. Murayama, M. E. Peskin, and X. Tata, Phys. Rev. D52, 1418 (1995).

[73] J. L. Feng and D. E. Finnell, Phys. Rev. D49, 2369 (1994).

[74] H. Baer, C. H. Chen, F. Paige and X. Tata, Phys. Rev. D53, 6241 (1996).

[75] H. Aihara et al., in Electroweak Symmetry Breaking and New Physics at the TeV Scale, T. Barklow, S. Dawson, H. Haber, and J. Seigrist, eds. (World Scientific, Singapore, 1996).

[76] M. Einhorn, in Physics and Experiments with Linear $e^{+} e^{-}$Colliders, F. A. Harris, S. L. Olsen, S. Pakvasa, and X. Tata, eds. (World Scientific, Singapore, 1993).

[77] J. Bagger, S. Dawson, and G. Valencia, Nucl. Phys. B399, 364 (1993).

[78] J. Wudka, contributed paper in this volume.

[79] E. N. Argyres, Nucl. Phys. B391, 23 (1993); J. Papavassiliou and K. Philoppides, Phys. Rev. D48, 4255 (1993).

[80] G. Couture et al., Phys. Rev. D38, 860 (1988).

[81] H. Hagiwara, S. Ishihara, R. Szalapski, and D. Zeppenfeld Phys. Lett. B283, 353 (1992)

[82] T. L. Barklow, in in Physics and Experiments with Linear Colliders, R. Orava, P. Eerola, and M.Nordberg, eds. (World Scientific, Singapore, 1992).

[83] Z. Ajaltouni et al., preprint hep-ph/9601233, to appear in the proceedings of the CERN Workshop on LEP II Physics.

[84] T. L. Barklow, in Proceedings of the International Symposium on Vector Boson SelfInteractions, U. Baur, S. Errede, and T. Müller, eds. (American Inst. Press).

[85] K. Riles, contributed paper in this volume.

[86] D. Choudhury and F. Cuypers, Nucl. Phys. B429, 33 (1994).

[87] S. J. Brodsky, T. G. Rizzo, and I. Schmidt, Phys. Rev. D52, 4929 (1995). 
[88] T. L. Barklow, S. Dawson, H. E. Haber, and J. L. Siegrist, in Particle Physics: Perspectives and Opportunities, R. Peccei, M. E. Zeller, D. G. Cassel, J. A. Bagger, R. N. Cahn, P. D. Grannis, and F. J. Sciulli, eds. (World Scientific, Singapore, 1995).

[89] M. Peskin, in Physics in Collisions IV, A. Seiden, ed. (Éditions Frontières, Gif-SurYvette, 1984).

[90] V. Barger, K. Cheung, T. Han, and R.J.N. Phillips, Phys. Rev. D52, 3815 (1995).

[91] V. Barger, J. F. Beacom, K. Cheung, and T. Han, Phys. Rev. D50, 6704 (1994).

[92] R. P. Kauffman, Phys. Rev. D41, 3343 (1990).

[93] M. Cvetic and S. Godfrey, in Electroweak Symmetry Breaking and New Physics at the TeV Scale, T. Barklow, S. Dawson, H. Haber, and J. Seigrist, eds. (World Scientific, Singapore, 1996); A. Djouadi, J. Ng, and T. G. Rizzo, ibid.

[94] T. G. Rizzo, contributed paper in this volume.

[95] C. A. Heusch, ed., Proceedings of the Electron-Electron Linear Collider Workshop, Int. J. Mod. Phys. 11, 1523 (1996).

[96] Proceedings of the Workshop on Gamma-Gamma Colliders, NIM 355, 1 (1995).

[97] J. F. Gunion and H. E. Haber, Phys. Rev. D48, 5109 (1993).

[98] J. Gunion and J. Kelly, Phys. Lett. B333, 110 (1994); S. Moretti, Phys. Rev. D50, 2016 (1994); M. Kramer et al., Z. Phys. C64, 21 (1994).

[99] J. Tandean, Phys. Rev. D52, 1398 (1995).

[100] H. Anlauf, W. Bernreuther, and A. Brandenberg, Phys. Rev. D52, 3803 (1995).

[101] D. L. Borden, D. A. Bauer, and D. O. Caldwell, Phys. Rev. D48, 4018 (1993).

[102] D. L. Borden V. A. Khoze, W. J. Stirling, and J. Ohnemus, Phys. Rev. D50, 4499 (1994).

[103] I. F. Ginzburg and V. G. Serbo, Phys. Rev. D49, 2623 (1994).

[104] G. V. Jikia, Phys. Lett. B298, 224 (1993), Nucl. Phys. B405, 24 (1993).

[105] O. J. P. Eboli, M. C. Gonzalez-Garcia, F. Halzen, and D. Zeppenfeld, Phys. Rev. D48, 1430 (1993); M. Baillargeon, G. Belanger, and F. Boudjema, Phys. Rev. D51, $4712(1995)$.

[106] T. Han, Int. J. Mod. Phys. 11, 1541 (1996).

[107] K. Hikasa, Phys. Lett. B164, 385 (1985). 
[108] T. Rizzo, Int. J. Mod. Phys. 11, 1563 (1996).

[109] J. F. Gunion, Int. J. Mod. Phys. 11, 1551 (1996).

[110] N. V. Dokholyan and G. V. Jikia, Nucl. Phys. B374, 83 (1992).

[111] S. J. Brodsky, in Physics and Experiments with Linear $e^{+} e^{-}$Colliders, F. A. Harris, S. L. Olsen, S. Pakvasa, and X. Tata, eds. (World Scientific, Singapore, 1993).

[112] F. Cuypers G. J. van Odenborgh, and R. Ruckl, Nucl. Phys. B409, 128 (1993).

[113] D. Choudhury and F. Cuypers, Nucl. Phys. B451, 16 (1995).

[114] K. Cheung, Phys. Lett. B323, 85 (1994).

[115] C. A. Heusch and P. Minkowski, Nucl. Phys. B416, 1 (1994).

[116] P. H. Frampton, Phys. Rev. Lett. 69, 2889 (1992).

[117] N. Leporé, B. Thorndyke, H. Nadeau, and D. London, Phys. Rev. D50, 2031 (1994).

[118] H. Nadeau and D. London, Phys. Rev. D47, 3742 (1993); G. Bélanger, D. London, and H. Nadeau, Phys. Rev. D49, 3140 (1994).

[119] F. Cuypers, preprint hep-ph/9508397.

[120] T. Barklow, Int. J. Mod. Phys. 11, 1579 (1996).

[121] D. Choudhury, F. Cuypers, and A. Leike, Phys. Lett. B333, 531 (1994).

[122] F. Cuypers, Int. J. Mod. Phys. 11, 1571 (1996), preprint hep-ph/9602426.

[123] F. Boudjema, A. Djouadi, and J. L. Kneur, Z. Phys. C57, 425 (1993).

[124] V. Telnov, NIM A355, 3 (1995).

[125] J. G. Heinrich, et al., SLAC Proposal E-144 (1991).

[126] P. Langacker and N. Polonsky, Phys. Rev. D47, 4028 (1993), Phys. Rev. D52, 3081 (1995).

[127] S. Bethke, Nucl. Phys. B Proc. Suppl. 39C, 198 (1995).

[128] K. Abe et al. (SLD Collaboration), Phys. Rev. D51, 962 (1995).

[129] S. Bethke, in Physics and Experiments with Linear $e^{+} e^{-}$Colliders, F. A. Harris, S. L. Olsen, S. Pakvasa, and X. Tata, eds. (World Scientific, Singapore, 1993).

[130] B. R. Webber, preprint hep-ph/9411384. 
[131] A. Brandenburg, L. Dixon, and Y. Shadmi, Phys. Rev. D53, 1264 (1996).

[132] K. Abe et al. (SLD Collaboration), Phys. Rev. Lett. 74, 4173 (1995).

[133] C. Carone and H. Murayama, Phys. Rev. Lett. 74, 3122 (1995); D. Bailey and S. Davidson, Phys. Lett. B348, 185 (1995).

[134] T. G. Rizzo, contributed paper in this volume.

[135] J. F. Gunion and P. C. Martin, contributed paper in this volume. 


\section{Chapter 3}

\section{A Zeroth-Order Design for the Next Linear Collider}

The Next Linear Collider consists of a set of subsystems-injectors, linacs, beam delivery lines, and interaction regions. These are responsible for creating intense and highly condensed beams of positrons and polarized electrons, accelerating them to high energy, focusing them to small spots, and colliding them in an environment that allows sensitive particle detectors to operate for physics. We introduce these various parts of the collider in this section, and then provide a more detailed discussion of each. A comprehensive design study of the NLC is given in Ref [1].

\subsection{Overview of Collider Systems}

A schematic of the NLC is shown in Fig. 3.1. The physical footprint of the collider complex is approximately $30 \mathrm{~km}$ in length and less than $1 \mathrm{~km}$ wide. This includes all beam transport lines in the injectors and linacs necessary to obtain $1 \mathrm{TeV}$ cms energy, and all space needed in the beam delivery sections to accomodate $1.5 \mathrm{TeV}$ cms energy. To reach $1.5 \mathrm{TeV}$, however, it may be necessary to extend the "trombones" in the layout of the collider to provide additional length for the main linacs. Rather simple beam-transport lines can be used to do this without moving the injectors or damping rings.

\subsubsection{Injectors and Damping Rings}

The electron injector system for the collider is copied from the system presently operating reliably at the SLC. This includes a polarized photocathode electron gun, and a bunching system that operates at a subharmonic of the main linac of frequency. The new challenge for the NLC injector is that it must produce trains of 90 bunches spaced by $1.4 \mathrm{~ns}$ at the machine pulse repetition rate of 120-180 $\mathrm{Hz}$. The bunched beam is accelerated in an S-band linac to $2 \mathrm{GeV}$, then injected into a damping ring designed to condense the transverse phase space of 


\section{RF Systems \\ (X) $11.424 \mathrm{GHz}$ \\ (S) $2.856 \mathrm{GHz}$ \\ (L) $1.428 \mathrm{GHz}$ \\ (UHF) $0.714 \mathrm{GHz}$}

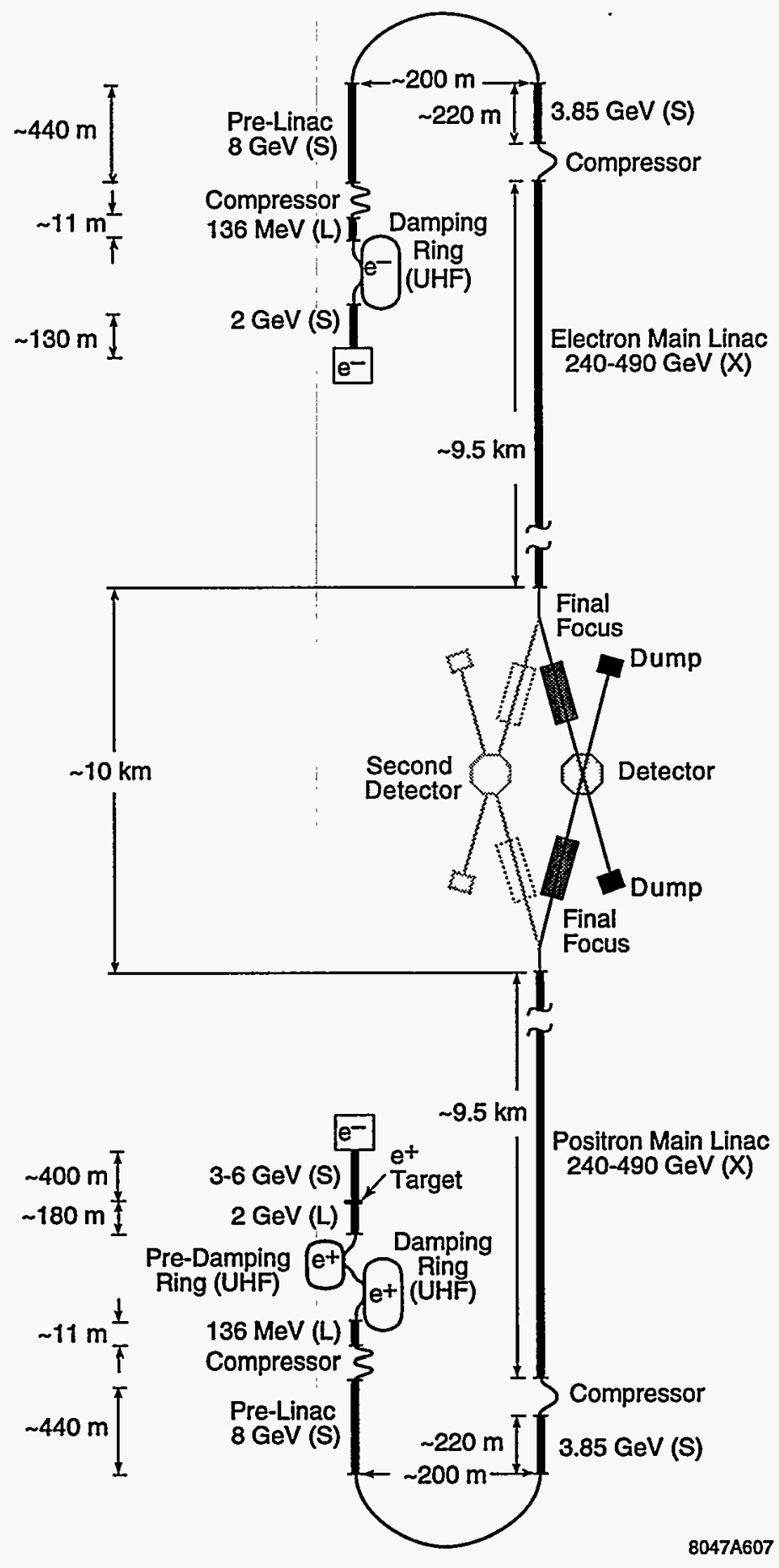

Figure 3.1: Schematic layout of NLC systems (not to scale). 
the beam through radiation damping. The required vertical emittance, $\gamma \varepsilon_{y}=3 \times 10^{-8} \mathrm{~m}-\mathrm{rad}$, is two orders of magnitude smaller than achieved in the SLC damping rings. This places tight constraints on the alignment of ring magnets, but many lessons have been learned from operation of the SLC damping rings, and several modern synchrotron light sources also provide valuable experience. Wiggler magnets, similar to that used in the SSRL ring at SLAC, are included in the NLC design to reduce damping times, and the vacuum and rf systems required for the NLC ring are similar in size and complexity to those of the Advanced Light Source at Lawrence Berkeley National Laboratory.

The techniques for positron production planned for the NLC are also largely copied from those in use at the SLC. Positrons are created in electromagnetic showers produced by targeting 3-6 GeV electrons onto a rapidly rotating metallic disk. The high-emittance positron beam is focused in an extended solenoid, and accelerated to $2 \mathrm{GeV}$ in a largeaperture L-band linac. This leads to a good capture efficiency. To further improve efficiency and ease operational tuning tolerances, the beam is cycled through a large-aperture predamping ring prior to injection into a main damping ring identical to that used for the electron beam. The overall layout also includes transport lines that will allow the drive electron beam to bypass the positron-production target and pre-damping ring (Fig. 3.2). This will allow the study of $e^{-} e^{-}$collisions (with both beams polarized).

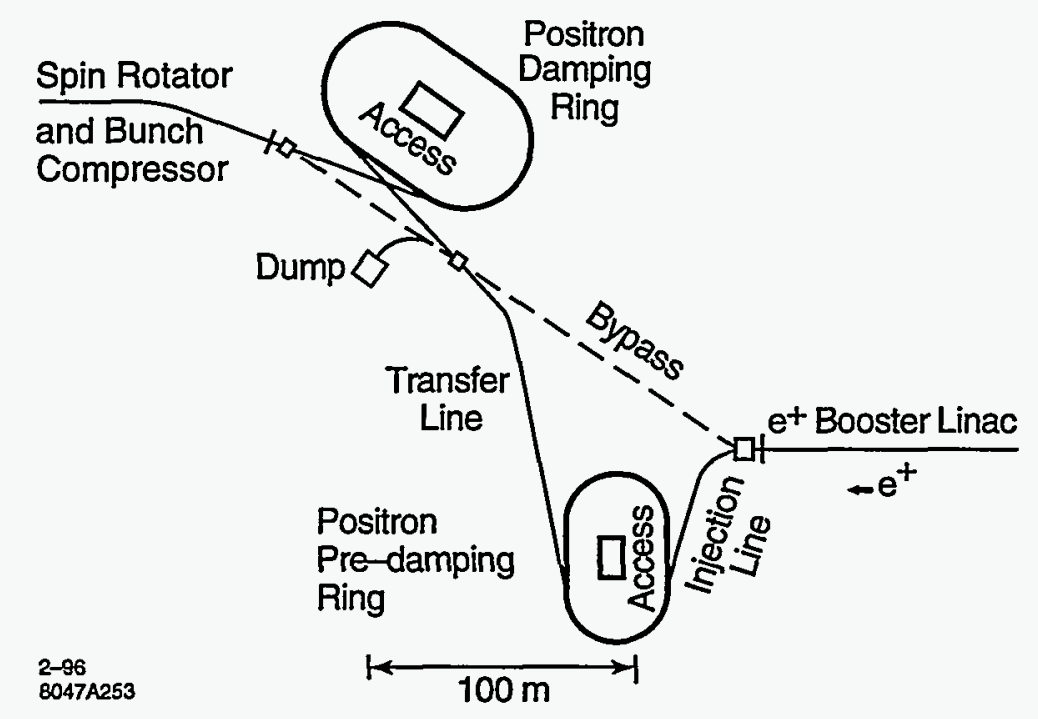

Figure 3.2: Layout of the positron target area and damping rings.

\subsubsection{Bunch Length Compressors}

The lengths of the electron and positron bunches become too great in the damping rings for the beams to be successfully accelerated in the main X-band linacs, so they must be reduced. This is done in stages (Fig. 3.1). A first compressor, located immediately after the damping 
rings, reduces the bunch length from $4 \mathrm{~mm}$ to $0.5 \mathrm{~mm}$ to optimize injection into an S-band linac that accelerates the beams to $10 \mathrm{GeV}$. The bunch lengths are then further compressed to $100-150 \mu \mathrm{m}$ in a two-stage compressor that also bends through a $180^{\circ}$ arc, reversing the direction of travel; this arc allows for future upgrades of the main linac length and permits feedforward of measurements of the bunch charge and trajectory made in the damping rings and compressors. This ability to preset the energy gain and injection orbit to the main linac is an important feature of the design.

\subsubsection{Main Linacs}

The main linacs of the NLC will use normal-conducting traveling-wave copper structures powered with $11.424 \mathrm{GHz}$ microwaves. The required power is generated by klystrons in 1.2$\mu$ s pulses which are then compressed into shorter $0.24-\mu$ s pulses of higher peak power in a passive rf transformer (SLED-II). A train of $\mathbf{9 0}$ electron (or positron) bunches is accelerated in the main linac by each rf pulse. While the number of particles in each of these bunches is less than that in the SLC, the total charge accelerated by each rf pulse is more than an order of magnitude greater. This multibunch design and the correspondingly larger fraction of energy transferred from the power sources to the beam is one of the important differences between the SLC and the NLC. The linac focusing lattice is designed to allow the centerof-mass energy to be increased from $300 \mathrm{GeV}$ to $1 \mathrm{TeV}$; further upgrade to $1.5 \mathrm{TeV}$ will require modifications. Diagnostic stations, located at five positions along each of the two main linacs, will include laser wire scanners to measure the transverse phase space, beambased feedbacks to correct for centroid shifts of the bunch train, multibunch BPMs and high-frequency kickers to measure and correct bunch-to-bunch position errors, and magnetic chicanes to provide noninvasive energy and energy spread measurements. These feedbacks must maintain bunch trajectories to within a few microns of a tuned reference orbit in order to prevent unacceptable emittance growth.

\subsubsection{The Beam Delivery System}

The beam delivery system consists of a collimation section, a switchyard, the final focus, the interaction region, and the beam extraction and dump. These are shown schematically in Figure 3.3.

After acceleration in the main linac, the beams enter collimation sections where particles at the extremes of the energy and transverse phase space are eliminated by collimators. Collimation is performed for both the horizontal and vertical planes at two betatron phases that differ by $90^{\circ}$ to effectively cut the beam in both position and angle. This primary collimation is then followed by secondary collimation in both planes and both phases to remove particles that are scattered by the edges of the primary collimators. These collimation sections are rather extensive, but our experiences at the SLC and FFTB have proven the need to perform this function well. 


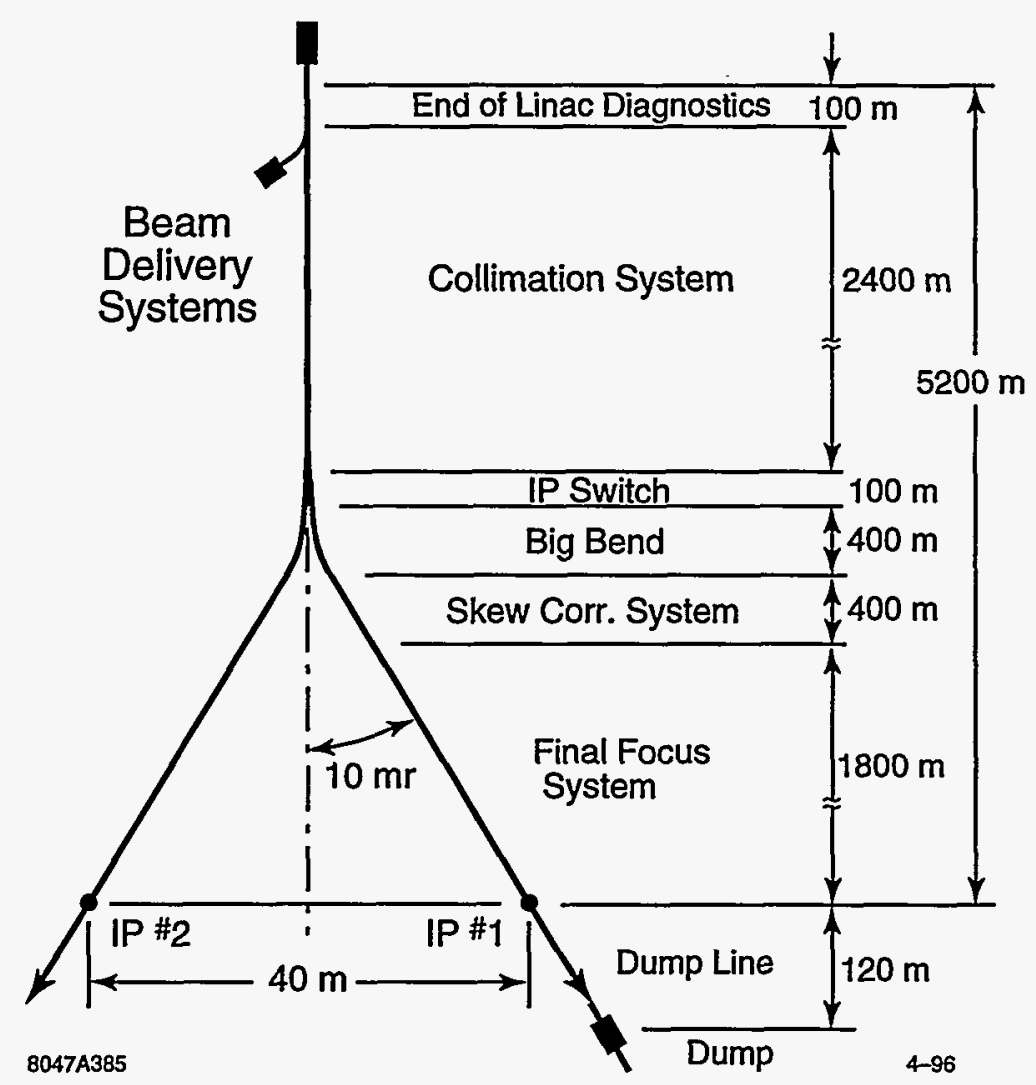

Figure 3.3: Schematic layout of the beam delivery system.

A passive switching section is used to direct the beams to one of two possible interaction points (IPs). The beam is deflected in the switchyard by $10 \mathrm{mrad}$ to produce a $20-\mathrm{mrad}$ crossing angle between the two beams at each IP. This deflection introduces little emittance growth, but greatly reduces detector backgrounds and provides the IP crossing angle required to collide the closely spaced bunches in each train.

The NLC final focus follows the design of the Final Focus Test Beam (FFTB) at SLAC. The optics consist of a matching section with appropriate instrumentation to measure the incoming beam phase space, horizontal and vertical chromatic correction sections, a final transformer, a final doublet, and a diagnostic dump line for the exiting beam. The final doublets require active stabilization to maintain beam collisions. Measurement of the final spot size is a particular challenge, but can be performed with an advanced laser fringe monitor similar to that used in the FFTB. In-situ tuning of the spot sizes and luminosity can also be done using measurements of the beam-beam deflections and other techniques developed at the SLC. 


\subsubsection{Interaction Region}

Two interaction regions are included in the layout of the NLC. These, of course, must share the available luminosity, but it will be possible to install two complementary experiments. As with all colliders, the interaction region will be a very crowded location. The final quadrupole magnets of the machine optics must be positioned as closely as possible to the collision point, and high-Z masking must be installed to protect elements of the experimental detector. The detector itself will require clear access to as much of the volume around the interaction point as possible. The design presented in this document includes quadrupoles $2 \mathrm{~m}$ from the interaction point, and complete access for detector elements at polar angles greater than 150 mrad. Calorimetric measurement of Bhabha scattering at smaller polar angles should also be possible, and is expected to provide a precise determination of the luminosity-weighted center-of-mass energy spectrum.

The NLC is designed to collide electrons and positrons at a $20-\mathrm{mrad}$ crossing angle. This prevents the tightly spaced bunches of one beam from being disturbed, as they approach the interaction point, by bunches in the opposing beam that are leaving the interaction point. To avoid loss of luminosity due to this crossing angle, it is necessary to use a pair of deflection rf cavities to "crab" the beams so that the bunches collide head on. This is a new task not encountered at the SLC, but a system with reasonable specifications has been designed. 


\subsection{Polarized Electron Injector}

The electron injector complex includes the polarized electron source, bunching system, and linac that accelerates the beam to $2 \mathrm{GeV}$.

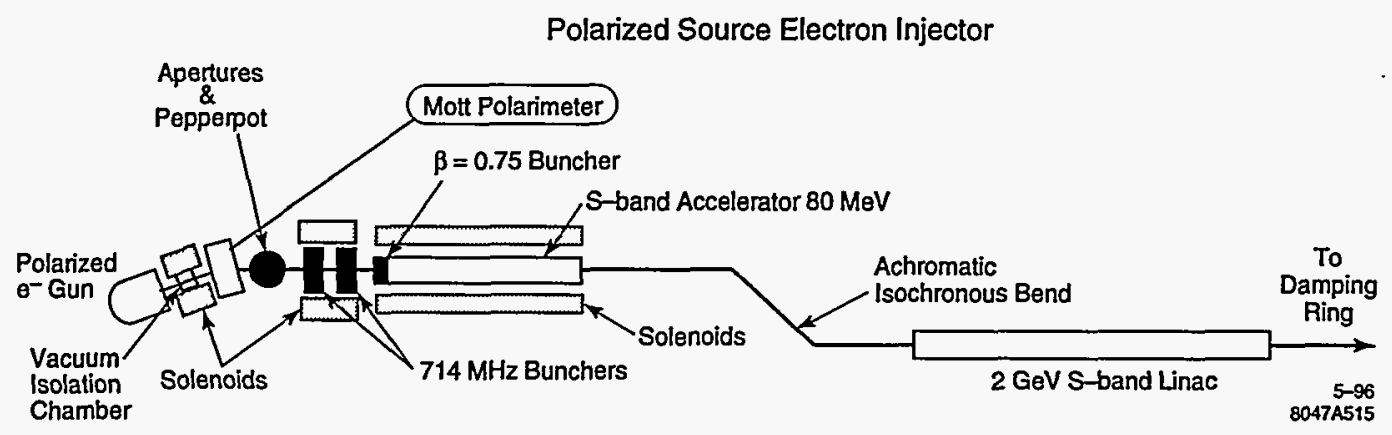

Figure 3.4: The polarized electron injector.

Figure 3.4 shows a schematic of the injector for the polarized electrons, and a summary of the corresponding design parameters is given in Table 3.1. The polarized electron source consists of a polarized high-power laser and a high-voltage DC gun with strained-lattice gallium-arsenide photocathode. Many of the performance requirements for the NLC injector are similar to those in the SLC, and the design of the NLC injector is based on the presently operating SLC injector. The main difference is that, while the SLC polarized electron injector produces one pair of electron bunches separated by $60 \mathrm{~ns}$ at $180 \mathrm{~Hz}$ repetition rate, the NLC injector must produce long trains of many bunches separated by only $1.4 \mathrm{~ns}$. This requires greater pulse currents to be extracted from the photocathode.

The SLC polarized source delivers high-intensity pairs of electron bunches with beam polarizations of $80 \%$ or better. With maintenance of ultra high vacuum conditions, cathode lifetimes have exceeded thousands of hours. System availability now approaches $99 \%$. The basic technologies of the SLC injector will be utilized for the NLC. Most importantly, the existing design of the polarized gun could be duplicated for the NLC with few changes.

The NLC requires two electron injectors-one to produce polarized electrons that will collide with positrons, and another to produce electrons that will be used to make positrons. An S-band linac accelerates the polarized electrons for collisions to $2 \mathrm{GeV}$ prior to injection into the damping ring, while a similar but longer linac accelerates the electrons for positronproduction to higher energy. The number of positrons needed to satisfy the luminosity requirements of the collider is greater at $1 \mathrm{TeV}$ cms energy than at $500 \mathrm{GeV}$, so the energy of the electrons for positron production is increased from $3.11 \mathrm{GeV}$ to $6.22 \mathrm{GeV}$ as the cms energy is increased. The positron injector beam line includes the space needed for this upgrade. The phase space of the $2-\mathrm{GeV}$ polarized electron beam is matched to the damping ring lattice in a special transition region, while the higher energy beam for positron production is transported to one of two target systems. (See Section 3.3.) The beam optics 
Table 3.1: NLC polarized electron injector parameters up to the damping ring.

\begin{tabular}{|c|c|c|c|c|c|c|c|c|}
\hline \multirow[b]{2}{*}{ Parameters } & \multicolumn{3}{|c|}{ NLG-I } & \multicolumn{3}{|c|}{ NLC-II } & \multirow{2}{*}{$\begin{array}{c}\text { Overhead } \\
20 \%\end{array}$} & \multirow{2}{*}{$\begin{array}{c}\text { SLC } \\
\text { achieved }\end{array}$} \\
\hline & $\mathrm{a}$ & $\mathrm{b}$ & c & $a$ & $\mathrm{~b}$ & c & & \\
\hline $\mathrm{N} /$ bunch at IP $\left(10^{10}\right)$ & 0.65 & 0.75 & 0.85 & 0.95 & 1.1 & 1.25 & 1.5 & \multirow{7}{*}{8.8} \\
\hline N/bunch at Gun & 1.2 & 1.4 & 1.6 & 1.75 & 2.0 & 2.3 & 2.8 & \\
\hline $\mathrm{I}_{\text {avg }}$ at gun $(\mathrm{A})$ & 1.4 & 1.7 & 1.9 & 2.0 & 2.4 & 2.7 & 3.2 & \\
\hline $\mathrm{N} /$ bunch at limiting aperture & 0.95 & 1.15 & 1.3 & 1.4 & 1.63 & 1.85 & 2.2 & \\
\hline $\mathrm{N} /$ bunch at $80 \mathrm{MeV}$ in $18 \mathrm{ps}$ & 0.83 & 0.97 & 1.1 & 1.2 & 1.4 & 1.6 & 1.96 & \\
\hline $\mathrm{I}_{a v g}$ at $80 \mathrm{MeV}(\mathrm{A})$ & 0.95 & 1.11 & 1.26 & 1.37 & 1.58 & 1.8 & 2.2 & \\
\hline N/bunch at damping ring & 0.75 & 0.88 & 1.0 & 1.1 & 1.28 & 1.45 & 1.7 & \\
\hline Parameters & \multicolumn{3}{|c|}{ NLC Required } & \multicolumn{4}{|c|}{ Simulation } & $\begin{array}{c}\text { SLC } \\
\text { no train }\end{array}$ \\
\hline Bunch train durations (ns) & \multicolumn{3}{|c|}{126} & \multicolumn{4}{|c|}{126} & \\
\hline Bunch separation (ns) & \multicolumn{3}{|c|}{1.4} & \multicolumn{4}{|c|}{1.4} & \\
\hline Pulse FWHM at gun (ps) & \multicolumn{3}{|c|}{700} & \multicolumn{4}{|c|}{700} & 2000 \\
\hline Pulse FW after bunching (ps) & \multicolumn{3}{|c|}{18} & \multicolumn{4}{|c|}{18} & 18 \\
\hline$\varepsilon_{\mathrm{n}, \mathrm{rms}}$ at $40 \mathrm{MeV}\left(10^{-4} \mathrm{~m}-\mathrm{rad}\right)$ & \multicolumn{3}{|c|}{1.0} & \multicolumn{4}{|c|}{0.5} & 1 to 1.3 \\
\hline$\Delta \mathrm{E} / \mathrm{E}_{\mathrm{FW}}$ at entr. to ring (\%) & \multicolumn{3}{|c|}{ \pm 0.6} & \multicolumn{4}{|c|}{ \pm 0.6} & \pm 0.6 to 0.8 \\
\hline Train-to-train intensity jitter (\%) & \multirow{2}{*}{\multicolumn{3}{|c|}{$<0.5 \%$}} & \multirow{2}{*}{\multicolumn{4}{|c|}{$\begin{array}{l}\text { negligible w/ intensity } \\
\text { jitter limiting aperture }\end{array}$}} & 0.8 at gun \\
\hline & & & & & & & & 0.5 bunch \\
\hline
\end{tabular}

of both electron injectors are identical up to the $2-\mathrm{GeV}$ point so that it is straightforward to replace the thermionic electron gun used for the positron drive beam with a polarized gun in the event that a second polarized electron source is needed for $e^{-} e^{-}$or $\gamma \gamma$ collisions.

The polarized electron source could use a conventional $120-\mathrm{kV}$ DC gun designed to produce a 126-ns-long train of 90 bunches spaced $1.4 \mathrm{~ns}$ apart with $2.8 \times 10^{10}$ electrons per bunch. Research efforts to develop a polarized rf gun are underway. If successful, it may be possible to benefit from the lower emittances produced by an $\mathrm{rf}$ gun to improve the performance of the injector complex.

A vacuum isolation region and a $20^{\circ}$ bend protect the polarized electron gun from the downstream environment (Fig. 3.4). The bend also allows the beam to be steered to a Mott scattering polarimeter for polarization measurements. The intensity jitter of the beam at the gun may be close to the allowed threshold of $0.5 \%$ rms from train to train due to the incoming laser intensity jitter on the photocathode. An aperture $24 \mathrm{~cm}$ downstream of the $20^{\circ}$ bend will be used to reduce the electron beam intensity jitter of the bunched beam. Two 714- $\mathrm{MHz}$ standing-wave subharmonic bunchers and an S-band buncher and accelerator section compress the beam from the gun such that $83 \%$ of the charge is captured in $18^{\circ}$ S-band (17.5 ps). Simulations with the computer code PARMELA were made of the beam 
optics from the gun through the second accelerator section, where the beam energy reaches $80 \mathrm{MeV}$. The energy spread, calculated without including the effect of beam loading in the long bunch train, is expected to be $\pm 0.6 \%$ for a single bunch.

Beam loading effects in the subharmonic bunchers are mitigated by using cavities with low $R / Q$, injecting the beam during the fill time of the cavity, and changing the phase of the input rf just before beam time. Axial magnetic field focusing will be used on the first two accelerator sections (up to $80 \mathrm{MeV}$ ) where the beam is expected to have larger energy spread than in the rest of the injector linac. At $80 \mathrm{MeV}$ there will be a doubly achromatic bend to connect the low energy beam line to the main injector beam line. This bend allows another low-energy injector beam line in the future which could be used for a polarized rf gun. Wire scanners on both sides of the achromatic bend will measure emittance in the low-energy beam line and at the entrance to the injector linac after the bend. Scrapers at the high-dispersion point will clip away tails and a wire scanner following the scrapers will measure energy spread. From $80 \mathrm{MeV}$ to $2 \mathrm{GeV}$, the linac will use 3-m-long accelerator sections with beam loading compensation. Each rf module will consist of four $3-\mathrm{m}$ accelerator sections powered by two S-band klystrons similar to SLAC 5045 klystrons. Quadrupole doublets between each section, and some wrapped around the first few sections, will be used to control the transverse size of the beam.

It is planned to test the design and technologies planned for in the NLC injector at the NLC Test Accelerator. Of particular interest will be tests of the beam loading compensation techniques intended to be used in both the subharmonic bunchers and the injector accelerator sections.

Our experience with polarized photocathodes leads us to expect that, with the average beam current required during the NLC bunch train, the beam polarization will be less than the $80 \%$ specified for the collider if no improvements are made to the characteristics of the SLC source. Tests of the cathodes in use at the SLC indicate that, if these cathodes were driven to produce the charge required for the NLC, the beam polarization would be no more than $60-65 \%$. The important factor is the charge density (per unit area) at the surface of the cathode. There are several straightforward ways to avoid this limit. The area of the cathode can be doubled or even tripled; however, the beam emittance will increase proportionally, requiring larger apertures in the bunchers. It is also possible, albeit somewhat inefficient, to simply build two guns and merge their beams at one of several points in the transport optics. More elegant solutions with super-strained photocathode materials are being investigated and have shown promising results. There remains work to be done, but beam polarizations of at least $80 \%$ will be available at the NLC. 


\subsection{Positron Source}

Positrons are created for the NLC by bombarding a thick high-Z metallic target with 3-6 $\mathrm{GeV}$ electrons. The design of the NLC positron system is based on the similar system used in the SLC, which has demonstrated excellent reliability over many years of operation. One of the key design goals for the NLC positron source is to produce a beam with twice the intensity required at the interaction point. The total number of positrons required for the bunch train is then an order of magnitude greater than the number of positrons in the single SLC bunch. The limiting factor in the design of the positron system is the survivability of the production target, and several steps are taken to assure that the required charge can be produced and successfully used.

The NLC positron source consists of three subsystems, a $3.11-\mathrm{GeV}$ accelerator for the drive electron beam (upgradable to $6.22 \mathrm{GeV}$ ), a positron production and collection system, and a positron linac to accelerate the positrons to $2 \mathrm{GeV}$. A layout of the NLC positron source is depicted in Fig. 3.5.

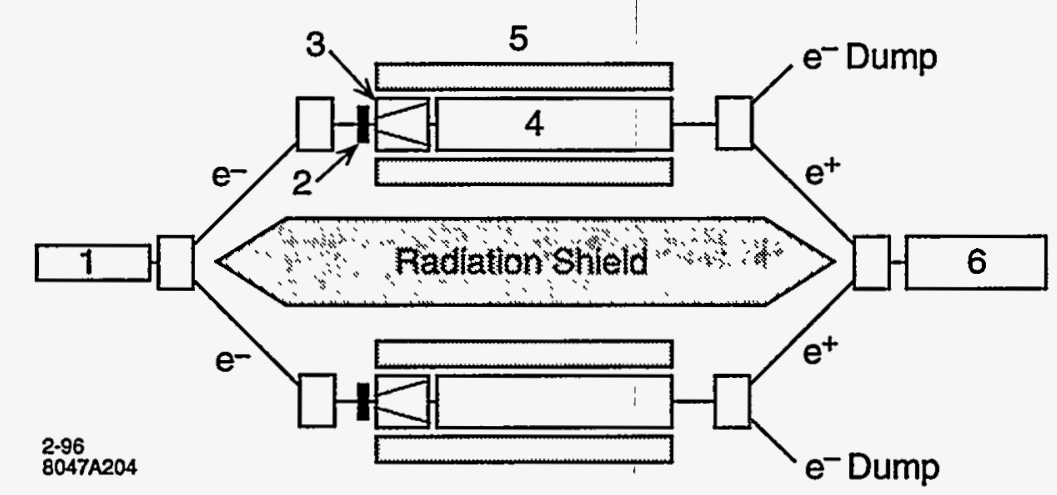

Figure 3.5: Layout of the NLC positron source with two side-by-side positron production and capture systems: (1) drive electron accelerator, (2) positron target, (3) flux concentrator, (4) L-band capture accelerator, (5) tapered-field and uniform-field solenoids, and (6) positron booster linac.

The source design is constrained by failure of the target material due to excessive stress generated by the impact of a single bunch train. To overcome this limit, we have chosen to use a tapered solenoid and large-aperture L-band linac to capture the produced positrons. This leads to a 16-fold increase in the transverse phase space acceptance relative to that of the SLC S-band design. The use of L-band also permits a larger-area drive-beam spot on the target. The net effect of these changes is to maintain the energy density in the target during the bunch train at values comparable to those in the SLC target. A summary of key parameters in the source design is given in Table 3.2.

A conceptual design of the NLC positron target system is shown in Fig. 3.6. The target material is chosen to be $W_{75} \mathrm{Re}_{25}$ because of its excellent thermo-mechanical properties, its 
high-Z characteristic, and its excellent performance in the SLC positron source. The target thickness is chosen to be four radiation lengths based on considerations of both the positron production yield and the single pulse energy deposition density in the target. The ringshaped target, with a radial thickness of $1 \mathrm{~cm}$ and and a medium radius of about $10 \mathrm{~cm}$, is rotated at a rate of $120 \mathrm{rpm}$ to distribute the beam pulse impacts around the target ring. A three-stage differential pumping assembly using radiation-resistant seals that rely on tight clearances ( $\mathrm{a}$ few microns) between rotating and stationary seal surfaces is used to seal the rotating target shaft for the target vacuum chamber. Cooling tubes are located in a copper casting in close contact with the inner ring face in order to adequately cool the target, which absorbs about $23 \mathrm{~kW}$ of beam power.

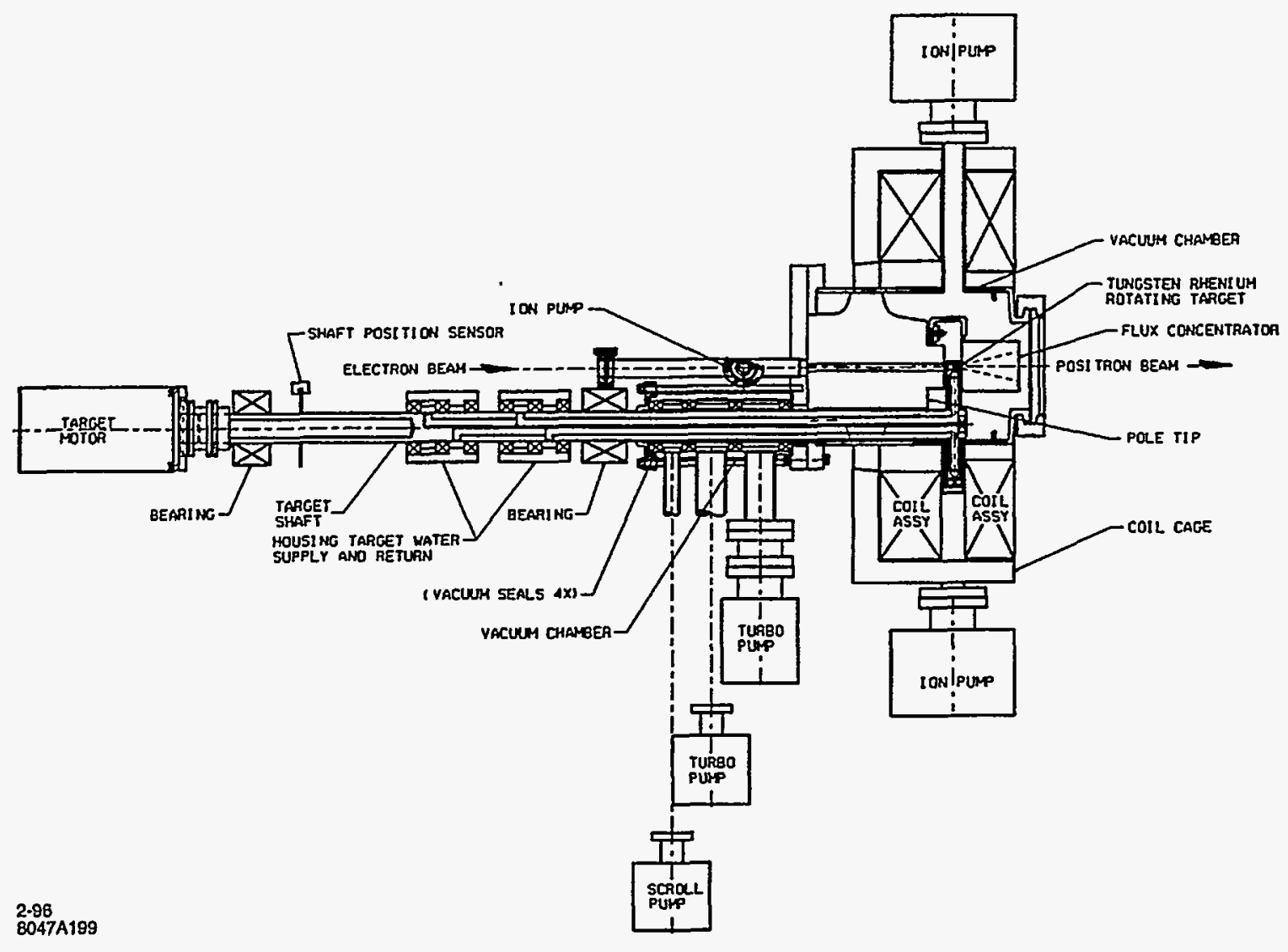

Figure 3.6: Schematic of the NLC positron target system.

For the drive beam accelerator, a thermionic gun coupled to two subharmonic bunchers and one S-band buncher injects a properly bunched electron beam into an S-band linac, which accelerates the electrons to $3.11 \mathrm{GeV}$ (or to $6.22 \mathrm{GeV}$ for higher cms energies). The drive beam is then directed into one of the two parallel beam lines for positron production and collection. The collection system includes a $240-\mathrm{MeV}$ high-gradient L-band accelerator 
Table 3.2: NLC Positron Source Parameters.

\begin{tabular}{|c|c|c|c|c|c|}
\hline Parameter & Unit & SLC 94 & $\begin{array}{r}\text { SLC max } \\
\text { design }\end{array}$ & $\begin{array}{r}\text { NLC-I } \\
(500 \mathrm{GeV}) \\
\end{array}$ & $\begin{array}{r}\text { NLC-II } \\
(1 \mathrm{TeV}) \\
\end{array}$ \\
\hline \multicolumn{6}{|l|}{ General Parameters: } \\
\hline Electrons per pulse at IP & {$\left[10^{10}\right]$} & 3.5 & 5 & 76.5 & 112.5 \\
\hline Bunches per pulse & & 1 & 1 & 90 & 90 \\
\hline Pulse duration & (ns) & 0.003 & 0.003 & 126 & 126 \\
\hline Bunch spacing & (ns) & - & - & 1.4 & 1.4 \\
\hline Repetition frequency & $(\mathrm{Hz})$ & 120 & 180 & 180 & 120 \\
\hline \multicolumn{6}{|l|}{ Drive Electron Beam: } \\
\hline Energy & $(\mathrm{GeV})$ & 30 & 30 & 3.11 & 6.22 \\
\hline Electrons per bunch & $\left(10^{10}\right)$ & 3.5 & 5 & 1.5 & 1.5 \\
\hline Electrons per pulse & $\left(10^{10}\right)$ & 3.5 & 5 & 135 & 135 \\
\hline Beam power & $(\mathrm{kW})$ & 20.2 & 47 & 121 & 161 \\
\hline RMS beam radius & (mm) & 0.8 & 0.6 & 1.2 & 1.6 \\
\hline Beam energy density per pulse & $\left(\mathrm{GeV} / \mathrm{mm}^{2}\right)$ & $5.2 \times 10^{11}$ & $13.3 \times 10^{11}$ & $9.3 \times 10^{11}$ & $10.4 \times 10^{11}$ \\
\hline \multicolumn{6}{|l|}{ Positron Production Target: } \\
\hline Material & & $W_{75} R_{25}$ & $W_{75} R_{25}$ & $W_{75} R_{25}$ & $W_{75} R_{25}$ \\
\hline Thickness & (R.L.) & 6 & 6 & 4 & 4 \\
\hline Energy deposition per pulse & $(\mathrm{J})$ & 37 & 53 & 126 & 188 \\
\hline Power deposition & $(\mathrm{kW})$ & 4.4 & 9 & 23 & 23 \\
\hline Steady-state temperature & $\left({ }^{\circ} \mathrm{C}\right)$ & 100 & 200 & 400 & 400 \\
\hline \multicolumn{6}{|l|}{ Positron Collection: } \\
\hline Accel. frequency & $(\mathrm{MHz})$ & 2856 & 2856 & 1428 & 1428 \\
\hline Accel. gradient & $(\mathrm{MV} / \mathrm{m})$ & 30 & 30 & 25 & 25 \\
\hline Minimum iris radius & $(\mathrm{mm})$ & 9 & 9 & 20 & 20 \\
\hline Edge emittance (allowing for & (m-rad) & 0.01 & 0.01 & 0.06 & 0.06 \\
\hline $2 \mathrm{~mm}$ clearance) & & & & & \\
\hline Positron yield per $e^{-}$ & & $2.5^{a}$ & $2.5^{a}$ & 1.4 & 2.05 \\
\hline Positrons per bunch & $\left(10^{10}\right)$ & 8.7 & 12.5 & 2.1 & 3.1 \\
\hline Positrons per pulse & $\left(10^{10}\right)$ & 8.7 & 12.5 & 189 & 279 \\
\hline
\end{tabular}

${ }^{a}$ The actual yield immediately following the capture section is 4 , but decreases to 2.5 after two $180^{\circ}$ bends and a $2 \mathrm{~km}$ transport line. 
embedded in a uniform-field solenoid and an adiabatic phase space transformer consisting of a flux concentrator and a tapered field solenoid. The capture system has an invariant transverse phase space admittance of $0.06 \mathrm{~m}$-rad. Yield simulations for the positron production and collection system are performed by using the EGS-4 and ETRANS codes. The positron beam is injected into a 1.75-GeV L-band linac consisting of 5-m-long detuned accelerating structures in a scaled FODO lattice, while the electrons that are generated and captured along with the positrons are dumped. Various types of beam diagnostic and control devices are installed at strategic places throughout the positron source for tuning and beam stabilization purposes.

Multibunch beam-loading in the positron source is compensated primarily by injecting the beam during the filling time of the accelerator structure. In the positron capture accelerator, where beam loading is particularly severe, the compensation can be performed by shifting the phase of the rf during the beam pulse, in addition to delaying the rf pulses relative to the beam.

The dual-vault positron source design shown in Fig. 3.5 aims to boost the operating efficiency of the positron source by providing an on-line spare such that NLC operation may be continued in the event of a component failure in the positron production and collection system. Without the on-line spare, failure of any component near the positron target during a physics run would cause extended machine downtime as the very high radiation levels would prevent prompt maintenance. 


\subsection{Damping Rings}

The NLC damping rings are designed to damp the incoming electron and positron beams to the small emittances needed for collisions. The rings have three purposes: (1) damping the incoming emittances in all three planes, (2) damping incoming transients and providing a stable platform for the downstream portion of the accelerator, and (3) delaying the bunches so that feedforward systems can be used to compensate for charge fluctuations.

To meet these goals, we have designed three damping rings: two identical main damping rings, one for the electrons and one for the positrons, and a pre-damping ring for the positrons. The pre-damping ring is needed because the emittance of the incoming positrons is much larger than that of the electrons. Each damping ring will store multiple trains of bunches at once. At every machine cycle, a single fully damped bunch train is extracted from the ring while a new bunch train is injected. In this manner, each bunch train can be damped for many machine cycles.

The parameters of the two main damping rings are similar to the present generation of synchrotron light sources and the B-Factory colliders that are being constructed in that they must store high-current beams $(\sim 1 \mathrm{~A})$ while attaining small normalized emittances. Table 3.3 compares the NLC ring parameters with those of the SLAC B-Factory Low-Energy Ring (PEP-II LER), the Advanced Light Source (ALS) at Lawrence Berkeley National Laboratory, and the Accelerator Test Facility (ATF) damping ring being constructed at KEK in Japan to verify many of the damping ring design concepts. In particular, the stored beam currents are less than half of what the PEP-II LER has been designed to store while the emittance, energy, and size of the rings are similar to those of the ALS and the ATF.

Table 3.3: Comparison of NLC main damping rings with parameters of other rings.

\begin{tabular}{|l|cccc|}
\hline \hline & NLC MDR & PEP-II LER & LBNL ALS & KEK ATF \\
\hline Energy (GeV) & 2 & 3.1 & 1.5 & 1.5 \\
Circumference (m) & 220 & 2200 & 200 & 140 \\
Current (A) & 1.2 & 3 & 0.6 & 0.6 \\
$\gamma \epsilon_{x}\left(10^{-6} \mathrm{~m}-\mathrm{rad}\right)$ & 3 & 400 & 10 & 4 \\
$\gamma \epsilon_{y}\left(10^{-6} \mathrm{~m}-\mathrm{rad}\right)$ & 0.03 & 16 & 0.2 & 0.04 \\
\hline \hline
\end{tabular}

Thus, these other rings will be able to test and verify many of the accelerator physics issues that will arise in the NLC damping rings. In particular, strong coupled-bunch instabilities will be studied in the high current B-factories while issues associated with the very small beam emittances, such as intrabeam scattering and ion trapping, will be studied in the ALS and ATF damping rings. In addition, the PEP-II LER and the Advanced Photon Source 
(APS) at Argonne National Laboratory will be able to study the photoelectron-positron instability that is thought to arise in positron storage rings.

These similarities with other rings have also simplified the design process and we have been able, and will continue, to benefit from experience at these other accelerators. For example: the damping ring rf system is based on those developed for the SLAC B-Factory and the ATF damping ring, the multibunch feedback systems are based upon the feedback systems which were designed for the SLAC B-Factory and successfully verified on the ALS, and the vacuum system is similar to that used by the ALS. Furthermore, the design uses "C" quadrupole and sextupole magnets similar to those designed at the ALS and the APS at Argonne National Lab, a high-field permanent magnet wiggler very similar to a design developed and installed at Stanford Synchrotron Radiation Laboratory (SSRL), and a double kicker system for extraction similar to one to be tested in the ATF.

The NLC damping ring complex is designed to operate with the parameters listed in Table 3.4. These design parameters exceed the requirements of all presently considered NLC upgrades. The rings produce electron and positron beams with emittances $\gamma \epsilon_{x} \leq 3 \times 10^{-6}$ $\mathrm{m}$-rad and $\gamma \epsilon_{y} \leq 3 \times 10^{-8} \mathrm{~m}$-rad, at repetition rates as high as $180 \mathrm{~Hz}$. The beams in the damping rings consist of multiple trains of 90 bunches with a maximum single bunch charge of $1.3 \times 10^{10}$. (This is $5 \%$ higher than the required charge at the IP to allow for beam loss due to collimation.) To provide operational flexibility, we have designed the rings to operate with a peak current roughly $20 \%$ higher than the nominal peak current. In addition, the rings have been designed to operate over an energy range of 1.8 to $2.2 \mathrm{GeV}$ with a nominal energy of $1.98 \mathrm{GeV}$. This will allow the damping rate to be increased if necessary.

Table 3.4: Requirements for NLC damping ring complex.

\begin{tabular}{|lc|}
\hline \hline$\gamma \epsilon_{x e q} / \gamma \epsilon_{x e x t}\left(10^{-6} \mathrm{~m}-\mathrm{rad}\right)$ & $3.0 / 3.0$ \\
$\gamma \epsilon_{y e q} / \gamma \epsilon_{y \text { ext }}\left(10^{-8} \mathrm{~m}-\mathrm{rad}\right)$ & $2.0 / 3.0$ \\
Charge/Bunch & $1.57 \times 10^{\mathbf{1 0}}$ \\
Bunches/Train & 90 bunches/train \\
Bunch Spacing (ns) & 1.4 \\
Repetition Rate $(\mathrm{Hz})$ & 180 \\
\hline
\end{tabular}

At present, most of the studies have concentrated on the main damping rings. Although relatively little of the detailed engineering has been performed, we have a good outline of the design and the problems we may encounter. In particular, we have identified and described solutions for the most difficult issues: these are the dynamic aperture, the vertical emittance, the impedance and instabilities, and the stability and jitter in both longitudinal and transverse phase space. As stated, much of the design rests on work being performed for the B-factories presently being constructed and the ATF damping ring. 
In most aspects, the pre-damping ring has relatively loose requirements. The emittance and damping time requirements are not severe. The required beam stability is not very significant. The two difficult items are attaining the dynamic aperture and the injection/extraction kicker systems. At this time, the design is still in a preliminary stage but we have addressed the difficult items just mentioned.

\subsubsection{Main Damping Rings.}

The NLC main damping rings are roughly $223 \mathrm{~m}$ in circumference and measure $50 \mathrm{~m}$ by 80 $\mathrm{m}$. They have a nominal energy of $1.98 \mathrm{GeV}$ and the required damping is achieved using both high field bending magnets and roughly $26 \mathrm{~m}$ of damping wiggler. The rings are designed in a racetrack form with two arcs separated by straight sections; the layout is illustrated in Figure 3.7.

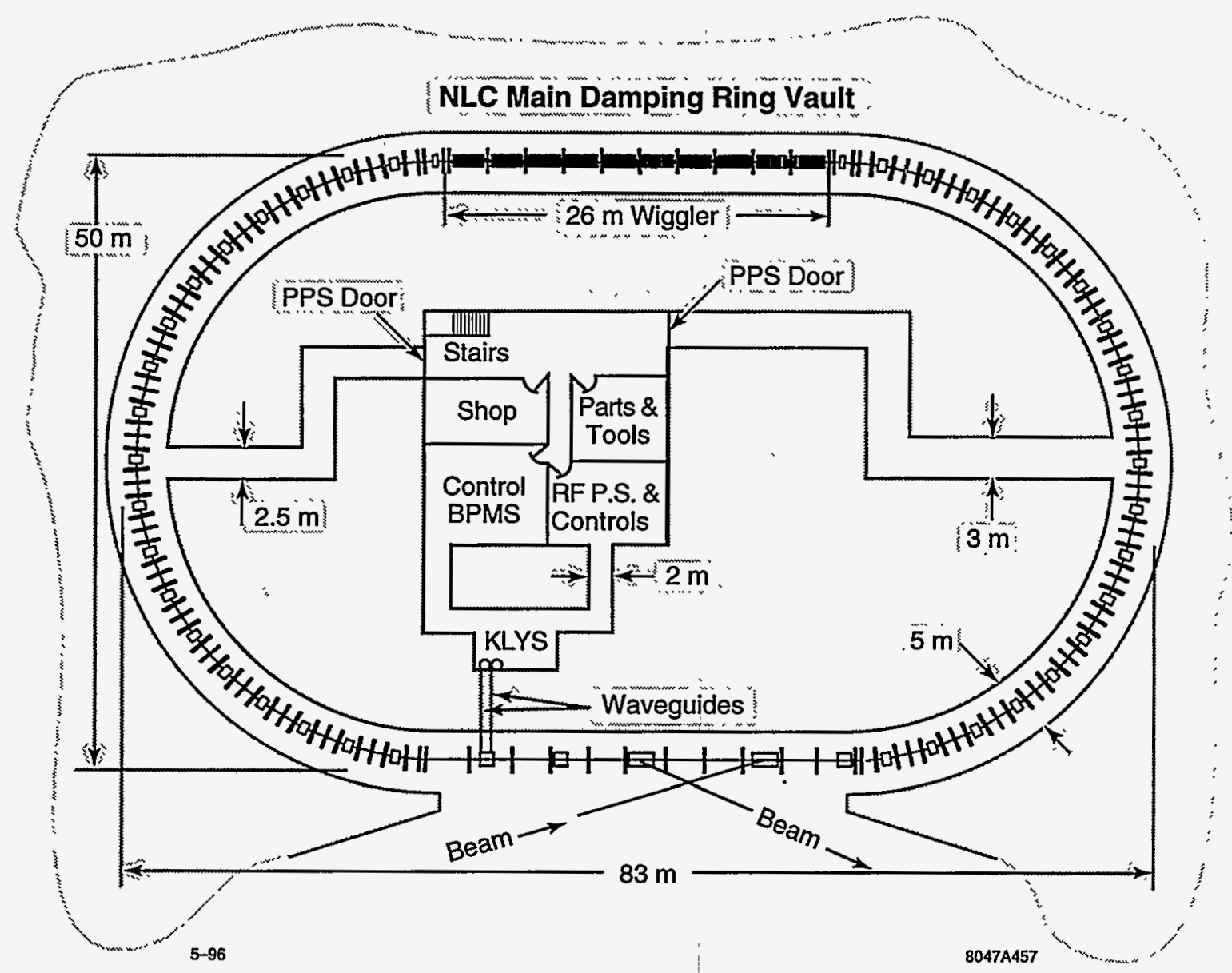

Figure 3.7: Layout of main damping ring.

The main damping rings are designed to damp beams with injected emittances $\gamma \epsilon_{x, y}=$ $1 \times 10^{-4} \mathrm{~m}$-rad to emittances of $\gamma \epsilon_{x}=3 \times 10^{-6} \mathrm{~m}$-rad and $\gamma \epsilon_{y}=3 \times 10^{-8} \mathrm{~m}$-rad. The rings 
will operate at $180 \mathrm{~Hz}$. They provide sufficient damping to decrease the injected emittance by over four orders of magnitude. These parameters are summarized in Table 3.5.

Table 3.5: Parameters for main damping rings and the pre-damping ring.

\begin{tabular}{|lcc|}
\hline \hline & MDR & PDR \\
\hline \hline Circumference (m) & 223 & 171 \\
Energy (GeV) & \multicolumn{2}{c|}{$1.8-2.2,1.98$ nominal } \\
Max. Current (A) & \multicolumn{2}{c|}{1.2} \\
Max. Rep. Rate (Hz) & \multicolumn{2}{c}{180} \\
Bunch Trains $\times$ Bunches per train & $4 \times 90$ & $3 \times 90$ \\
Train/Bunch Separation (ns) & $60 / 1.4$ & $64 / 1.4$ \\
$\nu_{x}, \nu_{y}, \nu_{s}$ & $23.81,8.62,0.004$ & $10.18,8.18,0.018$ \\
$\gamma \epsilon_{x e q}$ (m-rad) & 2.56 & 7.7 \\
$\gamma \epsilon_{x}, \gamma \epsilon_{y}$ (with intra-beam scatt.) & $3.1 \times 10^{-6}, 1.5 \times 10^{-8}$ & $4.5 \times 10^{-5}$ (coupled) \\
$\sigma_{\epsilon}, \sigma_{z}$ & $0.09 \%, 4.0 \mathrm{~mm}$ & $0.1 \%, 7.5 \mathrm{~mm}$ \\
$\xi_{x, \text { uncorr }, \xi_{y}, \text { uncorr }}$ & $-41.8,-22.4$ & $-13.9,-10.0$ \\
$\tau_{x}, \tau_{y}, \tau_{z}$ (ms) & $4.06,4.62,2.50$ & $4.44,6.15,2.73$ \\
$U_{S R}(\mathrm{kV} /$ turn) & 644 & 371 \\
$J_{x}$ & 1.14 & 1.39 \\
$\alpha_{p}$ & 0.00047 & 0.0051 \\
RF Voltage, Frequency & $1 \mathrm{MV}, 714 \mathrm{MHz}$ & $2 \mathrm{MV}, 714 \mathrm{MHz}$ \\
Lattice & $40 \mathrm{TME} \mathrm{Cells}$ & $30 \mathrm{FOBO}$ Cells \\
\hline \hline
\end{tabular}

The main damping ring lattice is based on detuned Theoretical Minimum Emittance (TME) cells which were chosen because of eased requirements on the combined-function bending magnets. The chromaticity is corrected with three families of sextupoles and the dynamic aperture is more than sufficient. The damping is performed using both high-field bending magnets and ten 2.5 -m sections of damping wiggler.

The rings operate with four trains of 90 bunches. The bunch trains are injected onto and extracted from the closed orbit using pulsed kickers and DC septa. To avoid coupled-bunch instabilities the rf cavities are based on those of the PEP-II B-Factory and a transverse bunch-by-bunch feedback system is used. As stated, the rings are designed to operate with maximum bunch charges of $1.57 \times 10^{10}$ particles; this is roughly $20 \%$ more than the maximum needed at the IP. In addition, the electron source has been designed to provide additional 
charge to allow for at least $10 \%$ losses during injection into the electron damping ring. Similarly, the positron source has been designed to produce at least $20 \%$ additional charge to provide for losses during injection into the pre-damping ring.

Finally, because the rings must generate extremely small beam emittances, there are tight jitter and alignment tolerances. Extensive effort has been made to include cancellations and tuning procedures in the design that will ease the tolerances to reasonable levels. In particular, all of the quadrupoles and all of the sextupoles will have independent power supplies and remote magnet movers. This will facilitate beam-based alignment as well as matching of the lattice functions.

\subsubsection{Positron Pre-Damping Ring}

The design of the pre-damping ring for positrons is similar to the SLC damping rings, except the lengths are scaled by a factor of five. The ring has a racetrack form with dispersion-free straight sections for injection and extraction. The ring is roughly 60 meters by 40 meters and is illustrated schematically in Figure 3.8.

In the design, the injection and extraction regions are in the straight sections on opposite sides of the ring. To reduce the requirements on these components, the systems are designed to occupy most of these regions. To minimize rf transients during injection and extraction, a new bunch train will be injected one half turn after a train is extracted. Furthermore, the rf cavities are placed downstream of the injection kicker and upstream of the extraction kicker so that the injection/extraction process will not interrupt the beam current seen by the $\mathrm{rf}$ cavities.

The positron pre-damping ring is designed to damp the large emittance positron beam from the positron source to an emittance of roughly $\gamma \epsilon_{x, y}=1 \times 10^{-4} \mathrm{~m}$-rad; these parameters are summarized in Table 3.5. At this point, the positrons are injected into the positron main damping ring where they are damped to the desired final emittances. The pre-damping ring allows us to decouple the large aperture requirements for the incoming positron beams from the final emittance requirements of the linear collider.

The pre-damping ring does not need to produce flat beams. Thus, to maximize the damping of the transverse phase space, the ring has horizontal damping partition number $\mathrm{J}_{x} \approx 1.4$, and operates on the coupling difference resonance. This increases the damping in both the horizontal and vertical planes. Furthermore, like the main damping ring, the ring damps multiple trains of bunches at once, the number of which is determined by the ring circumference.

The magnets and vacuum system are being designed to provide sufficient aperture to accept a $2-\mathrm{GeV}$ beam with an edge emittance of $\gamma \epsilon_{x, y}=0.09 \mathrm{~m}$-rad and momentum spread of $|\delta p / p| \leq 1.5 \%$ plus 2 -mm clearance for misalignments and mis-steering. Given the nominal injected edge emittance of $\gamma \epsilon_{x, y}=0.06 \mathrm{~m}$-rad, this provides a substantial margin for injection and internal mismatches. In addition, the injector specifications assume that roughly $20 \%$ of the delivered charge is lost at injection into the pre-damping ring. The pre-damping ring 


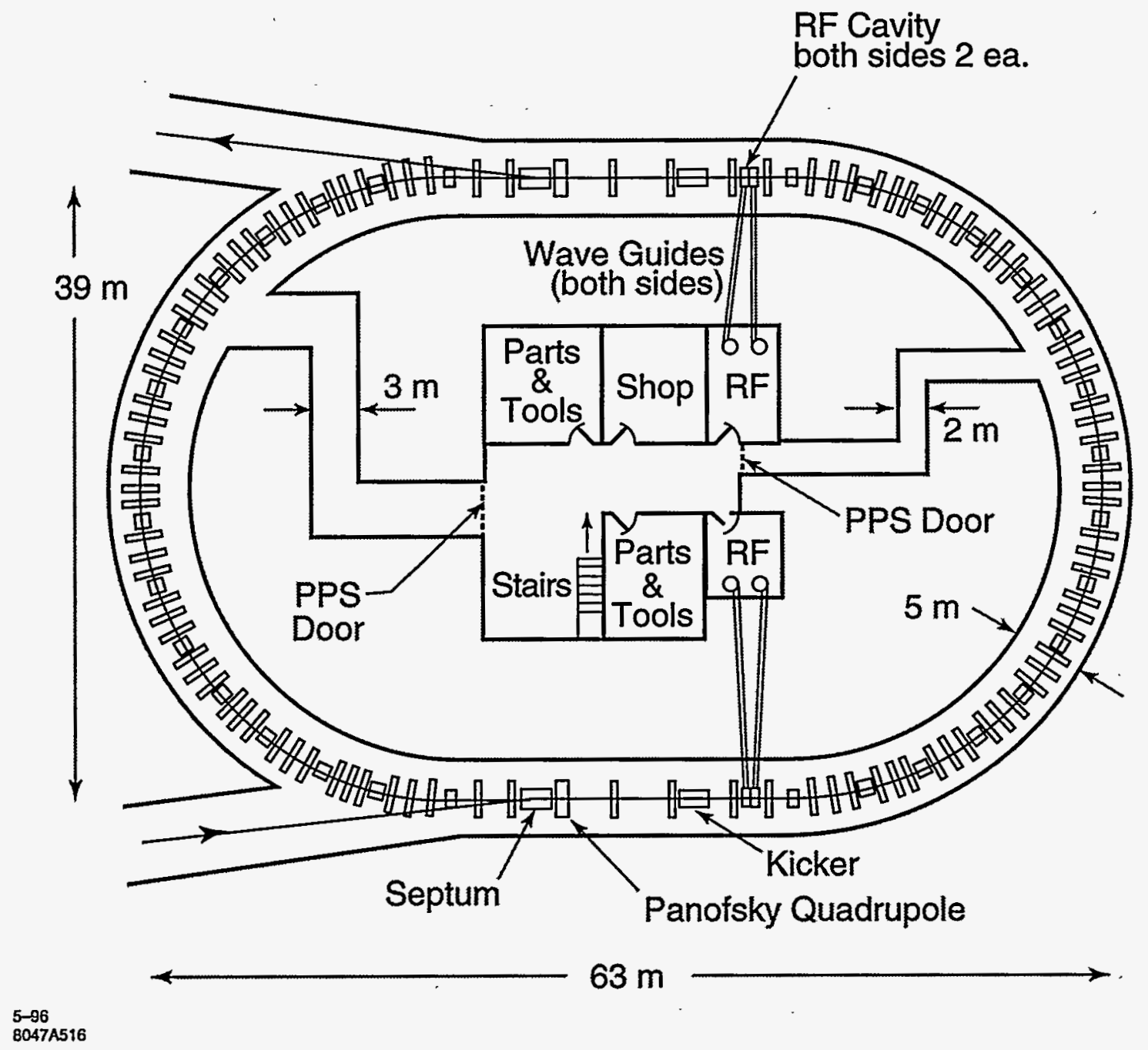

Figure 3.8: Layout of the positron pre-damping ring.

is designed to operate with a maximum bunch charge that is roughly $20 \%$ greater than the maximum required at the IP.

Like the main damping rings, all of the quadrupoles and all of the sextupoles will have independent power supplies and remote magnet movers. This will facilitate beam-based alignment as well as matching of the lattice functions which is especially important in the pre-damping ring because of the limited aperture. 


\subsection{Spin Rotators and Bunch Compressors}

The purpose of the spin rotators is rotate the polarization vector, which is vertically oriented in the damping rings, into any arbitrary direction so that the beam will be longitudinally polarized at the IP. The rotators are located at the exit of the damping rings and are then followed by the bunch compressors. A spin rotator will be installed on the electron side and space will be left for an installation on the positron side to allow for a future upgrade to polarized positrons or polarized electrons for $\gamma \gamma$ or $e^{-} e^{-}$collisions. The rotators are based on pairs of solenoids separated by a horizontal arc to allow full control over the spin orientation; this is similar in concept to the original SLC spin rotator system. Solenoids were chosen to control the spin because the other alternate, namely a snake, requires a vertical bending chicane which must be unreasonably long to prevent synchrotron radiation from increasing the vertical emittance. Of course, the problem with solenoids is that they couple the beam which, with flat beams, leads to an increase in the projected vertical emittance; this is the reason the original solenoid system is not presently used in the SLC. Our present design uses pairs of solenoids which are optically separated so that the coupling is fully cancelled. There are very tight tolerances on the quadrupole fields between the sextupoles but an extensive skew correction system has been included to ease the tolerances as well as correct any residual coupling from the damping rings.

The NLC bunch compressors must compress the bunches from the damping rings with 4-mm lengths to the 100-150 $\mu \mathrm{m}$ bunch lengths required in the main linacs and final foci. To perform the compressions, a two-stage compressor system has been designed: the first stage follows the damping rings and the second stage operates at a beam energy of $10 \mathrm{GeV}$ at the exit of $8-\mathrm{GeV}$ S-band prelinacs. The compressor system is illustrated schematically in Figure 3.9.

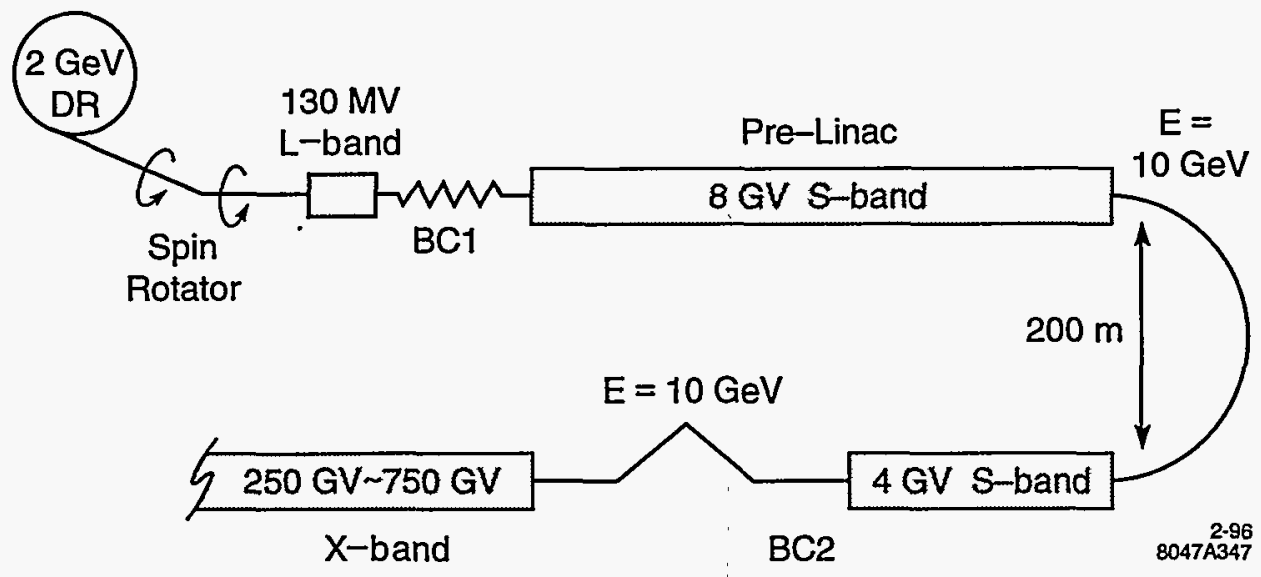

Figure 3.9: Schematic of the NLC bunch compressor system.

The bunch compressor system was designed with three primary goals:

- The compressor system must compress a bunch with length $\sigma_{z} \approx 4 \mathrm{~mm}$ from the 
damping rings to the $100-150 \mu \mathrm{m}$ length needed in the main X-band linacs and the final foci. This requires compressing the bunch length, and correspondingly increasing the energy spread, by a factor of $30-40$.

- The bunch compressor system should be able to compensate for bunch-to-bunch phase offsets induced by the beam loading in the damping rings; if not corrected, these phase errors would become energy errors at the end of the linacs. This requires that the compressor system rotate the longitudinal phase space by $\pi / 2$, translating the phase errors into injection energy errors.

- The bunch compressor system should provide a trombone-like arm, reversing the direction of the beam before injection into the main X-band linacs. This allows extension of the linac lengths to upgrade the beam energy at the IP without significant modification to the injection systems. It also allows for abort systems to dump the beam and feed-forward systems to correct the beam trajectory, phase, and energy before injection into the main linacs.

The two-stage system has a number of advantages over a single stage compressor. In particular, it keeps the rms energy spread less than roughly $2 \%$, it is optically more straightforward, and the bunch lengths are more logically matched to the acceleration rf frequency so that the energy spread due to the longitudinal wakefields can be cancelled locally. The disadvantage of the two-stage design is that it is more complex and lengthy than a singlestage compressor. The first stage rotates the longitudinal phase by $\pi / 2$ while the second stage performs a $2 \pi$ rotation. In this manner, phase errors due to the beam loading in the damping rings and energy errors due to imperfect multibunch energy compensation in the 8-GeV S-band prelinacs do not affect the beam phase at injection into the main linac.

Assuming an rms energy spread of $\sigma_{\delta}=1 \times 10^{-3}$, the low energy compressors compress the damping ring beam to a bunch length of $500 \mu \mathrm{m}$. The final bunch length is roughly independent of the incoming bunch length and the system has been designed to accept an initial bunch length of $5 \mathrm{~mm}$; this is $25 \%$ larger than expected from the damping ring. The bunch compressors consist of a 139-MV L-band (1.4-GHz) rf section followed by a long period wiggler which generates the $R_{56}$ needed for the bunch compression.

The high-energy bunch compressors follow the prelinacs. Assuming an rms bunch length of $\sigma_{z}=500 \mu \mathrm{m}$, they compress the beam to a bunch length between 100 and $150 \mu \mathrm{m}$ depending on the NLC parameters. As stated, they are telescopes in longitudinal phase space, rotating the longitudinal phase space by $2 \pi$. The bunch compressors consist of a $180^{\circ}$ arc which is followed by a 4-GeV S-band (2.8-GHz) rf section and a chicane. Parameters of the highenergy bunch compressors are listed in Table 3.6 for four different NLC parameter sets.

One of the driving philosophies behind the NLC compressor design has been to utilize naturally achromatic magnetic lattices wherever the beam energy spread is large. In particular, the optics is chosen so that quadrupoles are not placed in regions of large dispersion and strong sextupoles are not needed. This choice arises from experience with the SLC bunch 
Table 3.6: Parameters for $10-\mathrm{GeV}$ compressor.

\begin{tabular}{|l|cccc|}
\hline \hline & NLC-I a & NLC-I c & NLC-II a & NLC-II c \\
\hline Energy & \multicolumn{4}{|c}{$10 \mathrm{Gev}$} \\
Initial $\sigma_{z}, \sigma_{\epsilon}$ & \multicolumn{4}{|c}{$500 \mu \mathrm{m}, 0.25 \%$} \\
Final $\sigma_{z}$ & $100 \mu \mathrm{m}$ & $150 \mu \mathrm{m}$ & $125 \mu \mathrm{m}$ & $150 \mu \mathrm{m}$ \\
Final $\sigma_{\epsilon}$ & $1.5 \%$ & $1.2 \%$ & $1.3 \%$ & $1.4 \%$ \\
$180^{\circ}$ arc $R_{56}$ & 0.211 & 0.147 & 0.184 & 0.162 \\
Arc Length & \multicolumn{4}{|c}{$330 \mathrm{~m}$} \\
$V_{r f}(\mathrm{GV})$ & 3.85 & 3.50 & 3.73 & 3.61 \\
$f_{r f}$ & \multicolumn{4}{|c}{$2.8 \mathrm{GHz}$} \\
$L_{r f}$ & \multicolumn{5}{|c}{$250 \mathrm{~m}$} \\
Chicane $R_{56}$ & \multicolumn{5}{|c}{$100 \mathrm{~mm}$} \\
Chicane Length & \multicolumn{5}{|c}{} \\
\hline \hline
\end{tabular}

compressors which are based on second-order achromats where quadrupoles are located in dispersive regions and strong sextupoles are used to cancel the chromatic aberrations. Unfortunately, the SLC design is extremely difficult to operate and tune because of the large nonlinearities and the sensitivity to multipole errors in the quadrupoles; over the years additional nonlinear elements have been added (skew sextupoles and octupoles) to help cancel the residual aberrations but the tuning is still difficult. To further facilitate the tuning in the NLC design, we have explicitly designed orthogonal tuning controls and diagnostics into the system. We feel confident that these considerations will make the system relatively straightforward to operate and tune.

As discussed, the rf acceleration throughout the compressor system (including the 8$\mathrm{GeV}$ prelinac) is performed with relatively low frequency rf. In particular, the first bunch compressors use L-band $(1.4 \mathrm{GHz})$ and the prelinac and second bunch compressor use S-band $(2.8-\mathrm{GHz})$. Although these systems are, in general, longer and more expensive than higher frequency accelerator systems, we feel that their superior acceptances are needed at the lower beam energies. In particular, we have designed the systems so that they have small beam loading and relatively loose alignment tolerances. This is important to provide the reliability and stability that is desired in the bunch compressor system. Of course, such lightly-loaded systems are inefficient and could not be used to accelerate the beams to very high energy.

Finally, although the tolerances on components in the bunch compressor systems are not nearly as tight as in the main linacs or the final foci, we have chosen to adopt the same methods of beam-based alignment and tuning. In particular, to ease the alignment procedures, all of the quadrupoles will be mounted on remote magnet movers and will be 
powered by individual power supplies. In addition, each quadrupole will contain a BPM with a resolution of $1 \mu \mathrm{m}$. Similarly, all of the accelerator structures will be instrumented with an rf BPM to measure the induced dipole modes and each girder will be remotely movable; depending on location, the girders will support either one or two structures. 


\subsection{Main Linacs: Design and Dynamics}

The main linacs are designed to initially accelerate electrons and positrons from an energy of $10 \mathrm{GeV}$ to $250 \mathrm{GeV}$, and to be built so that with reasonably expected near-term improvements in the X-band rf system, the beam energy can be increased over time to $500 \mathrm{GeV}$. The infrastructure and layout of the collider is designed to allow the energy of the beam to be further increased to as much as $750 \mathrm{GeV}$ if suitably efficient rf sources, such as cluster klystrons, grided klystrons, or two-beam accelerators can be developed. The linac must accelerate trains of 90 electron or positron bunches with intensities that vary from $0.75 \times 10^{10}$ to $1.1 \times 10^{10}$ particles per bunch as the energy is increased. The spacing between bunches in a train is determined by the damping ring frequency to be $1.4 \mathrm{~ns}$. The repetition rate of the accelerator must be reduced from $180 \mathrm{~Hz}$ to $120 \mathrm{~Hz}$ as the energy is increased to maintain the linac AC power consumption below $200 \mathrm{MW}$.

There are two major design issues for the main linacs: the efficient acceleration of the beams using $\mathrm{X}$-band technology as described in the next section, and the preservation of the small beam emittances that are required to achieve the desired collision luminosity. In this section, we discuss the design and operation of the linacs emphasizing the beam dynamics issues.

The NLC linac design builds on the experience that has been gained from the operation of the SLC, in particular, the development of beam-based alignment, tuning, and feedback techniques. The NLC design also draws on the SLC operational experience in such areas as the transport of flat beams, the use of BNS damping, and linac energy management. In areas where we cannot directly verify NLC operational issues with the SLC, we have built test facilities. These include ASSET for X-band structure wakefield measurements, ASTA and NLCTA for X-band accelerator technology testing, and the FFTB for testing of components such as quadrupole movers that will be used in the main linacs as well as in the final focus. Of these facilities, the NLCTA will provide the most direct test of the basic linac design in its ability to accelerate multibunch beams and to control the effects of beam loading.

Parameters of the main linacs are listed in Table 3.7 for NLC-I, which has a $500 \mathrm{GeV}$ center-of-mass energy, and NLC-II, which has a $1 \mathrm{TeV}$ center-of-mass energy. The length of each of the two main linacs in NLC-I is $8.8 \mathrm{~km}$. This will increase only slightly in the upgrade to NLC-II, with the bulk of the energy gain coming from an increase in the unloaded gradient from 50 to $85 \mathrm{MeV} / \mathrm{m}$. This increase in gradient will be performed adiabatically by upgrading and doubling the number of klystrons which supply the rf power. It will be accompanied by an increase in beam charge which will leave the fractional beam loading roughly constant. Since the total linac length required for NLC-II is not much longer than that for NLC-I, we will probably construct the full NLC-II linac length initially and use the additional length in NLC-I to attain beam energies slightly higher than $250 \mathrm{GeV}$.

For both NLC-I and NLC-II, three different parameter sets are listed. These correspond to the same rf power and linac length, but assume different bunch charges and bunch lengths. The three parameter sets reflect a tradeoff in bunch charge versus beam size where the 
Table 3.7: NLC Linac Parameters

\begin{tabular}{|c|c|c|c|c|c|c|}
\hline & \multicolumn{3}{|c|}{ NLC-I } & \multicolumn{3}{|c|}{ NLC-II } \\
\hline & a & $\mathrm{b}$ & c & $\mathbf{a}$ & $\mathrm{b}$ & c \\
\hline$N\left[10^{10}\right]$ & 0.65 & 0.75 & 0.85 & 0.95 & 1.10 & 1.25 \\
\hline Bunches per Train & & 90 & & & 90 & \\
\hline Repetition Rate [Hz] & & 180 & & & 120 & \\
\hline$\sigma_{z}[\mu m]$ & 100 & 125 & 150 & 125 & 150 & 150 \\
\hline Unloaded Gradient [MV/m] & & 50 & & & 85 & \\
\hline Multibunch Loading [\%] & 25.5 & 29.4 & 33.34 & 22.0 & 25.5 & 28.9 \\
\hline Multibunch Loading $[\mathrm{MV} / \mathrm{m}]$ & -12.8 & -14.7 & -16.7 & -18.7 & -21.5 & -24.6 \\
\hline Single-bunch Loading* $[\mathrm{MV} / \mathrm{m}]$ & -0.2 & -0.3 & -0.4 & -0.4 & -0.5 & -0.6 \\
\hline Average $\phi_{R F}^{\dagger}\left[^{\circ}\right]$ & -15 & -15.5 & -16 & -7.5 & -8.2 & -11.2 \\
\hline BNS Overhead [\%] & 3 & 3 & 3 & 3.5 & 3.5 & 3.5 \\
\hline Feedback Overhead [\%] & 2 & 2 & 2 & 2 & 2 & 2 \\
\hline Repair Margin [\%] & 3 & 3 & 3 & 3 & 3 & 3 \\
\hline$L_{\mathrm{acc}}[\mathrm{m}]$ & & 8150 & & & 8834 & \\
\hline Number of sections in Structure & & 4528 & & & 4908 & \\
\hline$L_{\text {total }}[\mathrm{m}]$ & & 8807 & & & 9550 & \\
\hline$E_{\max }^{\ddagger}[\mathrm{GeV}]$ & 266.7 & 250 & 232.2 & 529 & 500 & 468 \\
\hline
\end{tabular}

${ }^{*}$ Single-bunch loading includes only HOM losses.

${ }^{\dagger}$ Average rf phase for $0.8 \%$ FWHM energy spread.

†Including $10 \mathrm{GeV}$ initial energy. 
luminosity is kept nearly constant. This is the main tradeoff that remains in a linear collider design when all constraints are taken into account. Larger beam sizes are desirable since they loosen many of the tolerances. However, increasing the beam charge to compensate the loss in luminosity produces problems related to beam power, and generally leads to tighter wakefield-related tolerances. The three parameter sets each represent compromises among these competing effects: together they span the range of operating conditions that we consider reasonable.

The design normalized emittance of the bunches entering the main linacs is $\gamma \epsilon_{x}=3.6 \times$ $10^{-6} \mathrm{~m}$-rad, $\gamma \epsilon_{y}=4.0 \times 10^{-8} \mathrm{~m}$-rad, and the energy is $10 \mathrm{GeV}$. During the acceleration of the bunches to $\sim 250 \mathrm{GeV}$ (NLC-I) or $\sim 500 \mathrm{GeV}$ (NLC-II), it is necessary to preserve the low transverse emittance and maintain the small final beam energy spread; these requirements are listed in Table 3.8. Note that we have allowed for over $100 \%$ vertical emittance growth. This lets us set fairly conservative tolerances on the accelerator structure alignment. However, we believe that we will do better than these tolerances as we gain more experience with X-band accelerator construction and operation.

Table 3.8: NLC Linac Emittance Requirements

\begin{tabular}{|c|c|c|c|c|c|c|}
\hline & \multicolumn{3}{|c|}{ NLC-I } & \multicolumn{3}{|c|}{ NLC-II } \\
\hline & $\mathrm{a}$ & $\mathrm{b}$ & c & $a$ & b & $\mathrm{c}$ \\
\hline $\mathrm{N}\left[10^{10}\right]$ & 0.65 & 0.75 & 0.85 & 0.95 & 1.10 & 1.25 \\
\hline$\sigma_{z}[\mu m]$ & 100 & 125 & 150 & 125 & 150 & 150 \\
\hline$\gamma \epsilon_{x i n j}\left[10^{-6} \mathrm{~m}-\mathrm{rad}\right]$ & & 3.6 & & & 3.6 & \\
\hline$\gamma \epsilon_{y i n j}\left[10^{-8} \mathrm{~m}-\mathrm{rad}\right]$ & & 4 & & & 4 & \\
\hline$\sigma_{\epsilon i n j}[\%]$ & & $<1.5$ & & & $<1.5$ & \\
\hline$\gamma \epsilon_{x e x t}\left[10^{-6} \mathrm{~m}-\mathrm{rad}\right]$ & & 4 & & & 4 & \\
\hline$\gamma \epsilon_{y e x t}\left[10^{-8} \mathrm{~m}-\mathrm{rad}\right]$ & 7 & 9 & 11 & 9 & 11 & 13 \\
\hline$\Delta E / E_{F W H M \text { ext }}$ single bunch [\%] & & 0.8 & & & 0.8 & \\
\hline$\Delta E / E_{F W H M \text { ext }}$ train $[\%]$ & & 1.0 & & & 1.0 & \\
\hline
\end{tabular}

\subsubsection{Linac Layout and Site Requirements}

Although the layout of the main linacs is fairly simple, an array of X-band accelerator structures interleaved with a FODO quadrupole lattice, one faces a number of challenges to accelerate low-emittance bunch trains without significantly degrading the beam phase space. The $\mathrm{X}$-band structures that provide a high-acceleration gradient also heavily load the beam, 
so careful control of the rf pulse shape will be needed to achieve the small beam energy spread that is required at the end of the linac. The X-band structures also have the drawback that strong transverse wakefields, both long-range (bunch-to-bunch) and short-range (intra-bunch), are generated when the bunch train passes off-axis through the structures. Although damping and detuning of the dipole modes of the structure will be used to suppress the long-range wakefield, and autophasing will be used to offset the short-range wakefield effect on betatron motion, the structures will still have to be aligned precisely to limit emittance growth from both short-range and long-range wakefield effects. The small bunch sizes also make the beam sensitive to other emittance growth mechanisms such as dispersion from quadrupole misalignments, focusing from the ions generated in the residual gas of the beam line vacuum chambers, and dilution from beam trajectory jitter, like that caused by vibrations of the quadrupole magnets.

Most of the deleterious effects will be limited by design. For example, the quadrupole magnet power supplies will be chosen to be stable enough not to cause significant beam trajectory jitter. For some of the effects, however, we will rely mainly on measurements of the beam properties to control emittance growth. In particular, beam trajectory measurements will be used to adjust the positions of the quadrupoles and structures to minimize the emittance growth caused by their misalignment. For this purpose, each quadrupole and structure in the linacs will contain a beam position monitor (BPM), and will be supported by movers that can be remotely controlled (e.g., like the magnet movers in the FFTB).

A beam-based approach will also be used in a trajectory feedback system to suppress low-frequency jitter of the bunch train as a whole, and if necessary, bunch-to-bunch position variations within the train (special multibunch BPMs will be used in this case). These feedbacks will be included in each of five instrumentation regions that will be located along the linacs. These regions will also include high-dispersion sections so that bunch energy and energy spread can be measured and used for if control feedback. As with the operation of the SLC, if the feedback and alignment algorithms fail to suppress emittance growth to the desired level, non-local types of tuning will be used, such as orbit bumps. To aid in this tuning, and to monitor the beam phase space, the instrumentation regions will also contain beam size monitors.

Near the beginning of each linac, there will be a quadrupole magnet after each structure pair, these components are shown in Fig. 3.10. The separation between quadrupoles will increase in two-structure increments, from one pair of structures to five pairs at the end of the linacs. The quadrupole lengths and pole tip fields are shown in Fig. 3.11, the vertical beta function in Fig. 3.12. The phase advance per cell ranges between about $70^{\circ}$ and $90^{\circ}$, with somewhat stronger focusing in the vertical plane. As part of the machine protection system, there will be a titanium spoiler after each structure. For normal beam operation, it will do little to prevent damage to the beam pipe, but for tuning, it allows a single high-emittance bunch to intercept the beam pipe without causing damage.

Since the main linacs are the largest components of the NLC, the impacts of the site characteristics on the cost and operation of the linacs will be the dominant technical factors 


\section{LINAC BEAMLINE COMPONENTS}

End View

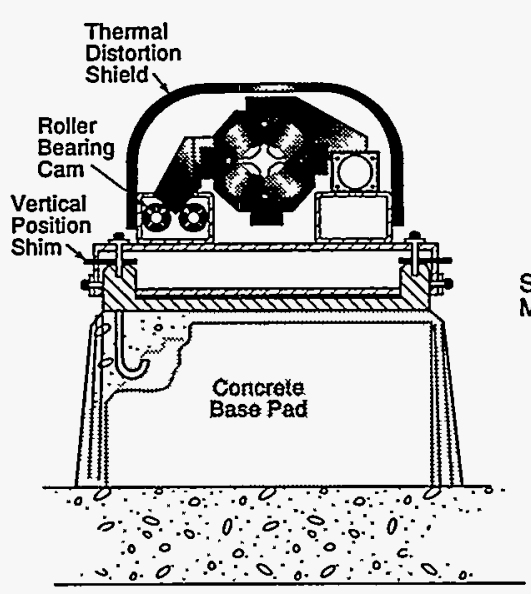

Side View

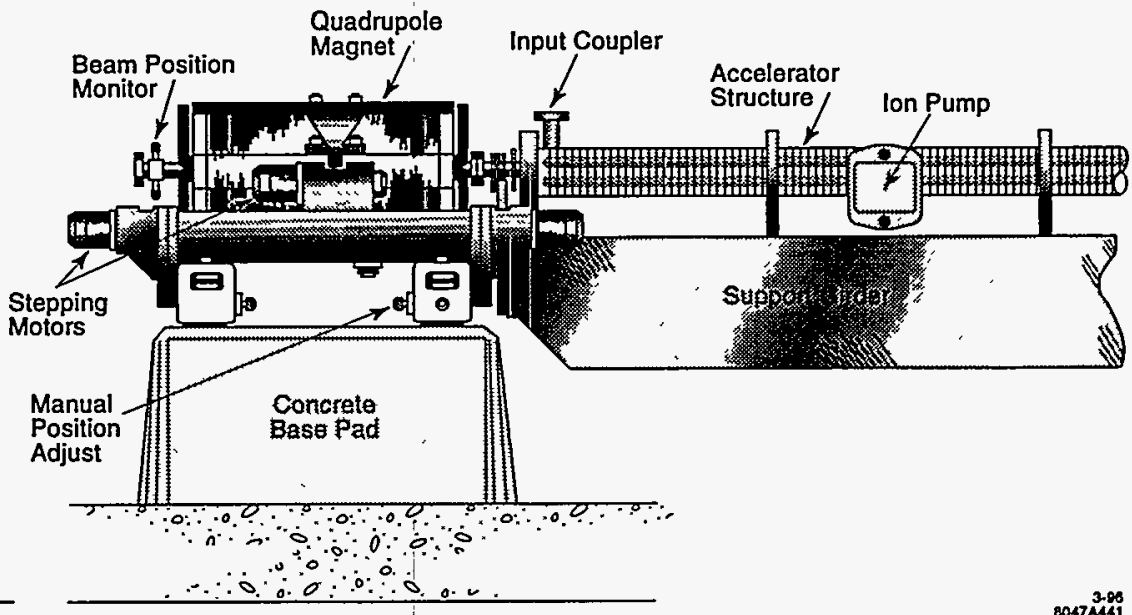

Figure 3.10: End view and side view of the beam line near the beginning of the linacs, where there is a quadrupole between each pair of accelerator components.

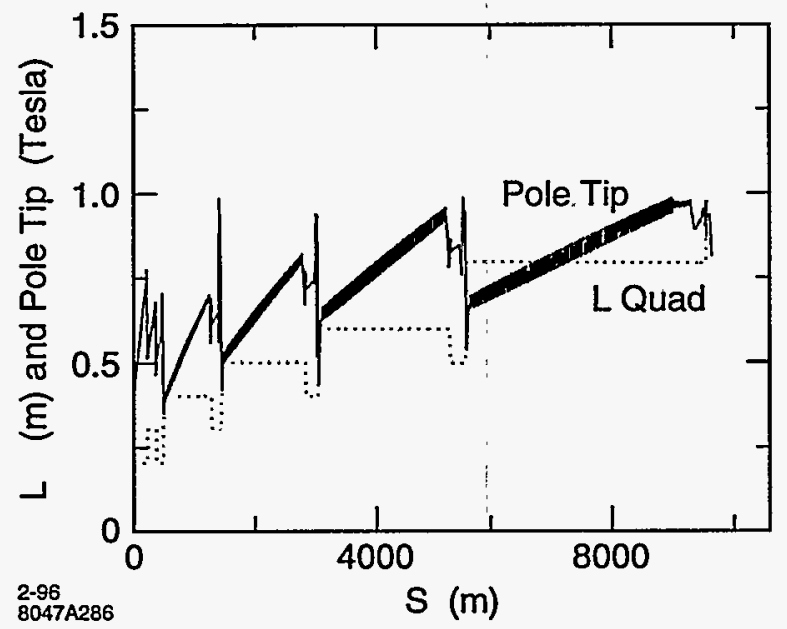

Figure 3.11: Quadrupole lengths (dotted) and pole-tip fields (solid) in NLC-IIb lattice. 


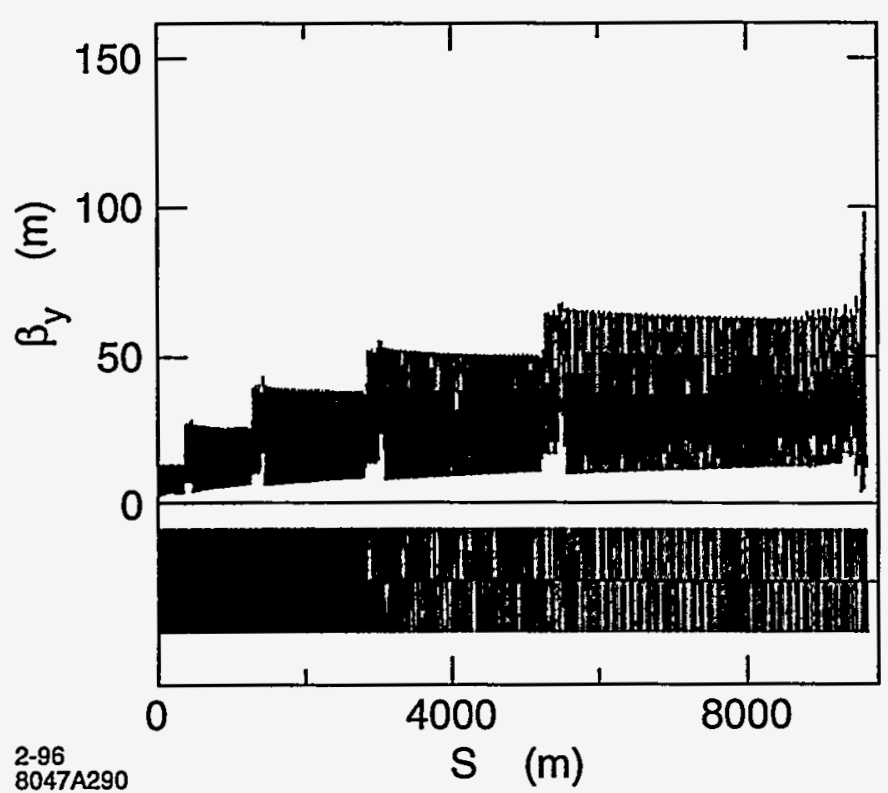

Figure 3.12: Vertical beta function in the NLC-II linacs.

in choosing a location to build the accelerator. As an example we have considered a layout similar to that of the present SLAC accelerator (Fig. 3.13). Placing the klystron gallery underground will help stabilize the gallery air temperature to within the $1^{\circ} \mathrm{C}$ required. In the beam line area, we want a $0.1^{\circ} \mathrm{C}$ stability, which should not be too difficult to achieve since the beam line tunnels will be well isolated. The beam line is best located at least $20 \mathrm{ft}$ below ground to provide natural radiation shielding. Access shafts to the surface will probably be required about every kilometer to provide cooling tower and substation connections, and to allow maintenance crews to reach the beam line. A flat terrain is desirable to minimize the depth of such access shafts and would also facilitate the establishment of a global coordinate system for alignment on the surface and the subsequent transfer of the reference system to the beam line. Hardness of the ground, seismic activity on all scales, and cultural noise such as that produced by traffic and heavy machinery, will all be factors in the site selection.

\subsubsection{Operation and Tuning of the Main Linacs}

Initial set up of the machine for operation includes creating an optimal single bunch energy spread profile for the BNS damping (or "autophasing") which is the introduction of a correlated energy spread along each bunch to offset the short-range wakefield effect on betatron motion. We wish to do this with minimum energy loss due to the phasing, and we also want the bunches at the end of the linac to have small enough energy spread to fit into the final focus bandwidth. The bunches are run behind the crest early in the linac, and then sufficiently in front of the crest later in the linac to remove enough energy spread to fit within the final focus bandwidth.

Another necessity for initial setup is multibunch energy compensation, since otherwise 


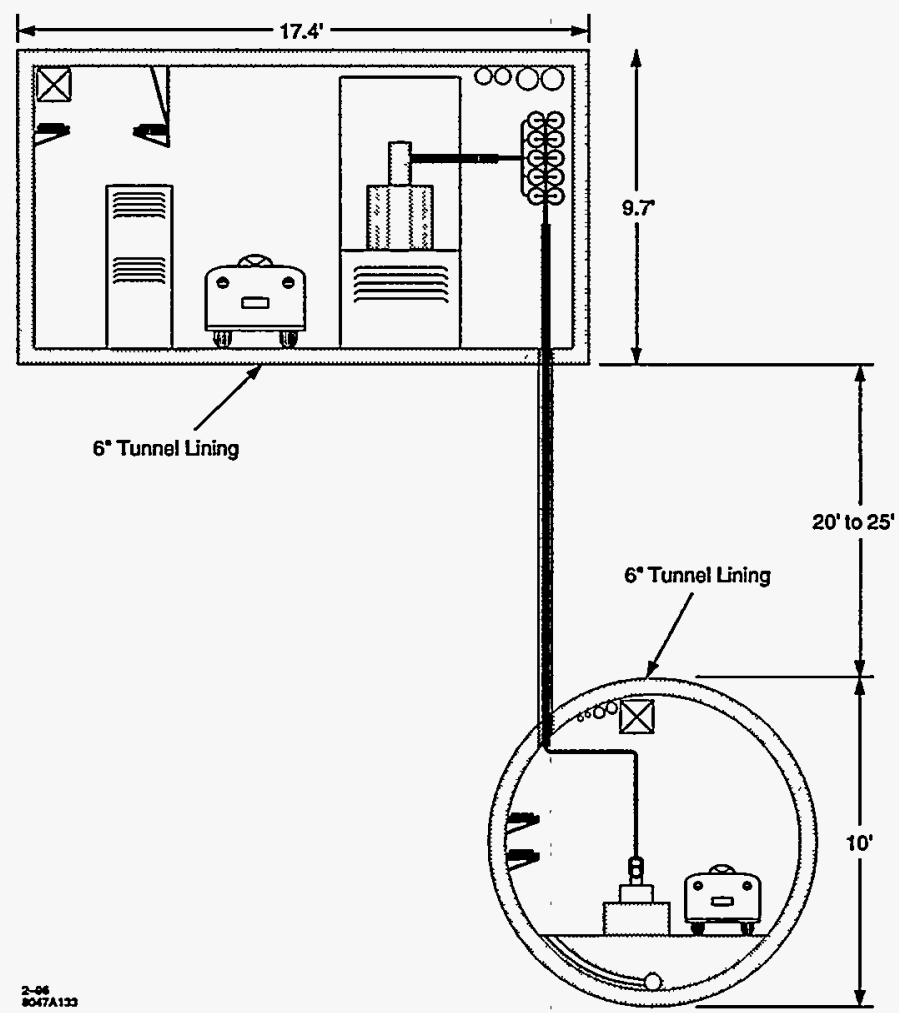

Figure 3.13: Klystron gallery and beam line tunnel layout ("SLAC type"). This is just one of several possible layouts for housing the accelerator and rf components. 
there would be a drop in energy of between 20 and $30 \%$ between the head and tail of the multibunch train. The method of compensation must keep the energies of all the bunches the same to within a few tenths of a percent. This will be done by pre-filling the accelerator structure with an rf pulse shaped to simulate the steady state beam-loaded rf field profile. This profile is then automatically maintained if the incoming rf field is kept constant during the passage of the train, assuming systematic variations of the bunch charges are kept small enough. This places fairly stringent tolerances on the injectors (and other upstream effects on the bunch charges).

To maintain high luminosity it will be required to keep both the emittance and beam position jitter small at the interaction point. Due to the tiny vertical beam size, pulse-topulse beam position jitter generated by the movements of the quadrupole magnets could potentially degrade the luminosity. The ground motion frequencies of concern are those above about $0.1 \mathrm{~Hz}$ where the feedback systems that will be used to stabilize the beam may not produce the suppression that is required. The spatial characteristics of the motion are important as well. The effect of quadrupole vibration on the beams is highly suppressed if the motion is correlated over distances longer than the betatron oscillation lengths of the beams.

Since few data exist on the spatial properties of ground motion, we did a series of seismic measurements at SLAC. We found that the motion can be well modeled by plane waves that are isotropic in direction. These surface waves are dispersive and the dependence of the phase velocity on frequency was inferred from the data by assuming the plane-wave model.

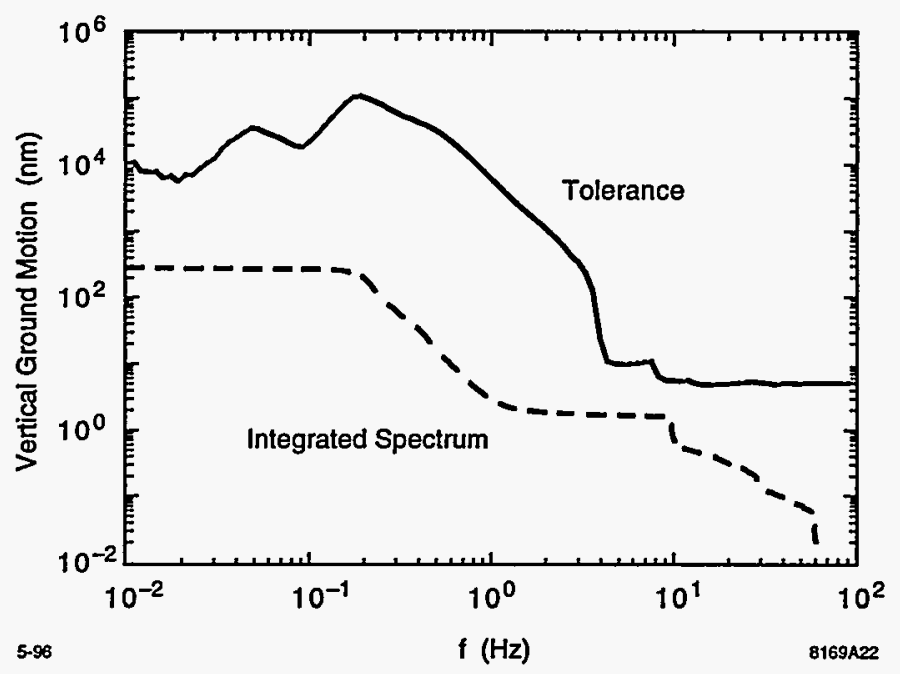

Figure 3.14: Integrated vertical ground motion spectrum (dashed) and tolerance on the vertical rms ground motion of the linac (solid) for a $1.5 \%$ luminosity reduction.

In addition to this wave-like motion, there can be an uncorrelated component which, as a worst case, we take equal to the electronic noise of the seismometers that we used for our measurements. The tolerance on ground motion as a function of frequency was 
then calculated, and is shown in Fig. 3.14 for waves plus the uncorrelated noise component. The tolerance includes the suppression expected from a trajectory feedback system with performance similar to that achieved at the SLC. The net effect on the luminosity is obtained by integrating the power spectrum weighted by the inverse square of the tolerance. For frequencies greater than $0.01 \mathrm{~Hz}$, this yields a luminosity reduction of about $0.1 \%$. Our conclusion is that ground motion in the linacs should not be a problem as long as care is taken to avoid introduction of substantial vibrations locally in the accelerator housing, (e.g. water pumps or other equipment.

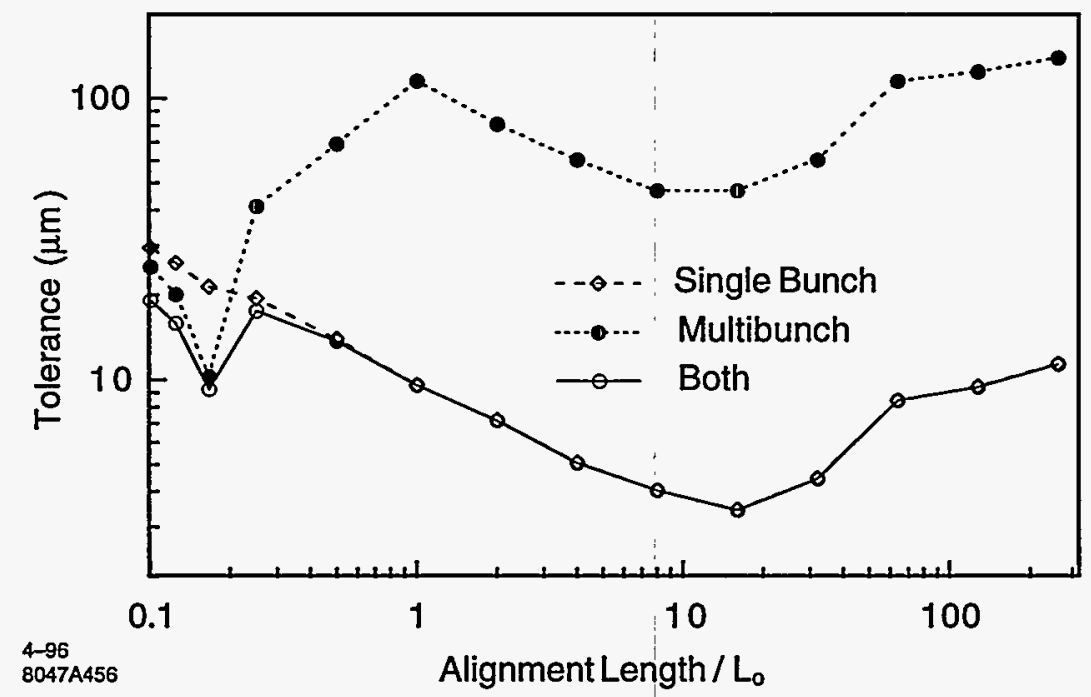

Figure 3.15: Tolerance on the structure alignment for a $50 \%$ emittance growth in the NLC-IIb linacs as a function of alignment length, when considering only single-bunch effects, only multi-bunch effects, and both effects together. $L_{0}$ is the $1.8 \mathrm{~m}$ structure length.

Quadrupole and accelerator structure alignment will be a key aspect of NLC operation, since quad offsets will lead to dispersive emittance growth and structure offsets will lead to wakefield related emittance growth. Initial alignment of the quadrupole magnets can be done with sufficient accuracy by conventional methods, but obtaining and maintaining the required beam quality will require additional beam-based techniques. Alignment procedures that use measurements of correlations between orbit trajectories and quadrupole strengths and mover settings have been developed at the SLC and FFTB. Adaptation of these techniques to the NLC linacs is straightforward, and can be used to determine the offsets between the beam position monitors and the quadrupoles, as well as the misalignments of the quadrupoles with respect to a smoothed line. The BPM trajectory readings can then be used to return the quadrupoles to smoothed locations at periodic intervals. The accelerator structures will be aligned by measuring the dipole wakefield signals generated by the passage of the beam. It is precisely these signals that must be nulled to eliminate wakefields that damage the beam phase space. Additional suppression of emittance growth in the linac is possible with the use 
of non-local correction methods; trajectory bumps, fast kickers, etc., can be used to cancel the emittance dilutions after they have accumulated. These are not preferred methods since they are sensitive to variations in beam transport properties between the sources of the dilution and the correction point, but they are in extensive use presently at the SLC.

Another major concern is the long-range wakefield in the accelerator structures, which if not heavily suppressed, will resonantly amplify the betatron motion of the bunch train by many orders of magnitude. Much work to date has been devoted to designing an accelerator structure that sufficiently suppresses the long range transverse wake fields. The outcome is the damped detuned structure (DDS), which incorporates both detuning and damping of the wakefield. This brings the amplification due to wakefields of incoming betatron oscillations under control, but still leads to rather tight structure alignment tolerances on some length scales. Figure 3.15 shows tolerances on the structure alignment as a function of alignment length scale, assuming the wakefield from the DDS. (For instance, 0.1 on the horizontal axis of this figure corresponds to the case where each group of $\sim 20$ cells in each of the $\sim 200$-cell accelerator structures is separately misaligned.) Non-local correction methods can be used to loosen these tolerances somewhat. As was noted earlier, we allow for a vertical emittance growth from all sources of over $100 \%$, even though we expect to do somewhat better than the tolerances that would lead to this amount of growth. The total emittance budget is given in Table 3.9 for the NLC-IIb linac design.

Table 3.9: NLC-IIb Linac Emittance Growth Budget

\begin{tabular}{|c|c|c|c|}
\hline Source & & $\Delta \epsilon_{y} / \epsilon_{y}$ & Dynamics \\
\hline Quad alignment & & $40 \%$ & Incoherent dispersion \\
\hline BPM resolution & $1 \mu m$ & & \\
\hline BPM to quad alignment & $2 \mu m$ & & \\
\hline Quad drift between alignment & & $10 \%$ & Coherent dispersion \\
\hline Steering period & $30 \mathrm{~min}$ & & + wakes \\
\hline \multicolumn{4}{|l|}{ Structure alignment } \\
\hline Internal alignment & $15 \mu m$ & $25 \%$ & Long-range wakes \\
\hline Beam measurement accuracy & $15 \mu m$ & $50 \%$ & Short-range wakes \\
\hline Other (e.g., quad roll, ion effects, and rf deflections) & & $50 \%$ & \\
\hline Total & & $175 \%$. & \\
\hline
\end{tabular}




\subsection{The RF System for the Main Linacs}

The basic design of the NLC main linacs rests on global experience gained from the design, construction, and 30 years of operation of the $3-\mathrm{km}$-long SLAC linac, which is powered at a frequency of $2.856 \mathrm{GHz}[2,3]$. Since its initial operation in 1966, the SLAC linac has been continuously upgraded for higher energy, higher intensity, and lower emittance.

The radio frequency (rf) system for the NLC main linacs is similar in character to the SLAC linac. The SLAC linac is currently energized by 240 high-power S-band klystrons. The klystron peak power and pulse duration are, respectively, $65 \mathrm{MW}$ and $3.5 \mu \mathrm{s}$. The power from each klystron is compressed by a SLED pulse compressor, and then split to feed four, 3 -m-long, constant-gradient, S-band accelerator structures operating in the $2 \pi / 3$ mode.

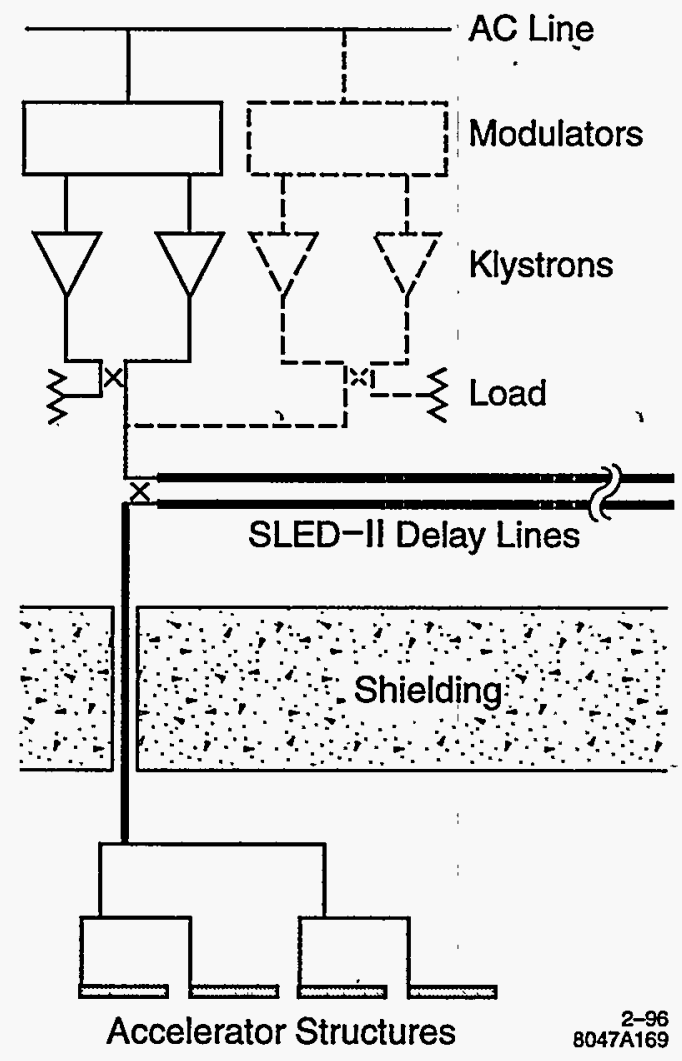

Figure 3.16: Schematic of one module of the high-power rf system for - the main linacs. Dashes indicate additions for the beam-energy upgrade from $250 \mathrm{GeV}$ to $500 \mathrm{GeV}$.

When the SLAC linac was built, the accelerating gradient was $7 \mathrm{MV} / \mathrm{m}$. The original design included a future upgrade path in which the number of klystrons would be quadrupled. The upgrades that were eventually implemented involved replacing each of the initial 24MW klystrons with a single higher-power klystron (first with 35-MW, XK-5 tubes and, later on, with 65-MW, 5045 tubes), and adding a SLED pulse compressor downstream of 
each klystron. For present-day SLC operations, fully upgraded with 240 SLEDed, 65-MW klystrons, the accelerating gradient has been tripled, to $21 \mathrm{MV} / \mathrm{m}$, and the maximum beam energy is $60 \mathrm{GeV}$ (for unloaded, on-crest operation).

The rf power system for the NLC's two high-gradient linacs that accelerate the electron and positron beams separately from $10 \mathrm{GeV}$ to $250 \mathrm{GeV}$ in the initial design, and to $500 \mathrm{GeV}$ or more after the upgrade, operates at $11.424 \mathrm{GHz}$. This system includes all the hardware through which energy flows, from the $\mathrm{AC}$ line to the accelerator structures. Figure 3.16 shows one module of the rf system schematically.

Electrical energy is transformed at each stage shown in the diagram: the modulator converts $\mathrm{AC}$ power into high-voltage pulsed DC, the klystron transforms pulsed DC into high peak power rf, the SLED-II pulse-compression system increases the peak power by about a factor of four (at the expense of a reduced pulse width), and the accelerator sections convert rf power into beam power. Because of the high average rf power required to drive the accelerator structures, it is important that the highest possible efficiency be maintained for the processing and transmission of energy at every stage of the rf system.

The primary technical choice for the rf system is the use of the 11.424-GHz frequency. This frequency, high in the X-band (8.2-12.4 GHz), is exactly four times the operating frequency of the existing SLAC $60-\mathrm{GeV}$ linac. The choice of such a high frequency, relative to existing high-energy linacs, allows higher accelerating gradient, shorter linac length, and lower $\mathrm{AC}$ power consumption for a given beam energy. Considering the size, weight, cost, and availability of standard microwave components, we have chosen a frequency in the Xband for a design that is upgradable from an initial $250-\mathrm{GeV}$ beam energy to $500 \mathrm{GeV}$ or more.

The general parameters of the high-power $\mathrm{rf}$ system and its major subsystems (klystrons, modulators, rf pulse compressors, and the accelerator structure itself) are specified in Table 3.10. The set of parameters has been optimized to provide high acceleration gradient $(35-64 \mathrm{MV} / \mathrm{m})$ for trains of bunches with a moderate charge per bunch $(1.2-1.8 \mathrm{nC})$. This optimization keeps single-bunch wakefields under control and reduces the beamstrahlung at the collision point to tolerable levels. The upgrade to $500-\mathrm{GeV}$ beam energy (1-TeV centerof-mass energy) is accomplished by doubling the number of modulators (as shown by dashed lines in Fig. 3.16), and by replacing each 50-MW klystron with a pair of 75-MW klystrons. The total active length of linac must also be increased from $16,300 \mathrm{~m}$ to $17,700 \mathrm{~m}$. The upgrade plan also includes improvements in the modulator and pulse compression systems to increase the rf system efficiency.

The design of the high-power X-band rf system for the NLC is based on specific experience gained from building $\mathrm{X}$-band prototypes and operating them at high power, and on an rf systems-integration test-the Next Linear Collider Test Accelerator (NLCTA)-which is currently under construction at SLAC. The goals of the NLCTA project $[4,5]$ are to integrate the technologies of X-band accelerator structures and high-power rf systems, to demonstrate multibunch beam-loading energy compensation and suppression of higher-order beam-deflecting modes, to measure any transverse components of the accelerating field, and 
Table 3.10: NLC main linac rf system parameters.

\begin{tabular}{|c|c|c|c|}
\hline & $\begin{array}{l}\text { NLCTA } \\
\text { Achieved }\end{array}$ & $\begin{array}{c}500-\mathrm{GeV} \\
\text { Design Goal }\end{array}$ & $\begin{array}{c}\text { 1-TeV } \\
\text { Upgrade }\end{array}$ \\
\hline \multicolumn{4}{|l|}{ General Parameters } \\
\hline Frequency $(\mathrm{GHz})$ & 11.4 & 11.4 & 11.4 \\
\hline Accel. Gradient (MV/m), Unloaded/Loaded & $67 /-$ & $50 / 35.3$ & $85 / 63.5$ \\
\hline Overhead Factor, ${ }^{[1]} \mathrm{F}_{O H}$ & & 1.20 & 1.15 \\
\hline Active Linac Length ${ }^{[2]}(\mathrm{m})$ & 1 & 16,300 & 17,700 \\
\hline Total Linac Length $=1.08 \times$ Active Length $(\mathrm{m})$ & & 17,600 & 19,100 \\
\hline Number of Modulators & & 2264 & 4908 \\
\hline Number of Klystrons & & 4528 & 9816 \\
\hline Peak Power per meter of Structure (MW/m) & & 50 & 145 \\
\hline Rf Pulse Length at Structure (ns) & 150 & 240 & 240 \\
\hline Repetition Rate $(\mathrm{Hz})$ & 60 & 180 & 120 \\
\hline Particles per Bunch $\left(10^{10}\right)$ & & 0.75 & 1.10 \\
\hline Number of Bunches per Pulse & & 90 & 90 \\
\hline Peak Beam Current (A) & & 0.86 & 1.26 \\
\hline Total Average RF Power at Structure ${ }^{[3]}$ (MW) & & 34.1 & 71.3 \\
\hline \multicolumn{4}{|l|}{ Klystron } \\
\hline Output Power (MW) & 50,75 & 50 & 75 \\
\hline Pulse Length $(\mu \mathrm{s})$ & $2.0,1.1$ & 1.2 & 0.96 \\
\hline Microperveance $\left(\mu A / V^{3 / 2}\right)$ & 1.2 & 0.6 & 0.75 \\
\hline Electronic Efficiency ${ }^{[4]}(\%)$ & 48 & 57 & 60 \\
\hline Beam Voltage $(\mathrm{kV})$ & 440 & 465 & 487 \\
\hline Focusing & Electromagnet & PPM & PPM \\
\hline Cathode Loading $\left(\mathrm{A} / \mathrm{cm}^{2}\right)$ & 7.4 & 7.4 & 7.6 \\
\hline \multicolumn{4}{|l|}{ Modulator (Blumlein PFN, transformer ratio $7: 1$ ) } \\
\hline PFN Voltage $(\mathrm{kV})$ & 48 & 68 & 71 \\
\hline Pulse Rise Time (ns) & 400 & 275 & 175 \\
\hline Net Modulator Efficiency (\%) & 58 & 72 & 75 \\
\hline \multicolumn{4}{|l|}{ RF Pulse Compression } \\
\hline System Type & SLED-II & SLED-II & BPC/DLDS \\
\hline Compression Ratio & $5-7$ & 5 & 4 \\
\hline Intrinsic Efficiency (\%) & $80-69$ & 80 & 100 \\
\hline Pulse Compression Efficiency (\%) & $73-64$ & 76.5 & 93 \\
\hline Power Transmission Efficiency (\%) & 90 & 94 & 94 \\
\hline Net Pulse-Compression Efficiency (\%), & & & \\
\hline Including Power Transmission Loss & $66-58$ & 72 & 87.5 \\
\hline Net Power Gain & $3.3-4.1$ & 3.6 & 3.5 \\
\hline \multicolumn{4}{|l|}{ Net RF System Parameters } \\
\hline Total AC Power (MW), Excl. Aux. & & 116 & 181 \\
\hline Rf System Efficiency (\%), Excl. Aux. & 19 & 29.6 & 39.4 \\
\hline Total Auxiliary Power $[5]$ (MW) & & 5.4 & 12 \\
\hline Total AC Power (MW), Incl. Aux. & & 121 & 193 \\
\hline Rf System Efficiency (\%), Incl. Aux. & & 28.2 & 37.0 \\
\hline Average Beam Power (MW), Excl. Injected & $\vdots$ & 9.3 & 18.6 \\
\hline AC-to-Beam Efficiency (\%) & & 7.7 & 9.4 \\
\hline
\end{tabular}

[1] Includes overhead for BNS damping, feedback, and stations off for repair.

[2] Active length $=\mathrm{F}_{O H}\left(\mathrm{E}_{0}-20 \mathrm{GeV}\right) /$ (Loaded Gradient).

[3] Assumes $3 \%$ of klystrons and modulators are off (repair margin) or running off beam time (on standby).

[4] Given by simulated efficiency less 5 percentage points for $500-\mathrm{GeV}$ design; equal to simulated efficiency for 1-TeV design.

[5] Includes klystron cathode heater power, thyratron heater and reservoir power, and power for modulators on standby (0.5\%). 
to measure the growth of the dark current generated by $\mathrm{rf}$ field emission in the accelerator.

The NLCTA's $160-\mathrm{kV}$ electron gun has been commissioned. The 70-MeV, X-band injector module and two of the three $130-\mathrm{MeV}$, X-band linac modules of the NLCTA are expected to become operational in 1996, with each module powered by a single 50-MW klystron. The first accelerator physics experiments are planned for 1996-1997. The last linac rf module (including the fifth and sixth 1.8-m-long X-band sections) is planned to be installed in 1997. It is expected that an upgrade from 50-MW klystrons to 75-MW klystrons will occur gradually as the new higher-power tubes become available through the klystron development program.

\subsubsection{Klystrons}
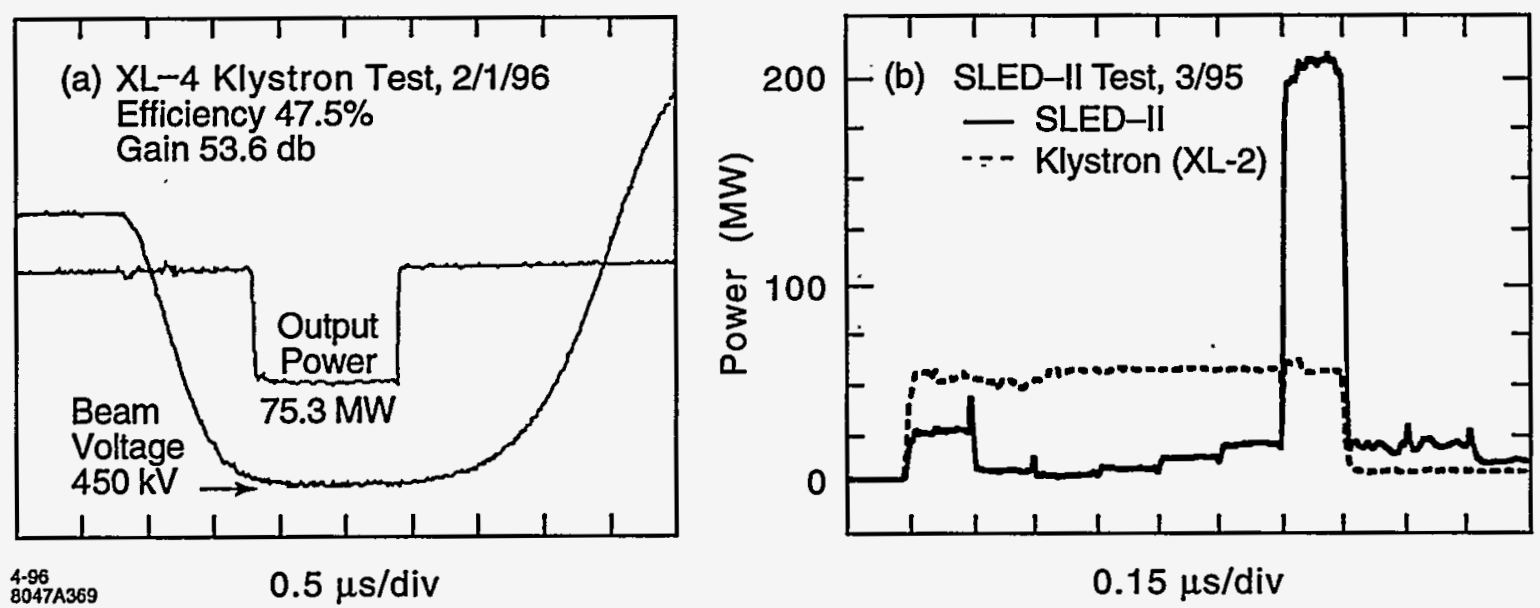

Figure 3.17: High-power tests of (a) an X-band klystron and (b) a SLED-II pulse compressor.

Obtaining the X-band peak power for the NLC has required the development of klystrons capable of delivering peak power significantly greater than previously achieved by commercially available X-band sources. Both the peak power and the pulse length required for the NLC design and upgrade have already been achieved by solenoid-focused X-band klystrons at SLAC. Four prototypes now produce 50-MW pulses, over 1.5 microseconds long, with performance characteristics that are correctly modeled by computer codes. The most recent prototype produces $75-\mathrm{MW}$ pulses, one microsecond long, and exhibits electronic efficiency of $48 \%$ (Fig. 3.17). These klystrons will be used to power the NLC Test Accelerator.

The solenoid which focuses the electron beam in the prototype klystrons has a weight of $750 \mathrm{~kg}$ and a power consumption of $20 \mathrm{~kW}$. Currently nearing completion is the first prototype of a $50-\mathrm{MW}$ klystron which is focused instead by a periodic permanent magnet (PPM) array of samarium-cobalt ring magnets weighing about $9 \mathrm{~kg}$. It is this klystron, which operates at a higher voltage and lower beam current for compatibility with PPM 
focusing, which is slated as the prototype for the NLC. Based on computer projections, the tube, designated X5011, is expected to operate at about $57 \%$ efficiency with $50 \mathrm{MW}$ of peak output power. This first, high-power PPM klystron, which is shown in Fig. 3.18, was fabricated and tested as a beam tester (a klystron without rf cavities) to validate the beam focusing. Beam transmission in excess of $99.9 \%$ was achieved without the magnet trimming which is customarily required for traveling-wave tubes which are PPM focused. The klystron prototype with rf cavities has been fabricated and is being readied for beam and $\mathrm{rf}$ tests.

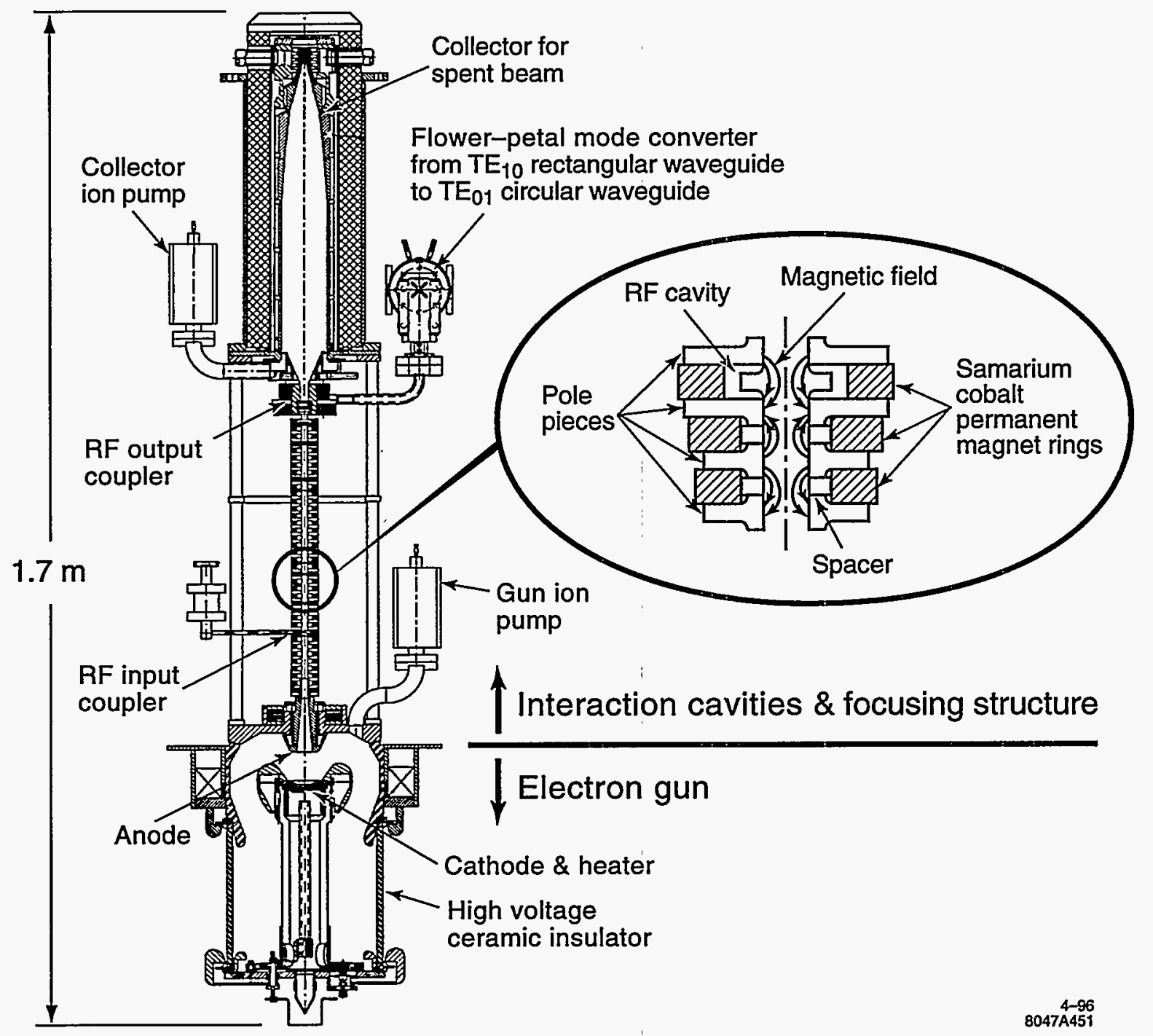

Figure 3.18: Preliminary layout of the X5011 PPM klystron, with water jacket removed. The insert shows one representative buncher cavity, to illustrate the use of oversize permanent magnets. 


\subsubsection{Modulators}

Each high-power rf station consists of a pair of PPM-focused klystrons (50 or $75 \mathrm{MW}$ ) and a pulsed-DC energy delivery system (modulator) that is tightly integrated in design with the electron guns of the klystrons. The pulsed-DC energy delivery system includes a singlethyratron switch, a Blumlein pulse-forming network (PFN), a high-efficiency power supply for charging the PFN's capacitance, and a pulse transformer. Using a Blumlein PFN allows for a relatively low transformer turns ratio (7:1), which yields a reasonably fast rise time $(0.3 \mu \mathrm{s})$, and hence, an improved efficiency.

The technology for standard line-type high power pulse modulators is very mature. The SLAC linac has been in operation for about 30 years using about 240 such modulators to drive the high-power klystrons. The efficiency of these modulators for converting wall-plug power into klystron beam power in the flat-top portion of the high voltage output pulse is about $60 \%$. Similar modulators have been constructed for the NLC Test Accelerator, and are currently under test. Parameter values for these modulators are listed under the "NLCTA Achieved" column in Table 3.10. The net efficiency for the NLCTA modulator is $58 \%$.

A key consideration for a modulator in a linear collider application is efficiency. A $1 \%$ relative decrease in modulator efficiency would result in an increase by between one and two megawatts in the $\mathrm{AC}$ line power required for the NLC rf system. It is clear that efficiencies for the SLC and NLCTA modulators are too low for consideration for the NLC.

An important component in determining the modulator efficiency is the pulse transformer. The pulse forming network (PFN) delivers a more-or-less rectangular pulse to the primary of the pulse transformer when the thyratron switch is triggered. The voltage pulse delivered to the klystron, however, contains a considerable amount of wasted energy in the rise and fall-time portions of transformer output pulse. In practical pulse transformer designs, it is observed that the rise time (and fall time) is determined in large measure by the transformer turns ratio: a high turns ratio implies a longer rise time, and vice versa. For the NLCTA modulator, a pulse transformer with a turns ratio of $21: 1$ is required to step up a $48-\mathrm{kV}$ PFN voltage to the $500 \mathrm{kV}$ required by the NLC klystron. This ratio can be reduced to about 14:1 by increasing the PFN charging voltage from 48 to $72 \mathrm{kV}$. This, of course, requires a thyratron with a higher voltage hold-off capability. A further reduction in transformer ratio to 7:1 can be achieved by using a voltage-doubling, or Blumlein, PFN configuration. Simulations show that a pulse-shape efficiency of about $80 \%$ can be achieved for this turns ratio. Combining this with an estimated efficiency of $93 \%$ for the DC power supply which charges the PFN, and assuming an additional $3 \%$ is lost in the voltage drop across the thyratron and other series resistances, the net efficiency for such a modulator design is estimated to be about $72 \%$.

The R\&D task for the NLC modulator group is to show that such an efficiency can be obtained in practice in a prototype modulator. Several possibilities are under consideration for the Blumlein PFN using both lumped capacitors and smooth transmission lines. Appropriate $R \& D$ is underway to design and construct prototype PFN configurations. Techniques for improving pulse transformer efficiency are also under investigation. 


\subsubsection{RF Pulse Compression and Power Transmission}

The 1.2- $\mu$ s-long X-band klystron pulses will be compressed by a factor of five-to $0.24 \mu \mathrm{s}-$ using the SLED-II rf pulse compression technique [6]. SLED-II is a modification of the SLED [7] compressor is used to increase the rf power into the $60-\mathrm{GeV}$ SLAC linac by a factor of about 3.

A SLED-II pulse compressor works by storing microwave energy in a pair of high- $Q$ resonant delay lines for most of the duration of the klystron pulse. The round-trip transit time of the $\mathrm{rf}$ in the delay lines determines the duration of the compressed pulse. Developmental SLED-II systems have been tested at SLAC with high-power X-band klystrons to validate the design and its components $[8,9,10]$. The peak power and efficiency needed for the NLC Test Accelerator have been demonstrated in a prototype system (Fig. 3.17). Three SLED-II systems are being manufactured for the NLCTA. Further validation of the SLED-II design at the higher power levels and efficiencies needed for the NLC will be performed in upgrades to the NLCTA. The rf pulse compression systems in the NLC and NLCTA are designed to accommodate future upgrades in which the peak rf power is nearly tripled, by replacing each 50-MW klystron with a pair of 75-MW klystrons.

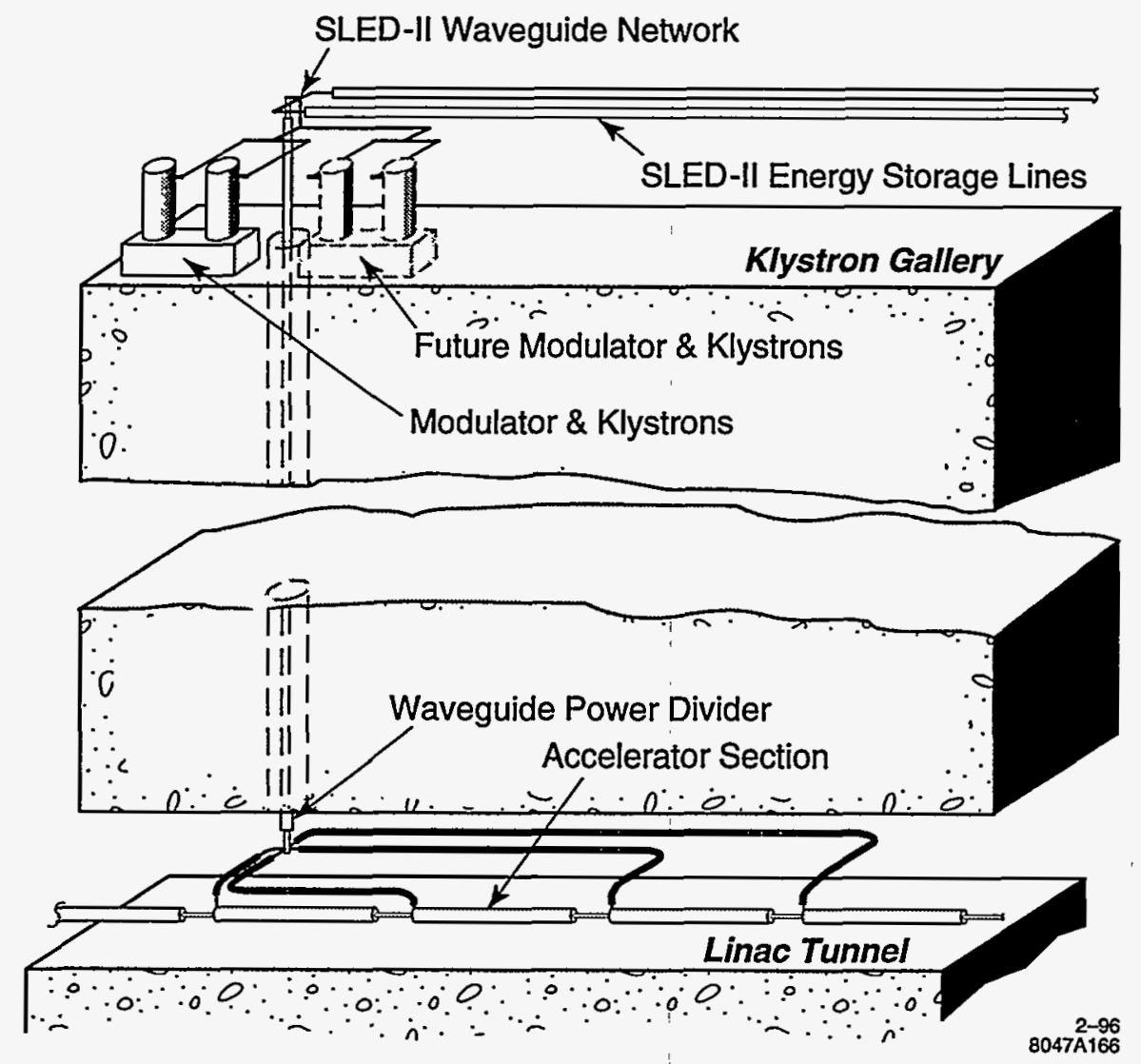

Figure 3.19: Physical layout of the waveguide network for rf pulse compression and power transmission. 
Figure 3.19 illustrates the physical layout of the waveguide network for rf pulse compression and power transmission to four 1.8-m-long linac sections in a two-tunnel (SLAC-type) configuration. The waveguide layout is sufficiently flexible to absorb the absolute motion of the accelerator sections due to their mechanical movers $( \pm 1 \mathrm{~mm}$ ) and the relative motion between the klystron gallery and the accelerator tunnel due to settling $( \pm 10 \mathrm{~mm})$. The SLED-II waveguide networks are located in the low-radiation environment of the klystron gallery so that the rf pulse-compression system may be modified, station by station, if necessary for upgrade to higher beam energies, simultaneously with colliding beam operations.

Since the round-trip delay time in the 4.75-in-diameter waveguide (at the group velocity, $0.964 c$ ) must equal the rf pulse duration $(0.24 \mu \mathrm{s})$, the physical length of the SLED-II delay lines is $35 \mathrm{~m}$. The physical layout can accommodate this delay-line length by spatially overlapping the delay lines from adjacent rf stations. Since the total length of linac fed by each rf station is approximately eight meters, each pair of delay lines will overlap parts of five other pairs of delay lines.

\subsubsection{Accelerator Structure}

The design of the X-band accelerator structures for the NLC is based on theoretical and experimental experience gained by modeling and building accelerator structures for the NLCTA and operating them at high gradients.

One of the main challenges is to suppress the deflecting modes that will otherwise cause severe multibunch emittance growth in the NLC linacs. Suppression of the transverse wakefield will be achieved through a combination of precision alignment and by detuning and damping higher-order modes. Another challenge in the design of the accelerator structures for the NLC is suppressing field emission at the high-surface field gradients encountered in these structures. This suppression, so far, has been achieved through machining, processing, and handling techniques that minimize surface roughness and eliminate contamination of the high-gradient surfaces. Other, additional methods may be adopted later.

The rf accelerator structure is designed to be very nearly a constant-gradient travelingwave structure. The design of the structure has been optimized to suppress the wakefield seen by trailing bunches. This has been accomplished by tailoring the cell-to-cell frequency distribution of the dominant deflecting mode to yield an initial Gaussian-like decay of the wakefield amplitude. On a longer time scale, the higher-order beam-induced modes of the structure will be damped by vacuum manifolds to which each cell of the structure is coupled. This structure is designated by the acronym DDS (damped detuned structure). The damping manifolds run parallel to the beam channel and are terminated into matched loads. (The slots that couple the cells to the manifolds are cut off to the fundamental accelerating mode.) This damping scheme will reduce the typical quality $(Q)$ factors of the deflecting modes to about 1000. The first prototype 1.8-m-long X-band accelerator section, which was detuned but not damped, was high-power tested up to a gradient of $67 \mathrm{MV} / \mathrm{m}$. The effect of the detuning in that first prototype section was demonstrated experimentally by using positron 
and electron bunches from the SLC damping rings as probe and witness beams, respectively. Another prototype 1.8-m section that is both damped and detuned is being manufactured and will be used for a similar test before it is installed in the NLCTA.

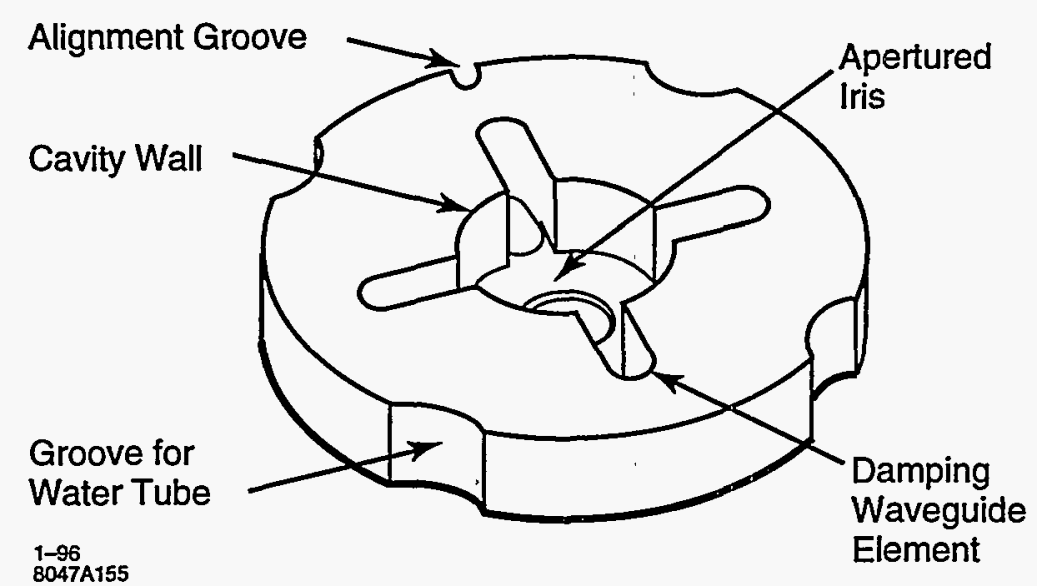

Figure 3.20: Basic cell design.

The basic features of the cell are shown in Fig. 3.20. The central portion is the conventional "cup" consisting of a cylindrical cavity wall and an apertured iris which, when stacked in a row with other cells, forms the disk-loaded waveguide accelerator structure. The diameter of the cavity wall, the thickness of the iris; and the diameter of its aperture vary progressively from cell to cell to "detune" the beam-deflecting dipole modes and suppress cumulative build-up of wakefields, while maintaining the quasi-constant-gradient characteristics of the fundamental accelerating mode. The central cavity is slot-coupled to four outer rectangular holes. When the cells are stacked, the holes form four waveguides which run parallel to the axis of the structure. When terminated in matched loads, the four waveguides become "damping manifolds." The slots (which are cut off for the fundamental accelerating mode) couple power from beam-excited dipole modes into these damping manifolds, lowering the $Q$ and suppressing long-range wakefield build-up. Microwave signals from the damping manifolds can be used to monitor the alignment of the structure with respect to the beam.

For a single detuned accelerator section, the fractional spacing for the fundamental dipole-mode frequencies (near the center of the gaussian distribution of frequencies) is about $3 \times 10^{-4}$. Machining precisions for conventional machining and diamond-point machining (obtained at KEK), and alignment tolerances of stacks of cells, are given in Table 3.11. Since the cell radius is about a centimeter, diamond-point machining should produce a random frequency error, for the fundamental dipole modes, somewhat less than the spacing between the frequencies. The systematic error should be significantly less than this.

Misalignment tolerances, on scales ranging from a few cells within a structure to several structures, were discussed in the preceding section. It was found that the tightest tolerances occurred for the alignment of groups of about 20-40 cells. Thus, great care must be taken in brazing together the subsections of complete structures after their initial assembly from 
individual cups.

The goal is an accelerator section 205 cells long (plus the input coupler) in which the axes of the individual cavities lie on a straight line to within $15 \mu \mathrm{m}$ (rms). To avoid a cumulative tolerance build-up which exceeds this goal, many other cell dimensions have to be held to tolerances in the 2 to $3 \mu \mathrm{m}$ range. This applies particularly to the diameter and coaxiality of the outer surface of the cell, since this has to be the reference surface used in stacking the cells prior to bonding. It also applies to the perpendicularity, flatness and parallelism of the cell faces. Errors here will cause "bookshelving" of the cells in the cell stack [11]. Equally important is the coaxiality of the iris aperture with respect to the cavity outer wall. These tight tolerances on cavity dimensions (including the thickness of the iris and the radius of its edge) are also necessary to achieve the design fundamental and dipole-mode frequency characteristics, because provision for tuning after assembly has been eliminated. Flatness of cell faces is also essential to achieve diffusion bonding (discussed below) over relatively large surface areas with a very low expected failure rate.

Table 3.11: Cell machining and assembly tolerances achieved (in microns).

\begin{tabular}{|c|c|c|c|}
\hline & $\begin{array}{c}\text { Detuned } \\
\text { 1.8-m } \\
\text { Section \#1 }\end{array}$ & $\begin{array}{c}\text { Detuned } \\
0.9-\mathrm{m} \\
\text { Sections \#1\&2 }\end{array}$ & $\begin{array}{c}\text { Detuned 1.8-m } \\
\text { Section \#2 } \\
\text { and DDS \#1 }\end{array}$ \\
\hline Machining Technique & Conventional & Conventional & Diamond-point \\
\hline Alignment Technique & Nesting & Vee-Block & Vee-Block \\
\hline Diameters & \pm 7 & \pm 7 & \pm 2 \\
\hline Concentricity & 10 & 10 & 1 \\
\hline Thickness & \pm 7 & \pm 7 & \pm 2 \\
\hline Parallelism & 10 & 10 & 0.5 \\
\hline Flatness & 10 & 10 & 0.5 \\
\hline Surface Finish & 0.4 & 0.4 & 0.05 \\
\hline \multicolumn{4}{|c|}{ Cell-to-cell Alignment of Outer Cylindrical Surfaces: } \\
\hline (a) Expected & 22 & 10 & 3 \\
\hline \multicolumn{4}{|c|}{ (b) Measured after diffusion bonding or brazing } \\
\hline & 10 & 6 & 4 \\
\hline
\end{tabular}

Single-crystal diamond-point machining is to be used on all surfaces of each cavity, the cell faces and outer periphery. The hardness, high thermal conductivity, and low thermal 
expansion of diamond result in a superior tool which, when used in a vibration-free lathe, yield mirror-like surfaces on copper which have a roughness of $0.1 \mu \mathrm{m}$ or less. This finish is necessary to obtain good diffusion bonding, high $Q$ factors, low dark current and high power-handling capability. The sharpness, mechanical stability, and minimal wear of the tool allow the dimensional tolerances to be met.

Table 3.11 illustrates what tolerances on dimensions and alignment have been achieved to date at SLAC, and what can reasonably be expected with the best technology available today. It can be seen that the most significant advances have been made by resorting to diamond-point machining and by using precision granite vee-blocks to align the stacks of cells prior to brazing or diffusion bonding. Cells designed to nest into each other have to fit loosely enough to permit assembly without galling, and this can result in unacceptable misalignment. Our present experience is that stacks of 38 cells can have a bow of a few tens of microns after diffusion bonding. This can be reduced to a few microns by setting the stack horizontally in cradles on a granite block and applying bending forces while monitoring movement with a precision coordinate measuring machine.

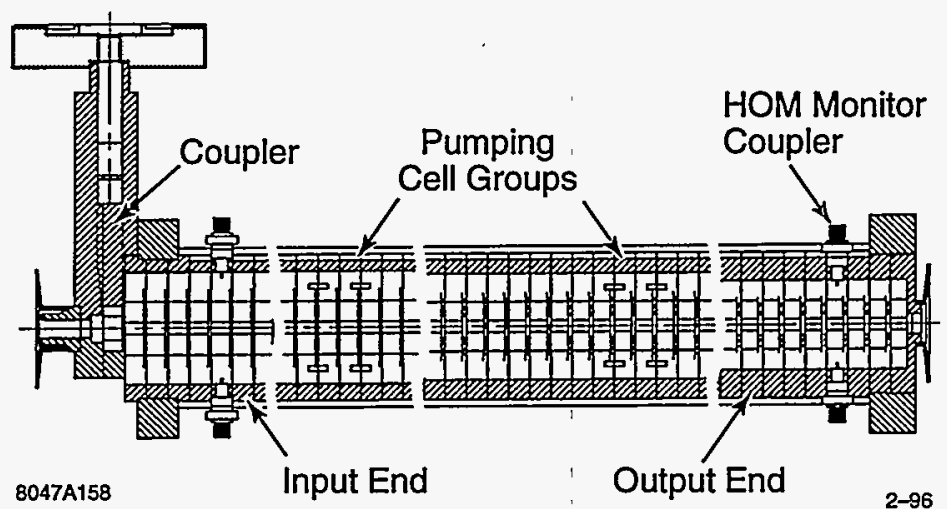

Figure 3.21: Longitudinal cross section of a 1.8-m long X-band accelerator section.

In addition to direct pumping along the beam-interaction region, the structure is pumped in parallel by the four damping waveguides. Two groups of four special pumping cells will be inserted into the structure, at points one-fourth of the section length from each end. A longitudinal cross section of the accelerator section is shown in Fig. 3.21. Each pumping cell group is connected to two pumps via short transverse manifolds.

Figure 3.21 shows no output coupler. The intention is to provide internal matched terminations for both the fundamental accelerating mode and the wakefield modes by depositing lossy coatings on the walls of the cavities and the damping manifolds in the last four cells. A coaxial loop loosely coupled to the last cavity will be used to monitor the fundamental-mode power. This signal will pass through a coaxial rf window in the cell wall, and will be used by the klystron phasing system. However, if satisfactory internal terminations cannot be developed, external loads and couplers will have to be used. 


\section{Mass Production of Accelerator Cells}

The two main linacs will contain about 1.8 million cells, which have to be produced in about three years. Assuming that a specialized plant set up to fabricate the cells operates 50 weeks per year at two shifts per day, then, at $80 \%$ efficiency, a production rate of about two cells per minute is required. A close estimate of the time taken to machine a single cell cannot be made without reference to specific machines and machining techniques. (See, for example, the study reports prepared for the CERN CLIC cells $[12,13]$.) However, very rough estimates may be drawn from those reports, noting that the surface area of the X-band DDS cell is about three times that of the CLIC cell. All the features of the DDS cell shown in Fig. 3.20 could be machined to conventional tolerances on the end of bar stock (leaving excess material on the critical surfaces to be diamond-point machined in subsequent operations), using a multipurpose $\mathrm{CNC}$ turning and milling machine. The cell could thus be cut from the bar using a parting-off tool, stress-relieved and delivered to a diamond-point lathe for finishing in two steps. In the first step, the cell could be gripped on the outside by a precision chuck while the back face is diamond turned. In the second step, the back face could be held in a vacuum chuck while the front face and cavity surfaces are diamond finished. Based on estimated operation times, the plant will need a minimum of 22 conventional CNC turning and milling machines and 8 diamond-point lathes, with appropriate numbers of all supporting equipment (automatic handling and transfer machines, stress-relieving ovens, cleaning, QC, and packing stations) to maintain the required throughput.

\section{High-power Tests and Dark Current Studies}

The theoretical and experimental studies on the behavior of copper accelerator structures under extremely high-rf fields have been carried out at SLAC for several years [14, 15]. We have studied in considerable detail the problems of $\mathrm{rf}$ breakdown and dark current generated by field emission at high gradient. The dark current may absorb rf energy, get captured, and produce undesirable steering effects and detrimental X-ray radiation. Many experiments have been done to measure the amplitude and energy spectrum of the dark current and to study phenomena related to rf breakdown such as outgassing, radiation, heating, etc. We have concluded that the dark current can be minimized by improving surface finish and cleanliness, and by rf processing.

High-power tests with an electron beam have demonstrated that the 1.8-m section is properly tuned to $11.424 \mathrm{GHz}$ and can accelerate a beam at a gradient of more than 67 $\mathrm{MV} / \mathrm{m}$. Dark current from this structure was measured in a Faraday cup as a function of average accelerating field (Fig. 1.8), and found to be negligible for an accelerating gradient of $50 \mathrm{MV} / \mathrm{m}$ was found to be negligible. At $85 \mathrm{MV} / \mathrm{m}$. The energy spectrum of the dark current was measured and found to be sufficiently low that the quadrupoles will overfocus and sweep the field-emission current away from the primary, high-energy beam.

Experiments performed with S-band accelerators at KEK $[16,17]$ have shown that the dark current produced by field emission from the accelerator disks is reduced by an order of 
magnitude when stringent precautions are taken to exclude dust during fabrication, assembly, processing and testing. These experiments indicate that the NLC accelerator cells should be cleaned in ultra-pure, dust-free chemical solutions and rinses, assembled and bonded under clean-room conditions, and perhaps given a final rinse in ultra-pure water before vacuum bakeout and installation. We expect application of these procedures to our X-band structures to yield equally significant improvements.

\subsubsection{RF Drive and Phasing Systems}

To achieve a highly monoenergetic multibunch beam pulse for the final focus, the beam loading induced by the bunch train must be compensated. The initial transient can be eliminated by pre-loading the sections by shaping the input rf pulse using an approximately linear rise of the field amplitude for one filling time of the structure. In this way, the first electron bunch will see a filled rf structure that appears to be in the steady state. This requires phase-agile control of the rf before it is amplified by the klystron.

The design of the rf drive and phasing systems for the NLC will be based heavily on experience gained from existing systems developed for the SLAC linac and its $60-\mathrm{GeV}$ upgrade for the SLC, with further extensions based on the design of the NLCTA. The challenges to be met for the NLC arise from its greater length, number of components that must be controlled, required tolerances, maintainability, and reliability. The requirements imposed on the rf drive and phasing systems are summarized below:

- The drive system must provide stable, adjustable, and reliable input power to 4528 klystrons (approximately 1-kW pulsed peak power per tube). The layout of the system must be such that individual subsystem failures do not cause the beam energy to decrease to less than $85 \%$ of its operating level. This requirement is dictated by potential collimator and other damage caused by off-energy beams.

- The amplitude of the drive power must be adjustable so as to individually saturate the high-power klystrons.

- The phase of the drive power must be adjustable in several ways:

- Slowly, to take care of phase drifts and drifts caused by length changes in equipment and terrain. Included here are the couplers, coax lines, intermediate amplifiers, high-power klystrons, SLED-II, waveguide components, and the changes in time at which the bunch trains are injected into the X-band linacs by the S-band bunch compressors.

- Quickly (within each 1.2- $\mu$ s klystron rf pulse), to create the proper resultant amplitude and phase profiles necessary to produce the desired fields in the accelerator structures and to place the bunches at the desired phase positions with respect to the X-band waves. These positions are dependent on bunch number and charge, 
which can change from pulse to pulse, and are dictated by BNS phasing, single bunch and multibunch beam loading, and other considerations.

- The phasing system design must also include initial adjustments (upon installation) of the waveguide runs which feed the four accelerator sections driven by one pair of klystrons in a station.

The phases of the high-power rf fields which act on bunch trains should be adjusted with an accuracy of about $0.5^{\circ}$ at $11.424 \mathrm{GHz}$. A schematic layout of the rf drive and phasing system for the NLC is shown in Fig. 3.22.

There are various schemes for providing the approximately $1 \mathrm{~kW}$ of pulsed $\mathrm{rf}$ needed to drive each high-power klystron. It is useful to have individual drivers because the various phase manipulations involved in the operation of the rf compressors are best done at the milliwatt level. Power-combining of the two klystrons on the single modulator is done by phase modulation of the two klystron inputs by equal amounts in opposite directions. This has the effect of sending the desired vectorial sum into the rf pulse compressor and dumping the vectorial difference into $a$ load.

There exists extensive Traveling Wave Tube (TWT) technology at this frequency and power level as a product of military applications. The available TWTs are reliable, but fairly complex to manufacture and operate. They have already been extensively cost-optimized, and are still very expensive.

Alternatively, a small X-band driver klystron of fairly narrow bandwidth and modest gain could be designed. In construction, it would be relatively simple compared to a TWT. It is possible that after the initial engineering models are tested, a modest program of redesign for automatic manufacturing could significantly reduce the unit cost.

\subsubsection{Upgrade to $1 \mathrm{TeV}$}

An essential goal of the NLC is that it be possible to increase the center-of-mass energy of the collider to a $\mathrm{TeV}$ or greater in an unobstrusive and adiabatic fashion with improvements that can be expected in the rf system of the main accelerators. The physical layout that we have chosen for the main linacs and their power sources (Fig. 3.19) provides access that allows upgrade of the rf system even as the collider is operating; for example, by taking one or a few stations off-line at a time. Our choice of X-band rf technology has been made to assure that accelerating gradients that approach $100 \mathrm{MV} / \mathrm{m}$ can ultimately be used without electrical breakdown or generation of unacceptable levels of dark current, and there are a number of possible advances in X-band technology that would lead to the $\mathrm{TeV}$ energy. We have based our design on what we believe to be improvements that are near-term and can reasonably be expected to occur in klystron and pulse compression technologies.

Tests with a prototype X-band klystron have already proven that output powers of 75 MW can be achieved, and a likely path to the TeV energy goal is taken by replacing and doubling the number of klystrons used in the main acelerator (dashed lines in Fig. 3.16). 


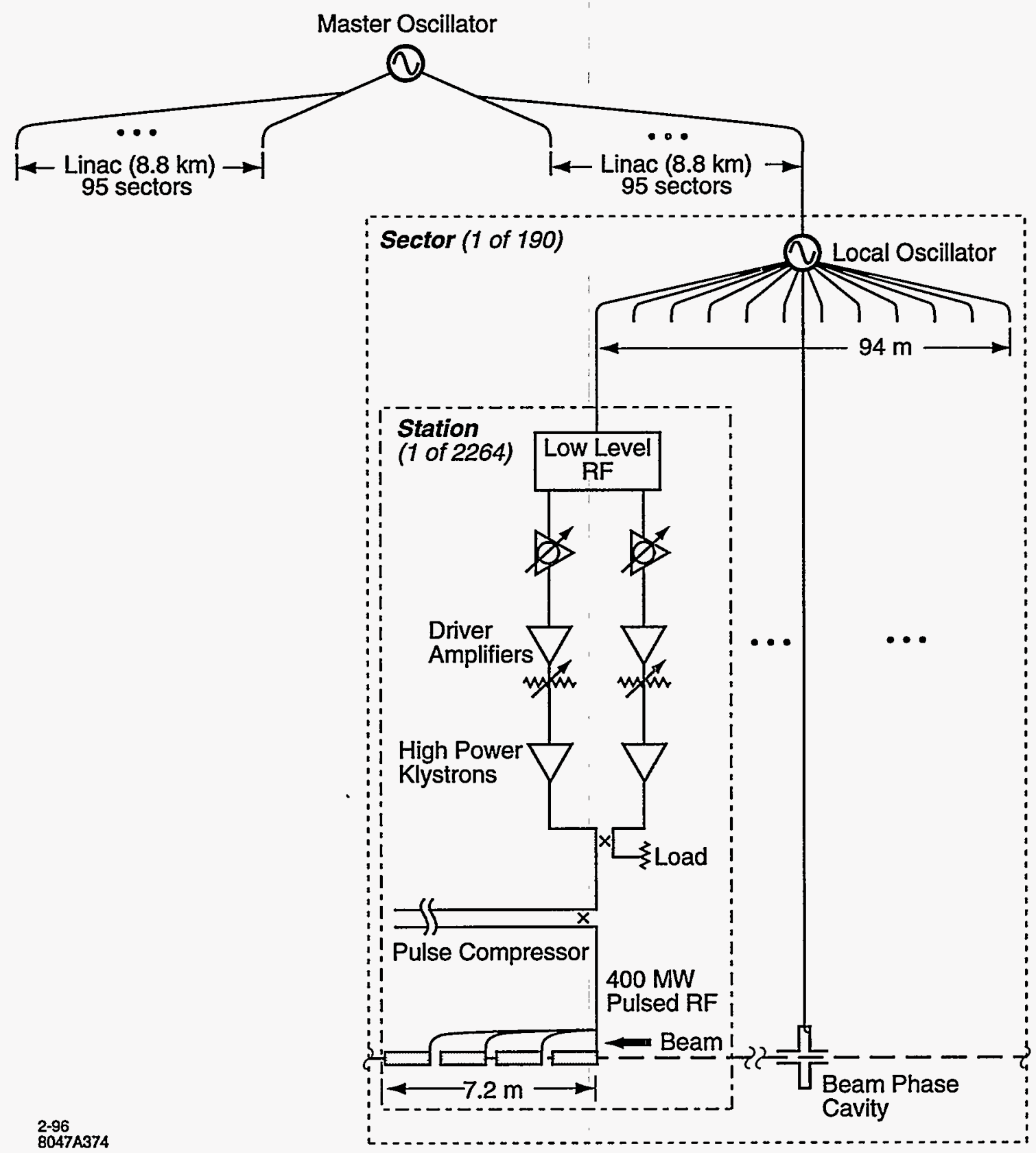

Figure 3.22: Schematic of rf drive system. The master oscillator provides phase-stable rf to local oscillators in each sector over a fiber distribution system. Local sources in each sector provide $11.424 \mathrm{GHz}$ to each rf station in the sector using coaxial lines. A beam-phase cavity uses the beam to determine the rf phase for optimum operation. Power levels are low (several milliwatts) until the final drive for the high-power klystrons. 
Although the klystron power rating necessary for the full $1 \mathrm{TeV}$ has been achieved, the power conversion efficiency of the present tubes (approximately $48 \%$ ) is not sufficiently high to maintain the total power consumed by the linac to an acceptable level (taken to be about $200 \mathrm{MW}$ ). Klystrons of the generation now being developed with PPM focusing are expected, according to computer modeling, to achieve conversion efficiencies of nearly $60 \%$.

There are additional improvements that we anticipate will occur in X-band technologies that will lead to still higher overall power efficiencies. The finite filling time of the energy storage technique used in the SLED-II pulse compressor wastes power and results in an intrinsic $20 \%$ loss that could be avoided with the development of new microwave switching techniques. Initial experimental investigations of an optically triggered silicon switch show promise, and effort is continuing to develop a usable device based on this idea [18, 19]. First tests of an alternate compression scheme, binary pulse compression (BPC), have also been carried out, and have proven that this technique, though more expensive than SLED-II, works well with intrinsic efficiency of $100 \%$. [20,21]. A nice feature of the BPC scheme is that it reuses nearly all of the hardware of the SLED-II systems, so that if conversion to $\mathrm{BPC}$ were necessary, then a station-by-station modification is mechanically reasonable.

Final tests of any of these upgrade paths remain to be done. It will be important to verify the power ratings and efficiencies of not only the power generating components, but also those of the entire system. This will be one of the primary goals of the experimental program of the NLCTA.

To obtain the full $1 \mathrm{TeV}$ energy and to maintain the linac power consumption below $200 \mathrm{MW}$, the klystron upgrade to $75 \mathrm{MW}$ and elimination of the intrinsic power loss of the SLED-II system must be accomplished. From our experience to date, we believe it is reasonable to expect the net efficiency of the rf system to be increased from the initial design value of about $30 \%$ to the $40 \%$ required for the upgrade. The total length of the main linacs must also be increased slightly, from 16.3 to $17.7 \mathrm{~km}$, but even if this additional length is not provided, the upgraded center-of-mass energy will still reach $925 \mathrm{GeV}$. This set of parameters is summarized in Table 3.10. Some of these potential improvements may, in fact, be ready in time to be included in the initial $500-\mathrm{GeV}$ machine. This would result in additional performance margin or cost reductions. The $500-\mathrm{GeV}$ parameters listed in Table 3.10 are conservatively based on experience with, and measurements on, prototypes which exist at the present time.

\subsubsection{Outlook}

The design of the rf system for the main linacs of the NLC is supported by existing and planned developmental prototypes, and by the NLCTA. Key NLC parameters such as the klystron power, acceleration gradient, and pulse-compression power gain have been exceeded in prototype systems. The next steps in the development program are completion of the NLCTA, the first damped and detuned structure, and the first PPM klystron prototype. The design of the NLC high-power rf system is mature and is now progressing toward detailed 
engineering considerations. Because of the magnitude of the project, special emphasis is being placed on designing for manufacturability and for overall system reliability. 


\subsection{Beam Delivery}

A schematic of the beam delivery and removal systems is shown in Fig. 3.3. These systems begin at the end of the linac and terminate at a post-IP beam dump. The total linac-to-IP length is $5.2 \mathrm{~km}$. They include a post-linac collimation system, an IP switch and big bend, a pre-final-focus diagnostic and skew-correction section, a final-focus system, and a post-IP beam line. The design of all of these systems has been strongly influenced by experience with similar systems at the SLC.

Figure 3.23 shows the lattice $\beta$ functions from the linac to the IP for the $1-\mathrm{TeV} \mathrm{cms}$ beam line. The first 100 meters contain a post linac diagnostic chicane, which is then followed by a $2.5-\mathrm{km}$ collimation system. The maximum $\beta$-function points in the collimation region correspond, for the most part, to the location of horizontal collimators and chromatic correction sextupoles. The horizontal $\beta$-function peak at $2.6 \mathrm{~km}$ marks the location of the IP switch. After that, up to the $3.0-\mathrm{km}$ marker, is a small $\beta$-function region that contains the big ( $10 \mathrm{mrad})$ bend, and following this, up to the $3.4-\mathrm{km}$ marker, is a small $\beta$-function region containing a pre-final-focus diagnostic and skew-correction region. In this region beam sizes in all phases and planes can be measured and the presence of coupling detected and corrected. The region from $3.4 \mathrm{~km}$ to $3.8 \mathrm{~km}$ contains the $\beta$ match into the final focus. Following the $\beta$ match, the first two peaks are the positions of the horizontal chromaticity compensation sextupoles. The last peak, in each plane, at the end of the beam line, is located at the position of the final-doublet elements.

We have studied beam delivery systems for center-of-mass energies from $350 \mathrm{GeV}$ to 1.5 $\mathrm{TeV}$, for a broad range of assumptions on beam and IP parameters, and have shown that it is possible to meet system specifications over this entire range. Within this energy span, the final-focus system design we have chosen requires two minor upgrades consisting of a transverse displacement of magnets by $20 \mathrm{~cm}$ or less and a change of the final doublet. The tunnel length allotted to collimation is adequate to collimate energy and the horizontal and vertical planes at both phases only one time at $1.5 \mathrm{TeV}$ cms energy. Since the centroid orbit of the collimation system differs only slightly from a straight line, it would be possible to allocate length at the end of the linac tunnel for a second IP phase collimation at this energy. It is sufficient to collimate the final doublet (FD) phase only one time.

\subsubsection{Collimation System}

The design of the post-linac collimation is strongly influenced by assumptions on incoming beam conditions. The number of particles needing to be collimated is difficult to predict precisely, because it depends on how well upstream systems have been tuned; we have relied on SLC experience for this estimate. Downstream, the number of particles that can be collimated in the final-focus system can be determined by edge-scattering and muon transport studies. The rough guideline that evolves from these considerations is that for each bunch train there may well be a few times $10^{10}$ particles per bunch train in the tails at the end of 

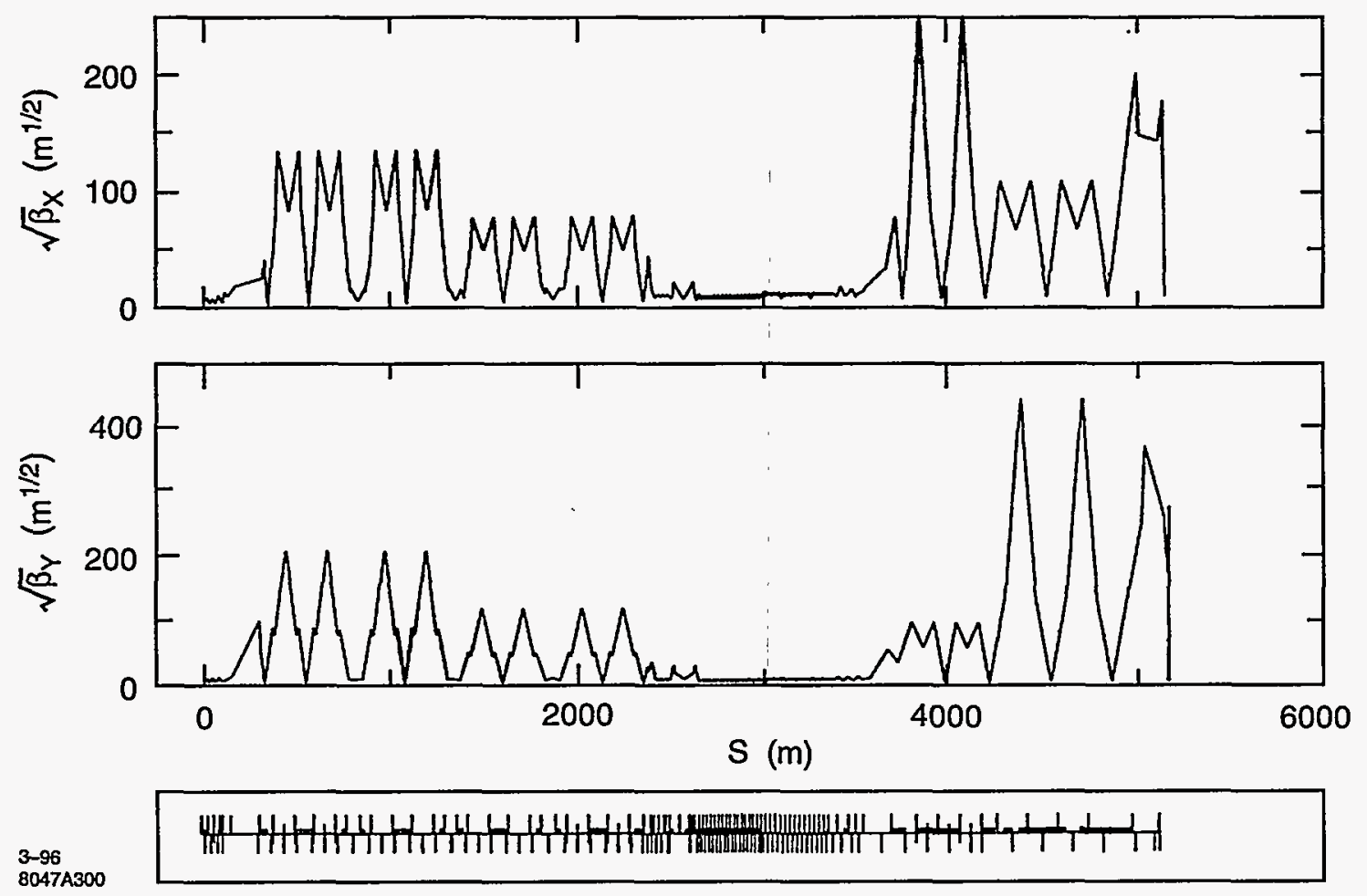

Figure 3.23: The $\beta$ functions from the linac to the IP for the $1-\mathrm{TeV}$ $\mathrm{cms}$ beam line.

the linac. This number needs to be reduced to a few times $10^{6}$ upon entry into the final-focus system.

Two types of collimation systems have been proposed: linear and nonlinear. In the former, beam enlargement at the collimators is achieved by traditional linear optics methods; in the latter strong sextupoles are used to blow up the beam. Since the sextupoles are exceedingly strong and system lengths are not reduced in the specific system proposals we have studied, we have opted to look in depth at a linear collimation scheme. It is not precluded that a nonlinear (or combination linear and nonlinear) system could be found that would be operationally superior and have a lower total cost. Our primary objective in the ZDR was to show that at least one collimation system exists that fulfills all functional requirements.

Since a small perturbation in upstream conditions could cause a complete bunch train of $10^{12}$ particles to be incident on the collimators of the collimation system, it is necessary in both the linear and nonlinear systems to rely on a primary collimator that is a spoiler, followed by a secondary collimator which is the absorber. The function of the spoilers is to increase the angular divergence of the beam, so that when the beam arrives at the absorber it has a much larger size ( $2.2 \mathrm{~mm}$ radius). The spoilers must be thermally rugged and very thin (1/4 radiation length). The best material we have found is a titanium alloy plated with titanium nitride for better electrical conductivity. The absorber, on the other hand, 


\section{Horizontal}

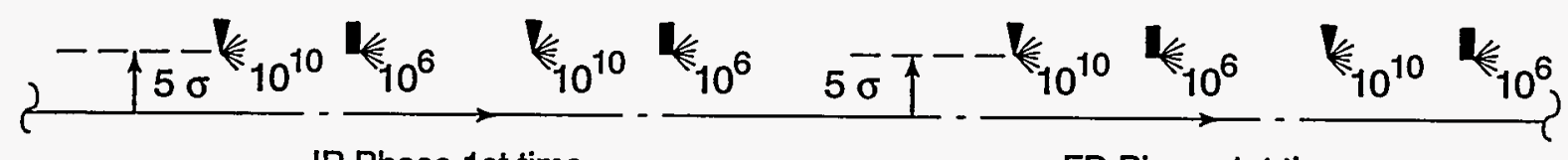

IP Phase 1st time

FD Phase 1st time

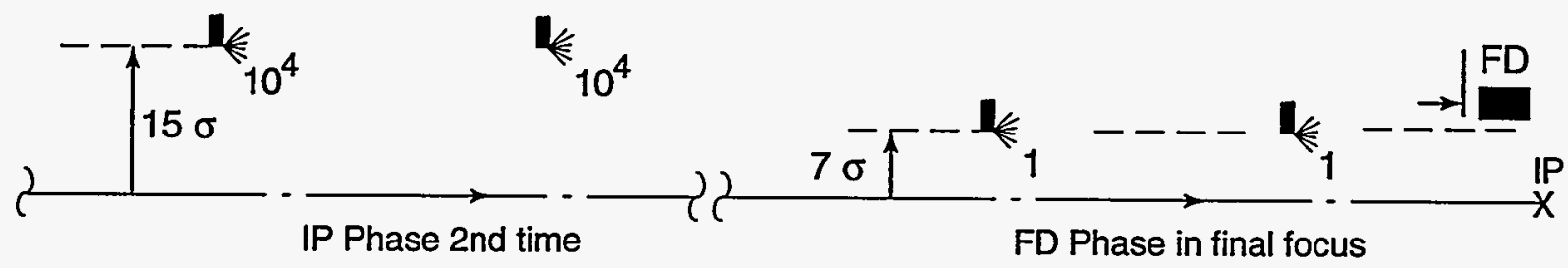

\section{Vertical}
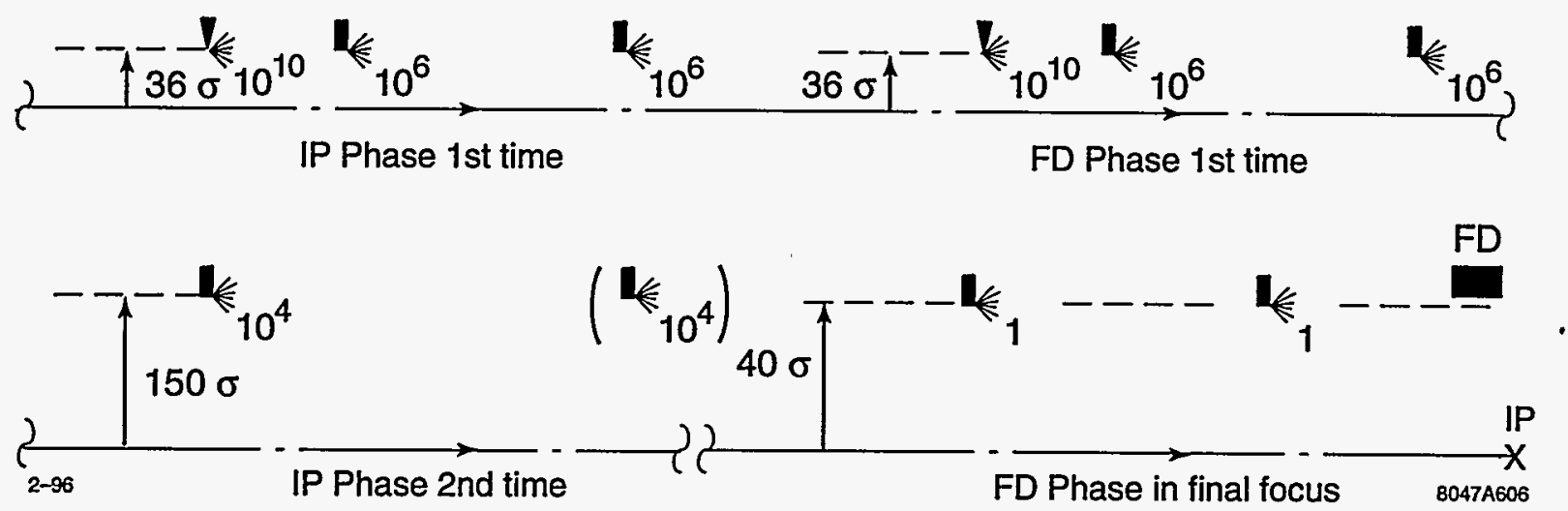

Figure 3.24: A schematic of the collimation system spoilers and absorbers. The apertures are shown as well as the estimated number of tail particles rescattered into the beam. 
must be able to routinely absorb and remove the full energy in the beam tail. For the 1 $\mathrm{TeV}$ cms parameters, $1 \%$ of the time-averaged beam power is $84 \mathrm{~kW}$. The preferred material for absorbers is copper. Figure 3.24 is a schematic of all spoilers and collimators in the beam delivery system. This figure shows the apertures and estimates for the number of tail particles rescattered into the beam.

The wake fields of the collimators can have a very deleterious effect on the beam core. To minimize the wakes, the beam pipe must be tapered before and after the collimator. A parallel-plane collimator will have a quadrupole wake even for an on-axis beam core, which can cause focusing of the core and alter the trajectory of particles in the tails. And beams that have been mis-steered close to the wall can experience very large wake-induced kicks. Because of the latter effect, the aperture of the second stage of IP-phase collimation must be quite large. This in turn places a requirement on the transmission quality of the beamline from the collimation system to the final-focus system, and on the dynamic aperture of the final-focus system. These have been verified with tracking studies.

Because of the large $\beta$-functions and strong focusing that arise when the beam is blown up with linear optics, there are important chromatic effects to compensate with sextupole pairs. And because it is necessary to collimate each transverse phase at least one time, there are very large $R_{12}$ and $R_{34}$ functions within the system. Large $R_{12}$ and $R_{34}$ functions lead to tight tolerances. Stability tolerances, that must be held between tunings of the waist knobs in the final-focus system, are looser than stability tolerances within the final-focus system. Though the particle backgrounds in the collimation system preclude the beambased stabilization methods contemplated for the final-focus system, beam changes can be monitored non-invasively in the skew correction system and aberrations arising from changes in the collimation system can be continuously compensated there.

Ground motion studies show that seismic ground motion in the beam delivery system has a negligible impact on beam collision offsets at the IP. As a result, vibration tolerances become tolerances between beam-line elements and the ground beneath them (FFTB quadrupoles mounted on movers have been shown to follow the ground $\pm 1 \mathrm{~nm}$ ), or tolerances on ground motion coming from cultural sources.

In the ZDR we have described the system specifications, discussed the relevant properties of materials, presented spoiler and edge-scattering distributions, clarified the relevant wake-field of tapered collimators and defined the optimum choice of collimator shape, quantified the impact of near-wall wakes for on-axis and mis-steered beam, designed lattices that implement the required functionality, calculated the position and strength tolerances of the magnetic elements, clarified the impact of ground motion, tracked the lattices to confirm their functionality, tracked spoiler distributions in the collimation system to determine power deposition, tracked edge-scattered distributions in the final-focus system to determine the probability of particle impacts on the final doublet, determined the extent of tail repopulation due to gas scattering, discussed potential operational problems, and addressed machine protection issues. To our knowledge all issues have been addressed with the satisfactory conclusion that it is possible to build and operate a collimation system for the proposed 
beam parameters from beam energies of $175 \mathrm{GeV}$ to $750 \mathrm{GeV}$, that will collimate the beam at the apertures required by the final-focus system. We believe that we have demonstrated an existence proof.

\subsubsection{IP Switch and Big Bend}

Following the collimation system are the IP switch, the big bend, and the skew correction and diagnostic section, as illustrated in Fig. 3.3. An NLC design with two IPs will require two IP switches and four big bends.

The IP switch follows the main linac and collimation section and allows slow switching between multiple IPs. It bends the beam a total of 1.5 mrad. Figure 3.25 shows the IP switch optics. The QS quadrupole is horizontally movable in order to switch between IPs. It is displaced by $\pm 3.25 \mathrm{~cm}( \pm 2.6 \mathrm{~cm})$ for the $500-\mathrm{GeV}$ per beam $(750-\mathrm{GeV}$ per beam) configuration.

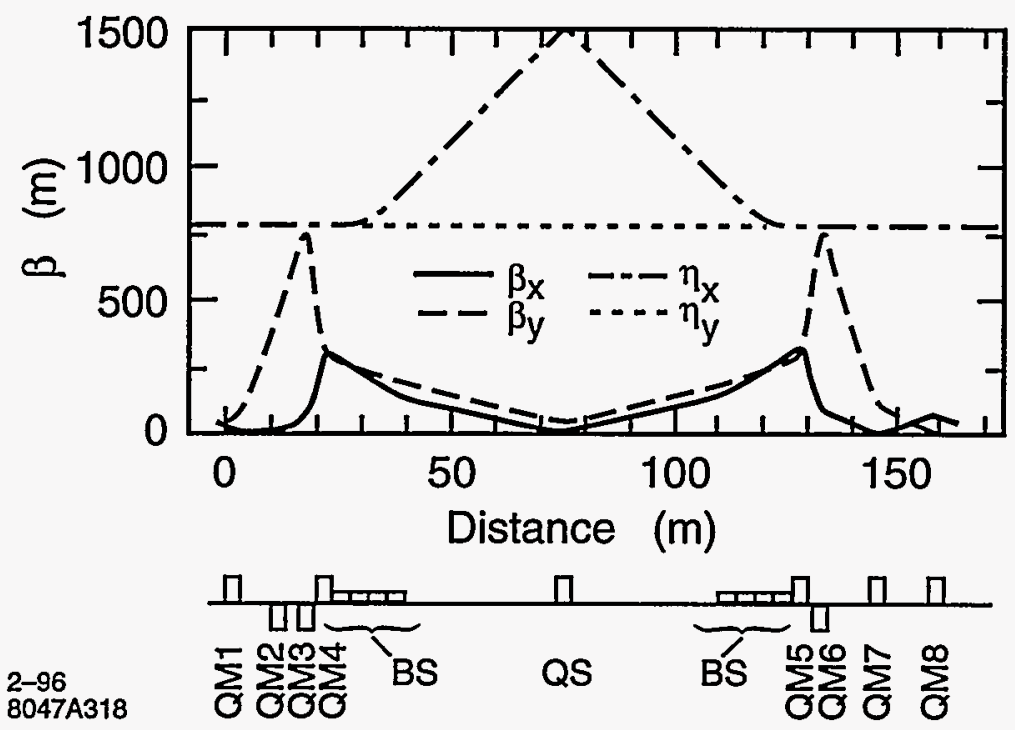

Figure 3.25: IP Switch Optics (500 GeV per beam).

The big bend is a low-angle arc after the main linac which provides detector muon protection, IP separation, and an IP crossing angle to facilitate extraction of the spent beams. The total bend angle (including 1.5-mrad IP switch angle) is $10 \mathrm{mrad}$ (20-mrad IP crossing angle) which provides approximately $40-\mathrm{m}$ spatial separation between the two IPs (approximately $700-\mathrm{m}$ transport to an approximately 1600 -m-long final focus). At 500-750 $\mathrm{GeV}$ per beam, the horizontal emittance growth due to SR sets lower limits on the system design length. The optical design of the big bend section was optimized for $500 \mathrm{GeV}$ per beam and 750-GeV per beam electrons (or positrons). The total emittance growth (in both IP switch and big bend) for the optimized design, due to chromatic filamentation and due to synchrotron radiation, is less than $2 \%$ vertically and $3 \%$ horizontally, for a beam energy of 
$500 \mathrm{GeV}$. The big bend rotates the spin orientation by about $550^{\circ}$ at $500 \mathrm{GeV}$. The relative depolarization is small: $0.06 \%$ for a $0.3 \%$ energy spread.

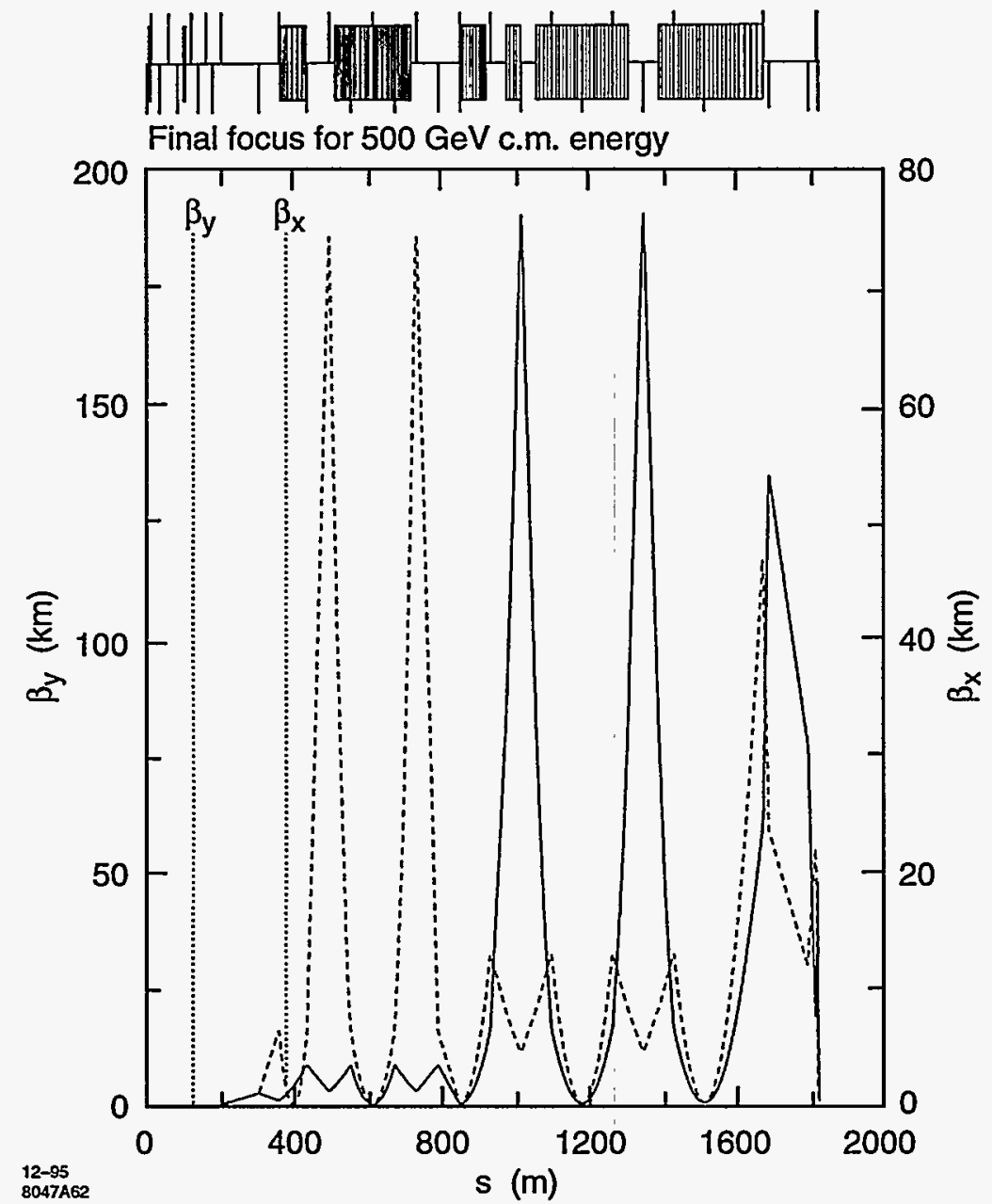

Figure 3.26: Horizontal and vertical beta functions for the $500-\mathrm{GeV}$ final focus.

\subsubsection{Final Focus}

The main purpose of the NLC final-focus system is to transport electron and positron beams of energy $180 \mathrm{GeV}$ to $750 \mathrm{GeV}$ from the end of the big bend to the IP, where the demagnified beams are collided with a typical spot size of 4-8 nm vertically and 200-300 nm horizontally. One must also remove the beam remnants cleanly in order to facilitate crucial post-IP diagnostics.

The basic layout of the final focus is very similar to that of the Final-Focus Test Beam (FFTB). The final focus is constructed from six functional modules. These are, in the order 
of their location: geometry-adjustment section (GAS), beta- and phase-matching section (BMS), horizontal chromatic correction section (CCX), beta-exchanger (BX), vertical chromatic correction section (CCY), and final transformer (FT). The total distance from the entrance of the GAS to the IP is about $1820 \mathrm{~m}$. A schematic of the magnet configuration is depicted in the top part of Figure 3.26. The figure also shows the beta functions corresponding to the largest IP beam divergences of the NLC parameter plane, for which the system was designed. This represents a worst case, since the effect of nonlinear aberrations, the aperture requirements, and the Oide effect (synchrotron radiation in the last quadrupoles) all become more severe for larger divergence. Located at the end of the final transformer is the final doublet, which focuses the beam to the design spot size at the IP. In the NLC, the final doublet is actually a quartet comprising four different quadrupoles: 2 conventional magnets, 1 superconducting and 1 permanent.

The chromaticity (energy-dependent focusing) of the final doublet is similar to that at the FFTB, and about 5 times larger than in the SLC. If the chromaticity were not corrected, the vertical IP spot size would increase by about a factor of 100 from the design value. The chromatic correction is accomplished in two separate beam-line sections, CCX and CCY, each of which accommodates a pair of sextupoles separated by an optical transform $(-I)$ that cancels geometric aberrations and second order dispersion. The momentum bandwidth of the final focus was optimized with three additional sextupoles and exceeds $\pm 0.6 \%$.

The NLC design calls for a 20-mrad crossing angle of the two beams at the IP, which requires two separate beam lines. Magnet apertures and focusing strengths in the two beam lines can be chosen independently, and are in fact quite different. The crossing angle, furthermore, requires rotation each beam prior to collision by $10 \mathrm{mrad}$ in the horizontal/longitudinal plane, by means of a 5-cm-long X-band crab cavity.

Placed about $150 \mathrm{~m}$ in front of the final doublet is a 70-m long soft bending section with a 12-G field, which serves to deflect the beam orbit at the final-doublet entrance by about 8 $\mathrm{mm}$ with respect to the hard photons radiated upstream, and, thereby, reduces the detector background substantially. A similar soft bend has proved invaluable at the SLC.

The final focus can operate in the entire cms energy range between $350 \mathrm{GeV}$ and $1.5 \mathrm{TeV}$. This flexibility is achieved with three slightly different geometries, illustrated in Fig. 3.27. A dedicated geometry-adjustment section (GAS) at the entrance to the final focus is used to adjust the incoming beam-orbit angle for the different geometries, such as to keep the IP position constant and the transverse magnet displacements small. Energy adjustability of the final doublet is provided by the superconducting quadrupole, whose field changes sign when the beam energy is raised from $250 \mathrm{GeV}$ to $500 \mathrm{GeV}$. Finally, the apertures of all magnets are consistent with a minimum vertical beam stay-clear of $55 \sigma_{y}$ at $500-\mathrm{GeV} \mathrm{cms}$ energy.

Higher-order optical aberrations, synchrotron radiation, residual uncorrected low-order aberrations, timing offsets and crab crossing errors all reduce the luminosity. A total luminosity loss of about $30 \%$ is included in the NLC design parameters. The low-order aberrations, such as dispersion, waist shift, skew coupling, etc., which are responsible for almost half of 

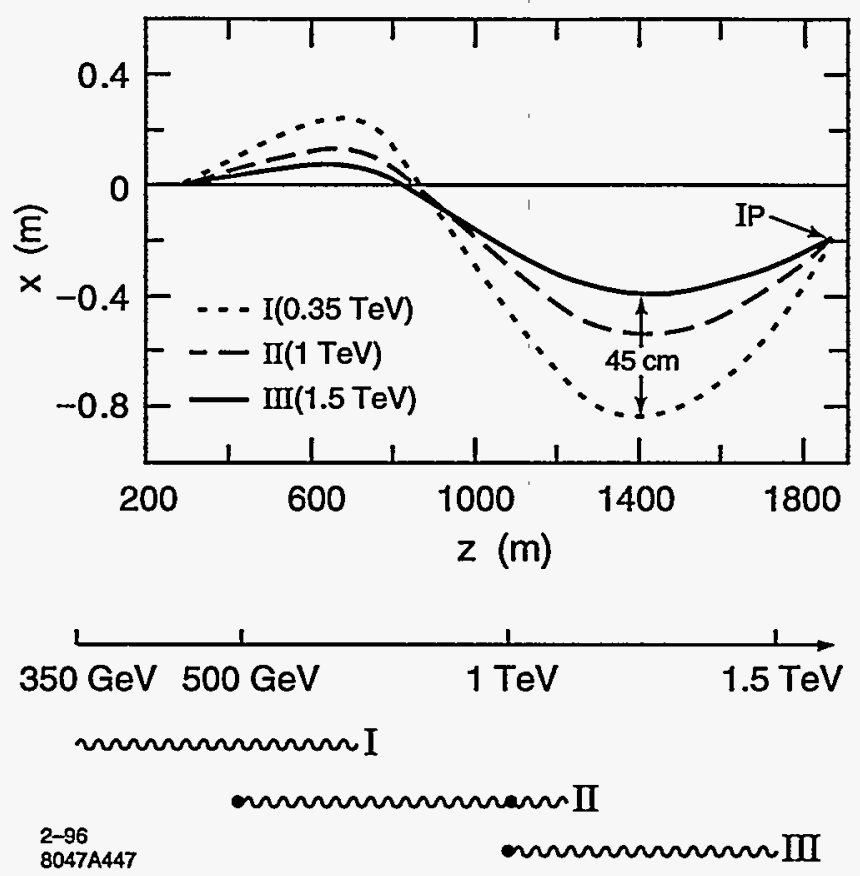

Figure 3.27: Top view of the final focus, and energy range of the three different geometries. Magnet displacements by at most $45 \mathrm{~cm}$ are necessary during an upgrade from $500(350) \mathrm{GeV}$ to $1.5 \mathrm{TeV}$, while the IP-orbit angle changes by about $1.5 \mathrm{mrad}$.

the total loss, need to be tuned and corrected at regular intervals, e.g., every few hours. An average luminosity loss of $1-2 \%$ was assumed for each low-order aberration. Actual tuning accuracy in the SLC is about $0.5 \%$.

The design luminosity is achieved, if, first, the aberrations stay small between tunings, and, second, the beams collide head-on at the IP (rms offset smaller than a quarter of half the beam size). The first condition translates into tolerances on magnet-position drifts over a 1-s time scale and on BPM stability over a few hours. The FFTB magnets and BPMs fulfill all the NLC tolerances. The second condition is met, when a) the effect of ground motion is not important and b) the magnet vibrations with respect to ground are reasonably small.

The ground motion measured above $0.01 \mathrm{~Hz}$ can be explained by isotropic plane ground waves, for which each frequency is associated with a certain wavelength; longer wavelengths correspond to lower frequencies. Figure 3.28 shows the response of the NLC final focus to a harmonic vertical displacement of quadrupoles as a function of wavelength. Plotted is the average squared ratio of the IP beam-beam offset and the ground-motion amplitude. The figure illustrates that the effect of ground motion is heavily suppressed at long wavelengths, i.e. below about $2 \mathrm{~Hz}\left(k \leq 0.01 \mathrm{~m}^{-1}\right)$. To calculate the expected beam-beam offset at the IP, one has to convert the curve of Fig. 3.28 into frequency domain, and, after multiplication with the ground-motion frequency spectrum and a typical SLC feedback response curve (see Fig. 3.29), to integrate over frequency. The total luminosity loss due to ground motion is then 


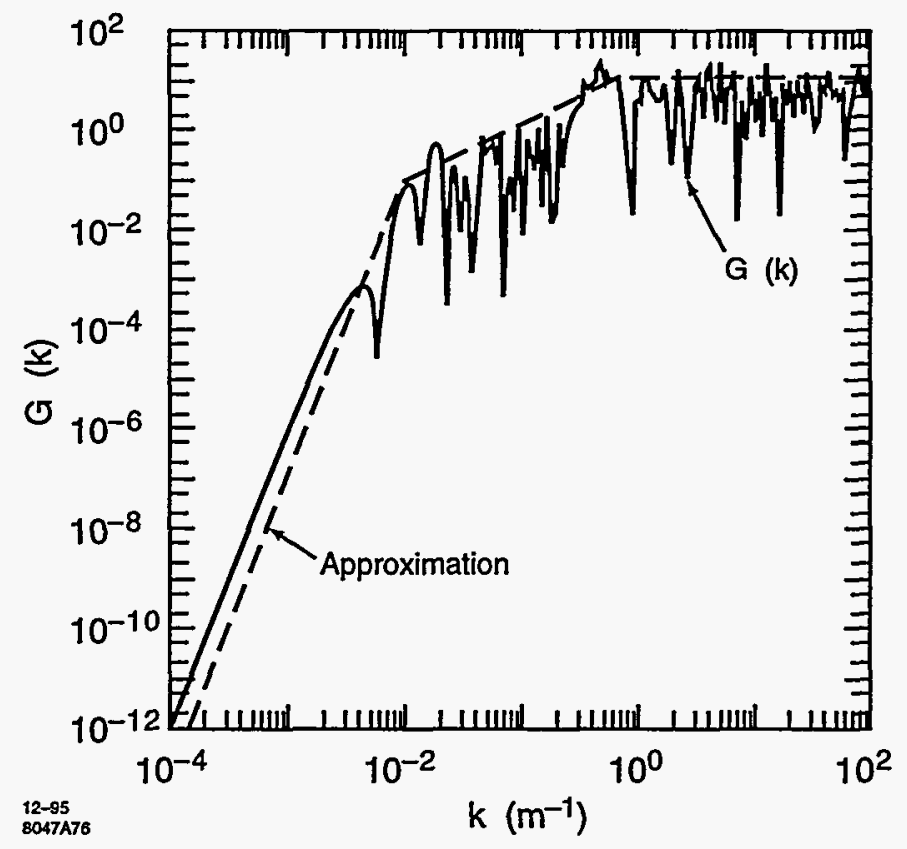

Figure 3.28: Lattice response function for the NLC final focus system.

predicted to be $0.8 \%$ on the SLAC site, and $0.02 \%$ at a quiet site (e.g. LEP tunnel). An upper bound on the luminosity loss due to any additional uncorrelated ground motion is $0.04 \%$. The conclusion is that one can use the ground (bedrock) as a reference for stabilization.

Thus magnet supports need to be designed which neither amplify nor damp the ground motion, but couple the magnet firmly to the ground. At DESY (SBLC-TF), the rms relative quadrupole-to-ground vibrations above $1 \mathrm{~Hz}$ were measured to be smaller than $1 \mathrm{~nm}$, which would meet all the NLC tolerances. Quadrupoles at the FFTB were found to vibrate by about $4 \mathrm{~nm}$ with respect to the ground, excited mainly by bad cooling pumps. These vibration amplitudes would still satisfy the tolerance criteria for all NLC magnets other than the final doublet. For the latter a special stabilization system based on an optical anchor and piezo-electric movers has been devised, which is discussed in the following chapter.

The beam extraction line behind the IP guides the disrupted beam onto a water-based beam dump, which must dispose of all the beam power (about $10 \mathrm{MW}$ ). Numerous diagnostics in the extraction line allow to control and stabilize beam orbit and energy, and also to monitor luminosity and polarization. A schematic is depicted in Fig. 3.30.

In conclusion, the NLC beam delivery system will not only produce small spot sizes at the IP, but it also promises redundant tunability, adjustability over a wide energy range, and a tolerable detector background. Most tolerances on magnet strength or position stability are not particularly tight, and have, in fact, already been achieved at the FFTB or at other places. Also natural ground motion is not thought to be a problem. However care is needed in the mechanical design of components and supports. 


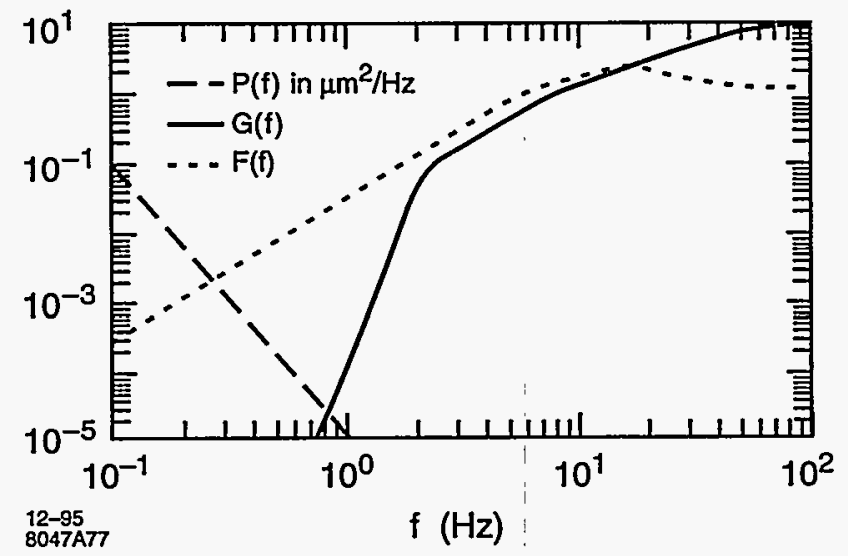

Figure 3.29: Three functions which determine the rms beam-beam separation due to plane-wave ground motion: $F(f)$ - the feedback response; $P(f)$-the local power density; $G(f)$-the lattice response function converted into frequency domain. The integral over the product of these functions gives the square of the rms beam-beam separation.

\section{Detector Legend}

a: BPM (deflection scan)

b: Wire Scan/Screen

c: Polarimeter

d: Beamstrahlung

e: Disrupted $e^{-}$

f: Pairs from IP1
Beam Legend

On-Energy $e^{-}$

Off-Energy $e^{-}, e^{+}$

- - Gammas

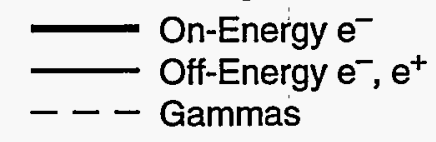

$b, e, f$

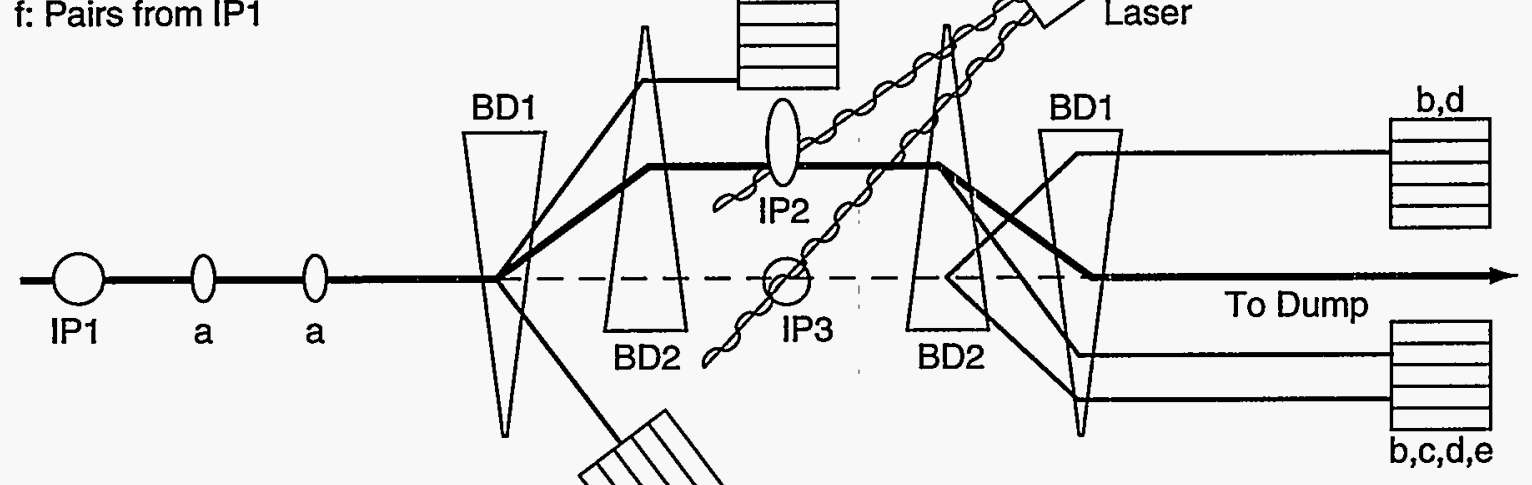

$3-96$
$8047 A 437$

b,f

\section{IP Legend}

IP1: $e^{+} e^{-}$

IP2: $e^{-}$Photon

IP3: Photon Gamma

Figure 3.30: NLC Extraction Line and Diagnostic Layout. 


\subsection{Interaction Region and Detector Backgrounds}

\subsubsection{Introduction}

The design of the interaction region (IR) of the $e^{+} e^{-}$collider involves an understanding of beam-beam effects, detector backgrounds, and the constraints required to maintain very small beam spot sizes at the interaction point (IP). Luminosity monitors with sufficient spatial and energy resolution can unfold the energy dependence of the beam-beam interaction's influence on the luminosity spectrum. With appropriate shielding around the IP a detector employing a high field solenoid and sufficiently granular tracking can be designed which is robust against backgrounds while allowing for excellent tracking and vertexing. Muon backgrounds in the detector are minimized by distancing the machine collimation system from the IP, introducing a $10 \mathrm{mrad}$ bend between the linacs and final focus systems, and installing simple shielding magnets in the tunnel.

Ground motion from geologic and man-made sources must not move the final quadrupole doublet by an amount more than the vertical beam spot size. The rapid fall off of naturally occurring ground motion with increasing frequency, the use of precision beam position monitor based feedback loops sampling at the machine cycle frequency $(120-180 \mathrm{~Hz})$ to correct the beam orbits at low frequency, and the coherence arising from the interplay between the machine's optical lattice and the wavelength of a ground motion disturbance all conspire to make seismic motions in bedrock negligibly important to NLC luminosity. Quadrupole motion above the level of the bedrock, due to laboratory sources (pumps, etc.) or amplification of the driving term by its mechanical support within the detector, can be minimized by optically referencing the doublet to bedrock with a LIGO-style interferometer. Feedback signals can then drive either piezoelectric actuators or magnetic beam-corrector elements to reduce motion back down to naturally occurring seismic levels. The selection of a geologically quiet site, the design of the physical lab plant to minimize vibration, and the design of a robust final doublet support will all aid in controlling the ground motion problem.

\subsubsection{Layout}

In the schematic layout of Figure 3.3 the electron and positron linac arms are each followed by a $3.2-\mathrm{km}$-long collimation section and two final focus tunnels at $\pm 10 \mathrm{mrad}$. These "Big Bends", $2.0 \mathrm{~km}$ from the interaction points (IP), protect the detectors from muons produced by the collimators, provide a $20 \mathrm{mrad}$ crossing angle between incoming and outgoing beams, and allow for two experimental halls with IPs separated by $40 \mathrm{~m}$. As trains consisting of 90 búnches of $0.65-1.1 \times 10^{10}$ particles separated by $1.4 \mathrm{~ns}$ will collide at $120-180 \mathrm{~Hz}$, a crossing angle is required so that a given bunch interacts with only its partner, and not with any other bunch still traveling to the IP.

Figure 3.31 shows a schematic of the masking and magnet layout in the interaction region. In the current final focus lattice the last quadrupole of the doublet (QFTA) ends $2 \mathrm{~m}$ from 


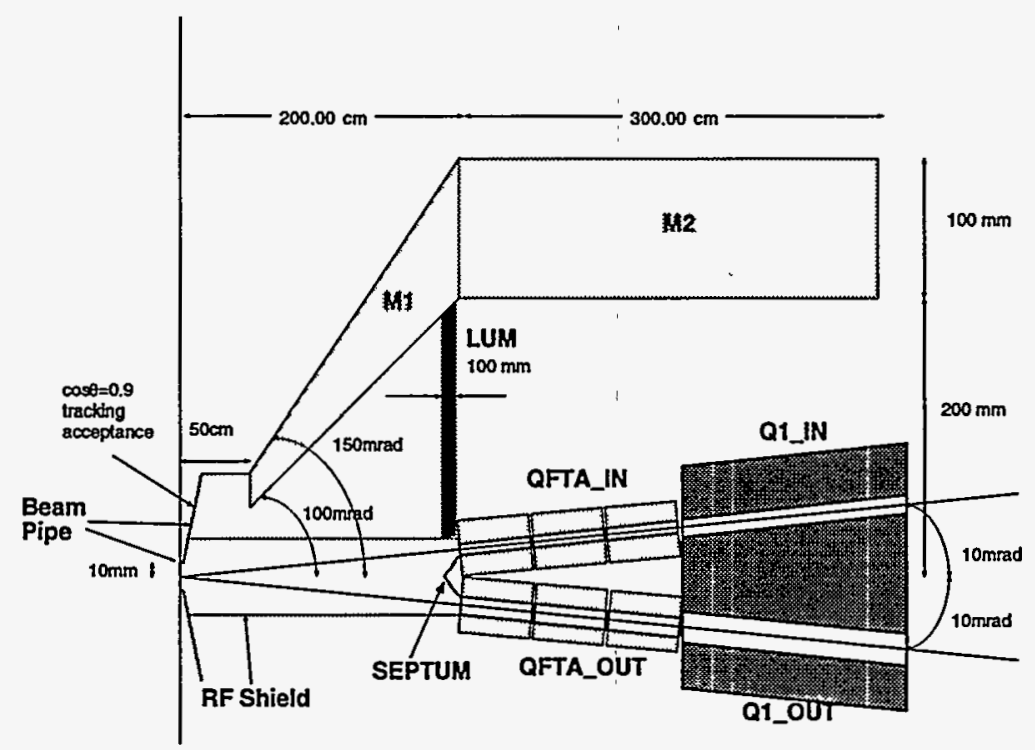

Figure 3.31: The interaction region masking and magnet layout.

the IP. Field strength and quality require that it be constructed of a machinable permanent magnetic material (samarium cobalt, for example). Its inner aperture radius is $4.5 \mathrm{~mm}$ and outer radius is $10.0 \mathrm{~cm}$. A similar doublet with a $6 \mathrm{~mm}$ inner aperture radius to transport the outgoing disrupted beam, together with any synchrotron radiation and beamstrahlung photons, is symmetrically positioned horizontally across from QFTA. These two magnets are followed by a twin bore superconducting magnet to complete the doublet. Each is surrounded by a superconducting coil which shields the detector's solenoidal field.

A "dead cone", within which the vast majority of the low $p_{t}, e^{+} e^{-}$pairs produced by the beam-beam interaction are confined, is defined by a conical tungsten mask which subtends the angular range from 100 to $150 \mathrm{mrad}$ and $0.5 \mathrm{~m}<z<2 \mathrm{~m}$. A cylindrical tungsten skirt, currently $10 \mathrm{~cm}$ thick, begins at $z=2.0 \mathrm{~m}$ and $r=20 \mathrm{~cm}$. Its purpose is to protect any exterior detector from photons produced when the pair electrons and positrons strike the from face of the quadrupoles or luminosity monitor. The beam pipe is assumed at this time to have a radius of $1.0 \mathrm{~cm}$ for the first $2.5 \mathrm{~cm}$ from the $I P$. This will accommodate the inner layer of a vertex detector with acceptance out to $\cos \theta=0.9$. The beam pipe then flares to a radius of $7.2 \mathrm{~cm}$, switching from beryllium to $1-\mathrm{mm}$-thick stainless steel once it begins to follow the contour of the M1 mask. Within the beam pipe is a thin rf shield and septum which ease the transition from the narrow apertures of the input and exit quadrupoles to the beam pipe radius.

\subsubsection{Backgrounds}

The backgrounds anticipated at the NLC are each described in the following subsections. The experience of the SLD detector at SLC is that, when the accelerator is working well, all 
expected backgrounds are small. High luminosity correlates strongly with low backgrounds. While the calculations outlined below indicate that backgrounds will not be a substantial problem at the NLC, the prudent experimenter will design a detector which can handle the oft-occurring non-standard running conditions and unexpected backgrounds which are in the nature of linear colliders.

The background levels that can be supported require that we assume a detector model. Experience with the SLD's pixel vertex detector, based on $22 \mu \mathrm{m} \times 22 \mu \mathrm{m}$ CCD pixels, is that hit density is the figure of merit. Integrating over its readout time of 19 bunch crossings, the SLD achieves $0.4 \mathrm{hits} / \mathrm{mm}^{2}$ in its innermost layer. Tracks found in an 80 layer drift chamber are projected back to the vertex detector and hits lying within a three $\sigma$ extrapolation error window of roughly $1 \mathrm{~mm}$ are added to the track. The traditional figure of merit for vertex detector backgrounds is thus taken to be $1 \mathrm{hit} / \mathrm{mm}^{2}$ per unit of time. Three to four layer self tracking silicon vertex detectors, with track extrapolation errors on the order of $10 \mu \mathrm{m}$, should be robust against backgrounds 10 to 20 times worse.

This section assumes that at the NLC the integration time for each detector is a train of 90 bunches. Depending on the technology actually used, this assumption may be overly pessimistic; nanosecond level timing and the ability to isolate background at the bunch level will certainly be a design goal for NLC detector components.

Raw occupancy is the figure of merit for a gas tracking chamber comprised of a relatively small number of wires each of which samples a large volume. The 5120 wire SLD chamber at $r=20 \mathrm{~cm}$ operates with $3-5 \%$ occupancy in good conditions. Simulations indicate that this is due to between one and ten thousand low energy photons produced from the secondary interactions of 2-MeV SR photons in the masking system. We take 1-10 thousand photons per train as the design goal for an NLC gas tracking chamber.

In SLD muons produced in the upstream collimators traverse the barrel calorimeter at the rate of one or two per beam crossing. In bad conditions there can be a factor of ten increase in the muon flux, at which point the schemes used to keep the muons from contributing to the trigger rate, and, to a lesser extent, the offline energy reconstruction offline, break down. The design goal of the muon protection system is that $1 \%$ of the beam can be scraped off in the collimation section and produce, on average, one muon per train of bunches that is transported into the detector.

\section{Muons}

Figure 3.32 shows the results of the muon background simulation. The black diamonds show the ordinate is the number of muons that can interact in a given collimator and produce one muon in a $8 \mathrm{~m}$ by $8 \mathrm{~m}$ square detector. The abscissa is the location of the collimator in the tunnel. The points to the left between 4 and $5 \mathrm{~km}$ from the IP correspond to the collimation system; the points between 1 and $2 \mathrm{~km}$ from the IP correspond to locations where it is planned that the clean up collimators remove the slightest of beam tails. The uppermost of the two curves corresponds to the calculation when four $9 \mathrm{~m}$ long tunnel filling 
toroids are added to the final focus to intercept and deflect errant muons. One sees that this spoiler system added three orders of magnitude of protection and that we are two orders of magnitude within the design goal (which would correspond to roughly $10^{12}$ ). Without the spoilers we have a marginal situation. With or without spoilers, we are much more sensitive to beam particles lost in the FF after the big bend. Figure 3.32 shows that $10^{6}$ particles lost in the final focus, which is one part per million per train, will produce one muon that reaches the detector.

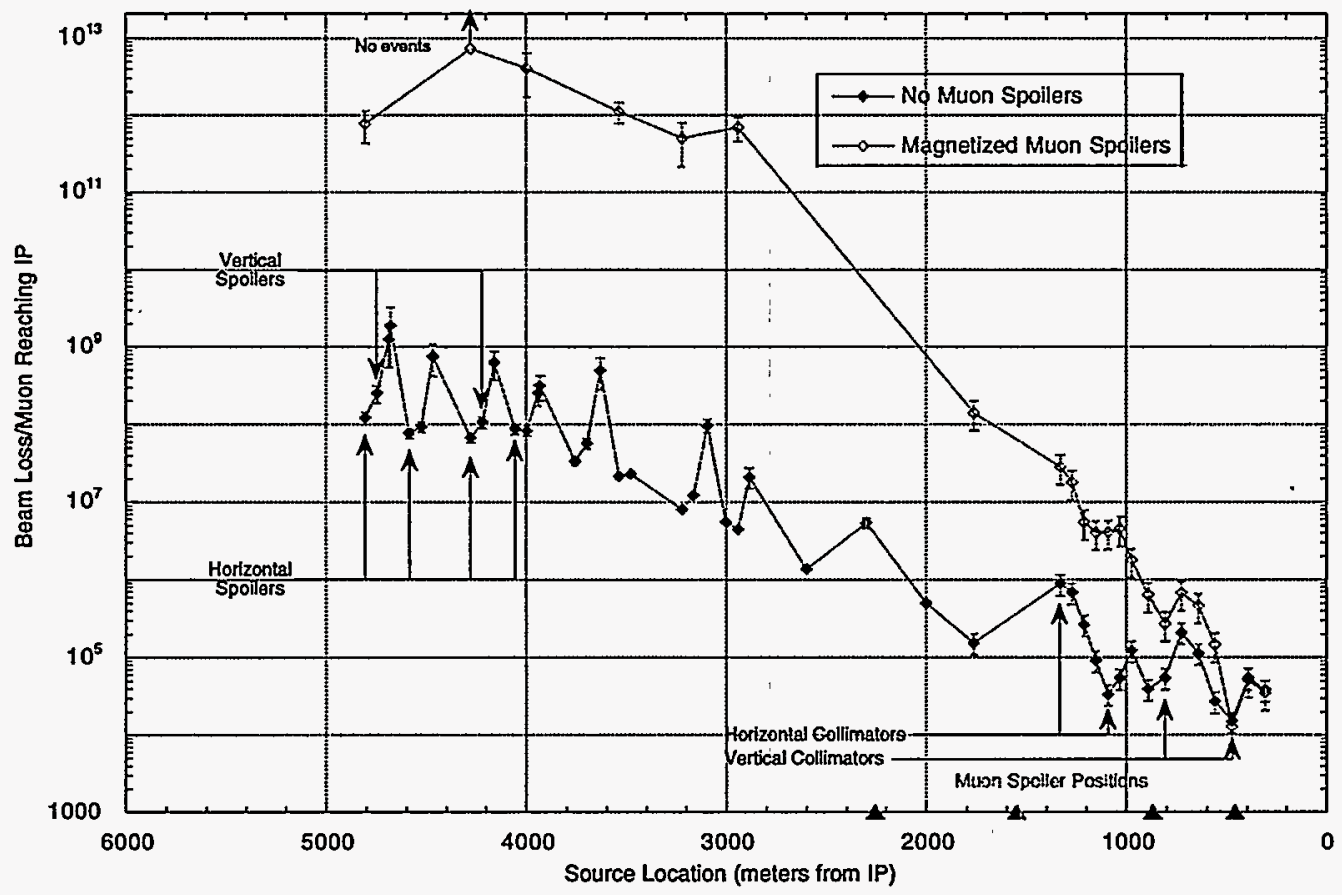

Figure 3.32: Muon backgrounds: the number of beam particles that can be lost on a given collimeter before producing one muon that strikes the detector.

\section{Pairs}

Roughly $10^{5}$ pairs will be produced by the beam beam interaction each bunch crossing, predominately through the Bethe-Heitler interaction of beamstrahlung photons and electrons or positrons. For the most part the pairs are produced with low intrinsic $p_{t}$; the same sign partner will tend to be focused by the opposing beam while the opposite sign partner will be deflected outside the beam envelope by the magnetic field of the bunch. The finite beam dimensions then result in a very hard kinematic edge in the $p_{t}-\theta$ distribution (Figure 3.33).

By introducing a strong solenoidal magnetic field all particles with $p_{t}<30 \mathrm{MeV}$ are curled up within the $5 \mathrm{~cm}$ minimum radius of the conical mask of Figure 3.31 . All particles with $\theta$ less than the dead cone are curled up. The number of particles falling outside these two cuts is relatively small and manageable. 


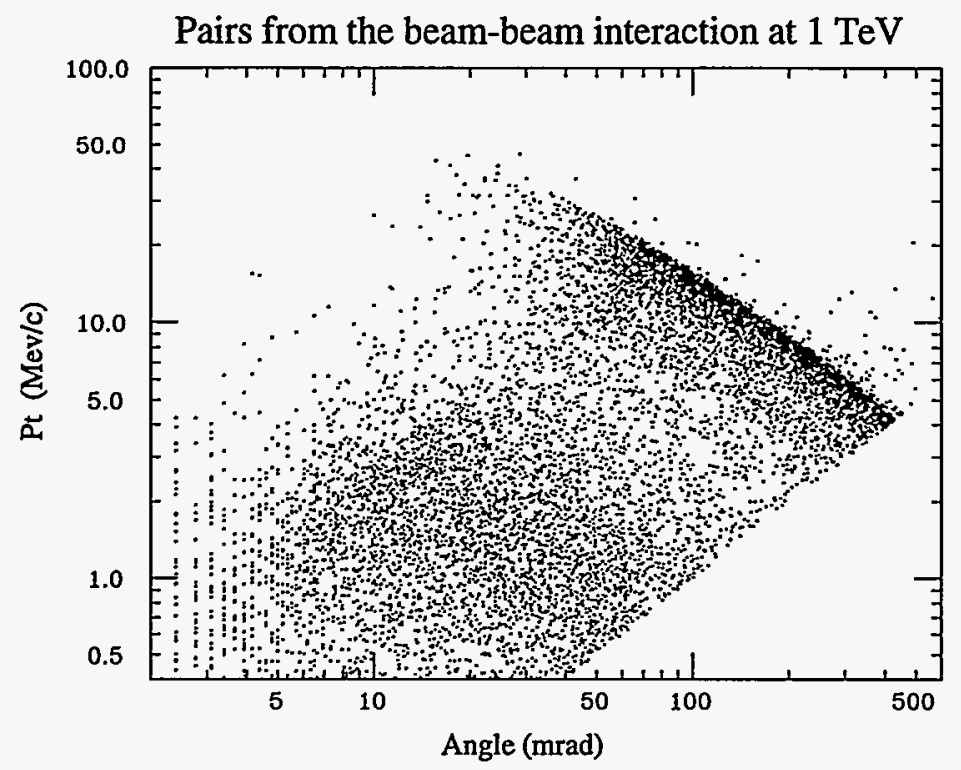

Figure 3.33: $p_{t}$ vs. $\theta$ distribution for pairs.

We have simulated the beam beam interaction at $1 \mathrm{TeV}$ with the ABEL program. Figure 2.51 in the "Design of the NLC Detector" section of this document shows the hit density expected at $r=2$ and $3 \mathrm{~cm}$ as a function of $z$ for a solenoidal field of 4 Tesla. At $r=2 \mathrm{~cm}$, as long as our VXD lies within $z=17 \mathrm{~cm}$, the hit density is manageable. Lowering the field would require that the innermost vertex layer be at correspondingly larger radius.

\section{Photons from Pairs}

Pairs will interact with the beam pipe, inner vertex detector layers, rf shield septum, and front faces of the entrance and exit quads. As they interact, photons will be produced which will form a secondary background in the VXD and in any tracking chamber at larger radius.

To study this problem we have simulated the IR of Figure 3.31 with EGS4 using the 1TeV ABEL ray files as input. Materials, thicknesses, and geometries have all been achieved at SLD. Figure 3.34 looks at the number of photons per train intercepting scoring planes with $\cos \theta=0.90$ acceptance. Photon-to-hit conversion efficiencies are expected to be about the same for silicon tracking or gaseous drift chambers, a couple of percent. The hit densities are all well below $1 / \mathrm{mm}^{2} /$ train, even before taking into account conversion efficiency. However, the raw number of photons crossing the $\mathrm{r}=30 \mathrm{~cm}$ plane is rather large, approximately what we have taken as our figure of merit. The situation, as indicated in the figure, is made better by going to higher field. It is also possible that more innovative masking schemes will help. It does seem however that low granularity tracking devices will be marginal in the NLC background environment. 
Average Photon Density as a Function of Radius

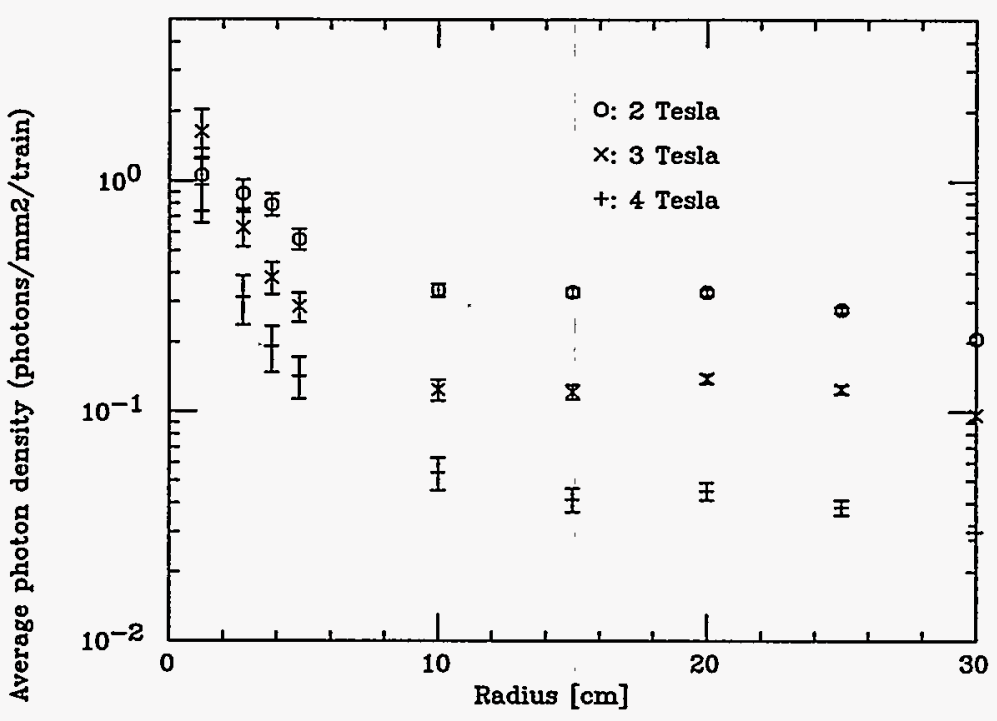

Figure 3.34: Photon hit density vs. radius.

\section{Synchrotron Radiation}

SR produced by the core of the beam in the soft dipole bend just upstream of the final doublet and SR produced by particles at the edge of the phase space allowed by the collimation system can cause backgrounds in the detector. Most of the radiation escapes through the output quadrupole aperture; photons striking the inside of the incoming quadrupole aperture near its IP-side tip are the biggest problem. The soft bend SR from the core can be masked far from the IP. Figure 3.35 shows the energy distribution of SR from the beam tail as calculated for the $1 \mathrm{TeV}$ lattice. As long as the QFTA aperture is large enough so that radiation produced immediately upstream in Q1 can escape the situation is not dramatic. If the QFTA aperture decreases below $4.3 \mathrm{~mm}$ radius or the tail collimation is loosened beyond $7 \sigma_{x}$ and $35 \sigma_{y}$ the energy deposited in the tip of QFTA increases dramatically.

Figure 3.36 shows the results of the EGS simulation using the SR energy distribution of Figure 3.35 as input. The charged particle hit density is tolerable for $r \geq 2 \mathrm{~cm}$ and $B=4$ Tesla, while the photon hit density is negligible in the region of the vertex detector. However, the photon density at $30 \mathrm{~cm}$ would correspond to having over 100,000 photons incident on a conventional drift chamber, a potentially worrisome figure were that technology chosen for the tracking system. Better masking, especially upstream in the lattice, and tighter collimation would reduce these numbers. Note also that the backgrounds would be much less for the $500 \mathrm{GeV}$ lattice. 
SR ENERGY DISTRIBUTION: QFTA INNER BORE

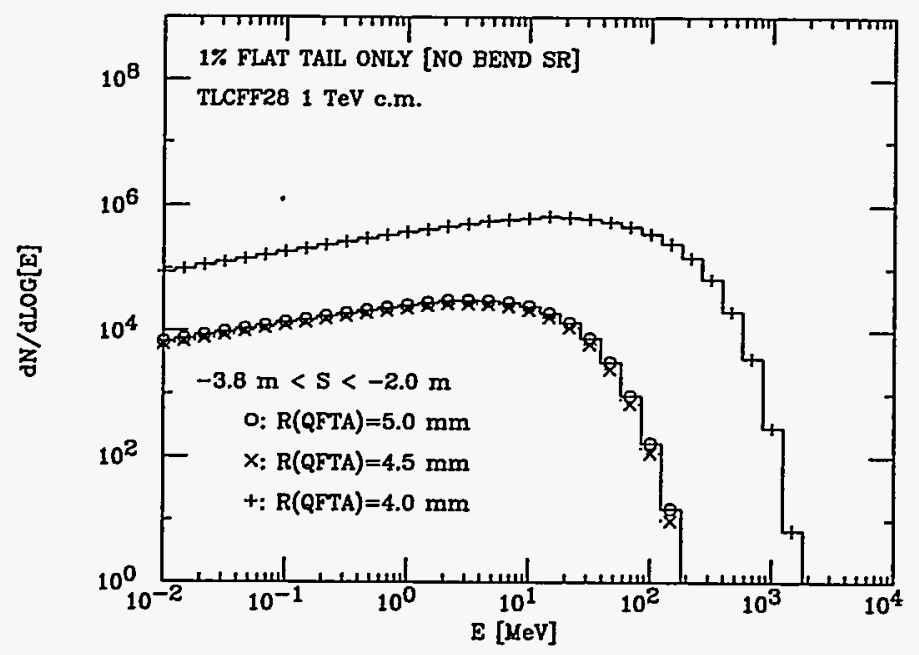

Figure 3.35: Synchrotron radiation energy distribution.

Average Hit Density as a Function of Radius

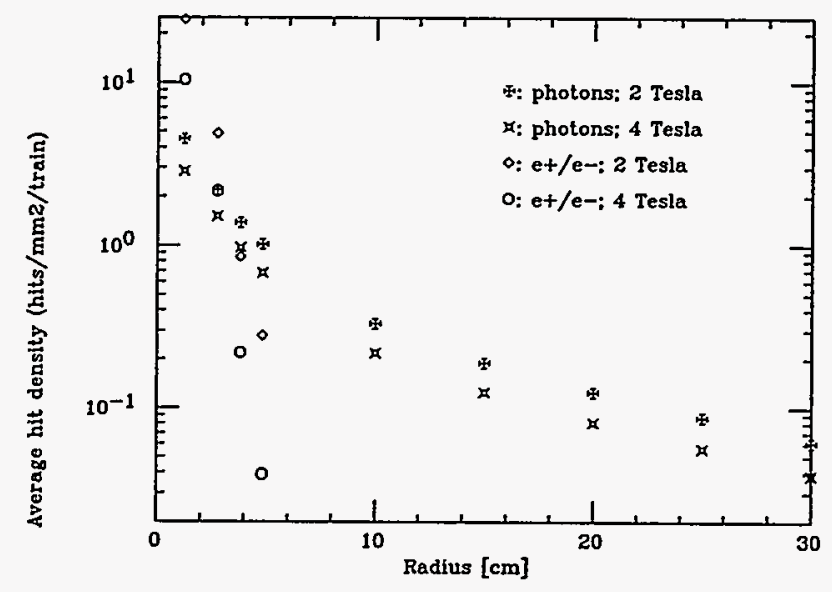

Figure 3.36: Synchrotron radiation hit distribution. 


\subsubsection{Quad Support and the Optical Anchor}

As outlined in the introduction, the issue of ground motion was once thought to be a potential "show stopper" for a machine with nanometer sized beams. Our current understanding of the relationship between beam feedback, the ground motion spectrum, and the coherence resulting from the machine lattice and the wave nature of the ground motion is that by minimizing external sources of vibration and by firmly anchoring the final quadrupoles to bedrock luminosity will be preserved.

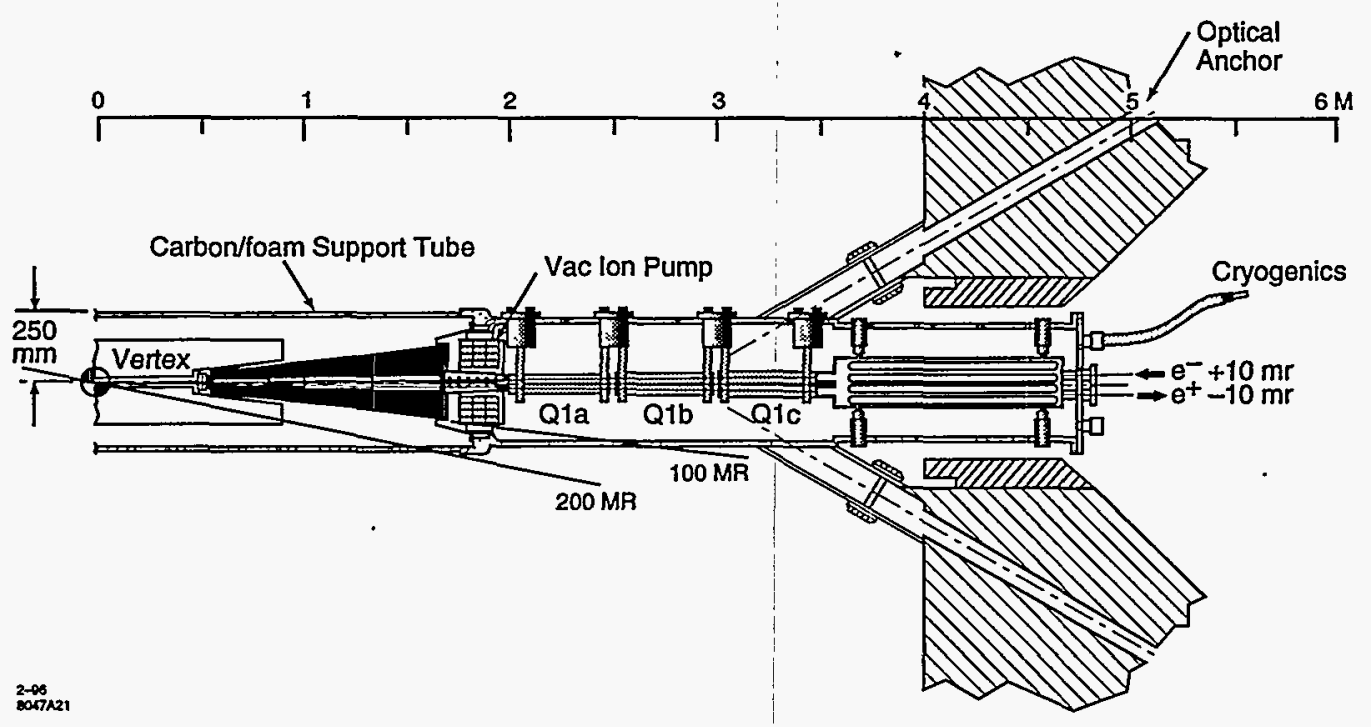

Figure 3.37: Current final doublet layout with 20-mrad crossing angle.

However, the final quads will need to be mounted inside a detector. Engineering the detector and the quad supports to avoid amplifying natural or cultural jitter sources is mandatory. Developing tools to remove the jitter that will nonetheless arise is highly prudent. Figure 3.37 shows an engineering drawing of our current ideas for the final quad layout and support.

In early tests at SLAC and DESY inertial seismometers driving pizeoelectric movers were used in feedback loops to inertially stabilize a magnet. A factor of three in amplitude reduction was achieved at each lab. Fears of using these devices within a particle detector magnetic field and a desire for larger reduction factors led to the search for optical solutions to the problem. The LIGO experiment, geophysicists, and astronomers have all addressed this problem through laser interferometry. The relative change in the length of two optical arms, $\ell_{1}$ and $\ell_{2}$, is related to an intensity change at a photodiode,

$$
\frac{\delta I}{I}=\delta \Phi=2 \pi \cdot \frac{\delta\left(\ell_{1}-\ell_{2}\right)}{\lambda}
$$

For $\lambda=633 \mathrm{~nm}$, and $\delta\left(\ell_{1}-\ell_{2}\right)=1 \mathrm{~nm}$, one will have $\delta \Phi=0.02$ and this will cause a $2 \%$ intensity change on the photodiode. 
While there are many constraints on the laser and optical system in this interferometer, including tolerances on the laser's intensity stability, frequency stability and power, as well as tolerances on pressure and temperature fluctuations in the optical transport arms, it appears that the solution is viable. Testing its performance and designing a detector which leaves room for optical lines of sight are what is required.

\subsubsection{Conclusion}

While more calculation and testing is required there does not appear to be any fundamental problem in designing an IR and detector which maintains the machine luminosity, can handle the backgrounds, and do the physics. 


\section{Bibliography}

[1] "Zeroth-Order Design Report for the Next Linear Collider," SLAC Report 474 (Stanford University, May 1996).

[2] J. Seeman, "The Stanford Linear Collider", Ann. Rev. Nucl. Part. Sci. (Annual Review, Inc., Palo Alto, California, 1991), Vol. 41, pp. 389-428.

[3] J. Seeman, "Accelerator Physics of the Stanford Linear Collider and SLC Accelerator Experiments Towards the Next Linear Collider", Advances of Acclerator Physics and Technologies (Vol. 12 of the Advanced Series on Directions in High Energy Physics), edited by Herwig Schopper (World Scientific, 1993), pp. 219-248.

[4] "Next Linear Collider Test Accelerator Conceptual Design Report", SLAC Report 411 (Stanford University, August 1993).

[5] R. D. Ruth et al., "The Next Linear Collider Test Accelerator" (SLAC-PUB-6252), Proceedings of the 1993 IEEE Particle Accelerator Conference, Washington, DC, May 17-30, 1003 (IEEE Catalog No. 93CH3279-7), pp. 543-545.

[6] P. B. Wilson, Z. D. Farkas, and R. D. Ruth, "SLED-II: A New Method of RF Pulse Compression" (SLAC-PUB-5330), Proceedings of the 1990 Linear Accelerator Conference, Albuquerque, New Mexico, September 10-14, 1990.

[7] Z. D. Farkas et al., "SLED: A Method of Doubling SLAC's Energy", (SLAC-PUB1453), Proceedings of the 9th International Conference on High Energy Accelerators (Stanford, California, May 2-7, 1974), pp. 576-583.

[8] C. Nantista et al., "High Power Rf Pulse Compression with SLED-II at SLAC" (SLACPUB-6145), Proceedings of the 1993 IEEE Particle Accelerator Conference, Washington, DC, May 17-20, 1993, (IEEE Catalog No. 93CH3279-7), pp. 1196-1198.

[9] A. E. Vlieks et al., "Accelerator and Rf System Development for NLC" (SLAC-PUB6148), Proceedings of the 1993 IEEE Particle Accelerator Conference Wahington, DC, May 17-20, 1003 (IEEE Catalog No. 93CH3279-7) pp. 620-622. 
[10] J. W. Wang et al., "High Gradient Tests of SLAC Linear Collider Accelerator Structures" (SLAC-PUB-6617), Proceedings of the 17th International Linear Accelerator Conference (LINAC 94), Tskuba, Japan, August 21-26, 1994.

[11] J. Seeman et al., "RF Beam Deflection Measurements and Corrections in the SLC Linac", Proceedings of the 1985 US Particle Accelerator Conference, Vancouver, Canada, May 1985 (IEEE NS32, No. 5), pp. 2629-2631.

[12] R.F.J. Read and W.J. Wills-Moren, Cranfield Precision Engineering, Ltd., Bedford, England, "Study Report for the Estimation of Unit Cost of Mass Producing Discs for the CLIC Accelerating Sections", 1993.

[13] "Technology and Costs for 2 Million Copper Discs of the CLIC Accelerator at CERN", Optische Werke G. Rodenstock, 1993.

[14] G. A. Loew and J. W. Wang, "RF Breakdown Studies in Room Temperature Electron Linac Structures" (SLAC-PUB-4647), Proceedings of the 13th International Symposium on Discharges and Electrical Insulation in Vacuum, Paris, France, June 27-30, 1988.

[15] J. W. Wang et al., "High Gradient Tests of SLAC Linear Collider Accelerator Structures" (SLAC-PUB-6617), Proceedings of the 17th International Linear Accelerator Conference (LINAC 94), Tsukuba, Japan, August 21-26, 1994.

[16] S. Takeda et al., "High Gradient Experiments by the ATF", 1991 IEEE Particle Accelerator Conference, San Francisco, California, May 6-9, 1001 (IEEE Catalog No. 91CH3038-7), pp. 2061-2063.

[17] H. Matsumoto et al., "Applications of Hot Isostatic Pressing (HIP) for High Gradient Accelerator Structure", 1991 IEEE Particle Accelerator Conference, San Francisco, California, May 6-9, 1991 (IEEE Catalog No. 91CH3038-7), pp. 1008-1010.

[18] S. G. Tantawi, R. D. Ruth, and A. E. Vlieks, "Active Ratio Frequency Pulse Compression using Switched Resonant Delay Lines" (SLAC-PUB-95-6748), Nucl. Instr. Meth. A370, 297 (1996).

[19] S. G. Tantawi, T. G. Lee, R. D. Ruth, A. E. Vlieks, and M. Zolotorev, "Design of a Multimegawatt X-Band Solid State Microwave Switch" (SLAC-PUB-95-6827), Proceedings of the 1995 IEEE Particle Accelerator Conference, Dallas, Texas.

[20] Z. D. Farkas, "Binary Peak Power Multiplier and Its Application to Linear Accelerator Deisgn", IEEE Transactions MTT-34 (1986), pp. 1036-1043.

[21] T. L. Lavine et al., "High-power Radio-frequency Binary Pulse Compression Experiment at SLAC" (SLAC-PUB-5451), Proceedins of the 1991 IEEE Particle Accelerator Conference, San Francisco, California, May 6-9, 1991 (IEEE Catalog 91CH3038-7), pp. 652-654. 


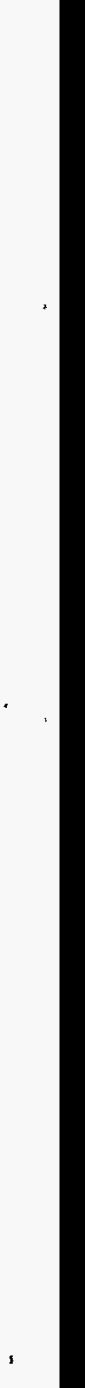




\section{Appendix: Papers Contributed to the NLC Physics Study}

1. T. G. Rizzo, "Probing Weak Anomalous Couplings with Final State Gluons at the NLC."

2. J. F. Gunion and P. C. Martin, "Prospects for and Implications of Measuring the Higgs to Photon-Photon Branching Ratios at the Next Linear $e^{+} e^{-}$Collider."

3. H. Baer et al., "Determination of Supersymmetric Particle Production Cross Sections and Angular Distributions at a High Energy Linear Collider."

4. R. Arnowitt and P. Nath, "Using Linear Colliders to Probe GUT and Post-GUT Physics."

5. J. Wudka, "The Meaning of Anomalous Couplings."

6. M. Gintner, S. Godfrey, and G. Couture, "Measurement of the $W W \gamma$ and $W W Z$ Couplings in the Process $e^{+} e^{-} \rightarrow \ell \nu q q^{\prime}$."

7. K. Riles, "Effects of Detector Resolution on Measurement of Anomalous Triple Gauge Boson Couplings."

8. T. G. Rizzo, "The Polarization Asymmetry and Triple Gauge Boson Couplings in $\gamma e$ Collisions at the NLC."

9. T. G. Rizzo, "Below Threshold $Z^{\prime}$ Mass and Coupling Determinations at the NLC."

10. T. G. Rizzo, "Anomalous Chromoelectric and Chromomagnetic Moments of the Top Quark at the NLC." 
1 
Probing Weak Anomalous Top Quark Couplings with Final State Gluons at the NLC

THOMAS G. Ruzzo'

Stanford Linear Accelerator Center

Stanford University, Stanford, California 94309 USA

\section{ABSTRACT}

The rate and corresponding gluon jet energy distribution for the process $e^{+} e^{-} \rightarrow t \bar{t} g$ are sensitive to the presence of anomalous dipole-like couplings of the top to the photon and $Z$ at the production vertex. For sizeable anomalous couplings of this type substantial deviations from the expectations of the Standard Model are likely. We explore the capability of the NLC to discover or place bounds on these types of top quark couplings. The resulting constraints are found to be quite complementary to those which arise from direct probes of the top quark production vertex.

\section{Introduction}

The Standard Model(SM) has provided a remarkably successful description of almost all available data involving the strong and electroweak interactions, In particular, the discovery of the top quark at the Tevatron with a mass[1], $m_{l}=175 \pm 9 \mathrm{GeV}$, close to that anticipated by fits to precision electroweak data is indeed a great triumph. However, the fact that $R_{b}$ (and perhaps $A_{b}$ ) remains[2] more than 3.3(1.8) $\sigma$ from SM expectations may be providing us with the first indirect window into new physics. In fact, this apparent deviation in b-quark couplings from the SM expectations could indicate that some new physics is interacting with the third family as a whole. Since the top is the most massive fermion, it is believed by many that the detailed physics of the top quark may be significantly different than what is predicted by the SM. This suggestion makes precision measurements of all of the top quark's properties mandatory.

Perhaps the most obvious and easily imagined scenario is one in which the top's couplings to the SM gauge bosons, i.e., the $W, Z, \gamma$, and $g$, are altered. This possibility, extended to all of the fermions of the third generation, has attracted a lot of attention over the last

'Work supported by the Department of Energy, contract DE-AC03-76SF00515. few years[3]. In the case of the electroweak interactions involved in top pair production in $e^{+} e^{-}$collisions, the lowest dimensional gauge-invariant operators representing new physics that we can introduce take the form of dipole moment-type couplings to the $\gamma$ and $Z$. The anomalous magnetic moment-type operators, which we can parameterize by a pair of dimensionless quantities, $x_{\gamma}$, are $C P$-conserving. The corresponding electric dipole moment terms, parameterized as $\bar{\kappa}_{\gamma} Z$, are $C P$-violating. The shift in the three-point $t \bar{t} \gamma$ and $t \bar{t} Z$ interactions due to the existence of these anomalous couplings can be written as

$$
\delta \mathcal{L}=\frac{i}{2 m_{t}} \bar{t} \sigma_{\mu \nu} q^{\nu}\left[e\left(\kappa_{\gamma}^{t}-i \bar{\kappa}_{\gamma}^{t} \gamma_{5}\right) A^{\mu}+\frac{g}{2 c_{w}}\left(\kappa_{Z}^{t}-i \bar{\kappa}_{z}^{t} \gamma_{5}\right) Z^{\mu}\right] t,
$$

where $e$ is the proton charge, $g$ is the standard weak coupling constant. $c_{w}=\cos \theta_{W}$, and $q$ is the $\gamma$ or $Z$ 's four-momentum. Gauge invariance will also lead to new four-point interactions involving two gauge bosons and the top, e.g., $t \bar{t}_{\gamma \gamma}, Z Z, Z_{\gamma}, W^{+} W^{-}$, but they will not concern us bere as we will only work to leading order in the electroweak interactions. In most cases gauge invariance will relate any $t \bar{t} Z, \gamma$ anomalous couplings to others involving the $t b W$ vertex. Escribano and Masso[3] have shown that in general all of the anomalous three-point couplings involving the neutral gauge bosons can be unrelated even when the underlying operators are SM gauge invariant. Thus in our analysis we will treat $\kappa_{\gamma, z}^{t}$ and $\bar{\kappa}_{\gamma, Z}^{t}$ as independent free parameters. (Of course, within any particular new physics scenario the anomalous couplings will no longer be independent.) As has been discussed in the literature[3], if any of the anomalous couplings are sufficiently large their effects can be directly probed by top pair production. The purpose of the present work is to consider the sensitivity of the process $e^{+} e^{-} \rightarrow t \bar{t} g$ to non-zero values of the $t \bar{t} Z, \gamma$ anomalous couplings.

\section{Analysis}

In the present analysis we consider how the 'normalized' gluon energy distribution,

$$
\frac{d R}{d z}=\frac{1}{\sigma\left(e^{+} e^{-} \rightarrow t \bar{t}\right)} \frac{d \sigma\left(e^{+} e^{-} \rightarrow t \bar{t} g\right)}{d z},
$$

where $z=2 E_{s} / \sqrt{s}$, can be used to constrain anomalous top couplings to the $\gamma$ and $Z$. (We work only to lowest order in $\alpha_{s}$ ) Note that the anomalous couplings will contribute to both the numerator and denominator of the expression of $d R / d z$. This implies that the sensitivity of $R$ to very large values(with magnitudes $\geq 1$ ) of the anomalous couplings is quite small. However, for the range of anomalous couplings of interest to us significan sensitivity is achieved. We follow the procedure given in Ref.[4] which also supplies the complete formulae for evaluating this gluon energy distribution.

In comparison to Ref.[4], the present analysis has been extended in two ways. (i) We allow for the possibility that two of the four anomalous couplings may be simultaneously non-zero. (ii) We lower the cut placed on the minimum gluon jet energy, $E^{\min }$, in performin energy spectrum fits. The reasons for employing such a cut are two-fold. First, a minimum 


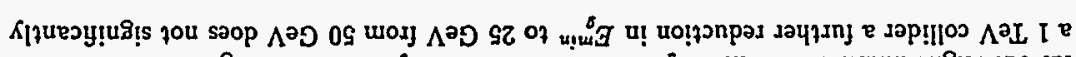

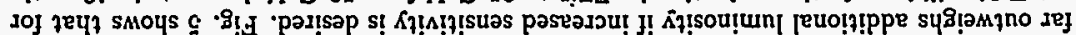

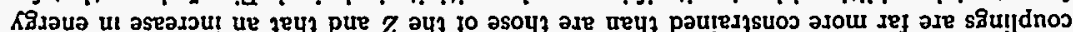

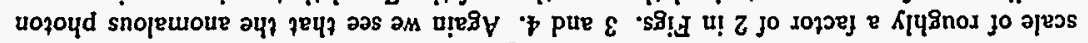

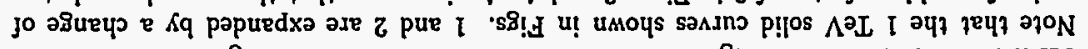

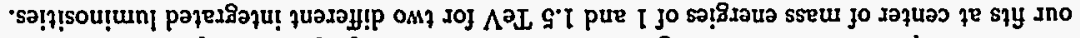

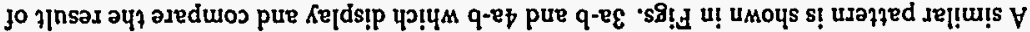

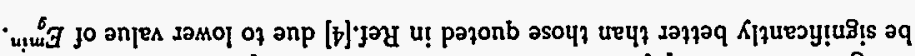

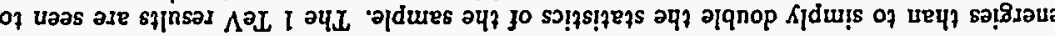

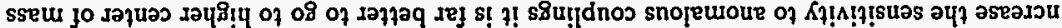

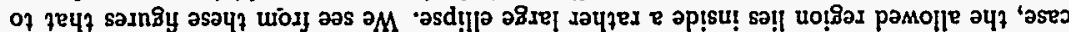

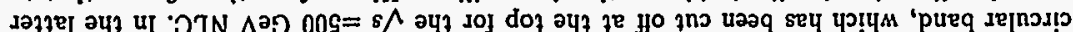

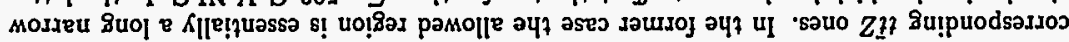

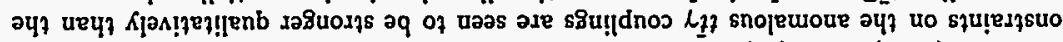

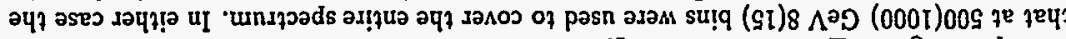

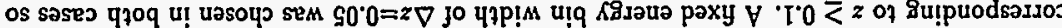

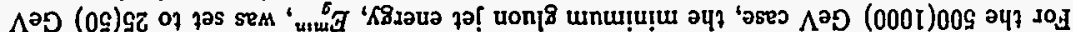

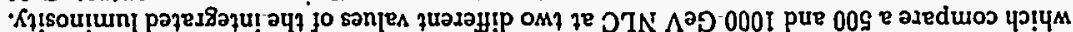

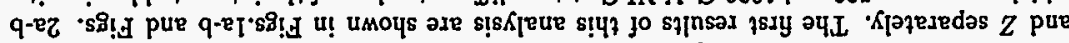

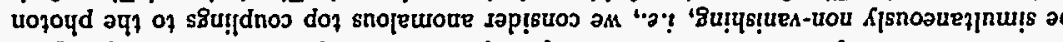

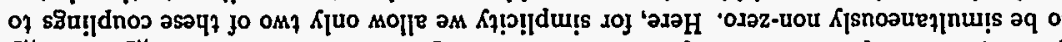

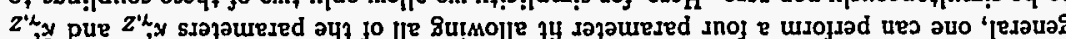

OTN $\Lambda^{\mathrm{a} D}(000 \mathrm{~T}) 00 \mathrm{~g}$

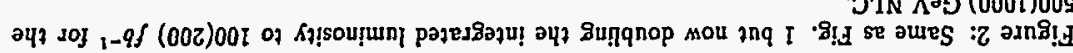
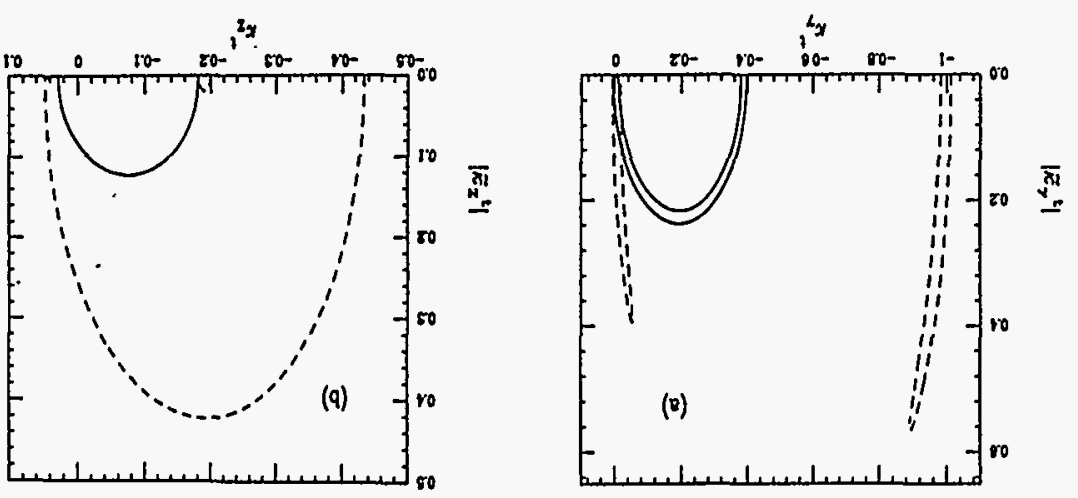

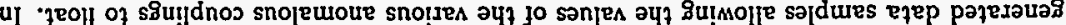

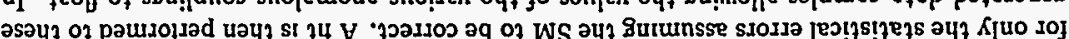

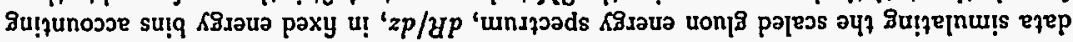

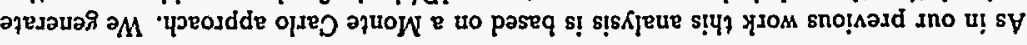

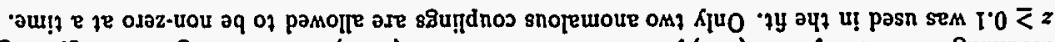

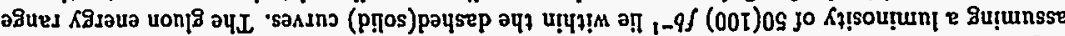

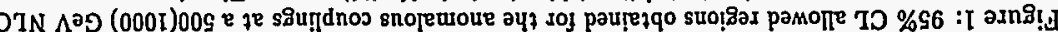
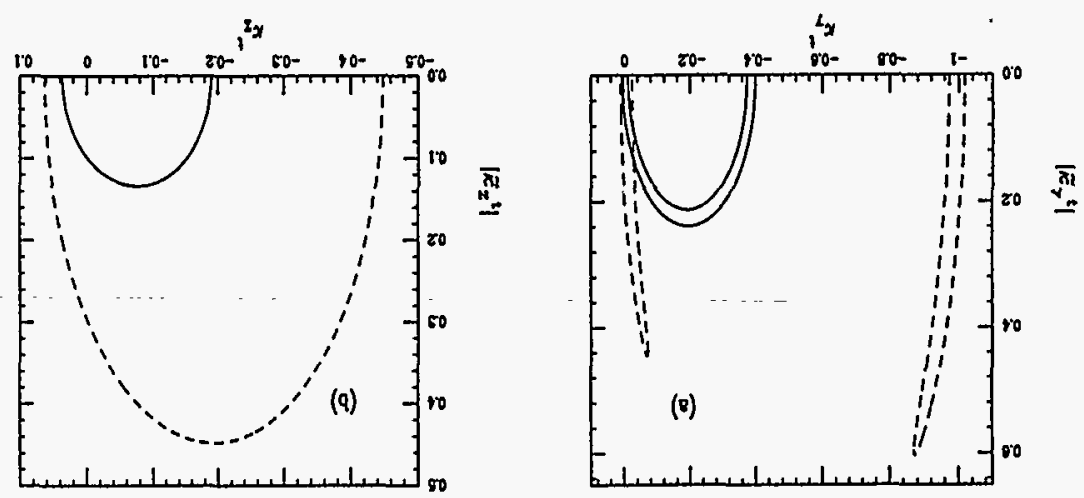

$\cdot[\hbar]$ s!s

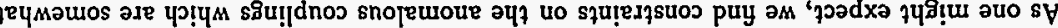

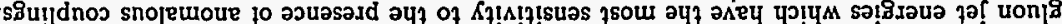

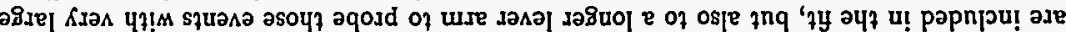

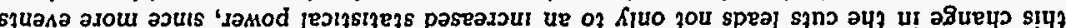

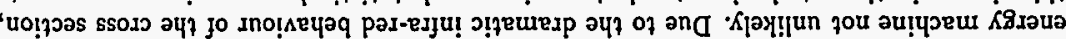

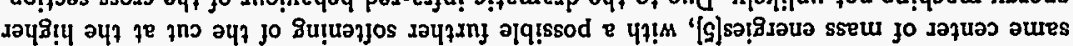

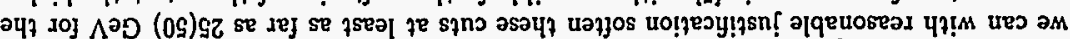

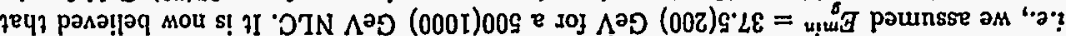

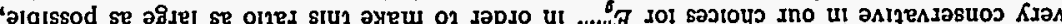
әدәм әм sts

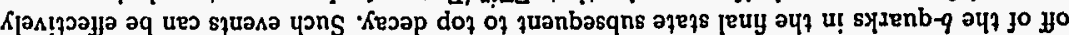

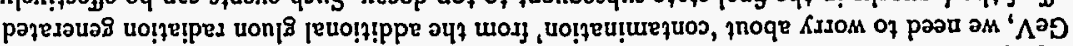

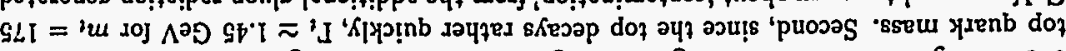

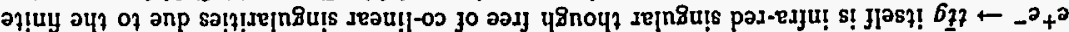

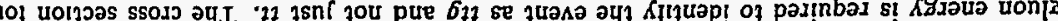



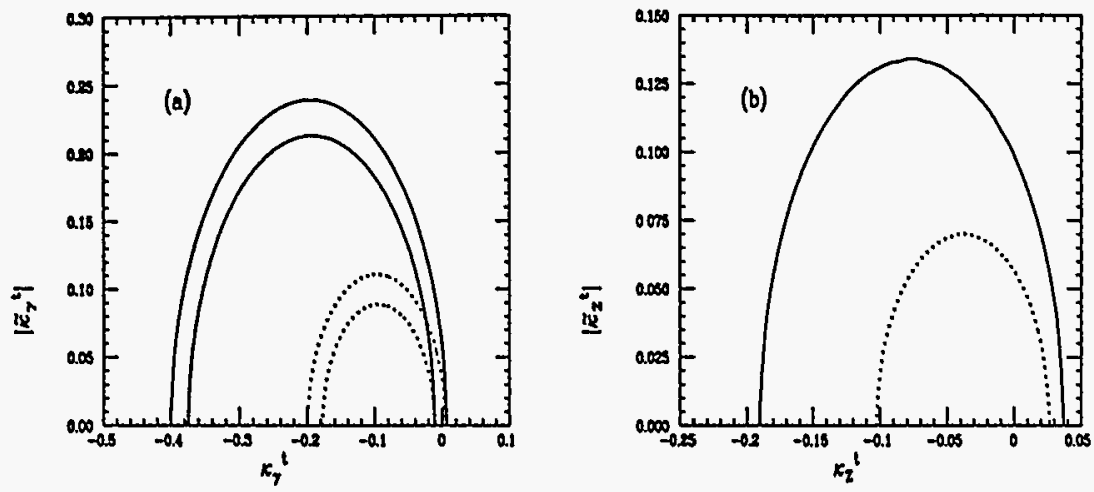

Figure 3: Same as Fig, 1 but now for a 1 (1.5) TeV NLC assuming an integrated luminosity of $100(200) f^{-1}$ corresponding to the solid(dotted) curve.

improve our anomalous coupling constraints for either $\gamma$ or $Z$.

\section{Discussion and Conclusions}

In this report we have shown that the process $e^{+} e^{-} \rightarrow t \bar{t} g$ can be used to obtain stringent limits on the anomalous dipole-like couplings of the top to both $\gamma$ and $Z$ through an examination of the associated gluon energy spectrum. Such measurements are seen to be complementary to those which directly probe the $t \bar{t}$ production vertex. By combining both sets of data a very high sensitivity to the anomalous couplings can be achieved.

\section{ACKNOWLEDGEMENTS}

The author would like to thank P. Burrows, A. Kagan and J.L. Hewett for discussions related to this work.

\section{References}

(1) H.H. Williams, talk given at the Workshop on High Energy Physics at the LHC, Fermilab, March 28-30, 1996.
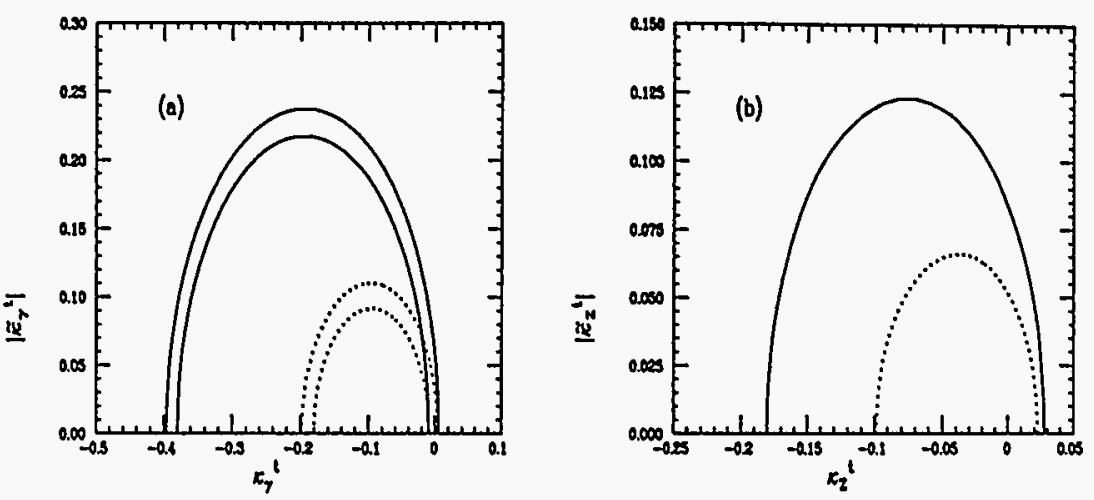

Figure 4: Same as Fig. 3 but now for luminosities of 200(300) $f b^{-1}$ at a $1(1.5) \mathrm{TeV}$ collider corresponding to the solid(dotted) curve.
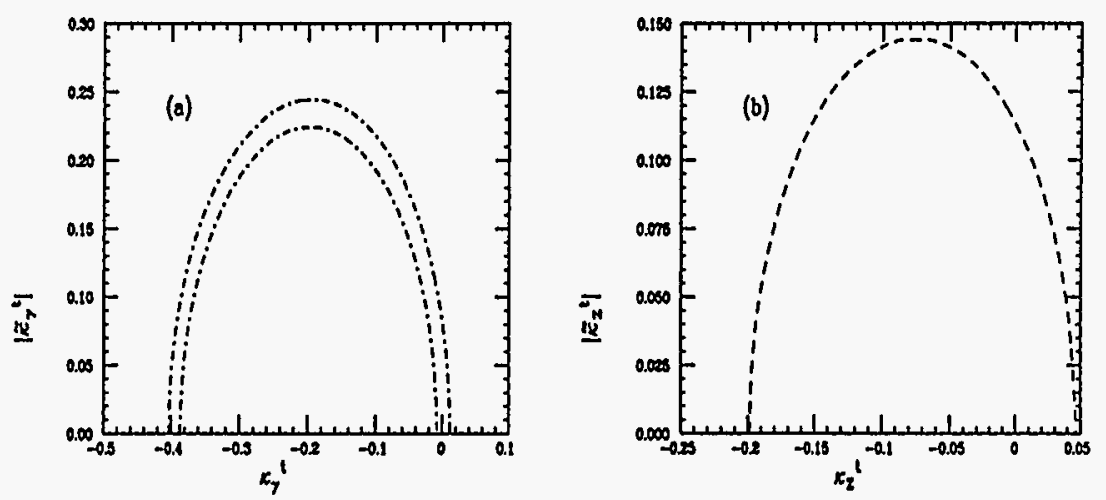

Figure 5: Same as Fig. 3 for the $1 \mathrm{TeV}$ NLC with a $100 \mathrm{fb}^{-1}$ of integrated luminosity but now with a gluon jet cut of $z \geq 0.05$. 
[2] See, for example, the LEP/SLD Working Group report LEPEWWG/96-01, March 1996.

[3] There has been an enormous amount of work in this general subject area; see for example: A. Grifols and A. Mendez, Phys. Lett. B255, 611 (1991) and erratum Phys. Lett. B259, 512 (1991); B. Ananthanarayan and S.D. Rindani Phys. Rev. Lett. 73, 1215 (1994); G. Köpp et al Z Phys, C65, 545 (1995); F. del Aguila and M. Sher, Phys. Lett. B252, 116 (1990); R. Escribano and E. Masso, Phys. Lett. B301, 419 (1993)and Nucl. Phys. 429, 19 (1994); W. Bernreuther, O. Nachtmann and P. Overmann, Phys. Rev. D48, 78 (1993); G. Couture, Phys. Lett. B305, 306 (1993) and Phys. Lett. B272, 404 (1991); G. Domokos et al., Phys. Rev. D32, 247 (1985); T.G. Rizzo, Phys. Rev. D51, 3811 (1995) and Phys. Rev. D53, 2326 (1996); J. Reid, M. Samuel, K.A. Milton and T.G. Rizzo, Phys. Rev. D30, 245 (1984). See also, P.D. Acton et al., OPAL Collaboration, Phys. Lett. B281, 305 (1992); D. Buskulic et al., ALEPH Collaboration, Phys. Lett. B297, 459 (1992); G. Kane, G.A. Ladinsky and C.P. Yuan, Phys. Rev. D45. 124 (1992); C.P. Yuan, Phys. Rev. D45, 782 (1992); D. Atwood, A. Aeppli and A. Soni, Phys. Rev. Lett. 69, 2754 (1992); M. Peskin, talk presented at the Second International Workshop on Physics and Experiments at Linear $e^{+} e^{-}$Collider, Waikoloa, HI, April 1993; M. Peskin and C.R. Schmidt, talk presented at the First Workshop on Linear Colliders, Saariselkä, Finland, September 1991; P. Zerwas, ibid.; W. Bernreuther et al., in Proceedings of the Workshop on $e^{+} e^{-}$Collisions at $500 \mathrm{GeV}$, The Physics Potential, (DESY, Hamburg) ed. by P. Igo-Kemenes and J.H. Kühn, 1992; A. Djouadi, ENSLAPP-A-365-92 (1992); M. Frigeni and R. Rattazzi, Phys. Lett. B269, 412 (1991); R.D. Peccei, S. Persis and X. Zhang, Nucl. Phys. B349, 305 (1991); D.O. Carlson, E. Malkawi and C.-P. Yuan, Phys. Lett. B337, 145 (1994); J.L. Hewett and T.G. Rizzo, Phys. Rev. D49, 319 (1994); T.G. Rizzo, Phys. Rev. D50, 4478 (1994); D. Atwood, A. Kagan and T.G. Rizzo, Phys. Rev. D52, 6264 (1995); K. Cheung, Phys. Rev. D53, 3604 (1996); P. Haberl, O. Nachtmann and A. Wilch, Phys. Rev. D53, 4875 (1996)

[4] T.G. Rizzo, SLAC-PUB-95-6758, to appear in Phys. Rev. D53.

[5] L. Orr, private communication. 
Prospects for and Implications of Measuring the Higgs to Photon-Photon Branching Ratio at the Next Linear $e^{+} e^{-}$Collider

\section{Jolnn F. Gunion and Patrick C. Martin}

Davis Institute for High Energy Physics

Department of Physics, University of California, Davis, CA 95616

\section{Abstract}

We evaluate the prospects for measuring $B R(h \rightarrow \gamma \gamma)$ for a StandardModel-like Higgs boson at the Next Linear $e^{+} e^{-}$Collider. We show that a rough measurement, with accuracy of $\sim \pm 20 \%$, is possible in three or four $L=50 \mathrm{fb}^{-1}$ years if $90 \mathrm{GeV} \leqslant m_{h} \leqslant 130 \mathrm{GeV}$, but only if the electromagnetic calorimeter has very excellent $\gamma \gamma$ invariant mass resolution. The value of even such a rough determination of $B R(h \rightarrow \gamma \gamma)$ in discriminating between the Standard Model and models with new physics is emphasized.

\section{Introduction}

One of the most important tasks of a Next Linear $e^{+} e^{-}$Collider (NLC) will be to detect and study Higgs boson(s). For any observed Higgs boson, extraction of its fundamental couplings and total width in a model-independent manner will be a primary goal. As we shall describe, $B R(h \rightarrow \gamma \gamma)$ is a crucial ingredient in this process in the case of a light Higgs boson with couplings similar to those of the Standard Model (SM) Higgs, $h_{S M}$, and therefore a total width that is too small to be directly observed. The measurement is challenging since $B R\left(h_{S M} \rightarrow \gamma \gamma\right)$ is at best of order a few times $10^{-3}[1,2]$.

In many models, deviations of the couplings and total width of the lightest Higgs boson from expectations for the $h_{S M}$ can be small. Thus, it will be important to estimate the error in the measurement of $B R(h \rightarrow \gamma \gamma)$ when the $h$ is SM-like and how this error propagates into errors in the determination of the couplings and total width. We shall see that it is very likely to be the dominant source of uncertainty. Dramatic deviations of $B R(h \rightarrow \gamma \gamma)$ from SM expectations are, however, also possible, even if the $h$ is very SM-like in its couplings to the SM particles. In particular, large effects can be caused by new particles (fourth generation, supersymmetric, etc.) in the one-loop graphs responsible for the $h \rightarrow \gamma \gamma$ coupling. Thus, the ability of the NLC to measure the $h \rightarrow \gamma \gamma$ branching ratio could prove to be quite critical.

To illustrate the difficulties and ambiguities that can be encountered without knowledge of $B R(h \rightarrow \gamma \gamma)$, let us suppose that the observed Higgs boson is the $h^{0}$ of a supersymmetric model. In grand unified (GUT) scenarios, this $h^{0}$ is light ( $m k^{\circ} \leqslant$ $130 \mathrm{GeV}$ in the minimal supersymmetric model, MSSNI) and decays primarily to $b \bar{b}$. As reviewed in Ref. [2], at the NLC it will be possible to measure $\sigma\left(Z h^{0}\right)$ and $\sigma\left(Z h^{0}\right) B R\left(h^{0} \rightarrow b \bar{b}\right)$ with reasonable precision, $\sim \pm 7 \%$ and $\sim \pm 3 \%$ respectively leading to a $\sim \pm 8-10 \%$ determination of $B R\left(h^{0} \rightarrow b \bar{b}\right)$. But, deviations in all these quantities relative to expectations for the $S M h_{S M}$ could be small even though the $b \vec{b}$ coupling of the $h^{0}$ is substantially enhanced. This could happen as follows. For modest values of the parameter $m_{A^{\circ}}$ ( $m_{A^{\circ}}$ being the mass of the CP-odd Higgs boson) and $\tan \beta>1$ ( $\tan \beta$ being the ratio of the vacuum expectation values of the two Higgs doublets) the MSSM predicts that $\sigma\left(Z h^{0}\right) \simeq \sigma\left(Z h_{S M}\right)$ and that the $h^{0} \rightarrow b \vec{b}$ coupling is enhanced. For example, if $m_{A^{0}} \sim 300 \mathrm{GeV}$ and $\tan \beta=3$ and the other MSSM parameters are chosen such that $m_{h^{0}}=110 \mathrm{GeV}, \Gamma\left(h^{0} \rightarrow b \bar{b}\right)$ is $50 \%$ larger than predicted for the $h_{S M}$ [2]. Nonetheless, $B R\left(h^{0} \rightarrow b \bar{b}\right)$ is only slightly larger than expected due to the fact that the numerator, $\Gamma\left(h^{\circ} \rightarrow b \bar{b}\right)$ and denominator, $\Gamma_{h \circ}^{\text {tot }}$, are both increased by similar amounts. Extra (supersymmetric

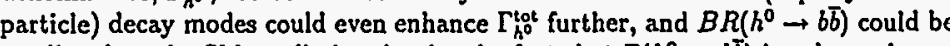
smaller than the SM prediction despite the fact that $\Gamma\left(h^{0} \rightarrow b \bar{b}\right)$ is enhanced.

To extract the $b \bar{b}$ coupling, or equivalently $\Gamma(h \rightarrow b \bar{b})=\Gamma_{h}^{\text {tot }} B R(h \rightarrow b \bar{b})$ we require a determination of $\Gamma_{b}^{\text {tot }}$. If $b \bar{b}$ is the dominant Higgs decay (as for $m_{h} \leqslant 140 \mathrm{GeV}$ in the SM), $\Gamma_{h}^{\text {tol }}$ is too small for direct measurement via mass peak reconstruction using the recoil mass technique in the $Z h$ mode. The only technique for extracting $\Gamma_{h}^{\text {tot }}$ using NLC data alone requires measuring $B R(h \rightarrow \gamma \gamma)$. The procedure is:

- Compute (accuracy $\pm 8-10 \%$, as noted above)

$$
B R(h \rightarrow b \bar{b})=\frac{\sigma(Z h) B R(h \rightarrow b \bar{b})}{\sigma(Z h)} ;
$$

- Measure at the associated $\gamma \gamma$ collider facility the rate for $\gamma \gamma \rightarrow h \rightarrow b \bar{b}$ (accuracy $\pm 5 \%$ ) proportional to $\Gamma(h \rightarrow \gamma \gamma) B R(h \rightarrow b \bar{b})$ and compute (accuracy $\pm 11-13 \%)$

$$
\Gamma(h \rightarrow \gamma \gamma)=\frac{\Gamma(h \rightarrow \gamma \gamma) B R(h \rightarrow b \bar{b})}{B R(h \rightarrow b \bar{b})} ;
$$

- Measure $Z^{\star} \rightarrow Z h$ followed by $h \rightarrow \gamma \gamma$, i.e. $\sigma(Z h) B R(h \rightarrow \gamma \gamma)$, and compute

$$
B R(h \rightarrow \gamma \gamma)=\frac{\sigma(Z h) B R(h \rightarrow \gamma \gamma)}{\sigma(Z h)} ;
$$

- Finally, compute

$$
\Gamma_{h}^{\text {tot }}=\frac{\Gamma(h \rightarrow \gamma \gamma)}{B R(h \rightarrow \gamma \gamma)}
$$


Our goal is to determine the accuracy associated with the measurement required in Eq. (3).

For a SM-like Higgs boson with $m_{h} \lesssim 130 \mathrm{GeV}, B R(h \rightarrow \gamma \gamma)$ can also be extracted from LHC data with a statistical accuracy of about $\pm 15 \%$ [2], assuming that ATLAS and CMS combined accumulate a total luminosity of $L=600 \mathrm{fb}^{-1}$ (corresponding to $\geq 3$ years of running at high instantaneous luminosity). If the accuracy of a measurement at the NLC is comparable to or better than the LHC accuracy, then correspondingly greater precision in extracting the total Higgs width and the Higgs couplings will be possible.

\section{Signal and Background in the Standard Model}

We consider SM Higgs masses in the range $50-150 \mathrm{GeV}$. (Note that the Higgs mass will be measured very precisely using the missing-mass technique in the $Z h_{S M}$ mode.) The $h_{S M} \rightarrow \gamma \gamma$ branching ratios for masses in this range are tabulated in Table 1 . We presume that the only background $(B)$ to the $e^{+} e^{-} \rightarrow Z h_{S M} \rightarrow Z_{\gamma \gamma}$ signal $(S)$ is the irreducible one, $e^{+} e^{-} \rightarrow Z_{\gamma \gamma}$, for which we employ an exact signal $(S)$ is the irreducible one, $e^{+} e^{-} \rightarrow Z \gamma \gamma$, for which we employ an exact
matrix element. ${ }^{1}$ Note that all decay modes of the $Z$ can be included without matrix element. 1 Note that all decay modes of the $Z$ can be included without introducing additional background by requiring that the mass reconstructed from the $\gamma_{1,2}$ four-momenta and incoming energy be within, say, $\pm 5 \mathrm{GeV}$ of $m_{z}$. We compute the $e^{+} e^{-} \rightarrow Z h_{S M} \rightarrow Z_{\gamma \gamma}$ signal rate for a small detector-res dependent (see later discussion) interval of $M_{r}$ centered on $m_{h_{S M}}$, chosen so as to maximize $S / \sqrt{B}$. Further cuts are specified in terms of $E_{\gamma_{1,2}}, p_{T}^{\gamma_{1,2}}$ and $y_{\gamma_{1,2}}$, the energies, transverse momenta and rapidities of the two photons in the $e^{+} e^{-}$ center-of-mass. For all values of $m_{h_{S M}}$, we required

$$
\left|y_{\gamma_{1}, 2}\right| \leq 2.5, \quad p_{T}^{\eta_{1}, 2} \geq \frac{m_{h}}{4}, \quad 0.1 \leq E_{\eta_{1}} / E_{\eta_{2}} \leq 10 .
$$

The cut on the rapidities of the individual photons turns out to be slightly more effective in maximizing $S / \sqrt{B}$ than a cut on the photon angles (whether applied in the overall center-of-mass or in the Higgs rest frame). The $p_{T}$ cut on the individual photons is designed to eliminate kinematic configurations in which the $Z_{\gamma \gamma}$ background is large due to a nearby soft or collinear photon singularity. The restriction on the ratio of energies is designed to eliminate kinematic configurations in which one photon is relatively soft while the other is fairly hard, as typical for the $Z_{\gamma \gamma}$ background. We then searched for additional cuts that maximize $S / \sqrt{B}$. We found that a particularly good choice is to require $p_{T}^{\gamma_{1}}+p_{T}^{\eta_{2}} \geq p_{T}^{\mathrm{min}}$.

In order to optimize $S / \sqrt{B}$ for the $Z h_{S M} \rightarrow Z_{\gamma \gamma}$ signal, both the energy, $\sqrt{s}$, th wich to run the collider and $p_{T}^{\mathrm{min}}$ must be adjusted as a function of $m_{h_{s}}$. For fixed $p$ in the background (which is largely radiative in nature and thus does not behave in the point-like $1 / s$ fashion) decreases slowly as $\sqrt{s}$ decreases. In contrast,

\footnotetext{
INote that beam polarization will not aid in suppressing this background relative to the signal.
}

the signal (which is proportional to the product of a threshold factor times the point-like $1 / \mathrm{s}$ behavior arising from the $Z$ propagator) tends to increase rapidly as $\sqrt{s}$ is decreased until reaching a maximum somewhat above threshold. We find that it is best to choose $\sqrt{s}$ to lie near this maximum. The rough optimal values, $\sqrt{s_{o p e}}$ are tabulated in Table 1 as a function of $m_{h_{S M}}$. For each $m_{h_{S M}}$ and its corresponding $\sqrt{3}_{\text {opt }}$ we then performed a rough scan for the $p_{T}^{\text {min }}$ that maximizes $S / \sqrt{B}$; these too are tabulated in Table 1 . We believe that any improvements in $S / \sqrt{B}$ that could be achieved by a more detailed search or further refinements in the cuts will be small.

Three different choices for electromagnetic calorimeter resolution are considered:

- I) Resolution like that of the CMS lead tungstate crystal [3], with $\Delta E / E=$ $2 \% / \sqrt{E} \oplus 0.5 \% \oplus 0.2 \% / E$;

- II) Resolution of $\Delta E / E=5 \% / \sqrt{E} \oplus 0.5 \%$; and

- III) Resolution of $\Delta E / E=10 \% / \sqrt{E} \oplus 1 \%$, an 'optimistic' limit of current preliminary design prototypes for the NLC and JLC detectors $[4,5]$.

With regard to III, we note that the NLC Physics summary report cited in Ref. [4] lists resolution of $\triangle E / E=12 \% / \sqrt{E} \oplus 1 \%$, somewhat worse than we employ; the $10 \% / \sqrt{E}$ is that appearing in the simulation package accessed via the WWW homepage. The resolution for the JLC-I detector design, Ref. [5], is $\Delta E / E=$ $15 \% / \sqrt{E} \oplus 1 \%$, substantially worse than we employ. Thus, case III is a somewhat optimistic version of current electromagnetic calorimeter plans. We shall see that resolution at least as good as that of case II will be required for the $Z h_{S M} \rightarrow Z_{\gamma \gamma}$ channel.

For each resolution and each $m_{h_{S M}}$ we have performed a rough scan for the $M_{\gamma}$ interval $\pm \frac{1}{2} \Delta M_{T}$ about $m_{h_{S M}}$ that maximizes $S / \sqrt{B}$. These intervals are given in Table 1. The optimal values for $\Delta M_{\gamma}$ are only slightly influenced by the previously discussed cuts. Note that as $m_{h_{S M}}$ increases past $m_{h_{S N}} \sim 90-100 \mathrm{GeV}$, where $B R\left(h_{S M} \rightarrow \gamma \gamma\right)$ rises to its maximum, it becomes advantageous to take a slightly smaller percentage of the total signal. In general, $S / \sqrt{B}$ varies rather slowly as $\Delta M_{\tau}$ is varied in the vicinity of the optimal value given.

In Fig. 1 a we plot $S / \sqrt{B}$ as a function of $m_{h_{S M}}$ as computed from the $L=$ $50 \mathrm{fb}^{-1} S$ and $B$ values given in Table 2. Fig. Ib gives the number of $L=50 \mathrm{fb}^{-1}$ years, $N_{\text {years }}$, required for $S / \sqrt{B}=5$. Results for all three calorimeter resolution choices are given. These plots make it clear that a high statistics signal is achieved within a reasonable number of years only if $90 \mathrm{GeV} \lesssim m_{h_{S M}} \leqslant 130 \mathrm{GeV}$ and only if the calorimeter has excellent resolution. Resolution choice (III), that is generically used in current discussions of the NLC detector, would not yield a $5 \sigma$ signal in less than 5 years for any Higgs mass. 
Table 1: We tabulate $\sqrt{s}_{\text {ops }}, B R\left(h_{S M} \rightarrow \gamma \gamma\right), p_{T}^{\min }(\mathrm{GeV})$, and $\Delta M_{\mathrm{r}}(\mathrm{GeV})$ as a function of $m_{h_{S M}}(\mathrm{GeV})$. Results for $\Delta M_{\pi}$ are given for all three resolution choices, I, II and III

\begin{tabular}{|c|c|c|c|c|c|c|}
\hline$m_{h_{S M}}$ & $B R\left(h_{S M} \rightarrow \gamma \gamma\right)$ & $\sqrt{s}_{\text {opt }}$ & $p_{T}^{\text {min }}$ & $\Delta M_{\gamma \gamma}(\mathrm{I})$ & $\Delta M_{\gamma \gamma}(\mathrm{II})$ & $\Delta M_{\gamma(}(\mathrm{III})$ \\
\hline 50 & $3.5 \times 10^{-4}$ & 155 & 45 & 1.00 & 1.00 & 2.00 \\
60 & $5.3 \times 10^{-4}$ & 165 & 55 & 1.20 & 1.20 & 2.40 \\
70 & $7.5 \times 10^{-4}$ & 175 & 65 & 1.12 & 1.40 & 2.80 \\
80 & $1.0 \times 10^{-3}$ & 190 & 70 & 1.28 & 1.60 & 2.88 \\
90 & $1.4 \times 10^{-3}$ & 200 & 75 & 1.44 & 1.44 & 3.24 \\
100 & $1.8 \times 10^{-3}$ & 215 & 90 & 1.60 & 1.60 & 3.60 \\
110 & $2.2 \times 10^{-3}$ & 225 & 100 & 1.32 & 1.76 & 3.52 \\
120 & $2.6 \times 10^{-3}$ & 240 & 105 & 1.44 & 1.92 & 3.84 \\
130 & $2.6 \times 10^{-3}$ & 250 & 120 & 1.56 & 2.08 & 4.16 \\
140 & $2.2 \times 10^{-3}$ & 265 & 125 & 1.68 & 2.24 & 4.48 \\
150 & $1.6 \times 10^{-3}$ & 275 & 140 & 1.80 & 2.40 & 4.80 \\
\hline
\end{tabular}

If the collider is not run at $\sqrt{s}$, observation of $h_{S M} \rightarrow \gamma \gamma$ at the $5 \sigma$ statistical level becomes significantly more diffcult. For example, consider $m_{h s u}=120 \mathrm{GeV}$ and suppose one insists on accumulating $L=50 \mathrm{fb}^{-1}$ while running at $\sqrt{s}=$ $500 \mathrm{GeV}$. Adopting resolution (I), the associated $\Delta M_{r \gamma}=1.44 \mathrm{GeV}$ (see Table 1), and the standard cuts of Eq. (5), we scanned in $p_{T}^{\min }$ to find the best $S / \sqrt{B}$ value. $S$ and $B$ decrease as $p_{T}^{\min }$ is increased in such a way that $S / \sqrt{B}$ slowly increases, reaching a maximum of $S / \sqrt{B} \simeq 2.53$ at $p_{T}^{\min }=210 \mathrm{GeV}$, with $S \simeq 3.25$ and $B \simeq 1.65$. At lower $p_{T}^{\mathrm{min}}$, the signal rate slowly becomes less marginal, but $S / \sqrt{B}$ declines; e.g. at $p_{T}^{\min }=125 \mathrm{GeV} S \simeq 4.7, B \simeq 7$ and $S / \sqrt{B} \simeq 1.7$. The above situation should be compared to the $S / \sqrt{B} \sim 4$ result achieved with $S \sim 17$ and $B \sim 20$ by running at $\sqrt{s}_{\text {opt }}=240 \mathrm{GeV}$ and taking $p_{T}^{\min }=105 \mathrm{GeV}$.

Since the $h_{S M}$ will already have been detected in other modes, the statistical significance of the signal is not as crucial as the accuracy in the determination of $B R(h \rightarrow \gamma \gamma)$. The accuracy (estimated as $\sqrt{S+B} / S$ ) achievable for the measurement of $\sigma\left(Z h_{S M}\right) B R\left(h_{S M} \rightarrow \gamma \gamma\right)$ after three $L=50 \mathrm{fb}^{-1}$ years is plotted as a function of $m_{h_{S M}}$ in Fig. 2 for each of the three calorimeter resolution choices. At best, a $20 \%$ measurement is possible after three to four years of running at $\sqrt{s}_{\text {opt }}$ for the excellent resolution cases (I) and (II) in the $90 \lesssim m_{h_{S M}} \leqslant 130 \mathrm{GeV}$ range. Since the statistical error for $\sigma\left(Z h_{S M}\right)$ is only of order $\pm 7 \%$, the error in $B R\left(h_{S M} \rightarrow \gamma \gamma\right)$ is dominated by the $Z h_{S M} \rightarrow Z_{\gamma \gamma}$ error and would then be of order $\pm 21 \%$. Given that the accuracy scales statistically, to achieve $\pm 10 \%$ would require an unacceptable $z 12$ years of running.

The $\pm 20 \%$ accuracy for $B R\left(h_{S M} \rightarrow \gamma \gamma\right)$ achievable with three to four years of running is not quite as good as the earlier-noted $\pm 15 \%$ statistical precision expected

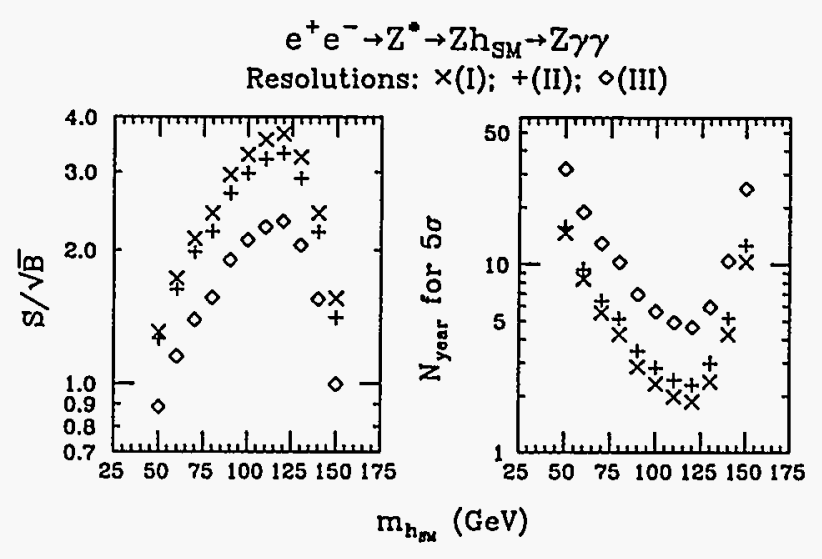

Figure 1: Results for $S / \sqrt{B}$ at $L=50 \mathrm{fb}^{-1}$ and for the number, $N_{\text {years }}$ of $L=50 \mathrm{fb}^{-1}$ years required for $S / \sqrt{B}=5$, as a function of $m_{h_{S M}}$.

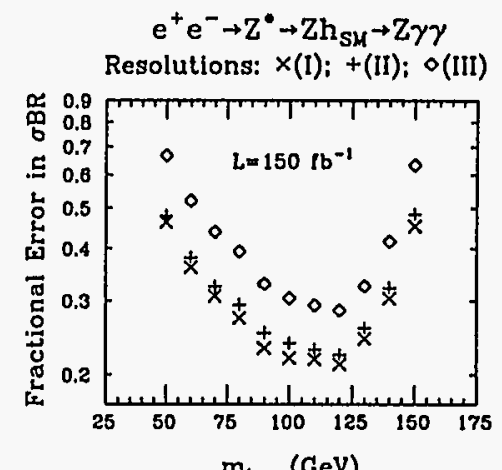

$\mathrm{m}_{\mathrm{h}_{\text {gex }}}(\mathrm{GeV})$

Figure 2: The fractional error in the measurement of $\alpha\left(Z h_{S M}\right) B R\left(h_{S M} \rightarrow\right.$ $\gamma \gamma$ ) as a function of $m_{h_{s y}}$, assuming three years of $L=50 \mathrm{fb}^{-1}$ yearly integrated luminosity at $\sqrt{s_{\text {opt }}}$. 
Table 2: For resolution choices I, II and III, we tabulate $S$ and $B$ as a function of $m_{h_{s M}}(\mathrm{GeV})$ for the $\sqrt{s}_{\mathrm{ops}}, \Delta M_{r y}$ and $p_{T}^{\mathrm{mnn}}$ choices of Table $1 . L=50 \mathrm{fb}^{-1}$ is assumed.

\begin{tabular}{|c|c|c|c|c|c|c|}
\hline$m_{h_{S M}}$ & $S(I)$ & $B(I)$ & $S(I I)$ & $B(I I)$ & $S(I I I)$ & $B(I I I)$ \\
\hline 50 & 17.6 & 179 & 16.9 & 179 & 16.8 & 359 \\
60 & 18.2 & 111 & 17.2 & 111 & 17.2 & 223 \\
70 & 16.1 & 57.6 & 16.7 & 72.0 & 16.7 & 144 \\
80 & 18.9 & 60.8 & 19.2 & 75.9 & 18.3 & 137 \\
90 & 21.7 & 53.9 & 19.7 & 53.9 & 20.9 & 121 \\
100 & 19.5 & 35.3 & 17.7 & 35.3 & 18.8 & 79.5 \\
110 & 16.0 & 20.3 & 16.7 & 27.1 & 16.6 & 54.2 \\
120 & 16.7 & 20.8 & 17.4 & 27.7 & 17.3 & 55.5 \\
130 & 12.0 & 13.7 & 12.4 & 18.3 & 12.4 & 36.5 \\
140 & 9.2 & 14.4 & 9.6 & 19.1 & 9.6 & 38.3 \\
150 & 4.9 & 9.8 & 5.1 & 13.1 & 5.1 & 26.2 \\
\hline
\end{tabular}

for $m_{h_{S M}}$ in the range $90-130 \mathrm{GeV}$ using LHC data. However, systematic errors in the LHC-based determination could be significant. This is because $B R\left(h_{S M} \rightarrow \gamma \gamma\right)$ is obtained from the $p p \rightarrow W h_{S M} \rightarrow W_{\gamma \gamma}$ event rate by using parton distribution is obtained from the $p p \rightarrow W h_{S M} \rightarrow W \gamma \gamma$ event rate by using parton distribution
function information to extract $\Gamma\left(h_{S M} \rightarrow W W\right) B R\left(h_{S M} \rightarrow \gamma \gamma\right)$ and then dividing by $\Gamma\left(h_{S M} \rightarrow W W-\right.$ - as determined at the NLC (from the $W W \rightarrow h_{S M}$ fusion and $Z h_{S M}$ cross sections). ${ }^{2}$ Thus, combining and/or comparing the NLC and LHC extractions of $B R\left(h_{S M} \rightarrow \gamma \gamma\right)$ could prove to be very useful.

\section{Final Remarks and Conclusions}

For a SM-like Higgs boson, $h$, we have evaluated the prospects for measuring $B R(h \rightarrow \gamma \gamma)$ in the $e^{+} e^{-} \rightarrow Z^{*} \rightarrow Z h \rightarrow Z \gamma \gamma$ mode at a future NLC. The measurement is difficult. A statistical accuracy of about $\pm 20 \%$ is the best that can be hoped for, and this can only be achieved for $90 \lesssim m_{h} \lesssim 130 \mathrm{GeV}$ after three to four years of $L=50 \mathrm{fb}^{-1} /$ year at the machine energy, $\sqrt{s}$ ope that maximizes the $Z h$ cross section, and only for excellent calorimeter resolution. In particular, the calorimeter resolution must be considerably better than that of resolution case III, which is on the optimistic end of what is currently being discussed for the NLC detector. The $\pm 20 \%$ error in $B R(h \rightarrow \gamma \gamma)$ will dominate the error in the calculation of $\Gamma_{h}^{\text {lot }}=\Gamma(h \rightarrow \gamma \gamma) / B R(h \rightarrow \gamma \gamma)$ (where $\Gamma(h \rightarrow \gamma \gamma)$ is determined using $\gamma \gamma \rightarrow h$ collisions) and, then again, the error in extracting the potentially revealing $h \rightarrow b \bar{b}$ partial width via $\Gamma(h \rightarrow b \bar{b})=\Gamma_{h}^{\text {tot }} B R(h \rightarrow b \bar{b})$.

${ }^{2}$ There are other techniques, but these are expected to have statistical error $z \pm 25 \%$ [2].
Despite this limited accuracy, it should be emphasized that the $90-130 \mathrm{GeV}$ mass range is quite possibly that in which the $h^{\circ}$ of the minimal supersymmetric model would be found. We have noted that if superpartners are heavy, $m_{10}$ is moderate in size $\left(m_{A^{\circ}} \leqslant 300 \mathrm{GeV}\right)$ and $\tan \beta$ is large then $\Gamma\left(h^{\circ} \rightarrow b \bar{b}\right)$ would be $50 \%$ or more larger than predicted for the $h_{S M}, a \geq 2.5 \sigma$ level of deviation for $\pm 20 \%$ error. We emphasized the fact that a less direct probe of the $h \rightarrow b \bar{b}$ coupling, such as the more easily measured $B R(h \rightarrow b \bar{b})$, would be subject to ambiguous interpretation.

Even a rough measurement of $B R(h \rightarrow \gamma \gamma)$ could be important. For example, if superpartners are light, the corresponding one-loop graphs contributing to the $h^{0} \rightarrow \gamma \gamma$ coupling could result in a large deviation of the branching ratio from the heavy superpartner prediction. A very dramatic deviation from SM or MSSM expectations would arise if there is a fourth generation; for a Higgs with SM-like couplings to $W W, B R(h \rightarrow \gamma \gamma)$ would be much smaller than anticipated due to increased cancellation between the $W$-loop and the sum of all the fermion loops.

As we have noted, LHC data for Wh production followed by $h \rightarrow \gamma \gamma$ can be combined with the predicted magnitude for $\sigma(W h)$ (as obtained by using parton distributions and NLC information on the $h W W$ coupling) to extract an independent value for $B R(h \rightarrow \gamma \gamma)$. For a SM Higgs in the $90-130 \mathrm{GeV}$ range, the statistical accuracy resulting from this procedure could be as small as $\pm 15 \%$, although systematic error might be significant. By combining this determination with that obtained directly at the NLC, increased accuracy for $B R(h \rightarrow \gamma \gamma)$ would be possible.

Thus, despite the apparent difficulty of measuring $B R(h \rightarrow-\gamma \gamma)$ with good accuracy at the NLC, the importance of this branching ratio is such that serious consideration should be given to upgrading the current plans for the NLC detector to incorporate a calorimeter with the very excellent resolution that would at least allow a rough measurement.

Finally, we note that if machine and/or physics considerations do not allow substantial luminosity to be accumulated at $\sqrt{s}$ opt after a light Higgs boson has been discovered, then one can also consider measuring $B R(h \rightarrow \gamma \gamma)$ with the $h$ produced via the $W W$-fusion production mechanism. At $\sqrt{3}=500 \mathrm{GeV}$, the $W W \rightarrow h$-fusion rate is larger than the $Z^{\star} \rightarrow Z h$ rate, and it could be that the accuracy achieved would be better than for the $Z h$ production mode. This is currently under study.

\section{Acknowledgements}

This work was supported in part by Department of Energy under grant No. DEFG03-91ER40674 and by the Davis Institute for High Energy Physics. 


\section{References}

[1] See J.F. Gunion, H.E. Haber, G.L. Kane and S. Dawson, The Higgs Hunters Guide, Addison-Wesley Publishing, and references therein.

[2] J.F. Gunion, A. Stange, and S. Willenbrock, Weakly.Coupled Higgs Bosons, preprint UCD-95-28 (1995), to be published in Electroweak Physics and Beyond the Standard Model, World Scientific Publishing Co., eds. T. Barklow, S. Dawson, H. Haber, and J. Siegrist.

[3] CMS Technical Proposal, CERN/LHCC 94-38, LHCC/P1 (1994).

[4] NLC Physics Summary Report, Physics Goals of the Next Linear Collider, in preparation; Simulation homepage: http:// nlc.physics.upenn.edu /nlc /software /software.html.

[5] JLC-I, JLC Group, KEK Report 92-16, as summarized by K. Fujii, Proceedings of the 2nd International Workshop on "Physics and Experiments with Lienar $e^{+} e^{-}$Colliders", eds. F. Harris, S. Olsen, S. Pakvasa and X. Tata, Waikoloa, HI (1993), World Scientific Publishing, p. 782. 
Determination of Supersymmetric Particle Production Cross Sections and Angular Distributions

at a High Energy Linear Collider

Howie Baer, Richard Dubois, Sal Fahey, Steve Manly,

Ray Munroe, Uriel Nauenberg, Xerxes Tata, David Wagner.

We have calculated the cross section of Standard Model processes and of the production of Supersymmetric (SUSY) particles as a function of the electron longitudinal polarization We have also calculated the angular differential cross section for an $80 \%$ right handed electron process. These calculations have been carried out for an $E_{c m}=0.5$ and $1.0 \mathrm{TeV}$. These curves, shown below, are to serve as the basis for our various analysis efforts to determine the ability of a linear collider and a reasonably designed detector to determine what is being observed These rates are calculated within the framework of the minimal supergravity model with gauge coupling unification and radiative electroweak symmetry breaking. This model has been imbedded within the event generator program ISAJET [1]. It assumes the Minimal Supersymmetric Standard Model (MSSM) Lagrangian to be the low energy $\left(E<M_{\text {Gur }}\right.$ ) effective theory which follows from some more fundamental Supergravity SUGRA theory, which may, in turn, be the low energy effective theory of a yet more fundamental one like string theory. Thus, we assume gauge coupling unification and radiative electroweak symmetry breaking, along with universal GUT scale soft SUSY breaking terms at a scale $M_{X}$ taken to be equal to the scale at which the $U(1)$ and $S U(2)$ gauge couplings unify. The parameters of the model which we use to determine the relevant quantitities are:

- $m_{0}$, the universal scalar mass

- $m_{1 / 2}$, the universal gaugino mass

- $A_{0}$, the universal trilinear term

- $\tan \beta$, the ratio of the Higgs field vaccum expectation value.

- the sign of the SUSY conserving Higgsino mixing term $\mu$.

Along with $m_{i}$, these parameters suffice to determine the weak-scale Supersymmetric particle (sparticle) masses and mixings, which in turn allow all sparticle and Higgs boson production cross sections and decay modes to be calculated. The cross sections and decay modes have also been embedded into the event generator ISAJET. As part of this project, we have included both Standard Model (SM) and SUSY cross sections in such a manner that the effects of longitudinally polarized beams on the production cross sections can be included.

The cross sections and angular distributions were calculated for a set of the supersymmetric parameters defined above. This was done in order to consider various experimental scenarios that would represent various degrees of difficulty in the observation and determination of the sparticle properties. Five SUSY cases are being studied with $\mathrm{m}_{t}$ set to $175 \mathrm{GeV}$; they are:
1. $\left(m_{0}, m_{1 / 2}, A_{0}, \tan \beta, \operatorname{sgn}(\mu)\right)=(400,200,0,2,-1)$ : dominantly chargino production.

2. $\left(m_{0}, m_{1 / 2}, A_{0}, \tan \beta, \operatorname{sgn}(\mu)\right)=(100,300,0,2,-1)$ : dominantly selectron production

3. $\left(m_{0}, m_{1 / 2}, A_{0}, \tan \beta, \operatorname{sgn}(\mu)\right)=(200,100,0,2,-1):$ mixed chargino/selectron/sneutrino production,

4. a non-SUGRA point with $\left(m_{\bar{g}}, m_{\tilde{q}}, m_{\tilde{q}_{i}}\right)=(900,1000,1000)$ and $\left(\tan \beta, \mu, m_{A}\right)=(10$. 100,200): chosen so that heavy, pseudo-scalar and charged Higgs production is accessible,

5. $\left(m_{0}, m_{1 / 2}, A_{0}, \tan \beta, \operatorname{sgn}(\mu)\right)=(300,150,-600,2,+1)$ : contains top squark production,

For reasons beyond our editorial control, the nomenclature for supersymmetric particles differs slightly between this article and the main text. The particle referred to here as $\widetilde{W}_{i}, \widetilde{Z}_{i}$, $H_{l}, H_{h}, H_{p}$, are refered to in Chapter 2 as $\tilde{\chi}_{i}^{+}, \tilde{\chi}_{i}^{0}, h^{0}, H^{0}$, and $A^{0}$, respectively.

In Fig. 1 we show the behavior of the cross section for Standard Model processes as a function of the longitudinal electron polarization. In Figs. 2,3, 4 we show the cross section of Supersymmetric particle production for various values of the electron polarization [3] in the five. regions of parameter space under study. The rate for the Standard Model processes $\mathrm{WV}^{+} \mathrm{W}^{-}$ and $e \nu \mathrm{W}$, which form the main background in our supersymmetric particle searches at $\mathrm{E}_{c m}=$ $500 \mathrm{GeV}$, can be significantly reduced by using a right-polarized electron beam. as can be seen, in the case of the $\mathrm{W}^{+} \mathrm{W}^{-}$process from Fig. 1 . As can be seen from Figs. 2, 3, 4. this can be accomplished with little loss of signal for right-handed sleptons, sneutrinos, and $\mathrm{ZH}_{\text {/ }}$ or stop accomplished with little loss of signal for right-handed sleptons, sneutrinos, and $\mathrm{ZH}_{\ell}$ or stop
production. Within the SUGRA framework, this strategy for reducing the background is not as effective for charginos, which are frequently gaugino like, and for $\bar{W}_{1} \bar{W}_{1}$ final states. since their cross sections are likewise reduced for right-handed electron beams [2].

Rate changes by an order of magnitude are possible by changing the helicity of the electron. This observation has major implications in our ability to interpret the signals being observed. Hence one of our main analysis thrusts is to understand the effect of having a longitudinally polarized electron. Crucial in our study is to understand the behavior of the background given by the main $S M$ processes which we consider to be $\mathrm{e}^{+} \mathrm{e}^{-} \rightarrow \mathrm{W}^{+} \mathrm{W}^{-}$, evW $\mathrm{Z}^{0} \mathrm{Z}^{0}$, and $f \bar{f}$. The cross sections for the background (SM) processes are usually larger than that for the supersymmetric processes. Nevertheless most of this difference is due to the $\mathrm{W}^{+} \mathrm{WW}^{-}$and $e \nu \mathrm{WW}$ (which has the same behavior as WW production) production process and, as can be seen from Fig. 1, this can be reduced subtantially by using right handed polarized electrons. Although presently we have only achieved $77 \%$ in the maximum of the magnitude of the longitudinal polarization, if we can reach $90 \%$ then the $\mathrm{W}^{+} \mathrm{W}^{-}$and $e \nu W$ cross sections can be reduced by an order of magnitude by using right handed electrons.

Understanding the angular differential cross section of the various processes is also important since the background and the supersymmetric signals have vastly different angular distributions. In Figs. 5 we show the differential cross-section for the main background processes $\mathrm{e}^{+} \mathrm{e}^{-} \rightarrow \mathrm{W}^{+} \mathrm{W}^{-}$and $\mathrm{Z}^{0} \mathrm{Z}^{0}$. We note that for both processes the final states appear mainly in the very forward and backward directions and, in particular, the $\mathrm{W}^{-}$is mainly in the direction 
of the incident electron. This is due to the fact that both of these processes are driven mainly by t-channel exchange of massless particles. In Figs. $6,7,8,9,10$ we show the differential cross section for supersymmetric particle production for points 1.5 in our parameter space, where we note that they are much less peaked than the background. Hence the angular distribution of the observed data may be helpful to separate the signal from the background.

We are currently engaged in examining features of the above scenarios and, in particular, how the signal to background varies in a realistic detector where the outgoing particle directions are smeared according to realistic resolutions and efficiencies.

\section{References}

11) F. Paige, S. Protopopescu, in Supercollider Physics, p. 41 ed. D. Soper (World Scientific 1986); H. Baer, F. Paige, S. Protopopescu, adn X. Tata, in Proceedings of the Workshop on Physics at Current Accelerators and Supercolliders, J. Hewett, A. White, and D. Zeppenfeld, (Argonne National Laboratory, 1993).

[2] HELAS: Helicity Amplitude Subroutines for Feynman Diagram Evaluations, H. Mu rayama, I. Watanabe and K. Hagiwara, KEK-91-11 (1992).

[3] The definition of $P_{L}$ is such that the amount of left handed polarization is given by $\left(1+P_{L}\right) / 2$. So, for example, if $P_{L}=-0.6$ we have the case where the electron beam is made up of $80 \%$ right-handed electrons and $20 \%$ left-handed.

[4] H. Baer, C. H. Chen, F. Paige and X. Tata, FSU-HEP-951215 (1995).

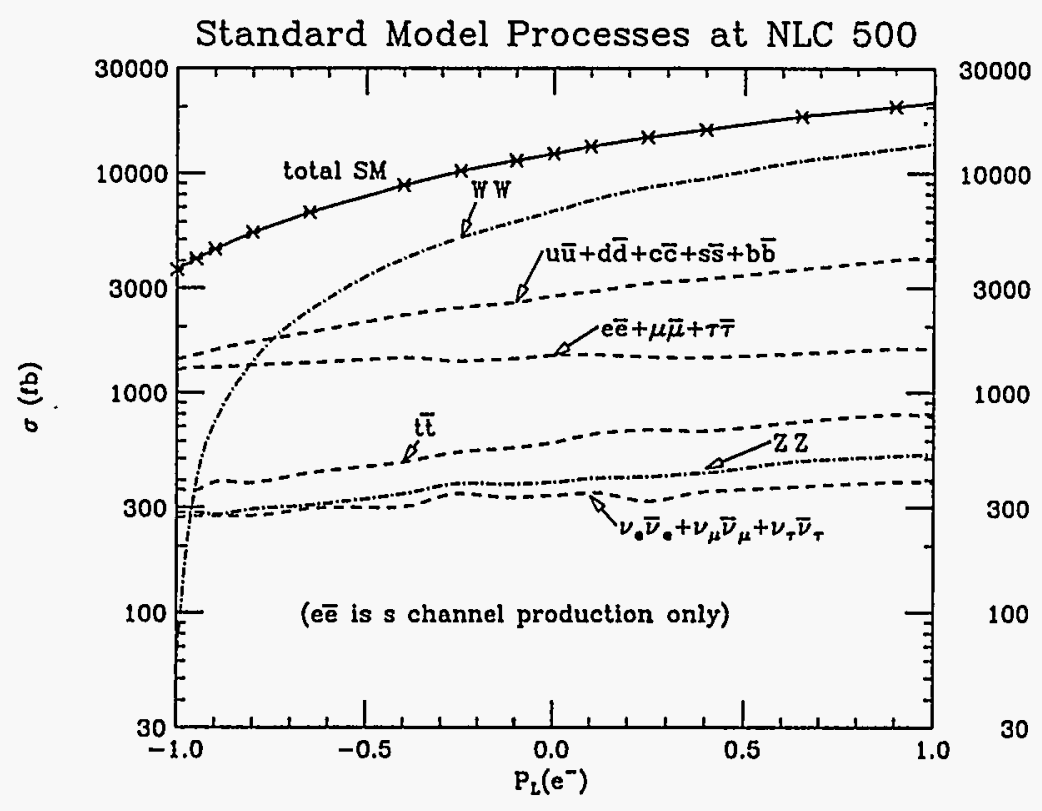

Figure 1: The cross section of Standard Model processes at $\mathrm{E}_{\mathrm{cm}}=0.5 \mathrm{TeV}$ as a function of the electron polarization. 


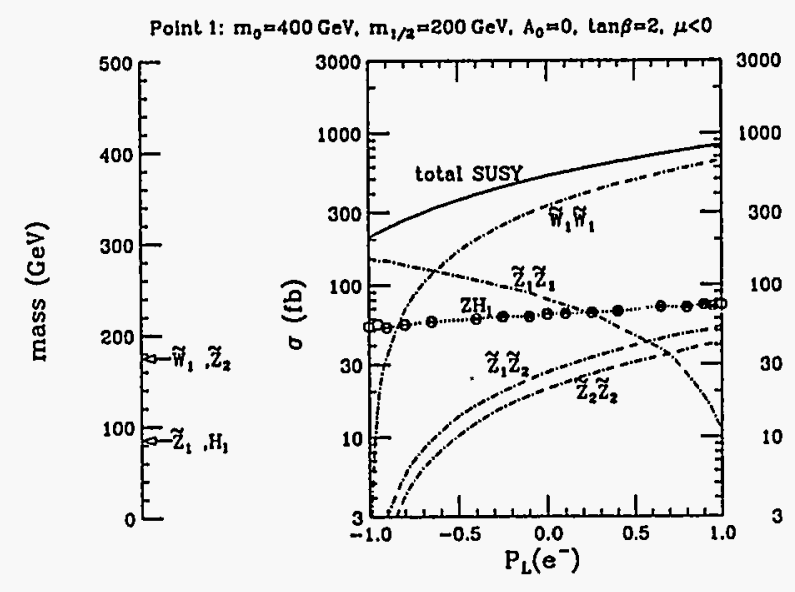

Point 2: $m_{0}=100 \mathrm{GeV}, m_{2 / 2}=300 \mathrm{GeV}, A_{0}=0, \tan \beta=2, \mu<0$

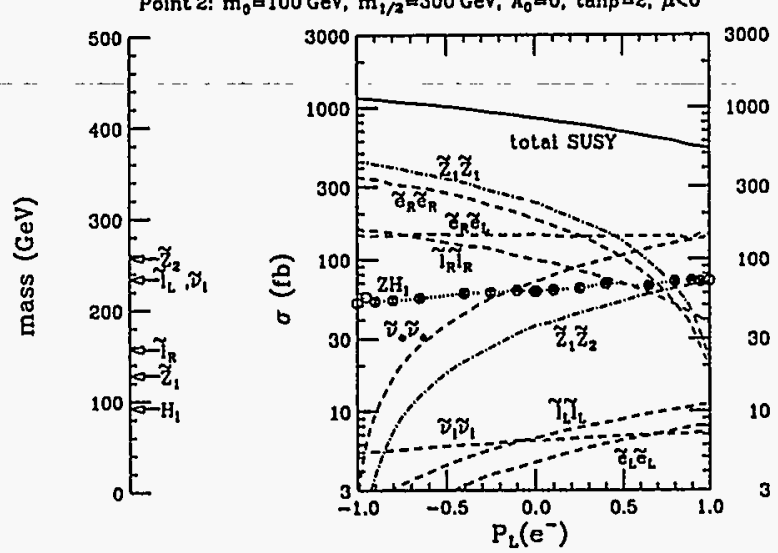

Figure 2: The cross section of Supersymmetric particle production at $E_{\mathrm{cm}}=0.5 \mathrm{TeV}$ as a function of the electron polarization for points 1,2 in our parameter space.
Point 3: $m_{0}=200 \mathrm{GeV}, m_{1 / 2}=100 \mathrm{GeV}, A_{0}=0, \tan \beta=2, \mu<0$

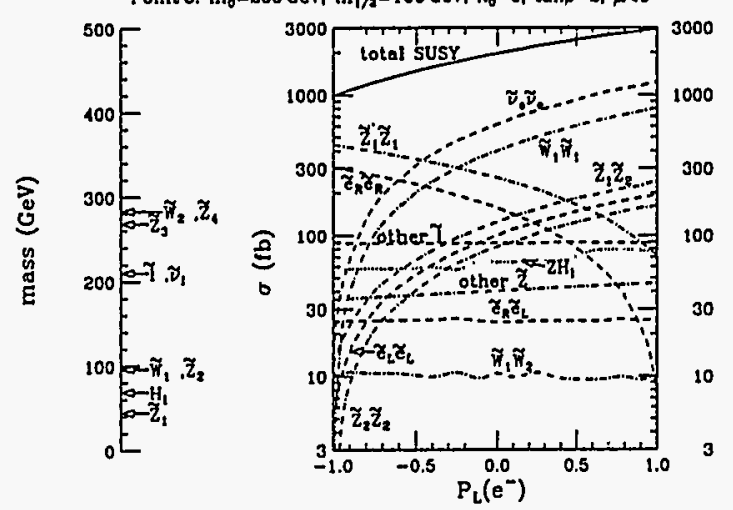

Point 4: $m_{q}=-A_{t}=1$ ToV, $\tan \beta=10, \mu=-100 \mathrm{GaV}, m_{\mu_{z}}=200 \mathrm{GeV}$

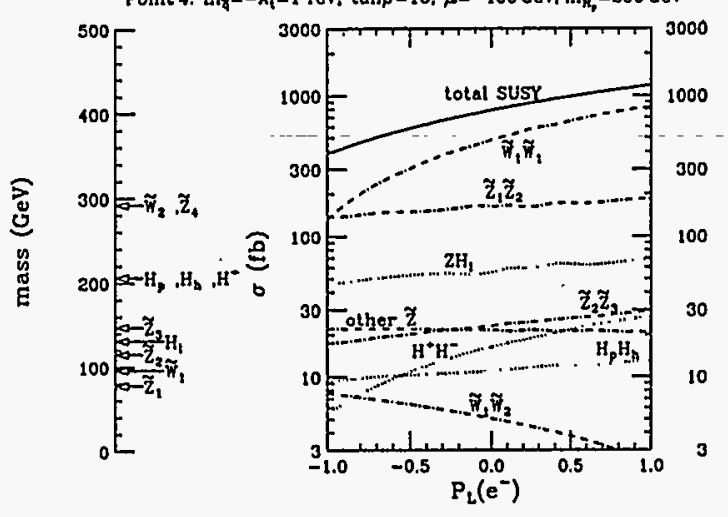

Figure 3: The cross section of Supersymmetric particle production at $E_{c m}=0.5 \mathrm{TeV}$ as a function of the electron polarization for points 3.4 in our parameter space. In point 3 . the line labeled "other $l^{n}$ is the sum of left and right handed pairs, hence the polarization dependent effects are not included. See Fig. 2 for their dependence on polarization. Also $m_{L_{L}}=216 \mathrm{GeV}$ and $\mathrm{m}_{i_{R}}=200 \mathrm{Gel}$ for all the sleptons. 


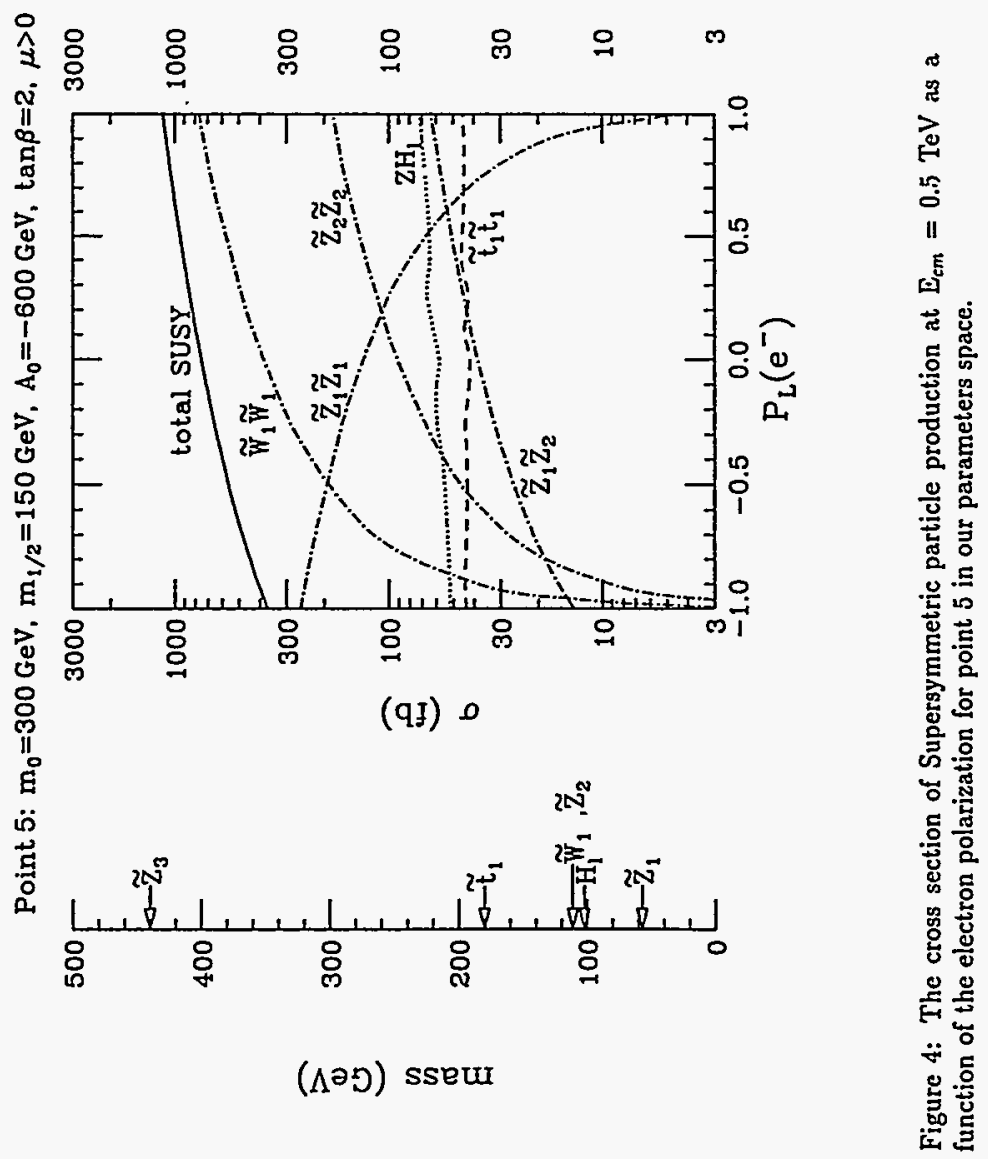



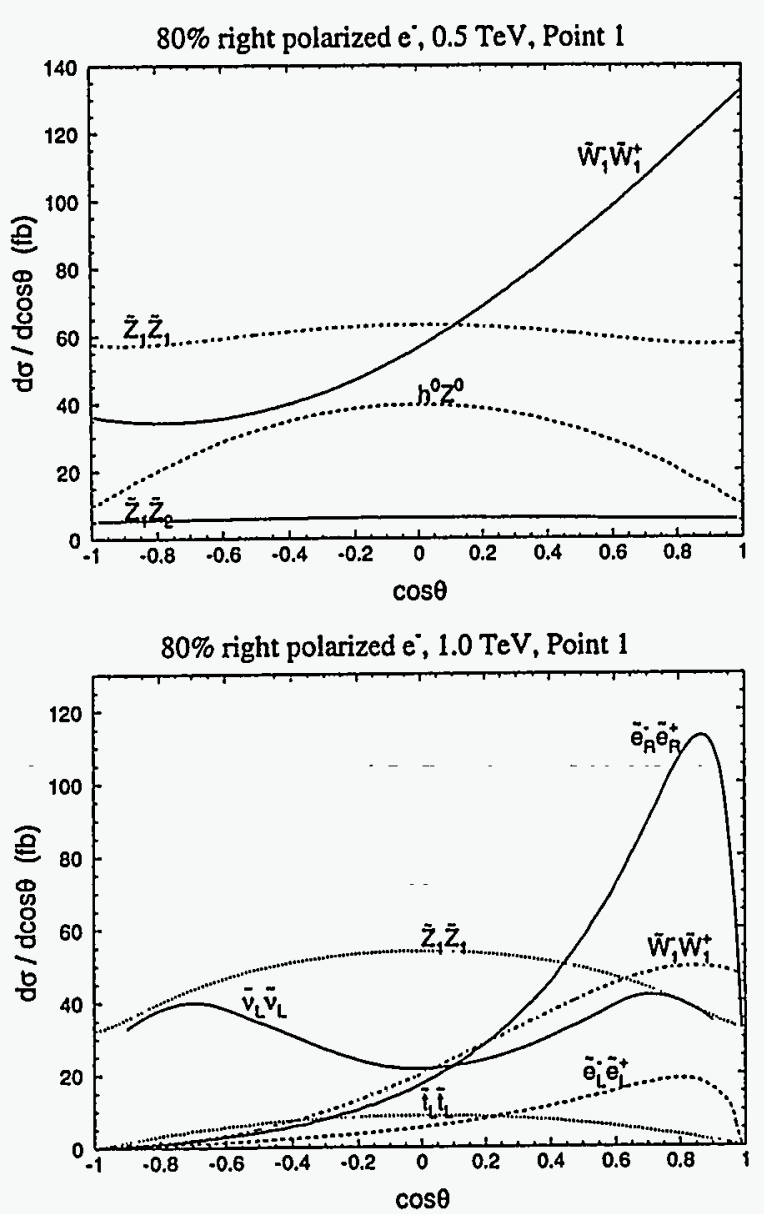

Figure 6: The differential cross section of Supersymmetric particle production for an $80 \%$ polarized right handed electron at $E_{c m}=0.5$ and $1.0 \mathrm{TeV}$ for point 1 in our parameters list.
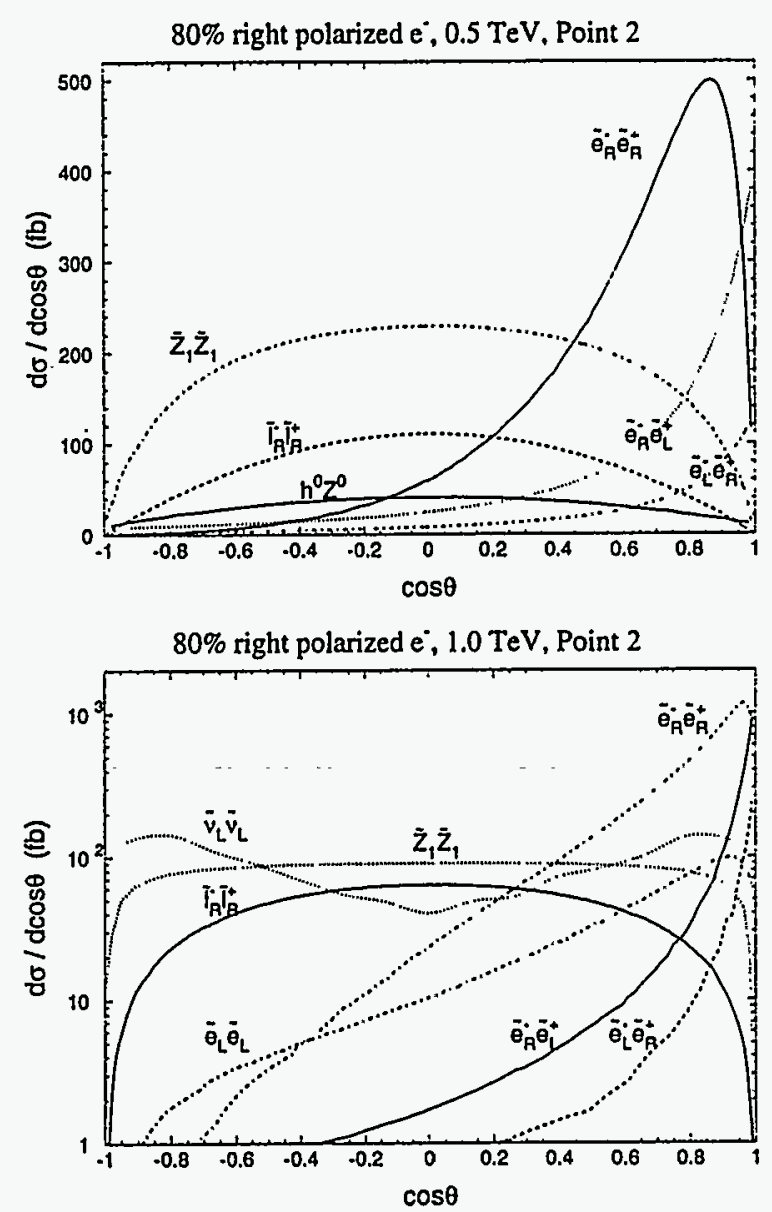

Figure $7:$ The differential cross section of Supersymmetric particle production for an $80 \%$ polarized right handed electron at $E_{c m}=0.5$ and $1.0 \mathrm{TeV}$ for point 2 in our parameters list. 


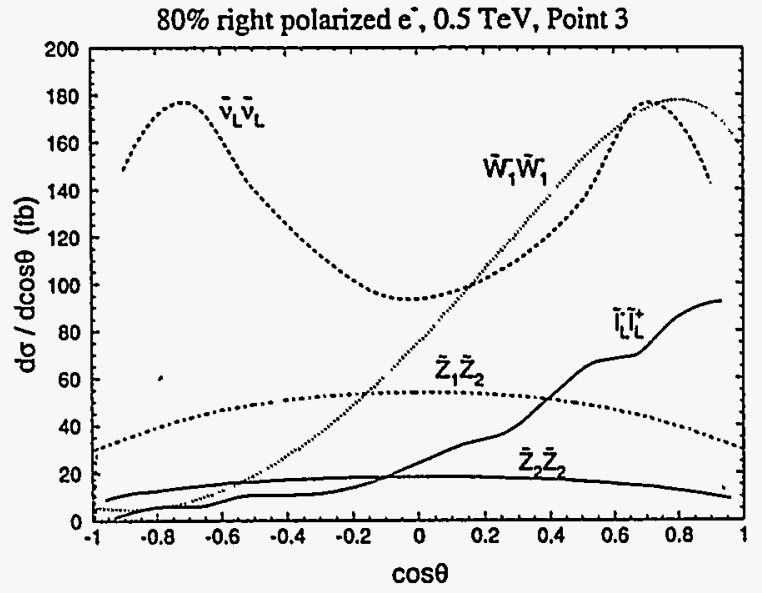

$80 \%$ right polarized $\mathrm{e}, 1.0 \mathrm{TeV}$, Point 3

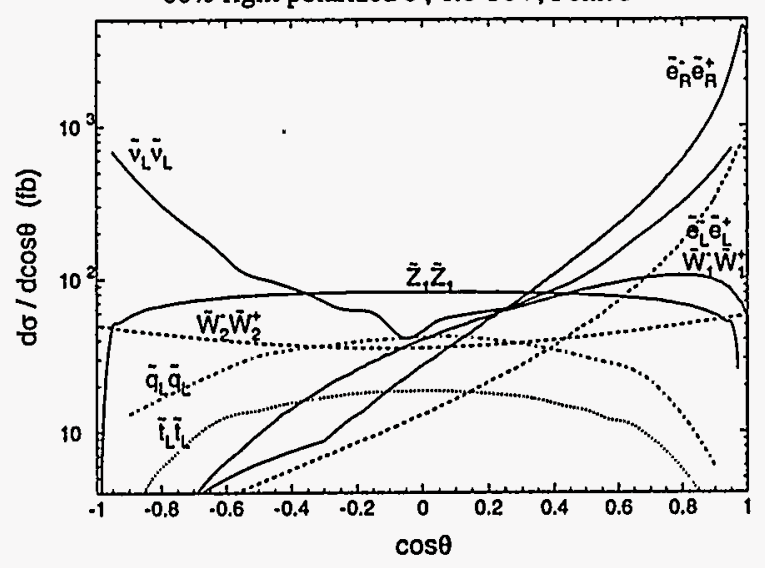

Figure 8: The differential cross section of Supersymmetric particle production for an $80 \%$ polarized right handed electron at $E_{c m}=0.5$ and $1.0 \mathrm{TeV}$ for point 3 in our parameters list.
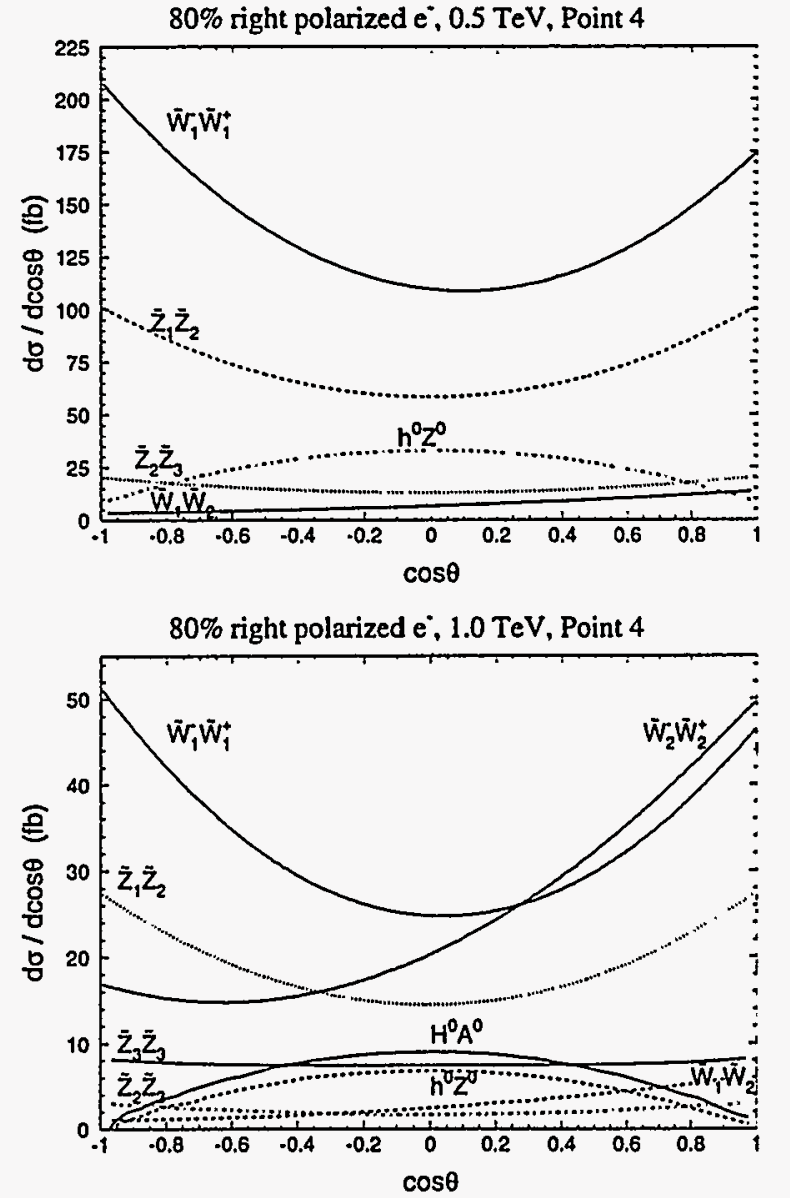

Figure 9: The differential cross section of Supersymmetric particle production for an $50 \%$ polarized right handed electron at $E_{c m}=0.5$ and $1.0 \mathrm{TeV}$ for point $t$ in our parameters list. 

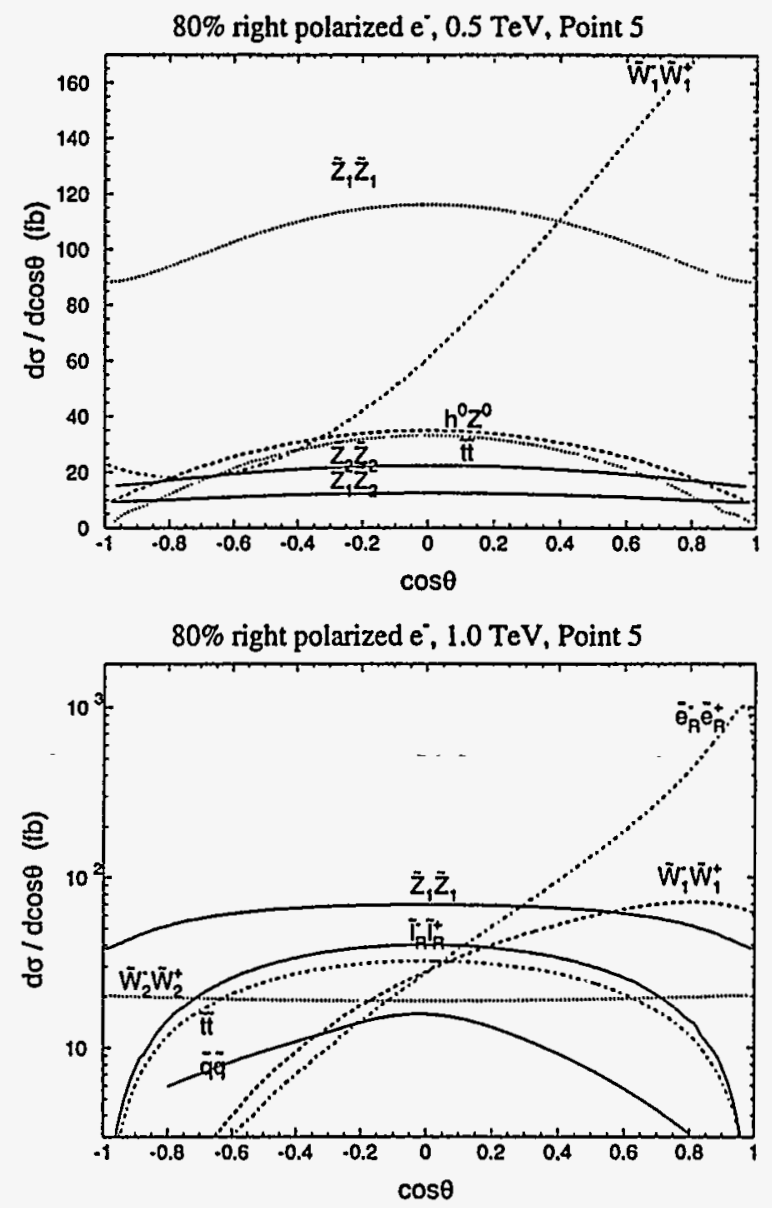

Figure 10: The differential cross section of Supersymmetric particle production for an $80 \%$ polarized right handed electron at $E_{\mathrm{cm}}=0.5$ and $1.0 \mathrm{TeV}$ for point 5 in our parameters list. 
USING LINEAR COLLIDERS TO PROBE GUT AND POST-GUT PHYSICS

\section{R. Arnowitt and Pran Nath}

${ }^{a}$ Center for Theoretical Physics. Department of Physics. Texas A\&M University, College Station, TX 77843-4242

${ }^{6}$ Department of Physics, Northeastern University, Boston. MA 02115

\section{Abstract}

The ability of linear colliders to test physics at the GUT scale $M_{G}$ and beyond is investigated. Using current estimates of measurements available in such accelerators, it is seen that soft breaking masses and their deviations from universal soft breaking can be measured with errors of about $(1-15) \%$. Thus linear colliders can be used to determine the grand unification group, and shed light on the nature of dynamics beyond $M_{G}$ up to the string scale $M_{\text {tsting }}$

\section{INTRODUCTION}

The usual view of a high energy collider is that it allows us to learn about physics below its energy reach, but physics above this energy remains unknown. In an absolute sense this is true: LEPI has determined the lowe bound on the Higgs mass to be $65 \mathrm{GeV}$, and one will have to wait until LEP 1.6 is turned on to learn more. However, two theoretical results has moderated this viewpoint. First the renormalization group equations (RGE) allow one to take data at one energy and extrapolate it to a higher energy to test theoretical ideas at this higher energy. A second, related, result is supersym metric grand unification. This implies that the upward extrapolation can be made over an enormous energy domain. i.e. not for $10 \mathrm{GeV}$ or $100 \mathrm{Gel}^{\circ}$ but for over $10^{14} \mathrm{GeV}$.

The fact that the three coupling constants $\alpha_{1} . \alpha_{2}$ and $\alpha_{3}$ unify to a GUT value $\alpha_{G} \cong 1 / 24$ at about $10^{16} \mathrm{GeV}$ was observed first in the 1990 LEP precision data [1], and has stood the test of time since then. Thus there has been refinements in the data and refinements in the theoretical treatment (i.e. inclusion of SUSY threshold effects at $M_{S} \simeq 100 \mathrm{GeV}-1$ $\mathrm{TeV}$ [2], GUT scale threshold effects [3.4], and possible small Planck scale effects [4]). It is possible that the unification of the three coupling constants at $M_{G} \cong 2 \times 10^{16} \mathrm{GeV}$ is purely a numerical accident, particularly since there is an adjustable parameter $M_{S}$. However, such an "accident" is not too easy to achieve for several reasons: thus there is only a narrow window of values for $M_{G}$ between about $5 \times 10^{15} \mathrm{GeV}$ (below which in most models too rapid proton decay $\left(p \rightarrow e^{+} \pi^{0}\right)$ would occur) and the "string" scale $5 x$ $10^{17} \mathrm{GeV}$ (above which gravitational effects become strong invalidating the analysis.) Further, unification does not occur for the Standard Model (S.I). and satisfactory SUSY unification occurs only for two Higgs doublets (the minimal number) and for no more than four families. Finally, the fine tuning problem requires that $M_{S} \lesssim 1 \mathrm{TeV}$, which indeed turns out to be the case.

In order to see whether grand unification is an accident of has deeper physical significance, it is necessary to have other measurements to experimentally test the idea. We will see below that linear colliders (LC) can indeed do this. and cannot only probe GUT scale physics but also physics that may be occurring above the GUT scale, and do this with a high degree of precision.

\section{SUPERGRAVITY MODELS}

To analyse grand unification we use here the supergravity models [5]. These have a number of positive attributes including the following: (1) They account for the unification of the coupling constant. (2) They allow for spontaneous breaking of supersymmetry at the GUT (or Planck) scale. (This is a crucial feature (which cannot be achieved satisfactorily in the low energy MSSM) for without this it is not possible to confront a theoretical model with experiment.] (3) Using the RGE to go to low energies, one finds that the spontaneous breaking of supersymmetry at energy $\geq ._{G}$ triggers the spontaneous breaking of $\mathrm{SU}(2) \times \mathrm{U}(1)$ at the electroweak scale $. M_{E W}=O\left(M_{z}\right)$ [6]. Thus supergravity models give an explanation of the Higgs phenomena (and as a consequence predicted a decade before its discovery that the top would be heavy). The above three items together imply a very predictive theory, and most of the phenomenological SUSY analyses make use of some or all of the constraints implied by supergravity. 
In order to understand the nature of the quantitites in GUT models that might be measured, we briefly summarize some of the structure of the supergravity models. These models depend on three functions of the scalar fields $\phi_{i}(\mathrm{x})$ (representing sleptons, squarks etc.): the superpotential $W\left(\phi_{i}\right)$ (which includes the Yukawa couplings). The gauge kinetic function $f_{a b}\left(\phi_{i}\right)$ (which enters as $f_{\alpha} \rho F_{\mu \nu}^{\alpha} F^{\mu \nu \rho}$ with $\alpha, \beta=$ gauge indices) and the Kahler potential $\mathrm{K}\left(\phi_{i}, \phi_{i}^{+}\right)$(which appears in the effective potential and elsewhere). These quantities have mass dimensions of (mass) ${ }^{3}$, (mass) ${ }^{0}$ and (mass) ${ }^{2}$ respectively. It is generally believed that if one expands these functions in a polynomial in $\phi_{i}$, those terms carrying the mass dimensions of $W, f_{\alpha \beta}$ and $\mathrm{K}$ are accessible to low energy discovery (their coupling constants are dimensionless) while higher terms are scaled by $\kappa$ where $\kappa^{-1}=M_{\text {Planck }}=2.4 \times 10^{18} \mathrm{GeV}$. These higher terms then represent Planck scale physics. Thus one writes

$$
W_{\text {phys. }}\left(\phi_{i}\right)=\lambda^{i j k} \phi_{i} \phi_{j} \phi_{k}+\kappa \lambda^{i j k l} \phi_{i} \phi_{k} \phi_{l}+\cdots
$$

$$
f_{\alpha \beta}\left(\phi_{i}\right)=c_{\alpha \beta}+\kappa c_{\alpha \beta}^{i} \phi_{i}+\ldots
$$

$$
K\left(\phi_{i}, \phi_{i}^{+}\right)=c_{j}^{i} \phi_{i} \phi_{j}^{+}+d^{i j} \phi_{i} \phi_{j}+\kappa c_{k}^{i j} \phi_{i} \phi_{j} \phi_{\kappa}^{+}+\ldots
$$

where $\lambda^{i j k}$ are the Yukawa coupling constants, and $c_{\alpha \beta}, c_{j}^{i}=O(1)$. We consider models where supersymmetry spontaneous breaks in a "hidden" sector at the Planck scale so that the total superpotential is a sum,

$$
W=W_{p h y s}\left(\phi_{i}\right)+W_{\text {hid }}(z), \quad .
$$

Here $z$ grows the VEV which breaks supersymmetry: $\langle z\rangle=O\left(\kappa^{-1}\right), \kappa\left\langle W_{h i d}\right\rangle$ $=O\left(\kappa^{-1} M_{z}\right)$. The large $z$ VEV then produces soft breaking masses of $=O\left(\kappa^{-1} M_{Z}\right)$. The large $z$ VEV then produces soft breaking masses of
$O\left(M_{z}\right)$ due to the interference between the physical and hidden sectors of the theory, with gravity playing the role of the messenger between them

The simplest of such models is the Minimal Supergravity Model (MSGM) which depends on four soft breaking parameters and one sign. These are $m_{0}$ (the universal scalar mass at $M_{G}$ ), $m_{1 / 2}$ (the universal gaugino mass at $M_{G}$ ), $A_{0}$ and $B_{0}$, (the cubic and quadratic soft breaking parameters at $M_{G}$ ) and the sign of $\mu_{0}$ (the Higgs mixing parameter in the term $\mu_{0} H_{1} H_{2}$ in the superpotential). (The MSGM might be compared to the low energy MSSM which has a possible 110 parameters.] For a simple GUT group one expects $m_{1 / 2}$ to be universal (except for perhaps a few percent corrections from Planck scale physics [4]). However, the universality of the $m_{0}$ masses is less clear.
In general $m_{0}$ has two contributions. one from the superpotential. which is indeed universal, and one from the Kahler potential, where universality depends upon the group symmetry of $K$. For example. consider the squark contribution in Eq. (3): $K=c_{j}^{i}(z) \tilde{q}_{i} \tilde{q}_{j}^{+}+\ldots$ where $\tilde{q}_{i}$ are the squark fields. $i, j$ generation indices and we've included super Higgs dependence in $c_{j}$. If there exists an $O(3)_{H}$ horizontal symmetry, then $c_{j}^{i}=\delta_{j}^{i} \mathrm{c}(z)$ and universal soft breaking masses develop when $z$ grows its VEV. Alternately an $\mathrm{SL}^{-}(2)_{H}$ symmetry [7] with the first two generations in a doublet (to suppress flavor changing neutral currents) will break universality.

The nature of the Kahler potential and the symmetries of its super Higgs interactions represent post-GUT (i.e. most likely Planck scale) physics. ITe will see that it is possible to test some of these possibilities with good accuracy at a linear collider.

\section{TESTING GUT SCALE PHYSICS}

Aside from small GUT scale threshold corrections, most physics below $U_{G}$ is insensitive to the nature of the GUT group. Thus relatively model independent tests of some GUT scale physics can be done. The universality of $\mathrm{m}_{1 / 2}$ at $M_{G}$ implies the well known relations for the U(1), SU(2) and $\mathrm{SC}^{-}(3)$ gaugino masses at the electroweak scale [8]

$$
M_{i}=\left(\alpha_{i} / \alpha_{G}\right) m_{1 / 2} ; \quad i=1,2,3
$$

with the gluino mass $m_{\dot{g}}$ given approximately by $M_{3}[9]$. The electroweak sector of this prediction can be well tested at the NLC, i.e. to about $5 \%[10]$ The LHC can measure $m_{\dot{g}}$ to perhaps $15 \%[11\}$, and hence a semi-quantitative test of the third relation $M I_{2} / M I_{3} \cong \alpha_{2} / \alpha_{3}$ is also available.

A second important prediction of supergravity models is the determination of $\mu^{2}$ at the electroweak scale:

$$
\mu^{2}=\frac{m_{H_{1}}^{2}-m_{H_{2}}^{2} \tan ^{2} \beta}{\tan ^{2} \beta-1}-\frac{1}{2} M_{Z}^{2}
$$

where $m_{H_{1,2}}$ are the running Higgs masses at the electroweak scale (including loop corrections) and $\tan \beta=\left\langle H_{2}\right\rangle /\left(H_{1}\right\rangle$. The masses $m_{H_{1,2}}$ are scaled by $m_{0}$ and $m_{j}$ whose ranges are $0<m_{0} \leq 1 \mathrm{TeV}, 170 \mathrm{GeV}<m_{j} \leq 1 \mathrm{TeV}$ (the upper bound being due to naturalness requirements). Hence over most of the parameter space $\mu$ is large and $\mu^{2} / M_{2}^{2}>1$. In this domain. a set of scaling laws hold for the neutralinos $\left(\dot{\chi}_{i}^{0}\right)$, charginos $\left(\dot{x}_{i}^{ \pm}\right)$and Higgs bosons (h. $\left.\mathrm{H}^{\circ}, \mathrm{H}^{ \pm}, \mathrm{A}\right)[12]$ 


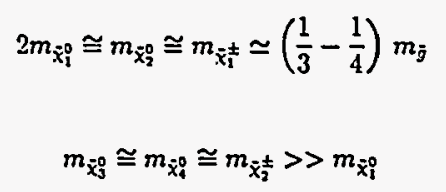

$m_{H^{\circ}} \cong m_{H^{ \pm}} \cong m_{A} \gg m_{h}$

At a linear collider (LC) the $\tilde{\chi}^{0}, \bar{\chi}^{ \pm}$masses can be measured to $\sim 1 \%[10,15]$ and probably good measurements of the Higgs masses will also be available. Thus if these relations were satisfied, it would be good circumstantial evidence for supergravity unification (though Eqs. (7-9) are not absolutely required by such models as $\mathrm{Eq}$. (6) also has solutions where $\mu=0\left(\mathrm{M}_{z}\right)$ ). It should be noted that many of the particles in Eqs. (7-9) may lie beyond the reach of the NLC, and so higher linear colliders will play an important role in testing such relations.

\section{TESTING POST-GUT PHYSICS}

As one goes to scales beyond $M_{G}$, the theory becomes more model dependent. It is sensitive to both the nature of the GUT group and the particle spectrum above $M_{G}$ (which may no longer be just that of the MSSM, i.e. what was needed to achieve grand unification at $M_{G}$ ). Linear colliders will be sensitive to both these types of model dependences, and hence help distinguish between different possibilities.

One interesting suggestion is that the symmetry of the Kahler potential, which as was seen controls the amount of universality, originates at a higher mass scale where supersymmetry breaks [13]. The highest scale that one can still treat phenomena field theoretically is the string scale, $M_{\text {str }} \simeq 5 \times 10^{17}$ $\mathrm{GeV}$. The RGE from $M_{\text {tr }}$ to $M_{G}$ would then lead to non-universal soft breaking contributions, even if universality held at $M_{\text {str. }}$. Such phenomena can then be tested at a LC since they produce effects at low energy. It is thus possible to experimentally explore the physics between $M_{G}$ and $M_{\text {tir }}$. To illustrate this, we consider several examples of possible GUT theories.

\section{(i) SU (5) GUT}

We assume here for simplicity the minimal particle content above $M_{G}$ i.e. that matter exists in three generations of $10=M_{i}^{X Y}$ and $\overline{5} \equiv \bar{M}_{i X}$ representations $(i=1,2,3)$, and there is a $\overline{5}=\mathcal{H}_{1 X}$, and $5=\mathcal{H}_{2}^{X}$ of Higgs (which contain the two Higgs doublets coupling to matter) and a $24=\sum_{Y}^{X}$ to break SU(5) to the SM. (X.Y $=1 \cdots .5$ are SU(5) indices.) The superpotential has the form

$$
\begin{aligned}
W & =\left[\frac{1}{4} h_{t} \epsilon_{N Y Z W U} M^{X Y} M^{Z W} \mathcal{H}_{2}^{\ell}+h_{b} M^{X Y} \cdot \bar{Y}_{X} \mathcal{H}_{Y Y}\right] \\
& +\left[M \operatorname{tr} \Sigma^{2}+\frac{1}{6} \lambda_{1} \operatorname{tr} \Sigma^{3}+\lambda_{2} \mathcal{H}_{1} \Sigma \mathcal{H}_{2}+\mu \mathcal{H}_{1} \mathcal{H}_{2}\right]
\end{aligned}
$$

If one were to assume that the soft breaking masses were universal at $M_{\text {trr }}$. then SU(5) invariance implies that there would be four soft breaking masses. at $M_{G}$ (which would then modify low energy phenomena). These are $m_{10}$ (which contains $\left.q \equiv\left(\tilde{u}_{L} \dot{d}_{L}\right), u \equiv \bar{u}_{R}, \mathrm{e} \equiv \bar{e}_{R}\right), m_{5}$ which contains $\ell \equiv\left(\dot{\nu}_{L}, \dot{e}_{L}\right)$. $\mathrm{d} \equiv \bar{d}_{R}$ ) and $m_{\mathcal{H}_{1,2}} \equiv m_{H_{1,2}}$ where $H_{1,2}$ are the two light Higgs doublets. (In addition one might consider generational splittings from e.g. an SU(2) ${ }_{H}$ symmetry at $\mathrm{M}_{s t r .}$ ) One may chose one of the soft breaking masses as the reference mass, $\tilde{m}_{0}$, and consider deviations of the other masses from $\dot{m}_{0} . A$ convenient choice is $m_{10} \equiv \dot{m}_{0}$ and we write

$$
m_{5}^{2}=\dot{m}_{0}^{2}\left(1+\delta_{5}\right): m_{H_{1.2}}^{2}=\dot{m}_{0}^{2}\left(1+\dot{\delta}_{1.2}\right)
$$

Using the dynamics of Eq. (10), one may calculate the deviations from universality that results at $\mathrm{M}_{\mathrm{G}}$. One finds that these are significant $(30-40 \%)$ though not enormous, and are sensitive to the parameters of the model.

One can in fact determine these parameters at a linear collider. and actually test the breakdown of universality occurring in the post-GUT regime experimentally. Thus using the RGE to take the masses down to the electroweak scale, one finds, for example [14]

$$
\check{m}_{0}^{2}=m_{e_{R}}^{2}-0.151 m_{1 / 2}^{2}+\sin ^{2} \theta_{w} \cdot V_{Z}^{2} \cos 23
$$

$$
\check{m}_{0}^{2} \delta_{5}=m_{\check{e}_{R}}^{2}-0.377 m_{1 / 2}^{2}+\left(\frac{1}{2}-2 \sin ^{2} \theta_{W}\right) \cdot V_{Z}^{2} \cos 2 \theta
$$

with $m_{1 / 2}=\left(\alpha_{G} / \alpha_{2}\right){ }_{1} W_{2}$. At the NLC one expects to be able to measure $m_{e_{B}} m_{\varepsilon_{L}}$ to about $1 \%, W_{2}$ to about $3 \%, \tan \beta$ to $10 \%[10.15 .16)$ and $\alpha_{G}$ to perhaps $3 \%$. As an example, consider the case where $m_{i_{L}}=240 \mathrm{GeV}, m_{i_{n}}$ $=200 \mathrm{GeV}, M_{2}=120 \mathrm{GeV}$ and $\tan \beta=5$. One finds then from Eqs. (12), (13) and the above estimated errors that

$$
\bar{m}_{0} \cong(18 \mathrm{i} \pm 3) \mathrm{GeV} ; \quad \delta_{5} \cong 0.256 \pm 0.031
$$


$(\eta) \cap \times(z) \cap S^{x}(\varepsilon) \cap S(! ! !)$

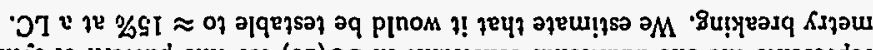

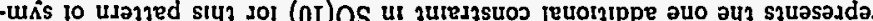

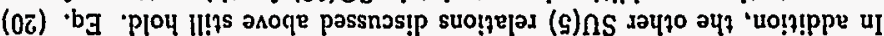

$$
t_{\rho}-z_{\rho}=s_{\rho}
$$

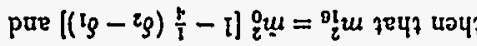

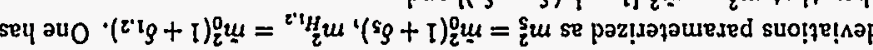

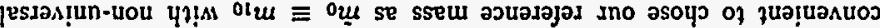

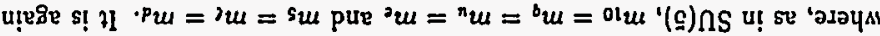

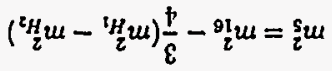

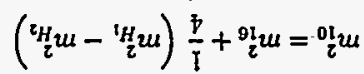

[0]] sey วu० OW วе บәYI

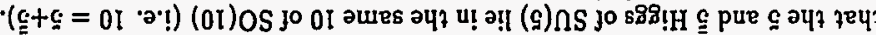

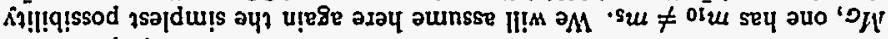

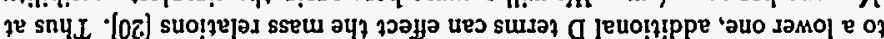
dnoss yues sәч

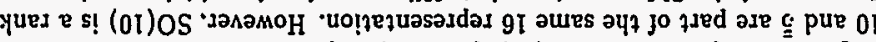

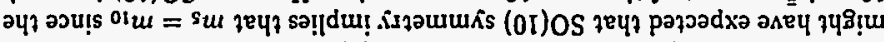

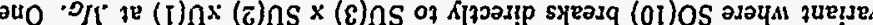

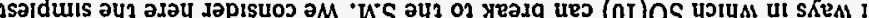

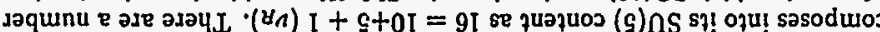

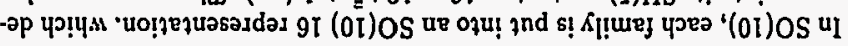

\section{LחD (or)OS (!!)}

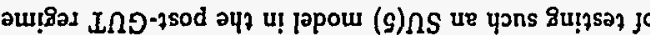

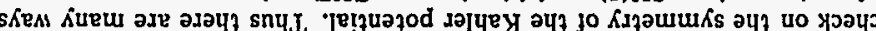

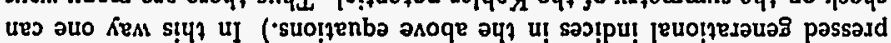

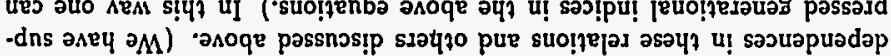

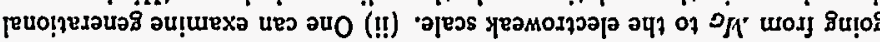

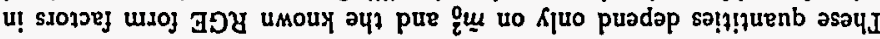

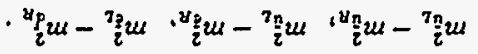

'ว"!

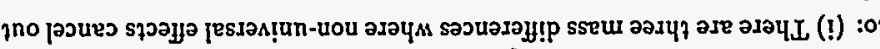

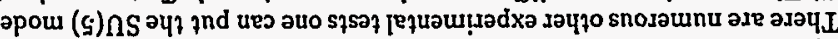

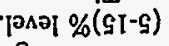

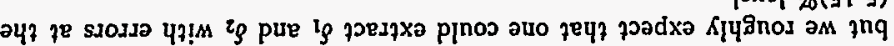

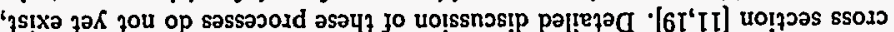

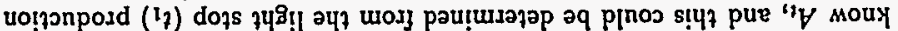

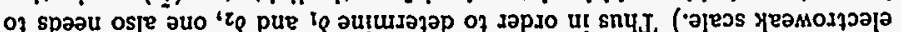

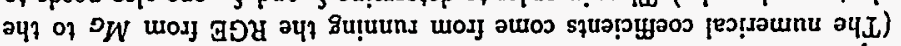

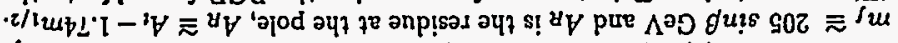

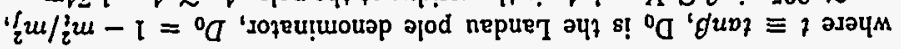

(9T)

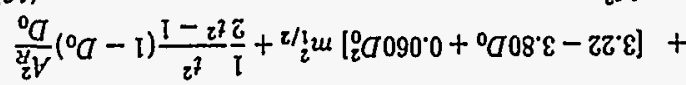

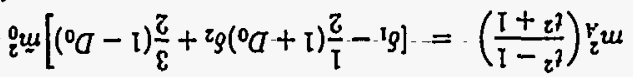

(sI)

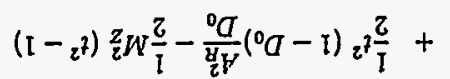

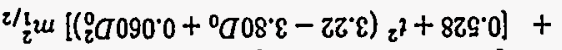

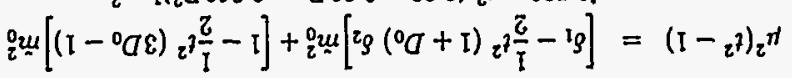

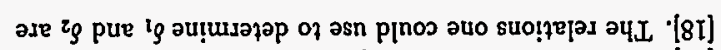

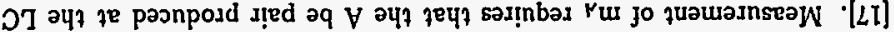

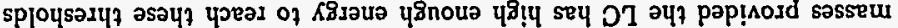

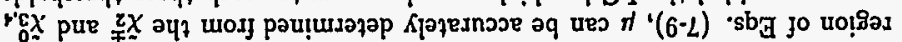

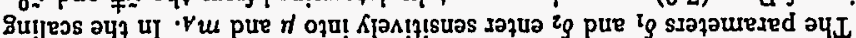

jppow (s)nS

әцุ jo Кำ

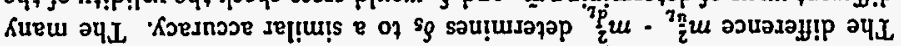

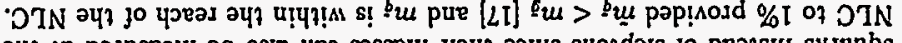

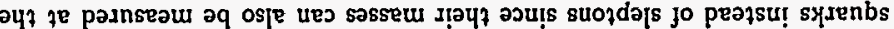

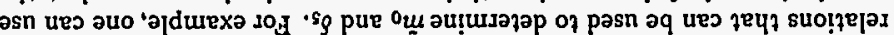

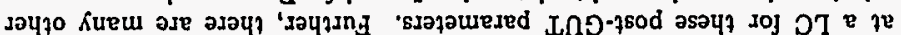

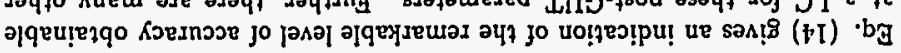


Some string models assume that the SM gauge group holds all the way up to the string scale. after which unification will occur at $M_{\text {str }}$ [21]. The RGE will then split the soft breaking masses at $M_{G}$. Choosing here $m_{q} \equiv \bar{m}_{0}$ as the reference mass one has

$$
\begin{aligned}
m_{u}^{2} & =\bar{m}_{0}^{2}\left(1+\delta_{u}\right) ; \quad m_{e}^{2}=\check{m}_{0}^{2}\left(1+\delta_{c}\right) \\
m_{d}^{2} & =\dot{m}_{0}^{2}\left(1+\delta_{d}\right) ; \quad m_{l}^{2}=\dot{m}_{0}^{2}\left(1+\delta_{l}\right) \\
m_{H_{1,2}}^{2} & =\dot{m}_{0}^{2}\left(1+\delta_{1,2}\right)
\end{aligned}
$$

with the notational $u=\tilde{u}_{R}$, e= $\tilde{e}_{R}$ etc. as in SU(5). There are a priori no relations between the different $\delta$ 's and hence mass differences that were universal for SU(5) or SO(10), i.e. Eq. (17), will no longer in general be universal. The different $\delta$ 's can, of course, be measured at a LC. Thus $m_{\tilde{u}_{L}}^{2}$ - $m_{\bar{u}_{R}}^{2}$ will determine $\delta_{u}, m_{\dot{e}_{R}^{2}}$ determines $\delta_{e}$, etc.

(iv) Distinguishing Post-GUT Groups

The LHC can give information about the nature of physics beyond the GUT scale, and in fact distinguish between different gauge groups that may hold beyond $M_{G}$. In the examples discussed above, one would conclude that if $\delta_{u, e} \neq 0$ or $\delta_{d} \neq \delta_{l}$, then SU(5) and SO(10) would not be valid GUT groups. but the SM group could still hold above $M_{G}$. But if, $\delta_{u}=0=\delta_{e}, \delta_{d}=\delta_{l}$. then both $S U(5)$ and $S O(10)$ would be consistent with this result. But if, $\delta_{5} \neq \delta_{2}-\delta_{1}$, then $S O(10)$ would be eliminated. Similar considerations can be carried out for other gauge groups and other symmetry breaking patterns.

\section{Concluding Remarks}

Linear colliders are ideal machines not only for the discovery of low energy SUSY, but also for exploring physics up past the GUT scale $M_{G}$ and even to the string scale $M_{\text {tr. }}$. Current estimates show that the soft breaking parameters can be measured with relatively good precision i.e. at the (1$15) \%$ level. The pattern of non-universal soft breaking masses would allow the determination of the GUT group and horizontal symmetries that may hold above $M_{G}$, and the magnitude of these parameters will shed light on the dynamics above $M_{G}$. The ability of linear colliders to do this represents one of the remarkable attributes of this class of acclerators.

However, the reach of the NLC, while good for studying light neutralinos, charginos and perhaps sleptons is likely to be insufficient for the heavier SUSY particles needed to give a full knowledge of what is happening at energies $\gtrsim M_{G}$. For example, if LEP 1.9 did not discover the lightest chargino $\dot{\chi}_{1}^{ \pm}$. then $m_{i \frac{1}{1}} \gtrsim 90 \mathrm{GeV}$. In the scaling domain, this would imply $m_{j} \approx 2 \pi 0 \mathrm{Giel}$. The $\bar{u}_{L}$ mass is given by $m_{\bar{u}_{L}}^{2} \cong \bar{m}_{0}^{2}+0.893 m_{\bar{j}}^{2}+\left(\frac{1}{2}-\frac{2}{3} \sin ^{2} \theta_{W V}\right) \cdot M_{Z}^{2} \cos 23$. which implies $m_{\tilde{u}_{L}}>250 \mathrm{GeV}$, with similar results for other squarks. Thus in order to sample the full SUSY spectrum, one needs colliders with $\sqrt{s}>1$ $\mathrm{TeV}$ (preferably up to $\sqrt{s}=2 \mathrm{TeV}$ ).

In view of the results obtained here, a more complete analysis of the accuracy at which linear colliders can measure parameters important for the study of grand unified models is indicated. In particular. one needs to examine more of the parameter space relevant to GUT models. so that a full picture is known of what precision experiments are available for these accelerators.

This research was supported in party by NSF grant numbers PHY-9+11.5.43 and $\mathrm{PHY}-19306906$.

\section{REFERENCES}

1. P. Langacker. Proc. PASCOS90, Eds. P. Nath and S. Reucroft (Wiorld Scientific. Singapore 1990); J. Ellis. S. Kelley and D. Y. . Nanopoulos. Phys. Lett. B249, 441 (1990), B260. 131 (1991); U. Amaldi. II. de Boer and H. Furstenau. Phys. Lett. B260. 447 (1991): F. Anselmo. L. Cifarelli, A. Peterman and A. Zichichi, Nuov. Cim. 104A. IS1i (1991); 115A. sS1 (1992).

2. J. Bagger. K. Mlatchev and D. Pierce, Proc. of Physics from Planck Scale to Electroweak Scale, ed. P. Nath, T. Taylor and S. Pokorski (World Scientific. Singapore 1995).

3. R. Barbieri and L.J. Hall, Phys. Rev. Lett. 68. 752 (1992); L.J. Hall and U. Sarid. Phys. Rev. Lett. 70, 2673 (1993).

4. D. Ring, S. Urano and R. Arnowitt, Phys. Rev. D52. 6623 (1995): T. Dasgupta, P. Mameles and P. Nath, Phys. Rev. D52, 5366 (1995).

5. A.H. Chamseddine, R. Arnowitt and P. Nath, Phys. Rev. Lett. 49. 970

(1982)). For reviews see P. Nath, R. Arnowitt and A.H. Chamseddine. "Applied $\mathrm{V}=1$ Supergravity" (World Scientific. Singapore 1984); H.P. Nilles, Phys. Rep. 100, I (1984); R. Arnowitt and P. Nath. Proc. VII Swieca Summer School, ed. E. Eboli (World Scientific, Singapore 1994).

6. K. Inoue et al.. Prog. Theor. Phys. 68, 927 (1982); L. Ibañez and G.G. Ross, Phys. Lett. B110, 227 (1982); L. Alvarez-Gaumé. J. Polchinski and M.B. Wise. . Vucl. Phys. B221, 495 
(1983); J. Ellis, J. Hagelin, D.V. Nanopoulos and K. Tamvakis, Phys. Lett. B125, 2275 (1983); L.E.Ibañez and C. Lopez. Phys. Lett. B128. 54 (1983); Nucl. Phys. B233, 545 (1984); L.E. Ibañez, C. Lopez and

C. Muños, Nucl. Phys. B256, 218 (1985).

7. M. Dine, R. Leigh and A. Kagan, Phys. Rev. D48, 4269 (1993).

8. P. Nath, A.H. Chamseddine and R. Arnowitt, Proc. of 1983 Coral Gables Conference on High Energy Physics, ed. Mintz, Perlmutter (Plenum Press, 1985); L. Alvarez-Gaumé et al. Ref. [6].

9. QCD corrections to this relation are given in S.P. Martin and M.T. Vaughn, Phys. Lett. B318, 331 (1993); D. Pierce and A. Papadopoulos. Nucl. Phys. B430, 278 (1994).

10. T. Tsukamoto et al., Phys. Rev. D51, 3153 (1995).

11. H. Baer et al., FSU-HEP-950204 (1995).

12. R. Arnowitt and P. Nath, Phys. Rev. Lett. 69, 725 (1992); P. Nath and R. Arnowitt, Phys. Lett. B289, 368 (1992).

13. N. Polonsky and A. Pomarol, Phys. Rev. D51, 6532 (1995).

14. A small correction of about $1 \%$ in size proportional to $\delta_{2}-\delta_{1}$ has been omitted from Eqs. (12) and (13). See e.g. Kawamura et al., Ref. 20.

15. J.Ĺ. Feng et al., Phys. Rev. D52, 1418 (1995).

16. M.M. Nojiri, Phys. Rev. D51, 6281 (1995); M.E. Peskin, talk at YKIS95, Kyoto (1995).

17. J.L. Feng and D.E. Finnell, Phys. Rev. D49, 2369 (1994).

18. H. Haber, Proc. of Beyond the Standard Model IV, ed. J. Gunion, T. Hans and J. Ohnemus (World Scientific, Singapore, 1995).

19. A. Bartl et al. hep-ph/9604221.

20. M. Drees, Phys. Lett. B181, 279 (1986); J.S. Hagelin and S. Kelley, Nucl. Phys. B342, 95 (1990); Y. Kawamura, H. Murayama and M. Yamaguchi, Phys. Lett. B324, 52 (1994).

21. K. Dienes, hep-th/9602045 and references therein. 


\section{Introduction}

During the last 15 years many papers have appeared which attempt to describe physics beyond the Standard Model in a model independent way using effective lagrangians [1]. Many of these papers present scenarios where enormous deviations from the Standard Model [2] are obtained. Given these situations one might reasonably ask whether there is any model that could generate such striking deviations. and. even in case where no such deviations are observed, what constraints on the underlying theory can be inferred from the experimental limits.

In this short note I want to describe how one can answer these questions. Since we have not observed yet any clear deviation from the Standard Model, the constraints on new physics are not completely unambiguous. Yet there are several statements that can be made irrespective of the kind of new physics which awaits us. The aim is to provide a sound recipe for extracting limits on the scale of new physics from the experimental bounds on the deviations from the Standard Model.

I will first motivate the results using electroweak physics as an example. Then I will discuss weakly and strongly interacting heavy physics concentrating on the interesting case of the vector-boson interactions.

\section{J. WUDKA *}

Department of Physics

University of California at Riverside

Riverside, California 92521-0413; U.S.A.

\section{ABSTRACT}

A prescription is presented for the interpretation of the coefficients in an effective lagrangian in terms of physical mass scales.

jose, wudkatoucredu
Most of the contents in this paper have appeared in various publications; my purpose is to present a summary of the results.

\section{Electroweak interactions as an example}

When considering the low-energy limit of a given theory one has (inevitably) to deal with effective interactions produced by virtual heavy physics effects [1]. Thus, when we consider the low-energy limit of the electroweak sector [3] of the Standard Model, one obtains Fermi's theory of the weak interactions. QED for all light fermions is also generated, together with a host of other interactions such as those describing the weak contributions to the fermion's anomalous magnetic moments, the $W$ and heavy quark contributions to the Euler-Heisenberg lagrangian, etc.

All these non-renormalizable interactions come with dimensional coefficients. For example, the term describing the weak contributions to the anomalous magnetic moment of the muon.

$$
\bar{\mu} \sigma^{\alpha \beta} \mu F_{\alpha \beta}
$$

has dimension five and will appear multiplied by a constant of mass dimension -1 . The four-fermion operators describing low-energy electroweak physics have dimension six and appear multiplied by a constant of mass dimension -2, etc.

When considering a specific theory such as the Standard Model all these dimensional coefficients can be calculated in terms of the gauge coupling constants. the $W$ and $Z$ masses, etc. Such interactions inherit the symmetries of the Standard Model: not all possible Lorentz invariant terms occur. 
With the benefit of hindsight, we may reinterpret the history of the Standard Model: when we were ignorant about the details of the electroweak theory what we did was write down the most general set of operators which

(i) Contained light fields (eg. leptons).

(ii) Respected the QED gauge symmetry (but not necessarily its global symmetries such as $P$ and $C !$ )

Such operators appeared multiplied by unknown coefficients which were constrained by the data. In this way we realized that the charged-current couplings were of the $\mathrm{V}-\mathrm{A}$ type and not something else. We were also able to get an idea of the order of magnitude of the scale of the new physics, and for this we used the fact that the four-fermion operators could be generated by some heavy particle through a process such as the one in the figure below.

light

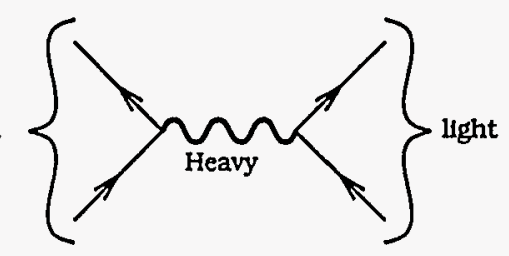

From such ideas-we-concluded that the coefficient-of the-four-fermion interactions would be-of-the form

$$
\left(\frac{\text { coupling constant }}{\text { heavy mass }}\right)^{2} \text {. }
$$

If we assume that the theory underlying the weak interactions is weakly coupled, so that (coupling constant ) $\leqslant 1$, we could get an estimate of the heavy mass from the observation of the processes mediated by the four-fermion interactions. We then designed colliders to probe physics at that energy.

Note also the two following points

- We did not expect the four-fermion interactions to be an accurate description of weak processes at energies close to the scale we just inferred. For example, the four-fermion theory cannot describe weak physics near the $Z$ pole.

- Not all weak effects are so amenable to observation. For example, the weak contributions to the anomalous magnetic moments are very small (at the $10^{-9}$ level) and only now are being probed at the Brookhaven experiment AGS $821[4]^{1}$. This is not because these weak contributions are accidentally suppressed, nor are they forbidden by some symmetry. The reason is simply that they are generated by loops, and thus are naturally small [6].

\footnotetext{
1 Extracting these effects from the data presents other problems, see [5] and references therein.
}

Thus when studying the weak interactions we took a very sensible approach: we selected those effects which are tree-level generated, and then restricted our interest to those processes forbidden by QED. By doing this we optimized the chances.of obtaining information about the interactions ultimately responsible for Fermi's effective theory. This is, of course, an unfair oversimplification of the history, but it does emphasize the following points

(a) When we want to discover new physics through the virtual processes which it induces, the most sensible thing to do is to select the effects that can be generated at tree level, and leave the study of loop-generated effects for later ${ }^{2}$.

(b) When describing the underlying theory through its low-energy manifestations, one should have an idea of which processes are responsible for the effective operators we are considering (eg. a $Z$ mediating $\left.e^{+} e^{-} \rightarrow \nu \bar{\nu}\right)$. In this way we can obtain rough estimates of the physical scales involved in the theory.

(c) A description of new physics in terms of effective operators has a limited range of validity. One can derive from the formalism the scale at which such description will fail [1]. Applying the formalism beyond such scale will give wrong results.

Even if we had been unlucky and found no deviations from QED. The above process would have provided a lower bound for the scale of new physics.

These considerations. though somewhat self-evident, are regularly ignored when considering physics beyond the Standard Model through effective operators. It is not true that "anything can happen beyond the-Standard-Model":- the fact that-the-Standard Model is'so well measured puts very strong restrictions on the kinds of new physics that could be responsible for the virtual effects we are attempting to measure. This is true even if we have not probed all possible processes: a strong deviation from the Standard Model in. for example, the $W I V Z$ coupling cannot occur in isolation, a host of other effects must be present concurrently which are constrained by current data.

The main restriction on the underlying theory is that it should respect the Standard Model gauge symmetries. If we assume that this is not the case then. even if the deviations from gauge invariance occur at a scale $\Lambda$, they will induce gauge variant terms to which existing data is sensitive [7]. In this case the many consequences of gauge invariance. such as lepton universality, would be nothing more than amazing coincidences. It is also important to note that in all models studied to date low energ: gauge invariance is respected by the underlying theory.

These restrictions do not extend to gloial symmetries. For example $S U(5)$ GUT does respect the Standard Model gauge symmetry but violates lepton and baryon numbers.

\footnotetext{
2 Though this is appropriate for weakly coupled theories similar considerations apply for strongly coupled ones, see
belorr.
} 


\section{Weakly coupled theories}

If the physics beyond the Standard Model were weakly coupled, and if we are interested in the virtual effects from the heavy particles, the relevant question is which of the manifold of terms generated at low energies could possibly be generated through tree-level graphs in the heavy theory. The list of such tree-induced processes is known [8] (provided we make the single mild assumption that the underlying theory is a gauge theory); the list of all terms of dimension 6 is also known [9]

All loop-generated terms are small, not necessarily unobservable, but harder to deal with. A reasonable strategy is to concentrate first on the observables that can get large contributions from the heavy physics.

To illustrate the consequences of these statements I will consider the possible modifications to the gauge-boson couplings induced by the heavy interactions.

3.1 VECTOR-BOSON INTERACTIONS.

Any interaction among vector bosons not contained in the Standard Model appears as an operator of dimension six or higher [9]. In unitary gauge such an operator might appear to have dimension 4, but this is because in this gauge (and ignoring Higgs interactions) the scalar doublet is replaced by a number $(=246 \mathrm{GeV})$.

All dimension 6 operators mediating vector-boson interactions are generated by loops, operators of dimension 8 and higher can be generated at tree level. Again I emphasize that such operators may appear as if they were dimension 4 operators in some gauges, but fundamentally they are not.

Because of their origin such operators get a coefficient

$$
\text { dimension 6: } \sim \frac{1}{16 \pi^{2} \Lambda^{2}} \quad \text { dimension } 8: \sim\left(\frac{v}{\Lambda}\right)^{4}
$$

where $\Lambda$ denotes a physical mass scale, i.e., the mass of a particle or other similar threshold.

The standard notation [2] is not derived from the effective lagrangian approach based on gauge invariant operators, but uses an effective Lagrangian restricted only through Lorentz and QED gauge invariances. Nonetheless the arguments described above can be used to interpret the couplings which appear in the standard approach.

Consider for example the $W W Z$ interaction [2]

$$
-i e \cot \theta_{w} \frac{\lambda}{M_{w}^{2}} W_{\alpha \beta}^{+} W_{\beta \mu}^{-} Z_{\mu \alpha}
$$

where $e$ denotes the proton charge, $\theta_{w}$ the weak-mixing angle, and $W_{\alpha \beta}^{ \pm}=\partial_{\alpha} W_{\beta}^{ \pm}-\partial_{\beta} W_{\alpha}^{ \pm}, Z_{\alpha \beta}=$ $\partial_{\alpha} Z_{\beta}-\partial_{\beta} Z_{\alpha}$ are the field strengths for the $W$ and $Z$ vector-boson fields. The coupling $\lambda$ is unknown and parametrizes a certain type of new physics; the gauge invariant formalism provides the estimates [6]

$$
\lambda \sim \begin{cases}\frac{6 M_{*}^{2} g^{2}}{16 \pi^{2}} \Lambda^{2} & \text { for } \operatorname{dim} \cdot 6 \text { operators, } \\ \frac{M^{4}}{\Lambda^{2}} & \text { for } \operatorname{dim}-8 \text { operators, }\end{cases}
$$

where, as before, $\Lambda$ denotes the mass of a heavy excitation, $g$ is the $S U(2)$ gauge coupling constant and $M_{w}$ the $W$ mass (the factor of 6 is due to combinatorics).

Using the above estimate one can understand what the experimental limits imply with respect to the underlying theory (corresponding to dimension 6 and 8 terms respectively)

$$
\lambda \sim \frac{1}{\left(100 \Lambda_{\mathrm{TeV}}\right)^{2}}, \quad \frac{1}{\left(12.5 \Lambda_{\mathrm{TeV}}\right)^{4}}
$$

where $\Lambda_{\mathrm{TeV}}$ denotes the scale of new physics in $\mathrm{TeV}^{V}$ units. Thus the statement $|\lambda|<0.1$ corresponds to $\Lambda>150 \mathrm{GeV}$ while $|\lambda|<10^{-4}$ implies $\Lambda>1 \mathrm{TeV}$.

This illustrates the power of the gauge invariant approach: we are able to interpret the results in terms of physical quantities and determine the implications on the scale of new physics ${ }^{3}$. For the case of the $W W Z$ interactions if we wish to probe physics at the $1 \mathrm{Tel}$ scale we must be able to measure $\lambda$ to to a precision of $\sim 10^{-4}$. This should be done, of course, at colliders whose $\mathrm{CM}$ energies lie below $1 \mathrm{TeV}$, otherwise the heavy particles would be produced directly and the effective operator formalism fails, just as the four-fermion theory should not be used at energies $\gtrsim S 0 \mathrm{Gel}$.

Similar considerations apply, for example, to the $Z_{\gamma \gamma}$ couplings. Here it is known [9] that the operators responsible for such couplings are of dimension 8 or higher and can be generated via tree graphs [8]. The coefficients are then expected to be of the form $1 / \Lambda^{4}$. The standard notation for this case [11] is based, again, on a lagrangian restricted only by Lorentz and QED gauge invariances. As an example consider the interactions

$$
i \frac{h_{3}^{Z}}{M_{z}^{2}}\left[\left(\square+M_{z}^{2}\right) Z_{\mu}\right] \tilde{F}^{\mu \nu} Z^{\nu}, \quad i \frac{h_{4}^{Z}}{M_{\Sigma}^{4}}\left[\left(\square+M Y_{z}^{2}\right) \partial_{\alpha} Z_{\mu \nu}\right] \tilde{F}^{\mu \nu} Z^{\alpha}
$$

denoting by $Z_{\alpha}$ the $Z$-boson field, $Z_{\mu \nu}=\partial_{\mu} Z_{\nu}-\partial_{\nu} Z_{\mu}$ and $\bar{F}_{\mu \nu}$ is the dual of the photon field strength. We then have the estimates

$$
h_{3}^{Z} \sim \frac{v^{2} M_{z}^{2}}{\Lambda^{4}} \simeq \frac{1}{\left(6.7 \Lambda_{\mathrm{TeV}}\right)^{4}}, \quad h_{4}^{Z} \sim \frac{M_{z}^{4}}{\Lambda^{4}} \simeq \frac{1}{\left(11 . \mathrm{TeV}_{\mathrm{Te}}\right)^{4}} .
$$

so that a bound $\left|h_{3,4}^{Z}\right|<1$ implies $\Lambda>150 \mathrm{GeV}$ and $\Lambda>92 \mathrm{GeV}$ respectively.

\footnotetext{
3 It has been shown argued [10] that any theory can be rendered gauge invariant by introducing spurious degrees of freedom. In this approach, however, all fermions (and scalars, when present) are assumed to be gauge singlets; nor is the gauge group uniquely fixed. Taking the Standard Model as an excellent spproximation to the low energy physics excludes this approach; for a discussion see J. Wudka in Ref. 1 .
} 


\subsubsection{Form factors}

It has been customary to use form factors to insure the theory does not violate unitarity. I will not do this here for the following reasons:

(i) The effective lagrangian approach should not be extended to scales close to a threshold. All attempts at modifying the formalism to this end are extremely model dependent and no general conclusions can be derived from them.

(ii) The form factors are usually chosen so that there are no poles in any physical process. This is unreasonable: even if in certain processes no poles occur, they will appear in the crossed channels.

As an example, the (expected) bounds $h_{3}^{Z} \leqslant 0.005, h_{4}^{Z} \leqslant 10^{-4}$ have been obtained for the LHC [12] using the $p \bar{p} \rightarrow Z \gamma \rightarrow e^{+} e^{-\gamma}$ reaction assuming that the $\mathrm{CM}$ energy was $14 \mathrm{TeV}$ while the scale of the form factor was $1.5 \mathrm{TeV}$. These values, however, imply that we have enough energy to observe directly the heavy physics $(1.5 \ll 14)$. The effective lagrangian approach breaks down in this region and no reliable information can be derived from this approach, but this is of little importance: the new physics would be directly observable.

Similar statements can be made for all form-factor modifications of effective couplings. In fact there is an example from low-energy hadron physics which illustrates the above statements. Consider the decay $K \rightarrow \pi e \nu$ which is characterized by two form factors [13] parametrized in the form $1+\lambda_{K} q^{2} / m_{\pi}^{2}$; $q$ is the difference between the $K$ and $\pi$ four-momenta, $\lambda_{k \pi} \sim 0.03$ is a constant and $m_{\pi}$ is the pion mass. This. to the same order in $q^{2}$ is equivalent to $1 /\left(1-q^{2} / n M^{2}\right)^{n}, M \simeq 800 \mathrm{GeV}$, which has poles at $q^{2}=n M^{2}$. Of course we do find "new" physics (i.e. physics beyond the lightest pseudoscalar mesons) around $M$. It is also true that one cannot simply replace the form factor by the expression $1 /\left(1-q^{2} / n M^{2}\right)^{n}$ in order to describe this new physics entirely.

\subsection{LARGE EFFECTS}

As I mentioned above, some operators are generated at tree level; for the corresponding processes we do not expect an a priori suppression. In this subsection I will give some examples. I will write all operators in the unitary gauge.

\subsubsection{Fermion-gauge-boson couplings}

Certain kinds of new physics can induce right-handed couplings of the $W$ to the quarks. The specific interaction is

$$
\frac{1}{\Lambda^{2}}(v+H)^{2} \bar{u}_{R} W^{+} d_{R}
$$

where $v=246 \mathrm{GeV}$ and $H$ denotes the Higgs field. Similar terms can be generated for the $b-t$ and the $c-s$ quark pairs. Certain kinds of physics also generate terms which mix generations. Some such interactions are probed by the Michel- $\rho$ parameter, as well as by the $W$ lifetime and branching ratios. All the bounds derived in this way are relatively weak: $\Lambda \gtrsim 500 \mathrm{GeV}$ (for the first and second generations only).
The couplings of the fermions to the $Z$ can be also modified. The bounds derived from the oblique $\rho$ parameter as well as from the LEP1 data are stronger than the ones above. implying $\Lambda \gtrsim 2 \mathrm{TeV}$ [14].

\subsubsection{Higgs couplings}

The presence of new interactions can modify the couplings of the Higgs to the fermions, the Higgs self couplings and the fermion- $V$-Higgs coupling $(V=W, Z)$. An example of the latter effects is the right handed current coupling described above.

\subsubsection{Four-fermion interactions}

Many different kinds of physics will generate four-fermion interactions, both CP violating and $\mathrm{CP}$ conserving. Such interactions are strongly bounded if they occur between first-family fermions. For the third family the bounds are generally weak (or non-existent).

The operators generated by vector exchange have been studied for NLC type of machines [15]. Similar studies exist for LHC and other hadron colliders [16]. I am not aware of a comprehensive study (including scalar and vector exchange possibilities) for the NLC.

\section{Strongly coupled theories}

When the underlying theory is strongly coupled ${ }^{4}$ the calculational reliability is reduced for quantitative predictions. It is still possible. however. to provide some reliable estimates [1i]. The idea is the following: let $\Lambda$ be the scale of new physics and assume that the interactions of the particles lighter than $\Lambda$ (the light excitations) is described by some effective theory which contains a series of (effective) coupling constants. Just as in other theories, one can use the effective theory to calculate the renormalization group evolution of these couplings. In the case of strong coupling one must work to all orders in perturbation theory, which is in general technically impossible. One can, however, estimate the renormalization group evolution and require that the running coupling constants do not diverge at lower energies. This yields upper bounds on the various coefficients of the terms in the effective lagrangian. It is interesting to note that the same estimates for the $W W Z, W W \gamma$ and $Z Z_{\gamma}$ couplings derived above are obtained, that is, the expressions (3.3) and (3.6) are valid also for stronsly coupled underlying theories.

These arguments can be further specialized if it is assumed that there are no light scalar particles. i.e. that the low energy spectrum corresponds to the Standard Model without the Higgs excitation. In

4 This possibly is usually associated with the assumption that there is no light Higgs, I will comment on this later.

5 In this argument it is assumed that no cancellations occur between various graphs, i.e. the theory is assumed to be natural $[18]-$ no fine tunings are required. 
this case the scale of new physics $\Lambda$ is constrained to be $\leqslant 4 \pi v \sim 3 \mathrm{TeV}[19,17]$ and some modifications occur which lead to refinements of the above bounds. For example (3.3) and (3.6) are replaced by

$$
\lambda \sim \frac{6 M_{w}^{2} g^{2}}{16 \pi^{2} \Lambda^{2}}, \quad h_{3}^{Z} \sim \frac{v^{2} M_{z}^{2}}{16 \pi^{2} \Lambda^{4}}, \quad h_{4}^{Z} \sim \frac{M_{z}^{4}}{16 \pi^{2} \Lambda^{4}}, \quad(\Lambda \lesssim 3 \mathrm{TeV}) ;
$$

note that $h_{3,4}^{Z}$ acquire a strong suppression factor, now a constraint $\left|h_{3,4}^{Z}\right|<1$ imply $\Lambda>40 \mathrm{GeV}, 26 \mathrm{GeV}$ respectively.

\section{Rigidity of the bounds}

I have argued above that there is a way of estimating the couplings which parametrize non-Standard Model effects using gauge-invariant effective lagrangians. The question is then how rigid are these bounds.

Consider first the weakly coupled theory. One can argue that under certain circumstances a given loop graph could be enhanced by having several particles in the loop. This gives an order of magnitude leeway in the above estimates (note that the same could be said about the tree-level graphs).

What one cannot say is that there could be hundreds of particles in the loop whose contributions cancel the $1 /(4 \pi)^{2}$ entirely. If this were the case the theory would be such that the one-loop effects would be as large as the tree-level ones and the theory would be, in fact, strongly coupled. One can study such situations in exactly-solvable toy models (J. Wudka, Ref. 1) and the result is that the effect of this type of effects significantly alters the theory: it is not possible (without significant fine-tuning) to maintain the scalar mass below the cutoff. But, if the Higgs is no longer in the light theory we must examine the model as a strongly coupled one. For this case we revert to the arguments given in section 4 above.

For strongly coupled theories the bounds, as I mentioned, are more qualitative. They are based on the assumption that no fine tuning should be required of the underlying theory. If one grants this, the bounds given hold (again with an order of magnitude uncertainty). I would also like to add that these arguments can be applied to the non-linear sigma model which describes low-energy hadron physics and they agree well with experiment [20].

For example, allowing for a factor of 10 enhancement in $\lambda$ would imply that, in order to probe physics at the $1 \mathrm{TeV}$ level one should measure $\lambda$ to a precision of $\lesssim 10^{-3}$. Similarly $h_{3,4}^{2}$ should be measured to a precision of $\lesssim 5 \times 10^{-3}$ and $\lesssim 7 \times 10^{-1}$ respectively.

While it is possible for some couplings to be thus enhanced, it is also possible for them to be suppressed, either accidentally or as a result of a symmetry. Thus a strong constraint on a given effective coupling might indicate either a large value of $\Lambda$ or the fact that the underlying theory suppresses the coefficient under consideration. If all effective couplings expected a priori not to contain the (small) factors of $1 /(4 \pi)$ are measured to be very small, the simplest possibility is that this is a result of large $\Lambda$, still all possible scenarios should be considered when analyzing the data.
If one allows for fine tuning several of these statements can be obviated. In this case, however. consistency would allow us to fine tune anything we want. such models contain no information. Of course one could say that Nature has chosen to fine tune just those interactions which we have not probed directly, and while this is a logical possibility, I will not consider it.

\section{Conclusion}

From the arguments given one can conclude that there is a reliable method for extracting information about the scale of new physics from the existing and expected data. In deriving the dependence of the measurements on this scale one can work in a model independent way using effective lagrangians. This does not mean, however, that the coefficients can in principle have any values whatever: general consistency requirements forbid their being too large and provide estimates for their value. Using this input one can then determine the reach into the realm of heavy physics that a given experiment has.

I have also strived to show that triple boson couplings are not the best place to look for deviations from the standard model. Despite the fact that one can write down lagrangians which appear to generate easily observable deviations for these couplings, such "models" cannot be derived from any consistent theory, weakly or strongly coupled, with or without light scalars. The point is that one cannot state. by the mere fact that a coefficient is measured to be small compared to one, that the corresponding experiment is a sensitive probe of new physics. For example. measuring the anomalous magnetic moment of the muon to $10^{-i}$ says nothing about non-Standard Model physics [6] (taking chiral symmetry to be natural [18]).

The formalism presented determines the constraints an experiment should satisfy in order to probe new physics up to a given scale. For example in order for a $500 \mathrm{GeV}$ collider to probe $W W Z$ physics beyond $1 \mathrm{TeV}, \lambda$ should be measured to a precision better than $\sim 10^{-4}$. Similar precision is required for the parameter $\Delta \kappa$ (related to the heavy physics contributions to the W magnetic and electric quadrupole moments [12]).

The processes which are worth measuring are those for which the coefficients of the effective operators are as large as possible. It is of course possible that the underlying theory will suppress precisely those couplings, but I believe it is better to look at these terms than to concentrate on terms which we are certain provide very small effects.

The interactions with the largest coefficients have been catalogued for the case of weakly-interacting heavy physics [8]. It is also possible to determine the kind of physics responsible for each of the treelevel generated operators. Examples of such interactions are the four-fermion interactions (generated by scalar of vector exchanges ${ }^{6}$ ), and the $Z$ couplings to fermions; the particular case of the $Z b \bar{b}$ vertex can be shown to receive its largest contributions through either $Z-Z^{\prime}$ mixing (where $Z^{\prime}$ denotes a heary

6 Tensor exchanges are reduced using Fierz identities. 
vector boson) or through mixing of $b$ with some heavy fermions. Both of these possibilities have been studied in the literature [21]; it can be shown using the results of [8] that these are the only possibilities: no other kind of heavy physics can alter this vertex as significantly.

Thus the effective lagrangian approach can not only estimate coefficients, but can also exhibit the culprits responsible for any operator. It is precisely the insistence that the underlying physics should be described by a consistent model (whatever the details) that imposes the various constraints discussed above. If such consistency requirements are foregone, the coefficients can indeed take any values, but in this case the underlying physics is not described by any consistent theory.

Acknowledgements: The author would like to thank M. Einhorn and $\mathrm{K}$. Riles for many useful comments. This work was supported in part through funds provided by the Department of Energy

\section{REFERENCES}

[1] See, for example, the reviews. H. Georgi, Ann. Rev. Nucl. Part. Science 43, 209 (1994). J. Polchinski, Lectures presented at TASI 92. Boulder, CO, Jun 3-28, 1992. A. Pich, Lectures given at 5th Mexican School of Particles and Fields, Guanajuato, Mexico, 30 Nov - 11 Dec 1992. G. Ecker, Prog. Part. Nucl. Phys. 35. 1 (1995). J. Wudka, Int. J. Mod. Phys. A9, 2301 (1994).

(2) K. Hagiwara et al., Nucl.Phys.. B282, 253 (1987). G.L. Kane et al., Phys. Rev. D39, 2617 (1989). U. Baur and E.L. Berger (Argonne), Phys. Rev. D41 1476 (1990). T. Helbig and H. Spiesberger, Nucl. Phys. B373, 73 (1992). X.-G. He and B. McKellar, Phys. Lett. B320, 165 (1994).

[3] G.-L. Lin et al., Phys. Rev. D49, 2414 (1994); ibid D44, 2139 (1991). H. Steger et al., Phys. Rev. Lett. 59, 385 (1987)

[4] V.W. Hughes, AIP conference proceedings no. 187, (1989) 326. M. May, AIP conference proceedings no. 176, (1988) 1168

[5] M.B. Einhorn, Phys. Rev. D49, 1668 (1994).

[6] C. Arzt. et al., Phys. Rev. D49, 1370 (1994).

[7] M. Veltman, Acta Phys. Polon. B12, 437 (1981).

[8] C. Arzt. et al., Nucl. Phys. B433, 41 (1995).

[9] C.J.C. Burges and H.J. Schnitzer, Nucl. Phys. B228, 464 (1983). C.N. Leung et al., Z. Phys. C31, 433 (1986). W. Buchmüller and D. Wyler, Nucl. Phys. B268, 621 (1986).

[10] C.P. Burgess and D. London, report MCGILL-92-04. e-Print Archive: hep-ph/9203215.

[11] U. Baur and D. Zeppenfeld, Phys. Lett. 201B, 383 (1988). U. Baur and E.L. Berger, Phys. Rev. D47, 4889 (1993).

[12] H. Aihara et al., Summary of the Working Subgroup on Anomalous Gauge Boson Interactions of the DPF Long-Range Planning Study, to be published in Electroweak Symmetry Breaking and Beyond the Standard Model, eds. T. Barklow, S. Dawson, H. Haber and J. Seigrist. e-Print Archive: hep-ph/9503425.
[13] L.B. Okun, Leptons and quarks, sect. 6.4 (North Holland, 1984). Review of Particle Properties, Phys. Rev. D50, 1177 (1994).

[14] B. Grzadkowski and J. Wudka, Phys. Lett. B364, 49 (1995).

[15] A. Djouadi et al., in proceeding of the Workshop on Physics and Experiments with Linear Colliders, Saariselka, Finland, Sep 9-14, 1991.

[16] See, for example, E. Eichten et al., Rev. Mod. Phys 56, 579 (1984), ERRATUM-ibid 58, 1065 (1986).

[17] S. Weinberg, Physica 96A, 327 (1979). H. Georgi and A. Manohar, Nucl. Phys. B234. 189 (1984). H. Georgi, Phys. Lett. B298, 187 (1993).

[18] G. 't Hooft, lecture given at Cargese Summer Inst., Cargese, France, Aug 26 - Sep 8, 1979.

[19] H. Georgi and A. Manohar, Nucl. Phys. B234. 189 (19S4). T. Appelquist and G.-H. Wu, Phys. Rev. D48, 3235 (1993).

[20] J. Gasser and H. Leutwyler, Nucl. Phys. B250, 465 (1985). R.S. Chivukula et al., Ann. Rev. Nucl. Part. Sci. 45, 255 (1995).

[21] E. Ma et al., Phys. Rev. D53, 2276 (1996). G. Bhattacharyya et al., report CERX-TH-95326 (unpublished). e-Print Archive: hep-ph/9512239. T. Yoshikawa et al., report HLPD-952S (unpublished). e-Print Archive: hep-ph/9512251. G. Altarelli et al, report CERN-TH-96-20 (unpublished). e-Print Archive: hep-ph/9601324. C.-H. V. Chang et al., report NHCU-HEP96-1 (unpublished). e-Print Archive: hep-ph/9601326. P. Bamert et al., report MCGILL-96-0t (unpublished). e-Print-Archive: hep-ph/9602438. K.S. Babu et al., report IASSNS-HEP-96-20 (unpublished). e-Print Archive: hep-ph/9603212. D. Comelli and J.P. Silva, report HEPPH9603221 (unpublished). e-Print Archive: hep-ph/9603221. 


\section{MEASUREMENT OF THE $W W \gamma$ and $W W Z$ COUPLINGS IN THE PROCESS $e^{+} e^{-} \rightarrow \ell \nu q \vec{q}^{+}$}

\section{MiKULÁŠ GinTNER, STEPHEN GOdFREY}

Ottawa-Carleton Institute for Physics

Department of Physics, Carleton University, Ottawa CANADA, K1S $5 B 6$

and

\section{Gilles Couture}

Département de Physique, Université du Québec à Montréal C.P. 8888, Succ. Centre-Ville, Montréal, Québec, Canada, H3C $3 P 8$

\section{ABSTRACT}

We studied the sensitivity of the process $e^{+} e^{-} \rightarrow \ell \nu q \bar{q}^{\prime}$ to anomalous trilinear gauge boson couplings of the $W W \gamma$ and $W W Z$ vertices at the center of mass energies $\sqrt{s}=500 \mathrm{GeV}$ and $1 \mathrm{TeV}$. The bounds for the couplings we obtained result from an analysis of a five dimensional angular differential cross section. In our calculations we included all tree level Feynman diagrams contributing to the final state as well as the finite widths of the vector bosons. Both unpolarized and polarized beams were considered. We found that the $500 \mathrm{GeV}$ measurements will be at the level of loop contributions to the couplings and may show hints of new physics while the $1 \mathrm{TeV}$ should be sensitive to new physics at the loop level. We also explored $\ell \nu q \bar{q}$ final states of the $W$ resonance and found that useful information could be extracted from this region of phase space.

\section{Introduction}

One of the important goals of the Next Linear Collider (NLC) is to make precision measurements of $W$ boson properties including $W$-boson interactions with the photon and $Z^{0}$. The measurements of the trilinear gauge boson vertices (TGV's) provide a stringent test of the gauge structure of the standard model[1,2]. The current measurement of these couplings performed by the CDF and DO collaborations [3] are rather weak. Although the measurements at the Large Hadron Collider should improve these limits considerably it is expected that measurements at high energy $e^{+} e^{-}$colliders will surpass those at the hadron colliders.

Probably the most useful of the $e^{+} e^{-}+W^{+} W^{-}$channels for these measurements is $e^{+} e^{-} \rightarrow \ell \nu q \vec{g}^{\prime}$. With only one unobserved neutrino this channel has several advantages: it can be fully reconstructed using the constraint of the initial beam energies, the $W^{+}$and $W^{-}$can be discriminated using lepton charge identification, it does not have the QCD backgrounds that plague the fully hadronic decay modes, and it offers much higher statistics than the fully leptonic modes.

In this paper we examine the sensitivity of this channel to anomalous $W W \gamma$ and $I V I W Z^{0}$ gauge boson couplings. We study this process for the centre of mass energies $\sqrt{s}=500$ and $1000 \mathrm{GeV}$ appropriate to the NLC. In our calculations we included all tree level diagrams to the four fermion final states using helicity amplitude techniques and we considered the finite widths of the vector bosons. To determine the sensitivity of the channel to anomalous gauge boson couplings we examined numerous distributions [4]. We also looked at the question of how important the non-resonant diagrams are in these calculations and how much information about the TGV's can be found in the off-resonance production. Using helicity amplitudes we are able to study the usefulness of initial state polarization in extracting the TGV's.

In the next section we discuss the effective Lagrangian parametrization and make introductory remarks on our calculations. In section 3 we present and discuss our results. We summarize our conclusions in section 4.

\section{Calculations}

\subsection{Parametrization}

In order to describe TGV's we use the most general parametrization possible that respects Lorentz invariance, electromagnetic gauge invariance and $C P$ invariance $[5,6, i]$. These constraints leave us with five free independent parameters for the $W W_{\gamma}$ and $W W Z$ vertices and the effective interaction lagrangian is given by

$$
\mathcal{L}_{W W \nu}=-i g_{\nu}\left\{g_{1}^{\prime \prime}\left(W_{\mu \nu}^{+} W^{-\mu}-W^{+\mu} W_{\mu \nu}\right) V^{\nu}+\kappa \nu W_{\mu}^{+} W_{\nu}^{-} V^{\mu \nu}+\frac{\lambda_{\nu}}{{ }_{M}^{2}} W_{\lambda \mu}^{+} W_{\nu}^{-\mu} V^{\nu \lambda}\right\}
$$


where the subscript $V$ denotes either a photon or a $Z^{0}, V^{\mu}$ and $W^{\mu}$ represents the photon or $Z^{0}$ and $W^{-}$fields respectively, $W_{\mu \nu}=\partial_{\mu} W_{\nu}-\partial_{\nu} W_{\mu}$ and $V_{\mu \nu}=\partial_{\mu} V_{\nu}-\partial_{\nu} V_{\mu}$ and $M_{W}$ is the $W$ boson mass. ( $g_{1}^{\gamma}$ is constrained by electromagnetic gauge invariance to be equal to 1.) At tree level the standard model requires $g_{1}^{Z}=\kappa_{V}=1$ and $\lambda_{v}=0$.

This approach has become the standard parametrization used in phenomenology making the comparison of the sensitivity of different measurements to the TGV's straightforward. Later in the paper we show bounds that could be imposed on these five parameters measuring the $e^{+} e^{-} \rightarrow \ell \nu q \bar{q}^{\prime}$ process at NLC machines. We also calculate the sensitivity to the $L_{9 L}$ and $L_{9 R}$ parameters [4] that appear in the chiral Lagrangian parametrization of the effective TGV's.

\subsection{Initial remarks and conditions}

There are 10 (20) diagrams contributing to the $\mu^{ \pm} \nu_{\mu} q \vec{q}^{\prime}\left(e^{ \pm} \nu_{e} q \vec{q}\right)$ final state [4]. Of these there are two (three) diagrams which contain TGV's in the muon (electron) mode of the reaction. To include finite width effects we used vector boson propagators of the form $\left(s-M M_{V}^{2}+i \Gamma_{V} M_{V}\right)^{-1}$ which yields a gauge invariant result. We used the CALKUL helicity amplitude technique [8] to obtain expressions for the matrix elements and performed the phase space integration using Monte Carlo techniques. To obtain numerical results we used the values $\alpha=1 / 128, \sin ^{2} \theta=0.23, M_{z}=91.187 \mathrm{GeV}, \Gamma_{z}=2.49 \mathrm{GeV}, M_{w}=80.22 \mathrm{GeV}$, and $\Gamma_{W V}=2.08 \mathrm{GeV}$. In our results we included two generations of quarks and took them to be massless. In order to take into account finite detector acceptance we require that the lepton and quarks are at least 10 degrees away from the beam and have at least $10 \mathrm{GeV}$ energy (with exception of Table 1).

\section{Results}

\subsection{Total cross section}

We calculate total cross sections as a function of $\sqrt{s}$ for both lepton modes [4]. The values for energies of $175 \mathrm{GeV}, 500 \mathrm{GeV}$, and $1 \mathrm{TeV}$ are given in Table 1 . In general the electron mode has a larger cross-section than the muon mode. The difference is small at $175 \mathrm{GeV}$ but becomes increasingly larger at higher energy as the t-channel photon exchange becomes increasingly important, reaching a factor of 5 at $1 \mathrm{TeV}$.

Imposing cuts on the invariant masses of the $\ell \nu$ and $q \vec{q}$ pairs we can assess contributions of the non-resonant diagrams to the total cross section. For the muon mode the invarian mass cuts reduce the cross-section by $10 \%$ to $20 \%$ depending on $\sqrt{s}$ and irrespective of whether the cut is on $M_{\ell \nu}$ or $M_{q \bar{q}}$. The relatively small effect of these cuts verifies the dominance of the resonant diagrams on the total cross section. For the electron mode the cross section with the cut on $M_{Q \rightarrow-1}$ is significantly larger than the cross section with the cut on
Table 1: Cross-sections for $e^{+} e^{-} \rightarrow \mu^{+} \nu_{\mu} q \vec{q}^{f}$ and $e^{+} e^{-} \rightarrow e^{+} \nu_{e} q \vec{q}$ including cuts on the

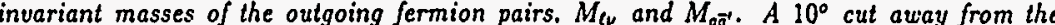
beam is imposed on charged final state fermions and no cut on their energy. The crosssections are given in $p b$.

\begin{tabular}{|c|c|c|c|c|c|}
\hline $\begin{array}{c}\sqrt{s} \\
(\mathrm{GeV})\end{array}$ & $\ell$ & no cut & $\left|M_{8 \bar{q}}-M_{W W}\right|<5 \mathrm{GeV}$ & $\left|M_{\mathcal{L V}}-M_{W}\right|<5 \mathrm{GeV}$ & both cuts \\
\hline \hline 175 & $\mu$ & 1.10 & 1.00 & 1.00 & 0.91 \\
& $e$ & 1.15 & 1.04 & 1.01 & 0.91 \\
\hline 500 & $\mu$ & 0.39 & 0.34 & 0.34 & 0.29. \\
& $e$ & 0.62 & 0.53 & 0.34 & 0.29 \\
\hline 1000 & $\mu$ & 0.077 & 0.063 & 0.064 & 0.052 \\
& $e$ & 0.44 & 0.39 & 0.064 & 0.052 \\
\hline
\end{tabular}

$M_{e v}$. This can be attributed to poles in the single $W$ production t-channel photon exchange diagrams. With appropriate kinematic cuts this can be used to study single-W production.

Despite the relative smallness of the off-resonance contributions to the muon mode they still contribute up to $30 \%$ of the cross section at $1 \mathrm{TeV}$. Clearly, they must be properly included when making high precision tests of standard model processes. For the electron mode they are even more important and are interesting in the context of single $H^{*}$ production.

\section{2 - Distributions}

We examined numerous distributions and applied various kinematic cuts to identify the observables and the regions of phase space that are the most sensitive to anomalous couplings. Details can be found in ref. [4]. Here we summarize the most interesting points and our conclusions.

Any disruption of the delicate gauge theory cancellations leads to large changes to the Standard model results. For $W_{L}$ production amplitudes the enhancements can be a factor of $\left(s / M_{W}^{2}\right)$. Because it is the longitudinal $W$ production which is the most sensitive to anomalous couplings, and because the cross section is dominated by transverse $I F$ production it is crucial to disentangle the $W_{L}$ from the $W_{T}$ background. The angular distributions of the $W$ decay products provide the most convenient tool for doing so.

To investigate the sensitivity of the $e^{+} e^{-} \rightarrow \ell^{ \pm} \nu_{\ell} q \bar{q}^{\prime}$ process to the anomalous couplings we used a combined distribution of five angular observables $(4,9,10]: \theta, \theta_{q q}, \dot{\theta}_{q q}, \theta_{\ell \nu}$, and $\phi_{\omega \nu}$, where $\theta$ is the $\mathrm{H}^{-}$scattering angle with respect to the initial $e^{+}$direction, $\theta_{q q}$ is the polar decay angle of the $q$ in the $W^{-}$rest frame using the $W^{-}$direction as the quantization axis, $\phi_{\theta g}$ is the azimuthal decay angle of the $q$ in the $W^{-}$rest frame, and $\theta_{\ell v}$ and $\dot{\phi}_{\ell v}$ are the analogous angles for the lepton in the $W^{+}$rest frame. There is an ambiguity in measurement 


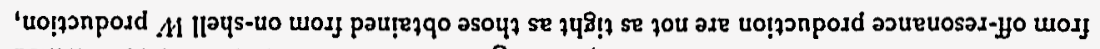

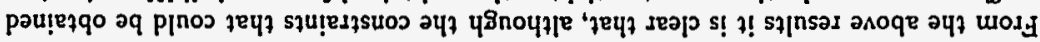

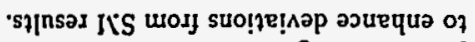

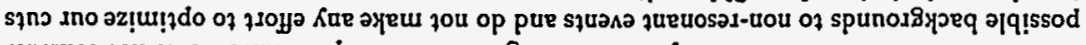

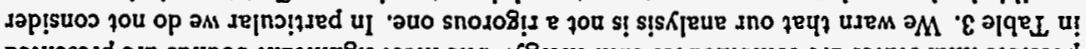

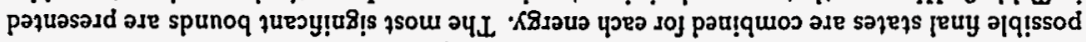

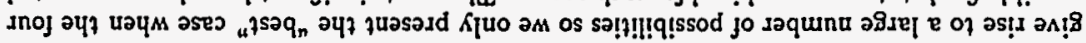

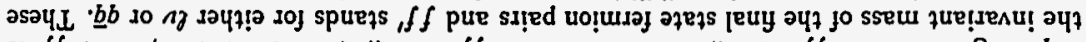

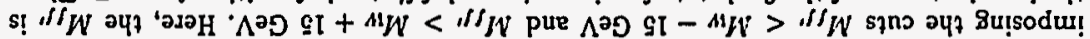

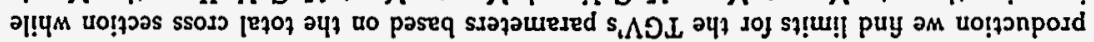

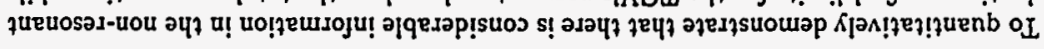

uo!jonpodd $M$

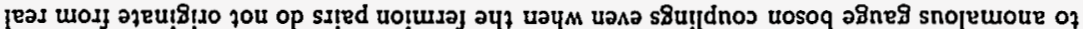

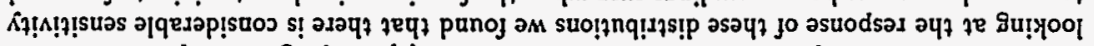

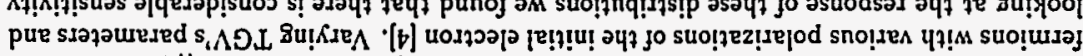

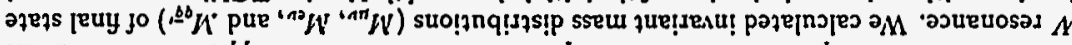

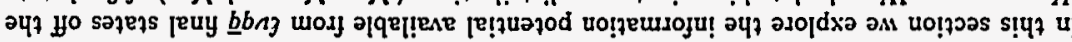

uo!ฺวnpoxd әวueuosəx-¥O $\varepsilon^{\cdot} \varepsilon$

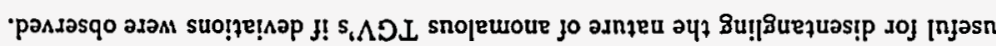

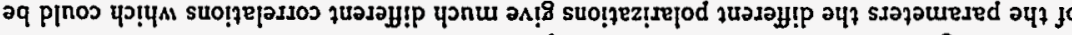
suolzeu!quos awos so

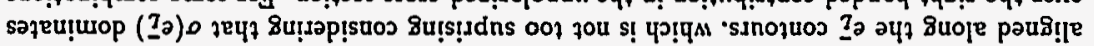

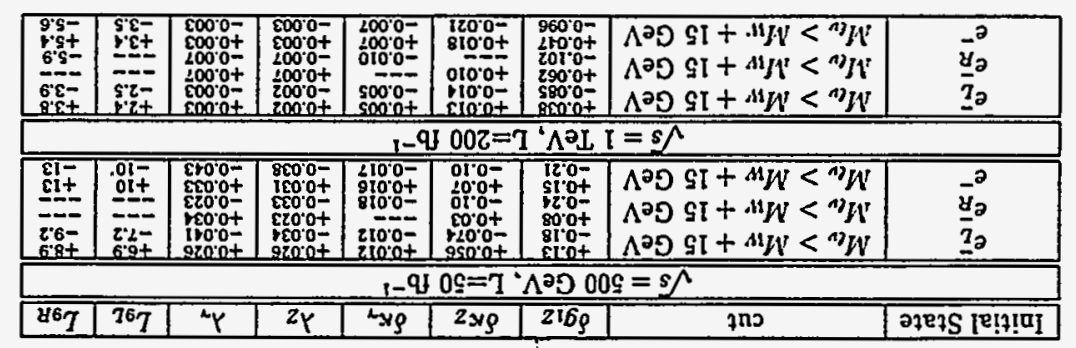

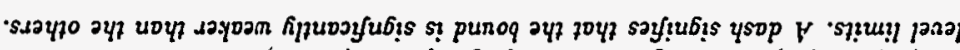

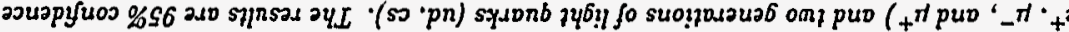

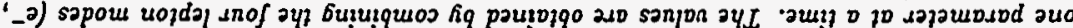

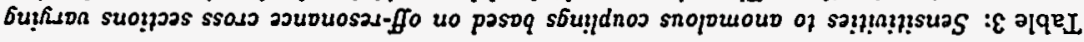

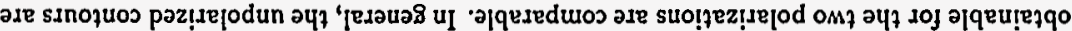

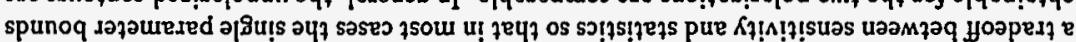

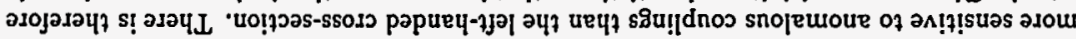

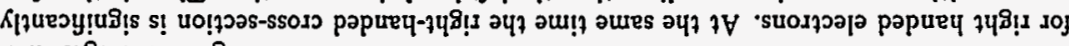

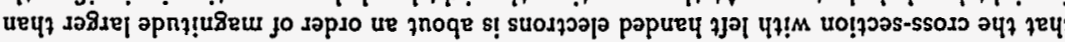
s! suo!̨ez!re

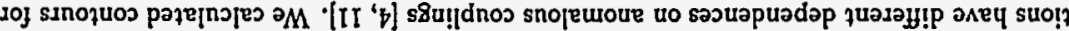

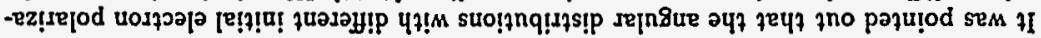

$\cdot[b]$ u! punoj aq ues sinozuos arow

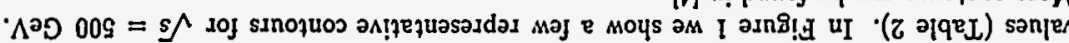

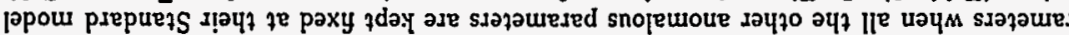

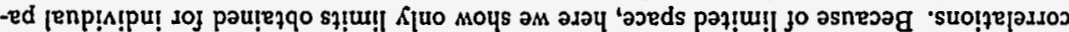

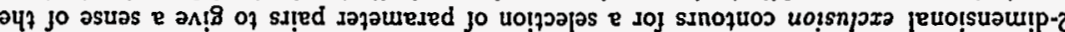

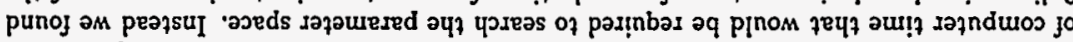

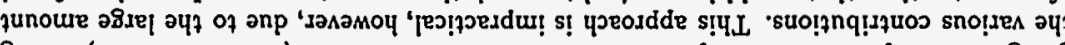

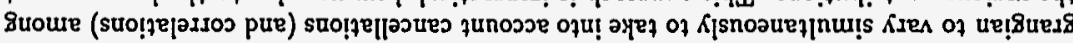

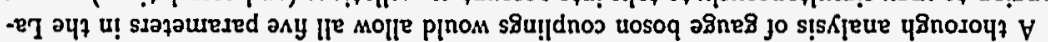

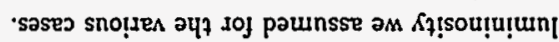

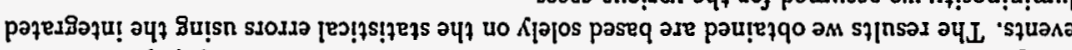

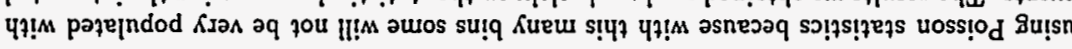

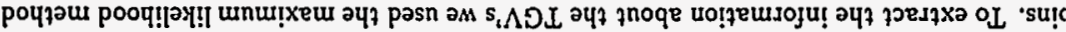

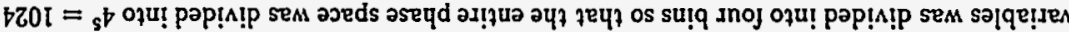

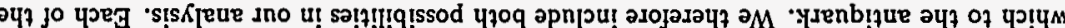

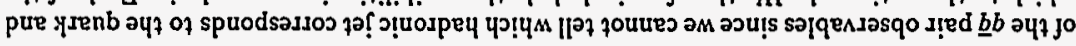

\begin{tabular}{|c|c|c|c|c|c|c|c|}
\hline 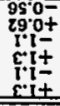 & $\begin{array}{l}87.07 \\
29.0- \\
1907 \\
290- \\
1900 \\
\end{array}$ & $\begin{array}{l}8000^{\circ} 0 \mp \\
200^{\circ} 0 \mp \\
200^{\circ} 0 \mp \\
\end{array}$ & $\begin{array}{l}8000^{\circ} 0 \mp \\
200^{\circ} 0 \mp \\
200^{\circ} 0 \mp \\
\end{array}$ & $\begin{array}{l}9000^{\circ} 0 \mp \\
100^{\circ} 0 \mp \\
100^{\circ} 0 \mp \\
\end{array}$ & $\begin{array}{l}\tau 00^{\circ} 0 \mp \\
200^{\circ} 0 \mp \\
200^{\circ} 0 \mp \\
\end{array}$ & $\begin{array}{c}1900^{\circ} 0 \mp \\
10^{\circ} 0 F \\
10^{\circ} 0 \mp \\
\end{array}$ & $\begin{array}{r}\text { pautquos } \\
2 \\
n \\
\end{array}$ \\
\hline \multicolumn{8}{|c|}{ 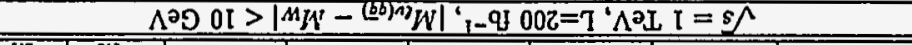 } \\
\hline 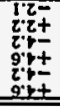 & 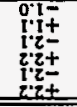 & $\begin{array}{l}9200^{\circ} 0 \mp \\
900^{\circ} 0 \mp \\
900^{\circ} 0 \mp \\
\end{array}$ & $\begin{array}{l}9800^{\circ} 0 \mp \\
900^{\circ} 0 \mp \\
900^{\circ} 0 \mp \\
\end{array}$ & $\begin{array}{l}9200^{\circ} 0 \mp \\
900^{\circ} 0 \mp \\
900^{\circ} 0 \mp \\
\end{array}$ & $\begin{array}{l}9800^{\circ} 0 \mp \\
\angle 00^{\circ} 0 \mp \\
200^{\circ} 0 \mp\end{array}$ & $\begin{array}{c}9600^{\circ} 0 F \\
020^{\circ} 0- \\
610^{\circ 0+} \\
070^{\circ} 0 F \\
\end{array}$ & $\begin{array}{r}\text { pəutquos } \\
2 \\
n \\
\end{array}$ \\
\hline \multicolumn{8}{|c|}{ 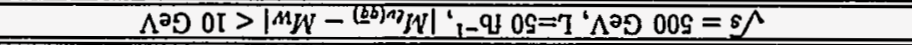 } \\
\hline 867 & $\overline{76} \mathrm{~T}$ & ${ }^{2} Y$ & $z_{Y}$ & +3y & $2 \times 9$ & ${ }_{2}^{1} 6 \rho$ & әрош \\
\hline
\end{tabular}

งา?u!n par

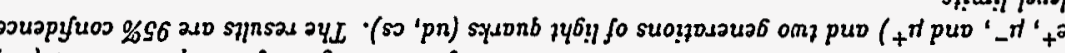

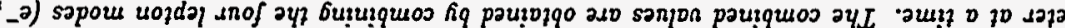

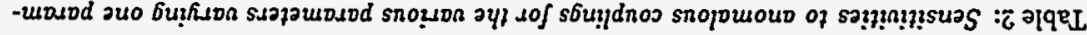



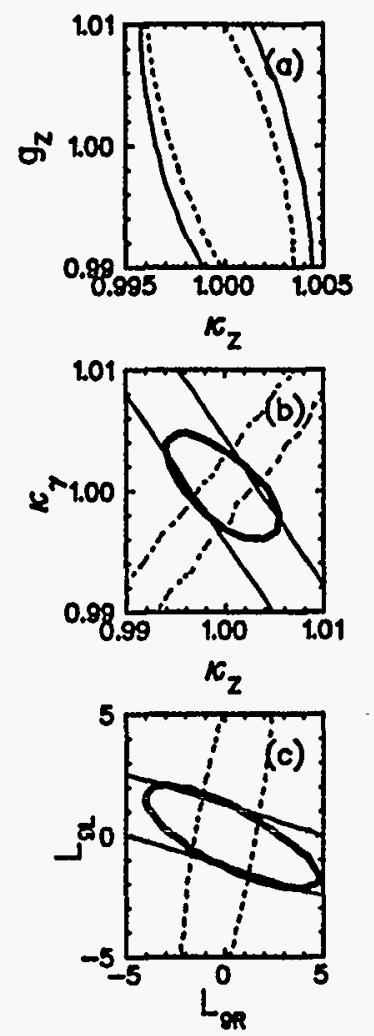

Figure 1: 95\% C.L. exclusion contours for sensitivity to anomalous couplings for unpolarized (the hcavy solid line) and polarized initial state electrons with $\sqrt{s}=500 \mathrm{GeV}$, combining all four lepton charge states. The solid curves are for $e^{-} e^{+}$, the dashed curves for $e^{-} e^{+}$. The integrated luminosity is $L=25 \mathrm{fb}^{-1}$ per polarization, for unpolarized electrons $L=50 \mathrm{fb}^{-1}$. there is nevertheless considerable information contained in these events. It appears to us that the method that makes optimal use of each event is to calculate the probability of each event, irrespective of where it appears in phase space, and compute a likelihood function for the combined probabilities. The only cuts that should be included are those that represent detector acceptance and that are introduced to eliminate backgrounds.

\section{Conclusions}

We performed a detailed analysis of the measurement of tri-linear gauge boson couplings in the process $e^{+} e^{-} \rightarrow \ell^{ \pm} \nu q \bar{q}$. To gauge the sensitivity of this process to anomalous gauge boson couplings we used the $W$ decay distributions as a polarimeter to distinguish the longitudinal $W$ modes, which are more sensitive to anomalous coupings, from the transverse modes. We implemented this through the use of a quintic differential cross section. with each angular variable divided into 4 bins, and then calculating the likelihood function of non-standard model couplings as compared to the standard model. The $500 \mathrm{GeV}$ NLC measurements are sensitive enough that they should be sensitive to loop contributions to the TGV's while the $1 \mathrm{TeV}$ will be able to measure such effects.

We studied the sensitivity of the off-mass shell cross sections to anomalous couplings by imposing kinematic cuts on the invariant mass distributions of the outgoing fermion pairs. A cursory analysis found that the off-resonance cross section is relatively sensitive to anomalous couplings and that useful information could be extracted from this region of phase space.

The optimal strategy to maximize the information contained in each event is to construct a likelihood function based on the four vector of each of the outgoing fermions on an event by event basis, putting them through a realistic detector simulation. This would make the best use of the information whether it be on the $W$ resonance or not. Kinematic cuts should only be introduced to reduce backgrounds. Since the precision of these measurements is beyond the level of loop induced radiative corrections it is crucial that radiative corrections are well understood and included in event generators used in the study of these processes.

\section{ACKNOWLEDGEMENTS}

The authors benefited greatly from many helpful conversations, communications, and suggestions during the course of this work with Tim Barklow, Genevieve Bélanger, Pat Kalyniak, Dean Karlen, and Paul Madsen. This research was supported in part by the Natural Sciences and Engineering Research Council of Canada and Les Fonds FCAR du Quebec. 


\section{References}

[1] For further recent reviews on TGV's see: I. Hinchliffe, To appear in the proceedings of the International Symposium on Vector Boson Self Interactions, UCLA, Feb 1-3, 1995, hep-ph/9504206; F. Boudjema, To appear in the proceedings of Beyond the Standard Model IV, Lake Tahoe, California, Dec. 12-16, 1994, hep-ph/9504409;

[2] For a recent comprehensive review of trilinear gauge boson couplings see: H. Aihara et al., To appear in Electroweak Symmetry Breaking and Beyond the Standard Model, eds. T. Barklow, S. Dawson, H. Haber and J. Siegrist (World Scientific), hep-ph/9503425.

[3] F. Abe et al. (CDF Collaboration), Phys. Rev. Lett. 74, 1936 (1995); J. Ellison et al. (D0 Collaboration), Proceedings of the DPF'94 Conference, Albuquerque, NM, August 1994; H. Aihara, to appear in the Proceedings of the International Symposium on Vector Boson Self-Interactions, UCLA, February 1995; T.A. Fuess (CDF Collaboration), Proceedings of the DPF'94 Conference, Albuquerque, NM, August 1994; F. Abe et Proceedings of the DPF'94 Conference, Albuquerque, NM, August 1994; F. Abe
al., (CDF Collaboration), Proceedings of the 27th International Conference on High Energy Physics, Glasgow, Scotland, July 20-27, 1994; F. Abe et al., (CDF Collaboration), FERMILAB-Pub-95/036-E, submitted to Phys. Rev. Lett.; S. Abachi et al., (D0 Collaboration), FERMILAB-Pub-95/101-E, hep-ex/9505007.

[4] M. Gintner, S. Godfrey, G. Couture, Phys. Rev. D52, 6249 (1995).

[5] K. Hagiwara et al, Nucl. Phys. B282. 253 (198i).

[6] K. Gaemers and G. Gounaris, Z. Phys. C1, 259 (1979).

[7] K.-i. Hikasa Phys. Rev. D33, 3203 (1986); K. Hagiwara et al, Nucl. Phys. B282, 253 (1987).

[8] R. Kleiss and W. J. Stirling, Nucl. Phys. B262, 235 (1985); Z.Xu D.-H.Zhang L. Chang Nucl. Phys. B291, 392 (1987).

[9] T. Barklow, Proceedings of the 1st Workshop on Physics with Linear Colliders, Saariselka, Finland, Sept. 9-14 (1992); T. Barklow, Proceedings of the DPF'94 Conference: 1994 Meeting of the Division of Particls and Fields of the APS, Albuquerque, NM, Aug2-6, 1994

[10] R.L. Sekulin, Phys. Lett. B338, 369 (1994).

[11] A.A. Pankov and N. Paver, Phys. Lett. B324, 224 (1994); B346, 115 (1995); A.A. Likhoded, A.A. Pankov, N. Paver, M.V. Shevlyagin and O.P. Yushchenko, UTS-DFT93-22 (1993). 
Effects of Detector Resolution on Measurement of

Anomalous Triple Gauge Boson Couplings

KEITH RULES

\author{
Randall Laboratory \\ University of Michigan \\ Ann Arbor, MI 48109-1120
}

\section{ABSTRACT}

This article describes a study of detector resolution effects in the measurement of anomalous gauge boson couplings, specifically the extraction of $W W Z$ and $W W \gamma$ parameters from the process $e^{+} e^{-} \rightarrow W^{+} W^{-}$with $W W \rightarrow \ell \nu q \bar{q}^{\prime}$. A computer program wwv, available on the World Wide Web, has been written to carry out this study and is described. Preliminary work indicates that determination of anomalous couplings does not depend critically upon detector performance, but more study is needed to understand how to correct for large effects due to initial state photon radiation. Determination of anomalous couplings is found to be insensitive to detector smearing over a broad range of assumed resolutions. The effects of initial state radiation, however, lead to substantial losses in efficiency from tinematic requirements or demand a detailed understanding of initial state radiation and beamsstrahlung photon energy spectra.

\section{Introduction}

Studies in recent years $[1,3]$ have demonstrated the tremendous potential of a high-energy $e^{+} e^{-}$collider for precise determination of anomalous triple gauge boson couplings. In particular, the process $e^{+} e^{-} \rightarrow W^{+} W-$ followed by $W W \rightarrow \ell \nu q \vec{q}^{\prime}$ promises to provide unmatched sensitivity to $W W V(V=\gamma Z)$ couplings[13]. This study addresses the question of whether realistic detector performance significantly degrades the expected precision in these couplings measurements. Important effects of initial state radiation and finite $W$ width are considered.

It is assumed that the reader is familiar with the conventional parametrization of Hagiwara et al.[4] in which $W W V$ couplings are defined in terms of parameters $\kappa_{V}, \lambda_{V}$, etc.. For the purposes of this study, the full HISZ[5] scenario will be used, in which only two parameters (taken to be $\Delta \kappa_{\gamma}, \lambda_{\gamma}$ ) are free to vary.

In keeping with the guidelines of the NLC workshop studies, no explicit detector simula. tion has been performed. Instead, 4-vectors representing momenta / energies of detectable particles (charged leptons, stable hadrons) have been smeared according to a standard prescription[12]. This smearing includes reduced acceptance in forward detector regions. One expects a priori that the most important resolution for $W W \rightarrow \mu \nu q \bar{q}^{\prime}$ events is that for reconstructed charged particle momentum, since the muon energy spectrum peaks near the beam energy. In addition, for both $W W \rightarrow e \nu q \vec{q}$ and $W W \rightarrow \mu \nu q \bar{q}^{\prime}$ one might expect the hadron calorimeter energy resolution for hadronic jets to be important. The "nominal" parameters describing these two resolutions (given below) are used as a benchmark against which both degraded and improved resolutions are compared. For reference, charged particle momentum resolution is taken to have the form (assuming a solenoidal magnetic field):

$$
\frac{\delta p_{\perp}}{p_{\perp}}=a \times p_{\perp}
$$

with $p_{\perp}$ in $\mathrm{GeV}$ and where $a=2.0 \times 10^{-4}$ (multiple scattering effects are unimportant in this measurement). Hadron calorimeter energy resolution is taken to have the form

$$
\frac{\delta E}{E}=\frac{A}{\sqrt{E}} \oplus B
$$

with $E$ in $\mathrm{GeV}$, where $A=45 \%$ and $B=50 \%$, and where the $A$ and $B$ errors are combined in quadrature. In addition to this nominal benchmark, two others were defined: a "perfect particle detector" for which the momenta of charged leptons and stable hadrons are detected with full efficiency and perfect resolutions and an "ideal daughter detector ${ }^{4}$ for which the momenta of $W$ daughter partons are measured perfectly (meaning measurement of neutrino / charged lepton / quark momenta and determination of lepton charge but not of quark charges):

The pythia Monte Carlo program[8] has been used for $e^{+} e^{-} \rightarrow W^{+} W^{-}$event generation: hadronization of quarks is carried out via the Lund algorithm. Although the pythia program has been shown in LEP II studies[7] to lack the precision in radiative corrections of other recent $e^{+} e^{-} \rightarrow W^{+} W^{-}$generators developed for LEP II, it serves as a useful and convenient platform for isolating the effects of detector resolution and the predominant effects of initial state radiation. For the studies described here only $e^{+} e^{-} \rightarrow W+W-$ events with $\left|\cos \theta_{W}\right|<$ 0.8 were generated. This restriction is motivated not only by the difficulty in accurately reconstructing $W$ 's in the forward direction, but also by the dominance of the cross section in forward regions by the $t$-channel $\nu_{\varepsilon}$ exchange diagram which is not affected by anomalous $W W V$ couplings. Restricting attention to the centrally produced $W$ 's has only a small effect on the statistical precision of extracted couplings.

In section 2, the procedure used in this study is described in detail. Results will be shown in section 3 , and section 4 contains a summary and some discussion.

The study described in this article was carried out using the wwv fortran program which can be accessed via the World Wide Web at the following URL:

$$
\text { http://wwu-personal. umich. edu/ kriles/wuv/uwvdoc.html }
$$


The source code is provided both in a patchy cards file and as a tar archive file containing extracted fortran code and common block files. In addition, programs for plotting differential cross sections and for fitting to idealized self-generated distributions are included in the patchy source code. Scripts for running these programs on a unix operating system are also provided.

\section{Procedure}

It is conventional and convenient to define the topology of a $e^{+} e^{-} \rightarrow W^{+} W^{-}$event via five angles (neglecting initial state radiation): polar production angle $\theta_{W}$ of the $W^{-}$; the decay polar and azimuthal angles $\theta_{i}^{*}, \phi_{i}^{*}$ of the charged lepton w.r.t. its parent W's lab-frame direction in the $W$ rest frame; and the analogous decay angles $\bar{\theta}_{\text {and }}$ and of one quark in its parent W's rest frame. No quark-charge tagging is assumed here; hence there is an ambiguity $\left(\vec{\theta}_{q} \leftrightarrow \pi-\vec{\theta}_{q}\right.$ and $\left.\bar{\phi}_{q} \leftrightarrow \bar{\phi}_{q}+\pi\right)$ that requires folding the hadronic decay angle distributions. The basic procedure used in the wrv program can be described quite simply:

- Loop over events..

- Identify $W$ pair candidate events and classify decays

- Reconstruct and store five $W$ production / decay angles per event

- Fit stored angular distributions to extract couplings parameters

The different steps in this procedure are detailed in this section.

\subsection{Event Selection and Reconstruction}

For this study, only very simple criteria have been used to select $W W \rightarrow q q^{\prime \prime} q^{\prime \prime} \bar{q}^{\prime \prime}$ events. This is largely because the events should in reality be easy to find with very little background. The leptons will generally be highly energetic and isolated. The hadronic jets are straightforward to reconstruct. Background from other four-fermion processes $e^{+} e^{-} \rightarrow \ell \nu q \bar{q}^{\prime}$ have been considered in ref.[3] and found to be controllable with invariant mass requirements on the reconstructed $\mathrm{W}$ candidates. No backgrounds have been considered in this study.

Charged leptons are subjected to the tracking / calorimeter efficiencies and resolutions described in ref[12]. In addition, this study requires the leptons be isolated by at least 15 degrees from any other charged particle with momentum greater than $1 \mathrm{GeV}$ and that the lepton energy lie between $90 \%$ of the kinematically allowed minimum and $110 \%$ of the kinematically allowed maximum for a lepton from $W$ decay when no initial state radiation is present and the $W$ width vanishes. The extra $10 \%$ of allowed energy range is meant to accommodate detector resolution and radiation and width effects.

Once the lepton candidate has been identified, additional requirements are imposed on the remaining detected particles to ensure consistency with the presence of hadronic $W$
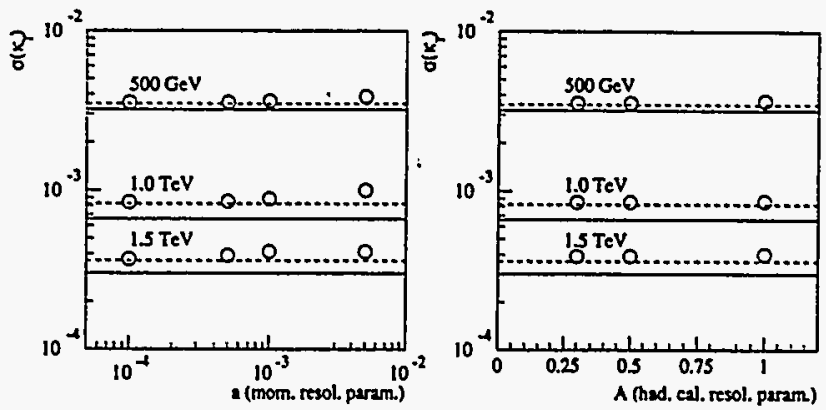

Figure 1: Idealized Case: Measurement precision for $\Delta \kappa_{\gamma}$ at various c.m. energies and for various detector resolution parameters, as explained in the text. All values are for 4000 accepted events with $\left|\cos \theta_{w}\right|<0 . S$. The solid lines at each energy represent the ideal W-daughter detector with the same angular binning, while the dashed lines represent a perfect particle detector. Events have been generated with no initial state radiation and with $\Gamma_{W}=0$.

decay. Aside from a single identified lepton, the event must contain at least 3 charged tracks. 3 neutral particles, charged particle energy greater than $5 \%$ of $\mathrm{E}_{\mathrm{CN}}$, total neutral energy greater than $2.5 \%$ of $\mathrm{E}_{\mathrm{CM}}$, and charged + neutral energy greater than $20 \%$ of $\mathrm{E}_{\mathrm{CM}}$. (These energies exclude the lepton's contribution.)

All detected particles, excluding the lepton, are subjected to a jet finding algorithm that forces two jets to be found. Two algorithms are available in wuv: the JADE[6] method with a varving $y_{\min }$ cutoff and a separation according to thrust axis hemispheres in the c.m. frame of the detected particles. The two algorithms are found to give nearly identical results. The thrust axis separation is used in what follows.

After the lepton and two hadronic jets have been identified, their four vectors are sub. jected to an algorithm to determine the five production / decay angles described above. A number of algorithm choices are available in wrv. The first is based on ref.[2] in which one assumes that the lepton momentum and the 3-velocities of the jets are measured perfectly. One also assumes that the leptonically decaying $W$ is on mass shell $\left(\Gamma_{W}=0\right)$ and that there is no initial state radiation present. A second algorithm is based on a least-squares constrained kinematic fit [9], using lepton and jet resolutions. A number of kinematic fitting options have been implemented. The two $W$ masses may vary independently, they may vary together, or they may be forced to equal the nominal $W$ mass. In addition, the fit may allow a massless photon radiated along the beam direction.

For generated events with no ISR and with $\Gamma_{W}=0$, the four different algorithms above (no photon assumed along beam direction) give very similar results over large ranges in 
detector resolution. In the study of these events, no photon radiation is assumed in the kinematic fit, and the $W$ masses float independently. For generated events with ISR and $\Gamma_{W} \neq 0$, the different algorithms vary considerably in effectiveness. In the study of these events, the $W$ masses float together, and a massless photon is assumed along the beam direction.

\subsection{Parameter Fitting}

Once the sets of five production / decay angles have been determined for all selected $W W \rightarrow \ell \nu q \bar{q}^{\prime}$ candidate events, the angles are binned into a 5-dimensional distribution for a subsequent maximum likelihood fit to a detector-smeared differential cross section that depends on anomalous couplings parameters. The differential cross section formulae are taken from ref.[4]. For convenience in comparing to previous studies, the HISZ[5] scenario is used here.

The treatment of detector smearing is non-trivial in the fit to the 5-dimensional angular distribution. The wwv program allows two approaches based on migration matrices that transform true production / decay angles into measured angles. The first approach assumes independent smearing among the five single-angle distributions, allowing a factorized transformation consisting of products of five two-dimensional migration matrices. The second approach does not assume independent smearing and uses a ten-dimensional migration matrix. This second approach was found to be less prone to biasing central values of fitted parameters and is used in what follows.

There is a considerable CPU/memory penalty, however, in using the unfactorized migration matrix. For the computer used in this study (Hewlett Packard 715/80 Workstation), memory was the more severe constraint. For 5-dimensional fitting the binning chosen was $\left.(10 \times 3 \times 3 \times 2 \times 2)^{2}\right)$ for the true / reconstructed variables $\theta_{W}, \cos \theta_{i}^{*}, \phi_{i}^{*}, \cos \vec{\theta}_{q}$, and $\bar{\phi}_{q}$, respectively. To reduce errors coming from non-linear variation of cross sections within a single bin, multiple sampling in uniform subdivisions is allowed for each dimension. In addition, since the differential cross sections are exactly quadratic in the couplings, full matrix element evaluation for each bin need only be done during initialization, after which a set of coefficients is calculated for each bin to allow rapid recalculation of differential contributions for arbitrary coupling values.

One other technical point concerns the polar production angle $\Theta_{W}$. The differential cross section formulae are provided in ref.[4] with respect to $\cos \Theta_{w}$. These formulae give very sharply peaked distributions in the forward direction. Consequently, to reduce non linearity across bins, the fitting is performed directly in $\theta_{w}$ instead of $\cos \theta_{w}$.

\section{Results}

Results are given below for two distinct cases. The first concerns analysis of idealized $e^{+} e^{-} \rightarrow$ $W^{+} W^{-}$events generated by the pythia program with no initial state photon radiation and

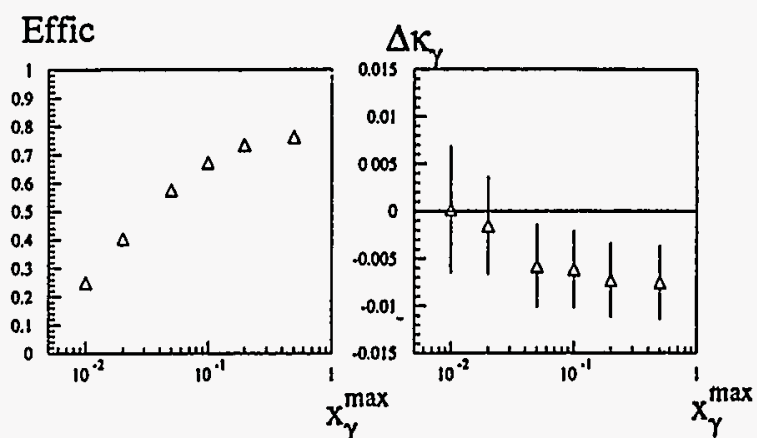

Figure 2: Efficiency and bias on fitted $\Delta \kappa_{\gamma}$ plotted vs the minimimum allowed true c.m. energy of the $W$ pair. The error bars indicate the statistical error from the fit to candidates in a sample of $4000 e^{+} e^{-} \rightarrow W^{+} W^{-}$events generated with initial state radiation and $\Gamma_{W} \neq 0$ at a nominal c.m. energy of $500 \mathrm{GeV}$.

with $\Gamma_{W}=0$. The second concerns the more realistic case with initial state radiation and finite $W$ width. In the first case. detector resolution and the details of the kinematic fittin play only a small role, thanks to the power of kinematic constraints. In the second case, on the other hand, detector resolution and the kinematic fitting approach both take on greater importance, as the kinematic constraints weaken.

\subsection{Idealized Case: $\Gamma_{W}=0$ and no ISR}

Consider first the idealized case of no initial state radiation and vanishing $W$ width. In this study $4000 e^{+} e^{-} \rightarrow W^{+} W^{-}$events were generated with $\left|\cos \theta_{w}\right|<0.8$ at c.m. energies of 500.1000 , and $1500 \mathrm{GeV}$. These correspond to integrated luminosities of approximately 10 , 50 . and $130 \mathrm{fb}^{-1}$, respectively. The events were kinematically fitted with equal floating $W$ masses and with no missing photon along the beam direction. The resulting distributions in production / decay angles were fitted to obtain $\Delta \kappa_{\gamma}$ in the HISZ scenario with $\lambda_{\gamma}$ fixed to zero. The procedure was repeated for a variety of charged particle momentum resolution and hadron calorimeter energy resolution parameters. The unfactorized 10-dimensional migration matrix with binning described above was used to account for detector smearing in the fit.

Figures la-b shows the resulting statistical errors on the fitted $\Delta \kappa_{\gamma}$ values for the different tracking and calorimeter resolutions. The dashed lines indicate what could be achieved with this procedure for a perfect particle detector. The solid lines indicate what could be achieved with an ideal $W$ daughter detector in which the charged lepton and initial quark moment are known exactly. The striking feature of Figure 1 is that the precision on $J \kappa_{\gamma}$ is quite 


\section{Effic}

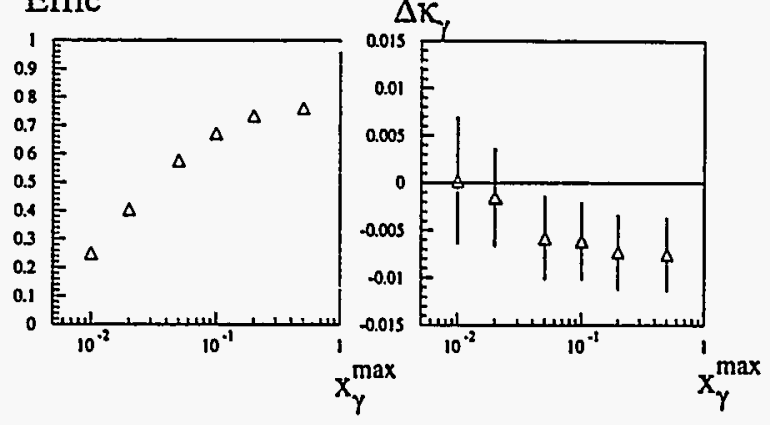

Figure 3: Efficiency and bias on fitted $\Delta \kappa_{\gamma}$ plotted $v s$ the maximum allowed fraction of c.m. energy obtained for the fitted energy of a photon undetected along the beam direction. The error bars indicate the statistical error from the fit to candidates in the sample of 4000 $e^{+} e^{-} \rightarrow W^{+} W^{-}$events generated with initial state radiation and $\Gamma_{W} \neq 0$ at a nominal c.m. energy of $500 \mathrm{GeV}$.

insensitive to detector resolution. This is an indication of the power of the constrained kinematic fitting when initial state radiation is not present.

\subsection{Inconvenient reality: $\Gamma_{W} \neq 0$ and ISR}

Introducing initial state radiation and finite $W$ width considerably spoils the idealized case above. The degradation comes from several sources. First, the Breit-Wigner spread in $W$ masses-detracts from an otherwise powerful kinematic constraint. Second, the emission of an undetected photon along the beam direction distorts the kinematics of the visible decay products. A kinematic fit assuming full energy/momentum conservation necessarily obtains incorrect production / decay angles. Third, the emission of the the photon leads to a reduced effective c.m. energy for the produced $W$ pair, leading to a distorted spectrum in actual production / decay angles from that expected at the nominal beam c.m. energy.

Since initial state radiation is strongly peaked toward zero photon energy, one can maintain a high efficiency by excluding specifically those events with c.m. energies far below the nominal value. Figure $2 a$ shows the efficiency obtained by the standard reconstruction / fitting procedure, including detector smearing effects, for 4000 events containing ISR and $\Gamma_{W} \neq 0$ at a nominal c.m. energy of $500 \mathrm{GeV}$. The efficiencies are plotted $v$ s the minimum allowed true c.m. energy of the $W$ pair. Figure $2 b$ shows the bias in the fitted $\Delta \kappa_{\gamma}$ value obtained from directly fitting these events. The error bars indicate the errors obtained in the associated fit to the passing candidates (not the error on the bias itself). As expected, one can eliminate bias in the central value with a modest loss of efficiency if one can cut

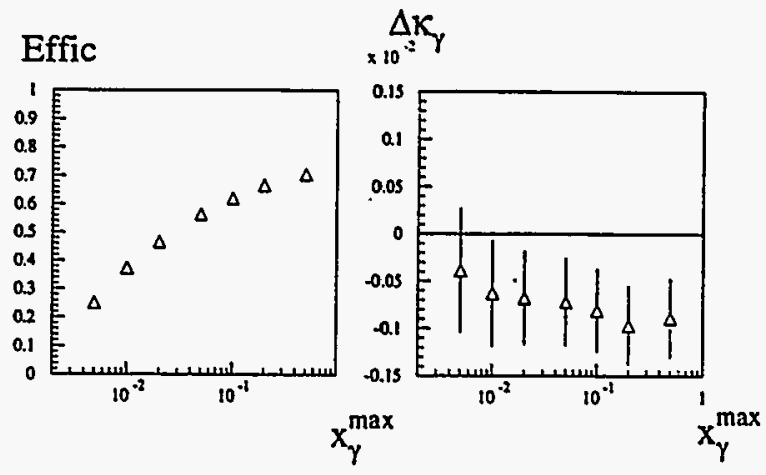

Figure 4: Same quantities as for Figure 3 but at a nominal c.m. energy of $1500 \mathrm{GeV}$. Notice that the vertical scale in the righthand plot is 10 times smaller than in Figure 3.

directly on the true $W$ pair c.m. energy.

The problem, of course, is making a cut based on reconstructed observables. Two approaches have been tried. One uses a " $x^{2 n}$ parameter that measures the deviation of the two reconstructed $W$ masses from nominal values. as described in ref.[2]. Another approach. which seems to give slightly better efficiency for the same bias and which is used in the following, comes from performing a kinematic fit to the event while allowing a missing photon along the beam direction. The fitted photon energy is then required to be less than a fraction $x_{\gamma}^{\max }$ of the nominal c.m. energy. Figure $3 a$ shows the efficiency obtained for the 4000 events at $500 \mathrm{GeV}$ plotted $v s x_{\gamma}^{\max }$. Figure $3 \mathrm{~b}$ shows the resulting biases in fitted $\Delta \kappa_{\gamma}$ values plotted $v s x_{\gamma}^{\max }$. One suffers a large efficiency loss to obtain a bias much smaller than the statistical error in these samples. Figures $4 \mathrm{a}-\mathrm{b}$ show the analogous quantities for a sample of 4000 generated events at a nominal c.m. energy of $1500 \mathrm{GeV}$. At the higher energy, it is more difficult to eliminate bias with modest efficiency loss.

In order to understand the effect of varying detector resolution on these biases and couplings errors, values of $x_{\gamma}^{\text {max }}=0.02(.01)$ were chosen for events at $500(1500) \mathrm{GeV}$, and the above fitting procedure was repeated for different track momentum and calorimeter energy resolution parameters. Figures $5 \mathrm{a}-\mathrm{b}$ show the resulting errors on $\Delta \kappa_{\tau}$ as the momentum and energy resolutions are varied over large ranges. In contrast to Figures la-b, detector resolution does matter. However, improving detector resolution beyond what has been chosen to be nominal for this workshop is not justified by these plots. 


\section{Summary and Discussion}

The effects of initial state radiation, finite $W$ width, and realistic detector resolution on measurement of anomalous gauge boson couplings in the process $e^{+} e^{-} \rightarrow W^{+} W^{-}$(with $\left.W W \rightarrow \ell \nu q \bar{q}^{\prime}\right)$ have been studied. The effects of initial state radiation are found to be most important. Although very poor charged-track momentum resolution can degrade the measurements of anomalous couplings, it appears that the nominal resolutions chosen for this workshop are adequate, even for a $1.5 \mathrm{TeV}$ linear collider.

The main difficulty in kinematically fitting the $W W \rightarrow \ell \nu q \vec{q}$ events with a photon along the beam direction is the inevitable degradation in photon energy resolution due to the presence of the undetected neutrino; attempting to fit for the photon energy event by event can have only limited success. This approach has been attempted in this study and found to lead to substantial efficiency loss for acceptably low bias. Another approach would entail fitting the observed differential cross sections to underlying $E_{C M}$-dependent differential cross sections weighted by the $E_{C M}$-dependent beam luminosity. This has the drawback of requiring accurate knowledge of beamsstrahlung effects, but recent work[10] indicates that precise luminosity monitors combined with fine-grained central calorimetery can give reliable estimates of integrated luminosity vs effective c.m. energy.

This study had considered only the $W W \rightarrow \ell \nu q \vec{q}^{\prime}$ channel. A recent preliminary study for this workshop [11] indicates that the four-jets $W W \rightarrow q \vec{q}^{\prime \prime} q^{\prime \prime} \bar{q}^{\prime \prime \prime}$ chanel may be more promising than first thought. The $W^{+} / W^{-}$separation is clean enough and the four-jets branching ratio product high enough that comparable statistical power to the $W W \rightarrow \ell \nu q \bar{q}^{\prime}$ channel is feasible, even when individual quark tagging is not reliable. It would be desirable to carry out a detailed study of the four-jets channel, including initial state radiation and detector smearing. With the greater kinematic information available in a four-jets event, fitting for the energy of a missing photon along the beam direction may prove more informative about true $W$ pair c.m. energy. Taking into account photon radiation, the $W W \rightarrow q \vec{q}^{\prime \prime} \bar{q}^{\prime \prime \prime}$ channel may in the end provide greater sensitivity to couplings than the $W W \rightarrow \ell \nu q \vec{q}$ channel.

\section{ACKNOWLEDGEMENTS}

The author wishes to thank T. Barklow, J. Brau, M. Einhorn, S. Godfrey, M. Pohl, and D. Strom for useful discussions.

\section{References}

[1] T.L. Barklow, in Physics and Experiments with Linear Colliders, eds. R. Orava et al., World Scientific, vol. 1, p 423.
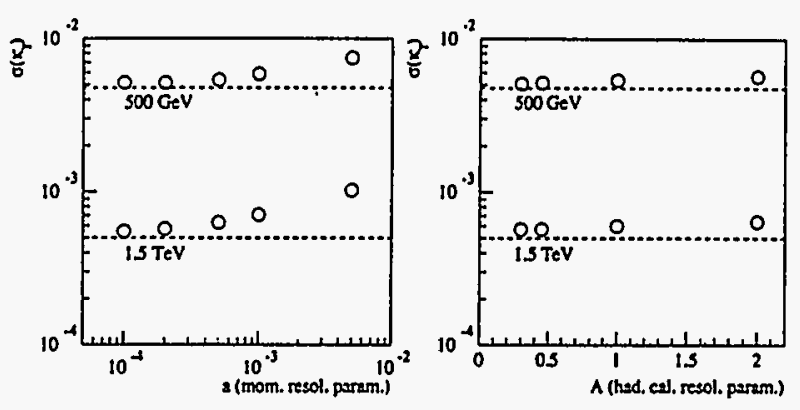

Figure 5: Measurement precision for $\Delta \kappa_{\gamma}$ at two c.m. energies and for various detector resolution parameters, as explained in the text. For events at nominal c.m. energy of 500 $\mathrm{GeV}$, the fraction of c.m. energy assigned to a kinematically fitted photon missing along the beam direction is required to be less than 0.02 . Foe events at $1500 \mathrm{GeV}$, the maximum fraction is 0.01 . Events have been generated with initial state radiation and with $\Gamma w \neq 0$.

[2] T.L. Barklow, in Proceedings of the International Symposium on Vector Boson SelfInteractions, eds. U. Baur, S. Errede, and T. Müller, Amer. Inst. Press, p. 307.

[3] M. Gintner, S. Godfrey, and G. Couture, contributed paper to these proceedings.

[4] H. Hagiwara, R.D. Peccei, and D. Zeppenfeld Nuc. Phys. B282, 253 (1987)

[5] H. Hagivara, S. Ishihara, R. Szalapski, and D. Zeppenfeld Phys. Lett. B283, 353 (1992)

[6] JADE Collaboration, W. Bartel et al., Z. Phys. C33 23 (1986).

[7] D. Bardin et al., in Physics at LEP II, V. 2, p. 3, CERN 96-01 (1996).

[8] H.U. Bengtsson and T. Sjöstrand, Comp. Phys. Comm. 43367 (1987).

[9] B.P. Roe, "Probability and Statistics in Experimental Physics", Springer-Verlag, New York 1992.

[10] D. Strom, presentation at SLAC meeting of this Workshop.

[11] D. Strom, presentation at BNL meeting of this Workshop.

[12] See chapters on detector parameters and simulations in the Workshop Summary Document

[13] See chapter on anomalous gauge boson couplings in the Workshop Summary Document 
The Polarization Asymmetry and Triple Gauge Boson Couplings in $\gamma e$ Collisions at the NLC

\section{Thomas G. Rizzo'}

Stanford Linear Accelerator Center

Stanford University, Stanford, California 94309 USA

\section{ABSTRACT}

We examine the capability of the NLC in the $\gamma e$ collider mode to probe the CP-conserving $\gamma W W$ and $\gamma Z Z$ anomalous couplings through the use of the polarization asymmetry. When combined with other measurements, very strong constraints on both varieties of anomalous couplings can be obtained.

\section{Introduction}

The Standard Model(SM) has so far done an excellent job at describing almost all existin data. One of the most crucial remaining set of tests of the gauge structure of the SM will occur at future colliders when precision measurements of the various triple gauge boson vertices(TGVs) become available[1]. If new physics arises at or near the TeV scale, then on rather general grounds one expects that the deviation of the TGVs from their canonical SM values, i.e., the anomalous couplings, to be at most $O\left(10^{-3}-10^{-2}\right)$ with the smaller end of this range of values being the most likely. To get to this level of precision, and beyond, for all of the TGVs a number of different reactions need to be studied using a variety of observables. Here we concentrate on the CP-conserving $\gamma W W$ and $\gamma Z Z$ anomalous couplings that can be probed in the reactions $\gamma e \rightarrow W \nu, Z e$ at the NLC using polarized electrons and polarized backscattered laser photons[2]. In the $\gamma W W$ case, the anomalous couplings modify the magnitude and structure of the already existing SM tree level vertex. No corresponding tree level $\gamma Z Z$ vertex exists in the SM, although it does appear at the one-loop level. One immediate advantage of the $\gamma e \rightarrow W \nu$ process over, e.g., $e^{+} e^{-} \rightarrow W^{+} W^{-}$is that the fWW vertex can be trivially isolated from the corresponding ones for the $Z W W$ vertex thus allowing us to probe this particular vertex in a model-independent fashion. To set the notation for what follows, the $\gamma W W$ and $\gamma Z Z$ anomalous couplings are denoted by $\Delta \kappa, \lambda$ and $h_{3,}^{0}[1]$, respectively. We will assume that the $\gamma W W$ and $\gamma Z Z$ anomalous couplings are unrelated; the details of our analysis can be found in Ref.[2].

'Work supported by the Department of Energy, contract DE-AC03-76SF00515.

\section{Analysis}

The use of both polarized electron and photon beams allows one to construct a polarization asymmetry, $A_{\text {pol }}$. In general the $\gamma e \rightarrow W \nu . Z \epsilon$ (differential or total) cross sections can be written schematically as $\sigma=\left(1+A_{0} P\right) \sigma_{\text {in }}+\xi\left(P+t_{0}\right) \sigma_{\text {ol }}$ where $P$ is the electron's polarization ( $>0$ for left-handed bèams). $-1 \leq \xi \leq 1$ is the Stoke's parameter for the circularly polarized photon, and $A_{0}$ describes the electron's coupling to the relevant gauge boson $\left[A_{0}=2 v a /\left(v^{2}+a^{2}\right)=1\right.$ for $W ' s$ and $\simeq 0.145$ for $Z$ 's, with $v, a$ being the vector and axial-vector coupling of the electron]. $\sigma_{\text {pol }}\left(\sigma_{u n}\right)$ represents the polarization (in)dependent contribution to the cross section, both of which are functions of only a single dimensionless variable at the tree level after angular integration. i.e.. $x=y^{2}=s_{r e} / M_{W, Z}^{2}$, where $\sqrt{s_{v e}}$ is the $\gamma-e$ center of mass energy. Taking the ratio of the $\xi$-dependent to $\xi$ independent terms in $\sigma$ gives us the asymmetry $A_{\text {pol }}$.
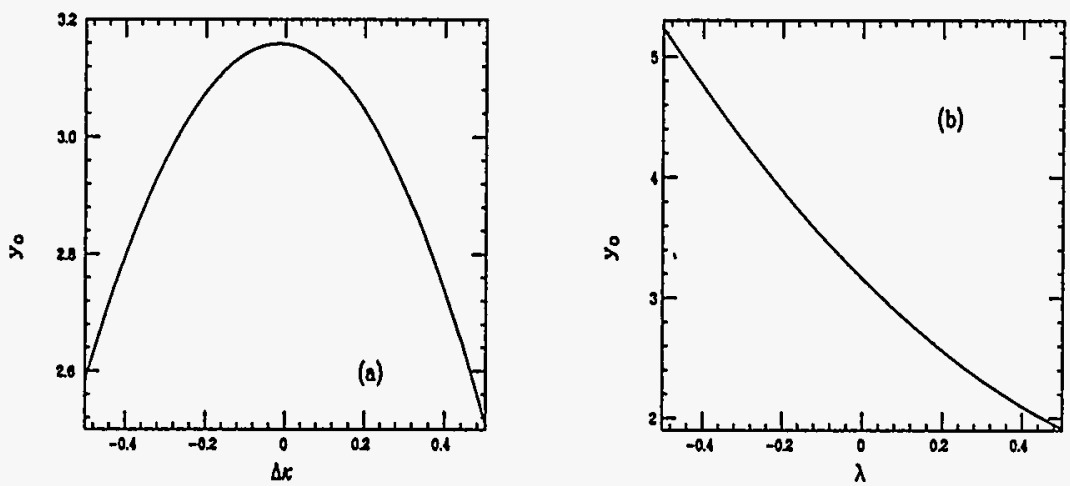

Figure 1: Separate $\Delta \kappa$ and $\lambda$ dependence of the value of $y_{0}$, the zero position for the proces $\gamma e-W \nu$.

One reason to believe a priori that $A_{\text {pol }}$, or $\sigma_{\text {pol }}$ itself. might be sensitive to modifications in the TGVs due to the presence of the anomalous couplings is the Drell-Hearn Gerasimov(DHG) Sum Rule[3]. In its $\gamma e \rightarrow W \nu, Z e$ manifestation, the DHG sum rule implies that

$$
\int_{1}^{\infty} \frac{\sigma_{p o l}(x)}{x} d x=0 \text {, }
$$

for the tree level SM cross section when the couplings of all the particles involved in the process are 'canonical', i.e., gauge invariant. That this integral is zero results from (i) the 
fact that $\sigma_{\text {pol }}$ is well behaved at large $x$ and (ii) a delicate cancellation occurs between the two regions where the integrand takes on opposite signs. This observation is directly correlated with the existence of a single value of $x$ (or $y$ ) where $\sigma_{\text {pol }}$ (and, hence, $A_{\text {pol }}$ ) vanishes. For the $W(Z)$ case this asymmetry 'zero' occurs at $\sqrt{s_{\gamma e}} \simeq 254(150) \mathrm{GeV}$, both of which are easily accessible at the NLC. As we will see, the inclusion of anomalous couplings not only moves the position of the zero but also forces the integral to become non-vanishing and, in the DHG Sum Rule directly. In the $W$ case, the zero position, $y_{0}$, is found to be far more sensitive to modifications in the TGVs than in the $Z$ case. The zero position as a function of $\Delta \kappa$ and $\lambda$ for the $\gamma e \rightarrow W \nu$ process is shown in Fig.1 whereas the corresponding $Z$ case is shown in Fig.2. In either situation, the position of the zero alone does not offer great sensitivity to the existence of anomalous couplings.(See Ref. 2.)

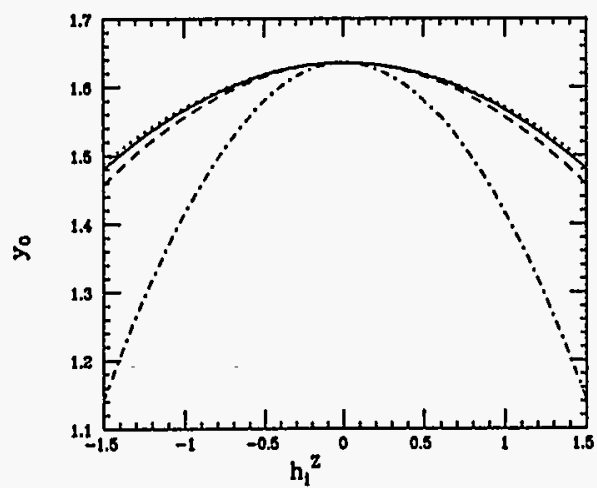

Figure 2: Position of the SM polarization asymmetry zero in $\gamma e \rightarrow Z e$ as a function of $h_{3,4}^{0}$ for $P=90 \%$ with a $10^{\circ}$ angular cut. The dotted(dashed, dash-dotted, solid) curve corresponds to the case $h_{4}^{0}=0\left(h_{3}^{0}=0, h_{3}^{0}=h_{4}^{0}, h_{3}^{0}=-h_{4}^{0}\right)$.

Our analysis begins by examining the energy, i.e., $y$ dependence of $A_{\text {pol }}$ for the two processes of interest; we consider the $W$ case first. For a $500(1000) \mathrm{GeV}$ collider, we see that only the range $1 \leq y \leq 5.4(10.4)$ is kinematically accessible since the laser photon energy maximum is $\simeq 0.84 E_{\text {e. }}$. Since we are interested in bounds on the anomalous couplings, we will asume that the SM is valid and generate a set of binned $A$ or data samples via electrons are $90 \%$ left-handed polarized as right-handed electrons do not interact through the $W$ charged current couplings. Our bin width will be assumed to be $\Delta y=0.1$ or 0.2 . We then fit the resulting distribution to the $\Delta \kappa$ - and $\lambda$-dependent functional form of $A_{\mathrm{pol}}(y)$ and subsequently extract the $95 \%$ CL allowed ranges for the anomalous couplings. The results of this procedure are shown in Fig. 3, where we see that reasonable constraints are obtained although only a single observable has been used in the fit.

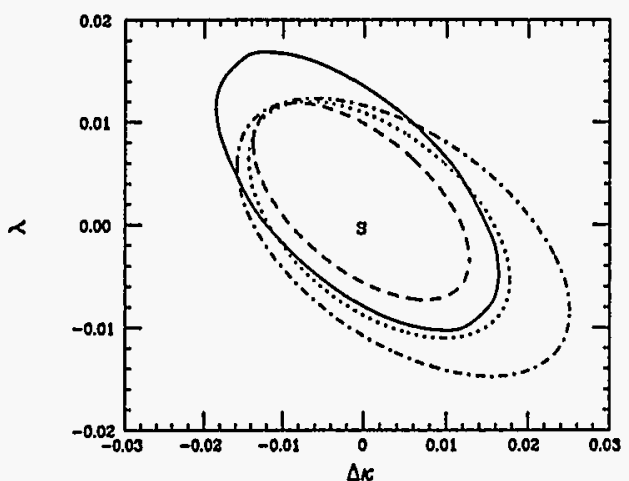

Figure 3: $95 \% \mathrm{CL}$ bounds on the $\mathrm{W}$ anomalous couplings from the polarization asymmetry. The solid(dashed, dash-dotted) curves are for a $500 \mathrm{GeV}$ NLC assuming complete $y$ coverage using 22 (22. $44)$ bins and an integrated luminosity per bin of $2.5(5,1.25) \mathrm{fb}^{-1}$. respectively. The corresponding bins widths are $\Delta y=0.2(0.2,0.1)$. The dotted curve corresponds to a $1 \mathrm{TeV}$.VLC using $+7 \quad y=0.2$ bins with $2.5 \mathrm{fb}^{-1} / \mathrm{bin}$. ' $\mathrm{s}$ ' labels the SM prediction.

Clearly, to obtain stronger limits we need to make a combined fit with other observables, such as the energy dependence of the total cross section. the $W$ angular distribution. or the $W$ polarization. As an example we show in Fig. 4 that the size of the $9.5 \% \mathrm{CL}$ allowed region shrinks drastically in the $1 \mathrm{TeV}$ case when the cross section data is included in a simultaneous fit together with the polarization asymmetry. As is well known, the cross section is highly sensitive to $\Delta x$ and thus the allowed region is highly compressed in that direction. We find that $\Delta \kappa$ is bounded to the range $-1.45 \cdot 10^{-3} \leq \Delta \kappa \leq 0.30 \cdot 10^{-3}$ while the allowed $\lambda$ range is still rather large. The addition of the angular distribution and $W$ polarization data to the fit is expected to reduce the size of this allowed region even further.

With these thoughts in mind. in the $Z$ case we will follow a similar approach but we will simultaneously fit both the energy dependence of $A_{\text {pot }}$ as well as that of the total cross section. (Later. we will also include the $\boldsymbol{Z}$ boson's angular distribution into the fit.) In this $Z$ analysis we make a $10^{\circ}$ angular cut on the outgoing electron and keep a finite form factor 


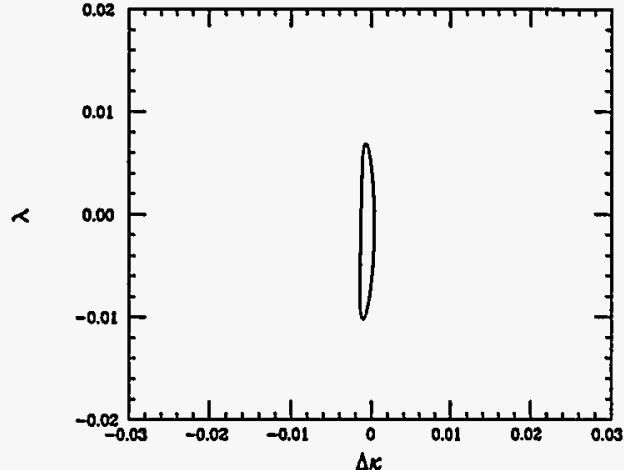

Figure 4: Same as the previous figure for a $1 \mathrm{TeV}$ NLC but now combined with the cross section data in a simultaneous fit. Only statistical errors are included.

scale, $\Lambda=1.5 \mathrm{TeV}$. so that we may more readily compare with other existing analyses. (The angular cut also gives us a finite cross section in the massless electron limit; this cut is not required in the case of the $W$ production process.) We again assume that $P=90 \%$ so that this analysis can take place simultaneously with that for the $W$. The accessible $y$ ranges are now $1 \leq y \leq 4.6(9.4)$ for a $500(1000) \mathrm{GeV}$ collider. Fig.5 shows our results for the 500 GeV NLC while Fig. 6 shows the corresponding $1 \mathrm{TeV}$ case. For a given energy and fixed total integrated luminosity we learn from these figures that it is best to take as much data as possible at the highest possible values of $y$. Generally, one finds that increased sensitivity to the existence of anomalous couplings occurs at the highest possible collision energies.

Even these anomalous coupling bounds can be significantly improved by including the $Z$ boson angular information in the fit. To be concrete we examine the case of a $1 \mathrm{TeV} \mathrm{NLC}$ with $16.8 \mathrm{fb}^{-1} /$ bin of integrated luminosity taken in the last $10 \Delta y$ bins (corresponding to the dash-dotted curve in Fig.6). Deconvoluting the angular integration and performing instead the integration over the $10 \Delta y$ bins we obtain the energy-averaged angular distribution. Placing this distribution into 10 (almost) equal sized $\cos 0$ bins while still employing our $10^{\circ}$ cut, we can use this additional data in performing our overall simultaneous $\chi^{2}$ fit. The result of doing this is shown in Fig.7 together with the anticipated result from the LHC using the $Z_{\gamma}$ production mode. Note that the additional angular distribution data has reduced the size of the 95\% CL allowed region by almost a factor of two. Clearly both machines are complementary in their abilities to probe small values of the $\gamma Z Z$ anomalous couplings. If the NLC and LHC results were to be combined, an exceptionally small allowed region would remain. The NLC results themselves may be further improved by considering measurements of the polarization of the final state $Z$ as well as by an examination of. $6 . g$. the complementary $e^{+} \epsilon^{-} \rightarrow Z_{\gamma}$ process: such studies are currently underway $\left.[-]\right]$

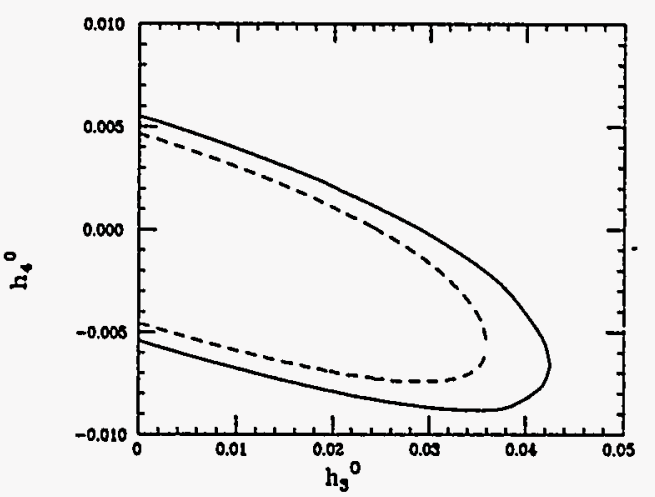

Figure 5: 95\%CL allowed region for the anomalous coupling parameters $h_{3}^{0}$ and $h_{4}^{0}$ from a combined fit to the energy dependencies of the total cross section and polarization asymmetry at a $500 \mathrm{GeV}$ NLC assuming $P=90 \%$ and an integrated luminosity of $3(6) \mathrm{fb}^{-1} / \mathrm{bin}$ corresponding to the solid (dashed) curve. 18 bins of width $\Delta y=0.2$ were chosen to cover the $y$ range $1 \leq y \leq 4.6$. The corresponding bounds for negative values of $h_{3}^{z}$ are obtainable by remembering the invariance of the polarization dependent cross section under the reflection $h_{3,4}^{0}--h_{3,4}^{0}$.

\section{Discussion and Conclusions}

The collision of polarized electron and photon beams at the NLC offers an exciting opportunity to probe for anomalous gauge couplings of both the $W$ and the $Z$ through the use of the polarization asymmetry. In the case of $y e \rightarrow W \nu$ we can cleanly isolate the - iWI vertex in a model independent fashion. When combined with other observables. extraordinary sensitivities to such couplings for W's are achievable at the NLC in the re mode. These are found to be quite complementary to those obtainable in $e^{+} e^{-}$collisions. In the case of the $\gamma Z Z$ anomalous couplings, we obtained constraints comparable to those which can be obtained at the LHC.

\section{ACKNOWLEDGEMENTS}


The author would like to thank S. J. Brodsky, I. Schmidt, J.L. Hewett. and S. Godfrey for discussions related to this work.

\section{References}

[1] For a complete set of references and a recent review of the physics of gauge bosons self-interactions, see H. Aihara et al, Report of the Subgroup on Anomalous Cantge Boson Interactions of the DPF Long-Range Planning Study, Fermilab report PUB95/031, 1995. See also G. Gounaris, J.-L. Kneur, and D. Zeppenfeld e-print archive hep-ph/9601233.

[2] S.J. Brodsky, T.G. Rizzo, and I. Schmidt, Phys. Rev. D52, 4929 (1995); T.G. Rizzo, SLAC-PUB-ז109, 1995.

[3] S. D. Drell and A. C. Hearn, Phys. Rev. Lett. 16, 908 (1966); S. Gerasimov, Yad. Fiz. 2, 598 (1965) [Sov. J. Nucl. Phys. 2, 430 (1966)]; L. I. Lapidus and Chou Kuang-Chao. J. Exptl. Theoretical Physics 41, 1545 (1961) [Sov. Phys. JETP 14. 1102 (1962)]; M. Hosada and K. Yamamoto, Prog. Theor. Phys. 36, 426 (1966). For a recent reviev of the empirical tests of the DHG sum rule see B. L. Ioffe, preprint ITEP-61 (1994): D. Drechsel. University of Mainz preprint, 1994. An analysis of the relationship of anomalous couplings to internal structure for spin-1 bound states is discussed in $F$. Schlumpf and S.J. Brodsky, SLAC-PUB-95-6860; S. J. Brodsky and I. Schmidt, SLACPLB 95-6761 (1995).

[4] D. Leung and J.L. Hewett, in preparation.

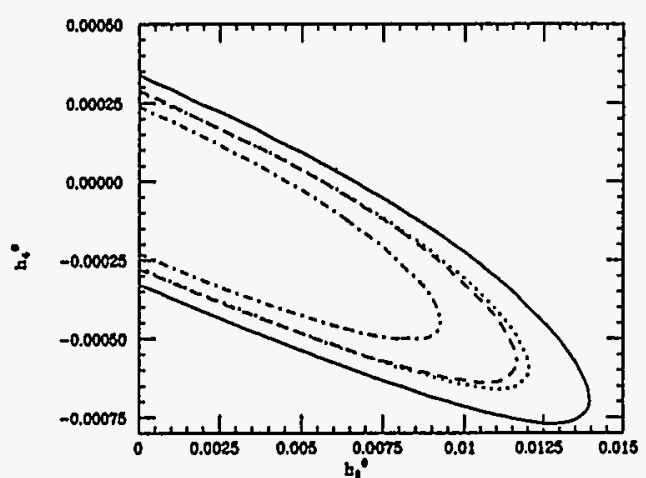

Figure 6: Same as Fig. 5 but for a $1 \mathrm{TeV}$.VLC. The solid(dashed) curre corresponds to a luminosity of $4(8) f b^{-1} /$ bin for 42 bins of width $\doteq y=0.2$ which covered the range $1 \leq y \leq 9.4$. The dotted curve corresponds to a luminosity of $8 \mathrm{fb}^{-1} / \mathrm{bin}$ but only for the last 21 bins. The dash-dotted curve corresponds to the case of $16.8 \mathrm{fb}^{-1} / \mathrm{bin}$ in only the last 10 bins.

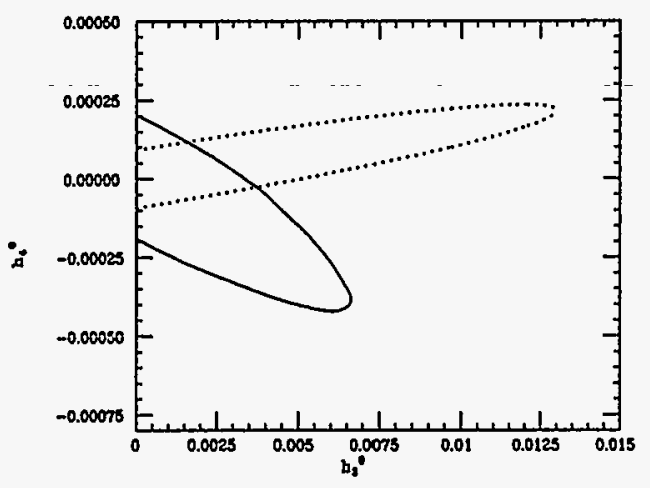

Figure 7: The solid curve is the same as dash-dotted curve in Fig. 6. but now including in the fit the $Z$ boson angular distribution obtained from the highest 10 bins in energy. The correspondin result for the $14 \mathrm{TeV}$ LIIC with $100 \mathrm{fb}^{-1}$ of integrated luminosity from the process $p p-Z \eta+$. is shown as the dotted curve. 
Below Threshold $Z^{\prime}$ Mass and Coupling Determinations at the NLC

THOMAS G. Rrzzo

Stanford Linear Accelerator Center

Stanford University, Stanford, California 94909 USA

\section{ABSTRACT}

We examine the capability of the NLC to determine the mass as well as the couplings to leptons and $b$-quarks of a new neutral gauge boson below production threshold. By using data collected at several different values of $\sqrt{s}$, we demonstrate how this can be done in a model-independent manner.

\section{Introduction}

A new neutral gauge boson, $Z^{\prime}$, is the most well-studied of all exotic particles and is the hallmark signature for extensions of the SM gauge group. If such a particle is found at future colliders the next step will be to ascertain its couplings to fermions. At hadron colliders, a rather long list of observables has been proposed over the years to probe these couplings-each with its own limitations[1]. It has been shown, at least within the context of $E_{6}$-inspired models, that the $\operatorname{LHC}\left(\sqrt{s}=14 \mathrm{TeV}, 100 \mathrm{fb}^{-1}\right)$ will be able to extract useful information on all of the $Z^{\prime}$ couplings for $M_{Z^{\prime}}$ below $\simeq 1-1.5 \mathrm{TeV}$. At the NLC, when $\sqrt{s}<M_{Z^{\prime}}$, a $Z^{\prime}$ manifests itself only indirectly as deviations in, e.g., cross sections and asymmetries from their SM expectations. Fortunately the list of useful precision measurements that can be performed at the NLC is reasonably long and the expected large beam polarization $(P)$ plays an important role. In the past, analyses of the ability of the NLC to extract $Z^{\prime}$ coupling information in this situation have taken for granted that the value of $M_{Z}$, is already known from elsewhere, e.g., the LHC|1]. Here we address the more complex issue of whether it is possible for the NLC to obtain information on couplings of the $Z^{\prime}$ if the mass were for some reason a priori unknown. In this case we would not only want to determine couplings but the $Z^{\prime}$ mass as well.

If the $Z^{\prime}$ mass were unknown it would appear that the traditional NLC $Z^{\prime}$ coupling analyses would become problematic. Given data at a fixed value of $\sqrt{s}$ which shows deviations

\footnotetext{
tWork supported by the Department of Energy, contract DE-AC03-76SF00515.
}

from the SM. one would not be able to simultaneously extract the value of.$V_{Z}$ as well as the corresponding couplings. The reason is clear: to leading order in $s / M Z_{Z}^{2}$, rescaling all of the couplings and the value of $M_{Z}$, by a common factor would leave the observed deviations from the SM invariant. In this approximation. the $Z^{\prime}$ exchange appears only as a contact interaction. Thus as long as $\sqrt{s}<M_{Z^{\prime}}$, the only potential solution to this problem lies in obtaining data on deviations from the SM at several. distinct $\sqrt{s}$ values and combining them into a single fit. Here we report on the first analysis of this kind, focussing on observables involving only leptons and/or $b$-quarks. In performing such an analysis, how many $\sqrt{s}$ values are needed? How do we distribute the luminosity $(\mathcal{L})$ to optimize the results? Can such an analysis be done while maintaining model-independence? In this initial study we begin to address these and some related questions.

\section{Analysis}

In order to proceed with this benchmark study, we will make a number of simplifying as. sumptions and parameter choices. These can be modified at a later stage to see how they influence our results. In this analysis we consider the following ten observables: $\sigma_{\ell, b}$. $A_{F B}^{\ell, b}$. $A_{L R}^{\ell, b}, A_{p o l}^{F B}(\ell, b),\left\langle P_{\tau}\right\rangle$, and $P_{\tau}^{F B}$. Other inputs and assumptions are as follows:

$$
\begin{array}{ll}
\text { e. } \mu, \tau \text { universality } & \text { ISR with } \sqrt{s^{\prime}} / \sqrt{s}>0.7 \\
P=90 \%, \delta P / P=0.3 \% & \delta \mathcal{C} / \mathcal{L}=0.25 \% \\
\epsilon_{3}=50 \%, \Pi_{b}=100 \% & |\theta|>10^{\circ} \\
\epsilon_{e, \mu, \tau}(\sigma)=100 \%, \epsilon_{\tau}\left(P_{r}\right)=50 \% & \text { Neglect } t \text {-channel exchange in } e^{+} e^{-} \rightarrow e^{+} e^{-}
\end{array}
$$

Of special note on this list are $(i)$ a $b$-tagging efficiency $\left(\epsilon_{b}\right)$ of $50 \%$ for a purity $\left(\Pi_{b}\right)$ of $100 \%$. (ii) the efficiency for identifying all leptons is assumed to be $100 \%$, although only $50 \%$ of $\tau$ decays are assumed to be polarization analyzed. (iii) a $10^{\circ}$ angle cut has been applied to all final state fermions, and (iv) a strong cut to events with an excess of initial state radiation(ISR) has also been made. In addition to the above, final state QED as well as QCD corrections are included, the $b$-quark and $\tau$ masses have been neglected. and the possibility of $Z-Z^{\prime}$ mixing has been ignored. Since our results will generally be statistics limited, the role played by the systematic uncertainties associated with the parameter choices above will generally be rather minimal.

To insure model-independence, the values of the $Z^{\prime}$ couplings, i.e., $(v, a)_{e . b}$, as well as $M_{Z^{\prime}}$, are chosen randomly and anonymously from rather large ranges representative of a number of extended gauge models. Monte Carlo data representing the above observables is then generated for several different values of $\sqrt{s}$. At this point, the values of the mass and couplings are not 'known' a priori, but will later be compared with what is extracted from the Monte Carlo generated event sample. Following this approach there is no particular relationship between any of the couplings and there is no dependence upon any particular $Z^{\prime}$ model. (We normalize our couplings so that for the SMI $Z, a_{\ell}=-1 / 2$.) Performing this 
analysis for a wide range of possible mass and coupling choices then shows the power as well as the limitations of this technique.

To get an understanding for how this procedure works in general we will make two case studies for the $Z^{\prime}$ mass and couplings, labelled here by I and II. There is nothing special about these two choices and several other parameter sets have been analyzed in comparable detail to show that the results that we display below are rather typical. To begin we generate Monte Carlo data at $\sqrt{s}=0.5,0.75$ and $1 \mathrm{TeV}$ with associated integrated luminosities of 70,100 , and $150 \mathrm{fb}^{-1}$, respectively, and subsequently determine the 5-dimensional $95 \% \mathrm{CL}$ allowed region for the mass and couplings from a simultaneous fit using the assumptions listed above. This 5-dimensional region is then projected into a series of 2-dimensional plots which we can examine in detail. Figs. 1 and 2 show the results of our analysis for these two case studies compared with the expectations of a number of well-known $Z^{\prime}$ models[1]. Several things are immediately apparent-the most obvious being that two distinct allowed regions are obtained from the fit in both cases. (The input values are seen to lie nicely inside one of them.) This two-fold ambiguity results from our inability to make a determination of the overall sign of one of the couplings, e.g., $a_{\ell}$. If the sign of $a_{\ell}$ were known, only a single allowed region would appear in Figs. $1 \mathrm{a}-\mathrm{b}$ and $2 \mathrm{a}-\mathrm{b}$ and $\mathrm{a}$ unique coupling determination would be obtained. Note that this same sign ambiguity arises in SLD/LEP data for the SM $Z$ and is only removed through the examination of low-energy neutrino scattering. Secondly, we see that the leptonic couplings are somewhat better determined than are those of the $b$-quark, which is due to the fact that the leptonic observables involve only leptonic couplings, while those for 6 -quarks involve both types. In addition, there is more statistical power available in the lepton channels due to the assumption of universality and the leptonic results employ two additional observables related to $\tau$ polarization. Thirdly, we see from Figs. 1a-b the importance in obtaining coupling information for a number of different fermion species. If only the Fig. la results were available, one might draw the hasty conclusion that an $E_{6}$-type $Z^{\prime}$ had been found. Fig. $1 \mathrm{~b}$ clearly shows us that this is not the case. Evidently neither $Z^{\prime}$ corresponds to any well-known model. Lastly, as promised, the $Z^{\prime}$ mass is determined in both cases, although with somewhat smaller uncertainties in case II. We remind the reader that there is nothing special about these two particular cases.

Of course, the clever reader must now be asking the question 'why use 3 different values of $\sqrt{3}$, why not 2 or 5?' This is a very important issue which we can only begin to addres here. Let us return to the mass and couplings of case I and generate Monte Carlo 'data' for only $\sqrt{s}=0.5$ and $1 \mathrm{TeV}$ with $\mathcal{L}=100$ and $220 \mathrm{fb}^{-1}$, respectively, thus keeping the total $\mathcal{L}$ the same as in the discussion above. Repeating our analysis we then arrive at the '2point' fit as shown in Fig. 3a; unlike Fig. 1a, the allowed region does not close and extends outward to ever larger values of $v_{\ell}, a_{\ell}$. The corresponding $Z^{\prime}$ mass contour also does not close, again extending outwards to ever larger values. We realize immediately that this is just what happens when data at only a single $\sqrt{s}$ is available. For our fixed $\mathcal{L}$, distributed as we have done, we see that there is not enough of a lever arm to simultaneously disentangle the $Z^{\prime}$ mass and couplings. Of course the reverse situation can also be just as bad. We now

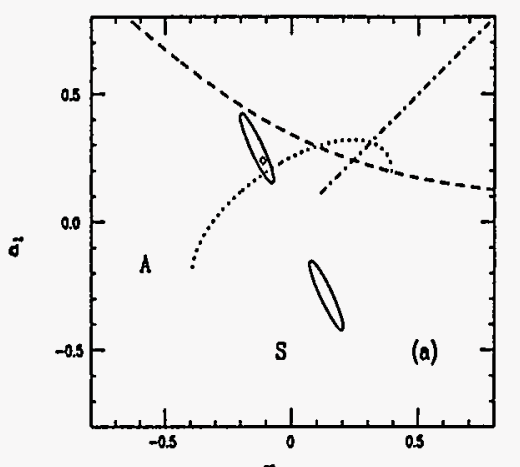

$\nabla_{1}$

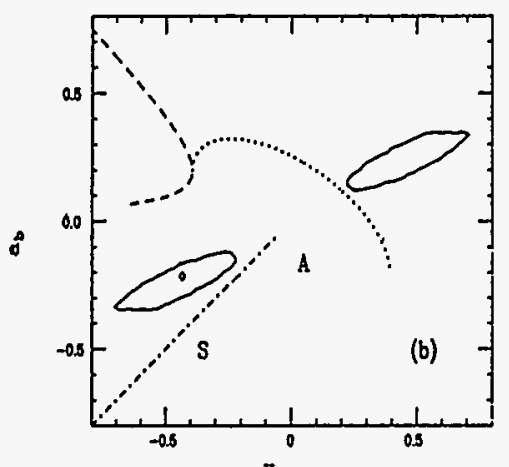

$\nabla_{b}$

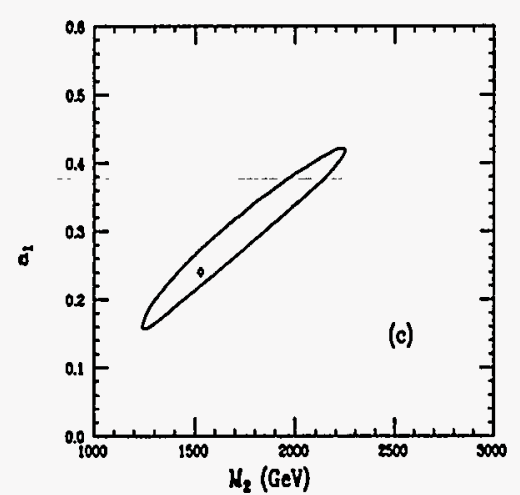

Figure 1: 95\% CL allowed regions for the extracted values of the (a) lepton and (b) b-quark couplings for the $Z^{\prime}$ of case I compared with the predictions of the $E_{6}$ model(dotted). the Left-Right Model(dashed), and the Un-unified Model(dash-dot), as well as the Sequential SM and Alternative LR Models(labeled by ' $S$ ' and ' $A$ ', respectively.) (c) Extracted $Z$ ' mass; only the $a_{\ell}>0$ branch is shown. In all cases the diamond represents the corresponding input values. 

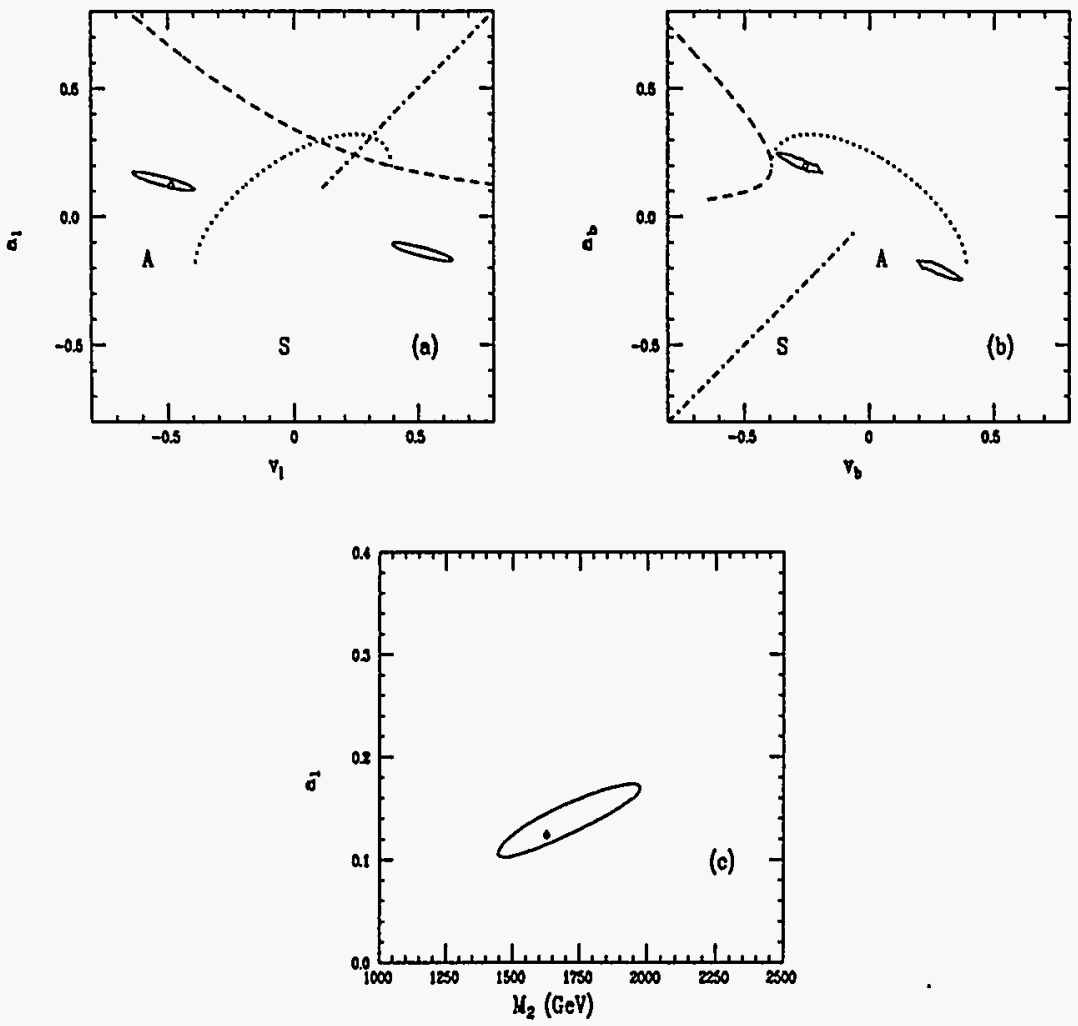

Figure 2: Same as Fig. 1 but for a different choice of $Z^{\prime}$ mass and couplings referred to as case II in the text. generate 'Monte Carlo 'data' for the case I mass and couplings in $100 \mathrm{GeV}$ steps in $\sqrt{s}$ orer the 0.5 to $1 \mathrm{TeV}$ interval with the same total $\mathcal{L}$ as above but now distributed as 30.30 . 50. 50,60 . and $100 \mathrm{fb}^{-1}$, respectively. We then arrive at the '6-point' fit shown in Fig. 3b which suffers a problem similar to Fig. $3 \mathrm{a}$. What has happened now is that we have spread the fixed $\mathcal{L}$ too thinly over too many points for the analysis to work. This brief study indicates that a proper balance is required to simultaneously achieve the desired statistics as well as an effective lever arm to obtain the $Z^{\prime}$ mass and couplings. It is important to remember that we have not demonstrated that the '2-point' fit will never work. We note only that it fails with our specific fixed luminosity distribution for the masses and couplings associated with cases I and II. It is possible that for 'lucky" combinations of masses and couplings a 2-point fit will suffice. Clearly, more work is required to further address this issue.
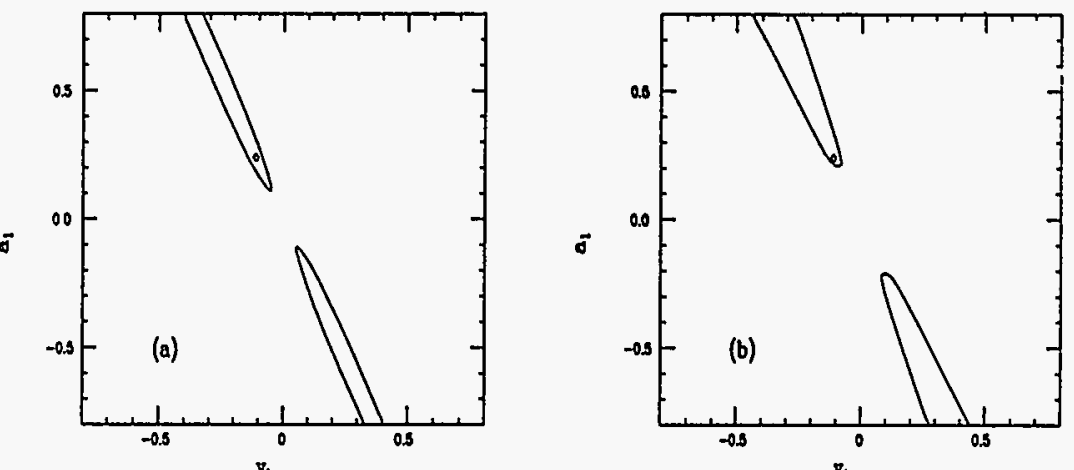

Figure 3: Failure of the method in case I when data is taken at (a) too fow ('2-point" fit) or (b) too many (' 6 -point' fit) different center of mass energies for the same total integrated luminosity as in Figs. 1 and 2. The luminosities are distributed as discussed in the text.

How do these results change if $M_{Z^{\prime}}$ were known or if our input assumptions were modified? Let us return to case I and concentrate on the allowed coupling regions corresponding to a choice of negative values of $v_{e}$, these are expanded to the solid curves shown in Figs, ta and 4c. The large dashed curve in Fig. 4a corresponds to a reduction of the polarization to $80 \%$ with the same relative error as before. While the allowed region expands the degradation is not severe. If the $Z$ ' mass were known, the 'large' ellipses shrink to the small ovals in Fig. ta; these are expanded in Fig. $4 \mathrm{~b}$. This is clearly a radical reduction in the size of the allowed region! We see that when the mass is known, varying the polarization or its uncertainty orer a reasonable range has very little infuence on the resulting size of the allowed regions. From Fig. tc we see that while knowing the $Z^{\prime}$ mass significantly reduces the size of the allowed 
region for the $b$ couplings, the impact is far less than in the leptonic case for the reasons discussed above. Figs. $5 \mathrm{a}$ and $5 \mathrm{~b}$ show that case $I$ is not special in that similar results are seen to hold for case II.

What happens for larger $Z^{\prime}$ masses or when data at larger values of $\sqrt{s}$ becomes available? Let us assume that the 'data' from the above three center of mass energies is already existent, with the luminosities as given. We now imagine that the NLC increases its center of mass energy to $\sqrt{s}=1.5 \mathrm{TeV}$ and collects an additional $200 \mathrm{fb}^{-1}$ of integrated luminosity. Clearly for $Z^{\prime}$ masses near or below $1.5 \mathrm{TeV}$ our problems are solved since an on-shell $Z^{\prime}$ can now be produced. Thus we shall concern ourselves with $Z^{\prime}$ masses in excess of $2 \mathrm{TeV}$. Figs. 6a-d show the result of extending our procedure-now using 4 different $\sqrt{s}$ values, again for two distinct choices of the $Z^{\prime}$ mass and couplings. These ' 4 -point' results are a combined fit to the data at all four center of mass energies. (Only one of the allowed pair of ellipses resulting from the overall sign ambiguity is shown for simplicity.) Note that the $Z^{\prime}$ input masses we have chosen are well in excess of $2 \mathrm{TeV}$ where the LHC may provide only very minimal information on the fermion couplings[1]. Clearly by using the additional data from a run at $\sqrt{s}=1.5 \mathrm{TeV}$ this technique can be extended to perform coupling extraction for $Z^{\prime}$ masses in excess of $2.5 \mathrm{TeV}$. The maximum 'reach' for the type of coupling analysis we have done is not yet known. It seems likely, based on these initial studies, that the extraction of interesting coupling information for $Z^{\prime}$ masses in excess of $3 \mathrm{TeV}$ seems possible for a reasonable range of parameters.

\section{Outlook and Conclusions}

In this paper we have shown that it is possible for the $N L C$ to extract information on the $Z$ couplings to leptons and $b$-quarks even when the $Z^{\prime}$ mass is not a prioriknown. The critical step for the success of the analysis was to combine the data available from measurements performed at several different center of mass energies. For reasonable luminosities the specific results we have obtained suggest, but do not prove, that data sets at at least 3 different energies are necessary for the procedure to be successful.

\section{ACKNOWLEDGEMENTS}

The author would like to thank J.L. Hewett and S. Godfrey for discussions related to this work.

\section{References}

[1] For a complete set of references and a recent review of the physics of new gauge bosons see, M. Cvetic and S. Godfrey, Carleton University report OCIP/C-95-2. 1995, a part of
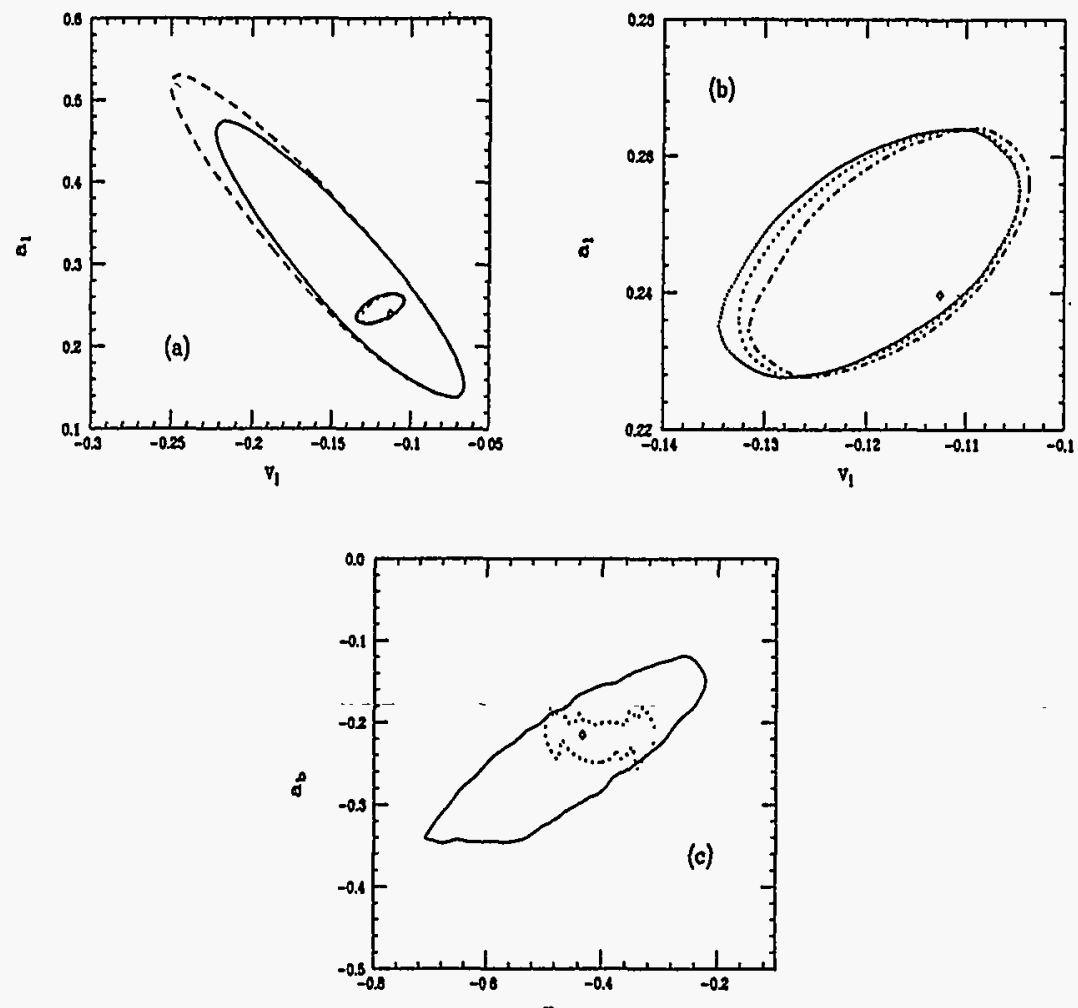

Figure 4: (a) Expanded lobe(solid) from Fig. 1a; the dashed curve shows the same result but for $P=80 \%$. The smaller ovals, expanded in (b) apply when the $Z$ ' mass is known. Here, in (b) $P=90(80) \%$ corresponds to the dash-dot (dotted) curvo while the case of $P=90 \%$ with $\delta P / P=5 \%$ corroponds to the squaredoted curve. (c) Expanded lobolid from $P$ ib deted curve corresponds to the case when $M_{z}$ is $\mathrm{known}$. 

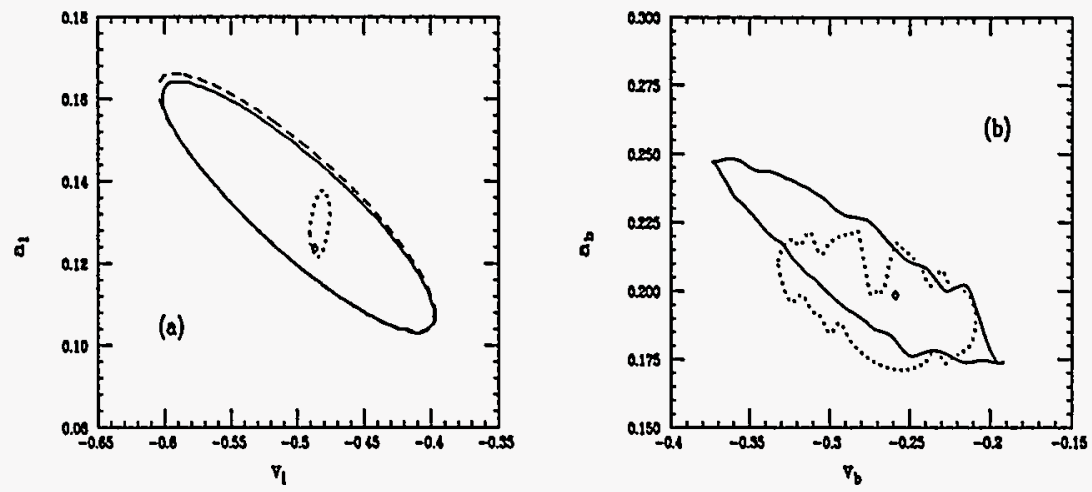

Figure 5: (a) Expanded lobe(solid) from Fig. 2a: the dashed curve shows the same result but for $P=80 \%$. The smaller dotted oval, applies when the $Z^{\prime}$ mass is known and $P=90 \%$. (b)

Expanded lobe(solid) from Fig. $2 b$; the dotted curve corresponds to the case when $M_{Z}$ is known.

the DPF long-range planning study to be published in Electroweak Symmetry Breaking and Physics Beyond the Standard Model, eds. T. Barklow, S. Dawson, H. Haber, and J. Seigrist (World Scientific 1996). See also, A. Djouadi, talk given at the Workshop on Physics and Experiments with Linear Colliders, Morioka-Appi, Japan, Sept. 8-12, 1995, hep-ph/9512311; J. Layssac et al., hep-ph/9602327; A. Leike and S. Riemann, to appear in the Proceedings of the Physics with $e^{+} e^{-}$Linear Colliders Workshop, AnnecyGan Sasso-Hamburg, 1995, ed. P. Zerwas, hep-ph/9604321. For a summary of $Z^{\prime}$ search limits, see the companion article in the NLC ZDR by K. Maeshima, K. McDonald and T.G. Rizzo.
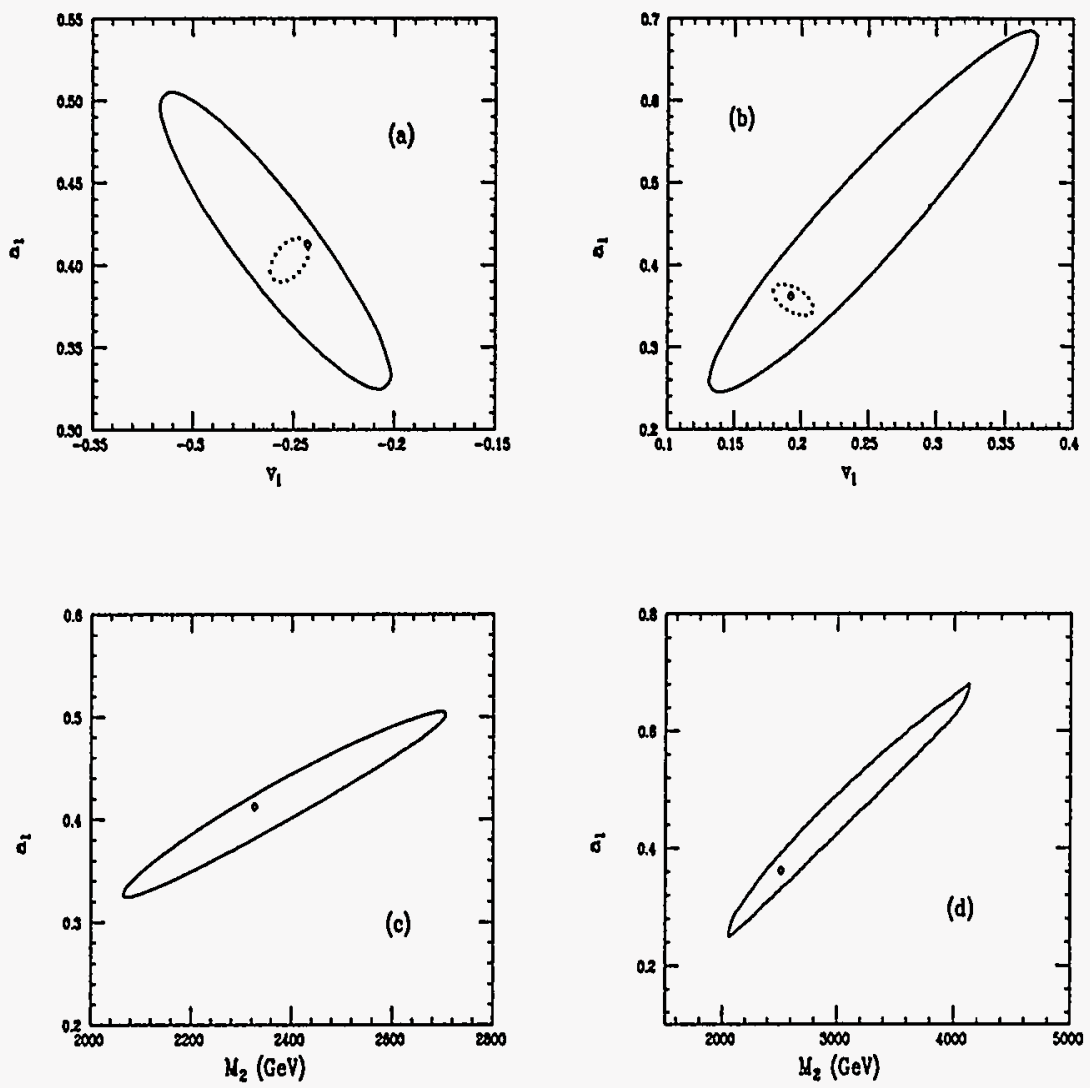

Figure 6: Lepton coupling determination for $Z^{\prime \prime}$ 's with masses of (a) $2.33 \mathrm{TeV}$ and (b) $2.51 \mathrm{TeV}$ when the mass is unknown(solid) and known(dotted). (c) and (d) are the corresponding mass determinations which result from the five-dimensional fit. These results include an additional 200 $f b^{-1}$ of luminosity taken at a center of mass energy of $1.5 \mathrm{TeV}$. 
Anomalous Chromoelectric and Chromomagnetic Moments of the Top Quark at the NLC

THomas G. Rizzo 1

Stanford Linear Accelerator Center

Stanford University, Stanford, California 94309 USA

\section{ABSTRACT}

The production of top quark pairs in association with a hard gluon in $e^{+} e^{-}$ collisions at the NLC provides an opportunity to probe for anomalous $t \bar{t} g$ couplings, e.g., the chromoelectric and chromomagnetic moments of the top. We demonstrate that an examination of the energy spectrum of the additional gluon jet can yield strong constraints on the size of these two anomalous couplings. The results are shown to be quite sensitive to the cut on the minimum gluon jet energy needed to remove events where the final state $b$-quark radiates strongly. The possibility of using additional observables to improve the sensitivity to anomalous couplings is briefly discussed.

\section{Introduction}

The discovery of the top quark with a mass consistent with the expectations from precision electroweak measurements is a major triumph for the Standard Model(SM). However, due to the fact that it is so heavy, $m_{t}=175 \pm 9 \mathrm{GeV}[1]$, the top itself has been proposed as a window for new physics beyond the SM. One obvious scenario is a modification of the interactions of the top quark with the conventional gauge bosons, i.e., the $W . Z, \gamma$, and $g$, whose couplings to top may differ in detail from those anticipated by the SM. This possibility has lead to a substantial effort over the past few years[2] investigating potential anomalous couplings of the top as well as other third generation fermions. In the case of the strong interactions of the top, the lowest dimensional gatuge-invariant operator representing new top quark plysics and conserving $C P$ that we can introduce is the anomalous chromomagnetic moment, which we can parameterize via a dimensionless quantity $\kappa$. On the otherhand, the corresponding chromoelectric moment, parameterized by $\bar{n}$, violates $C P$ and arises from an operator of the

\footnotetext{
${ }^{1}$ Work supported by the Department of Energy, contract DE-AC03-i6SF00515.
}

same dimension. In this modified version of QCD for the top quark. the tree-level three-point $t \bar{t} g$ interaction Lagrangian takes the form

$$
\mathcal{L}=g_{s} I T_{a}\left(\gamma_{\mu}+\frac{i}{2 m_{t}} \sigma_{\mu \nu}\left(\kappa-i \bar{\kappa} \gamma_{s}\right) q^{\nu}\right) t G_{2}^{\mu} .
$$

where $g_{\mathrm{a}}$ is the strong coupling constant, $m_{\mathrm{t}}$ is the top quark mass. $T_{\mathrm{z}}$ are the color generators, $G_{a}^{\mu}$ is the gluon field and $q$ is the outgoing gluon momentum. Due to the non-Abelian nature of QCD, a four-point, dimension-five $t \bar{t} g g$ interaction is also generated. but this will not concern us in the present work since it only contributes to the process of interest at higher order. As has been discussed in the literature, if either or both of $k . \dot{k}$ are sufficiently large in magnitude their effects can be probed through top pair production processes at both $e^{+} e^{-}[3]$ and hadron[4] colliders. The purpose of the present work is to consider the sensitivity of $i \bar{t} g$ production in $e^{+} e^{-}$collisions at the NLC to non-zero values of $k$ and $\dot{k}$.

\section{Analysis}

In our analysis we will only consider how the top quark anomalous couplings can modify the energy distribution of the extra giuon jet associated with top pair production. In principle. other observables may be available that are also sensitive to these couplings; these are generally beyond the scope of the present work, but one example will be discussed later. The basic cross section formulae and analysis procedure can be found in Ref.[3]. Here. we go beyond this initial study in several ways: (i) we generalize the form of the $t \bar{t}_{g}$ coupling to beyond this initial study in several ways: $(i)$ we generalize the form of the $t \bar{t} g$ coupling to
allow for the possibility of a sizeable chromoelectric moment. $\dot{k}$. The incorporation of $\bar{x} \neq 0$ into the expressions for the differential cross section in Ref.[3] is rather straightforward and can be accomplished by the simple substitution $\kappa^{2} \rightarrow \kappa^{2}+\tilde{\kappa}^{2}$ made universally. Note that since a non-zero value of $\dot{x}$ produces a $C P$-violating interaction it appears only quadratically in the expression for the gluon energy distribution since this is a $C P$-conserving observable. Thus, in comparison to $x$, we anticipate a greatly reduced sensitivity to the value of able. Thus, in comparison to $\kappa$, we anticipate a greatly reduced sensitivity to the value
$\bar{\kappa}$. (ii) We use updated expectations for the available integrated luminosities of the NLC at various center of mass energies as well as an updated efficiency $(\simeq 100 \%)$ for identifying top-quark pair production events. Both of these changes obviously leads to a direct increase in statistical power compared to Ref.[3] (iii) We lower the cut placed on the mínimum gluon jet energy, $E_{m}^{\min }$, in performing the energy spectrum fits. The reasons for having such a cut are two-fold. First, a minimum gluon energy is required to identify the event as $t \bar{t} g$. The cross section itself is infra-red singular though free of co-linear singularities due to the finite top quark mass. Second, since the top decays rather quickly: $\Gamma_{\mathrm{l}} \simeq 1.45 \mathrm{GeV}$, we need to worry about 'contamination' from the additional gluon radiation of of the b-quarks in the final state. Such events can be effectively removed from our sample if we require that $E_{\mathrm{sin}}^{\min } \Gamma_{1} \gg 1$. In our past analysis we were overly conservative in our choices for $E_{n}^{\min }$ in order to make this ratio as large as possible, i.e., we assumed $E_{3}^{\min }=50(200) \mathrm{GeV}$ for an NLC with a center of mass energy of $500(1000) \mathrm{GeV}$. It is now believed that we can with 
reasonable justification soften these cuts to at least as low a value as $25(50) \mathrm{GeV}$ for the same center of mass energies[5], with a potential further softening of the cut at the higher energy machine being possible. Due to the dramatic infra-red behaviour of the cross section. this change in the cuts leads not only to an increased statistical power but also to a longer lever arm to probe events with very large gluon jet energies which have the most sensitivity to the presence of anomalous couplings. Combining all these modifications, as one might expect, we find constraints which are substantially stronger than what was obtained in our previous analysis[3].

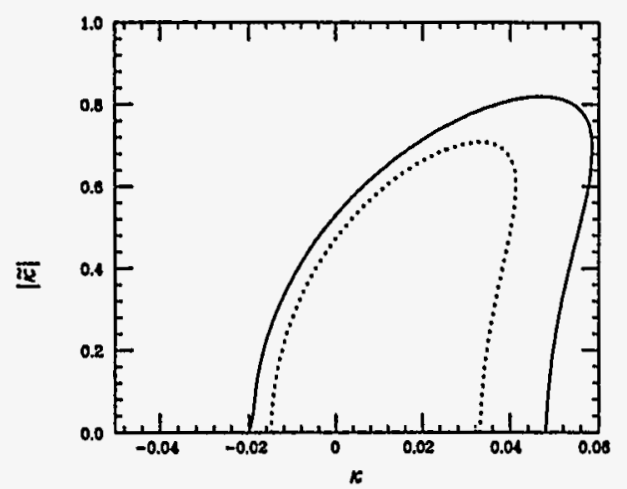

Figure 1: $95 \% \mathrm{CL}$ allowed region in the $\kappa-\bar{\kappa}$ plane obtained from fitting the gluon spectrum above $E_{f}^{\min }=25 \mathrm{GeV}$ at a $500 \mathrm{GeV}$ NLC assuming an integrated luminosity of 50 (solid) or 100 (dotted) $f^{-1}$.

As in Ref.(3), our analysis follows a Monte Carlo approach employing statistical errors only. For a given $e^{+} e^{-}$center of mass energy, a binned gluon jet spectrum is generated for energies above $E_{g}^{\min }$ assuming that the $S M$ is correct. The bin widths are fixed to be $\Delta z=0.05$ where $z=2 E_{g} / \sqrt{s}$ for all values of $\sqrt{s}$, with the number of bins thus determined by the values of the top mass $\left(m_{\mathrm{t}}=175 \mathrm{GeV}\right), \sqrt{3}$ and $E^{\mathrm{min}}$. All calculations are performed only in the lowest order. As an example, at a $500 \mathrm{GeV}$ NLC with $E_{g}^{\min }=25 \mathrm{GeV}$, there are 8 energy bins for the gluon energy spectrum beginning at $z=0.10$; the last bin covers the range above $z=0.45$. After the Monte Carlo data samples are generated, we perform a fit to the general expressions for the $\kappa-\bar{\kappa}$ dependent spectrum and obtain the $95 \%$ CI, allowed region in the $\kappa-|\bar{\kappa}|$ plane. (Note that only the absolute value of $\tilde{\kappa}$ occurs due to the reasons described above.)

Fig. 1 shows the results of this procedure for a $500 \mathrm{GeV}$ NLC with a cut of $E_{s}^{\mathrm{min}}=25$
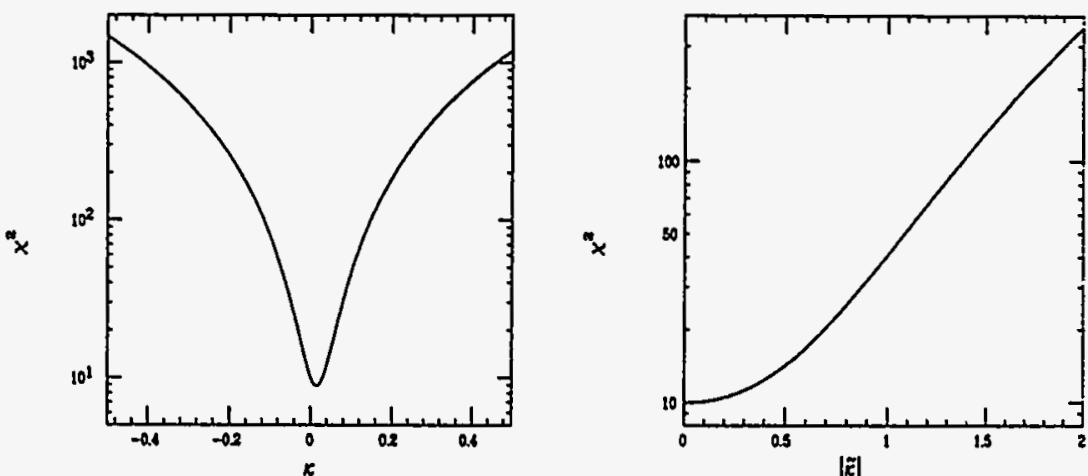

Figure 2: $\chi^{2}$ plots for the $500 \mathrm{GeV}$ NLC with a luminosity of $50 \mathrm{fb}^{-1}$ corresponding to Fig. 1 . Only one of $x$ or $\bar{k}$ is assumed to be non-zero at a time.

$\mathrm{GeV}$ for two different integrated luminosities. As expected. excellent constraints on $x$ are now obtained but those on $\tilde{x}$ are more than an order of magnitude weaker. A doubling of the integrated luminosity from 50 to $100 \mathrm{fb}^{-1}$ decreases the size of the allowed region by about $40 \%$. We note that in the previous study only extremely poor constraints on $k$ were obtained at a $500 \mathrm{GeV} e^{+} e^{-}$collider, $-1.9 \mathrm{~s}^{\circ} \leq \kappa \leq 0.44$. due to the presence of a degenerate minima in the $\chi^{2}$ distribution. Now. with the both the increased luminosity and top-tagging efficiencies, as well as the longer lever arm in energy. these previous difficulties are circumvented. This is explicitly shown by the $l^{2}$ plots in Fig. 2. In the case of a non-zero $\kappa$ a very sharp minimum is found whereas the rise in $x^{2}$ is slow when $\tilde{\kappa}$ is non-zero. In neither case is there any evidence for a second minimum. This lack of degeneracy is found to hold for all the other cases we have considered.

Going to a higher energy leads to several simultaneous effects. First, since the cross section approximately scales like $\sim 1 / s$ apart from phase space factors. a simple doubling of the collider energy induces a reduction in statistics unless higher integrated luminosities are available to compensate. Second, the sensitivity to the presence of non-zero anomalous couplings is enhanced at higher energies, roughly scaling like $\sim \sqrt{s}$ for $\kappa$ and, correspondingly: like $\sim s$ for $\tilde{\kappa}$ assuming the same available statistics at all energies. In Fig. 3 we show the results of our analysis at a $1 \mathrm{TeV} \mathrm{NLC} \mathrm{for} E^{\mathrm{min}}=50 \mathrm{GeV}$ : the corresponding case where we maintain the jet energy cut of $E_{3}^{\mathrm{min}}=25 \mathrm{GeV}$ is shown in Fig. 4 . (Note that in our previous analysis, we obtained the $95 \%$ CL bound $-0.12 \leq k \leq 0.21$ for this center of mass energ! and an integrated luminosity of $200 \mathrm{fb}^{-1}$.) For $E_{g}^{\min }=50(25) \mathrm{GeV}$, the energy range is divided into $15(16) \Delta z=0.2$ bins beginning at $z=0.10(0.05)$ with the last bin covering the range 


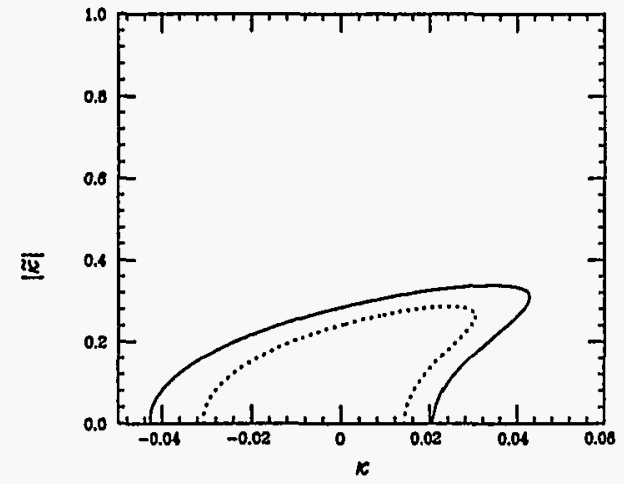

Figure 3: Same as Fig. 1, but for a $1 \mathrm{TeV}$ collider with $E_{g}^{\text {min }}=50 \mathrm{GeV}$ and luminosities of 100 (solid) and 200 (dotted) $\mathrm{fb}^{-1}$. Note that the allowed region has been significantly compressed downward in comparison to Fig. 1.

$z \geq 0.80$. We see from these figures that by going to higher energy we drastically compress the allowed range of $\bar{\kappa}$ while the improvement for $\kappa$ is not as great. Lowering the energy cut is seen to lead to a far greater reduction in the size of the $95 \%$ CL allowed region than is a simple doubling of the integrated luminosity. This demonstrates that if improvements to the present analysis are to be made it is very important to explore just how low the $E^{\text {min }}$ can be placed and still remove the contamination from gluon radiation off of final state $b$-quarks. Comparing the $200 \mathrm{fb}^{-1}$ result in Fig. 4 with the corresponding case of $50 \mathrm{fb}^{-1}$ in Fig. 1 , we see that our approximate scaling laws are observed to hold rather well.

As a final example, we consider a $\sqrt{s}=1.5 \mathrm{TeV}$ NLC with $E_{s}^{\min }=75 \mathrm{GeV}$. This requires $16 \Delta z=0.05$ bins beginning at $z=0.10$ to cover the entire gluon jet energy spectrum the final bin covers the range $z \geq 0.85$. The results of this analysis are shown in Fig. assuming integrated luminosities of 200 and $300 \mathrm{fb}^{-1}$. The $95 \% \mathrm{CL}$ allowed region is seen to be further compressed in the vertical direction, i.e., to smaller values of $\tilde{x}$, than that found in the $1 \mathrm{TeV}$ NLC case with either $E^{\mathrm{min}}=25$ or $50 \mathrm{GeV}$. However, the overall size of the allowed region for the $1.5 \mathrm{TeV}$ collider with $E_{g}^{\min }=75 \mathrm{GeV}$ is comparable to that of the $\mathrm{TeV}$ NLC with $E_{g}^{\min }=25 \mathrm{GeV}$. Softening the $E_{g}^{\min }$ cut at the $1.5 \mathrm{TeV}$ collider will yield even stronger constraints.

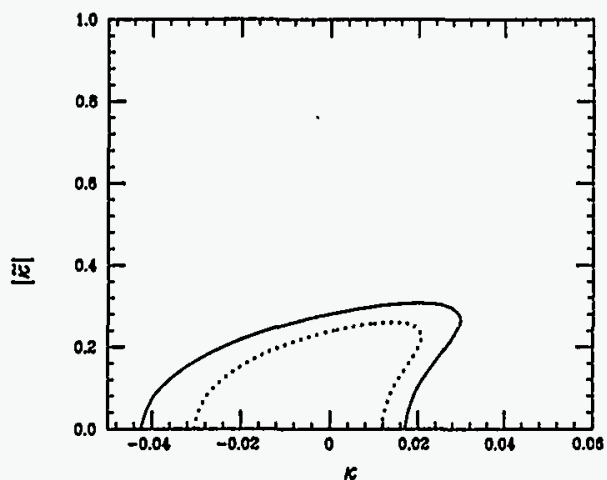

Figure 4: Same as Fig. 3 but with $E_{g}^{\mathrm{min}}=25 \mathrm{Ger}$.

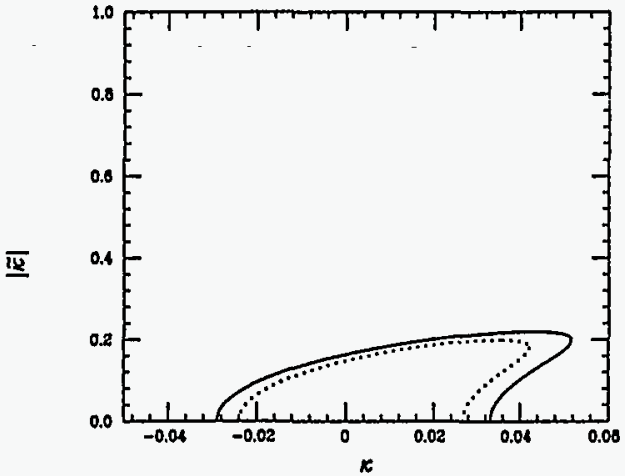

Figure 5: Same as Fig. 1 but for a $1.5 \mathrm{TeV}$ NLC with $E_{g}^{\min }=75 \mathrm{GeV}$ for luminosities of 200(solid) and 300 (dotted) $\mathrm{fb}^{-1}$. 


\section{Discussion and Conclusions}

We have presented an updated analysis of the constraints imposed on the chromoelectric and chromomagnetic moments of the top quark by detailed measurements of the gluon jet energy spectrum associated with the process $e^{+} e^{-} \rightarrow t \bar{g} g$ at the NLC for various center of inass energies. The value of the cut on the gluon energy was shown to play a key role in obtaining strong bounds on these anomalous couplings. These results may be strengthened in the future if we find that the $E_{g}^{\min }$ cut can be further softened.

If we are to improve the sensitivity to anomalous couplings we must introduce additional observables. The large initial electron beam polarization, $P=90 \%$, may play an important role in this regard. To get an idea of how this might work, we can construct an asymmetry using the Monte Carlo data generated above by asking how the number of events in a fixed energy bin is allered when one changes from left-handed to right-handed polarization. i.e... $\left.A(z)=\left(N_{C}(z)-N_{R}(z)\right) / N_{L}(z)+N_{R}(z)\right)$. $A(z)=\left[N_{L}(z)-N_{R}(z)\right] /\left[N_{L}(z)+N_{R}(z)\right]$. For a $\sqrt{s}=500 \mathrm{GeV}$ :NLC. $A$ is found to be approximately $z$ independent with a value near $39 \%$ in the SM and will in general be $\kappa, \dot{\kappa}$ dependent if anomalous couplings are present. We thus can repeat our analysis for the 500 $\mathrm{GeV}$ case now including the values of $A$ in the fit. This is shown in Fig. 6. Unfortunately including $A$ has not drastically reduced the size of the $95 \%$ CL allowed region as we might must seek out other observables, particularly those which are sensitive to the $C P$-violating chromoelectric moment interactions. This analysis is currently underway.

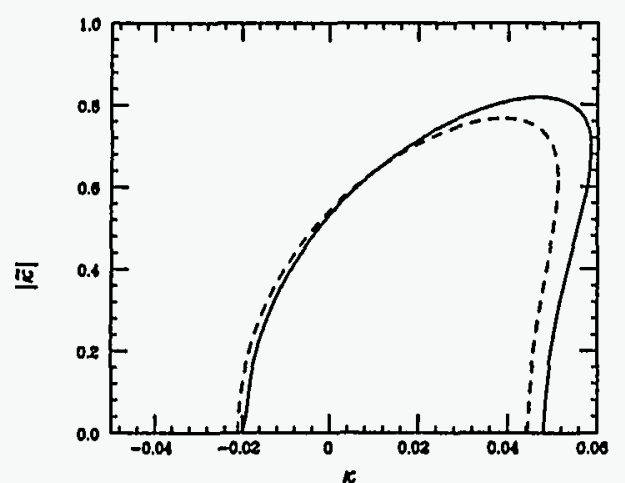

Figure 6: The solid curve is the same as in Fig. 1 for a $500 \mathrm{GeV}$ NLC with $E^{\mathrm{min}}=25 \mathrm{GeV}$ and a luminosities of $50 \mathrm{fb}^{-1}$. The dashed curve included the polarization asymmetry into tlie fit assuming an initial beam polarization of $90 \%$.

\section{ACKNOWLEDGEMENTS}

The author would like to thank P. Burrows. D. Atwood. A. Kagan and J.L. Hewett for discussions related to this work.

\section{References}

[1] H.H. Williams, talk given at the Workshop on High Energy Physics at the LHC. Fermilab. March 28-30, 1996.

[2] There has been an enormous amount of work in this general subject area: see for example: T.G. Rizzo, Phys. Rev. D51, 3811 (1995) and Phys. Rev. D53. 23:26 (1996): A. Grifols and A. Mendez, Phys. Lett. B255. 611 (1991) and erratum Phys. Lett. B259. 512 (1991); B. Ananthanarayan and S.D. Rindani Phys. Rev. Lett. 73. 1215 (1994): G. liöpp et al, 2 Phys. C65, 545 (1995); F. del Aguila and U. Sher, Phys. Lett. B252. 116 (1990); R. Escribano and E. Masso. Phys. Lett. B301. 419 (1993)and .iucl. Phys. 429. 19 (1994); W. Bernreuther, O. Nachtmann and P. Overmann, Phys. Rev. D48. is (1993); G. Couture, Phys. Lett. B305. 306 (1993) and Phys. Lett. B272. 404 (1991): G. Domokos et al., Phys. Rev. D32. 247 (1985); J. Reid. .I. Samuel. K... . Milton and T.G. Rizzo, Phys. Rev. D30. 245 (1984). See also. P.D. Acton el al.. OP.AL C'ollaboration. Phys. Lett. B281, 305 (1992); D. Buskulic et al. ALEPH Collaboration. Phys. Lett. B297, 459 (1992); G. Kiane. G.A. Ladinsky and C.P. Yuan. Phys. Rer. D45. 124 (1992); C.P. Yuan, Phys. Rev. D45, 782 (1992); D. Atwood. A. Aeppli and A. Soni. Phys. Rev. Lett. 69, 2754 (1992); .I. Peskin. talk presented at the Second Internationnl Workshop on Physics and Experiments at Linear $e^{+} e^{-}$Collider, Waikoloa. HI. April 1993; M. Peskin and C.R. Schmidt, talk presented at the First Workshop on Linear Colliders. Saariselkä, Finland, September 1991: P. Zerwas, ibid. IV. Bernreuther at al. in Proceedings of the Workshop on $\epsilon^{+} e^{-}$Collisions at $500 \mathrm{Gel}$. The Physics Potential, (DESY, Hamburg) ed. by P. Igo-Kemenes and J.H. Kühn. 1992; A. Djoutadi. ENSLAPP-A-365-92 (1992); M. Frigeni and R. Rattazzi, Phys. Lett. B260, 412 (1991): R.D. Peccei, S. Persis and X. Zhang, Nucl. Phys. B349, 305 (1991); D.O. Carlson. E. Malkawi and C.-P. Yuan, Phys. Lett. B337, 140 (1994); J.L. Hewett and T.C. Rizzo. Phys. Rev. D49, 319 (1994).

[3] T.G. Rizzo, Phys. Rev. D50, 4478 (1994).

(t) D. Atwood, A. Kagan and T.G. Rizzo, Phys. Rev. D52, 620t (1995); T.G. Rizzo. SLAC. PUB-95-6758, to appear in Phys. Rev, D53; K. Cheung, Phys. Rev. D53. $3604(1946)$ P. Haberl, O. Nachtmann and A. Wilch. Phys. Rev. D53. 1875 (1996).

[5] L. Orr, private communication. 
